\title{
Idaho National Laboratory Annual Report FY 2013 LDRD Project Summaries
}

March 2014

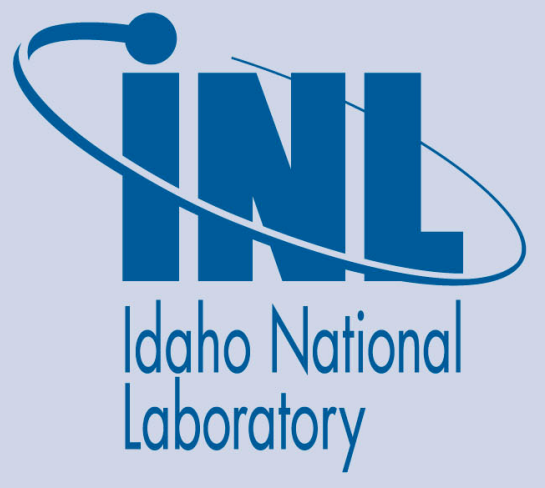

The INL is a U.S. Department of Energy National Laboratory operated by Battelle Energy Alliance 
INL/EXT-13-30443

\title{
Idaho National Laboratory Annual Report FY 2013 LDRD Project Summaries
}

March 2014

\author{
Idaho National Laboratory \\ Idaho Falls, Idaho 83415
}

http://www.inl.gov

Prepared for the

U.S. Department of Energy

Through the INL LDRD Program

Under DOE Idaho Operations Office

Contract DE-AC07-05ID14517 
Idaho National Laboratory ANNUAL REPORT FY 2013

\section{LDRD PROJECT SUMMARIES}
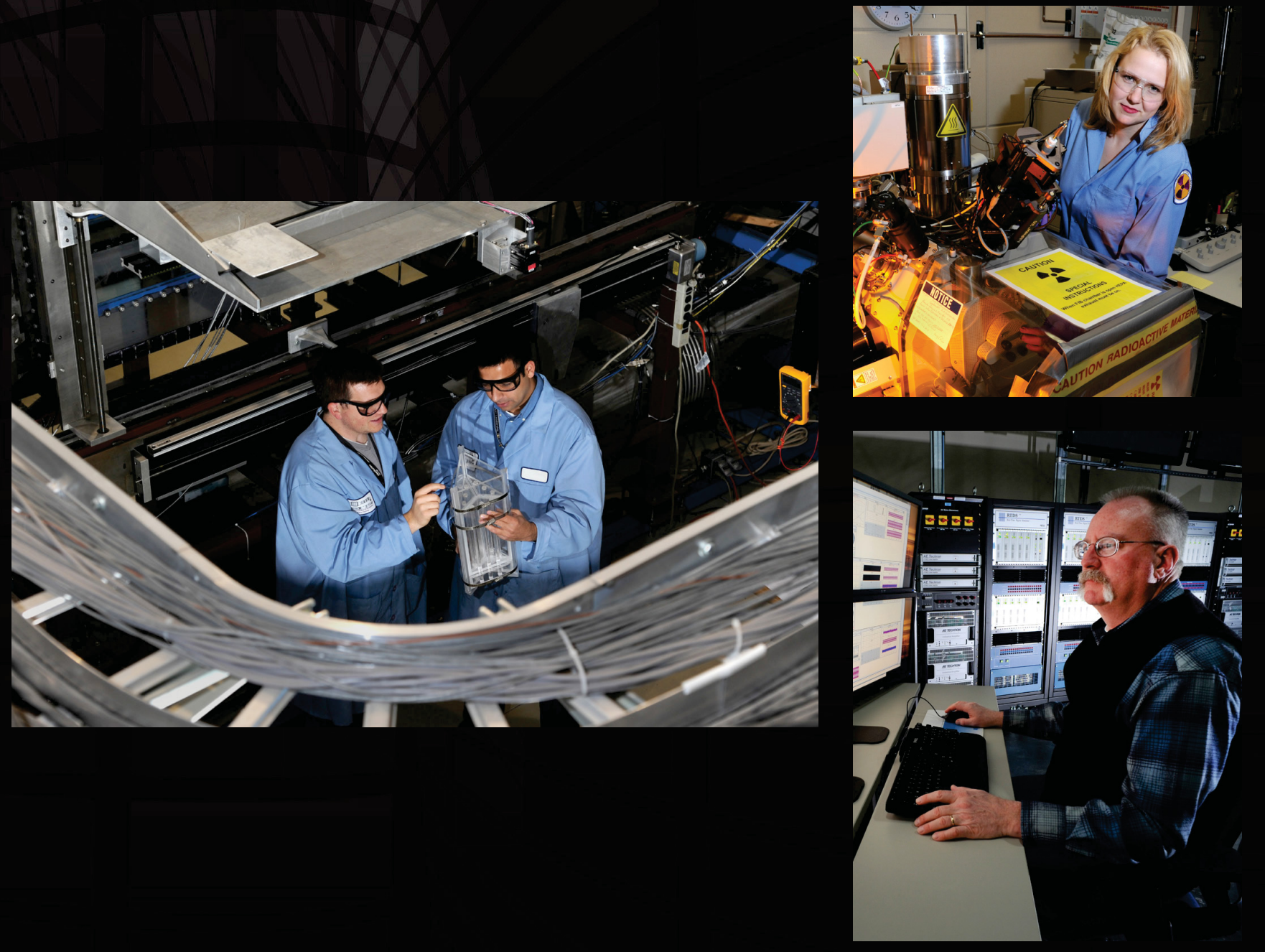

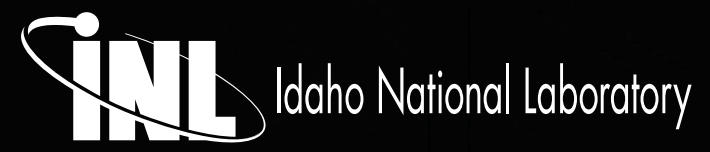




\title{
Acknowledgments
}

This annual report provides an overview of the Laboratory Directed Research and Development (LDRD) Program at Idaho National Laboratory (INL). Summarized in this report are 89 technical research projects conducted in FY 2013. The LDRD Program at INL is managed for the Laboratory by John J. Grossenbacher, Laboratory Director; Todd Allen, Deputy Laboratory Director for Science and Technology; and Anita Gianotto, LDRD Program Manager.

Many people contributed to this report; however, particular thanks go to the principal investigators who conduct the cutting-edge research so important to this laboratory.

\author{
Managing Editor: Dena Tomchak \\ Technical Writing and Quinn Grover \\ Editing: Riley Goodwin \\ Text Processing: Cheryl Priest \\ Cover Design: Kris Burnham
}

Prepared for the U.S. Department of Energy

Director, Nuclear Energy, Science \& Technology

Under DOE Idaho Operations Office

Contract Number: DE-AC07-05ID14517

\begin{abstract}
Disclaimer
This report was prepared as an account of work sponsored by an agency of the United States Government. Neither the United States Government nor any agency thereof, makes any warranty, express or implied, or assumes any legal liability or responsibility for the accuracy, completeness, or usefulness of any information, apparatus, product, or process disclosed, or represents that its use would not infringe privately owned rights. Reference herein to any specific commercial product, process, or service by trade name, trademark, manufacturer, or otherwise does not necessarily constitute or imply its endorsement, recommendation or favoring by the United States Government or any agency thereof. The views and opinions of authors expressed herein do not necessarily state or reflect those of the United States Government or any agency thereof.
\end{abstract}




\section{CONTENTS}

\section{Section 1 Nuclear Science and Technology}

11-000 - Haptic Tele-Operated End Effector for Hazardous Environments ..... 2

11-005 - Speciation Behavior of Americium Oxidation States for the Separation of Americium from

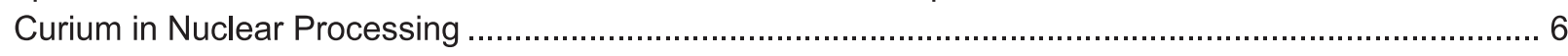

11-023 - Development of a Simplified Soft Donor Technique for Trivalent Actinide/Lanthanide Separations ......... 9

$11-051$ - Alternative Processing for Improved $\mathrm{UO}_{2}$ Based Fuels …..................................................... 13

11-055 - Spatially Resolved Positron Analysis of Hydride Formation in LWR Fuels with GE-Global

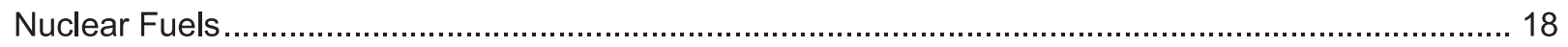

11-061 - Advanced Modeling and Simulation Concepts for ATR ............................................................ 21

12-031 - 2-D and 3-D EBSD Technique Development and Microstructure Reconstruction for Phase Field

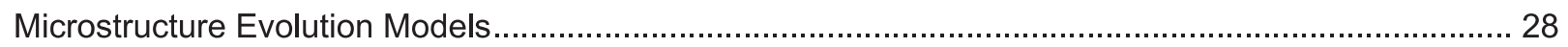

12-045 - Development of an Uncertainty Quantification Method for MIR Facility ........................................ 31

12-061 - Development of a High Burnup Zr-Based Fuel Cladding for LWRs .............................................. 35

12-062 - Development of Diamond Based Sensors for User Facility Capabilities at INL Advanced

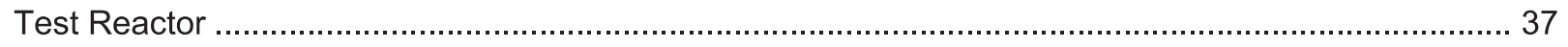

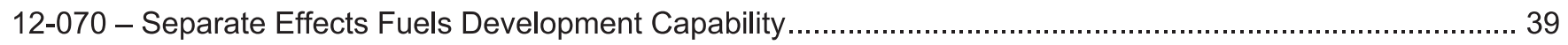

12-112 - Reactor Coolant Pump Alternate Can Material Development Program............................................ 42

12-117 - Feasibility of a Broad Application Test Reactor Using LEU ...........................................................4 44

13-013 - Development of a Micromechanistic Phase-Field Modeling Approach for Life Estimation and

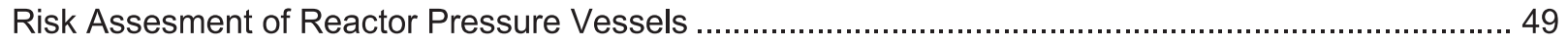

13-014 - Aluminium Electroplating from Low Temperature Molten Bromide Salts ....................................... 52

13-023 - Characterization and Modeling of High Burn-up Mixed Oxide Fuel .............................................. 54

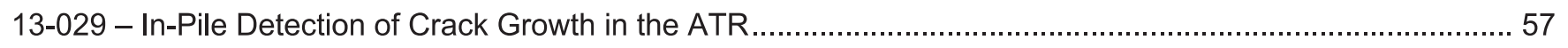

13-032 - Experimental and Computational Analysis of Hydride Microstructures in Zirconium in Dry

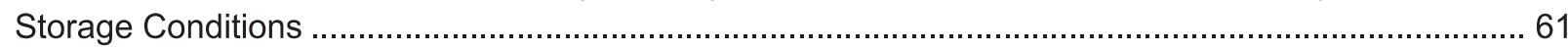

13-039 - Induction Based Fluidics Mass Spectrometry for Characterizing Radioactive Extraction Solvents.........63

13-040 - Epistemic Uncertainty Quantification in Dynamic Probabilistic Risk Assessment .................................6 65

13-050 - Concurrent Atomistic to Macroscale Modeling of Materials Under Irradiation Using the Phase

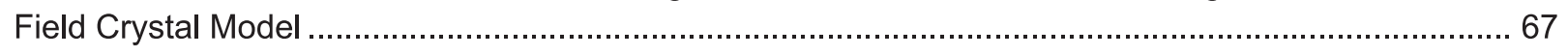

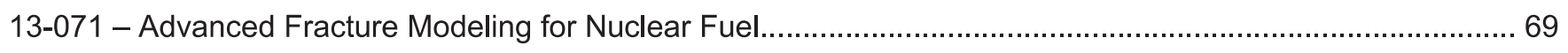

13-097 - MOOSE Capability Extension In Support of Full Core Modeling ................................................. 71

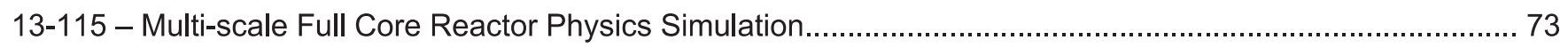


13-121 - Advanced In-Situ Measurement Techniques in TREAT

13-123 - Experimental Verification of Predicted Chemical Transmutation of Cesium-137 to Barium-137 via Beta Decay in $\mathrm{CsCl}$ Resulting in the Radioparagenetic Formation of Rocksalt $\mathrm{BaCl}$......

Section 2 national and Homeland Security

11-042 - Experimental Cyber Security: Vulnerability Prediction, Discovery, and Mitigation 83

11-059 - Unconventional Radiation Detection Methods for Nuclear Nonproliferation, Safeguards, and Treaty Verification.

11-060 - SMC Advanced Armor Materials and Systems R\&D 90

12-001 - Long Term Evolution Wireless Cyber Security. .92

12-017 - Polymer Components in Laminate Armor Systems for Ballistic Protection ........................................ 94

$12-050$ - Cross Section Representations for Nuclear Nonproliferation ..................................................... 96

$12-054$ - Treaty Verification with Photo-Induced Prompt Signatures .......................................................... 98

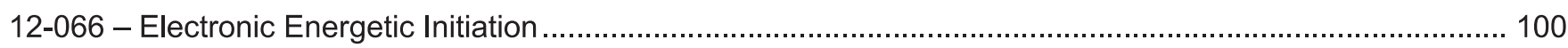

12-073 - A Nonproliferation Focused Study and Comparison of the GEANT4 Simulation Toolkit and MCNP Functionality, Accuracy, and Development Needs ...................................................... 102

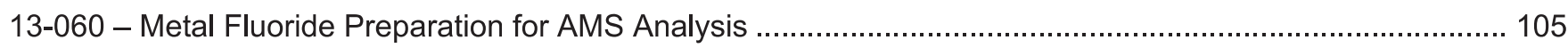

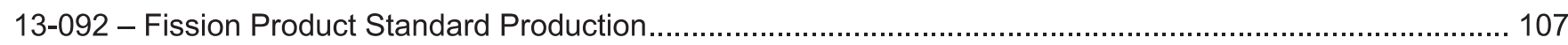

13-093 - Spectrum Allocation and Communications in Dynamic Spectrum Access Channels ......................... 109

13-118 - Geomagnetic Disturbance Field Coupling Measurements on INL Power Grid ............................... 111

\section{Section 3 Energy \& EnVironment SCIEnCE AND TeChNology}

11-007 - Integration of Renewable Energy Resources through Energy Storage and SuperGrid.

12-093 - Development of Unique Nanomaterials for Selective Replacement of Strategic and Critical Materials

12-096 - Isolation and Purification of Critical Metals Using Ionic Liquid Based Solvent Extraction 123

12-099 - Designing Algal Biofuel Consortia for Increased Function and Reduced Nutrient Costs

12-108 - Bakken Hydraulic Fracturing Water Needs and Potential Impacts to the Fox Hills/ Hell Creek Aquifer

12-113 - Coupled-Smoothed Particle Hydrodynamics and Discrete Element Model (SPH-DEM) Simulation of Hydraulic Fracturing and Shale Gas Production

12-118 - A Leap Ahead in Energy Storage - An Integrated Approach to an Inorganic High Energy Lithium Battery.

12-120 - Modeling Guided Development of Nanostructured High Energy Electrode Materials for Li-ion Batteries . 
13-027 - Diagnostics of Advanced Energy Storage Materials 139

13-045 - Direct Solar Hydrogen Production Using High Temperature Solid Oxide Photoelectrochemical Cells 142

13-059 - Application of Facilitated Transport Membranes Using Novel Stabilized Metal Complexes 144

13-065 - Multi-domain Modeling, Simulation, and Integration Tools for the Dynamic Analysis and Optimization of Hybrid Energy Systems ....

13-074 - A Nanobioreactor for Cellulose Degradation Using In Vitro Synthetic Biology ..... 151

13-079 - Diverse Biological Factories for Sustainable Manufacturing...... 153

13-100 - An Efficient System for the Production and Harvesting of Lipids from Photosynthetic Microbes 155

13-114 - Multiphase Fluid Trapping in Propped Hydraulic Fracture. 158

13-122 - Examination of the Impacts on Water Resources from Commercialization of Fossil Energy Development in an Arid Western Basin Using Integrated Hydrologic Modeling 161

\section{Section 4 Institute for Nuclear Science and Technology}

11-006 - Supercritical Fluid Extraction of Actinides from TRISO Reactor Fuels 163

11-041 - In-situ Micro-raman Spectroscopy and Modeling of Breakaway Oxidation of Zircaloy Cladding 165

11-043 - Characterization of Nanomechanical Response of Material to Determine Key Variables in Stress Corrosion Cracking

12-007 - Uncertainty Quantification of Safety Codes Using a Bayesian Approach with Data from Separate- and Integral-Effects Tests

12-026 - Protectiveness and Stability of the Zirconium Oxide in Early-Phase Corrosion of Zirconium Alloys Predictive Relations to Surface Structure and Composition

12-043 - Science-Based Simulation Model of Human Performance for Human Reliability Analysis 176

12-056 - Recovery of Precious Metals from Used Nuclear Fuel and Electronic Waste Using Sulfur-Impregnated Nanoscaffolds

12-059 - Chemistry and Flowsheet Development for Lanthanide Separations 182

12-077 - Observation of Zirconium Oxidation at Atomic Level Using Non-linear Optical Spectroscopy

12-090 - Development of Emissivity-tuned, Robust Encapsulated Radioisotope Heat Sources for an Advanced Radioisotope Thermo-Photovoltaic (RTPV) Power System .

13-095 - Development and Validation of a Societal-Risk Goal for Nuclear Power Plant Safety

13-105 - Micro/Nano Scale AFM-based Thermal Conductivity Measurement and Atomistic Modeling for Oxide Fuel: the Effects of Grain Boundary, Fission Gas, and Radiation Damage.

13-106 - Building Organic-Inorganic Hybrid Materials to Protect Metal lon Sequestering Agents from Radiation-Induced Oxidative Damage.

13-107 - Application of Chemical Heat Pumps for Temperature Amplification in Nuclear Hybrid Energy Systems for Synthetic Transportation Fuel Production 
13-110 - Nuclear-Renewables-Oil Shale Hybrid Energy System 198

13-112 - High-Temperature Nuclear Co-Electrolysis for Spacecraft Cabin Air Revitalization \& Liquid Fuel Production 200

\section{Section 5 Center for Advanced Energy Studies}

11-065 - Development and Demonstration Project for National Fuel Efficiency 203

11-066 - Targeted Energy Management Toolset for Comfort and Savings Based on Advanced Computational Intelligence Techniques 208

12-086 - Alert/Alarm Dashboard 212

13-011 - Integrated Approach to Algal Biofuel, Bio-power, and Agricultural Waste Management 217

13-033 - Magnetic Separation Nanotechnology for Spent Nuclear Fuel Recycle 221

13-035 - Development of New Molten Salt Sensor Technology for Application to Safeguarding Pyroprocessing

13-068 - Cooling In Fractured Geothermal Reservoirs: Analyses of Long-Term Cooling In Typical Geothermal Reservoirs and Application to Geothermal Resource Potential Assessments

\section{Section 6 Center for Advanced Modeling and Simulation}

10-031 - Fickian and Thermal Diffusion in Nuclear Materials from Linear Response Theory and Multiscale Simulations

11-074 - Uncertainty Analysis for Multiscale Models of Nuclear Fuel Performance

11-076 - Exploratory Nuclear Reactor Safety Analysis and Visualization via Integrated Topological and Geometric Techniques

\section{SECtion 7 InStRUmEntATION, CONTROL AND INTELLIGENT SyStemS}

11-046 - Known Secure Sensor Measurements for Detecting Unauthorized Process Manipulation and Falsification of State 242

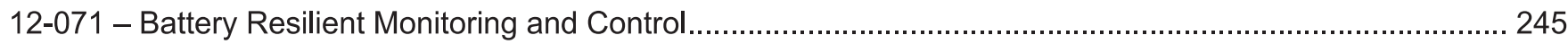

12-082 - Resilient Monitoring, Adaptation, and Control (ReMAC) System............................................... 248

12-089 - Multi-agent Analysis to Characterize Complex Infrastructure Interdependencies .............................2251

12-121 - State Awareness for Smart Grid Electrical Load Balancing, Distribution, and Security ..................... 256

13-120 - Improved Control System Resilience via Secure Machine Learning ..............................................2259

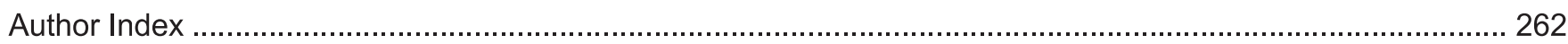




\section{NUCLEAR SCIEN Gañ̄ TECHNOLoor}




\title{
11-000 - Haptic Tele-Operated End Effector for Hazardous Environments
}

\author{
Anthony Crawford and Jeff Molitor
}

Natural movements and force feedback are important elements in using tele-operated equipment if complex and rapid manipulation tasks are to be accomplished in remote and/or hazardous environments (e.g., hot cells, glove boxes, decommissioning, explosive disarmament, and space). In order to execute such complex and rapid manipulation tasks, this project has developed an anthropomorphic robotic hand with radiation tolerant technologies and a corresponding admittance-type, exoskeleton-like, multi-fingered, haptic hand user interface that secures the user's palm and provides 3-D force feedback to the user's fingertips. The human hand form was chosen because the users of the current state-of-the-art in tele-operated manipulators report that task speed would improve by a factor of 5 to 10 were they able to use their own hands to execute a task. The project is also being used to expand the Pl's engineering aptitudes by contributing to his master's degree in electrical engineering at the University of Idaho. This will greatly supplement his master's and Ph.D. education in mechanical engineering.

\section{Summary}

This LDRD project has successfully developed a haptic hand user interface, a corresponding robotic hand with radiation tolerant technologies (Figure 1), the associated haptic controller hardware/algorithms, and a moveable platform to centralize all components. The haptic hand user interface has been designed, prototyped, and subjected to proof of concept testing. The user interface has been shown to have sufficient flexibility (degrees of freedom and range of motion) and speed to allow the user to move their fingers throughout their full range of motion without impedance. The device also has sufficient strength to communicate the necessary levels of force such that the user can appropriately interpret the environment and use that information to execute the intended task, whether that is manipulation or grasping. This is contrary to other hand user interfaces which are generally limited to effectively executing either manipulation or grasping activities but not both.
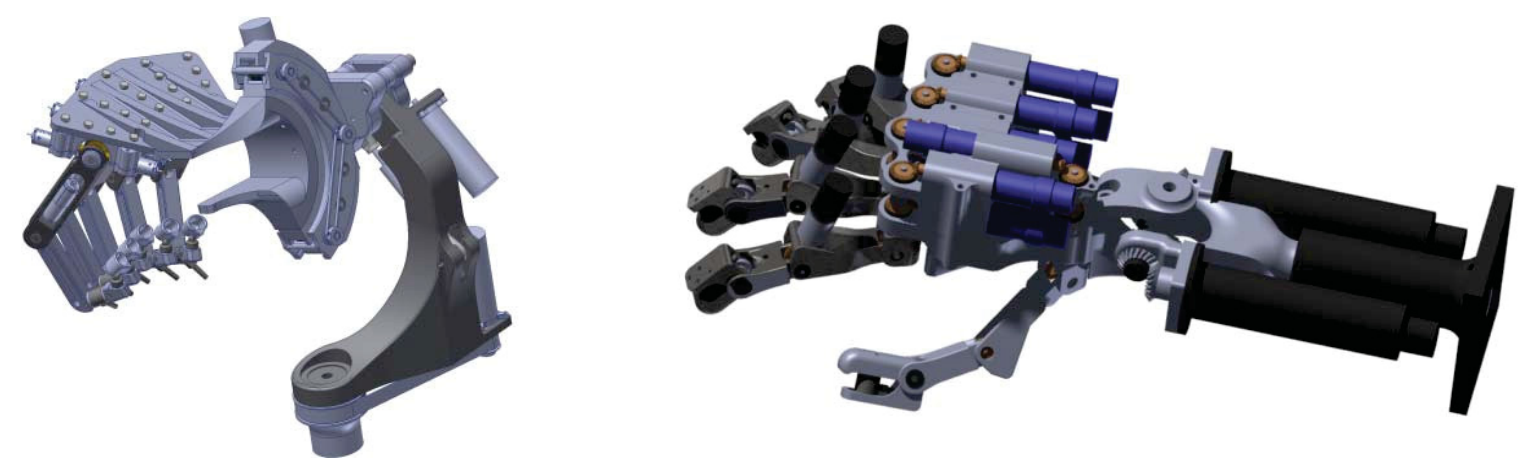

Figure 1. Image of haptic user interface and corresponding robotic hand. 
In addition to the device's unique form being specifically developed to provide sufficient manipulation dexterity and grasping force, this research has also developed a unique, nonlinear force profile relating the force experienced by the virtual environment (or robotic hand to the user) interface (Figure 2). The profile can be essentially divided into Region 1 , which ranges between $0 \%$ and $35 \%$ of the maximum applied force (manipulation tasks), and Region 2, which ranges between $35 \%$ and $100 \%$ of the maximum applied force (grasping tasks).

The difference in the slopes associated with each of the regions correlates to a desired level of force sensitivity and were devised based on six aspects which demonstrate the importance that force feedback has in the execution of manipulation tasks versus grasping tasks. These aspects include:

- Task complexity-Manipulation tasks are much more complex and delicate than grasping tasks and require more employed degrees of freedom. An article in IEEE Transactions on Robotics and Automation by Cutkosky explains that the delicate nature of the manipulation tasks indicate that they are more susceptible to perturbations (which argues the need for a suitable level of force feedback), while grasping tasks are less susceptible to perturbations (which argues for their allowance for reduced levels of force feedback).

- Task frequency-The compiled results of a study presented at the IEEE International Conference on Robotics and Automation by Zheng, Rosa, and Dollar that examined the typical hand tasks executed by a machinist and a maid revealed that an average of $72.5 \%$ of the executed hand tasks could be considered to be manipulation tasks while the remainder were more robust grasping in nature.

- The human's ability to sense just noticeable force differences-In "Human Factors for the Design of Force-Reflecting Haptic Interfaces," Tan demonstrates that the human sensory system begins to sense a force difference at $7 \%$ of the applied force. This indicates that light manipulation tasks desire much greater force sensitivity than higher force robust grasping.

- Fatigue-Hands become fatigued more rapidly if they consistently have to exert forces in excess of those executed during manipulation tasks. Thus, reducing the overall force communicated by a user interface will also reduce fatigue.

- Number of dominant muscles-A poster by Kutch and Valero-Cuevas along with a study conducted by Ehrsson and his colleagues show that as the hand transitions from manipulation to grasping, the force generating capability of some of the muscles active during manipulation actually saturate and no longer contribute to additional force generation during grasping (e.g., the flexor digitorum muscle). Thus their influence is much less utilized during grasping, arguing the importance to provide sufficient force feedback to the muscle in order to place it in the proper position prior to grasping initiation. 
- Associated brain activity-That same study by Ehrsson showed that more areas of the brain are activated during a precision grasp (viewed as similar in complexity to manipulation) vs. a power grasp. Therefore, the brain is processing more information in order to monitor the precision grasp and it is more important to supply more sensitive force information to perform this task.

Manipulation and grasping experiments were performed to test the developed nonlinear force profile as applied by the haptic device. Very condensed results are shown in Table 1 and Table 2. Table 1 shows the performance of a user to follow a circular pattern on a virtual surface while maintaining an applied force below $8 \mathrm{~N}$. In the table $T$ is the time, $r e$ is the distance error between the actual radius of the virtual circle and the measured radius, $F z$ is the applied force, up is the \% of time the user lifts their finger off the surface, down is the amount of time the user exceeds the $8 \mathrm{~N}$ applied force, and mid is the amount of time the user stayed within the desired 0-8N range. Table 2 shows the performance of the user in grasping a virtual plastic cup and maintaining force control, without collapsing the cup, as it is filled with a virtual fluid (affecting its weight and surface stiffness). The percentages in the table indicate what percentage of time the user is allowing the cup to slip through their fingers as the weight causes the user to progress through Region 1 and Region 2. The bold values in both tables indicate the increased performance generated by the nonlinear profile in both manipulation and grasping.

Table 1. Interface manipulation study results.

\begin{tabular}{|c|c|c|c|c|c|c|c|}
\hline & \multirow{2}{*}{$\begin{array}{c}k \\
(\mathrm{~N} / \mathrm{m})\end{array}$} & \multirow{2}{*}{$\begin{array}{c}\mathrm{T} \\
(\mathrm{s})\end{array}$} & \multirow{2}{*}{$\begin{array}{c}\text { re avg } \\
(\%)\end{array}$} & \multirow{2}{*}{$\begin{array}{c}\text { Fz avg } \\
(\mathrm{N})\end{array}$} & Up & Down & Mid \\
\hline & & & & & \multicolumn{3}{|c|}{$(\%)$} \\
\hline $\mathrm{L}$ & - & 7.5 & $7 \%$ & -6.3 & 2 & 32 & 65 \\
\hline $\mathrm{NL}$ & - & 6.8 & $10 \%$ & -4.1 & 6 & 9 & 85 \\
\hline
\end{tabular}

Table 2. Interface grasp study results.

\begin{tabular}{|c|c|c|}
\hline \multirow{2}{*}{} & $\begin{array}{c}\text { Region 1 } \\
\text { Below Limit }\end{array}$ & $\begin{array}{c}\text { Region 2 } \\
\text { Below Limit }\end{array}$ \\
\cline { 2 - 3 } & \multicolumn{2}{|c|}{$(\%)$} \\
\hline $\mathrm{L}$ & 38 & 34 \\
\hline $\mathrm{NL}$ & 42 & $\mathbf{1}$ \\
\hline
\end{tabular}

\section{Benefits to DOE}

DOE benefits from this research via the insight generated into how the human hand interacts with an environment and how one can employ that insight to improve the efficiency and effectiveness of remote handling devices. The product provides a proof of concept for increasing the quality and speed of conducting energy, nuclear, environmental, and science activities that require remote handling in a hazardous environment. The increased speed also reduces the time that contents of an experiment are exposed to theft or unapproved examination. The results also testify the scientific difference between manipulation and grasping tasks, how the human hand accommodates such differences, and how to modify force feedback parameters in a device to accommodate these characteristics.

\section{Publications}

Crawford, A., "Nonlinear Master-Slave Force Profile Control to Manage Haptic User Interfaces," Int. J. Robot. Res., Submitted September 2013.

Crawford, A., "User Interface for a Tele-Operated Robotic Hand System," Patent Application 15,586,116, April 23, 2013, Pending. 
Crawford, A., "Nonlinear Force Profile Used to Increase the Performance of a Haptic User Interface for Teleoperating a Robotic Hand," Proceedings of NPIC \& HMIT, San Diego, CA, 2012.

Crawford, A., "Force Control and Nonlinear Master-Slave Force Profile to Manage an Admittance Type Multi-Fingered Haptic User Interface," Proceedings of ISRCS 2012, Salt Lake City, UT, August 2012.

\section{Presentations}

Crawford, A., "Haptics," ASME Early Career Forum, Idaho Falls, ID, August 22, 2012. 


\title{
11-005 - Speciation Behavior of Americium Oxidation States for the Separation of Americium from Curium in Nuclear Processing
}

\author{
Catherine Riddle, Ken Czerwinski, ${ }^{1}$ and Patricia Paviet-Hartmann
}

Many countries are currently expanding nuclear energy as a method of safely generating carbon (C) free energy. Closed fuel cycle technologies may be key to waste reduction and a sustainable nuclear energy future. In support of this effort, a fundamental understanding of actinide chemistry, including americium (Am), is at the forefront of separations research. Higher oxidation states of Am can be used to separate Am from lanthanides and curium $(\mathrm{Cm})$, but this has never been done in an industrial process. Advances in controlling the oxidation state and speciation of Am in the solution and solid states are needed to develop such processes. This report describes some preliminary work to understand structure and speciation of complexes of $A m(V)$ and $A m(V I)$ especially using X-Ray Absorption Fine Structure (XAFS) spectroscopy and x-ray methods.

$\mathrm{Am}$, in the formal oxidation states of $\mathrm{Am}(\mathrm{V})$ and $\mathrm{Am}(\mathrm{VI})$, has been prepared by oxidation of $\mathrm{Am}(\mathrm{III})$ in acid solution using sodium bismuthate $\left(\mathrm{NaBiO}_{3}\right)$ and the solution species characterized by XAFS at the Advanced Photon Source (APS) at Argonne National Laboratory. Only a limited number of studies on Am(III) species using XAFS have been performed. We report here on the first studies of $\mathrm{Am}(\mathrm{V})$ and $\mathrm{Am}(\mathrm{VI})$ solution species with XAFS methods. A systematic analysis of the effect of complex formation during the oxidation/reduction phase has been conducted and provides new information on the behavior of higher oxidation states of Am. XAFS was used to examine number, type, and distances of atoms in close proximity to the $\mathrm{Am}(\mathrm{V})$ or $\mathrm{Am}(\mathrm{VI})$.

Limited data has been reported on single crystals of $A m(V I)$ compounds, and there is no reported work on single crystals of $A m(V)$ compounds. Various methods for the preparation of stable crystalline complexes of $A m(V)$ and Am(VI) were investigated. Sodium acetate $\left(\mathrm{C}_{2} \mathrm{H}_{3} \mathrm{NaO}_{2}\right)$ was the most promising compound and was used to produce a sodium americyl(V)acetate crystal which was evaluated using single crystal $\mathrm{x}$-ray crystallography.

The data from both XAFS and the crystallography work are currently being developed using density functional theory as a computational characterization method, adding further information on the behavior of oxidized Am. The speciation understanding provided by this work will help advance actinide separation technology. This work also serves as the Pl's dissertation research for a Ph.D. in radiochemistry from the University of Nevada, Las Vegas (UNLV) with defense planned for winter 2014.

\section{Summary}

Initial baseline research on oxidized Am using XAFS was accomplished at Argonne National Laboratory's APS during the first and second year of this project. These data showed a distinct increase in energy, proving that characterization of $\mathrm{Am}(\mathrm{V}, \mathrm{VI})$ oxidation states with XAFS is possible. However, background noise produced by low concentrations of Am-243 in the samples provided no useful data in the Extended XAFS region (Figure 1) and thus showed inconclusive results. Refined experiments, which are the result of what was learned from the baseline experiment, were designed to enhance the oxidized Am signal by substantially increasing the Am-243

1. University of Nevada, Las Vegas 


\section{NUCLEAR SCIENCE \& TECHNOLOGY}

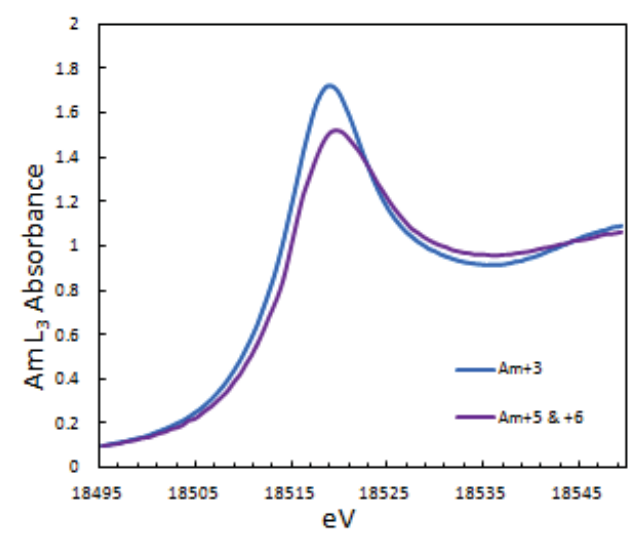

Figure 1. XAFS analysis from APS at Argonne National Laboratory showing the noise in the upper Extended XAFS region. concentration, thereby minimizing the background noise within the Extended XAFS spectra. In addition to the noise problem found during the APS experiment, a new problem associated with the oxidizing agent was discovered during preparation for the next XAFS experiment. A key research development resulted from the analysis of ultraviolet-visible spectra of the Am/bismuthate solutions prior to the samples being sent for XAFS analysis to the Stanford Synchrotron Radiation Light Source (SSRL). The data showed no oxidation of Am in the samples, and the experiment using XAFS was put on hold until the problem could be identified and resolved. The redox problem, identified late in FY 2012, was due to peroxides (a common reducing agent) in the $\mathrm{NaBiO}_{3}$ stock chemical. These pockets of peroxide have the effect of randomly reducing the Am so the results show either complete oxidation, partial oxidation, or no oxidation of Am in any given experiment. This problem was remedied by using purified $\mathrm{NaBiO}_{3}$, in which the peroxides have been completely removed. XAFS beam time was rescheduled for

SSRL through our university collaborator, UNLV, and the oxidized Am samples (using peroxide free bismuthate) were prepared at INL by the PI. Beam time at SSRL was in collaboration with Los Alamos National Laboratory (LANL) through a newly established collaborative effort between UNLV, INL, and LANL for XAFS work. The XAFS data from SSRL showed both $\mathrm{Am}(\mathrm{V})$ and $\mathrm{Am}(\mathrm{VI})$ oxidation states in solution without the noise issues experienced during the APS run, as shown in Figure 2. However, it was not possible to separate the two oxidation states from each other, making it difficult to use density functional theory on the data.

A second key development in the project was the experimental design and subsequent synthesis of a sodium americyl(V)acetate single crystal, analogous to acetate uranyl, neptunyl, and plutonyl crystals. An extensive literature search showed that this is the first time $\mathrm{Am}(\mathrm{V})$ crystals have been produced. Crystals of uranyl acetate were first synthesized as a surrogate to Am crystals using perchloric acid $\left(\mathrm{HClO}_{4}\right)$. The same experimental procedure that produced oxidized Am in solution for XAFS with bismuthate was used as the stock solution for the crystal synthesis in dilute $\mathrm{HClO}_{4}$. The analysis of the americyl(V)acetate crystal was accomplished with the assistance of UNLV and our collaborators at LANL.

The experimental work accomplished in the past three years has advanced the Pl's Ph.D. dissertation research as well as gaining her doctoral candidacy, achieved in May 2012. Defense of the Pl's dissertation for the radiochemistry Ph.D. program at UNLV has been scheduled for March 2014 with graduation in May 2014.

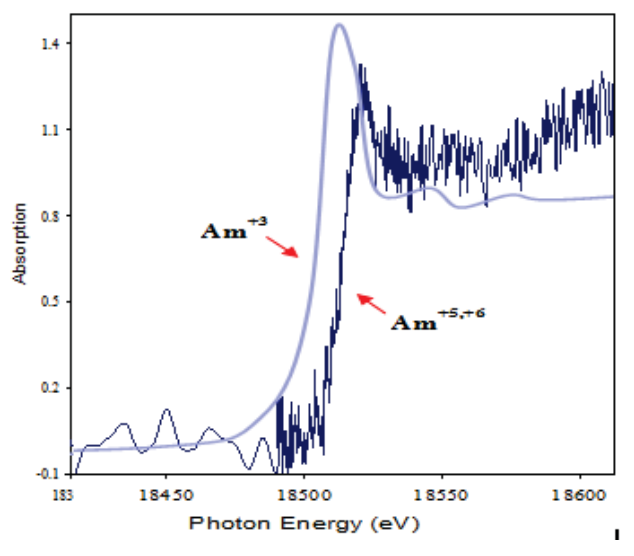

Figure 2. XAFS analysis from SSRL showing $A m(V, V I)$ mixture and noise elimination.

Overall, this LDRD research has exceeded goals set by the PI through successful collaborations with UNLV and LANL, which give INL access to important advancements, including: knowledge in XAFS measurements for actinides and a door for usage of XAFS instruments at APS and SSRL, single crystal x-ray crystallography 
analysis of actinides, and density functional theory analysis. The expertise and long term collaborations gained from this project will result in increased capability at INL and meet current and future needs of DOE.

\section{Benefits to DOE}

This work introduced the potential for new separation processes, with respect to the separation of Am from lanthanides and $\mathrm{Cm}$, which could vastly improve processes currently under development within DOE-NE. This work benefits the R\&D objective for advancing the deployment of secure and sustainable energy systems. This research will lead to a better understanding of the behavior of this actinide species in both complexed and uncomplexed forms in the stages prior to, during, and post separation as well as the separation of trivalent actinides from the lanthanides. The involvement of UNLV will open more avenues for targeted funding agencies and enhance the training of the future workforce. The collaborations with other national laboratories, such as LANL, will foster new funding avenues as well as promote future areas of research.

This project's research was the basis for the Pl's dissertation in partial fulfillment of a Ph.D. in radiochemistry from UNLV and will ultimately benefit both INL and DOE by producing a new Ph.D. in radiochemistry. The PI will be the first student to graduate from a newly formed distance learning program in which chemists working at a national laboratory with a master's degree can complete their Ph.D. in radiochemistry from UNLV.

\section{Publications}

Riddle, C., "Characterization of Bismuthate Oxidized Americium (V,VI) in Acidic Solution Using X-ray Absorption Fine Structure Spectroscopy," J. Nucl. Mater., In preparation.

Riddle, C., "Crystal Structure of Americium Acetate $\left[\left(\mathrm{AmO}_{2}\right)_{2}\left(\mathrm{OOCCH}_{3}\right)_{2}\left(\mathrm{H}_{2} \mathrm{O}\right)\right] \cdot 2 \mathrm{H}_{2} \mathrm{O}$," Inorg. Chem., In preparation.

Riddle, C., "Relativistic Density Functional Theory Modeling of Americium (V) Molecules," J. Chem. Phys., In preparation.

\section{Presentations}

Riddle, C., et al., "Speciation Behavior of Americium Higher Oxidation States for the Separation of Americium from Curium," ANS Global Conference, Salt Lake City, UT, September 30-October 4, 2013.

Riddle, C., et al., "Speciation Behavior of Americium Higher Oxidation States for the Separation of Americium from Curium," American Chemical Society $242^{\text {nd }}$ National Meeting \& Exposition, Denver, CO, August 28September 1, 2011. 


\title{
11-023 - Development of a Simplified Soft Donor Technique for Trivalent Actinide/Lanthanide Separations
}

\author{
Leigh Martin, Louise Natrajan, ${ }^{1}$ and Clint Sharrad ${ }^{1}$
}

The aim of this project was to develop an understanding of the various chemical processes that may lead to the successful separation of the trivalent lanthanides from the trivalent actinides using an amino acid buffer system and/or short chain peptides. By exploring such a novel extraction system, we have developed a simple system that, unlike the favored current option for actinide/lanthanide separation (TALSPEAK), can be thermodynamically modeled. Finally, to give a global package for the performance of this type of separation system, the research also addressed the radiolysis effects on the separation performance.

\section{Summary}

In the initial stages of the project, the research team extensively investigated amino acids as buffers for the separation of the actinide and lanthanide elements. Solvent extraction experiments to study the separation of a series of lanthanide elements (lanthanum [La], cerium [Ce], prasedymium [Pr], neodymium [Nd], samarium [Sm], europium [Eu], gadolinium [Gd], terbium [Tb], dysprosium [Dy], holmium [Ho]) from americium (Am) were performed to determine the kinetics of extraction of the lanthanides, the effects of amino acid concentration, and the effects of an aqueous phase complexant. Figure 1 summarizes the results for the L-alanine buffered system. With diethylenetriaminepentaacetic acid as a holdback complexant for the actinides, it was found that this separation worked effectively over the $\mathrm{pH}$ range $\sim 1.5-3$. Further, this work has identified that there is little buffer concentration effect between 1.0M and $0.5 \mathrm{M}$ alanine concentration. The kinetics of extraction were found to be improved over the traditional TALSPEAK formulation. One of the constant problems

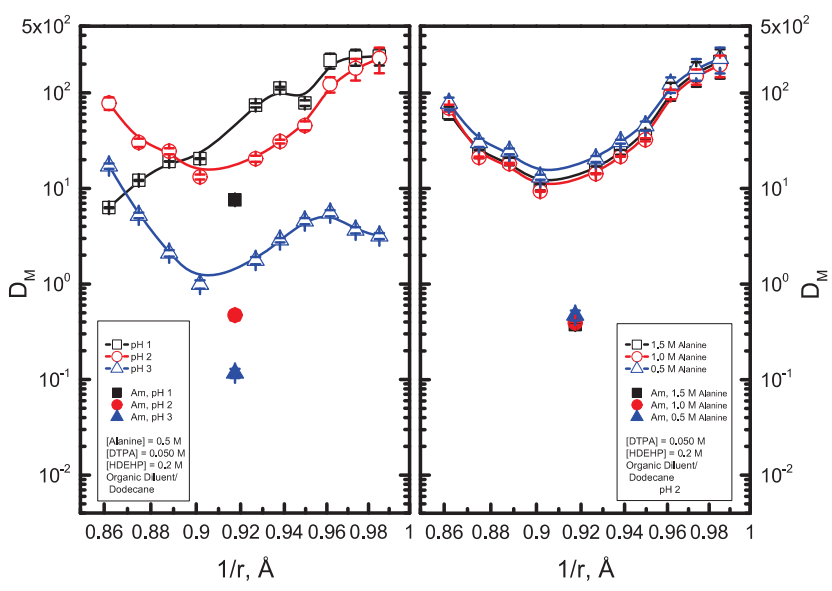

Figure 1. Comparison of trivalent lanthanide/Am ${ }^{3+}$ extraction using $L$-alanine buffered TALSPEAK at $\mathrm{pH} 1$, $\mathrm{pH} 2$, and $\mathrm{pH} 3$ (left). Demonstration of the successful separation of $\mathrm{Ln}^{3+}$ from $\mathrm{Am}^{3+}$ at varying concentrations of L-alanine buffer, the aqueous acidity is adjusted to $\mathrm{pH} 2$ (right). Open symbols represent the lanthanide series, closed americium. with the original formulation of the TALSPEAK process is that the experimental data could not be modeled from the literature thermodynamic constants. The amino acid buffered separation was modeled using Equation (1) using an internally consistent set of thermodynamic data.

$D=\frac{\overline{M(D E H P \cdot H D E H P)_{3}}}{\left[M^{3+}\right]+\left[M_{A l a^{2+}}\right]+\left[M R^{2-}\right]+\left[M H R^{-}\right]}$

1. University of Manchester 
The results obtained from the model demonstrated good agreement between the experimental and calculated theoretical values. Experimental distribution ratios for both $\mathrm{Eu}^{3+}$ and $\mathrm{Am}^{3+}$ begin to deviate from the calculated distribution ratios when the $\mathrm{pH}$ is $\sim 3.1$. The thermodynamic model (Equation [1]) can accurately predict the distribution ratios over a range of $\sim 1.5 \mathrm{pH}$ units.

Following the success of the L-alanine buffered separation process, two approaches were employed to study the effects of radiolysis on the alanine based separation system: pulsed radiolysis and $\gamma$-radiolysis. Pulsed radiolysis was used to study the ${ }^{\circ} \mathrm{OH}$ radical reaction kinetics with L-alanine as a function of $\mathrm{pH}$ and temperature. Solvent extraction experiments using $\gamma$-irradiated solvents were performed to study the effects of radiolysis on the actinide/lanthanide separation factors. In traditional TALSPEAK, it has been identified that lactic acid/lactate ion protect the diethylenetriaminepentaacetic acid ligand responsible for the separation. It has been shown that the ${ }^{\bullet} \mathrm{OH}$ radical reacts more slowly with L-alanine than with lactic acid (room temperature rate constant $\sim 4.30 \times 10^{7}$ ). The activation energy for the reaction was found to be $\sim 13.8 \mathrm{~kJ} \mathrm{~mol}^{-1}$, this is higher

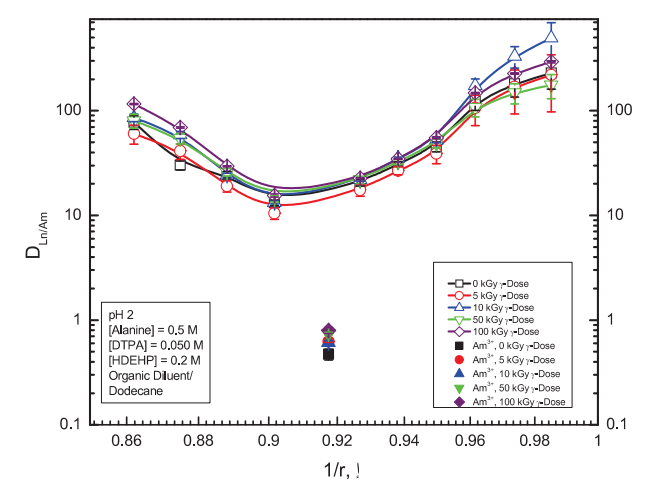

Figure 2. Effect of increasing $\gamma$-dose on $A n^{3+} / L n^{3+}$ separation. than that reported for the lactate ion. It was expected these results would negatively affect the separation performance of the L-alanine based TALSPEAK. The $\gamma$-radiolysis solvent extraction experiments were performed as a function of $\mathrm{pH}(2-3)$, dose (0-100 kGy) and buffer concentration $(0.5-1.5 \mathrm{M})$. In all cases, there were only small changes to the separation factors, as all of these variables were changed (Figure 2). These results are encouraging as this separations system can be considered as radiolysis resistant.

The modeling exercises highlighted a lack of thermodynamic data in the literature for the amino acids L-arginine, L-histidine, and L-methionine. The direction of the LDRD shifted in the final year to the determination of stability constants for $\mathrm{Ln}^{3+} / \mathrm{An}^{3+}$ amino acid complexes to complete the modeling iterations for all amino acid systems. Several techniques have been employed to attempt to measure these constants: potentiometric titration, ultraviolet-visible spectroscopy, or fluorescence spectroscopy. Unfortunately-due to the weak nature of the amino acid complexation with metal ions-when employing potentiometric titrations, no change was observed in ligand, only titrations and ligand and metal titrations. As such, this method can only be used to determine the amino acid pKa's.

Overall, measuring stability constants using fluorescence spectroscopy was successful. However, there was a large emission centered around $500 \mathrm{~nm}$ which is currently attributed to an emission from the ligand that complicated the analysis. It has been determined that, in the future, researchers should use recrystallized ligands in an attempt to eliminate fluorescence signatures due to impurities in the solutions under interrogation. 
Performing ultraviolet-visible titrations of metal ions with alanine has proven to be the most successful technique investigated so far. The lanthanide absorption spectra are sensitive enough to the ligand binding, and there is no interfering absorbance from the alanine ligand. However, this technique is limited to the few lanthanides that exhibit an absorbance signal. The stability constants for Nd:Alaninate and Eu:Alaninate are listed in Table 1. The stability constants in Table 1 are reduced by about a factor of two compared to the $2.0 \mathrm{M}$ ionic strength data in the open literature.

\section{Table 1. Stability constants for Nd:Alaninate and Eu:Alaninate.}

\begin{tabular}{|c|c|c|c|}
\hline Complex & $\beta_{111}$ & $\begin{array}{c} \pm 3 \sigma \\
\text { error }\end{array}$ & $\begin{array}{c}\text { lonic Strength } \\
(\mathrm{M})\end{array}$ \\
\hline Nd:Alaninate & 2.40 & 0.01 & 1.0 \\
\hline Eu:Alaninate & 2.04 & 0.02 & 1.0 \\
\hline
\end{tabular}

\section{Benefits to DOE}

This work has contributed towards the design of an advanced fuel cycle that is proliferation resistant, contributing to DOE's mission of nuclear security. The study of amino acids to buffer separations systems for minor actinide/lanthanide separations has provided previously unavailable data on the long term performance of these reagents in separations applications.

\section{Publications}

Grimes, T., R. Tillotson, and L. Martin, "Trivalent Lanthanide/Actinide Separation Using Aqueous-Modified TALPSEAK Chemistry," Solvent Extr. Ion Exc., Submitted.

Martin, L., et al., "Alanine Radiolysis and its Effects on Lanthanide Actinide Separations," Solvent Extr. Ion Exc., In preparation.

\section{Presentations}

Martin, L., et al., "Radiation Induced Degradation Kinetics of Next Generation Actinide/Lanthanide Separations Buffers," Global 2013 International Nuclear Fuel Cycle Conference, Salt Lake City, UT, September 29-October 3, 2013.

Langford-Paden, M., et al., "Development of a Simplified Soft Donor Technique for Trivalent Actinide/Lanthanide Separations," Global 2013 International Nuclear Fuel Cycle Conference, Salt Lake City, UT, September 29October 3, 2013.

Grimes, T. and L. Martin, "Determination of Stability Constants for the Complexation of Lanthanides and Actinides by Amino Acids," Global 2013 International Nuclear Fuel Cycle Conference, Salt Lake City, UT, September 29-October 3, 2013.

Grimes, T. and L. Martin, "Trivalent Lanthanide/Actinide Separation Using Aqueous-Modified TALSPEAK Chemistry," Actinides 2013, Karlsruhe, Germany, July 21-26, 2013.

Langford-Paden, M., et al., "Development of a Simplified Soft Donor Technique for Trivalent Actinide Lanthanide Separations," Actinides 2013, Karlsruhe, Germany, July 21-26, 2013. 
Grimes, T., R. Tillotson, and L. Martin, "Determination of Stability Constants for the Complexation of Lanthanides and Actinides by Amino Acids," $37^{\text {th }}$ Actinide Separations Conference, Spokane, WA, June 24-27, 2013.

Langford, M., et al., "Development of a Simplified Soft Donor Technique for Trivalent Actinide Lanthanide Separations," 245 ${ }^{\text {th }}$ American Chemical Society National Meeting, New Orleans, LA, April 7-11, 2013.

Langford, M., et al., "Development of a Simplified Soft Donor Approach to Trivalent Actinide/Lanthanide Separations," Atalante 2012, Montpellier, France, September 2-7, 2012, Invited.

Grimes, T., R. Tillotson, and L. Martin, "Radiolytic Stability of a Modified TALSPEAK Process," $67^{\text {th }}$ Northwest Regional Meeting of the American Chemical Society, Boise, ID, June 24-27 2012.

Martin, L., R. Tillotson, and T. Grimes, "Radiation Effects on An3+/ Ln3+ Separation in Amino Acid Buffered TALSPEAK," Actinide Separations Conference, Chattanooga, TN, May 21-24, 2012.

Grimes, T., R. Tillotson, and L. Martin, "Lanthanide/Actinide Separation Using Aqueous-Modified TALSPEAK Chemistry," Actinide Separations Conference, Chattanooga, TN, May 21-24, 2012.

Martin, L., et al., "Development of a Simplified Soft Donor Approach to Trivalent Actinide/Lanthanide Separations," $17^{\text {th }}$ Symposium on Separations and Technology for Energy Applications, Gatlinburg, TN, October 23-27, 2011. 


\title{
11-051 - Alternative Processing for Improved $\mathrm{UO}_{2}$ Based Fuels
}

\author{
Paul Lessing and W. Roger Cannon
}

This research project is improving the physical properties and hence, in-reactor performance of sintered uranium oxide-based fuels. This has been accomplished by developing a science-based understanding of alternative and advanced processing methods as they apply to depleted uranium oxide $\left(\mathrm{UO}_{2}\right)$. The science basis has been to develop a fundamental understanding of the chemical and physical factors that affect the ceramic processing of the $\mathrm{UO}_{2}$ surrogate of $\mathrm{CeO}_{2}$. The final physical properties have been enhanced by using the developed fundamental knowledge to develop improvements to the fabrication and sintering methods. Emphasis was placed on dry processing methods that are suitable for inert atmosphere glove box operations. This has included the study, selection, and performance-quantification (e.g., pellet strength and fracture toughness) of radiationresistant binders (e.g., for TRU-MOX fuels). The binders have included both water alone and various classes of organic (polymer) binders. Rounded-granules formed from powders + binders were homogeneously consolidated using isostatic pressure in a "Dry Bag Isostatic Press" and then pressureless-sintered to the desired final shape and density.

\section{Summary}

During FY 2013, processing studies were performed to demonstrate tighter dimensional control (reducing the amount of fuel pellet machining), production of more uniform green pellets (creating more uniform sintering shrinkage and allowing machining in the green state rather than in the sintered state), and adjusted moisture content of the green pellets during storage (reducing rejects due to chipping).

Improvements to the processing of nuclear fuel pellets were primarily made by converting to dry bag isostaticpressing as a replacement to metal-die pressing. A "dry" processing procedure was developed which led to diameter uniformity in dry bag isostatically-pressed rods. It was necessary to develop a process which produced high density, flowable granules without the use of the traditional time-consuming step of "slugging." Slugging is expensive and produces non-round granules. The developed process also includes an improved method for packing granules in the dry bag. Additional studies were performed to find methods to reduce waste and discharge of dust during nuclear fuel fabrication.

\section{Dimensional Control}

Dimensional control studies used $\mathrm{CeO}_{2}$ as a surrogate for ceramic fuel. Processing for precise dimensional control requires very reproducible dimensions in the green part prior to sintering. The three conditions necessary to produce very consistent dimensions are:

1. Consistency in the fuel powder source

2. Uniform powder packing in the dry bag

3. A consistent pressing schedule.

Condition three is programmed into the dry bag isostatic press and was therefore not a variable in our study. The effort during FY 2013 concentrated primarily on criterion number two: uniform and economical packing of the granules in the dry bag. Commercial powder was passed through a spinning riffler to make it completely homogenous. 
The production of rounded granules which will flow evenly into the "parts bag" is critical to uniform packing. Several different binders, plasticizers, and methods for producing granules were studied. All the methods were economical and scalable with few processing steps. Figure 1 contains micrographs of the granules produced by three of these essentially "dry" methods for two different sources of powder, ceria powder from Tianjao International (TJTM) and Inframat Advanced Materials (IAM). In Figure 1, essentially "dry" processed granules using $0.2 \mathrm{wt}$ \% PEG 8000 and 0.2 wt. \% zinc stearate $\left(\mathrm{C}_{36} \mathrm{H}_{70} \mathrm{O}_{4} \mathrm{Zn}\right.$ ) as binders: (a) (TJTM-w) ${ }^{1}: 8.8 \%$ water was added yielding angular granules. Fine granules were sieved out. Time to flow through standard Hall funnel: 51 seconds. (b) TJTM- $d^{2}$ processed entirely dry, but granules are screened to give them a more round morphology. Though fine granules were sieved out, many adhered to the granular surface. Time to flow through standard Hall funnel: 92 seconds. (c) IAM processed entirely dry and passed through a spheronizer. Time to flow through standard Hall funnel: 46 seconds. The granular size distribution was wider. Granular size distribution and granular surface properties were found to be most important in improving the flow rate of granules.

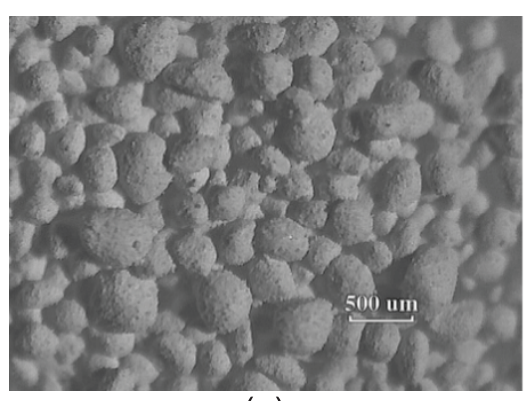

(a)

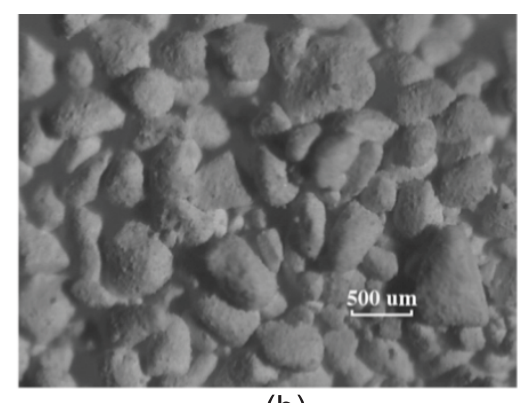

(b)

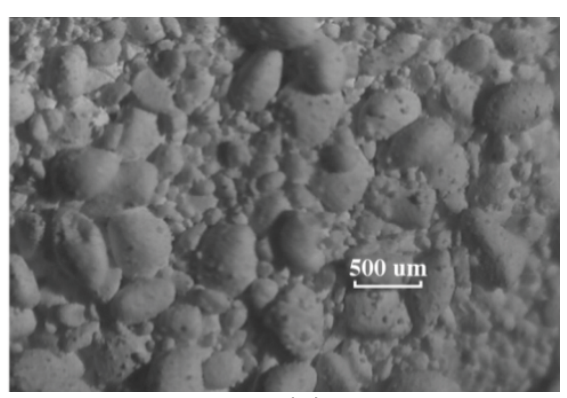

(c)

Figure 1. Granules produced by three different dry methods.

Packing was monitored using tap density curves (i.e., packing fraction vs. number of taps using a tap density instrument in accordance with American Society for Testing and Materials standard B527-93). Figure 2 (next page) shows that packing fractions as high as 0.44 ( 1.0 being theoretical density) can be achieved through mechanical tapping of the granular bed. Packing fractions of at least $0.25-0.35$ are usually found under ceramic manufacturing conditions. High flow densities (i.e., packing densities before tapping) are achieved with all three granular sets shown in Figure 3 (next page), as compared to the baseline. Figure 3 indicates that after approximately 1000 taps, packing factors no longer increased and are, therefore, considered the ultimate packing for the granules. At this point, intergranular porosity is at a minimum. Intragranular porosity depends on the granulation method and the powder source. Higher surface area powders generally contain more intragranular porosity.

\footnotetext{
1. w stands for water.
}

2. d stands for dry. 


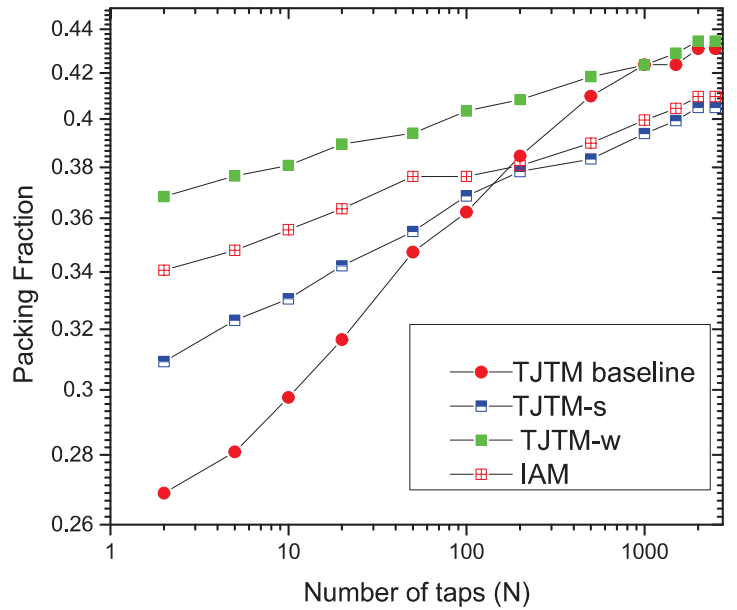

Figure 2. Tap density curves illustrating the ability of granules to pack into the parts bag. Packing fraction is the fraction of theoretical density of solid $\mathrm{CeO}_{2}$. TJTM baseline granules were roll compacted and granulated with no attempt to round the granules or eliminate fines.

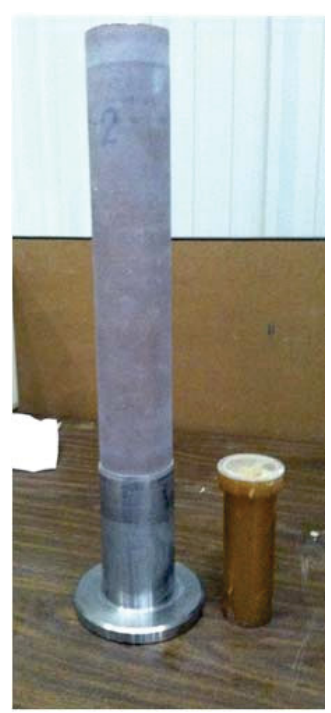

Figure 3. Larger bag tool assembly with smaller, inner parts bag. (The powder is poured into this bag which is then inserted into the assembly prior to isostatically pressing the rod.)

Figure 4 (next page) shows the dry bag tool assembly to the left and the parts bag, in which the rods are pressed, to the right. In order to ensure ultimate packing and minimization of the intergranular porosity, the parts bag was filled by pouring the granules through the standard Hall funnel. The parts bag was then placed on the tap density instrument and tapped 1500 times. This resulted in very uniform, consistent packing. Rods were then pressed in the dry bag isostatic press at $207 \mathrm{MPa}(30,000 \mathrm{psi})$. After being removed from the parts bag, the diameters of the rods were measured at three different rotations and averaged. Figure 5 compares the consistency of dimensions of three different rods pressed from TJTM-w granules as a function of distance from the bottom of the rod. With the exception of the "elephant feet" at either end of the rod, the dimensions are reproducible to less than $75 \mu \mathrm{m}$ (0.003 in.). "Elephant feet" are commonly observed during commercial isostatic pressing, and redesign of the bag can correct for this, as shown in the patent "Mold for Isostatic Pressing" developed by Bewlay and Dalpe. Figure 6 compares the diameter profiles of the other granule types. Though dimension reproducibility is exceptionally good through this method, according to a report for the DOE written by Stoll, dimensional tolerance for fuel pellets is $\pm 10 \mu \mathrm{m}$, and machining may still be necessary, but discharge of grinding-dust will be greatly reduced. 


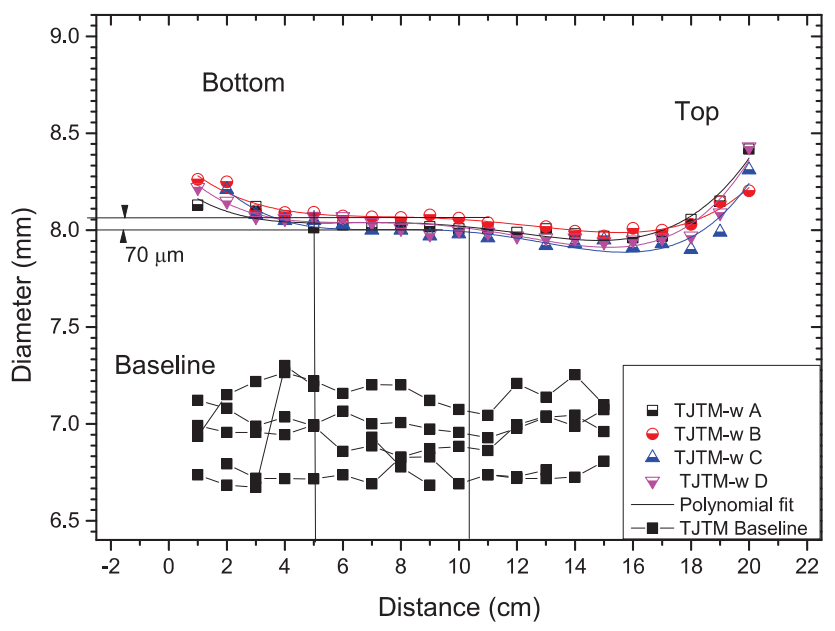

Figure 4. Average diameter of rods as a function of distance from the bottom of the rod. The larger diameters at the ends of the rods are called "elephant feet" by the dry bag isostatic pressing industry and are usually cut away.

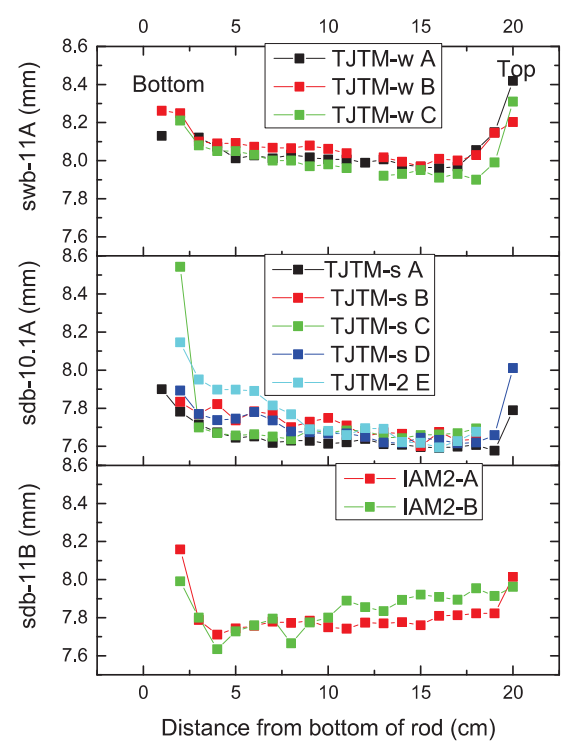

Figure 5. Left: Comparision of diameter profile for three different granular sources. Inframat Advanced Materials (IAM2) was differentiated from IAM because it came from a different lot of IAM ceria powder.

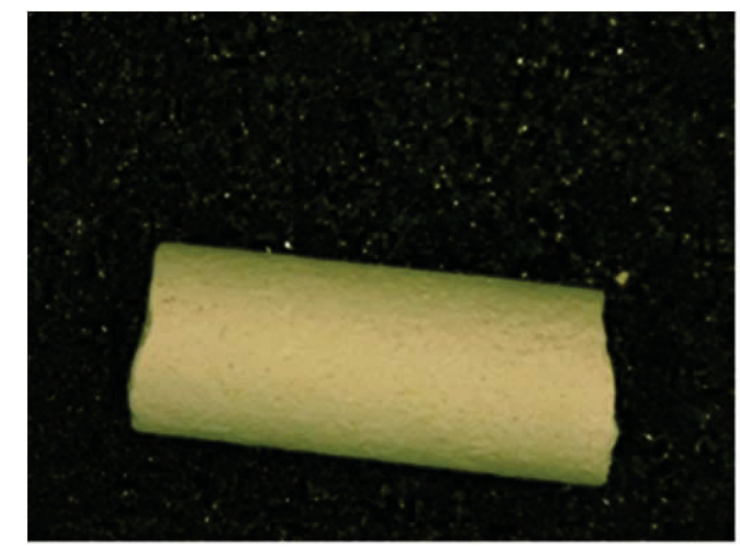

Figure 6. Right: Portion of sintered $\mathrm{CeO}_{2}$ rod originated from a dry bag isostatic-pressed, long "green" rod.

This "dry" processing can be applied to the fabrication of a variety of improved and accident-resistant light water reactor fuel pellets. We are well on the way to developing a process that leads to better-performing, homogeneous pellets that can be fabricated to a "near-net shape" with very minimal distortion of dimensions during the firing process. Figure 6 shows a segment of iso-pressed rod that was subsequently fired/sintered to its final high density. 


\section{Moisture Effects on the Strength of Binderless Pellets}

The Nuclear Fuel Cycle: from Ore to Waste by Wilson states that binderless processing is being increasingly used to fabricate fuel pellets. Fracture strength of the green pellets is an issue with binderless processing and leads to an increased rejection rate. Humidity control during storage should help. Fracture strength of green TJTM pellets was studied at two different levels of storage humidity. After 10 day storage at a relative humidity of $74 \%$, the mean fracture strength, measured by diametral compression, was $527 \pm 35 \mathrm{kPa}$, whereas, after 10 day storage at a relative humidity of $24 \%$, the mean fracture strength was $453 \pm 23 \mathrm{kPa}$. Although it may seem obvious that increased moisture adsorption in the pellet would lead to higher strength, the best accepted theoretical modelexplained in several articles from the Journal of the American Ceramic Society, American Ceramic Society Bulletin, Agglomeration, and Special Ceramics 8-claims capillary pressure from moisture to be insignificant compared to Van der Waals forces.

\section{Benefits to DOE}

Variations on our new fabrication techniques will prove to be innovative and valuable to ceramic fuel pellets for industrial light water reactors, including mixed oxide fuel and inert matrix fuel, in three ways: (1) the results can be directly applied to the enhanced fabrication of $\mathrm{UO}_{2}$-based fuels of interest to DOE-NE, such as mixed oxide fuel $\left(\mathrm{UO}_{2}+\mathrm{PuO}_{2}\right)$, transuranic mixed oxide fuel from recycled fuel $\left(\mathrm{UO}_{2}+\mathrm{PuO}_{2}+\right.$ actinides $)$, and enriched $\mathrm{UO}_{2}$, (2) the project builds INL equipment capability in fabricating $\mathrm{UO}_{2}$ and other powder-processing based fuels, and (3) the knowledge can be applied to other fuel compositions under development, such as $\mathrm{U}_{3} \mathrm{Si}_{2}$ and $\mathrm{UN}$, for potential use in accident tolerant fuel systems.

\section{Publications}

Hoggan, R., et al., "Processing of Surrogate Nuclear Fuel Pellets for Better Dimensional Control with Dry Bag Isostatic Pressing," 2013, to be submitted.

\section{Presentations}

Lessing, P.A., "Alternative Processing of Improved $\mathrm{UO}_{2}$-Based Fuels," to an INL external peer review committee, Idaho Falls, ID, June 23, 2011. 


\title{
11-055 - Spatially Resolved Positron Analysis of Hydride Formation in LWR Fuels with GE-Global Nuclear Fuels
}

\author{
Douglas Akers, Mark Drigert, Maria Okuniewski, Paul Cantonwine, ${ }^{1}$ and Tony Hill ${ }^{2}$
}

Hydride formation in commercial nuclear fuel Zircaloy cladding is one of the critical issues limiting the lifetime of commercial nuclear fuel designs to about $80 \mathrm{MWd} / \mathrm{kgU}$. General Electric-Global Nuclear Fuels (GE-GNF) has developed with the INL a CRADA to develop the INL's Spatially Resolved Positron Annihilation (SRPA) technology to provide in-situ, nondestructive measurements of hydride formation in nuclear fuel cladding and components following irradiation. The SRPA technology is a novel technology and has received an INL patent and was nominated for an R\&D 100 Award. This technology provides an improved fundamental understanding of the atomic-level microstructure of GE-GNF reactor fuel cladding and the effects of hydride formation on the lifetime of the cladding when exposed to long-term irradiation. In addition to developing an understanding of hydride damage effects, it provides a basis extending current reactor fuel life times, minimizing waste and developing new reactor fuels.

Verification and testing of the SRPA method for use in the characterization of nuclear fuel cladding for hydride production is being performed using surrogate samples containing hydrogen at the INL, development of a strain measurement capability to assess strain effects in cladding, along with irradiated cladding measurements at the GE Vallecitos facility. In addition, comparison studies have been performed on specimens using Transmission Electron Microscopy and Scanning Electron Microscopy. The project includes a strong cross correlation component that correlates the SRPA data, ex-situ measurement data currently used for hydride characterization, and the effects of strain and irradiation damage on the cladding. These data will be used to develop the design of a pool side SRPA system that can be used by GE-GNF to perform in-situ characterization of fuel cladding for hydride content at GE fuel storage facilities. In addition to the direct application of the SRPA technology to the GE-GNF's current and advanced light water reactor fuels programs, the SRPA technology has specific applications to other nuclear fuel-related issues such as fuel cladding interaction and stress corrosion cracking.

\section{Summary}

Progress during the final year of this project (2013) included:

- Development and testing of a SRPA scanning system suitable for use in high radiation fields and on irradiated Zircaloy specimens (see Figure 1)

- Using the SRPA scanning to perform measurements on a range of irradiated Zircaloy specimens at the GE Vallecitos facility in a higher radiation environment

- Cross correlation of the SRPA data with ex-situ measurement techniques such as optical metallography and Scanning Electron Microscopy performed at INL and Idaho State University

- Development and testing of a SRPA based strain measurement system that allows direct measurement of strain phenomena in the cladding at levels as low as $0.05 \%$ strain and segregation of the strain results from those associated with hydride damage (Figure 2)

1. General Electric-Global Nuclear Fuels

2. Idaho State University 
- Development of initial conceptual designs for both hot cell and pool side basin scanning systems for use at GE-GNF facilities.

Extensive SRPA measurements have been performed on prepared specimens with known concentrations of hydrogen induced in Zircaloy specimens provided by GE-GNF and the Electric Power Research Institute. SRPA measurements have also been completed on a range of irradiated Zircaloy specimens at the GE Vallecitos facility using the radiation hardened SRPA system shown in Figure 1. Results of these measurements are being correlated with GE data on irradiation exposure and cladding damage.

Figure 2 shows the SRPA based microstrain measurement system developed for assessing the effects of strain on the atomic structure of Zircaloy and the relative effect of strain versus hydride effects on the microstructure of the Zircaloy. The SRPA microstrain measurement system allows in-situ SRPA measurements of strain while the specimen is under load. The system has been demonstrated to provide direct measurement of strain effects as low as $0.05 \%$ strain. It was demonstrated that induced strain effects in GE Zircaloy components produces a much less significant effect on the Zircaloy microstructure than the effect of the induced hydrides.

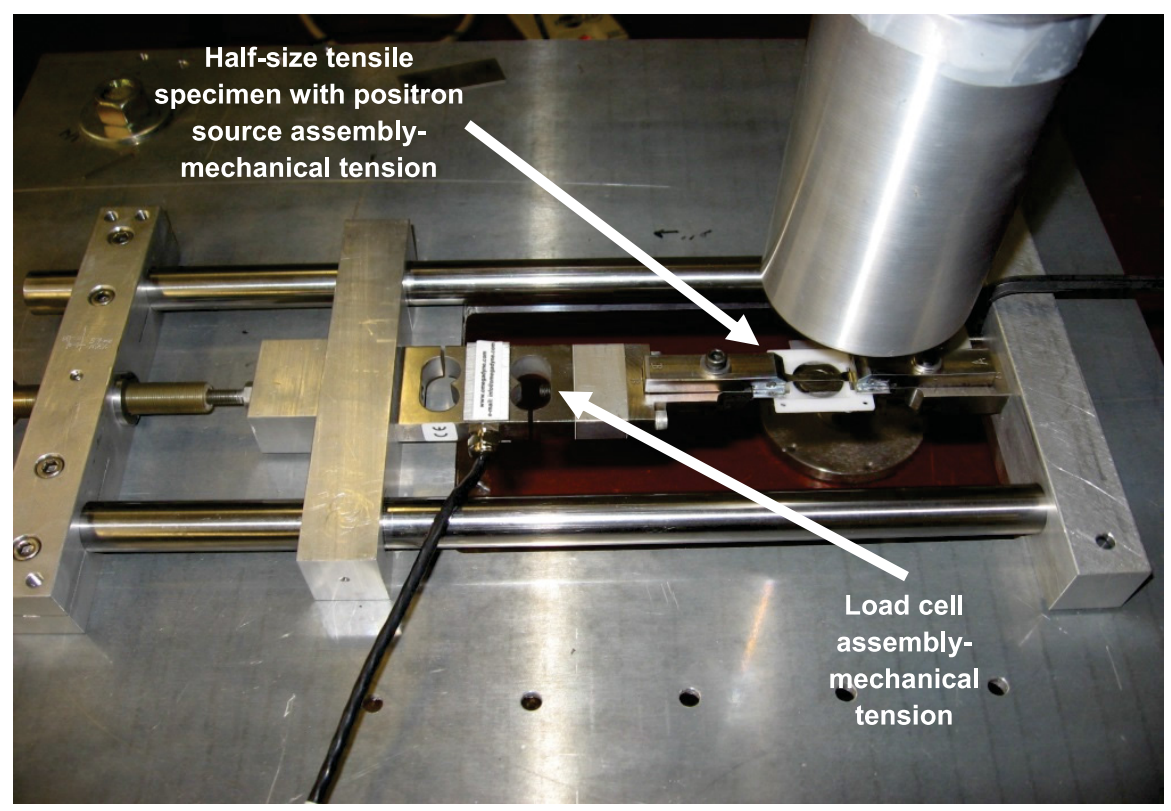

Figure 2. SRPA Microstrain Measurement System.

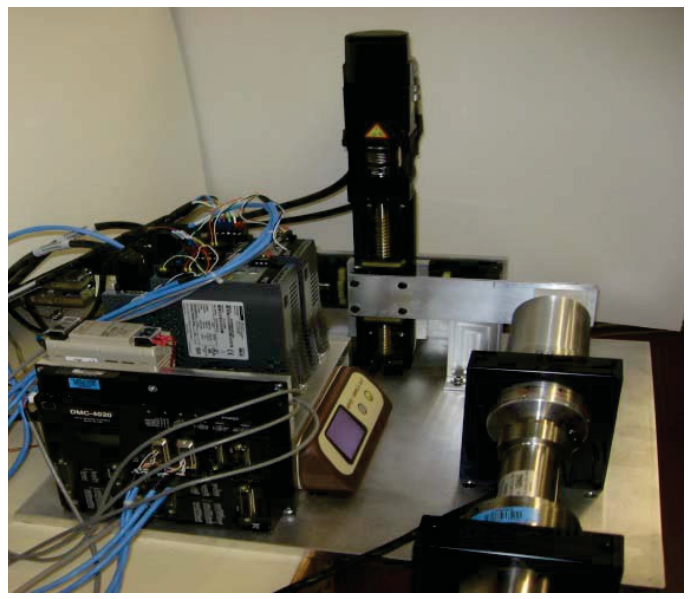

Figure 1. SRPA Rad-Hardened Scan Bed Used at GE Vallecitos. 
Figure 3 shows the conceptual design of the hot cell scanner system to be used in a hot cell with highly-irradiated specimens containing hydrogen or other types of cladding or fuel damage. This design is being developed with GE as the basis for a pool side scanner that will operate on Zircaloy rods removed from boiling water reactor fuel assemblies or assembly components. The actual system will be a modification of an existing GE scanning system that can be adapted for use to perform SRPA measurements. The end goal is to determine the effects of hydride and other damage effects on the remaining life of fuel assemblies and to determine if life extension is possible. Further, the SRPA measurement technology and the data obtained can be used in the development of new cladding materials and in the assessment of remaining life for these materials.

In addition to the scanning systems shown, an initial conceptual design of a pool-side SRPA scanning system for use on intact fuel assemblies and other components has been developed. This system is considered to be of importance not only to GE-GNF but to Electric Power Research Institute for use at reactor sites world-wide. Initial

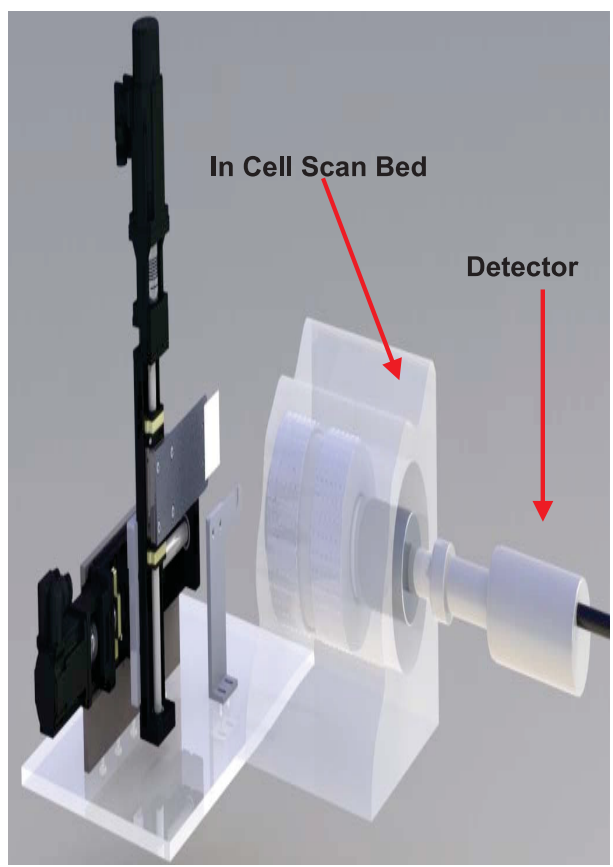

Figure 3. SRPA Hot Cell Scanner Conceptual Design.

work has begun with GE-GNF on integrating the physics measurement systems with current pool scanning systems in use at GE facilities.

\section{Benefits to DOE}

Implementation of the SRPA measurement system with GE-GNF through the INL CRADA process addresses a number of the current critical issues for the U.S. nuclear power industry and demonstrates a good government/commercial partnership and the utilization of "science-based" research to address real world problems. This project supports the INL mission of supporting the development of current and future reactor fuels optimized reactor systems. Further, it provides capabilities for the development of new reactor fuels and in the minimization of wastes by extending the life of fuels.

\section{Presentations}

Akers, D.W., "SRPA Hydride Research Overview (proprietary)," GE, November 2012.

Akers, D.W., "SRPA Hydride Research Overview (nonproprietary)," EPRI and PNNL, September 7, 2011.

Akers, D.W., et al., "SRPA Hydride Research and Results (proprietary)," GE Wilmington, August 13, 2011.

Akers, D.W., et al., "SRPA Hydride Research and Results (proprietary)," GE Vallecitos, July 26, 2011. 


\title{
11-061 - Advanced Modeling and Simulation Concepts for ATR
}

\author{
Richard Martineau, Ray Berry, Kord Smith, ${ }^{1}$ Barry Ganapol, ${ }^{2}$ and David Nigg
}

The overreaching goal of LDRD 11-061 is to develop the multiphysics modeling and simulation methodology necessary to predict the behavior of the highly heterogeneous and complex 3-D geometric configuration of INL's ATR. Accomplishing this goal will require the development and application of advanced, implicit numerical algorithms for a nonlinearly coupled power profile and multiphase conjugate heat transfer. There are three main development deliverables for this LDRD:

1. Development and implementation of the seven-equation, two-phase flow model into a MOOSE-based application called BIGHORN.

2. Development and implementation of a strongly coupled conjugate heat transfer algorithm into BIGHORN.

3. Development and implementation of a nonlinear diffusion acceleration approach to couple a power profile solution to the conjugate heat transfer into a MOOSE-based application called Rattle $S_{N}$ ake.

\section{Summary}

\section{Two-Phase Flow Research}

In order for hyperbolic flow equation systems like the compressible Euler equations or the seven-equation, twophase model to be numerically approximated to produce a stable solution able to capture discontinuities with a minimum of nonphysical oscillations, the numerical system must include an artificial viscosity. This artificial viscosity usually occurs in two forms: (1) "Explicit artificial viscosity," wherein viscous terms are added explicitly to the discretized equations with coefficients that can be set to control the artificial viscosity effects, and (2) "Implicit artificial viscosity," wherein the effects of artificial viscosity result from some artifact of the discretization itself, such as the truncation error of the discretization method or from the numerical definition of the flux functions. Most modern solution approaches are of the latter form and use methods to represent the flux terms in the flow equations that are variants of numerous approximate Riemann solvers. Because monotonic capture of physical features such as discontinuities cannot be achieved with methods higher than first order, accurate higher order methods must necessarily be nonlinear (hybrid) methods. These sophisticated methods produce a high order solution where the numerical solution is smooth and limits the solution locally by forcing it to revert to a first order solution in the vicinity of discontinuities or regions with a propensity to oscillate. Researchers have spent their careers developing such sophisticated methods with countless journal papers and books being published to document their approaches. These efforts continue unabated today, with continual improvements, because of the high financial value that results from more efficient and accurate solutions of flow systems ranging, for example, from aerospace to automotive to nuclear reactor coolant systems.

These modern nonlinear methods, which use "smart" and automatic adjustment of artificial viscosity, are expensive with regard to computing resources, costing perhaps twice as much or even more than the simpler method's "dumb" artificial viscosity. In addition, these modern methods can be very difficult to implement with more complex equation systems (such as the seven-equation, two-phase model used in Bighorn and RELAP-7)

1. Massachusetts Institute of Technology

2. University of Arizona 
and/or with other discretization schemes of multi-physics (like the continuous finite element method of the INL MOOSE framework). With this LDRD, we have revisited the potential of using these older, simpler artificial viscosities but with the prospect of making them just a little "smarter," just enough to produce acceptable solutions with a minimum of added complexity and expense. Artificial viscosities of this intermediate type include Lapidus velocity based, second-order pressure based, and third-order pressure based. We have also been developing another intermediate type of artificial viscosity model that has the potential of inexpensively offering solutions with fidelity rivaling those of modern high-order limiter methods-the entropy viscosity method. The entropy viscosity method is conceptually simple. Explicit artificial viscosities are added to the governing equation system along with another partial differential equation for the entropy. The artificial viscosity coefficients are automatically adjusted locally, based on the entropy production, which results in an optimally smoothed and stabilized solution. The entropy viscosity method has been developed and implemented for the 1-D Euler equations with variable area, with source terms, and with two equations of state (ideal gas and stiffened gas for compressible liquid) within the MOOSE framework using a continuous finite element discretization. Typical transient Riemann (shock tube) problems and steady state nozzle problems demonstrate the correct behavior of the method. Initial development and implementation for multi-dimensional Euler equations and for the seven-equation, two-phase model have been conducted with development continuing toward achievement of the same robustness.

The entropy viscosity method is demonstrated here for the Euler equations of compressible flow in 1-D for two important classes of problems: transient and steady state. For the transient class, several cases of classical shock tube (uniform area) problems were run and reported. Because of space limitations, only one case will be shown. For the steady state class, the nozzle with the variable crosssectional area was run for compressible water/vapor and reported. For every simulation, the continuous finite element method of MOOSE was used to discretize the conservation equations. For gas and compressible liquid, the stiffened gas equation of state was used. The simulations were integrated implicitly in time with the BDF2 formula, and the required preconditioner of the Jacobian Free/Newton Krylov method was selected to be the Jacobian matrix for all cases.

Toro's "shock tube test 2" begins with a tube and a length of 1.0 filled with an ideal gas and having an initial discontinuity located at $x_{0}=0.5$ with $u_{\text {left }}=-2, p_{\text {left }}=0.4, T_{\text {left }}=0.4$, and $u_{\text {right }}=2, p_{\text {right }}=0.4, T_{\text {right }}=0.4$. For times $t>0$, a low pressure (and low internal energy) occurs in the center, the extent of which grows with time to both the right and left as the waves spread in those directions. The solution at time $t=0.15$ is shown in Figure 1 along with the various viscosity coefficients. For this test, the solution profiles are smooth,

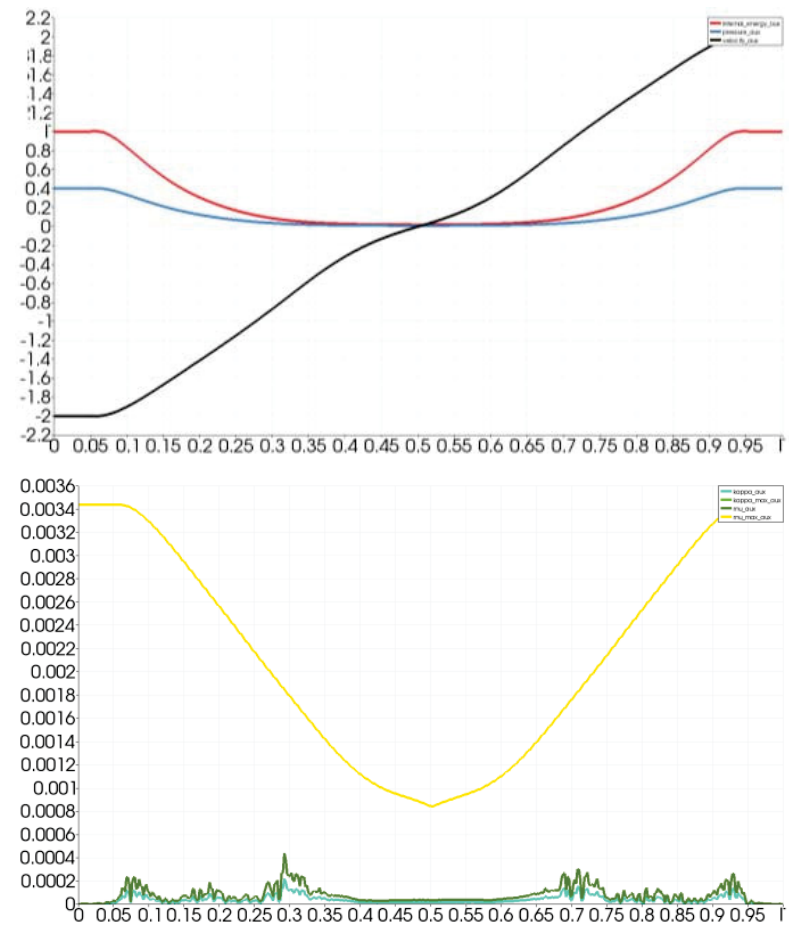

Figure 1. Top: Shock tube solution profiles at $t=0.15 s$ for pressure (red), internal energy (blue), and velocity (black). Bottom: viscosity coefficients $\mu$ (green), $\kappa$ (cyan), $\mu_{\max }$ and $\kappa_{\max }$ (yellow). 
and the second order viscosities are small compared to the first order ones. In comparison, several discrete schemes display a solution with an erroneous peaked region in the internal energy profile around $x=0.5$. This artifact is not present here with the entropy viscosity method.

The flow simulation of either compressible liquid water (low Mach) or vapor (medium Mach) through a convergingdiverging cosine area nozzle is next used to test the entropy viscosity method for steady state solutions. The stiffened gas equation of state is used for both phases with corresponding parameters given. A stagnation pressure boundary condition with $p_{0}=1.0 \mathrm{MPa}$ and $T_{0}=453 \mathrm{~K}$ is applied at the inlet and a static pressure boundary condition with $p_{b}=0.5 \mathrm{MPa}$ applied at the outlet. Velocity and temperature are initially set uniformly everywhere at 0 and $453 \mathrm{~K}$, respectively, and pressure is initialized with the steps $p_{\text {left }}=p_{0}$ and $p_{\text {right }}=p_{b}$. Steady state solutions are obtained for the vapor and liquid phases by time-marching the transient solutions until changes cease. The liquid phase was run with 50 elements, since the steady state solution is smooth and does not exhibit shocks or discontinuities that need spatial resolution, and a time step of 0.001 . The steady state solution for the liquid phase is shown in Figure 2 and appears as a smooth, symmetric profile in all variables, reminiscent of a venturi tube solution (with the exception of density, which is constant for the venturi solution). For the steady state vapor phase solution, the fluid transitions from subsonic to supersonic at the throat $(x=0.5)$ then shocks to subsonic flow in the divergent region at approximately $x=0.8$ and remains subsonic to the flow exit. This simulation was run with 400 elements to resolve the steady state shock, and the time step was correspondingly set to $10^{-4} \mathrm{~s}$. The steady state solutions are shown in Figure 3 . The second order viscosity coefficients $\mu$ and $\kappa$ are seen to only saturate (or be limited) to the first order viscosity coefficients $\mu_{\max }$ and $\kappa_{\max }$ in the shock region. Some instabilities can be noticed in the viscosity profiles only in the vicinity of the sonic point $(x=0.5)$ where the flow transitions from subsonic to supersonic, but they do not seem to affect the numerical solution of the physical variable profiles in velocity, pressure, and density. This small instability can be removed by effectuation of a smoothing of the viscosities over three cells.

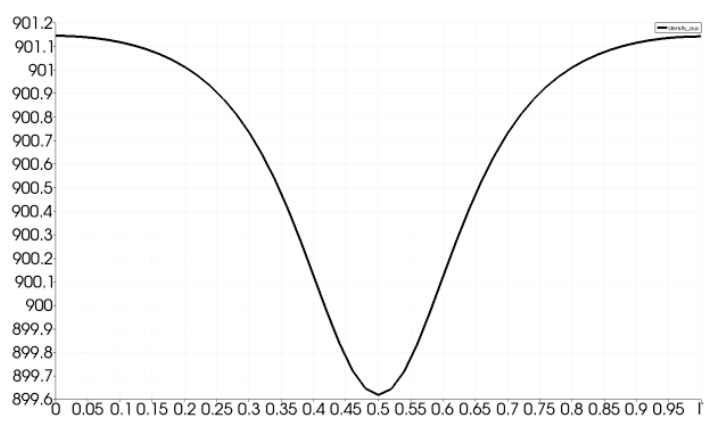

(a) Density profile

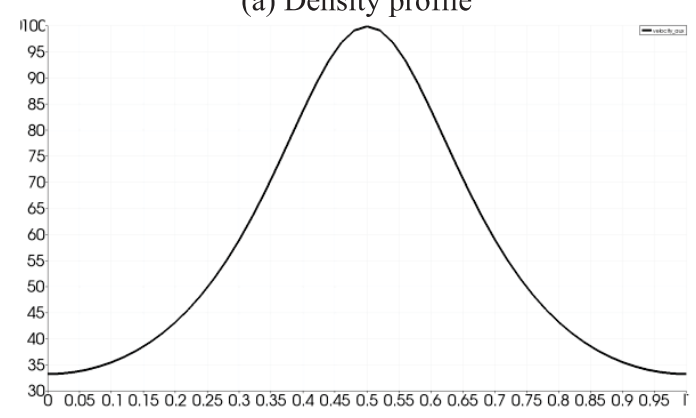

(c) Velocity profile

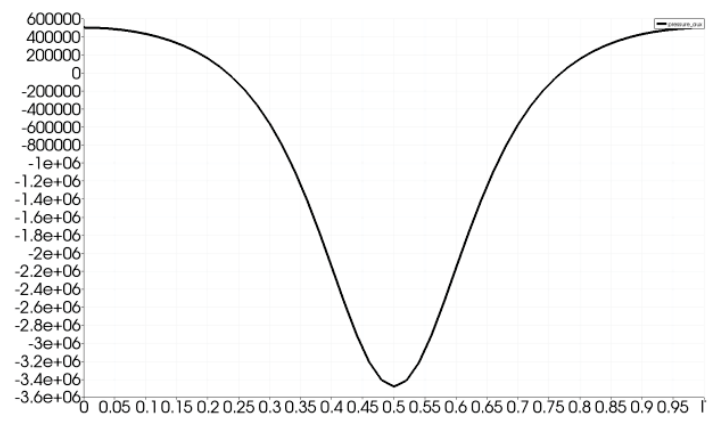

(b) Pressure profile

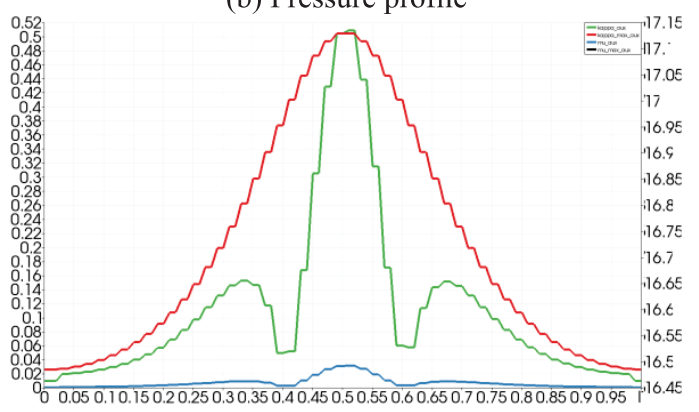

(d) Viscosity coefficients. Left axis is for $\boldsymbol{\mu}$ (blue) and $\boldsymbol{\kappa}$ (green), right axis for $\boldsymbol{\mu}_{\max }$ and $\boldsymbol{\kappa}_{\max }$ (red). 
Figure 2. Steady state results for liquid phase with 50 elements.

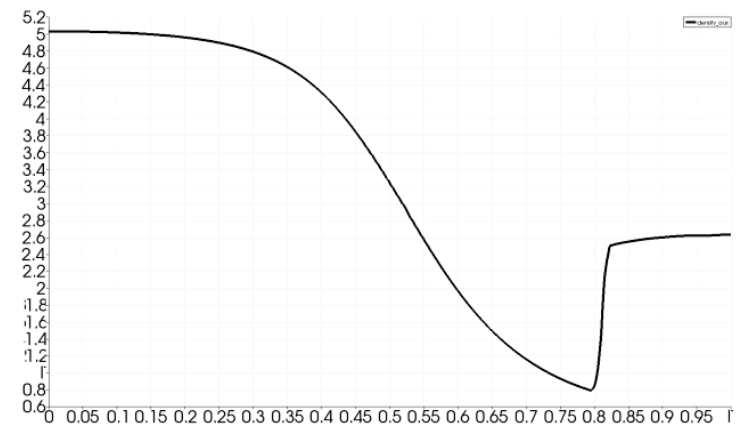

(a) Density profile

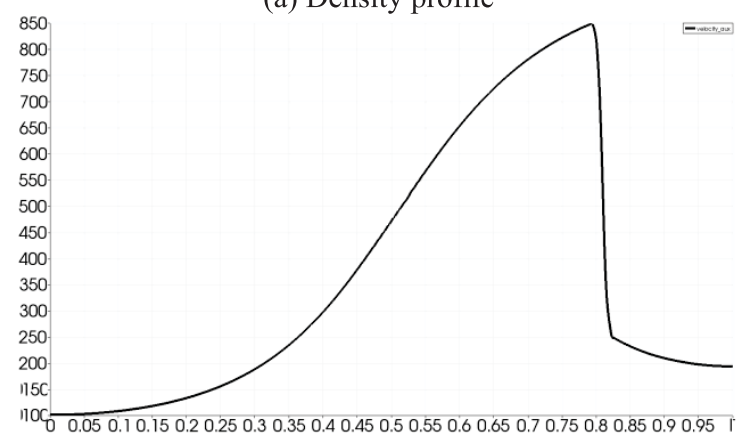

(c) Velocity profile

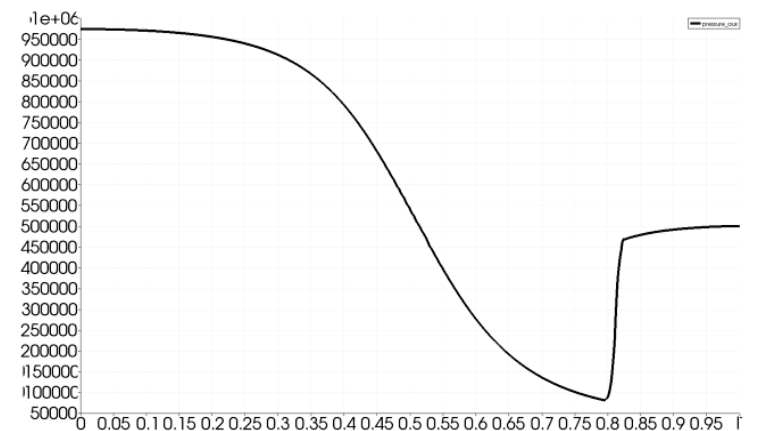

(b) Pressure profile

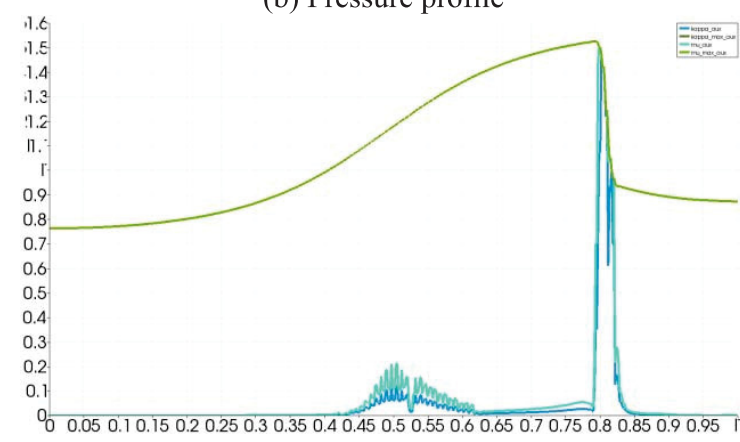

(d) First and second order viscosity coefficients

Figure 3. Steady state results for vapor phase with 400 elements.

\section{Necessary Improvements Added to the MOOSE Framework as a Result of the Research}

1. Discontinuous Galerkin methods (DG methods)

DG methods are numerical methods used for solving partial differential equations with a dominant first order part (e.g., fluid mechanics, electromagnetics, and plasma physics). They combine features of finite element and finite volume frameworks and have been successfully applied to hyperbolic, parabolic, and elliptic equations. Like the continuous Galerkin (CG) method, the DG method is a finite element method using a weak formulation of a particular model system. Unlike CG, the DG method works over a trial function space that is piecewise discontinuous.

\section{Mass/energy consistent boundary normal}

The need for global conservation of mass and energy in fluid flow problems is essential since it highly affects the quality of the solution. This principle is used in order to compute the outward normal in a unique manner, allowing it to apply essential boundary conditions consistently.

3. Explicit time integration schemes (of arbitrary order)

Originally, the MOOSE framework was designed to solve transient problems only using implicit time integration schemes like implicit Euler, BDF2, or Crank-Nicolson. The time integration subsystem within the framework was re-designed to allow users to plug in their own time integrators and, at the same time, was enhanced by adding two explicit time integrators: explicit Euler and two step Runge-Kutta 2 method. 
4. Mass lumped matrix

5. Portable, Extensible Toolkit for Scientific Computation options (CG), Left/Right preconditioning

Preconditioning is a very important technique in solving systems of linear equations in form $A x=b$ using an iterative solver like the generalized minimal residual method, CG, etc. The purpose of a preconditioner is to make solving of the system easier in such a way that $P^{-1} A$ has smaller condition number than the original matrix $A$.

Instead of solving the original system, one may solve the right preconditioned system (which was already available in the MOOSE framework):

$A P^{-1} P A x=b$

or, the left preconditioned system (which was made available for applications):

$P^{-1}(A x-b)=0$

6. 1-D-3-D mortar finite element method

The mortar finite element method is a discretization method for solving partial differential equations. It allows us to tie non-conforming meshes together in a mathematically elegant way. Typically it is implemented using Lagrange multipliers.

For demonstration purposes, we will show a solution to a simple diffusion problem with a known solution in order to verify that the method gives the correct answer. The problem being solved is:

Find $(u, \lambda) \in(X, M)$ such that

$\begin{array}{lr}a(u, v)+b(v, \lambda)=(f, v), & v \in X \\ b(u, \mu)=0, & \mu \in M\end{array}$

where $^{a(u, v)=\int_{\Omega} \nabla u \cdot \nabla v d \Omega}$ and $b(v, \mu)=\int_{\Gamma} v \cdot \mu d \Gamma$.

Boundary conditions are chosen as follows:

$u=g, \quad$ on $\Gamma$

Functions $f$ and $g$ are chosen in such a way that the exact solution is $u=y$ (for 2-D example) and $u=x+y+z$ (for 3-D example). The physical domain is $\Omega=(0,1)^{2}$ for the 2-D case and $\Omega=(-1,1)^{3}$ for the 3-D one. 
Because the 3-D case is harder to visualize (one has to see "inside" the solve domain), we made one subdomain transparent in order to see the non-conforming mesh of the interface. For the same reason, the mesh has been created with quite large elements, which has no effect on the solution since it is only first order and lies in the discretization space. For the verification, we computed the discretization error in L2 norm, which yielded $1.621932 \times 10-15$ for the $2-D$ solution (Figure 4 ) and $6.317485 \times 10-16$ for the $3-D$ case

(Figure 5).

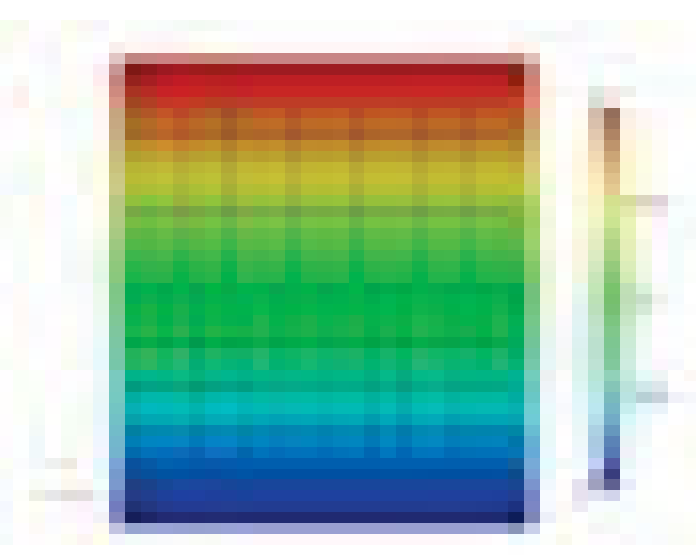

Figure 4. 2-D example.

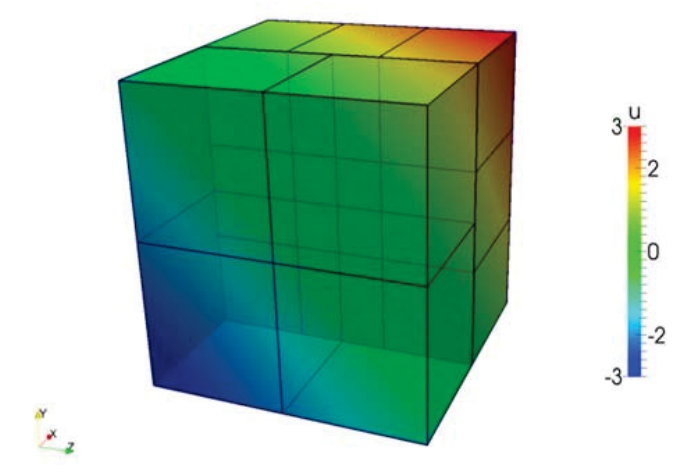

Figure 5. 3-D example.

7. Artificial viscosities

Second-order schemes for high-speed compressible flow problems lead to unavoidable high frequency oscillations in the vicinity of shocks. To overcome this deficiency, many shock capturing schemes were introduced in the literature. The BIGHORN code now has three methods available:

- Lapidus (velocity based artificial dissipation)

- Second-order pressure based artificial dissipation

- Third-order pressure based artificial dissipation.

\section{Benefits to DOE}

This LDRD is part of a longer-term goal of using ATR as a platform for verification and validation of advanced modeling and simulation and as tool for light water reactor performance enhancement. It will build upon the Phase 1 upgrade of ATR modeling and simulation to current state-of-the-art computational methods which use ATR as a test bed for applications and demonstrations of science-based methods for performance and safety analysis, which include but are not limited to: (1) Complex heterogeneous geometries, (2) the use of flexible geometric configuration for nuclear heated core multiphase flow experiments as well as core physics benchmarks, and (3) extension of the validation data base of Phase 1. 


\section{Presentations}

Berry, R.A., "A Logical Progression of Steps for Implementation and Testing of the 7-Equation, Two-Phase Model into a Computational Framework," International Conference on Math, and Computational Methods Applied to Nuclear Science \& Engineering, Sun Valley, LaGrange, IL, May 5-9, 2013.

Delchini, M.O., J.C. Ragusa, and R.A. Berry, "Entropy Viscosity Method Applied to Euler Equations," International Conference on Math, and Computational Methods Applied to Nuclear Science \& Engineering, Sun Valley, LaGrange, IL, May 5-9,2013.

Berry, R.A., "Modeling Challenges for Multi-scaled, Two-Phase Flow (in Light Water Nuclear Reactors)," Pacific Northwest Numerical Analysis Conference, Boise, ID, October 27, 2012, invited lecture. 


\section{2-031 - 2-D and 3-D EBSD Technique Development and Microstructure Reconstruction for Phase Field Microstructure Evolution Models}

Melissa Teague, Michael Tonks, Stephen Sintay, ${ }^{1}$ and Brian Gorman ${ }^{2}$

Electron Back-Scatter Diffraction (EBSD) scans provide a detailed description of a microstructure that can be reconstructed to define initial microstructures for mesoscale models. While reconstruction methods exist for collecting EBSD scans from non-radiological samples, method development is needed to perform these types of experiments on radiological samples. Additionally, no current method exists for utilizing these reconstructed 2-D and 3-D microstructures in phase field models. This project aims to develop a method to reconstruct realistic microstructures from EBSD scans of un-irradiated and irradiated $\mathrm{UO}_{2}$ and Zircaloy cladding for use in INL's finite, element-based phase field code MARMOT. This includes developing experimental techniques for collecting 2-D and 3-D EBSD data from radiological samples and the methodology to reconstruct and import this data directly into mesoscale phase-field models.

\section{Summary}

The focused ion beam, along with its attached EBSD detector, was used to characterize 3-D microstructure and chemistry of irradiated oxide fuel. Both the ability to serial section and perform EBSD on irradiated fuel are unique, first-of-its-kind research performed at INL; several journal articles have been generated, and the international community is displaying great interest in the work. Having the ability to study the crystal structure, chemistry, and microstructure of irradiated fuel in 3-D is critical to understanding the fundamentals of fuel performance.

In addition, the framework was developed to reconstruct 2-D structural data obtained from these experiments for use in INL's mesoscale code MARMOT. Preliminary models of fission gas evolution were developed using the reconstructed microstructure (see Figure 1).
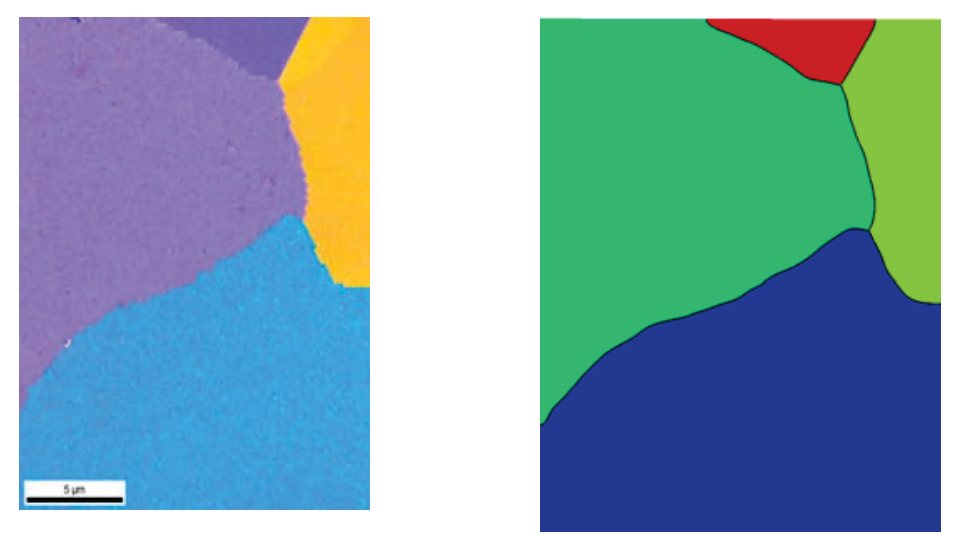

Figure 1. Microstructure reconstruction in MARMOT of a high burnup MOX fuel, where the EBSD scan is shown on the left and the reconstruction is shown on the right.

1. Los Alamos National Laboratory

2. Colorado School of Mines 
Additionally, a Ph.D. student has been hired through this project that brings a strong background in microstructure reconstruction and EBSD experimental practice to the laboratory. The LDRD will support the research for the student's Ph.D. through Washington State University. His research will develop methods for 3-D reconstruction of microstructures in MARMOT. He will also develop methods to create statistically similar microstructures. On the experimental side, he will work with the PIs to develop improved sample preparation methods for use with the EBSD detector.

\section{Benefits to DOE}

DOE benefits from the development of the unique, industry leading capability to analyze radiological materials utilizing 2- and 3-D EBSD as well as the ability to utilize this experimental information in phase field models, both of which are accomplished through this project. The capability to examine radiological samples with advanced characterization techniques such as EBSD is critical to the understanding of current fuel performance and behavior in spent nuclear fuel storage. The ability to reconstruct the experimental data and utilize it in phase field modeling efforts provides critical synergy between experimental efforts and lower length-scale modeling.

\section{Publications}

Teague, M., B. Gorman, and B. Miller, "EBSD and TEM Characterization of High Burn-up Mixed Oxide Fuel," J. Nucl. Mater., Accepted.

Teague, M., et al., "Microstructural Modeling of Thermal Conductivity of High Burn-up Mixed Oxide Fuel," J. Nucl. Mater., In press.

Teague, M. and B. Gorman, "Utilization of Dual-Column Focused Ion Beam and Scanning Electron Microscope for Three Dimensional Characterization of High Burn-Up Mixed Oxide Fuel," Prog. Nucl. Energ., September 12, 2013, In press, Available online.

Teague, M., M. Tonks, and S. Novascone, "Microstructural Characterization and Modeling of High Burn-up Mixed Oxide Fuel," 2013 LWR Fuel Performance Meeting/TopFuel, Charlotte, NC, September 15-19, 2013.

\section{Presentations}

Teague, M., et al., "Microstructural Characterization and Modeling of High Burn-up Mixed Oxide Fuel," 2013 LWR Fuel Performance Meeting/TopFuel, Charlotte, NC, September 15-19, 2013.

Fromm, B., et al., "Microstructure Reconstruction for Phase Field Modeling of Reactor Materials," $12^{\text {th }}$ U.S. National Congress on Computational Mechanics, Raleigh, NC, July 22-25, 2013.

Teague, M. and B. Gorman, "Utilization of Focused Ion Beam for 3D Characterization of High Burn-up Mixed Oxide Fuel," EMR-S 2013 Spring Meeting, Strasbourg, France, May 27-31, 2013.

Teague, M., "Characterization and Microstructural Modeling of High Burn-up Oxide Fuel from the Fast Flux Test Facility," Fuel Cycle Technologies Annual Review Meeting, Chicago, IL, October 30-31, 2012. 
Tonks, M.R., et al., "Multiscale Fuel Performance Modeling: Coupling Atomistic, Meso and Continuum Scale Simulations," 2012 ANS Annual Meeting, Chicago, IL, June 24-28, 2012.

Teague, M., et al., "Microstructural and Chemical Characterization of High Burn-up Mixed Oxide Fuel," TMS 2012, Orlando, FL, March 11-15, 2012. 


\title{
12-045 - Development of an Uncertainty Quantification Method for MIR Facility
}

\author{
Piyush Sabharwall, Carl Stoots, Barton Smith, ${ }^{1}$ and Pavlos Vlachos ${ }^{2}$
}

Currently, computational fluid dynamics (CFD) is widely used in the nuclear thermal-hydraulics field for design and safety analyses. However, validation of this method is still an unresolved issue. In order to validate CFD codes, high-quality, multi-dimensional flow field data is essential. The Matched-Index-of-Refraction (MIR) Flow Facility at INL has a unique capability to contribute to the development of validated CFD codes through the use of Particle Image Velocimetry (PIV). The significance of the MIR facility is that it permits non-intrusive velocity measurement techniques, such as PIV, through complex models without requiring probes and other instrumentation that disturb the flow. The MIR was modeled after a typical wind tunnel and was fabricated with a stainless steel frame. The facility consists of several components, including a settling chamber, square contraction, and test section. In the MIR facility, uncertainty quantification is a challenging task due to the use of optical measurement techniques. This study will develop a reliable method and computer code to analyze uncertainty and sensitivity of the measured data.

The main objective of this study is to develop a well-established uncertainty quantification method for the MIR Flow Facility, which consists of many complicated uncertainty factors. In this study, the uncertainty sources will be resolved in depth by categorizing them into uncertainties from the MIR flow loop and PIV system (including particle motion, image distortion, and data processing). Then, each uncertainty source will be mathematically modeled and adequately defined. Finally, this study will provide a method and procedure to quantify the experimental uncertainty in the MIR Flow Facility with sample test results. The uncertainty quantification method developed in this study will lead to high-quality MIR data with accurate and reliable uncertainty quantification, and it will be a tremendous asset to the ongoing efforts to validate advanced computational methods.

\section{Summary}

The MIR facility has tremendous potential as a user facility for basic and applied research by government, industry, and academia where accurate and reliable uncertainty quantification is essential for producing high-quality data for computer code validation. Despite extensive research in this area, there is no accepted methodology for quantifying the uncertainty associated with individual vector evaluations. The PIV measurement uncertainty is affected by the numerous elements present in the instrumentation chain, and, in the vast majority of the cases, those uncertainties are interconnected and coupled.

The main objective was to develop and test uncertainty estimation techniques based on the uncertainty surface method, shown in Figure 1, and correlation plane method, shown in Figure 2, with the help of Utah State University and Virginia Tech University, which are both team members of this project. These methodologies have been applied to synthetic data sets with excellent results. Currently, both methodologies are being compared with each other for a given experimental data set. Prior to this work, such uncertainty quantification was not possible. INL showed early leadership in the field of PIV uncertainty by funding the first PIV uncertainty workshop,

\footnotetext{
1. Utah State University

2. Virginia Tech University
} 
which was organized by the PIs. The LDRD work described here capitalizes on that start and places INL at the forefront of PIV uncertainty research.

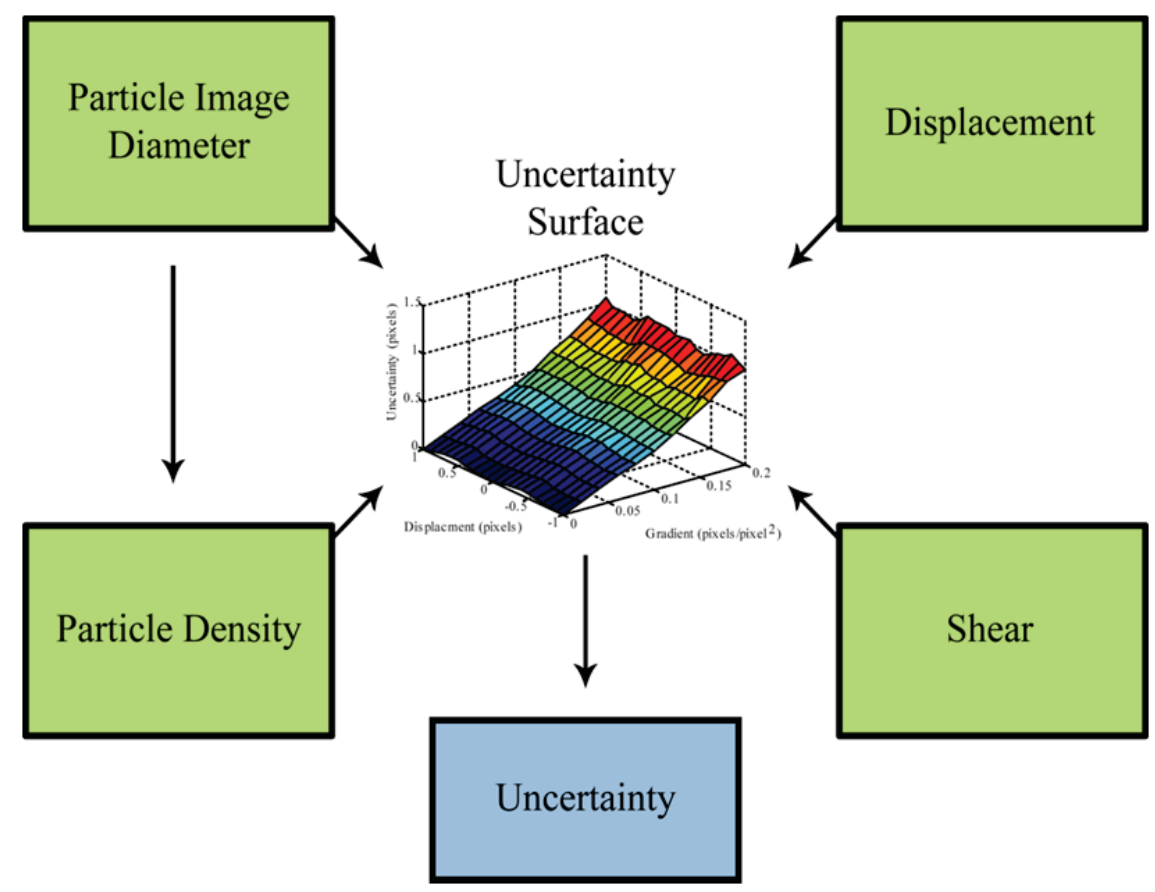

Figure 1. Uncertainty surface being developed by contribution from four different uncertainty sources (particle image diameter, particle density, displacement, and shear).

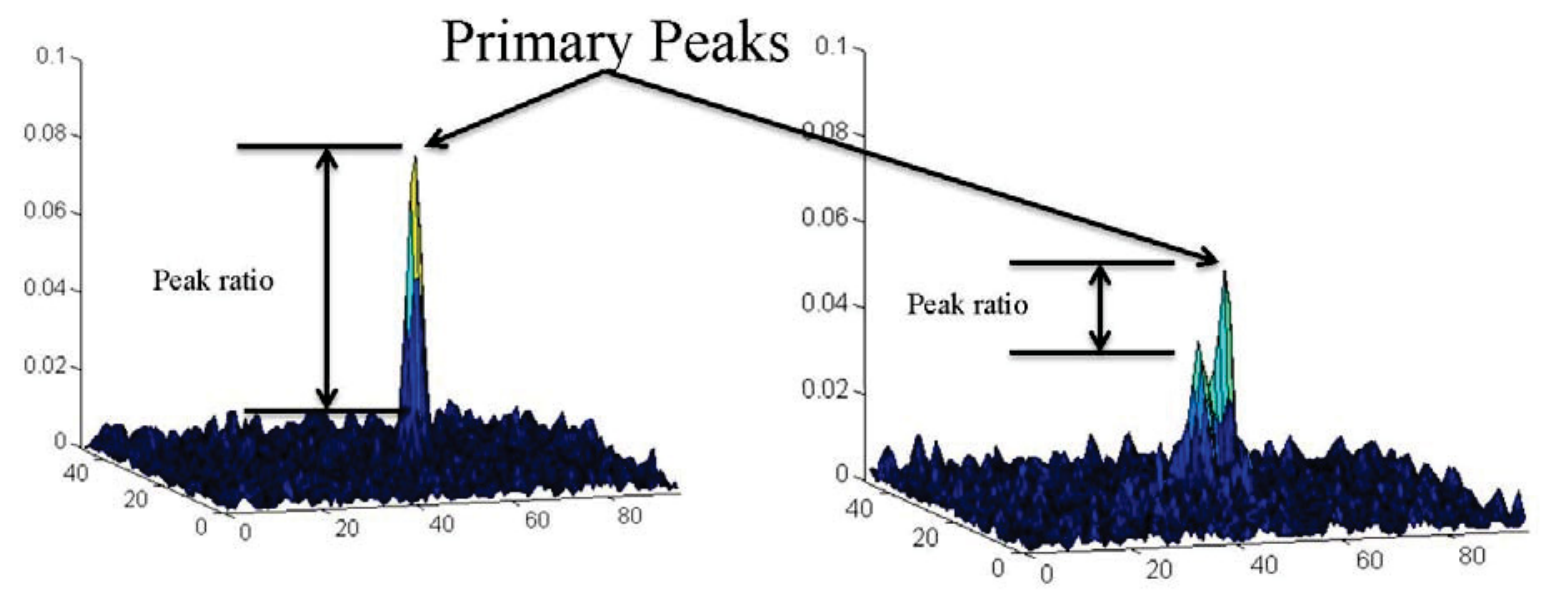

Figure 2. Estimation of uncertainty bounds for individual PIV measurements from the correlation plane. 


\section{Benefits to DOE}

This work will contribute to the DOE mission by working to quantify PIV uncertainty, which can be used to ensure high-quality data for the validation of CFD codes used for nuclear reactor design and safety analysis. Additionally, this work will directly contribute to the accomplishment of DOE goals by helping to develop the CFD tools necessary for Nuclear Energy programs and by helping to develop and characterize PIV uncertainty estimates used to validate advanced computer codes. Better quantitative understanding of the data will lead to better understanding of the physical phenomena and more reliable design for advanced reactors, which in turn will help address energy needs.

\section{Publications}

Sabharwall, P., et al., "Matched-Index-of Refraction Flow Facility at Idaho National Laboratory," Global Gas Turbine News, ASME Gas Turbine Division, January 2014, Ongoing.

Warner, S.O. and B.L. Smith, "Autocorrelation-Based Estimate of Particle Image Density for Diffraction Limited Particles," Meas. Sci. Tech., August 2013, Submitted.

Charonko, J.J. and P.P. Vlachos, "Estimation of Uncertainty Bounds for Individual Particle Image Velocimetry Measurements from Cross-Correlation Peak Ratio," Meas. Sci. Technol., Vol. 24, doi:10.1088/09570233/24/6/065301, June 2013.

Wilson, B.M. and B.L. Smith, "Uncertainty on PIV Mean and Fluctuating Velocity Due to Bias and Random Errors," Meas. Sci. Tech., Vol. 24, doi:10.1088/0957-0233/24/3/035302, March 2013.

Wilson, B.M. and B.L. Smith, "Taylor-Series and Monte-Carlo-Method Uncertainty Estimation of the Width of a Probability Distribution Based on Varying Bias and Random Errors," Meas. Sci. Tech., Vol. 24, doi:10.1088/0957-0233/24/3/035301, March 2013.

Charonko, J.J. and P.P. Vlachos, "Estimation of Uncertainty Bounds from Cross Correlation Peak Ratio for Individual PIV Measurements," Proceedings of 2012 ASME FEDSM, Puerto Rico, July 8-12, 2012.

\section{Presentations}

Sabharwall, P., "Development of an Uncertainty Quantification Method for MIR Facility-Status Report," INL Peer Review Meeting, Idaho Falls, ID, July 23, 2013.

Smith, B.L., "PIV Uncertainty," Seminar at University of New Mexico Mechanical Engineering, Albuquerque, NM, October 2, 2012.

Harris, J., B.M. Wilson, and B.L. Smith, "Investigation of Relative Importance of Some Error Sources in Particle Image Velocimetry," ASME Fluids Engineering Conference, Puerto Rico, July 8-12, 2012.

Sabharwall, P., "Development of an Uncertainty Quantification Method for MIR Facility," INL Peer Review Meeting, Idaho Falls, ID, June 20, 2012. 
Wilson, B.M. and B.L. Smith, "Propagation of Instantaneously Varying Systematic and Random Uncertainties into the Measurement Mean, Variance, and Covariance," Bulletin of the American Physical Society APS March Meeting 2012, Boston, MA, February 27-March 2, 2012.

Smith, B.L., S. Beresh, and P.P. Vlachos, "PIV Uncertainty Roadmap," Bulletin of the American Physical Society APS March Meeting 2012, Boston, MA, February 27-March 2, 2012.

Warner, S., B.L. Smith, and P.P. Vlachos, "Autocorrelation Based Estimate of Particle Image Density in PIV," Bulletin of the American Physical Society APS March Meeting 2012, Boston, MA, February 27-March 2, 2012.

Jones, K. and B.L. Smith, "Method to Determine the Minimum Random Uncertainty in PIV-Based on Real Images," Bulletin of the American Physical Society APS March Meeting 2012, Boston, MA, February 27March 2, 2012. 


\title{
12-061 - Development of a High Burnup Zr-Based Fuel Cladding for LWRs
}

\author{
Robert Mariani, James Cole, and Randall Fielding
}

A new oxide dispersion-strengthened (ODS) zirconium (Zr)-based alloy is sought for improved safety, reliability, and burnup in light water reactor applications. However, ODS alloys have high prices that arise from traditional mechanical alloying that involves energy-intensive, time-consuming, and labor-intensive processes. To be cost-effective, an ODS Zr-based cladding alloy would therefore require new materials and methods that eliminate traditional powder metallurgical methods. Novel ODS agents were therefore conceived that could be used in a way that eliminates traditional powder metallurgy processes, such as mechanical alloying, and permit large batch and/or continuous castings.

Proposed material additions to the $\mathrm{Zr}$ alloy are based on thermodynamics, experimental data, a simple transport model, and prior modeling experience with other ODS-type materials. Niobium ( $\mathrm{Nb}$ ) (tantalum $[\mathrm{Ta}]$ or vanadium [V]) is added for its property in which the hydrogen permeability increases with decreasing temperatures, thereby avoiding or mitigating hydride precipitation. A second additive such as molybdenum (Mo) may be used, but may not be needed, at a level to induce phase-segregation of $\mathrm{Nb}$ from the bulk $\mathrm{Zr}$ and induce $\mathrm{Nb}$ precipitation on grain boundaries (since $\mathrm{Nb}$ is soluble in $\mathrm{Zr}$ ), which helps prevent increasing hydride levels with thermal cycling. A third additive, such as $\mathrm{Nd}_{2} \mathrm{O}_{3}$, is used to control grain-size and inhibit microstructural changes to the cladding that can result from irradiation at elevated temperature and thermal cycling with outages. The third additive can also facilitate rapid formation of the more mechanically stable cubic $\mathrm{ZrO}_{2}$ corrosion layer on the cladding, as compared to its other phases.

The initial focus of the research effort is development of the nano-scale ODS agents for alloy fabrication. A contract with a small business was established to produce, for the first time ever, nano-spheres of Mo with a nano-scale coating of neodymia $\left(\mathrm{Nd}_{2} \mathrm{O}_{3}\right)$. If successful, this technology would improve performance and reliability of the current fleet of nuclear power plants, with potential for increased burnup, uprates, and safety margin for wet and dry storage. Applications of this research are much broader than Zr-based alloys and include iron (Fe)-based alloys for extreme service involving high temperatures and corrosive environments that require resistance to thermal cycling fatigue, creep deformation, and surface wear. As a result, successful development and deployment hold high potential for significant advances in science and technology and pose significant commercial value for ODS alloys.

\section{Summary}

Under a subcontract, Nanomaterials Company (Malvern, PA) produced nanoscopic Mo powder for the first time. The primary particle size (5-30 nm) was determined using line-broadening x-ray diffraction and using laser light scattering. Fair agreement was found for the two methods considering laser light scattering and will indicate agglomerated primary particles ( $20 \mathrm{~nm}$ median size) while the $\mathrm{x}$-ray diffraction $(10 \mathrm{~nm})$ may be a better indicator of the primary particle size even with necking between particles (initial agglomeration). Samples submitted to INL were examined using a transmission electron microscope, and their size characteristics were consistent with sample aging (mild agglomeration) of the as-produced powder that was characterized by line broadening $\mathrm{x}$-ray diffraction (Figure 1). 
Nanomaterials Company found some clogging of their proprietary process machine; only a small amount of nanoscopic Mo was produced. Their machine was modified during FY 2013, and Mo particles coated with $\mathrm{Nd}_{2} \mathrm{O}_{3}$ are expected to be delivered to INL in early FY 2014. Their characteristics will then be examined under transmission electron microscope examination.
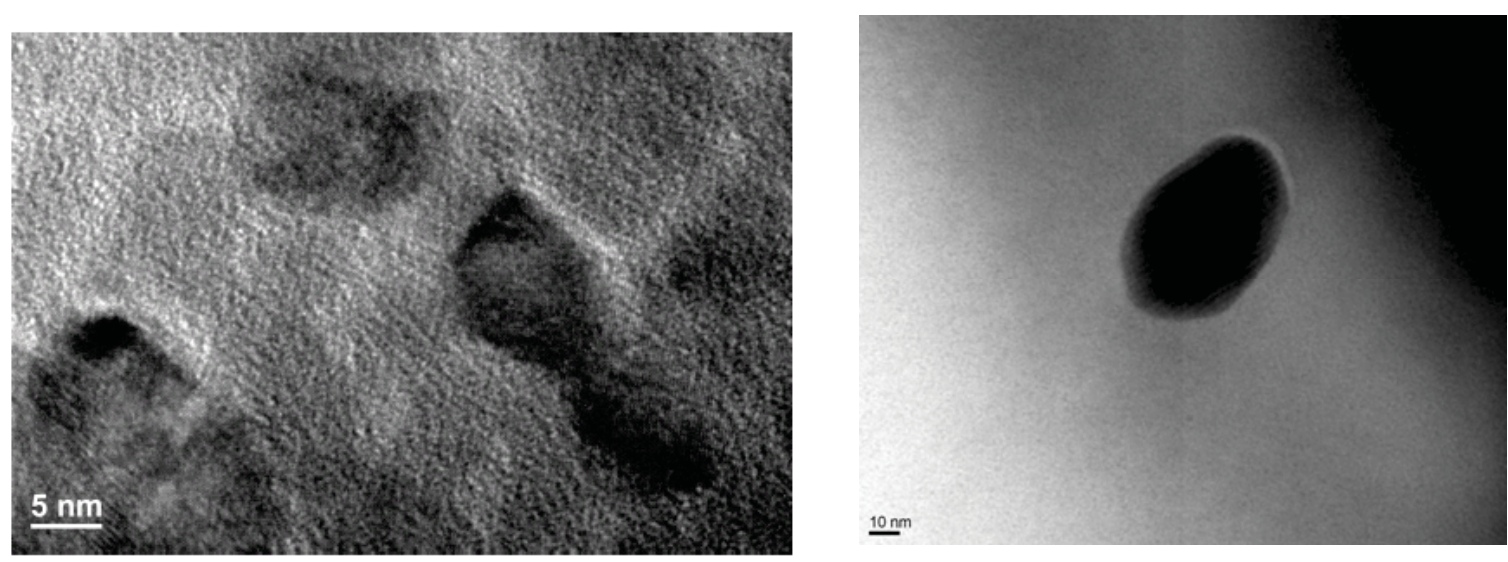

Figure 1. Transmission electron microscope images of Mo nanoparticles. Left: nanoparticles mounted in epoxy, aged approximately six weeks under toluene. Right: nanoparticles mounted in tin (Sn), aged approximately three months.

\section{Benefits to DOE}

This research will deliver the materials and process necessary to prepare Zr-based alloys resistant to creep and hydriding in light water reactor applications, offering higher burnups, uprates, and improved safety margins for wet and dry storage for DOE. The research will deliver new fabrication methods for the ODS alloy that eliminate timeconsuming, labor- and energy-intensive traditional methods such as mechanical alloying. A new conceptual model for hydriding has already been published that can be leveraged for research on in-reactor and storage performance improvements. Though initially focused on Zr-based cladding applications and improvements, broad application of this technology would overcome a 100-year old science and technology barrier, bring significant energy savings in the production of ODS alloys, and pose huge commercial value for steels. Favorable peerreview comments indicated the research is truly out-of-the-box thinking.

\section{Publications}

Mariani, R.D., J.I. Cole, and A. Aitkaliyeva, "A Novel Zr-1Nb Alloy and a New Look at Hydriding," Proceedings of the LWR Fuel Performance Meeting, TopFuel 2013, American Nuclear Society, Charlotte, NC, September 15-19, 2013 (peer-reviewed paper and presentation).

Mariani, R.D., "Zirconium-Based Alloys, Nuclear Fuel Rods and Nuclear Reactors Including Such Alloys, and Related Methods," U.S. Patent Application 13/021,480, February 4, 2011. 


\section{2-062 - Development of Diamond Based Sensors for User Facility Capabilities at INL Advanced Test Reactor}

David Chichester, James Johnson, and Scott Watson

This project is evaluating the performance of chemical vapor deposited (CVD) diamond-film semiconductor radiation detectors for use as multienergy neutron detectors and assessing their potential use as in-core neutron flux monitors to support ATR-NSUF research activities. CVDdiamond detectors operate like other semiconductor radiation detectors, but they have rather unique physical and electrical properties ideally suited for use in harsh environments. A schematic layout of a typical diamond semiconductor detector is shown in Figure 1. lonizing radiation creates electron-hole pairs in the diamond as it traverses the material (13-ev per electron-hole pair); an applied high-

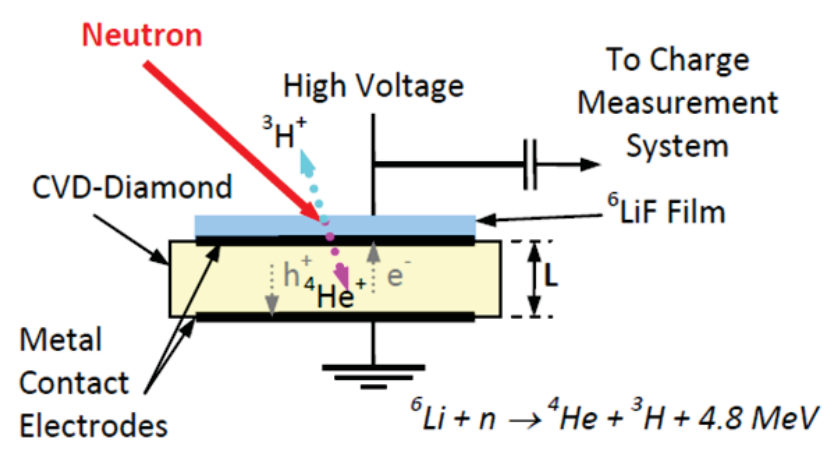

Figure 1. Schematic layout of a standard metalinsulator-metal diamond semiconductor with a ${ }^{6} \mathrm{LiF}$ coating designed for thermal neutron detection. voltage creates an electric field within the crystal which sweeps the charge signals to the electrodes, resulting in a current pulse measurable in an external measurement circuit. The thickness $(L)$ of the diamond layer ranges from as thick as $0.5 \mathrm{~mm}$ to thinner than $0.05 \mathrm{~mm}$; thinner layers are possible. Applied voltages of 500 to $1000 \mathrm{~V}$ are typical. Fast neutron detection can be made using these detectors directly, as a result of neutron capture in carbon. For thermal neutron detection, a conversion material must be used to generate an ionizing particle; conversion film examples include ${ }^{6} \mathrm{LiF}$, polyethylene, and uranium (U)-235.

\section{Summary}

Work for this project started in FY 2012 with the purchase of two CVD-diamond detectors from a commercial vendor. Upon receiving the detectors, we began evaluating their performance, using on-hand electronic data acquisition equipment and radiation sources available at INL's North Yellowstone Complex Laboratory, INL's Active Neutron Interrogation Laboratory, and the Massachusetts Institute of Technology Nuclear Research Reactor (MITR) in Cambridge, Massachusetts. We measured the detector's fast-fission spectrum (a bare californium [Cf]-252 source) intrinsic detection efficiency to be approximately $2-5 \times 10^{-14}$. For a near-thermal neutron spectrum (the MITR thermal beam port) the intrinsic detection efficiency for the ${ }^{6} \mathrm{LiF}$ detector was estimated to be approximately $(2.6 \pm 0.4) \times 10^{-4}$. Also, this signal is separable from the CVD-diamond detector fast-neutron response, indicating that the detectors may be able to provide multi-energy spectral information when used in high-flux environments.

In FY 2013, work for this project focused on evaluating the performance of CVD-diamond detectors as a function of temperature and on the development of a high-speed, high-throughput data acquisition system. Also in 2013 , INL began collaborative work with the Italian National Agency for New Technologies, Energy, and Sustainable Economic Development (ENEA) and Rome "Tor Vergata" University, for continuing research in this area. In relation to this, ENEA sent two additional CVD-diamond neutron detectors to INL for study. Photographs of one setup for these temperature effects test are shown in Figure 2 with a plot of the linear relative gain performance of 
the ENEA detector versus temperature. For FY 2014, a second experiment campaign is being planned for execution at MITR (an ATR-NSUF partner facility). This campaign will use one of the MITR ex-core beam ports, where thermal neutron flux levels are on the order of $10^{12} \mathrm{n} \mathrm{cm}^{-2} \mathrm{~s}^{-1}$ to $10^{13} \mathrm{n} \mathrm{cm}^{-2} \mathrm{~s}^{-1}$. Tests will be performed to study CVD-diamond detector performance at high counting rates and at elevated temperatures.
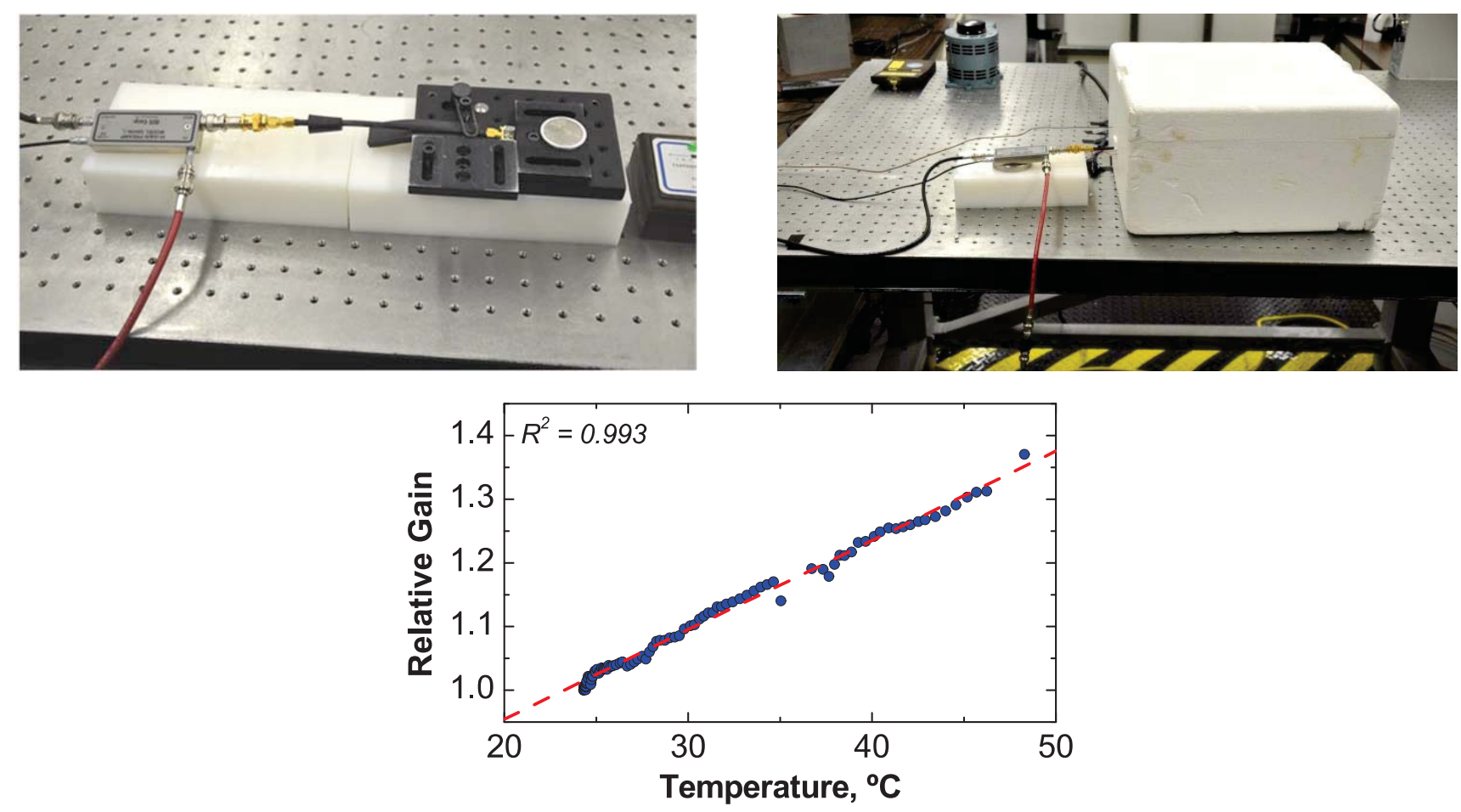

Figure 2. (Upper-left photograph) The ENEA, Rome "Tor Vergata" University, CVD-diamond detector mounted on the thermal test stand without the insulating box; (upper-right photograph) shows the same scene with the insulating box. The figure at the bottom shows the detector's relative gain, using a fixed alpha-emitting check source, as a function of the CVD-diamond detector temperature.

\section{Benefits to DOE}

Advanced thermal- and fast-neutron detection methods are directly relevant to the DOE national security mission in support of future nuclear energy systems, as described in this report. More generally, these detectors are ideally suited for use in extreme environments and, as such, may prove useful for stockpile stewardship, nuclear nonproliferation, nuclear forensics, and used fuel monitoring.

\section{Presentations}

Robinson, M., "Investigation of CVD-Diamond Detectors for Neutron Radiation Monitoring," DOE NNSA Stewardship Science Graduate Fellowship Annual Program Review, Santa Fe, NM, June 25-27, 2013. 


\title{
12-070 - Separate Effects Fuels Development Capability
}

\author{
J. Keith Jewell, Tony Hill, and Eric Burgett ${ }^{1}$
}

Separate effects testing, including growth, irradiation, and monitoring of these materials, encompasses the full science-based approach for fuels development, from the nanoscale to the mesoscale behavior of the sample material and other defects driven by the modeling and simulation efforts of INL. The goal is to develop the capability to deliver an exponential increase in the available data on the time evolution of microstructural changes in fuel samples specifically engineered for isolated effects study. This data can be used in the iterative development of science-based modeling and simulation that is accurately validated and benchmarked against experimental data. The Separate Effects Fuels Development Capability LDRD supports the development of a transformational separate effects testing program to perform in-situ irradiation studies and characterization of the nanoscale to mesoscale behavior of nuclear fuel materials under a wide variety of in-pile conditions.

Following the successful FY 2012 deliverable of an engineering design report that presented the modeling and simulation effort used to evaluate the feasibility and practicality of an accelerator-driven, fuel-boosted neutron irradiation facility, focus in FY 2013 turned to the other two necessary components of a complete separate effects testing capability: advanced detection and sample fabrication.

A major focus of the FY 2013 effort was in advancing detection and imaging necessary for collecting the microstructure data in prototypic reactor environments. Precision high-fidelity data at the microstructure scale is necessary for developing the phase-field modeling that describes fuel evolution during irradiation and heating cycles. However, collecting high-resolution data in extreme reactor environments presents difficulties for obvious reasons. In an effort to simulate the high neutron, gamma, alpha, and beta fields seen in a reactor, specially made samples of californium (Cf)-252 were produced on scanning electron microscope (SEM) sample holders. The deposition density of the Cf-252 was controlled such that the fission yield within the SEM field of view was comparable to typical reactor fission rates.

The second focus of the FY 2013 effort was in fabrication of microstructure test samples specifically engineered for examining the microstructure evolution. Typical fuel sample fabrication relies on bulk processes, such as hot isostatic press and spark plasma sintering techniques designed to produce fuel pin sized quantities. The amorphous, heterogeneous composition of "reactor-sized" quantities makes it impossible to study the underlying microstructure that drives phenomena such as defect migration, fission gas mobility, crack propagation, swelling, creep, and fuel-clad interactions. Instead, the project has developed a metalorganic chemical vapor deposition system to grow epitaxial crystalline materials in any arbitrary crystalline orientation with extreme control over the thickness and orientation of the layers. In addition, the metalorganic chemical vapor deposition system allows for fine control of minor components for doping studies. This allows for interfacial/surface layers or uniform, intralayer distributions of isotopes of interest to be incorporated into the grown crystalline or amorphous layers. Bulk single crystal growth capabilities are being developed as well. These capabilities will allow for the creation of large single crystals, bi-crystals, tricrystals, and the basis for materials with fixed void fractions, porosity, or alloyed materials. These capabilities do not exist nation-wide currently. These crystals can be cut and polished for diffusion, thermal conductivity, and grain restructuring studies.

1. Idaho State University 


\section{Summary}

The LDRD achieved significant progress in utilizing SEM technology for imaging microstructure phenomena in an irradiation environment. Prior to this investigation, it was unknown what impact the fission rate per unit area for a nominal fuel during irradiation would have on the signal to noise ratio of the SEM. The impact of activation of the exposed SEM parts was also unknown. To address the first question, a special Cf-252 sample was electroplated directly to an aluminum (Al) stem with enough Cf-252 per unit area to mimic the areal fission rate of a fuel under nominal reactor irradiation. Figure 1 is a mosaic of the results. There does not seem to be any impact on the SEM resolution due to the fission fragments. However, further testing is going to be carried out, which includes adding layers of gold over the deposit to provide a large sea of electrons between the fissions and the SEM sensors and to mill standard image acuity markers in the gold layer using a focused ion beam to accurately determine the resolution at each step.

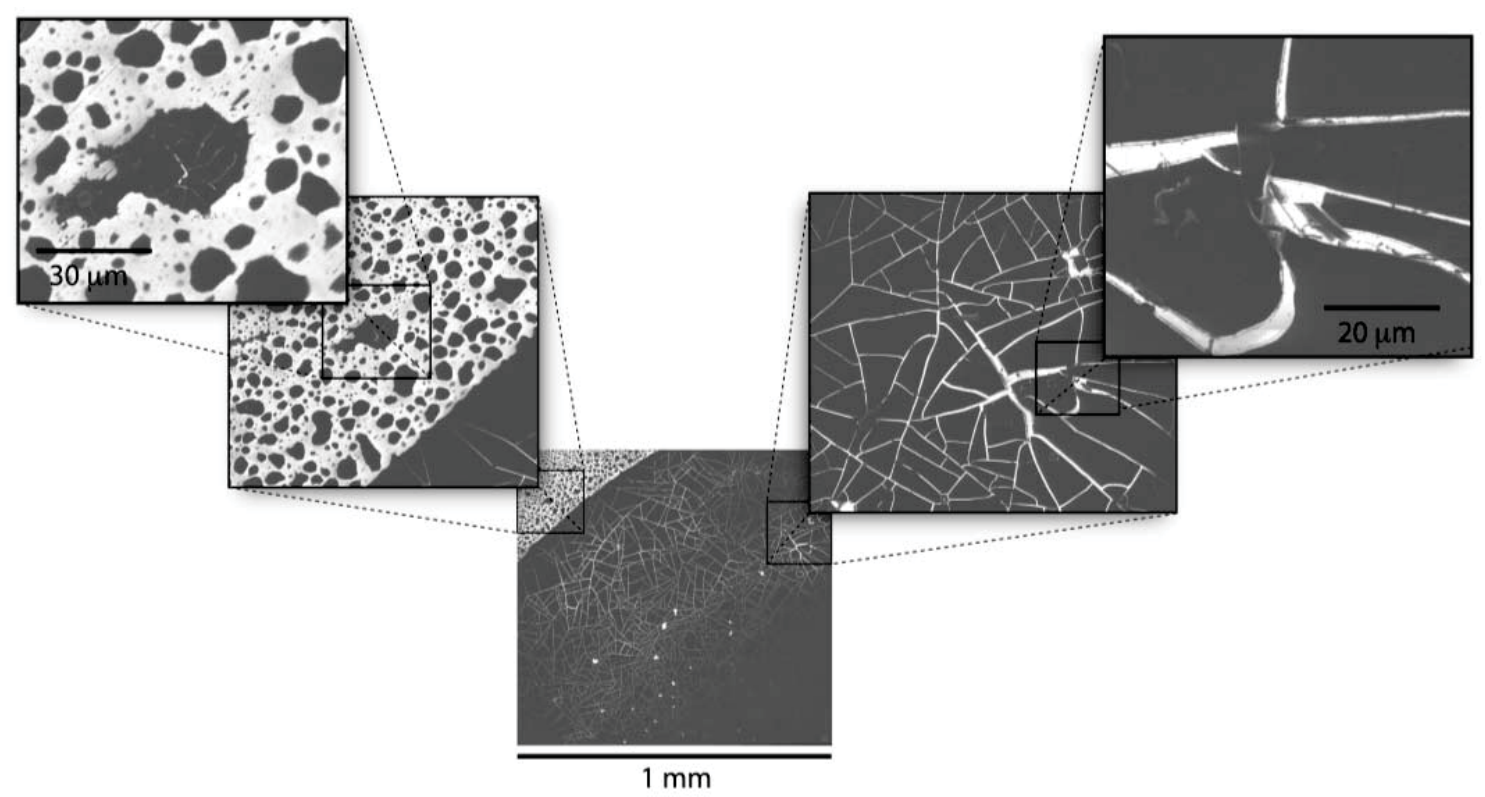

Figure 1. High-resolution SEM images of Cf-252 sample electroplated to a SEM stem. The Cf-252 was concentrated into an area such that the fission rate in the field of view is the same as a fuel inside a reactor.

The activation issue will also be looked at in FY 2014. Most of the materials at the business end of a SEM are radiation hardened and relatively insensitive to gamma radiation, but the impact of signal to noise will need to be studied. However, it is not expected to be a showstopper. Any future SEM that would be designed and engineered for in-situ work will certainly be driven by the desired performance specs and would include substitute materials and removing sensitive circuits to a shielded volume.

The LDRD is successfully developing all three phases (irradiation, fabrication, and detection) of an isolated effects testing capability. Advances in imaging and fabrication this year, combined with the irradiation capability from the FY 2012 engineering design report, present a recommended, optimal path forward for realizing a significant advance in the study of microstructural behavior of fuel material under realistic reactor conditions. This capability would bring a science-based understanding of the microstructural, time-dependent phenomena that drives the macroscopic fuel performance and would be a tremendous complement to the well-established irradiation cycle 
and post-irradiation examination experimental paradigm. A wealth of new information that informs advanced modeling and simulation is, at this time, a feasible and realistic goal for exponential advances in fuels and materials development.

\section{Benefits to DOE}

The work funded by this LDRD is in line with the larger strategy by DOE-NE to shift the paradigm of advanced nuclear fuels development to ensure the nation's energy security in the future. The historical approach of relying on experiential models and extensive qualification testing does not allow for the rapid advances necessary for replacing the light water reactor fleet in this country. Separate and isolated effects testing will inform new, first principle models to develop a predictive performance capability as fuels research moves forward. This project focuses on the three components (irradiation, fabrication, and detection) necessary for game-changing advances in nuclear fuels development, with the promise of shaving decades off the development time for new fuels needed in the next generation of U.S. nuclear power. 


\title{
12-112 - Reactor Coolant Pump Alternate Can Material Development Program
}

\author{
Mark Carroll, Ross Klein, ${ }^{1}$ Robert Ladefian, ${ }^{1}$ and Richard Wright
}

A significant amount of reactor coolant pump (RCP) efficiency is being lost through parasitic electrical fields developed in the powerful induction motors. To address this issue, a customized alloy system is being developed that will have a measurable effect on increasing RCP efficiency while meeting the substantial number of additional performance characteristics necessary in elevated temperature and controlled chemistry nuclear environments. A cooperative development program was initiated to develop a tailored alloy system suitable for specific components in RCPs produced by the lone U.S. based manufacturer, Curtiss-Wright Flow Control Company. Considering the extremely long service lives and the enormous power requirements of the RCPs, a materials selection strategy that results in even fractional decreases in parasitic field losses was the overarching focus of this development program. A combination of the nuclear expertise and materials science and engineering capabilities of the INL along with the industrial and power plant production expertise of Curtiss-Wright was envisioned as a cooperative capability that would enhance the overall effort, as opposed to addressing the specific issues alone by either party. The collaborative effort in the Reactor Coolant Pump Alternate Can Material Development Program was carried out under CRADA 12-CR-09.

\section{Summary}

The initial alloy selection/development effort had a specific focus on the development of a tailored nickel (Ni)-based alloy, which has increased electrical resistance properties and also exhibits the necessary quality of retaining a stable microstructure for longservice components. Through this program, the capability to execute high-purity vacuum induction melting of small alloy "buttons" (Figure 1) roughly $30 \mathrm{~g}$ in mass has been refined in order to meet the rapid turnaround necessary to satisfy the comprehensive test matrix of customized Ni-chromium (Cr)-based compositions, with elemental fractions selected through the generation of ThermoCalc-based phase field ternary models of the specific ranges of interest. The proven capabilities of Ni-based alloys in harsh or high-temperature systems makes them an ideal matrix material for further improvement through controlled elemental additions in order to engineer idealized properties.

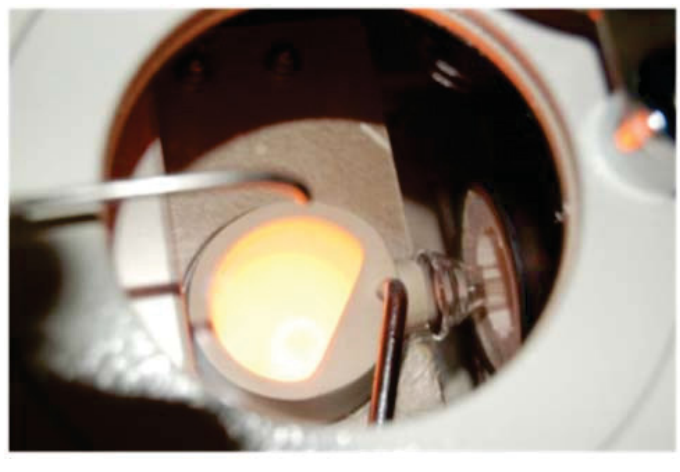

Figure 1. Vacuum-induction melting buttonsized alloy compositions for rapid evaluation of properties.

The geometric limitations of the experimental castings necessitated the development of an accurate and repeatable measurement technique for the principal physical property of the new alloys being evaluated. Figure 2 shows the refined technique that utilized high-resolution equipment and precision machining of test cylinders from the experimental buttons to measure electrical resistivities from over 35 individual castings from targeted composition ranges.

1. Curtiss-Wright Flow Control Company 


\section{NUCLEAR SCIENCE \& TECHNOLOGY}
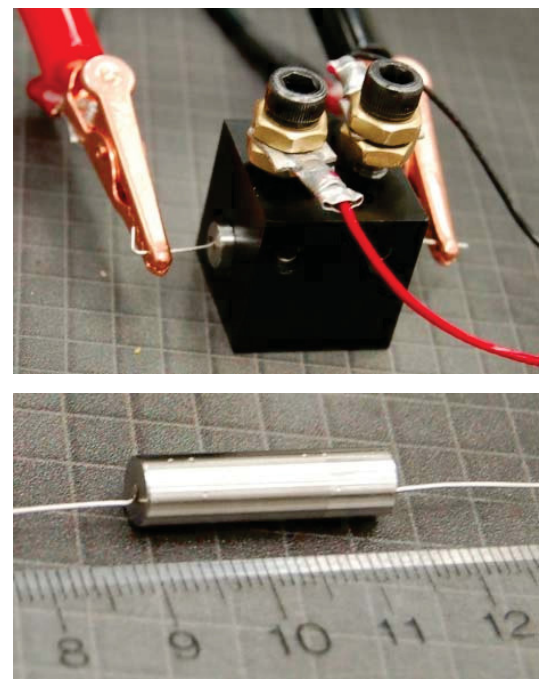

Figure 2. Resistivity measurements on specimens much smaller than prescribed by ASTM International standards.
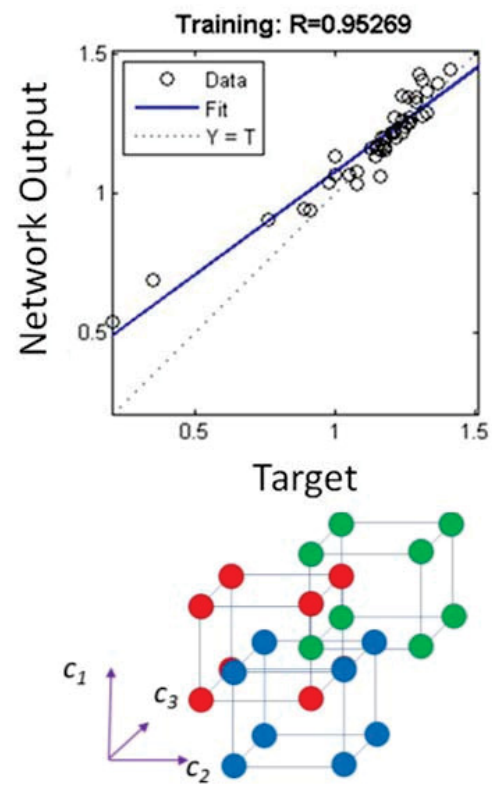

Figure 3. Neural network relationships based upon overlapping experimental composition matrices.
Results from the casting trials were used to develop predictive capabilities for critical electrical properties of interest through the generation of an optimized neural network (Figure 3). Elemental alloying additions were used as input parameters to predict the electrical resistivity of theoretical alloy compositions. Commercial alloys were included in conjunction with the experimental cast alloys, populating the network with over $80 \mathrm{Ni}$-based alloys in order to ensure robustness of the network. The challenge of developing an accurate neural network is fundamental to this project, as targeting high resistivity values outside the range of commercially available alloy compositions is key to the program's success.

Full-size "industrial" castings using base material composition ranges were cast by a commercial vendor and further processed into thin sheets in order to provide the industrial partner with suitable material for advanced welding trials. These trials were carried out using weld conditions developed for the present stateof-the-art alloy so that microstructural response and stability in the heat-affected zone of the weld could be fully evaluated for secondary phase nucleation and growth.

\section{Benefits to DOE}

This developmental effort allows DOE to employ an integrated scientific approach to directly address critical issues facing the nuclear industry through technology-driven solutions. The ability to rapidly respond to application-specific needs in mission-critical areas through tailored alloy development is a crucial capability for INL to foster. With regard to this particular program, increased plant efficiency will allow proven designs to continue to be utilized in modern plants, as well as to provide critical additional efficiency ratings to future small modular reactor designs - all of which are envisioned to play important roles in energy generation that extend beyond the common need of supplying power to the electrical grid. Incremental improvements to the efficiency of RCPs through tailored material systems will ensure that this type of design keeps U.S. based manufacturing competitive in the growing international nuclear power sector. 


\section{2-117 - Feasibility of a Broad Application Test Reactor Using LEU}

Hans Gougar, Michael Pope, and John Ryskamp ${ }^{1}$

INL's ATR has been the world's most powerful irradiation facility since 1967. Although the fuel and structural components of ATR can, presumably, be indefinitely replaced, the cost of doing so under an increasingly stringent regulatory regime may exceed that of a replacement facility. This was the motivation behind a previous INL project which yielded the conceptual designs of two possible replacements for ATR. This project primarily serves the Laboratory R\&D Objective No. 2, "Improve reactor designs so reactor systems are more affordable, safe, effective, and efficient." It explores the feasibility of using low enriched uranium (LEU) fuel to drive a materials test reactor to meet the irradiation needs of current and future ATR customers as the useful life of ATR comes to a close. The project leverages the findings of an earlier effort, the Broad Application Test Reactor (BATR) project, in which two test reactor concepts fueled with high enriched uranium were identified and analyzed. The result was the conceptual design of the MATRIX, the Multiple Application Thermal Reactor for Irradiation experiments (Figure 1).

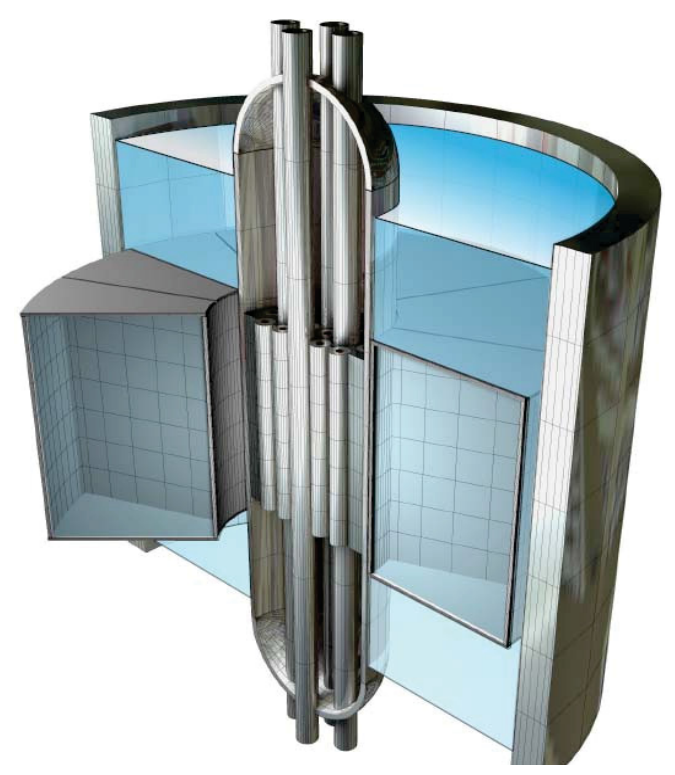

Figure 1. Artist's conception of the MATRIX.

Unlike ATR, a new test reactor may be asked to accommodate a wide range of missions and customer requirements beyond fuel and material testing in pressurized coolant loops. One objective was to therefore identify the missions of high flux reactors in North America and gather their performance and operational requirements. The performance and operational requirements of these missions will be used to assess compatibility and to identify conflicts that would prevent dual or multiple use. The first and second objectives were met in the first year of the project.

The conversion of ATR to LEU fuel is constrained by the needs and design of the existing facility. The design of a new test reactor is not so constrained. The flexibility to efficiently serve a broad array of irradiation needs as well as those of the Naval Reactor programs will require creativity and investment in features such as standard instrumented leads, in-core instrumentation, and online test placement, and reconfigurable and replaceable core components. The first step, however, is to determine whether the basic neutronics of the core will support the different objectives. Another objective was to explore design features that would both mitigate the effects of using LEU and allow the facility to pursue missions other than the primary one of controlled chemistry loop irradiations. This objective was met in the second FY.

1. Private Consultant 


\section{Summary}

Two leading concepts from the BATR project were examined using the SERPENT code. The so-called modularhexagonal design featured an array of fuel assemblies and separate large flux traps inserted into a primary beryllium $(\mathrm{Be})$ reflector. This core region was surrounded by a single pressure vessel separating the core from a secondary heavy water reflector. It was found to have a large amount of excess reactivity by virtue of this reflector. However, the separation of the fuel from the test locations by said Be carries a significant penalty in fast flux in the test locations. For this reason, the modular-hexagonal concept in its original form was dropped from consideration.

The so-called multiple annular core featured fuel assemblies surrounded by individual pressure boundary tubes (PBTs) rather than a single large pressure vessel. These tubes were suspended in a heavy water reflector (no $\mathrm{Be}$ ). It displayed much better performance with regard to fast flux in test locations and very high thermal flux was available outside the PBTs. The stainless steel PBTs, however, carry a significant reactivity penalty, which could be overcome by use of aluminum (AI) or Zircaloy. The necessary American Society of Mechanical Engineers code case, however, is an open question for using Al for this application. Also, layout of the primary loop structures as well as access to fuel assemblies, control elements, and test trains was considered an open question in the multiple annular design.

Analysis performed using the SERPENT code largely confirmed those conducted in the earlier INL study. The performance of the core using LEU fuel was also found to be comparable to the highly enriched uranium (HEU) fueled concepts.

\section{Multiple Applications and Users}

Potential uses for, and associated requirements of, a high flux test reactor with the functionality currently served by a handful of high flux reactors in the United States (ATR, High-Flux Isotope Reactor, Missouri University Research Reactor) were explored. The needs were gathered through a survey and group discussions in March 2012. Survey results were documented in the second of three technical reports (Publications 1,2, and 3) issued during the first year. Assuming that the primary mission was achieved with loops and high density fuel, isotope production was found to be possible for certain isotopes with online insertion and removal. Neutron scattering in a beam tube to support interrogation of nuclear fuels and materials is also a possibility; however, having more than one beam tube would severely limit reflector irradiation volume.

Most of the secondary missions identified for this test reactor are better served by large volumes of high-thermal neutron flux regions. All variants investigated were equipped with large secondary reflector tanks surrounding the core. Because penetrating the pressure vessel is not required for these test locations, placing them outside the pressure boundary provides easier access to the test locations. Nominally, these tanks are specified to contain heavy water $\left(\mathrm{D}_{2} \mathrm{O}\right)$, which provides the highest thermal neutron flux of any materials considered. However, because $\mathrm{D}_{2} \mathrm{O}$ would require sealing and some means of handling the tritium produced, Be was also considered. Though the secondary tank of any of these variants could be made to accommodate most of the identified secondary missions, some of them have higher neutron flux than others.

The cost of high neutron flux in a region outside the pressure boundary is, naturally, rapid accumulation of neutron damage on the vessel material. All of the reactor variants investigated would have to accommodate this. It is likely that the vessels or PBTs would need to be designed for replacement after a certain number of years. 
Alternatively, for some of the variants, the pressure vessel could be moved away from the fuel, which would lower the neutron flux on the vessel but also lower the neutron flux outside of it. The feasibility of using Al rather than steel for a pressure vessel or PBT has a significant impact on the neutron flux levels in the secondary tank and, in some variants, reactivity of the core. The additional licensing cost of such a design choice should be evaluated in future work.

\section{High Performance with LEU}

The concepts identified in the BATR project were shown to yield a satisfactory (comparable to or better than ATR) irradiation capability. Highly enriched uranium allows for longer cycles in a smaller volume than LEU. For MATRIX, some creativity was required to achieve the same level of performance with the lower grade fuel.

Different moderator and vessel materials and fuel/core geometries were explored and produced some promising candidates.

Of the four variants for which detailed neutronic analysis were performed, one (identified as the Cylindrical design-see Figure 2) was found to yield superior neutronic performance comparable to (or better than) ATR with regard to achievable fast flux in the primary irradiation holes and cycle length. The volume of test space in the InPile Tubes is larger in MATRIX with comparable magnitude of neutron flux. In addition to the InPile Tubes, the Cylindrical MATRIX concept features test spaces at the centers of fuel assemblies where very high fast flux can be achieved. This magnitude of fast flux is similar to that achieved in the ATR A-positions; however, the available volume having these conditions is greater in the MATRIX design than in the ATR.

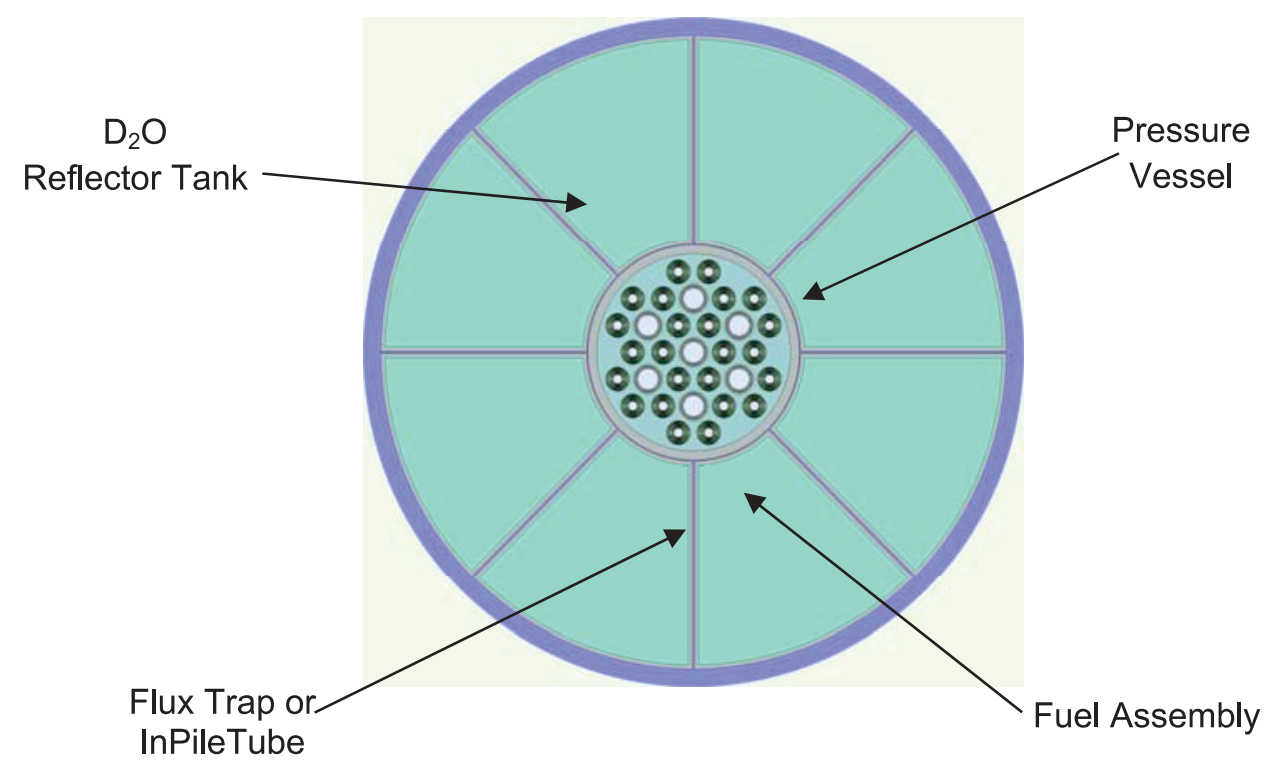

Figure 2. Baseline Cylindrical core model with Heavy Water Reflector Cans.

Perhaps the most significant advantage of the Cylindrical MATRIX design over the ATR is the large reflector tank located outside the pressure vessel. Constructed from Al and located close to the fuel elements, the low parasitic capture pressure vessel facilitates a very high thermal neutron flux in the reflector tank (see Figures 2 and 3 ). This concept is borrowed both from some of the original BATR designs and from the Jules Horowitz reactor under construction in France. Nominally, the reflector tank would be an atmospheric (or near-atmospheric) pressure $\mathrm{D}_{2} \mathrm{O}$ 
volume to serve as a high thermal flux test space. In this work, an alternative concept was introduced wherein $\mathrm{Al}$ cans containing $\mathrm{D}_{2} \mathrm{O}$ are placed in an open light water pool. From preliminary neutronic studies, this concept appears to perform well.

A 'White Paper' was prepared (Publication 5) which summarizes the status of the MATRIX baseline concept and outlines the near term trade studies, optimization, and further design work that should be performed as part of a formal conceptual design process.

\section{Benefits to DOE}

There is concern about how to continue meeting the increasing demand for irradiation services given: (1) the national security imperative to reduce the inventory and use of HEU in civilian facilities, and (2) the difficulty and expense of maintaining and repairing the aging high flux test reactors operated by the federal government. It has been suggested that HEU-to-LEU conversion of ATR and High-Flux Isotope Reactor may not be the most costeffective way to do this and that a new high flux test reactor may be required. It is also recognized that replacing both ATR and High-Flux Isotope Reactor in the current fiscal climate will be difficult. Replacing one of them will be less of a challenge. It is therefore reasonable to explore whether one new facility can serve the missions currently served by multiple reactors. This will require extensive evaluation and discussions among DOE and laboratory personnel to evaluate the attributes of the existing reactors and to define and compare their mission requirements. It will also require some understanding of the different features of test reactors that lend themselves to certain missions. The work performed on this LDRD specifically addressed each of those issues. A technical article has been drafted and will be submitted to a peer-reviewed journal for publication.

In FY 2013, DOE established the NA-NE Research Reactor Working Group (WG), with members from the national Nuclear Security Administration and the Office of Nuclear Energy, "to identify the capabilities needed for the United States research reactor complex of the future and coordinate related efforts across the Department to ensure that these needs are met within the broader context of DOE and other U.S. Government policy. The WG will engage other DOE Program Offices and key stakeholders as appropriate. The WG will compile the necessary technical data and evaluate options to inform and support DOE leadership's policy decisions on an efficient and effective path forward." The requirements report generated from the project in FY 2012 provided important information to the WG in the early stage of their deliberations. The White Paper has also been provided to the WG.

The PI of this project was invited to participate in a consultancy meeting held by the International Atomic Energy Agency in June 2013. The International Atomic Energy Agency is constructing a catalog of capabilities and capacities of research reactors toward the deployment of innovative nuclear energy systems and technologies which will be published in the near future. The PI provided the input (in the form of a 12 page report) on ATR based upon information compiled under this LDRD. 


\section{Publications}

Pope, M.A., H.D. Gougar, and J.M. Ryskamp, "Design Studies for a Multiple Application Thermal Reactor for Irradiation Experiments (MATRIX)," Nucl. Eng. Des., to be submitted.

Gougar, H.D., M.A. Pope, and J.M. Ryskamp, MATRIX - The Next Step to a $21^{\text {st }}$ Century High Flux Test Reactor, INL/EXT-13-30137, September 2013.

Pope, M.A., H.D. Gougar, and J.M. Ryskamp, Evaluation of Concepts for the Multiple Application Thermal Reactor for Irradiation Experiments (MATRIX), INL/EXT-13-30045, September 2013.

Gougar, H.D. and M.A. Pope, Requirements, Supporting Technologies, and Recommended Scoping Investigations for a New Test Reactor Fueled with Low-Enriched Uranium, INL/LTD-12-27111, September 2012.

Pope, M.A., et al., Evaluation of Multi-Mission Test Reactor Concepts, INL/LTD-12-27125, September 2012.

Pope, M.A., H.D. Gougar, and J.M. Ryskamp, Evaluation of Broad Application Test Reactor Concepts with Low Enriched Uranium Fuel, INL/LTD-12-25788, August 2012.

\section{Presentations}

Pope, M., "Complementary Analyses in ATR Physics," MATRIX Project Review \& Strategic Planning, Idaho Falls, ID, July 29, 2013.

Pope, M., "Review of Baseline and Variant Physics and T/H," MATRIX Project Review, Idaho Falls, ID, September 10, 2012.

Pope, M., "Preliminary Results for the LBATR," New LEU-Fueled Test Reactor Complementary Work and Preliminary Analyses, Idaho Falls, ID, March 2, 2012.

Ryskamp, J., "Summary of BATR Project: Targets, Analyses, and Concepts," New LEU-Fueled Test Reactor User Requirements, Idaho Falls, ID, March 1, 2012.

Gougar, H., "Results of Requirements Survey and Cross Check with Other Users," New LEU-Fueled Test Reactor User Requirements, Idaho Falls, ID, March 1, 2012. 


\title{
13-013 - Development of a Micromechanistic Phase-Field Modeling Approach for Life Estimation and Risk Assesment of Reactor Pressure Vessels
}

\author{
S. Bulent Biner, Michael Tonks, Paul Millett, and Yongfeng Zhang
}

For life assessment of current light water reactors, it appears the only viable option is predictive modeling methodologies for the reactor pressure vessel steel's embrittlement along with limited experiments. Our modeling approach aims at development of a micromechanically-based phase-field modeling algorithm in which co-evolution of microstructure due to radiation effects (e.g., precipitation of late blooming phases and hardening) and damage evolution (e.g., change in the fracture morphology and shift in ductile brittle transition temperature curves) can be simulated in a unified framework for aging reactor pressure vessel steels.

\section{Summary}

The following briefly summarizes our technical progress achieved during FY 2013:

For quantitative modeling, a thermodynamic database (1-3) for an iron (Fe)-copper (Cu)-manganese (Mn)nickel $(\mathrm{Ni})$ alloy system was incorporated into the 3-D phase-field algorithm. With this implementation, the simulation correctly predicts, as in experiements (4-6), the evolution of $\mathrm{Mn}-\mathrm{Ni}$ rich ring/shell structure around the Cu precipitates with the body-centered cubic (bcc) to face-centered cubic $(f c c)$ phase transformation in spinoidal regime during thermal aging, as shown in Figure 1.

The segregation/precipitation behavior for a dilute Fe-1.5@\%Cu-0.5@\%Mn-0.5@\% in the presence of lattice defects: self intersitial atom loops, (SIA), vacancy loops, and ordinary dislocations was parametrically studied, as shown in Figure 2. For similar size defect loops, the vacancy loops provide enhanced precipitaton kinetics. (A manuscript based on these results has been submitted for a journal publication and workshop presentation.)

Evolution of dislocations (e.g., generation, annihilation, and formation of junctions) in the bcc lattice was efficiently implemented into a 3-D phase-field model code, and their interactions with precipitates of $\mathrm{Cu}, \mathrm{Mn}$, and $\mathrm{Ni}$ (i.e., late-blooming phases) are currently being investigated, as shown in Figure 3.
Time: 1.0
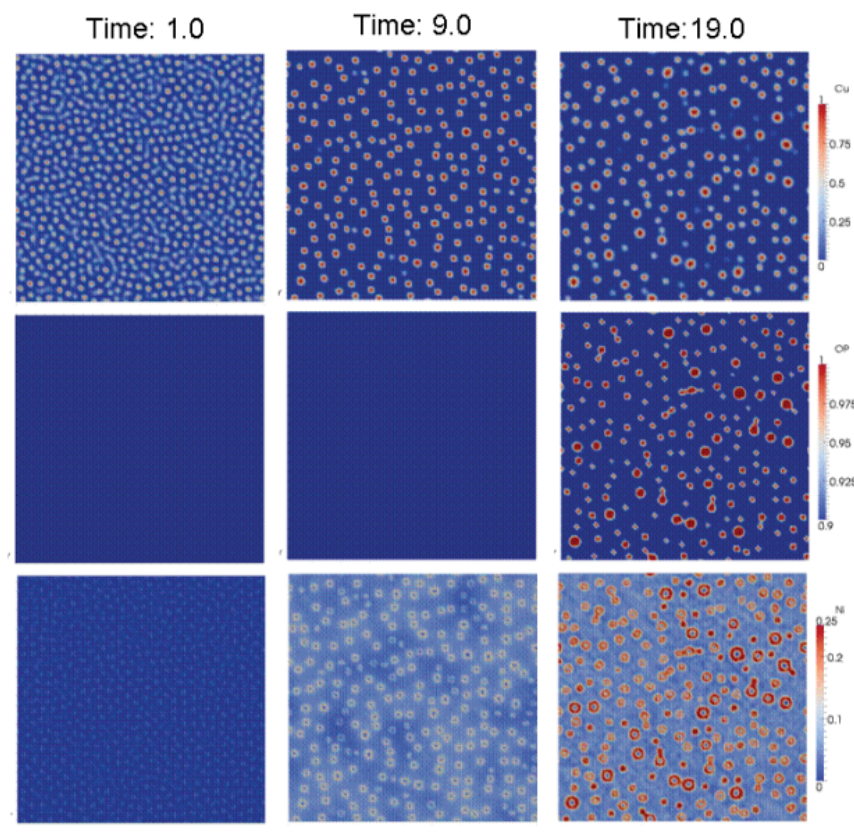

Figure 1. Isothermal phase transformation and microstructural evolution in Fe-15@\%Cu-1@\%Ni at $873^{\circ} \mathrm{K}$. The top row, is time evolution of $\mathrm{Cu}$ precipitation, middle row, the structural bcc to fcc phase transformation of Cu precipitates, and bottom row is the time evolution of Ni segregation. 


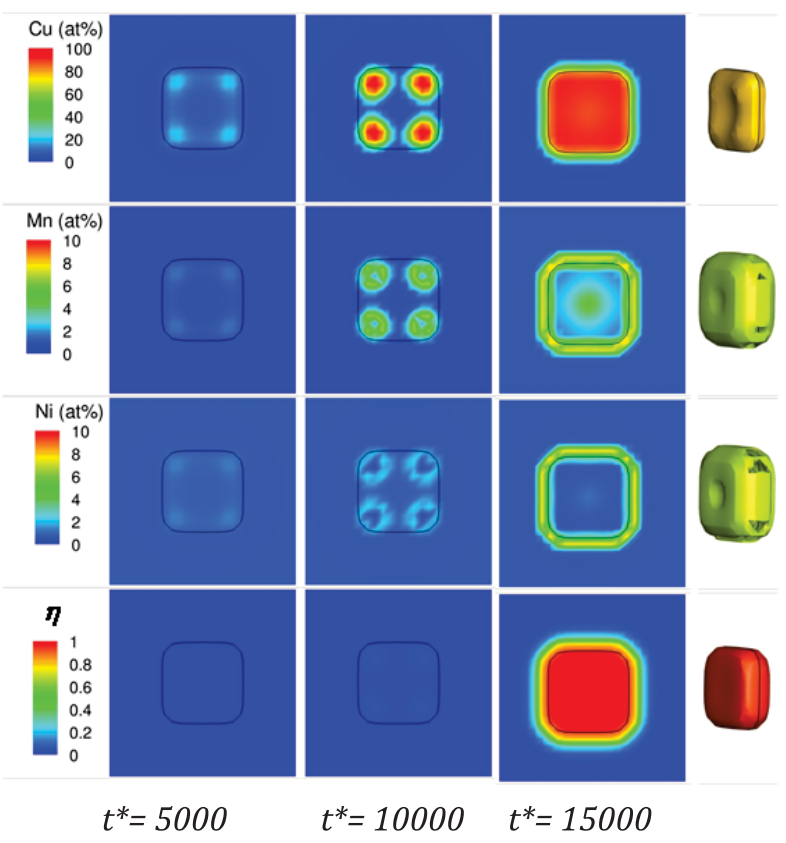

Figure 2. Time evolution of concentration profiles and bcc to fcc phase transformations around a square vacancy loop with $10 \mathrm{~b} \times 10 \mathrm{~b}$ and [100] (100) character, $b$ is the length of the Burgers vector. The iso-surfaces in the last column correspond to CCu=80@\%,CMn=7@\%,CNi=7@\% and $\eta=0.9$, respectively.
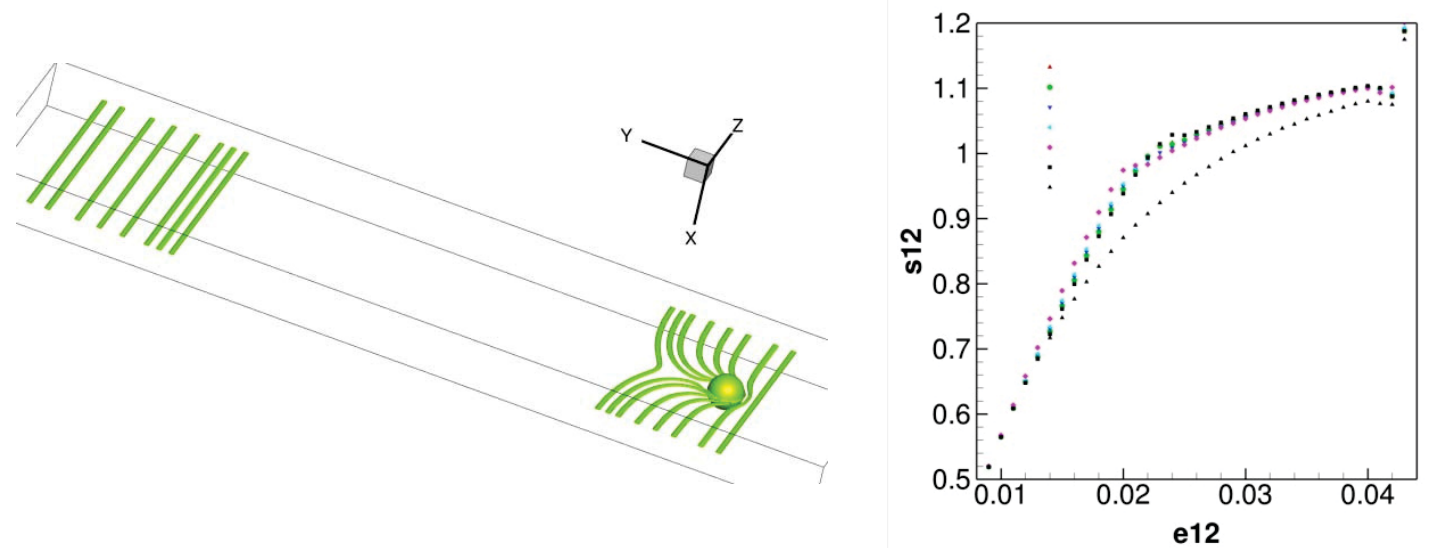

Figure 3. Right, Phase-field simulation of interaction of an edge dislocation with a Cu precipitate in Fe resulting in an increase in the flow stress (hardening).

\section{Benefits to DOE}

The project develops science-based modeling and simulation tools for microstructure evolution and resulting mechanical behaviors under irradiation conditions. These predictive tools will be greatly useful in ranging from life extension programs of LWR to long-term storage of spent nuclear fuels. 


\section{Publications}

Rao, W., Y. Zhang, and S. Biner, "The Role of Lattice Defects on Segregation/Precipitation Behavior of a Dilute Fe-Cu-Mn-Ni Alloys: A Phase-Field Approach," Journal of Modeling and Simulation in Mater. Sci. Eng., Submitted.

\section{Presentations}

Rao, W., Y. Zhang, and S. Biner, "The Role of Lattice Defects on Segregation/Precipitation Behavior of a Dilute Fe-Cu-Mn-Ni Alloys" International Workshop on Structural Materials for Innovative Nuclear Systems, Idaho Falls, ID, October 7-10, 2013, Submitted. 


\title{
13-014 - Aluminium Electroplating from Low Temperature Molten Bromide Salts
}

\author{
Prabhat Tripathy and Eric Dufek
}

There is an increasing need for the development of substitute coating materials for cadmium (Cd) and hexavalent chromium ( $\mathrm{Cr}$ ) in order to mitigate corrosion of steel- and aluminium (Al)-based structural components used in aerospace and other industries. The objective of the proposed project was to develop an alternative molten-saltbased electrolyte system for electroplating metallic Al onto a variety of substrate materials. Although both chloride and bromide salts provide attractive features of a typical plating bath, the former suffers from disadvantages in terms of: (1) the higher volatility of $\mathrm{AlCl}_{3}$ resulting in significant loss during electroplating, and (2) limited scale up possibilities. $\mathrm{AlBr}_{3}$, on the other hand, offers twin advantages, viz. lower melting point and higher sublimation temperature, and is hence expected to provide better results over $\mathrm{AlCl}_{3}$. The eutectic composition of a ternary bromide melt ( $\mathrm{LiBr}-\mathrm{KBr}-\mathrm{CsBr}$ ) offers the possibility of low temperature operation. It was therefore decided to use the combination of $\mathrm{AlBr}_{3}$ and the ternary melt to: (1) prepare Al electroplated stainless steel and other metallic components, and (2) characterize as well as evaluate the coatings by using a combination of scanning electron microscopy with energy dispersive $\mathrm{x}$-ray attachment and preliminary corrosion tests.

\section{Summary}

The detailed experimental work along with subsequent characterization and preliminary corrosion tests suggest that the bromide melt offers significant advantages over the coatings that can be obtained from the chloride melts. These advantages include: (1) preparation of thick, dense, and adherent Al coating on the substrates, (2) significantly less loss of $\mathrm{AlBr}_{3}$ during the coating process, and (3) possibility of obtaining very good bonding between the substrate and the coating (something which has not been reported to take place in a coating obtained from the chloride melt). The preliminary corrosion tests of the coated materials strongly suggest that either a sufficiently thick electrodeposited $\mathrm{Al}$ layer or a combination of metallic $\mathrm{Al}$ and $\mathrm{Al}_{2} \mathrm{O}_{3}$ layer on the stainless steel substrate will perhaps be able to mitigate the stress corrosion cracking of the stainless steel structural components that are routinely being used in nuclear reactors. The ternary bromide electrolyte seems to provide an ideal plating bath in order to accomplish this objective.

\section{Benefits to DOE}

The outcome of the project suggests potential benefits for the aerospace, shipping, wind energy, and oil (both onand off-shore) industries. The proposed project has clearly fulfilled the initial requirement of the possibility of obtaining a thick, dense, and adherent coating on a variety of substrate materials that include stainless steel, mild steel, copper, and brass from a bromide melt, something which has not been reported in open literature. This coating process has also been found to be quite effective in terms of providing complete coverage on complex substrates such as metallic screws and tool drill bits. Moreover, as the coating was prepared at a higher temperature (than the coating that can be obtained from a chloride melt), very good bonding could be obtained between the substrate and the Al coating by way of the formation of a metallic interlayer. The project has shown the potential to be beneficial in terms of obtaining an effective solution to the mitigation of stress corrosion cracking of stainless steel structural components that are routinely being used in reactor environments. Further, the experimental work suggests that either a sufficiently grown Al layer or a combination of layers of $\mathrm{Al}_{\text {and }} \mathrm{Al}_{2} \mathrm{O}_{3}$, prepared from the bromide melt, can potentially protect the structural components (made of mild steel, stainless 
steel, high strength low alloy steel, and Al alloys) from corrosion under a variety of conditions that include corrosive and marine environments.

\section{Publications}

Tripathy, P.K., et al., "Aluminium Electroplating on Steel from a Fused Bromide Electrolyte," Surf. Coat. Technol., To be submitted. 


\title{
13-023 - Characterization and Modeling of High Burn-up Mixed Oxide Fuel
}

\author{
Melissa Teague
}

Improved nuclear fuels have the potential for increased efficiency and security. One way to realize such advances is to develop ways of combining traditional experimental methods with advanced computational models. The objective of this work was to enhance the fundamental understanding and modeling of fuel performance by combining experimental data collection and finite element modeling. The experimental efforts included utilizing INL's one-of-a-kind advanced characterization, post-irradiation equipment. Finite element modeling was performed to evaluate the thermal conductivity of high burn-up, fast-reactor mixed oxide fuel. Input parameters and experimental microsctructure for the models were developed by applying chemical/phase information to optical micrographs previously obtained as part of Fuel Cycle R\&D.

\section{Summary}

This project supported analysis of uranium $(\mathrm{U})$ and plutonium $(\mathrm{Pu})$ mixed oxide fuel with simulated fission products using atom probe tomography, the first ever atom probe tomography (APT) of Pu bearing material (Figure 1). Demonstration of the ability to study transuranic materials using APT is ground breaking in the scientific community. Additionally the project supported APT analysis of U-55Pu-20Zr transmutation fuel, the analysis of revealed non-uniform oxidation depth for the different elements. The depth of oxidation within the sample was found to be greatest for $\mathrm{Zr}$, followed by $\mathrm{U}$, and then $\mathrm{Pu}$. This variable oxidation within the sample was unexpected; however, the ordering is in-line with the oxygen affinity of the samples. Other samples taken from near the edge of the U-55Pu-20Zr sample showed a large fraction and precipitation of silicon ( $\mathrm{Si}$ ). $\mathrm{Si}$ contamination from the mold during casting has been suspected, but this is the first observation of the form it takes.

- $\quad$ APT of MOX fuel with simulated fission products, first ever APT of transuranic-bearing material

- $\quad$ APT of U-55Pu-20Zr transmutation fuel samples

- Preferential oxidation of fuel components observed, microstructural evidence of Si contamination from mold during casting.

The modeling component of the LDRD demonstrated a new approach to fuel performance modeling that incorporated the advanced characterization data directly into advanced modeling tools. Radially and temperature-dependent models for effective thermal conductivity were developed utilizing optical micrographs of high burn-up mixed oxide fuel. The micrographs were employed to create finite element meshes with the OOF2 software. The meshes were then used to calculate the effective thermal conductivity of the microstructures utilizing INL's cutting edge MOOS/BISON fuel performance code (Figure 2). The new thermal conductivity models were used to calculate thermal profiles at end of life for the fuel pellets. These results were compared to thermal conductivity models from the literature, and comparison between the new finite element-based thermal conductivity model and the Duriez-Lucuta model was favorable

- Tightly coupled experimental and modeling approach to understanding fuel performance 
- New microstructure-based modeling approach for effective thermal conductivity of high burn-up oxide fuel developed and demonstrated.

- Critical insights into fuel cladding gap evolution and composition gained from modeling efforts.

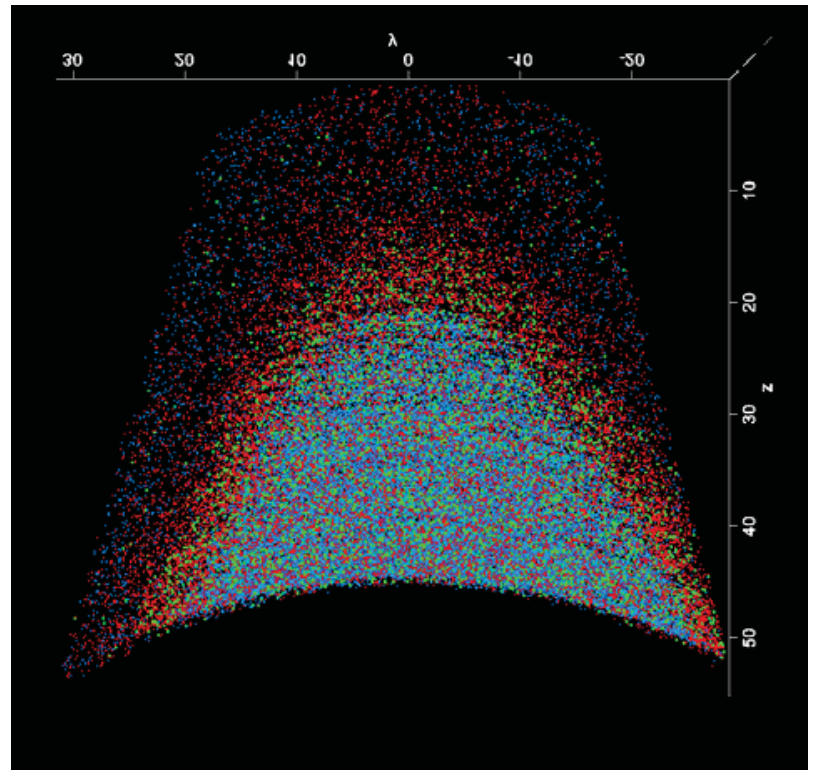

Figure 1. APT from U-55Pu-20Zr samples, showing oxide penetration depth. Zr blue, Pu Red, U Green.

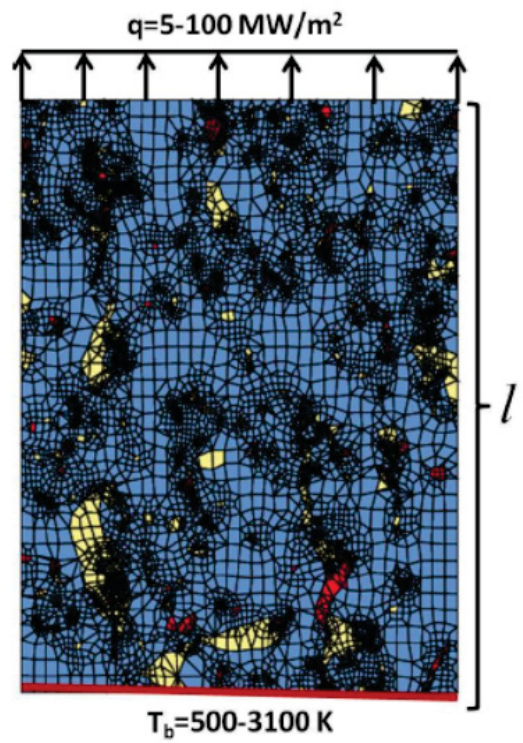

Figure 2. Microstructural effective thermal conductivity model, red is metallic precipitates, yellow porosity, and fuel is light blue. The effective thermal conductivity of the microstructure is calculated using the following equation $\frac{\partial T}{\partial x} \approx \frac{\bar{T}_{t}-T_{b}}{l}$. 


\section{Benefits to DOE}

The project enhances the international reputation of DOE-NE as a pioneer in technique development for postirradiation exam methods. The demonstrated modeling methods are providing new insights into the behavior of ultra-high burn-up mixed oxide fuel and new emphasis and basis for experimental conductivity measurements.

\section{Publications}

Teague, M., et al., "Microstructural Modeling of Thermal Conductivity of High Burn-up Mixed Oxide Fuel," J. Nucl. Mater., Vol. 444, 161-169, January 2014, In press.

Teague, M., M. Tonks, and S. Novascone, "Microstructural Characterization and Modeling of High Burn-up Mixed Oxide Fuel," 2013 LWR Fuel Performance Meeting/TopFuel, Charlotte, NC, September 15-19, 2013.

\section{Presentations}

Teague, M. and M. Tonks, "Characterization and Modeling of High Burn-up Mixed Oxide Fuel" LDRD Annual Review, Washington, D.C., July 11, 2013.

Teague, M., "Characterization and Microstructural Modeling of High Burn-up Oxide Fuel from the Fast Flux Test Facility," Fuel Cycle Technologies Annual Review Meeting, Chicago, IL, October 30-31, 2012. 


\section{3-029 - In-Pile Detection of Crack Growth in the ATR}

Joe Palmer, Sebastien Teysseyre, Joy Rempe, Gordon Kohse, ${ }^{1}$ and Steinar Solstad ${ }^{2}$

A key component in evaluating the ability of light water reactors to operate beyond 60 years is characterizing the degradation of materials exposed to radiation and various water chemistries. Of particular concern is the response of reactor materials to Irradiation Assisted Stress Corrosion Cracking. Some test reactors outside the United States, such as the Halden Boiling Water Reactor, have developed a technique to measure crack growth propagation during irradiation (Figure 1). This technique incorporates a compact loading mechanism to stress the specimen during irradiation. A crack in the specimen is monitored using a reversing Direct Current Potential Drop method, which consists of introducing a precisely controlled current across a loaded specimen and measuring the drop in voltage across the crack mouth (which changes as a function of crack growth). This three year project applies the techniques used at the Halden Boiling Water Reactor to develop and evaluate a more robust test rig design

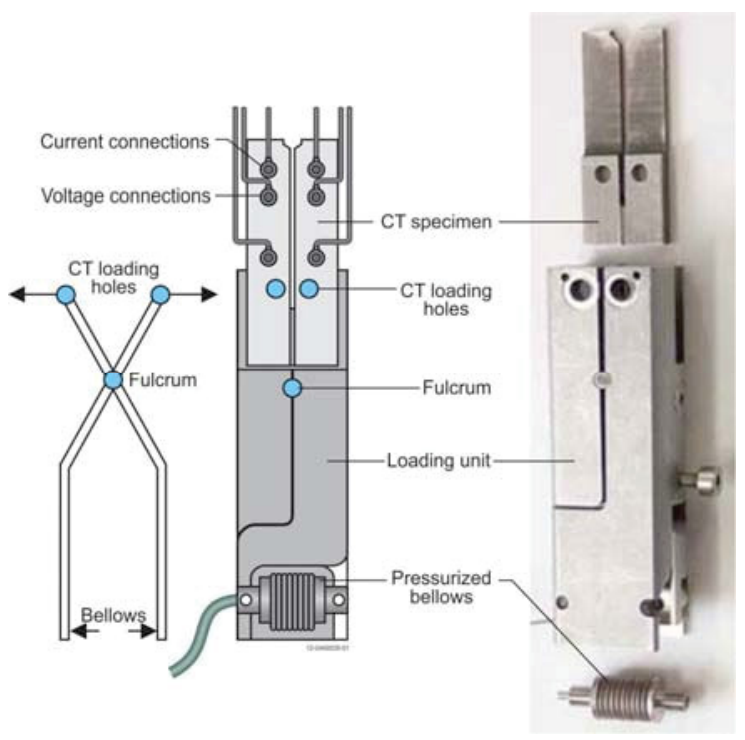

Figure 1. Schematic of loading mechanism with Direct Current Potential Drop connections.

that can survive the higher flux conditions at U.S. material testing reactors, such as INL's ATR and the Massachusetts Institute of Technology (MIT) Research Reactor.

\section{Summary}

The first year of the proposed three year project was devoted to designing, analyzing, fabricating, and bench top testing a mechanism capable of applying controlled stress to specimens while they are irradiated in a pressurized water loop (simulating pressurized water reactor conditions). During the second year, the mechanism will be tested in a high pressure, high temperature water environment outside of a reactor in representative water chemistries. In addition, necessary documentation and safety reviews for testing in a reactor environment will be completed. In the third year, the assembly will be tested in the MIT Research Reactor, and Post Irradiation Examinations will be performed.

During the first year, two specimen loading mechanisms were constructed. INL first constructed an initial prototype from stainless steel. Then, using the INL design as a starting point, the MIT experiment design group completed a full 3-D finite elements analysis to identify requirements for an enhanced mechanism. Based on the results of their analysis, the MIT mechanism was made from aircraft grade titanium (Ti). Use of Ti material had three advantages: (1) lower induced radioactivity after irradiation, (2) higher strength, and (3) essentially, immunity to corrosion.

1. Massachusetts Institute of Technology

2. Halden Reactor Project 
The operating force for both mechanisms is generated by pressurizing a miniature bellows, as shown in Figure 1. Bench top testing was completed on the INL stainless steel and MIT Ti versions to verify that the load applied to the specimen could be accurately predicted for various pressures supplied to the bellows. In these tests, each mechanism was attached to a pre-calibrated force gauge, which took the place of the specimen, as shown in Figure 2.

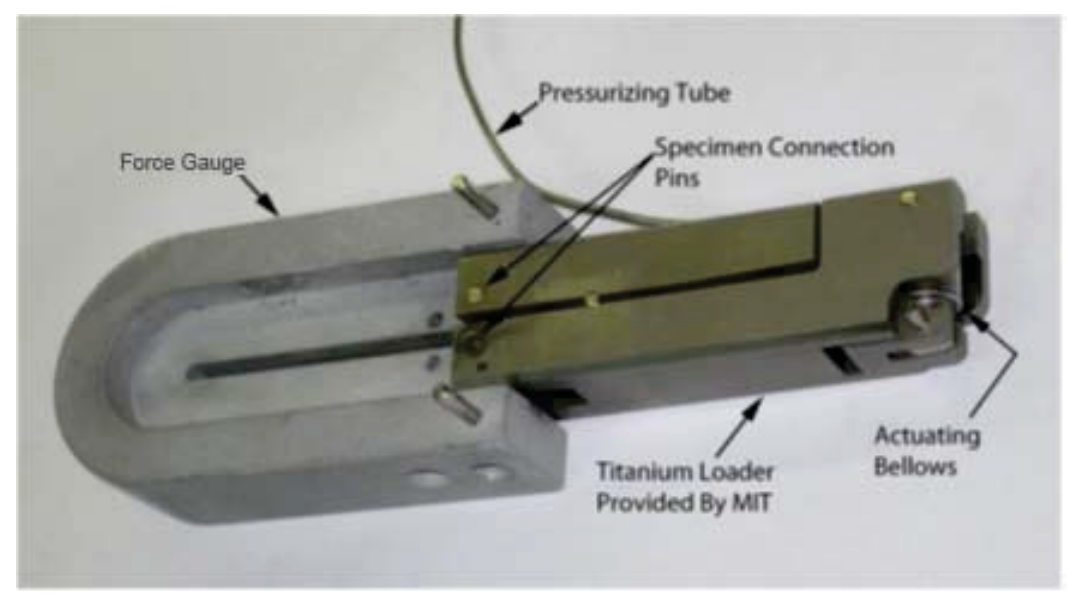

Figure 2. Ti loader connected to force gauge for bench top testing.

Test results (Figure 3 ) demonstrated that the force applied to the specimen pins for a given pressure was very predictable and repeatable while the pressure was ramped up or held constant. As the pressure increased, the deviation between calculated and measured force was less than $4 \%$. However, some hysteresis was evident as the pressure decreased (maximum deviations of $\sim 25 \%$ were observed). Shown on the right side of Figure $3 a$ and $3 \mathrm{~b}$ are data points taken at $1600 \mathrm{psig}(10.9 \mathrm{MPa}$-gauge) after the pressure had been cycled up and down. For both the stainless steel and Ti loaders, resulting forces at these points matched the calculated force within $2 \%$ and nearly duplicated forces obtained during the increasing pressure portion of the cycle.

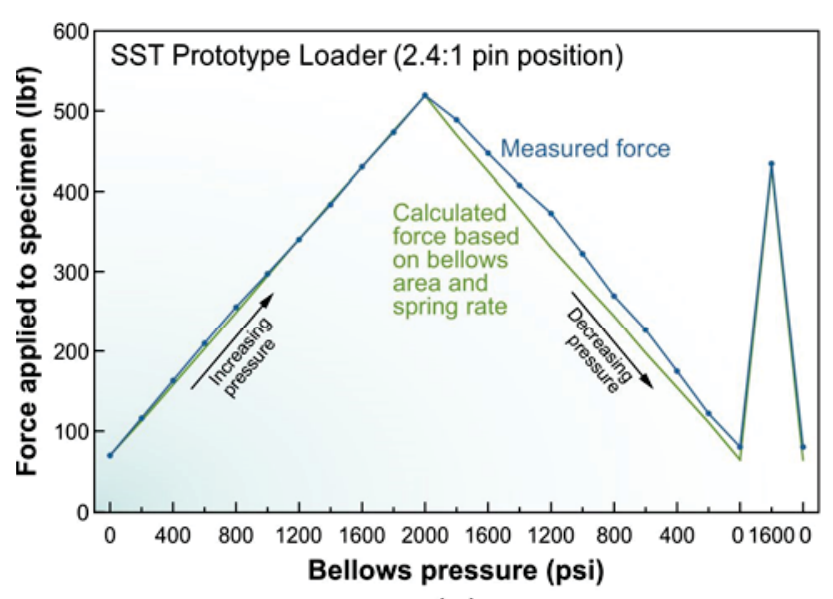

(a)

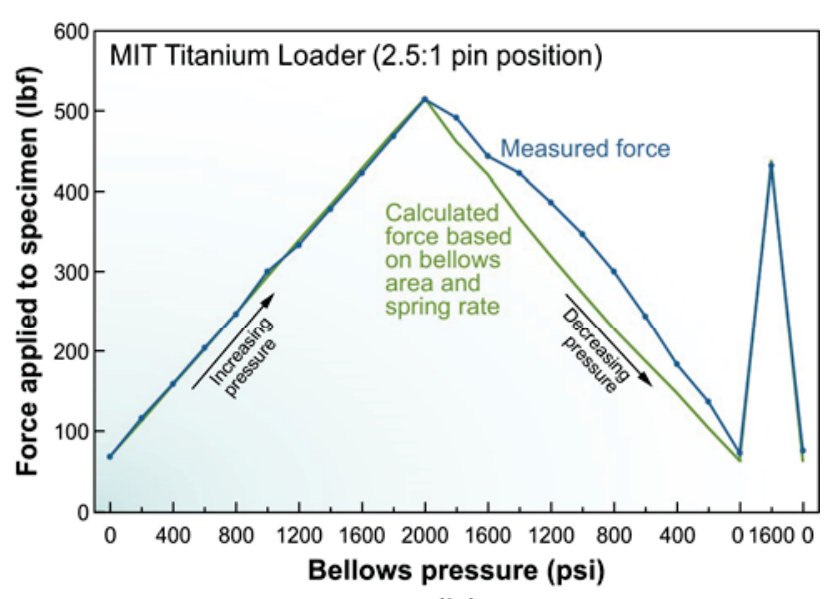

(b)

Figure 3. Force applied to specimen versus pressure: (a) stainless steel loader, and (b) Ti loader. 
Results from bench top testing are very encouraging. During in-pile testing, the pressure applied to the bellows will typically be ramped up and held at a constant pressure. Hence, the method of applying pressure during irradiation should result in a very predictable and stable force on test specimens. It is expected that the pressure to the bellows will be cycled periodically, and bench top tests indicate that as the full pressure is restored, the force will reliably return to the calculated value.

The FY 2013 work also included efforts to develop enhanced techniques for attaching leads to the specimens that will survive in a radiation environment. Halden Reactor experience indicates that the electrical connections needed in the Direct Current Potential Drop method must be extremely robust. During FY 2013, INL developed and fabricated Compact Tension specimens with "ears" attached to the top (Figure 4). Electrical leads attached to the specimen ears consist of mineral insulated cable. This mineral insulated cable is laser welded to an intermediate boss, and the boss is resistance welded to the specimen ears.

This research also funded thesis research for the PI's Ph.D. from the University of Idaho.

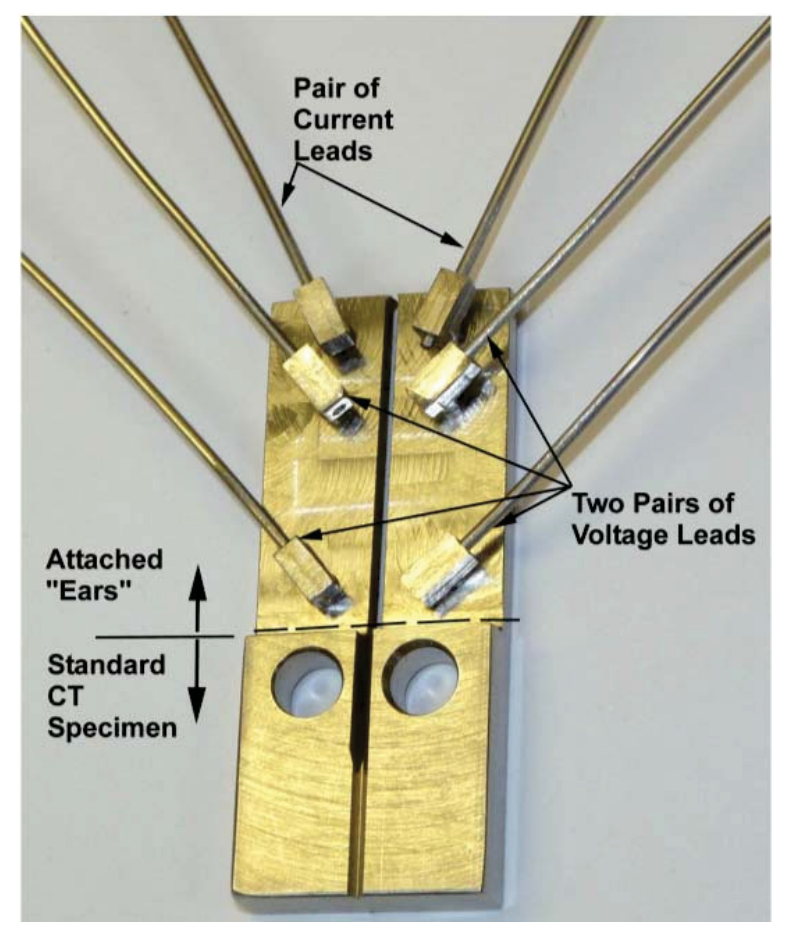

Figure 4. Compact specimen with leads dry fit.

\section{Benefits to DOE}

Development of advanced in-core instrumentation is critical to DOE's energy security mission to provide worldclass facilities for advancing nuclear science and technology. Successful completion of this effort will result in a proven crack growth test rig that can be used by DOE-NE researchers and industry organizations to obtain realtime data for crack growth during irradiation testing in U.S. high flux material testing reactors. 


\section{Publications}

Rempe, J.L., et al., "Advanced In-pile Instrumentation for Materials Testing Reactors," ANIMMA 2013 Special Edition, IEEE Transactions on Nuclear Science, July 2013, Accepted.

\section{Presentations}

Rempe, J.L., et al., "Advanced In-pile Instrumentation for Materials Testing Reactors," 2013 Conference on Advancements in Nuclear Instrumentation, Measurements Methods (ANIMMA 2013), Marseilles, France, June 23-27, 2013. 


\title{
13-032 - Experimental and Computational Analysis of Hydride Microstructures in Zirconium in Dry Storage Conditions
}

\author{
Michael Tonks, Mark Carroll, Laura Carroll, S. Bulent Biner, Jason Hales, and Richard Williamsom
}

The formation of the $\delta-\mathrm{ZrH}_{1.5}$ hydride structure in zirconium $(\mathrm{Zr})$ alloy cladding of reactor fuel has a significant impact on the fuel performance during reactor operation and can have an even larger impact on cladding integrity during fuel storage. One of the most important variables with respect to cladding integrity for used fuel disposition is the orientation of the hydride distribution in the cladding. For this reason, the objective of this project is to quantify $\mathrm{Zr}$ hydride microstructural evolution in pure $\mathrm{Zr}(\alpha-Z r)$ and $Z r-4$ under dry storage conditions. This is being accomplished using closely coordinated experimental analysis and advanced computational simulation to predict the reorientation of hydrides at mesoscopic length scales and, subsequently, to relate these characteristics to a physics-based material model of embrittlement that will be critically useful to dry storage design.

\section{Summary}

As this was the first year of this project, all of the reported progress occurred in FY 2013. Our work was split between the development of a quantitative phase field model of $\delta-\mathrm{ZrH}_{1.5}$ formation and the development of the necessary experimental expertise and materials to hydride $\mathrm{Zr}$ samples, drive the hydride reorientation with applied stress, and evaluate the resultant mechanical properties.

The first step of our model development was to establish the conditions experienced by the cladding through an entire life cycle, including both reactor operation and storage. To accomplish this, the BISON code was modified to include decay heat and convection boundary conditions appropriate for storage environments. With these capabilities, a 10 pellet fuel rodlet was modeled through life in the reactor, 12 years in spent pool storage, a five day vacuum drying period, and 10 years in dry storage. The calculation established the temperature, stress, and deformation history throughout the process.

With the conditions from the BISON simulation, we developed a phase field model of the formation of $\delta$ - $\mathrm{ZrH}_{1.5}$ in pure $\mathrm{Zr}$ (Figure 1a). To ensure that the model is quantitative, we employed the $\mathrm{Zr}$-hydrogen $(\mathrm{H})$ free energy from the ThermalCalc database. We also used accurate measures of the lattice mismatch between the $\mathrm{Zr}$ and the hydride phase. With this information, we have developed a model that can predict hydride nucleation and growth in a single crystal. Future work will include adding the impact of an externally applied stress and investigating the growth in a polycrystal.

The experimental component of this work is divided into three main objectives that were developed in order to support the fundamental modeling of hydride behavior: (1) procure $\alpha-\mathrm{Zr}$ and $\mathrm{Zr}-4$ material and perform hydrideeliciting process treatments, (2) drive the reorientation of the hydrides through the application of a uniaxial stress, and (3) evaluate the mechanical properties that result from varying hydride fractions and orientations. During the first year, an effective experimental setup and process to introduce hydrides into $\mathrm{Zr}-4$ was developed and refined (Figure 1b). Comprehensive scoping studies were effectively carried out that determined the specific conditions necessary to achieve hydride bulk fractions under multiple exposure conditions. Additionally, a fundamental understanding of the hydride response in a specific crystal orientation will be possible based upon the development of a technique to slowly grow large grains in Zr-based materials and effectively cool them through the $\alpha / \beta$ transformation temperature, allowing the extraction of large single crystals of $\alpha-Z r$. 

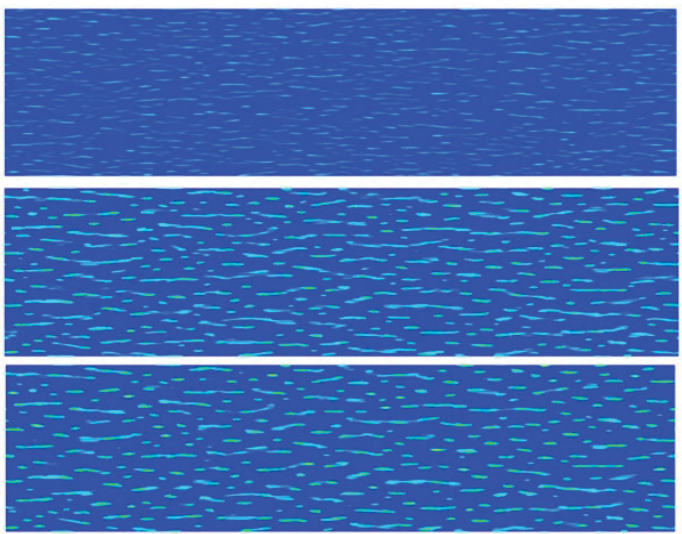

(a)

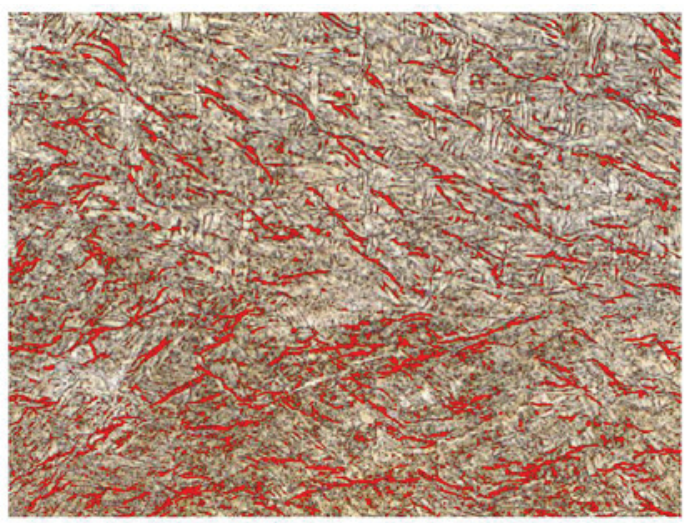

(b)

Figure 1. $\mathrm{ZrH}_{1.5}$ investigation, where: (a) shows the formation of hydride microstructure in a $\mathrm{Zr}$ single crystal predicted by our quantitative phase field method, and (b) is an optical image of $\mathrm{Zr}-4$ hydrided in a $100 \% \mathrm{H}_{2}$ environment, with the hydride precipitates highlighted in red.

The detailed characterization of microstructures decorated with extensive hydride distributions will be possible in FY 2014 by utilizing a specialized heating stage that was procured under this program to allow in-situ transmission electron microscopy on $\mathrm{ZrH}_{2}$ up to and beyond the hydride dissolution temperatures. This suite of equipment and capabilities will be utilized extensively to apply the conditions that enable quantifiable hydride redistribution.

\section{Benefits to DOE}

This project benefits DOE's mission of ensuring our energy security by providing the foundation to accurately obtain new understanding of the thermo-physical conditions of high-burnup fuel cladding during dry fuel storage extending beyond the current 40 year storage norm. 


\title{
13-039 - Induction Based Fluidics Mass Spectrometry for Characterizing Radioactive Extraction Solvents
}

\author{
Gary Groenewold, Gracy Elias, and Bruce Mincher
}

Electrospray ionization (ESI) mass spectrometry (MS) is a powerful approach for the analysis of solutions employed in solvent extraction processes used to separate metal cations found in solutions of used nuclear fuel. Accordingly, there is a strong motivation to use ESI-MS to analyze solutions that may have residual radioactivity, however, this is impractical because of inefficient transfer of sample from solution into the mass spectrometer, which results in loose contamination on the exterior surfaces of the ESI instrument. New technology referred to as induction-based fluidics (IBF) was recently shown to be capable of delivering nanoliter-sized droplets onto laser desorption-MS targets that enabled measurement of minute samples without losses. It was hypothesized that an IBF device fitted to an ESI source would be capable of launching nanoliter droplets into the ESI aperture without losses, eliminating the problem of loose contamination of ESI-MS instrumentation. This would allow for ESI-MS analysis of a much wider range of sample types, including those that contain radioactive contamination.

The approach used in the project was to procure an IBF device from Nanoliter LLC, which was founded by the inventor of IBF, Dr. Drew Sauter. Collaborative research interfaced an IBF to two different ESI-MS instruments, one a quadrupole ion trap MS, and the other a time-offlight MS. Demonstration experiments were conducted using both instruments that analyzed solutions containing an organic ligand used in metal solvent extraction processes, an ionic surfactant typical of an ionic liquid, and lanthanide coordination complexes. Abundant signals were generated from droplets as small as 20 nanoliters (Figure 1), which indicated that the IBFESI-MS approach will function well with radioactive solutions, provided an instrument is relocated within a radiological buffer area. Instrument relocation is planned for FY 2014 as part of the scope of the project.

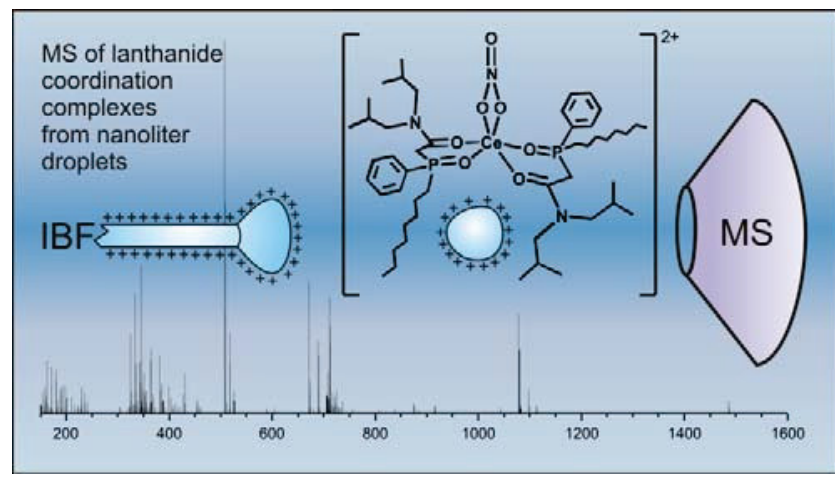

Figure 1. Schematic diagram of the IBF source, launching a nanoliter droplet toward the mass spectrometer aperture. The mass spectrum shows cerium (Ce) coordination complexes with the organic extractant ligand $\mathrm{CMPO}$, for example [Ce $\left.\left(\mathrm{NO}_{3}\right)(\mathrm{CMPO})_{2}\right] 2^{+}$at $\mathrm{m} / \mathrm{z} 508$ (shown by the chemical structure).

\section{Summary}

The IBF source was initially interfaced to a quadruple ion trap mass spectrometer, which is an MS platform that is small, relatively inexpensive, and easily situated in a radiation buffer or contamination area. Analysis of dilute solutions of organic compounds used in solvent extraction (specifically carbamoylmethyl-phosphine oxide [CMPO] derivatives) showed facile, sensitive measurement of the solutes in droplets having volumes ranging from 20 to 100 nanoliters. CMPO concentrations in these experiments were in the micromolar range, and the droplets were delivered to the mass spectrometer without apparent losses. Subsequent experiments using the ion trap with the IBF source demonstrated detection of ionic surfactants (tetraalkylammonium salts) at very dilute concentrations $\left(1 \times 10^{-9} \mathrm{M}\right)$, which corresponded to observation of an absolute mass of 35 femtograms $\left(35 \times 10^{-15} \mathrm{~g}\right)$ from a 30 nanoliter droplet. These results strongly suggested that the combination of the IBF source with the ion trap 
mass spectrometer is indeed capable of analyzing radioactive samples without seriously contaminating the ESI source.

The ion trap experiments were limited by the need to precisely time the arrival of the solute ions from the nanodroplet with the ion trapping period; the ion trap functions by trapping ions delivered from the ion source for a short period of time and then scanning them out in a fashion that enables measurement of their mass-to-charge ratio. During the course of the initial experiments, reproducible synchronization of the ion arrival from the IBF source and ion trapping was not achieved, which resulted in droplet-to-droplet imprecision that was overcome by signal averaging. The result suggested that if synchronization were improved, even more impressive measurement results would be achieved.

An alternative solution to the synchronization problem is to utilize a mass spectrometer having a faster duty cycle. To accomplish this, the IBF source was interfaced to an ESI mass spectrometer equipped with a time-of-flight analyzer, which was operated at about 10 scans per second. Analysis of 20 nanoliter-sized droplets containing micromolar concentrations of lanthanide metals and CMPO produced highly reproducible spectra with excellent signal-to-noise. The IBF source was also operated at a fast duty cycle, delivering a drop every 0.8 seconds, which indicated that rapid analysis of multiple, nanoliter-sized samples was a viable option.

\section{Benefits to DOE}

There is an ongoing need for chemical measurement of vanishingly small sample volumes across three DOE mission areas: nuclear energy, national security, and sustainable energy. The need is especially acute for scenarios in which the sample size is small or poses risks from toxicity or radioactivity. ESI-MS provides an excellent level of information on the solution solutes but is not compatible with radioactive samples because the majority of the sample winds up deposited on the exterior surfaces of the source, where it constitutes loose contamination. The IBF project produced a high performance ESI-MS approach that provides detailed molecular information for organic and organometallic compounds from nanoliter-sized droplets, without contaminating the exterior surfaces of the ESI equipment. This opens the door for analysis of a wide range of radioactive solutions not previously compatible with ESI-MS. When the instrumentation is relocated in a radiological buffer area, it will constitute a capability that is rare in the analytical chemistry and radiochemistry communities and would significantly enhance INL's competitive position in the fuel cycle separations area. In addition, the capability would open new avenues for scientific and technological advancement, particularly in process control, since the proposed technology is capable of solution analysis using exceedingly small volumes.

\section{Publications}

Groenewold, G.S., A.D. Sauter, Jr., and A.D. Sauter III, "Rapid Analysis of Single Droplets of Lanthanide-Ligand Solutions by Electrospray lonization Mass Spectrometry Using an Induction Based Fluidics Source," Anal. Chem., Vol. 85, 6398-6404, July 2, 2013. 


\title{
13-040 - Epistemic Uncertainty Quantification in Dynamic Probabilistic Risk Assessment
}

\author{
Joseph Nielsen, Akira Tokuhiro, ${ }^{1}$ and Robert Hiromoto ${ }^{1}$
}

Dynamic Probabilistic Risk Assessment (DPRA) is a powerful tool that provides a more rigorous analysis of complex dynamic systems as opposed to classical risk assessment tools such as fault tree/event trees. DPRA methods allows for coupling to simulation tools such as RELAP5, which allows for capturing the actual timing of events rather than utilizing an expert knowledge-based approach that results in grouping multiple transients together. Uncertainty quantification (UQ), both aleatory and epistemic, can be evaluated concurrently within the simulation. Aleatory uncertainty refers to random or stochastic uncertainty, which is inherent to the system under study. Epistemic uncertainty refers to the uncertainty of the state of limited knowledge about the system on the part of the analyst. Epistemic uncertainty can be narrowed or eliminated with additional knowledge.

DPRA methods have a disadvantage resulting from combinatorial or state explosion. As the number of potential system states grows, the size of the simulation grows exponentially. Solution methods utilize either a Monte Carlo-based approach or Discrete Dynamic Event Trees (DDET). We are evaluating the use of DDET with the use of the branch-and-bound algorithm and linear programming methods to solve the DDET efficiently and limit the potential for state explosion. This allows us to focus on scenarios and timing of events that lead to failure. In many cases, with regard to failure of equipment or systems, failure rates are extremely small (approximately $1 \times 10^{-6} / \mathrm{hr}$ ). The algorithm can direct the simulation to high probability failure scenarios and conditions for efficient UQ with regard to both epistemic and aleatory uncertainty. Further, epistemic uncertainty can be quantified and ranked with regard to where additional information pertaining to modeling fidelity and validation experiments can yield the greatest amount of risk reduction.

\section{Summary}

The first year of this proposed two year project was devoted to implementation of the optimization algorithm and methods to establish constraints and bounding functions. The branch-and-bound algorithm is an optimization algorithm intended to find the maximum (or minimum) value of a function associated with combinatorial explosion problems. In collaboration with the University of Idaho, we have co-developed and integrated a set of tools in a consistent manner; in particular, a potentially practical coupling of RELAP5, a 'controller tool' to start and stop the time-dependent calculation, definition of sets per translation of expert/experiential knowledge of reactor transients, linear programming as a means to establish linked conditions and constraints on event 'branches,' and a branchand-bound (programming) approach to 'trim' branches yielding desirable outcomes (such as cold shutdown condition) when the interest is the opposite (i.e., events leading to potential core meltdown). Furthermore, we have adopted the LENDIT metrics to essentially reduce the system characterization in a consistent manner. LENDIT metrics, which represent Length, Energy, Number, Distribution, Information, and Time, can be used with S2R2 (State, System, Response, Resource) sets. Linking of these elements is facilitated by utilization of expert/experiential knowledge referred to as 'knowledge base mining.' This allows us to establish constraints and bounding functions to support the branch-and-bound algorithm. The use of LENDIT metrics for the reduction of system characterization has been evaluated to be consistent with the operator splitting techniques employed in the RAVEN framework.

1. University of Idaho 
Within the framework of the object-oriented programming paradigm, the University of Idaho programming members have formulated a first draft DPRA event tree object-oriented programming design. The design specifications are detailed in the Unified Modeling Language, which is a general-purpose modeling language used extensively in software engineering applications. The branch-and-bound method fits naturally within this objectoriented programming construct, and the structure also exposes the potential parallelism that can be adapted in software compatible for RAVEN/MOOSE integration.

In addition to the work performed this year, we were able to implement the branch-and-bound algorithm to evaluate the station blackout transient of a Westinghouse pressurized water reactor. A dynamic event tree was created and coupled with the RELAP5-3D thermal-hydraulic code. Using these methods, we were able to achieve over $33 \%$ reduction in simulation time in finding the highest probability of core damage. The current algorithm relies on a depth first approach to searching the tree, where a combined breadth and depth search would yield greater efficiency in the simulation. The efficiency achieved by the algorithm is dependent upon specific scenarios; it is anticipated that significant reductions in simulation time will be achieved.

Work to begin implementation of these methods within the RAVEN/MOOSE framework is currently underway. These methods can now be incorporated with sensitivity analysis and UQ methods. Methods developed within the RAVEN framework, or tools such as DAKOTA from Sandia National Laboratory, can be used concurrently to provide Sensitivity and Uncertainty Quantification (SU/UQ) during the DDET simulation. These tools will provide more information, such as covariance data to response functions, to support developing constraints within the optimization algorithms.

\section{Benefits to DOE}

The methods and algorithms developed during this research can have a significant benefit to DOE's nuclear energy mission. Improving the capability to quantify risk with regards to modeling and simulation can result in significant cost savings. Validation experiments can be efficiently designed to provide data with regard to risk reduction. Implementation of these algorithms can begin using object-oriented methodology within the RAVEN framework, which is under development as part of the MOOSE project. This will allow for implementation of the methods regardless of the simulation tool.

\section{Publications}

Nielsen, J.W., et al., "Method to Branch and Bound Large SBO State Spaces under Dynamic Probabilistic Risk Assessment via Use of LENDIT Scales and S2R2 Sets," J. Nucl. Sci. Technol., Submitted.

\section{Presentations}

Nielsen, J., A. Tokuhiro, and R. Hiromoto, "Branch and Bound Algorithm Applied to Discrete Dynamic Event Trees," Transactions of the American Nuclear Society 2013 Winter Meeting, Washington, D.C., November 10-14, 2013, Paper accepted.

Nielsen, J., A. Tokuhiro, and R. Hiromoto, "Method to Branch and Bound Large SBO State Spaces under Dynamic Probabilistic Risk Assessment via Use of LENDIT Scales and S2R2 Sets," Probabilistic Safety Assessment and Management Conference, Tokyo, Japan, April 15-18, 2013. 


\section{3-050 - Concurrent Atomistic to Macroscale Modeling of Materials Under Irradiation Using the Phase Field Crystal Model}

Michael Tonks, Yongfeng Zhang, Katsuyo Thornton, ${ }^{1}$ S. Bulent Biner, Richard Williamson, Derek Gaston, and Dmitry Karpeev

Radiation damage has a large impact on material behavior, leading to material swelling and embrittlement as well as significant changes in material properties such as elastic constants. In this project, we are developing a multiscale model that bridges the atomistic to the macroscale in order to capture the critical mechanisms occurring during the early stages of irradiation. This is being accomplished by extending our existing multiscale framework to the atomistic-scale by implementing the phase field crystal (PFC) model within INL's MARMOT code. The PFC model uses free energy equations to define the behavior of periodic variables, providing atomisticscale resolution at areas of interest (grain boundaries, dislocations, etc.) but with diffusive time scales.

In this project, we are developing a PFC model of a single-component body-centered cubic (BCC) metal that captures the effects of radiation damage by randomly introducing vacancies and interstitials at a controlled rate. We will expand the model to be truly multiscale, with atomic resolution around defects and mesoscale resolution within the perfect lattice, and implement it in MARMOT. Then, this model will be coupled to BISON, where the temperature and neutron flux will be passed down to MARMOT, and the elastic constants will be passed back to BISON. Thus, we will obtain a first-of-its-kind multiscale capability that bridges the atomic to the macroscale.

\section{Summary}

As this was the first year of this project, all of the reported progress occurred in FY 2013. There were two main tasks for the project this year: first, implementing the PFC model in the MARMOT code and second, enhancing the PFC model to capture material-specific behavior for a BCC metal. The first task was completed at INL with help from Dmitry Karpeev at Argonne National Laboratory, while Professor Thornton and her team completed the second at the University of Michigan (UM).

We successfully implemented the PFC model in MARMOT for a BCC iron (Fe) material, using the formulation from, "Thermodynamics of BCC Metals in Phase-Field-Crystal Models," by Jaatinen et al. However, solving this model, which contains a sixth-order gradient term, was a difficult task. In order to use traditional finite elements, we introduced two substitutional variables, giving three secondorder residual equations rather than a single sixth-order equation. Using this approach, we have modeled solidification and evolution in
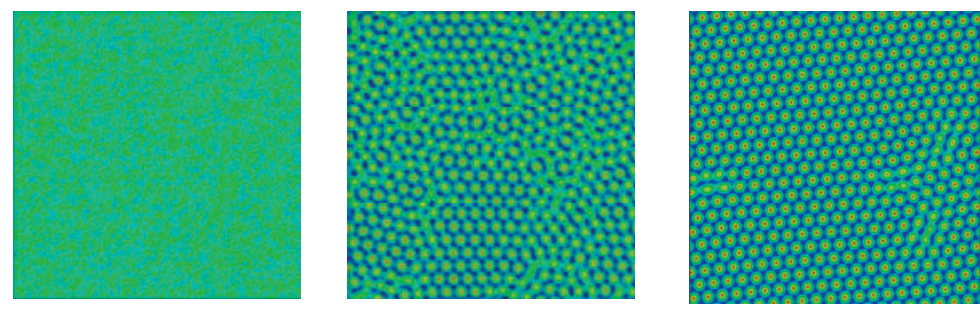

Figure 1. Results from a 2-D PFC simulation of solidification in Fe using MARMOT. The figures show the probability of finding an atom, with the initial liquid (left), the polycrystal structure after some time (center), and the final, nearly single crystal, structure (right). 
This represents the first implementation of the PFC model based on classical density functional theory using traditional finite element method and the first application of the MOOSE framework to model atomistic phenomena.

Our collaborators at UM are developing a PFC model that has the capability to model specific materials with the full two-body direct correlation function in real space, as described in Physical Review E by Pisutha-Arnold et al., called the rational function fit form. The rational function fit form of the PFC model uses rational functions to fit the two-body direct correlation function in Fourier space; in real space, the rational function fit becomes a series of second-order Helmholtz equations that replaces the higher-order gradients of the classical density functional theory-based PFC model by Jaatinen et al. At INL, we have begun to implement it in MARMOT and are working with Dmitry Karpeev to determine the best methods with which to solve the Helmholtz equations.

Research at UM has also focused on determining the correct approach to determine the effective elastic constants using the PFC model. This capability is essential for our multiscale development work since the effective elastic constants calculated at the atomic scale will be passed up to BISON. However, in existing work in the literature, elastic constants are usually calculated using an approach that has been shown to not conserve mass. Consequently the results obtained have been shown inconsistent with those defined from the theory of thermoelasticity of stressed materials. As a correction of this, a new approach which conserves mass during deformation has been proposed by our UM collaborators in the paper "Calculations of Isothermal Elastic Constants in the Phase-Field Crystal Model" by Pisuth-Arnold et al. Using this new approach, calculations of the elastic constants are currently ongoing with both the classical density functional theory-based and the rational function fit PFC models.

In addition to the technical goals of this project, we are also dedicated to educating a new generation of nuclear scientists. To this end, a postdoctoral researcher was hired to work on this project at UM in Professor Thornton's group. In addition, a student who recently graduated with a bachelor's degree in physics is working on implementing the PFC model within MARMOT at INL.

\section{Benefits to DOE}

This project will benefit the DOE missions of energy resources and science by developing the capability to model radiation damage concurrently from the atomistic to the macroscale. This will provide a better fundamental understanding of radiation damage, allowing for the design of accident tolerant reactors.

\section{Publications}

Butterfield, A., et al., "Scalable Solving of Phase Field Crystal Models Using Finite Element Method Based Solver," 2014, In preparation.

\section{Presentations}

Chan, V., et al., "Phase-Field Crystal Model and Thermodynamics of Elastically Stressed Solids," Materials Science \& Technology 2013 Meeting, Montreal, Canada, October 29, 2013.

Butterfield, A., et al., "Obtaining Elastic Constants Using Phase Field Crystal Modeling," 2014 American Nuclear Society Annual Meeting, Reno, NV, June 15-19, 2014, In preparation to be submitted. 


\title{
13-071 - Advanced Fracture Modeling for Nuclear Fuel
}

\author{
Benjamin Spencer, Hai Huang, Jason Hales, Derek Gaston, and John Dolbow ${ }^{1}$
}

Fracture in ceramic nuclear fuel plays an important role in its thermal and mechanical behavior. Currently employed techniques for fracture modeling in fuel have well-known mesh dependencies. Improved fracture modeling for nuclear fuel is essential for predictive modeling of fuel behavior in conditions such as accidents, which are outside the range of applicability of current fuel behavior models. This project applies two fracture modeling techniques to fuel performance: the extended finite element method (XFEM), and the discrete element method (DEM). Both of these techniques are well-known to overcome weaknesses of methods used in current practice, but have had limited applications to multiphysics problems, and to our knowledge, have not been applied to nuclear fuel modeling. This project is making these methods available in the BISON nuclear fuel performance modeling code and demonstrating their applicability to nuclear fuel simulation. This will strengthen our ability to predict fuel behavior in normal and accident conditions.

\section{Summary}

This project, which just completed its first year, consists of concurrent efforts to apply the XFEM and DEM fracture modeling techniques to nuclear fuel. In XFEM, the standard finite element method is enhanced to permit it to model discontinuities in solution fields, such as those that occur across a crack. The standard continuous finite element basis functions are enriched with discontinuous functions that enable cracks or other material interfaces to be dynamically inserted at arbitrary locations in the mesh, which do not have to coincide with element boundaries.

The focus for the XFEM effort has been to implement this method in the MOOSE finite element framework, upon which the BISON fuel modeling code is based. XFEM has been implemented in BISON using the phantom node technique, which is a variant of XFEM in which elements containing discontinuities are split into two specially treated elements, and the mesh is connected in a way that properly represents discontinuities of the solution fields across the interface and continuity of fields on either side of the interface. An algorithm to perform this mesh cutting was developed following the work of Richardson et al. (2011), with several improvements. MOOSE-based codes can now use this algorithm to prescribe discontinuities on a mesh. Because MOOSE is an inherently multiphysics computational platform, adding this mesh-cutting algorithm enabled a first-of-its-kind capability to solve arbitrary multiphysics problems with discontinuities represented with XFEM. Figure 1 shows a demonstration of this capability in BISON on a tightly

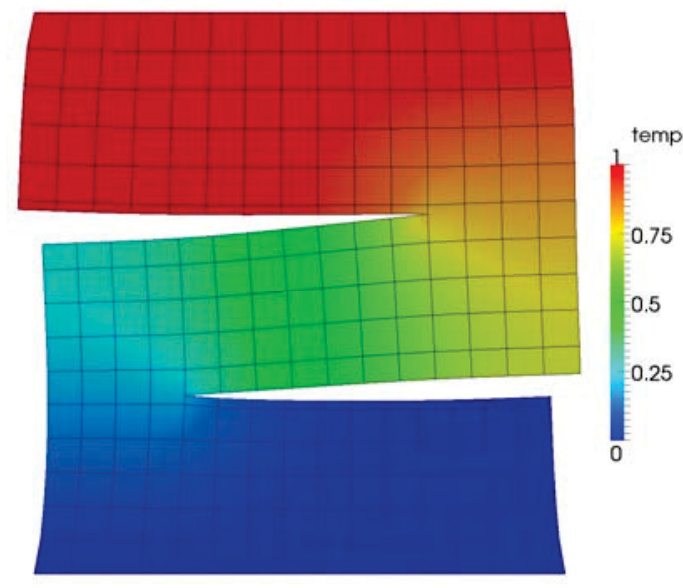

Figure 1. Tightly coupled, thermal/mechanical BISON simulation of a cracked domain. Two cracks are defined at arbitrary locations independent of the mesh using XFEM. Effects of the cracks are shown on the temperature field (shown with contours), and the displacement field (shown with deformed mesh).

1. Duke University 
coupled, thermal/mechanical model of a domain cut by two arbitrarily defined cracks. This will be extended to permit arbitrary crack propagation and applied to fuel fracture simulations in BISON.

DEM is a numerical modeling technique that represents a solid volume with a network of particles connected by mechanical bonds. A major strength of this method is that it is naturally able to represent arbitrary fracture by breaking those bonds once a failure criterion has been met. In this project, a series of proof of concept 2-D DEM simulations of fracture in nuclear fuel were performed using an existing research code. These simulations demonstrated the ability of a coupled thermal/mechanical DEM model to realistically model the formation of radial cracks in nuclear fuel during an initial power-up. Figure 2 shows the crack patterns predicted by the DEM model at various power levels. The DEM model also predicted circumferential crack pattern formation during powerdown. This work is documented in a recently submitted journal publication. An initial, limited DEM capability has also been implemented in the BISON code. This will be expanded to permit DEM modeling of fuel to include the full set of fuel material and behavior models available in BISON.

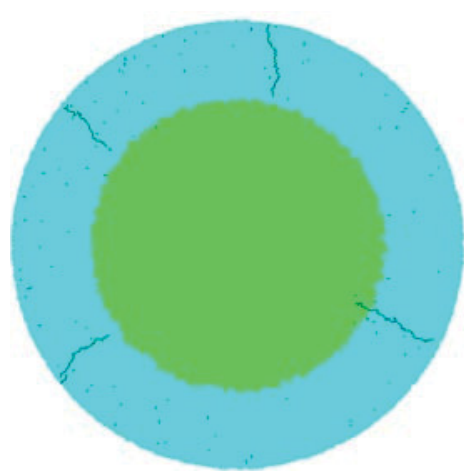

(a) Peak power=10 kW/m

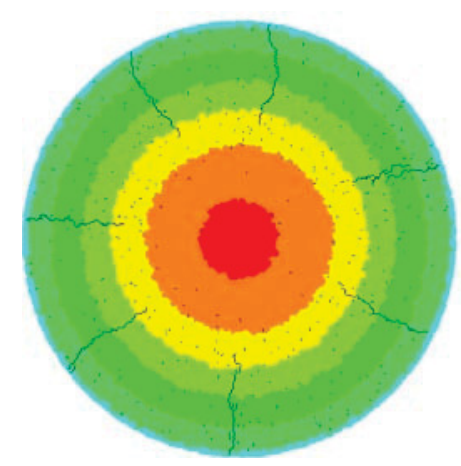

(b) Peak power=25 kW/m

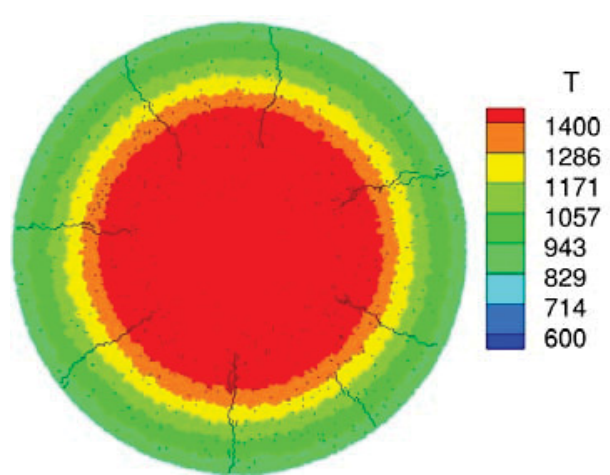

(c) Peak power=40 kW/m

Figure 2. Results of 2-D DEM simulations of a fuel pellet cross section showing temperature $(K)$ and crack patterns (black lines) following initial power ramp.

\section{Benefits to DOE}

This project has already demonstrated improved capabilities for fracture modeling in nuclear fuel. This will provide a stronger basis for predictive simulation of fuel designs and postulated accident scenarios that are outside the realm of applicability of methods currently in use by DOE. The fracture modeling techniques developed in this work are also directly applicable to modeling fracture in other nuclear power plant components, such as reactor pressure vessels and containment vessels. This will improve the safety and efficiency of nuclear power generation.

\section{Publications}

Huang, H., B. Spencer, and J. Hales, "Discrete Element Method for Simulation of Early-Life Thermal Fracturing Behavior in Ceramic Nuclear Fuel Pellets," Nucl. Eng. Des., Submitted. 


\title{
13-097 - MOOSE Capability Extension In Support of Full Core Modeling
}

\author{
Derek Gaston, Cody Permann, David Andrs, John Peterson, and Dmitry Karpeev ${ }^{1}$
}

The MOOSE framework is currently being utilized by multiple projects to simulate physics related to nuclear reactor core modeling. This project is focused on adding capability to MOOSE to provide the foundation for solving multiple coupled physical systems (such as fluid flow, neutronics, and fuels performance) simultaneously. Utilizing this capability, it will be possible to use existing MOOSE-based applications, without modification, to model nuclear reactor cores. This will enable INL to enhance their class-leading status for multiphysics simulation capabilities.

\section{Summary}

This LDRD funded extremely significant development in the initial capability towards modeling a full reactor core including microstructure, fuel performance, neutronics, and thermal-hydraulics, all simultaneously. This was accomplished by meeting our milestones throughout the year:

1. Preliminary support for the Portable, Extensible Toolkit for Scientific Computation time integration has been added, and some demonstration cases have been performed. Better time integration will give more accurate solutions for complicated multiphysics problems.

2. Preliminary mortar finite element support was added giving us a new and unique capability for modeling gap heat conduction, solid mechanical contact, fluid structure interaction, etc.

3. Two new systems were added to MOOSE: Transfers and MultiApps. These two systems work in tandem to link together multiple MOOSE-based solves (including separate MOOSE-based applications), giving us a flexible capability for loose-coupling.

Utilizing these new capabilities, we were able to link together multiple existing MOOSE-based applications (without modification) to achieve a proof of concept simulation of a nuclear reactor core (Figure 1). This activity has catapulted INL modeling and simulation well into the lead of simulation of nuclear reactors and has already had a large impact on funding decisions from national programs such as Nuclear Energy Advanced Modeling and Simulation and Consortium for Advanced Simulation of Light Water Reactors. Further, industry has taken notice, with both Westinghouse and General Electric now partnering with us. A presentation on this capability was delivered as a plenary at the 2013 American Nuclear Society Mathematics \& Computation meeting in Sun Valley, Idaho. In addition, Assistant Secretary for Nuclear Energy Pete Lyons requested a personal presentation detailing this

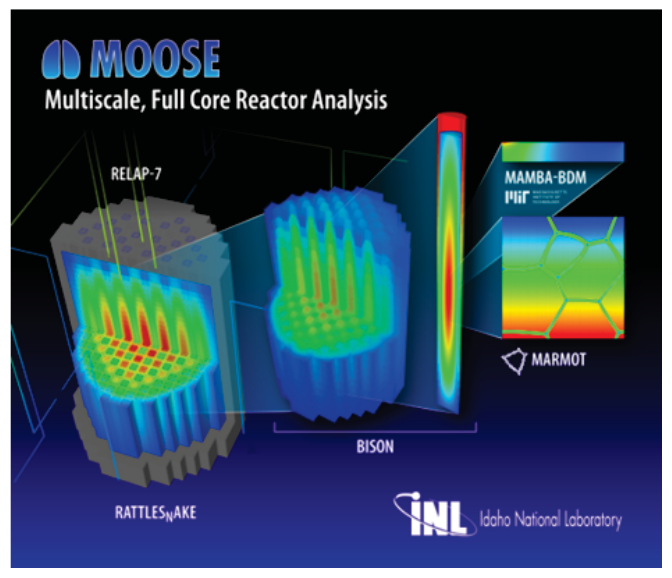

Figure 1. Multiscale Westinghouse AP1000 simulation performed utilizing new capabilities developed by this LDRD. 
capability, prompting him to take a trip to Idaho. The Idaho chapter of the American Nuclear Society invited Derek Gaston to speak on this topic at a special summer dinner event. Derek also spoke on this topic as the closing plenary lecture at American Nuclear Society Global 2013. An article detailing the new capability and initial results is currently being prepared for publication in a special edition of the Annals of Nuclear Energy American Nuclear Society journal.

Going forward, there are still many tasks left before MOOSE can be a complete platform enabling full core reactor simulation (and other problems of that nature, including underground used nuclear fuel storage). The MultiApp system needs many enhancements including the ability to perform fixed-point iterations. Transfers need to be completely overhauled and redesigned. While the current design met the present goals, it needs to evolve to allow transfers to deal with two very important capabilities: ParallelMesh (enabling better memory use through domain decomposition) and full coupling across different meshes. The mortar method implementation shows great promise on small example problems but will need to continue to gain a lot of capability (including working with moving meshes and mesh adaptivity) before it can be utilized for a production problem.

\section{Benefits to DOE}

DOE's national security mission of energy resource utilization is a direct benefactor of this research as a result of eventual improvements in reactor design and operation (such as optimization of fuel shuffling patterns). Nuclear security is enhanced by this project through the ability to design safer fuel and reactors. An eventual usage of this capability is planned for modeling spent nuclear fuel storage, enhancing environmental quality.

\section{Publications}

Gaston, D.R., et al., "Continuous Integration for Concurrent Computational Framework and Application Development," Workshop on Sustainable Software for Science: Practice and Experiences, Denver, CO, November 17, 2013, Accepted.

Gaston, D.R., et al., "Massive Hybrid Parallelism For Fully Implicit Multiphysics," Proceedings of the International Conference on Mathematics and Computational Methods Applied to Nuclear Science and Engineering, Sun Valley, ID, May 5-9, 2013.

\section{Presentations}

Gaston, D.R., et al., "Advanced Simulation of LWRs using MOOSE," American Nuclear Society Global, Salt Lake City, UT, September 29-October 3, 2013.

Gaston, D.R., et al., "Multiphysics, Multiscale Algorithms for Nuclear Power Applications," American Nuclear Society Mathematics \& Computation, Sun Valley, ID, May 5-9, 2013.

Gaston, D.R., et al., "Massive Hybrid Parallelism for Fully Implicit Multiphysics," American Nuclear Society Mathematics \& Computation, Sun Valley, ID, May 5-9, 2013. 


\title{
13-115 - Multi-scale Full Core Reactor Physics Simulation
}

\author{
Mark DeHart, Yaqi Wang, Kord Smith, ${ }^{1}$ Jim Morel, ${ }^{2}$ Cristian Rabiti, Hongbin Zhang, Richard Martineau, and
} Barry Ganapol ${ }^{3}$

Historically, experiments have been performed to understand fuel performance and for all aspects of fuel design; the 52 reactors built at INL since 1949 are a testament to that history. In today's budget-restricted environment, the cost of data acquisition through experimental testing severely limits the design and construction of experimental reactors and even measurements in existing reactors. However, such data are still needed to improve reactor design and provide the ability to advance the state-of-the-art. Hence, it is necessary to develop alternate tools that can be used to test the potential feasibility of design concepts before proceeding with fabrication. The concept of a virtual reactor provides such a capability, and provides the means to substantially reduce the cost of experimentation by allowing virtual experiments before any hardware development is initiated. Because the ATR is designed to drive a wide range of experimental phenomena, it is the perfect vehicle for demonstration of the concept of a virtual reactor. Hence, the objective of this research is to develop a prototypic, multi-scale, multiphysics virtual reactor based on ATR. Specifically, the research team is working to develop a neutron transport solver with RattleS $S_{N}$ ake (further explained in the publications produced by this project), a neutron transport application built upon INL's MOOSE framework (first demonstrated in 2009 by D.R. Gaston, C. Newman, G. Hansen, and D. Lebrun-Grandie). In this research, a three scale solution is proposed: fuelresolved, plate-homogenized, and element-homogenized scales. This approach allows the user to focus computational effort on the region of the core that is of most interest, while still providing coupling to other regions of the core that affect the region of interest. This multi-scale approach will make a full, three-dimensional virtual reactor model tractable on existing computational platforms. The resulting capability is to be used to support the mission of performance improvements together with continued safe and productive operation of the ATR. In addition, the technology developed here will ultimately be of value for support of the existing fleet of experimental and power reactors, and assist in the design to future reactor types.

\section{Summary}

Multiple scales require multiple levels of resolutions corresponding to different discretization schemes.

RattleS $S_{N}$ ake currently solves the radiation transport equation in the Self-Adjoint Angular Flux (SAAF) formulation discretized with a multigroup approximation in energy, a continuous finite element method (CFEM) in space, and a discrete ordinates $\left(S_{N}\right)$ approximation in angle, demonstrated in an upcoming article by $Y$. Wang, $H$. Zhang, and R.C. Martineau in Nuclear Science and Engineering. Two new transport schemes have been developed: the spherical harmonics expansion method $\left(P_{N}\right)$ and the least-squares method with CFEM and $S_{N}$.

The $P_{N}$ method provides an alternative discretization scheme created particularly for the plate-homogenized and element-homogenized scales. The $\mathrm{P}_{\mathrm{N}}$ implementation takes advantage of the components already present in Rattle $S_{N}$ ake and is still based on the SAAF formulation with CFEM. This provides strong continuity of both the even and odd parity expansion moments with SAAF, which is different than the traditional parity-based $P_{N}$ methods. To our knowledge, this is the first multi-dimensional implementation of the $P_{N}$ method for the SAAF

1. Massachusetts Institute of Technology

2. Texas A\&M University

3. University of Arizona 
transport equation. The implementation of a $\mathrm{P}_{\mathrm{N}}$-based solver allows for novel comparisons, verification, and analysis between the $S_{N}$ and $P_{N}$ implementations as well as comparisons between the $P_{N}$ and analytical benchmarks. The implementation has been tested with benchmark problems, including the assemblyhomogenized International Atomic Energy Agency 2-D pressurized water reactor benchmark. Convergence of the solution with increasing $\mathrm{P}_{\mathrm{N}}$ order has been observed, as illustrated in Figure 1. Note that the stair-stepping error pattern between consecutive N's is caused by an effective de-coupling of the spherical harmonic moments on the problem interior.

Although the existing discretization scheme (SAAFCFEM-S $S_{N}$ ) can treat void regions, it introduces an extra stabilization term that destroys the symmetry of the streaming and collision operator. A new form of a secondorder self-adjoint transport equation has been developed that has an advantage relative to SAAF-CFEM- $S_{N}$ in that the streaming and collision operator is symmetric positive definite. This equation is closely related to the standard least-squares or normal form of the transport equation. However, unlike the standard least-squares form of the transport equation, our least-squares equation is compatible with source iteration. This derivation has been implemented in Rattle $S_{N}$ ake with support from Texas A\&M University, as explained by J.B. Hansen and J.E. Morel in the presentation, "A New Least-Squares Transport Equation Compatible with Voids." In future work, we will investigate more effective solvers to take full advantage of the symmetric positive definite streaming and collision operator.

One of the objectives in this research is to couple a high-order transport solver with the low-order diffusion solver provided by RattleS ${ }_{N}$ ake through nonlinear diffusion acceleration. Current efforts have used the OpenMC Monte Carlo code to provide the high-order solution. The OpenMC project within the Computational Reactor Physics Group at the Massachusetts Institute of Technology (MIT) aims to provide a fully-featured Monte Carlo particle transport code based on modern methods. The nature of the high-order/low-order coupling provides RattleS ${ }_{N}$ ake with access to spatially averaged data available from the rigorous transport capabilities made possible by OpenMC. This provides RattleS ${ }_{N}$ ake with appropriately weighted cross sections from the continuous energy OpenMC transport calculation. INL has derived consistent nonlinear diffusion acceleration equations and identified the parameters to be passed between OpenMC and Rattle $S_{N}$ ake. From this work, a generic interface has been developed. This generic interface has been used for SAAF-CFEM- $S_{N}$ calculations described by Yaqi Wang at the International Conference on Mathematics, Computational Methods \& Reactor Physics.

Research this year has also explored the best practice for pin-homogenized calculations with the Benchmark for Evaluation and Validation of Reactor Simulations benchmark developed at MIT, which is a detailed pressurized water reactor benchmark containing real plant data for assessing the accuracy of reactor physics simulation tools. 
This benchmark has been used to assess Rattle $S_{N}$ ake performance for pin-homogenized calculations. Rattle $S_{N}$ ake results have been found to be in excellent agreement with benchmark results and partially validate the methods and coupling mechanisms that have been completed to-date. Compared to the 35-group CASMO-5 Method of Characteristics calculations using a Cartesian barrel representation, the maximum difference of assembly powers is $-2.7 \%$, located in the outer assemblies with the core baffle on two sides. The root mean square of assembly power differences is $0.96 \%$. Figure 2 illustrates the RattleS ${ }_{N}$ ake results for the benchmark calculation.

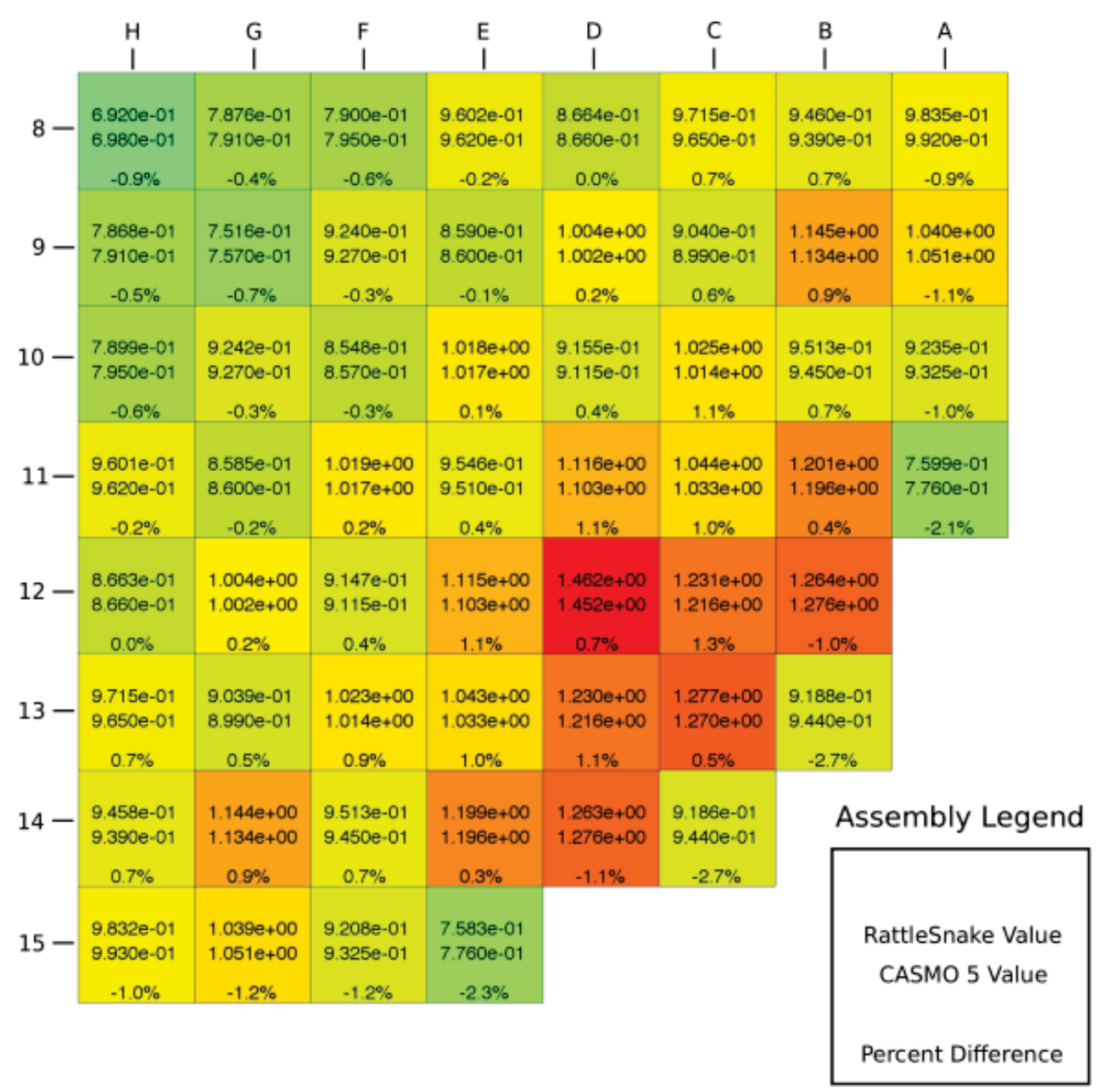

Figure 2. Rattle $S_{N}$ ake results for MIT's Benchmark for Evaluation and Validation of Reactor Simulations pressurized water reactor benchmark. 


\section{Benefits to DOE}

These multi-scale methods are laying the groundwork for next generation multi-physics analysis of ATR for improved performance, safety, and economics. This will, in turn, improve the efficacy of the ATR mission in support of Naval Reactors, NE, and National Nuclear Security Administration needs. Upon demonstration of the applicability of this approach to ATR, the technology could and should be extended to light water reactors and next generation reactor designs, consistent with DOE Office of Science and NE programs.

\section{Publications}

Wang, Y., H. Zhang, and R.C. Martineau, "Diffusion Acceleration Schemes for the Self-Adjoint Angular Flux Formulation with a Void Treatment," Nucl. Sci. Eng., 2013, Accepted.

\section{Presentations}

Gleicher II, F.N., Y. Wang, D. Gaston, and R.C. Martineau, "The Method of Manufactured Solutions for RattleS ${ }_{N}$ ake, an $S_{N}$ Radiation Transport Solver Inside the MOOSE Framework," T. Am. Nucl. Soc., Vol. 106, 372-374, 2012.

Wang, $Y$., "Nonlinear Diffusion Acceleration for the Multigroup Transport Equation Discretized with $\mathrm{S}_{\mathrm{N}}$ and Continuous FEM with RattleS ${ }_{N}$ ake," Proceedings, International Conference on Mathematics, Computational Methods \& Reactor Physics, Sun Valley, ID, May 5-9, 2013. 


\section{3-121 - Advanced In-Situ Measurement Techniques in TREAT}

Tony Hill, J. Keith Jewell, and Eric Burgett

An analysis that included neutronics modeling and simulation was performed to determine the feasibility and baseline requirements for high-fidelity imaging and microstructure characterization of fuel samples within the modified Transient Reactor Experiment and Test Facility (TREAT) configuration and line-of sight access to the pile. The analysis produced a framework for the detection and imaging requirements that can be implemented alongside nominal TREAT operations with improved functionality for science-based in-situ studies. In this way, both the macroscopic and the microstructure behavior of the fuel can be studied simultaneously under the same power profile and take full advantage of the significant "off-cycle" time between transients when TREAT is in a low power steady state.

This LDRD explores TREAT configurations that can facilitate high-resolution, in-situ measurements on smaller, specially prepared samples and on the feasibility of mating current micro-structure measurements technologies and techniques to the new experimental environment. This work can be accomplished without impacting the TREAT restart or the nominal mission of the TREAT program. This effort will define a possible set of experimental conditions/configurations and begin to delineate a path forward for instrumentation needs. During the first funding year, the focus was on establishing a baseline configuration for utilizing TREAT for science-based microstructure studies in a way that would not impact the core mission of transient fuel testing for qualification. This effort is a starting point and proof of concept for delivering advanced science-based information on in-situ fuel performance and underlying phenomena.

Development of these capabilities will make INL a leader in the science-based characterization of the time evolution of microstructural fuel behavior during irradiation. The proposed work leverages the one-of-a-kind TREAT facility and moves the facility into the realm of science-based understanding for fuels development. Successful completion of the project would position the TREAT facility as the premier test site for both beginning of life microstructure fuels testing as well as end of life transient testing.

\section{Summary}

The TREAT reactor was modeled in an unmodified configuration (shown in Figure 1) to compare the impact of the proposed irradiation cavity on the base operations of the TREAT reactor. The graphite nature of the TREAT reactor makes it difficult to isolate the standard TREAT operating conditions from any modifications, such as the science-based irradiation cavity. Fourgroup cross section neutron contour maps were produced as a base model for comparison with science-based irradiation systems integrated into the TREAT thermal column located on the east side of the reactor.

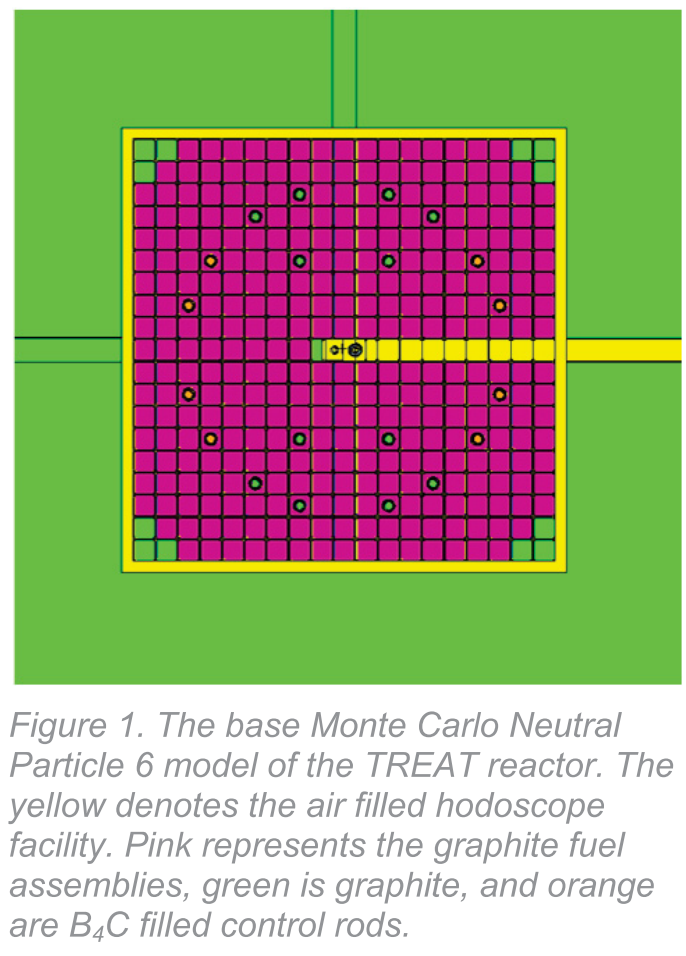


Both low-enriched uranium (U) and highly enriched $U$ (HEU) irradiation cavities were investigated to optimize the utility of the science-based microstructure characterization capability while minimizing the impact to TREAT transient testing. It was determined that the previously unused "thermal column" on the east side of the reactor vessel offered sufficient neutron flux and access for sample movement and imaging equipment to develop a parasitic microstructure characterization component to TREAT operations. There is adequate space for a specially designed scanning electron microscope (SEM) system in this location. With a lowenriched $U$ designed system, an integrated fast neutron flux of $E 12 \mathrm{n} / \mathrm{cm}^{2} / \mathrm{s}$ is achievable while operating TREAT in the $100 \mathrm{~kW}$ steady state mode. Impact is minimized to the hodoscope mission specific parameters and the flux contour map of the operating TREAT reactor, as shown in Figure 2.

A similar neutronics analysis was performed for an HEUboosted irradiation cavity in the thermal column in order to achieve an $\mathrm{E} 13 \mathrm{n} / \mathrm{cm}^{2} / \mathrm{s}$ integrated flux in the microstructure cavity while operating TREAT in the $100 \mathrm{~kW}$ steady state mode. This results in a slight flux tilt to the reactor but provides the largest fast neutron spectrum available for the science-based irradiation capability. Further modifications were explored, such as a water-cooled jacket around the HEU designed system in order to mimic a light water reactor neutron spectrum.

Full TREAT core flux profiles were produced for a number of different microstructure characterization irradiation scenarios. In addition, a safety envelope was produced to ensure that normal transient testing would not be limited by the inclusion of the irradiation cavity in the east thermal column. It was determined that the irradiation cavity can operate indefinitely in the steady state mode, and cooling was designed to ensure that the hottest fuel element can survive a 0.5 second $16 \mathrm{GW}_{\text {th }}$ pulse in the transient operation. Using an adiabatic boundary with conduction to the coolant flow only, a computational fluid dynamics code that is part of the Fluent and Solidworks package was used to model the hottest fuel element, showing a $53^{\circ} \mathrm{C}$ temperature rise in the hottest spot.

Understanding the dynamics of fuel materials during the first six hours of irradiation is a long-range goal for the science-based fuels program. The TREAT science cavity can be used to deliver unprecedented data along these lines by taking advantage of the TREAT operational flexibility and improvements in SEM-based technologies. The HEU science cavity provides a flux of $\sim 2 \times 10^{13} \mathrm{n} / \mathrm{cm}^{2} / \mathrm{s}$ during TREAT steady state running, which is 500 times less flux than a nominal fast reactor. It would take the best part of a year to expose a science sample in that cavity to an equivalent total dose. The upside is that the evolution of the materials would be high resolution in both spatial and temporal dimensions. If instead one operates the TREAT reactor in pulse mode, one can decrease the experiment time at an expendable loss of temporal information. The HEU cavity provides $1 \times 10^{15} \mathrm{n} / \mathrm{cm}^{2} / \mathrm{s}$ when TREAT is operated at less than $10 \mathrm{MW}$, which it can do for a maximum of approximately 200 seconds, then it needs to cool for $\sim 60$ minutes. The equivalent fast reactor dose can be delivered in less than five days with images collected at the end of each pulse. The programmable pulse of the TREAT reactor is a perfect complement to an in-situ SEM system in a parasitic irradiation cavity. The SEM can be programmed to image 
many different samples during the image collection periods, thus increasing the experimental throughput by potentially two orders of magnitude or better, depending on the resolution requirements of each experiment.

\section{Benefits to DOE}

The work funded by this LDRD is in line with the larger strategy by DOE-NE to shift the paradigm of advanced nuclear fuels development to ensure the nation's energy security for the future. The historical approach of relying on experiential models and extensive qualification testing does not allow for the rapid advances necessary for replacing the light water reactor fleet in this country. Separate effects and isolated effects testing will inform new first principle models to develop a predictive performance capability as fuels research moves forward. This project, in particular, leverages the necessary TREAT investments for fuel qualification and takes advantage of the enormous advances in computing methods and resources. 


\section{3-123 - Experimental Verification of Predicted Chemical Transmutation of Cesium-137 to Barium-137 via Beta Decay in $\mathrm{CsCl}$ Resulting in the Radioparagenetic Formation of Rocksalt $\mathrm{BaCl}$}

Rory Kennedy, Chris Stanek, ${ }^{1}$ Mitch Meyer, Richard Martineau, and Jian Gan

For over 30 years, crystalline ceramic nuclear waste forms have been considered for the encapsulation of specific fission products. Faced with the necessity to predict the long-term behavior of ceramic waste forms, researchers have focused on candidate compositions that exhibit: (1) geological analogues, thus suggesting long-term stability (i.e., Synroc), (2) resistance to radiation damage (either using accelerated isotopes or by ion irradiation), and (3) leaching resistance. However, these studies have not yet adequately accounted for the chemical and physical changes that may occur to the waste form during fission product transmutation (i.e., the conversion of one element or isotope into another, in this case during radioactive decay). Indeed, transmutation effects are critical to understanding the performance of a waste form. The candidate waste form selection criteria discussed above may adequately describe the performance of a waste form very early in its life but lack the predictability required to evaluate the performance of the waste form far into the future. The objective of this project is to perform experiments on unique samples in order to demonstrate how chemical transmutation may significantly and non-intuitively modify the structure and property relations that govern waste form performance. These experiments will be used to validate first-principle calculations that have already been performed.

The experiment proposed focuses on an important short-lived fission product, cesium (Cs)-137, which, with strontium (Sr)-90, is responsible for much of the heat load in a geologic repository. Recall that Cs-137 decays via $\beta$ - to barium $(\mathrm{Ba})-137$, with a half-life of 30.07 years. The chemical differences between the parent radioactive isotopes (Cs-137 and Sr-90) and the final stable isotopes (Ba-137 and zirconium [Zr]-90) are significant. For example, the charge state and size of the starting and final cations are different enough to expect variation in the structure-property relations that govern waste form stability. In fact, we have performed

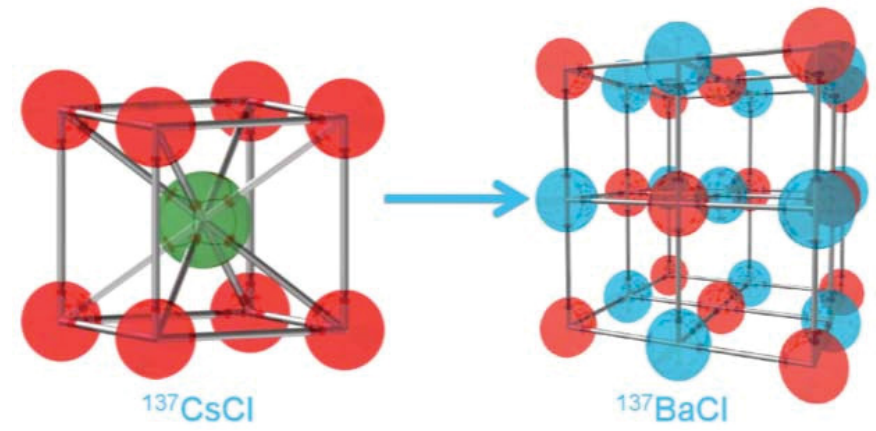

Figure 1. Depiction of chemical transmutation of Cs-137 to $\mathrm{Ba}-137$ via $\beta$ - decay in $\mathrm{CsCl}$, resulting in the radioparagenetic formation of rocksalt $\mathrm{BaCl}$. Red atoms denote chlorine $(\mathrm{Cl})$, green $\mathrm{Cs}$, and blue $\mathrm{Ba}$. density functional theory calculations that predict the meta-stability of rocksalt $\mathrm{BaCl}$ if formed via the radioactive decay of rocksalt $\mathrm{CsCl}$, see Figure 1 . $\mathrm{BaCl}$ in the rocksalt structure is counterintuitive and has never been observed. However, our hypothesis is that transmutation provides a unique synthesis route for unconventional materials. From a waste disposal perspective, understanding how the material properties evolve as a function of time (and composition) is critical to ensure the reliability of the waste package. However, once we understand how the structure evolves over time, we can backward design candidate waste forms. That is, we can design a waste form from the end of their life to the

1. Los Alamos National Laboratory 
beginning, rather than vice versa, which is the standard current approach. Our ultimate goal is to develop the ability to design a waste form with predictable and acceptable performance for the duration of service, perhaps including a crystal structure that actually becomes more stable over time.

Critical to this is validation of the calculations performed. A 49-year-old CsCl sample in the INL hot cells provides a unique opportunity to determine the effects of transmutation on a crystalline material for a fission product of interest. The age of the sample is particularly noteworthy. Furthermore, we can compare to complementary transmission electron microscope experiments done at Los Alamos National Laboratory on $\mathrm{Lu}_{2} \mathrm{O}_{3}$ (which is not a fission product of interest, but rather is using an accelerated approach for an isotope with a six-day half-life).

\section{Summary}

$\mathrm{CsCl}$ sample material was identified and inspected in the INL Hot Fuel Examination Facility hot cells. Estimations of dose versus particle size were performed. Eleven particles ranging in size from $\sim 50 \mu \mathrm{m}$ on one side to $\sim 300 \mu \mathrm{m}$ on another side were selected and individually packaged in sample containers by remote operation in the hot cell. The samples were transferred out of the hot cells to the Analytical Laboratory hot cells. The samples were then transferred to the Fuels and Applied Science Building and positioned in an inert atmosphere glove box to be prepared for operation in the Focused Ion Beam for transmission electron microscope sample preparation in the Electron Microscopy Laboratory. Scheduling conflicts on the Electron Microscopy Laboratory instruments did not allow the Focused lon Beam or transmission electron microscope work to be performed in FY 2013.

Due to the anticipated high gamma activity of the samples, many practice runs were performed on non-radioactive $\mathrm{CsCl}$ samples in order to optimize the handling techniques to minimize personnel dose exposure.

\section{Benefits to DOE}

Success in the project will represent an important discovery and advance the science of materials research in discovering a new chemical form $(\mathrm{BaCl})$ and its stable crystal structure. This information will provide insight into the long term chemical behavior of nuclear and radioactive waste forms, furthering DOE's energy resources mission. 


\section{NATIONAL AND HOMELANSECuRiry}




\section{1-042 - Experimental Cyber Security: Vulnerability Prediction, Discovery, and Mitigation}

Miles McQueen, Jason Wright, Jason Larsen, Lawrence Wellman, Milos Manic, ${ }^{1}$ and Dumidu Wijayasekara ${ }^{1}$

Even though the quantity and diversity of an information technology's or control system's vulnerabilities are related to the system's security, there are few effective ways of predicting the density, discovery, reporting, and patch development of software vulnerabilities. We are limited in our understanding of how effectively vulnerabilities are being identified and mitigated. Consequently, we cannot easily determine or predict the degree to which a system is vulnerable to cyber attack. Critical infrastructure cyber security design, assessment, and measurement must take into account that software vulnerabilities exist in the system, and that the vulnerabilities require different levels of effort by an attacker to discover and exploit. This research has initiated the development of controlled experimentation methods and observational studies to establish standards for software vulnerability prediction, discovery, identification, and mitigation. INL's research project establishes prediction and mitigation standards that are notably absent in many current cyber attack evaluations and technologies; this will create a safer and more secure national infrastructure.

In conjunction with the goal of establishing standards for experiments in cyber security, two significant investigations were performed in 2011 with the potential to be of significant interest to the general cyber security community and to produce experimentally verifiable predictions about future vulnerabilities which will aid situational awareness and anticipation of attacks. In 2012, resources were devoted to updating the initial work performed in 2011 with a particular emphasis on firm establishment of the misclassification of software bugs as "not vulnerabilities." In addition, we furthered the development of an automated bug classifier to aid software products development and maintenance teams in more accurate identification of those bugs likely to be vulnerabilities. In 2013, a Computational Intelligence-based automated classifier was developed that ranks bugs in the order of likelihood of the bug being a vulnerability. The developed classifier uses multiple algorithms, multiple training sets, and multiple variables in order to reduce the probability of over-fitting. In addition, a novel algorithm to identify an optimal number of variables for automatic classification of bugs was developed.

\section{Summary}

The vulnerability research organizations Rapid7, Google Security team, and Zero Day Initiative imposed grace periods for public disclosure of vulnerabilities. The grace periods ranged from 45 to 182 days, after which disclosure might occur with or without an effective mitigation from the affected software vendor. In 2011, we found no direct evidence that the shorter grace periods of 45 and 60 days were justifiable. However, there was evidence that the recently announced Zero Day Initiative grace period of 182 days yielded some benefit in speeding up the patch creation process and could be practical for many software products. In 2012, we updated the analyses and demonstrated that the early research results were holding at predicted levels and that vendors were now consistently meeting the 182 day grace period deadline (Figure 1). 


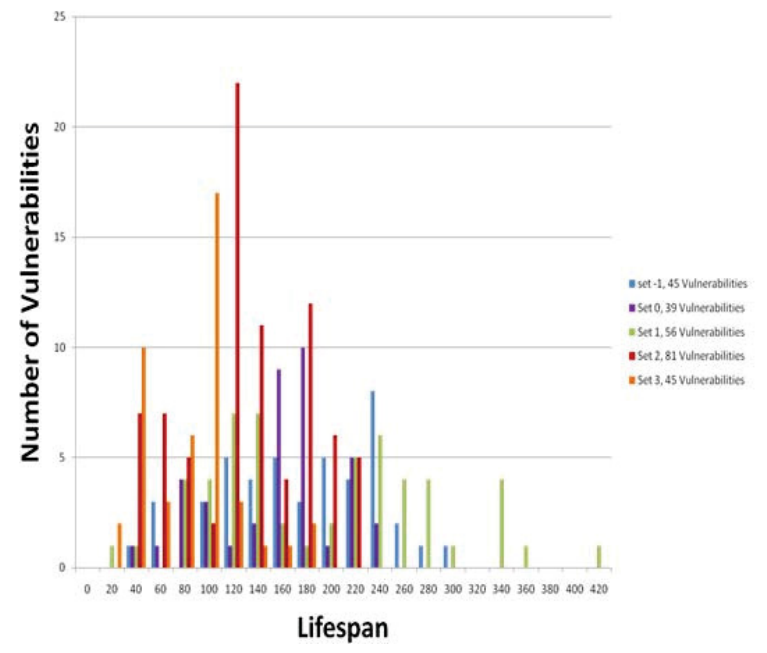

Figure 1. Vulnerability lifespans before 182 day grace period was imposed (Set -1, Set 0, and Set 1) and after the grace period was imposed (Set 2 and Set 3).

We proposed two new vulnerability exposure metrics with the end-user in mind. Both metrics, "Vulnerability Free Days" and "Average Active Vulnerabilities" per day, depend not just on vulnerability lifespans of a product but also on the rate of vulnerability reporting. In 2011, we demonstrated the metrics in a case study using four browsers: Safari, Chrome, Firefox, and Internet Explorer. Based on the derived metric values for each browser, there were large differences in vulnerability exposure, with Safari having the lowest exposure (Figure 2). In 2012, we enhanced our case study through improved simulation and further demonstrated the earlier results. A paper was written and published about this effort.

In 2011, we initiated experiments into software vulnerability density and conceptualized classifiers to more effectively and accurately identify those bugs more likely to be vulnerabilities. In 2012, we clearly demonstrated that for the experiment's chosen software product, the number of misclassified bugs is significant. In 2013, we demonstrated, with $95 \%$ confidence, that there are somewhere between 499 and 587 bugs (mean value of 543 ) that are vulnerabilities but are not recognized as such (Figure 3 ). This compares to a total of 76 known vulnerabilities for the given software product versions under evaluation. Due to the significance of this result, many of the past decade's research results related to software vulnerabilities and, based on known vulnerabilities, are now called into question.

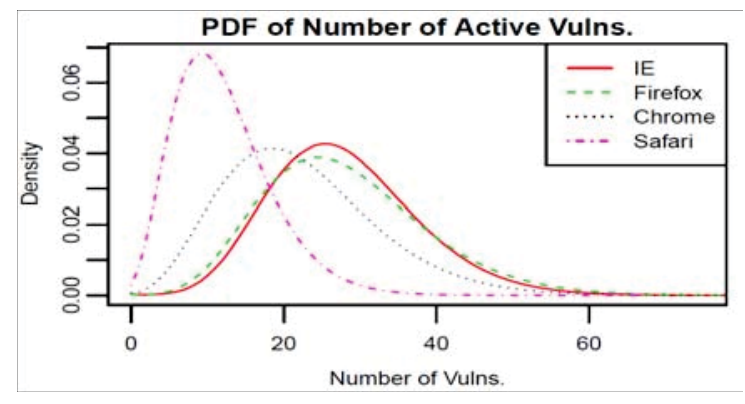

Figure 2. Average Active Vulnerabilities in four browsers.

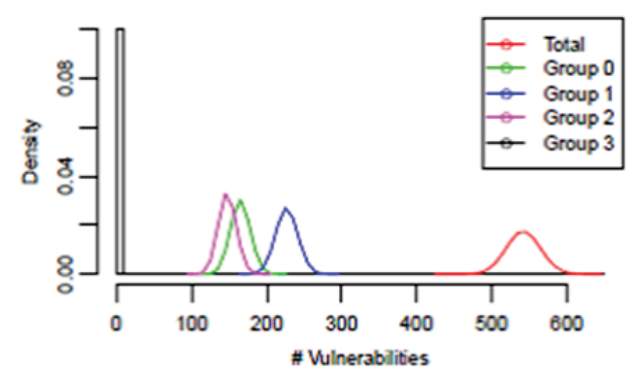

Figure 3. 95\% confidence interval, per each score bin, for the number of bugs misclassified as not vulnerabilities in the chosen software product. 
In 2012, we furthered the development of the automated bug classifier started in 2011 for identifying Hidden Impact Bugs. We have some very promising early results with the Artificial Neural Network classifier providing a 69.73\% truepositive rate and a $2.5 \%$ false-positive rate (Figure 4 ). In 2013, the automated classifier was further improved to accommodate a larger number of dimensionality and bugs. The classification was performed using multiple algorithms and results are shown in Figure 5. A Bayesian detection rate analysis was performed that identified the potential workload on a vulnerability identification team (Figure 6). In addition, a novel algorithm that identifies the optimal set of variables for improved text-based classification of bugs was developed. Using the developed algorithm, the classification of bugs as vulnerabilities was further improved (Figure 7).

\begin{tabular}{|l|c|c|c|c|c|c|}
\hline \multirow{2}{*}{ Classifier } & \multicolumn{2}{|c|}{ True Positives } & \multicolumn{2}{c|}{ True Negatives } & \multicolumn{2}{c|}{ False Positives } \\
\cline { 2 - 8 } & Rate & Number & Rate & Number & Rate & Number \\
\hline Naïve Bayes (NB) & $91.8 \%$ & 67 & $45.7 \%$ & 2741 & $54.3 \%$ & 3259 \\
Naïve Bayes Multinomial (NBM) & $80.8 \%$ & 59 & $89.6 \%$ & 5377 & $10.4 \%$ & 623 \\
Decision Tree (DT) & $28.8 \%$ & 21 & $99.5 \%$ & 5969 & $0.5 \%$ & 31 \\
\hline
\end{tabular}

Even the lowest rate (28\%) is an order of magnitude better than random guess (1.2\%)

\begin{tabular}{|l|c|c|}
\hline & $\begin{array}{c}\text { Classified as } \\
\text { Hidden Impact } \\
\text { Bugs }\end{array}$ & $\begin{array}{c}\text { Classified as } \\
\text { Normal Bugs }\end{array}$ \\
\hline True Hidden & $69.73 \%$ & $30.26 \%$ \\
Impact Bugs & $(53)$ & $(23)$ \\
\hline True Normal & $2.5 \%$ & $97.4 \%$ \\
Bugs & $(25)$ & $(974)$ \\
\hline \multicolumn{2}{|c|}{ May contain unidentified vulnerabilities }
\end{tabular}

Figure 4. Confusion matrix for the Artificial Neural Network classifier, $69.73 \%$ true positives and $2.5 \%$ false positives.
- May contain HIBs that are yet to be identified

- Large number of False Positives may overwhelm True Positives

Figure 5. Classification results for multiple classifiers using 6,000 bugs.

\begin{tabular}{|l|c|c|}
\hline Classifier & $\begin{array}{c}\text { Number of bugs } \\
\text { classified as HIBs per day }\end{array}$ & $\begin{array}{c}\text { Probability that one } \\
\text { is an HIB }\end{array}$ \\
\hline Naïve Bayes (NB) & 80.4 & 0.02 \\
Naïve Bayes Multinominal (NBM) & 16.5 & 0.09 \\
Decision Tree (DT) & 1.3 & 0.40 \\
\hline
\end{tabular}

Figure 6. Bayesian detection rate analysis for each classifier tested.

\begin{tabular}{|l|c|c|c|}
\hline \multicolumn{1}{|c|}{ Parameter } & $\begin{array}{c}\text { No } \\
\text { Dimensionality } \\
\text { Selection }\end{array}$ & $\begin{array}{c}\text { Conventional } \\
\text { Dimensionality } \\
\text { Selection }\end{array}$ & $\begin{array}{c}\text { Novel IG Based } \\
\text { Dimensionality } \\
\text { Selection }\end{array}$ \\
\hline $\begin{array}{l}\text { True Positive } \\
\text { Rate }\end{array}$ & 0.671 & 0.881 & 0.912 \\
\hline $\begin{array}{l}\text { True Negative } \\
\text { Rate }\end{array}$ & 0.908 & 0.915 & 0.931 \\
\hline $\begin{array}{l}\text { Bayesian Detection } \\
\text { Rate }\end{array}$ & 0.085 & 0.116 & 0.143 \\
\hline
\end{tabular}

Figure 7. Classification results using the novel dimensionality selection algorithm. 
In 2011, we held the first Experimental Security Panoramas Workshop with leading researchers as speakers. This Experimental Security Panoramas workshop brought together technical leaders to discuss their experiences and challenges in designing and executing credible cyber security experiments. The workshop was intended to provide a research forum and aid development of a research community for improved scientific experimentation related to all aspects of the cyber security eco-system. Based upon the success of this workshop, INL organized a follow-up workshop in 2012, which included another group of leading vulnerability researchers and had a significantly increased number of attendees.

\section{Benefits to DOE}

This research furthers the security of DOE information systems and achieves further recognition of DOE and its laboratories as technical leaders in cyber security research and development. The results of this project have established foundational science and experimental bases for cyber security vulnerability prediction, discovery, and mitigation that are notably absent in many current evaluations and technologies. Previously, cyber security evaluations and designs fell short in part due to a lack of a strong, experimental science basis of the underlying system security state and were often dependent upon personal experience, belief, and limited empirical evidence. With continued, scientifically-based improvement in our understanding, prediction, and identification of software vulnerabilities, the risk management and security of DOE networks and information systems, as well as our nation's critical infrastructure, will be significantly enhanced.

\section{Publications}

Wijayasekara, D., M. Manic, and M. McQueen, “Vulnerability Identification via Text Mining Bug Databases," In preparation.

Wright, J., J. Larsen, and M. McQueen, "Estimating Software Vulnerabilities: A Case Study Based on the Misclassification of Bugs in MySQL Server," $8^{\text {th }}$ International Conference on Availability, Reliability, and Security, Regensburg, Germany, September 2013.

Wijayasekara, D., M. Manic, and M. McQueen, "Information Gain Based Dimensionality Selection for Classifying Text Documents," Proceedings of IEEE Congress on Evolutionary Computation, Cancun, Mexico, June 20-23, 2013.

Wright, J., M. McQueen, and L. Wellman, "Analyses Of Two End-User Software Vulnerability Exposure Metrics," $7^{\text {th }}$ International Conference on Availability, Reliability and Security, Prague, Czech Republic, August 2012.

Wijayasekara, D., et al., "Mining Bug Databases for Unidentified Software Vulnerabilities," $5^{\text {th }}$ International Conference on Human System Interaction, Perth, Australia, June 2012. 


\section{1-059 - Unconventional Radiation Detection Methods for Nuclear Nonproliferation, Safeguards, and Treaty Verification}

David Chichester, Scott Watson, Scott Thompson, James West, and James Johnson

Technical challenges that are not currently met by today's nuclear instrumentation technology include the need for wide-area, continuous radiation monitoring for safeguards monitoring inside centrifuge enrichment halls, wide-area radiation monitoring in higher throughput spent nuclear fuel handling and processing facilities, noncontact sensing methods capable of working within the high radiation fields, and high data rate signal processing for active interrogation screening systems. The work outlined in this report highlights accomplishments of a three-year LDRD project intended to be an exploratory set of R\&D activities aimed at trying advanced unconventional radiation detection methods to address these and other shortfalls. In some cases, these approaches are unconventional simply because they branch out beyond traditional sensor reliance on neutron and gamma rays. In other cases they are unconventional because they seek to adapt measurement approaches developed in one field for use in a different way for very different applications. The project seeks to develop innovative and enhanced diagnostic tools and spectroscopic capabilities for use in nuclear science and for practical use in advance nuclear system instrumentation, nuclear safeguards, environmental radiation monitoring, and treaty verification. Our objective is that these new diagnostic tools will overcome challenges presented by current instrumentation.

\section{Summary}

A small exploratory effort examined the use of near-ultraviolet imaging as a means for locating and assessing ionizing radiation in air (Figure 1). One particularly interesting application for this approach is the assessment of alpha-emitting radionuclides, but the capability also has the potential for investigating fluorescence induced via lower-linear energy transfer quanta as well. A peer-reviewed article was published from this effort.
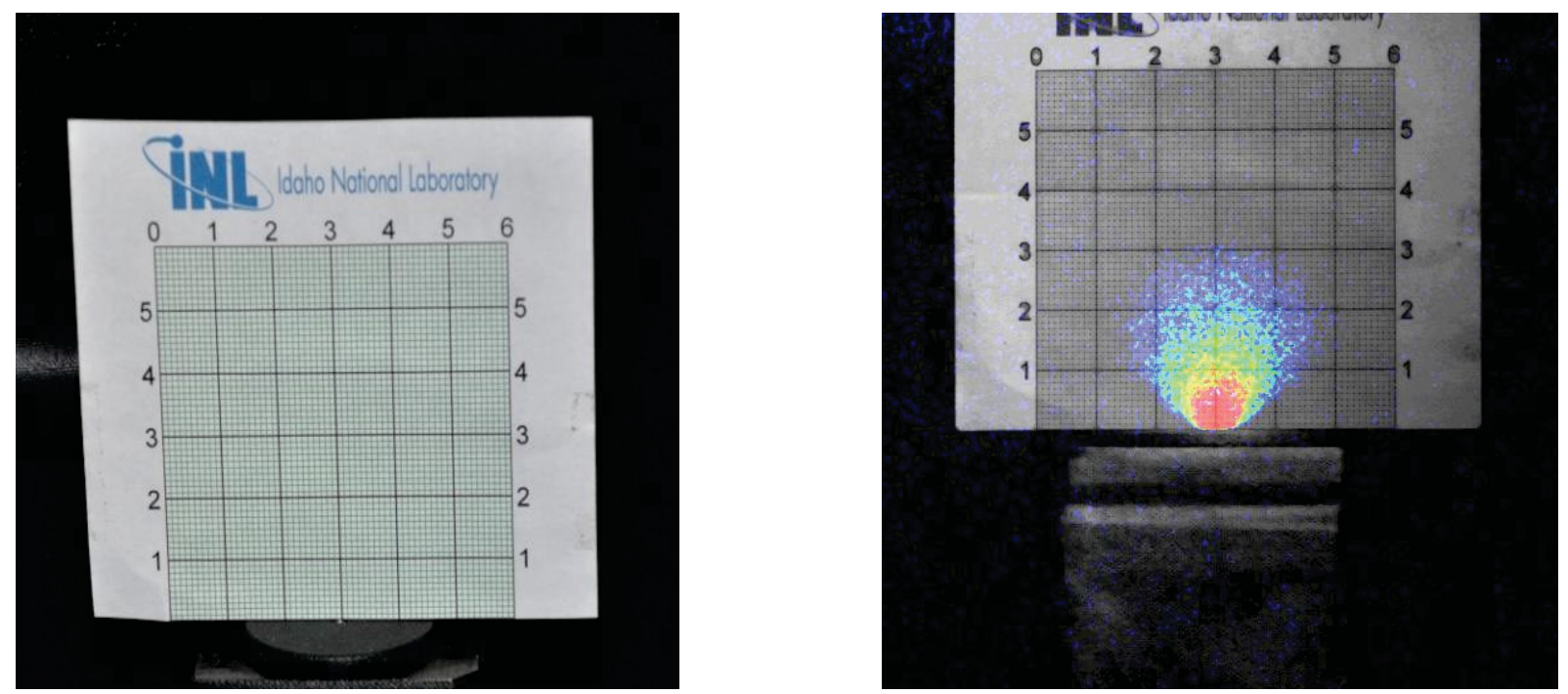

Figure 1. A close-up photograph of an americium-241 alpha-emission composite view of the near-ultraviolet light generated by the source overlaid on the visual image (right). 
Thermographic imagery was studied as a method for detecting and assessing shielded radiation sources, exploiting radiative decay heat as a detection signature. With this specific application in mind, a proof of principal experiment was conducted using a midrange $(7.5-13 \mu \mathrm{m})$ infrared thermal imaging camera (FLIR Systems $A B$, model SC325). Power levels of $0.02 \mathrm{~W}$ and above were clearly discernible over background levels in laboratory testing. Field trial measurements of concepts developed in this study have subsequently been sponsored by another INL program.

A small exploratory effort to study how low-technology, low-cost radiation detectors could be incorporated into a distributed sensor network for wide-area radiation field monitoring was conducted. This activity focused on the use of commercial off-the-shelf detector equipment and a commercial off-the-shelf open-source, electronic prototyping platform. This project was transitioned to external funding by a Work for Others customer, resulting in a sensor system deployed for humanitarian monitoring outside the United States.

A small effort examined the feasibility of adapting already-fielded equipment commonly used by law enforcement and military personnel to function as radiation sensors for emergency applications. The result from this task led to the submission of an INL invention disclosure record.

Additional research studied the use of long-length scintillating fiber bundles for radiation detection. Time-of-flight analysis techniques were used to record signal arrival times at each end of the bundles, and a Lagrangian operator was developed to transform signal response for image construction. In-house designed instrumentation was developed, and a customized data acquisition software tool was written to support data acquisition and interpretation. A separate development project was initiated from this effort to support INL post-irradiation examination research at MFC.

\section{Benefits to DOE}

Unconventional radiation detection methods contribute to a number of DOE missions, including advanced nuclear technology for the next-generation of nuclear power. This research supports the development of new diagnostic tools and spectroscopic capabilities for (a) use in basic nuclear science and engineering R\&D and (b) practical use in advance nuclear system instrumentation and control, transformational nuclear safeguards, radiological safety and dosimetry, and environmental radiation monitoring and compliance verification.

\section{Publications}

Chichester, D.L., S.M. Watson, and J.T. Johnson, "Comparison of BCF-10, BCF-12, and BCF-20. Scintillating Fibers for Use in a 1-Dimensional Linear Sensor," IEEE Trans. Nucl. Sci., Vol. 99, 2013, In press.

Chichester, D.L. and M.T. Kinlaw, "The MARVEL Assembly for Neutron Multiplication," App. Rad. Iso., Vol. 80, 42-48, October 2013.

Chichester, D.L. and S.M. Watson, "Multispectral UV-Visual Imaging as a Tool for Locating and Assessing lonizing Radiation in Air," IEEE Trans. Nucl. Sci., Vol. 58, 2512-2518, October 2011. 


\section{Presentations}

Chichester, D.L., S.M. Watson, and J.T. Johnson, "Comparison of BCF-10, BCF-12, and BCF-20 Scintillating Fibers for Use in a 1-Dimensional Linear Sensor," IEEE Nuclear Science Symposium and Medical Imaging Conference, Anaheim, CA, October 29-November 3, 2012.

Chichester, D.L. and M.T. Kinlaw, "Development of a New Multiplying Assembly for Research, Validation, Evaluation, and Learning," IEEE Nuclear Science Symposium and Medical Imaging Conference, Anaheim, CA, October 29-November 3, 2012.

Chichester, D.L. and S.M. Watson, "Multispectral UV-Visual Imaging as a Tool for Locating and Assessing Ionizing Radiation in Air," IEEE Nuclear Science Symposium and Medical Imaging Conference, Knoxville, TN, October 30-November 6, 2010. 


\title{
11-060 - SMC Advanced Armor Materials and Systems R\&D
}

\author{
Henry Chu, Michael Bakas, Benjamin Langhorst, Jeffrey Lacy, Thomas Zagula, and \\ Robert O'Brien
}

DOE, Department of Defense, and Department of Homeland Security are in need of advanced armor materials and innovative armor systems that provide enhanced physical protection solutions. INL has completed research on three novel concepts which would develop these materials and systems. These concepts included:

(1) confirmation of ballistic performance of the INL-developed bainite armor steel, which can be produced at significantly lower cost than current heavy armor material; (2) exploration of spark plasma sintering (SPS) of SiC, which can greatly improve the current manufacturing techniques used to produce armor-quality SiC; and (3) investigation of kinetic-physical interactions between a shaped charge (SC) and a ferroelectric $\mathrm{LiTaO}_{3} \mathrm{Crystal}_{\text {, }}$ which may help defeat the lethal effects of an SC on U.S. military personnel and vehicles.

\section{Summary}

Current manufacturing processes for armor-grade steel alloys use expensive alloying elements such as nickel, vanadium, and cobalt with the rapid quenching process. The bainite steel experimentation intended to advance the development of a new lower cost armor alloy. Previously, the INL team developed an alloy composition with lower-cost elements that could be air cooled. During FY 2013, multiple 4 in. $\times 4$ in. $\times 1$ in. bainite steel tiles were produced, machined, and heat treated to serve as large-scale bainite targets. These tiles were then tested against a lethal 50 caliber M2 armor piercing round. For comparison purposes, current standard High Hardness armor steel (MIL-STD-46100) and Hardox steel (an inexpensive alternative armor steel) were also tested. Based on the experimental data, the INL novel bainite steel showed equivalent performance to the standard High Hardness armor steel and has substantially better performance over the Hardox. Images of the impact as well as behind armor behavior were captured using high speed video and flash x-ray, respectively.

$\mathrm{SiC}$ is a high-performance, low-density armor ceramic that is difficult and expensive to sinter to full density and High Hardness. The goal of the SiC experimentation was to establish whether or not SPS is a viable means of producing armor quality $\mathrm{SiC}$. Since $\mathrm{SiC}$ is in considerable demand because of its low-density and high-ballistic performance, a method capable of increasing production quality and efficiency is of value. The initial SPS production goal was to produce a large SiC tile of $\geq 98 \%$ density. After developing an SPS process that produced samples of $25 \mathrm{~g}$ and $40 \mathrm{~mm}$ in diameter with nearly $100 \%$ density, samples of $200 \mathrm{~g}$ and 3 in diameter were produced. The best density achieved in these larger samples was $97 \%$. While the goal of $98 \%$ density was not achieved, these tiles represent some of the largest and densest SiC tiles produced by SPS. Analyses of the microstructure of the SPS SiC revealed that the density of the large samples may be improved with modifications to the SPS process.

The study of the impact of an $\mathrm{SC}$ through a ferroelectric $\mathrm{LiTaO}_{3}$ crystal was intended to understand the potential of these crystals to discharge a large electrical current upon disruption by an SC. Conceptually, electromagnetic armor is designed to defeat SCs by discharging a large electrical current that causes the jet to lose its penetrating power. Past research established that a sudden phase change from high pressure can cause ferroelectrics to discharge intrinsically-stored electrical energy. The project goal was to impact ferroelectric material $\mathrm{LiTaO}_{3}$ with a shape charge to determine whether any electrical discharge from the crystal would produce a dynamic response 
on the SC's copper jet. A flash x-ray imaging system was used to measure any changes to the penetration of the copper jet capability due to the presence of ferroelectric crystals.

$\mathrm{LiTaO}_{3}$ crystals were tested in five different material placement configurations in combination with a stack of armor steel witness plates. Flash x-ray images were collected for all tests. Analysis of the experimental data is in progress.

\section{Benefits to DOE}

Advanced and innovative armor systems will enhance the performance and security of DOE/National Nuclear Security Administration and Department of Defense personnel, vehicles, and facilities. This project focused on novel concepts for advanced manufacturing of heavy armor materials and substituting lighter, more effective materials to improve protection performance.

\section{Publications}

The SPS SiC result will be presented and published at the peer-reviewed American Ceramic Society symposium and proceeding. An Invention Disclosure Record has been filed, BA-799 2362, Large Scale Spark Plasma Sintering of Silicon Carbide Plate, Michael Bakas, et al., August 20, 2013.

The formula, process method and test results of the bainite armor steel will be published in peer-reviewed material science and metallurgy journals. An Invention Disclosure Record is already on file. BA-458 1699, Bainitic Steel Alloy for Armor Applications, Michael Bakas, et al., October 22, 2009. BA-625 2040, Cost Effective Steel Alloy for Armor Applications, Michael Bakas et al., April 25, 2011.

The ferroelectric test data is expected to be published in the International Journal of Impact Engineering or Journal of Applied Physics. No intellectual property is anticipated. 


\title{
12-001 - Long Term Evolution Wireless Cyber Security
}

\author{
Kurt Derr, Sam Ramirez, and Jose Loera
}

In the next few years, Long Term Evolution (LTE) technology will become pervasive in industry, government, and intelligently controlled infrastructure, as LTE offers a high-speed, high-bandwidth telecommunication option that enables new human to machine and machine to machine applications. As development of LTE compatible mobile devices moves forward, more software vulnerabilities will be discovered and impact asset owners and end users. This research is developing the capabilities to enable the nation to discover vulnerabilities that exist in the latest data communications protocols and secure LTE-enabled devices from cyber attacks. The objectives of this LDRD were: (1) investigate, analyze, and test new fourth generation (4G) LTE devices to determine the degree to which cyber security vulnerabilities of $3 G$ systems are resolved by new $4 G$ protocols, as well as the degree to which vulnerabilities exist in the design and implementation of $4 G$ protocols; and (2) mitigate any vulnerabilities discovered, to the extent possible, in LTE protocol implementations.

\section{Summary}

A lab-scale LTE R\&D system (Figure 1) was designed and constructed to conduct research experiments on cellular security and cellular communications issues. This system is capable of interfacing to WiMAX, WiFi, 2G, $3 G$, and LTE systems. The research performed this year utilized the LTE and WiFi network interfaces to demonstrate: (1) applications for voice, video, text, and file transfer; (2) Android to Android smart phone communications using video and voice; and (3) Android smart phones as user equipment (UE) to demonstrate handovers from WiFi to LTE and LTE to WiFi.

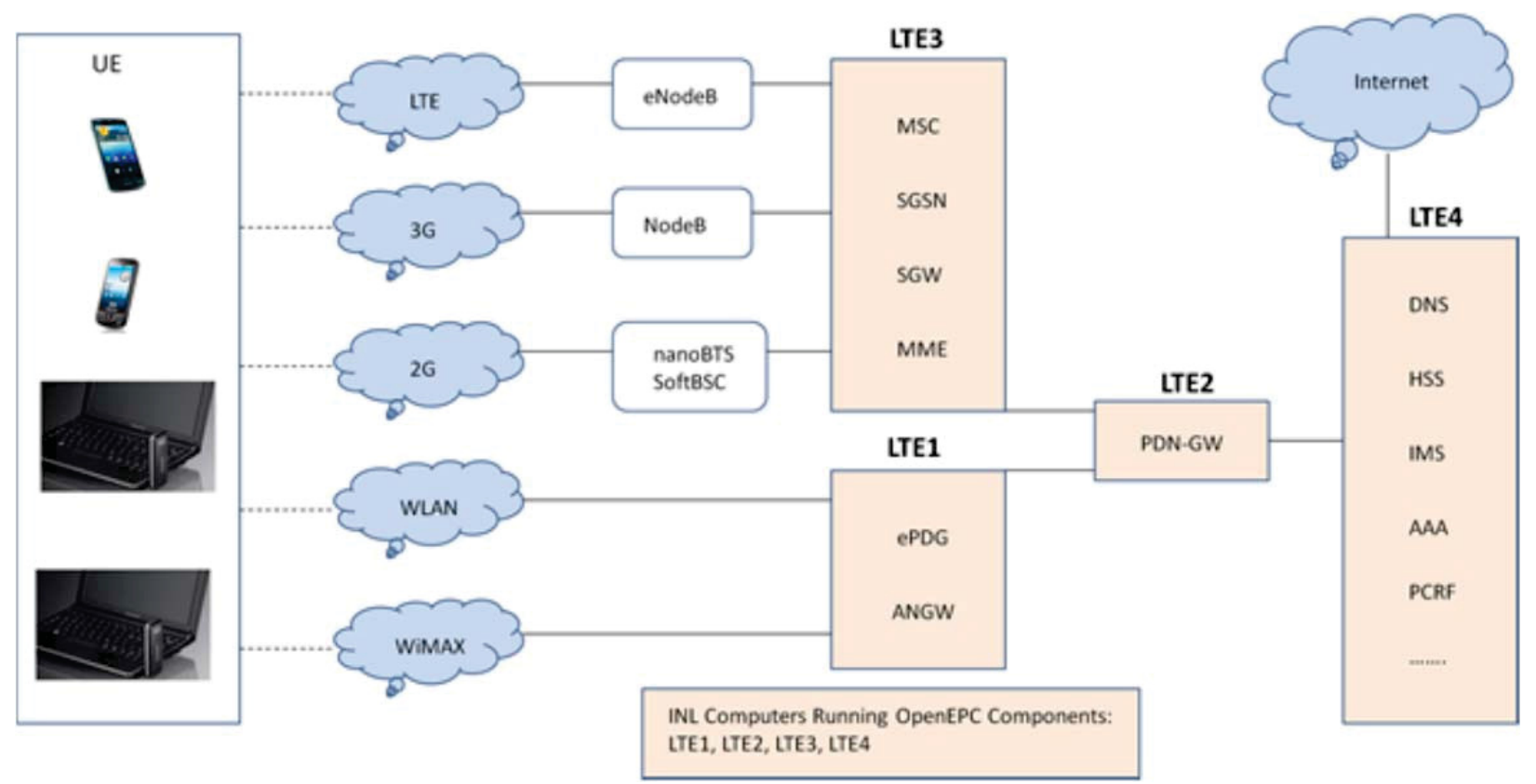

Figure 1. LTE experiment system designed to include LTE1, LTE2, LTE3, and LTE4 computers and legacy wireless communication technologies. 
The approach to vulnerability research included: (1) identifying UE applications/use cases that exercise the communications functionality of the LTE system, (2) running UE applications while monitoring communications in the LTE system, and (3) utilizing protocol fuzzing or other techniques to discover vulnerabilities while running UE applications. Other experiments involved code modifications to packets in the LTE stack to evaluate Denial of Service threats and signal jamming to determine the potential for both Denial of Service and performance degradation. Spoofing threats were also realized by creating packets representing an adversary posing as another user during multimedia communications between two users.

The risks associated with the discovered vulnerabilities were characterized using the Microsoft DREAD method (Damage potential, Reproducibility, Exxploitability, Affected users, and Discoverability). Signal jamming techniques required the least effort to exploit the system, while vulnerabilities discovered with protocol fuzzing techniques were the most difficult to discover and required the most effort to carry out.

\section{Benefits to DOE}

This research initiative contributes directly to DOE's goal of securing critical infrastructure from cyber attacks. The LTE experimentation laboratory provides a national capability for wireless communications system research that was previously not available for evaluation of the potential cyber vulnerabilities of commercial off-the-shelf vendor LTE systems. The project research results indicate that LTE systems require more cyber vulnerability research before being employed in sensitive environments or before implementation as a standard system for all government and public safety communications. 


\title{
12-017 - Polymer Components in Laminate Armor Systems for Ballistic Protection
}

\author{
Benjamin Langhorst and Henry Chu
}

As armoring performance grows more important due to increased explosives and ballistic threats, the performance of low-density materials, such as polymers, needs to be evaluated as an alternative to more traditional protective materials such as metals and ceramics. To perform these evaluations, INL researchers completed experiments to characterize the performance benefits of using polymers in future light-weight armor concepts that will protect military, law enforcement, and emergency response vehicles. The development of lightweight vehicle armor with improved protection also requires a balance of vehicle mobility, safety, and performance (e.g., sensing, communications, and occupant comfort).

\section{Summary}

In FY 2012, a novel test method was developed to make ballistic performance testing of polymers more efficient and affordable. A "Residual Mass" method was conceptualized and demonstrated to be more informative than traditional pass/no-pass armor methods, while also requiring $50-75 \%$ fewer experiments (see Figure 1). This method compares the projectile erosion achieved by different armor test groups. Experimental results validated that this method will enable the performance of different target groups to be statistically compared to provide researchers with performance rankings and associated confidence levels. A description of this new method is pending publication and was used in subsequent

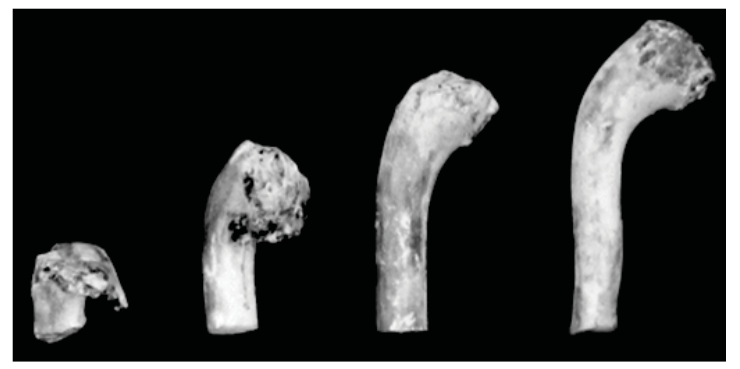

Figure 1. Residual projectiles that were captured after perforating targets were analyzed using the Residual Mass method. polymer armor experiments.

Since the thermo-mechanical properties of polymers are very different than those of traditional armor materials, polymer armor designs will need to consider the thermal stability of the polymers integrated into an armor system. In another experiment in FY 2012, researchers measured the in-situ temperature in and around an impact region during a ballistic impact. These measurements indicated that polymers are subjected to near-instantaneous temperature rises of $5-10^{\circ} \mathrm{C}$ above the ambient temperature. The experimental data was compared with numerical simulations to validate the numerical methods so that further simulations could be used to identify impact regimes at which polymer components within armor are at risk of melting.

Experiments during FY 2013 measured improvements in mass efficiency that could potentially be achieved by integrating polymers into reactive armor systems. For one of these experiments, polymer-based energetic material was sandwiched between thin steel plates to form a semi-energetic reactive armor system that would react under high-velocity impact. The performance of this system was compared to a traditional non-energetic reactive armor system featuring rubber sandwiched between thin steel plates. Testing indicated that the semi-energetic system functioned as intended and reaction rates of the energetic material can be measured. The methods and data from this study will be used to engineer and test future armor systems. 


\section{NATIONAL \& HOMELAND SECURITY}

Also during FY 2013, the potential of polymers to disperse ballistic shock was investigated (see Figure 2). Experiments included different integrated system prototypes that featured equivalent-thickness inter-layers of air, high-impedance polymer, and low-impedance polymer. After these targets were impacted by projectiles with $67-75 \mathrm{~kJ}$ of kinetic energy, the back surface of the targets were analyzed using a 3-D laser profiler. A statistical analysis of the data revealed that ballistic shock dispersion is significantly affected by the number of steel layers that the target is divided into but not significantly affected by the polymer materials used between the steel layers.

\section{Benefits to DOE}

This program benefits DOE's mission by enhancing the protection of critical infrastructure, including the security of nuclear facilities and materials, along with providing technology solutions. Enhancing the blast and ballistic
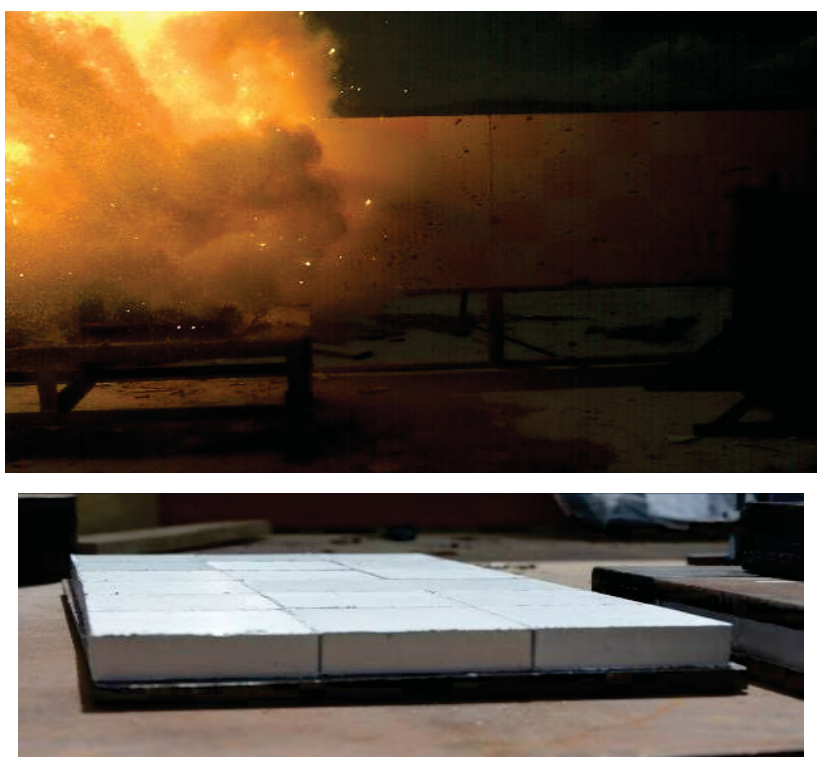

Figure 2. Semi-energetic targets were ignited by the impact of an incoming projectile. protection of protective vehicles also will benefit other government agencies like the Department of Defense. These foundational studies advanced the body of knowledge in the general armor development community by introducing more efficient armor testing methods and developing the tools needed to accurately predict the performance of polymers that are subjected to ballistic impact.

\section{Publications}

Langhorst, B., T. Lillo, and H. Chu, "A Residual Mass Ballistic Testing Method to Compare Armor Materials or Components," ASTM J. Testing and Evaluation, Vol. 42:3, 2014.

Robinson, A., N. Rasmussen, and B. Langhorst, Non Energetic Reactive Armor (NERA) and Semi Energetic Reactive Armor (SERA) FY 13 Final Report, INL/EXT 13 29988, 2013.

\section{Presentations}

Langhorst, B., T. Lillo, and H. Chu. "Novel Ballistic Testing Method to Compare Armor Materials or Components."

Langhorst, B. and J. Lacy, "Impact-Induced Temperature Rise in Polymer-Steel Laminate Armor Targets." 


\section{2-050 - Cross Section Representations for Nuclear Nonproliferation}

\section{Ryan Clement}

As the nation's premier nuclear energy laboratory, INL maintains capabilities to analyze, evaluate, and advise on the full life cycle of nuclear fuel, from initial post-mine separations and chemistry to long-term, permanent storage and/or transmutation. A cornerstone of this ability is the availability of accurate and up-to-date nuclear data combined with technical skills to create new data when the currently available evaluations are insufficient. Because of this, INL is developing tools to evaluate and enhance the theoretical and experimental nuclear cross section data available for nuclear energy researchers.

\section{Summary}

This project's early work in reviewing several key nuclear decay quantities resulted in submission and acceptance of four "errata" and an additional accepted submission to a peer-reviewed nuclear engineering journal using the improved data. The research then changed focus from NJOY/ACE file representation to mobile nuclear data representation in order to assist nuclear researchers in transitioning from printed materials to ubiquitous and freely available modern electronic distribution platforms. This work created two mobile applications: one for Apple's iOS and the other for Google's Android mobile device operating system. These two mobile applications are based on the National Nuclear Data Center's (NNDC) (located at Brookhaven National Laboratory) Nuclear Wallet Cards. This work was performed in collaboration with Dr. Jagdish Tuli of the NNDC.
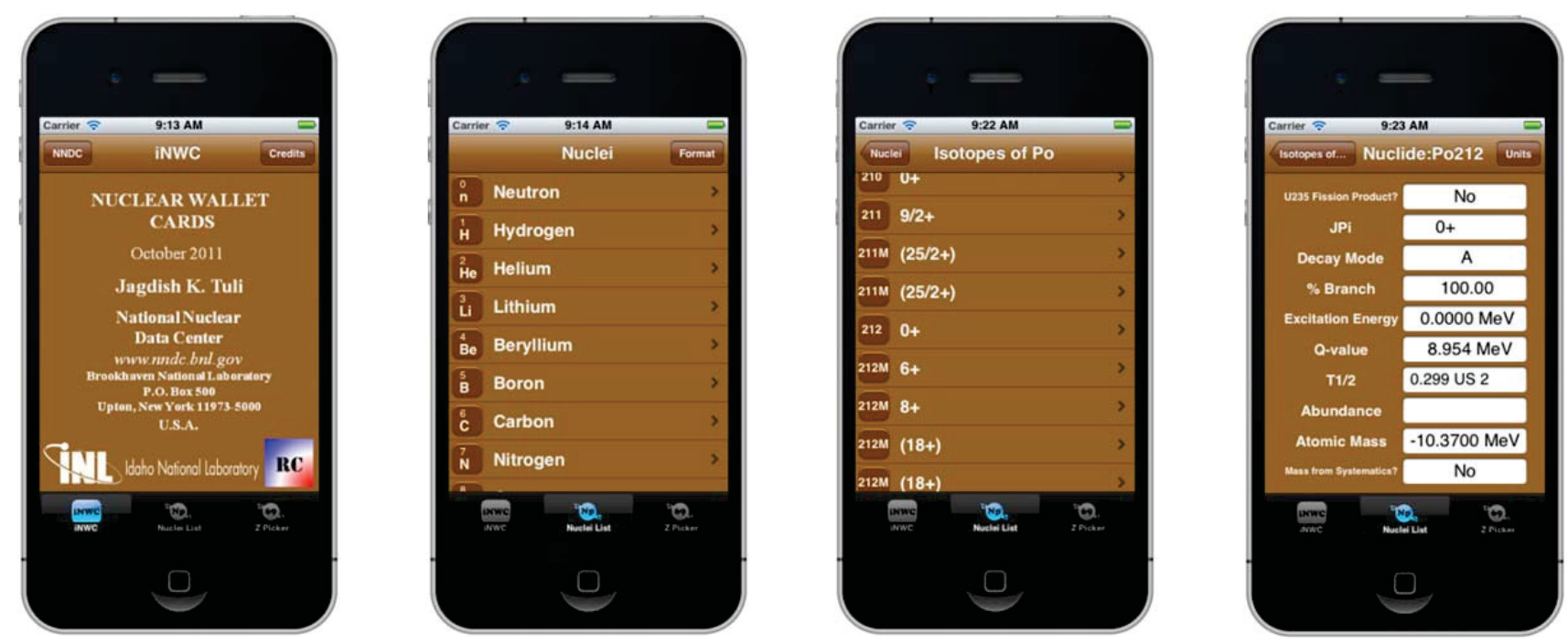

Figure 1. Series of screen shots that a user may utilize. In this example, the user who had selected the iNWC app on an iPhone 4 would see the "splash screen" shown on the far left.

The user would then select the "Nuclei List" from the bottom menu to bring up the nuclei list shown on the middle left screen shot. If the user was looking for an isotope of polonium they would then see the image on the middle right. Finally, the user finds the exact isotope and spin state they are looking for and the available data for the $0+$ state of polonium-212 is displayed. 
The iOS work has led to a peer-reviewed iPhone/iPad application on Apple's App Store and iTunes. NNDC has communicated the INL-created iPhone application at nuclear physics/data community meetings and on their web page. The application is on the NNDC web page at http://www.nndc.bnl.gov/wallet/ (Figure 1). The Nuclear Wallet Cards for Android app is designed to work on any size of android device, adapting its user interface to fit any screen orientation, pixel density, and physical dimensions available.

\section{Benefits to DOE}

DOE and National Nuclear Security Administration laboratories and key stakeholders benefit from the corrections to nuclear data in fission related quantities in several nuclear databases by reducing potential errors in future nuclear work. The nuclear data mobile applications benefit the nuclear scientific community by providing a popular data resource in a usable, accessible, mobile device format. The iOS application has gained a good deal of attention and has initiated discussions with the Defense Threat Reduction Agency for future work. The continued efforts in the nuclear data field have resulted in a National Nuclear Security Administration nuclear data project.

\section{Publications}

Piet, S.J., et al., "Fuel Cycle System Analysis Implications of Sodium-Cooled Metal-Fueled Fast Reactor Transuranic Conversion Ratio," Nucl. Technol., Vol. 181, 427-458, March 2013.

Browne, E. and J. Tuli, Erratum to "Nuclear Data Sheets A=232," Nucl. Data Sheets, Vol. 107, 2579-2648, October 2006, Submitted.

Singh, B. and J. Tuli, Erratum to "Nuclear Data Sheets A=233," Nucl. Data Sheets, Vol. 105, 109-222, May 2005, Submitted.

Browne, E. and J. Tuli, Erratum to "Nuclear Data Sheets A=215, 219, 223, 227, 231," Nucl. Data Sheets, Vol. 93, 763-1061, 2001, Submitted.

Browne, E. and J. Tuli, Erratum to "Nuclear Data Sheets A=230," Nucl. Data Sheets, Vol. 69, 155-208, 1993, Submitted.

iNWC, Available on Apple's iTunes and App Store, December 2013. 


\title{
12-054 - Treaty Verification with Photo-Induced Prompt Signatures
}

\author{
Mathew Kinlaw, Alan Hunt, ${ }^{1}$ Scott Thompson, and Edna Cárdenas ${ }^{1}$
}

INL is developing technologies to detect, identify, and image items of interest for nonproliferation and national security missions. This LDRD seeks to extend imaging capabilities to collect high- and low-resolution images that emergency first responders and nuclear inspectors can use to quickly identify the geometric arrangement and isotopic composition of suspect items. The principal methodology uses a moderate-energy photon beam to induce photoatomic and photonuclear reactions in inspected materials. By exploiting the resulting emission signatures, based on their temporal characteristics, an inspected item's contents can be localized and identified based on their effective atomic numbers and densities. Composite signatures enable further identification of nuclear materials. The resulting 3-D images enable individual internal features to be resolved and can be high- or lowresolution, depending on a particular application.

\section{Summary}

This research focused on proof-of-concept measurements to assess the efficacy of combining the primary signatures to enable 3-D images of targets of interest to be generated. By exploiting the signatures' time dependence, target localization of multiple targets was demonstrated at various locations along the inspection beam. Taking advantage of the atomic number and density dependence of the induced reactions, the locations and arrangements of high- $Z$ materials were able to be identified and imaged. Using a composite signature, which incorporates an additional, more-conventional prompt fission signature, contents consisting of nuclear material were further discerned from those consisting of high-Z, non-nuclear material.

Measurements were made on non-nuclear materials to evaluate the technique's fidelity using a standard $155 \mathrm{~mm}$ artillery shell (Figure 1). In the resulting image, the empty interior of the shell was evident, as was the shell's tapered, increased wall thickness. These measurements also illustrated a built-in, inherent information barrier that may be of interest in treaty verification applications. By limiting the overall timing resolution of the system, specific dimensions (or other geometric characteristics) are unobtainable from the data. However, with specific enhancements to the overall timing resolution of the system, the technique also could discern individual walls of the shell. For applications where high fidelity is sought, as may be desired for emergency response-type applications, this level of enhanced spatial resolution may be required.

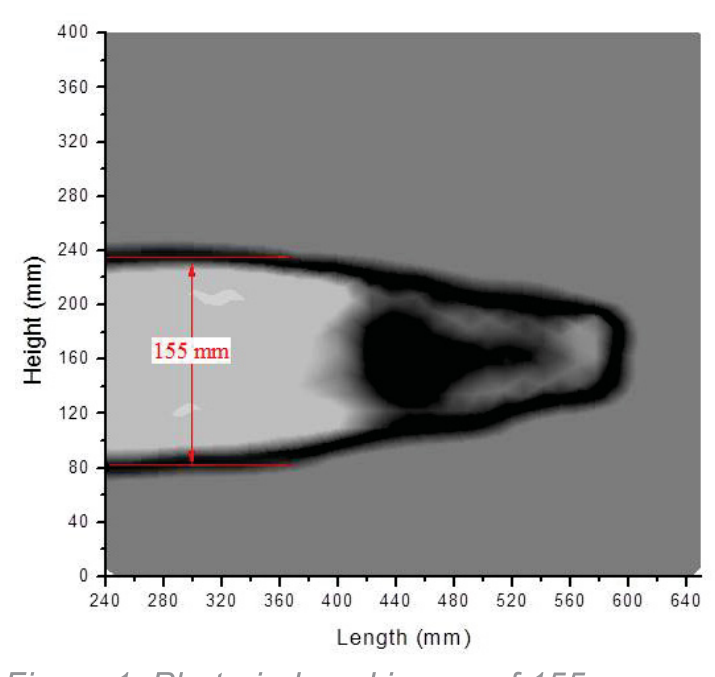

Figure 1. Photo-induced image of $155 \mathrm{~mm}$ artillery shell.

1. Idaho State University 
Calculations were completed to determine the dominant reaction, or combination of reactions, produced for a given inspection beam energy distribution. Basic assumptions resulting from preliminary calculations quickly become convoluted when subsequent factors are included (e.g., detector filtering). To address this, data were collected from multiple sets of targets comprised of medium- and high-Z materials dissolved in solutions. The resulting signature responses appeared to increase linearly with concentration, however, this relationship was not consistent with the mass increase. These results support the hypothesis that elemental volume information can be extracted from the signature generation and subsequent attenuation of both the incident bremsstrahlung beam and return signal. In support of modeling efforts, an analysis software package was also developed to simulate the responses of typical scintillation detectors. This software converted neutron and photon scattering events occurring in a detector cell into a distribution of light pulses, based on the detector material's properties, detection thresholds, and additional parameters. Options to eliminate nearest-neighbor coincidences (i.e., cross-talk) were also incorporated, as well as automated time-of-flight algorithms.

\section{Benefits to DOE}

This research will provide a technical solution applicable to DOE mission objectives for arms control and treaty verification applications. The ability to generate high- or low-resolution 3-D images, in addition to unique implementation of radiation signatures, can enable confirmation that treaty limited items are as declared. The same detection technology could contribute to confirming inspected item types and/or attributing disassembled components and is applicable to nuclear facility safeguards, cargo screening, and improvised explosives device detection.

\section{Publications}

An INL Invention Disclosure Record is under review for patent application.

\section{Presentations}

Kinlaw, M.T., A.W. Hunt, and S.J. Thompson, "Treaty Verification with Photo-Induced Prompt Signatures," presented to the Strategic Advisory Committee, Idaho National Laboratory, Idaho Falls, ID, June 2012.

Kinlaw, M.T., A.W. Hunt, and S.J. Thompson, "Treaty Verification with Photo-Induced Prompt Signatures," presented to the Strategic Advisory Committee, Idaho National Laboratory, Idaho Falls, ID, July 2011.

Kinlaw, M.T., A.W. Hunt, and S.J. Thompson, "Treaty Verification with Photo-Induced Prompt Signatures," presented to the BEA S\&T Board of Directors, Idaho National Laboratory, Idaho Falls, ID, July 2011. 


\title{
12-066 - Electronic Energetic Initiation
}

\author{
Michael Daniels, Nikki Rasmussen, Reston Condit, and Michelle Pantoya ${ }^{1}$
}

INL is developing systems that can safely and reliably ignite composite energetic materials used in energetic reactions. Development of such systems requires engineered solutions that prevent inadvertent initiation and assure reliable ignition when desired. Focusing on understanding the fundamental principles of the initiation energies for various igniter compositions as well as the component designs capable of assuring reliable and stable ignition, this research project seeks to develop initiation materials (e.g., first-fire mixtures or ignition triggers) and technologies that provide full, electronic control of energetic material initiation safely and reliably for the DOE, Department of Defense, and emergency responders. Concepts for initiators were explored, including critical parameters influencing electrical arc ignition, electrical resistivity, and conductivity of particulate mixtures. INL collaborated with Texas Tech University to explore the fundamental principles of the initiation process and to evaluate how the initiation materials could be tailored to meet the project goals.

\section{Summary}

Through the course of this LDRD, scientific understanding was expanded for arc-sensitivity of materials based on factors such as bulk electrical conductivity, particle shape, particle size, and packing factor. We discovered ways to make energetic materials less sensitive to electrostatic discharge. We successfully developed new technology that lessens the need for this material and showed the feasibility of novel initiators.

\section{Benefits to DOE}

This research resulted in innovations that will increase the safety of using energetic materials for experimental and field applications relevant to the DOE, Department of Defense, and emergency responders. Energetic materials are key materials relative to industry applications within critical infrastructure systems, and safe and reliable initiation of these materials is vital for continued use. Deployment of the initiator technologies developed in this LDRD has the potential to significantly increase the reliability of initiation, extend the shelf-life of components, reduce transportation shipping costs and hazards, and minimize the dispersal of hazardous toxic metals.

\section{Publications}

Poper, K., et al., "Tailoring the Electrostatic Ignition Sensitivity of an Energetic Composite Using Nanofillers," Pending submission to Appl. Phys. Lett. upon approval from INL Patent Office.

Collins, E., et al., "Ignition Sensitivity and Electrical Conductivity of a Composite Energetic Material with Conductive Nanofillers," Pending submission to Nanotechnology upon approval from INL Patent Office.

Weir, C., M. Pantoya, and M. Daniels, "The Role of Aluminum Particle Size in Electrostatic Ignition Sensitivity of Composite Energetic Materials," Combust. Flame, Vol. 160, 2279-2281, October 2013.

Collins, E., et al., "Synthesizing Aluminum Particles Towards Controlling Electrostatic Discharge Ignition Sensitivity," Submitted to the J. Electrostat., Manuscript ID: ELAST-D-13-00148, September 2013.

1. Texas Tech University 
Collins, E., et al., "Piezoelectric Ignition of Nanocomposite Energetic Materials," J. Propul. Power, In press September 2013.

Weir, C., et al., "Electrostatic Discharge Sensitivity and Electrical Conductivity of Composite Energetic Materials," J. Electrostat., Vol. 71, 77-83, February 2013.

\section{Books}

Weir, C., M. Pantoya, and M. Daniels, Electrostatic Discharge Sensitivity of Composite Energetic Materials, Saarbrucken: Lambert Academic Publishing (an imprint of AV Akademikerverlag GmbH \& Co.), 2012.

\section{Patent Applications}

Daniels, M., et al., "Energetic Materials and Methods of Tailoring Electrostatic Discharge Sensitivity of Energetic Materials," Attorney Docket 2939 11946US (BA 793).

Daniels, M., et al., "Hybrid Exploding Bridge Wire Energetic Material Initiator," Attorney Docket 2939-P11963US (BA-772). 


\title{
12-073 - A Nonproliferation Focused Study and Comparison of the GEANT4 Simulation Toolkit and MCNP Functionality, Accuracy, and Development Needs
}

\author{
Scott Thompson, Ryan Clement, and Brian Castle
}

According to published works by both Brown and Mckinney, the Monte Carlo Neutral Particle (MCNP) family of codes is currently the most widely used Monte Carlo tool for modeling fission emissions and their subsequent material interactions. However, for validation and benchmarking reasons it is very desirable to have another internationally recognized and widely used Monte Carlo simulation tool to support nonproliferation research and development. This LDRD will contribute to national nuclear security through the development of new detectors and detection systems for nuclear nonproliferation and counterproliferation measurements. These new nuclear detection methods will increase the efficiency of advanced safeguards, nuclear forensics, emergency response, and nuclear material detection. The GEANT4 simulation toolkit is a clear choice because of both its extreme flexibility and its abilities to model visible light generation and transport in scintillators, and to more-closely mimic signal generation in solid-state and gaseous radiation detectors, as explained in more detail in the article "GEANT4-A Simulation Toolkit" by Agostinelli. GEANT4 has an exceptionally strong history and user base in the high-energy physics regime, yet it has only recently become a commonly used tool in the nonproliferation community.

This project was a nonproliferation-themed, head-to-head comparison of the GEANT4 simulation toolkit and the MCNP family of codes. A series of measurements with well-documented results were modeled in parallel with both of these Monte Carlo-based simulation packages. The measurements modeled highlight physical processes that are inherent to nuclear material detection, imaging, and radiation dose measurement, along with other nonproliferation, safeguards, and security based applications.

\section{Summary}

Several measurement campaigns performed at INL were modeled, all of which incorporated standard helium (He)-3 based proportional counters. These measurements emphasized physical phenomena pertinent to nonproliferation detection methods. Examples of studied observables included fission reaction emissions (both prompt fission neutron die-away and delayed emissions), neutron thermalization rates, and physical detector response. The modeled measurements incorporated electronic neutron generators (both $[\mathrm{d}, \mathrm{d}]$ and $[\mathrm{d}, \mathrm{t}]$ reaction generators that produce $2.5-\mathrm{MeV}$ and $14.1-\mathrm{MeV}$ neutrons respectively) as well as californium (Cf)-252 isotopic spontaneous fission source. These sources span the energy range applicable to nonproliferation and nuclear safeguards measurements.

A sample geometry for an active neutron interrogation measurement that was modeled as a part of these efforts is shown in Figure 1. In this geometry, a large challenge pallet comprised of solid shielding material with a compartment at its center for holding special nuclear material is irradiated with neutrons. The detectors positioned adjacent to the pallet monitor for the emission of fission signatures that indicate the presence of fissionable material. This figure also demonstrates the advantage of using GEANT4 visualization capabilities to illustrate and communicate problem geometries. The orange tracks in this geometry denote neutron trajectories, while the blue represent those of gammas. Yellow dots indicate particle interaction points. 


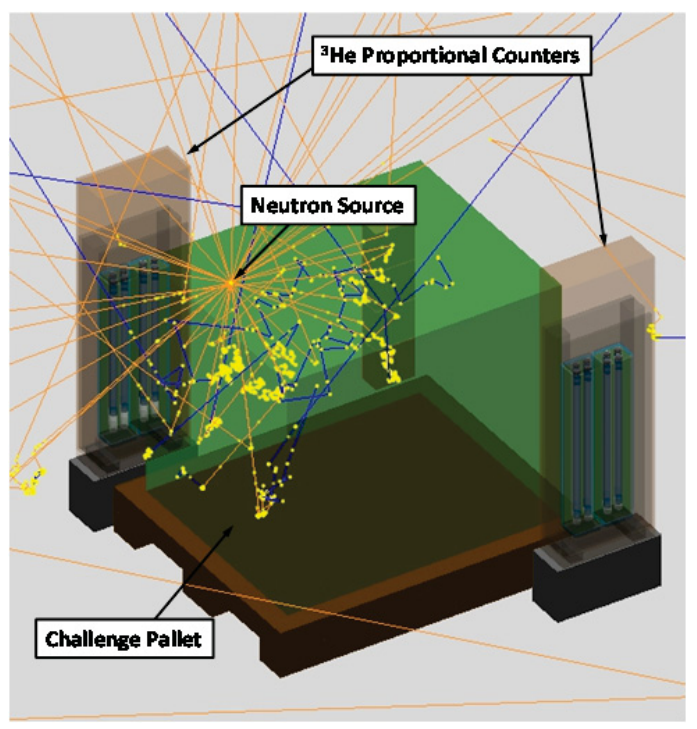

Figure 1. An example of a modeled measurement geometry.
The results from a series of measurements and simulations that monitored neutron count rates in $\mathrm{He}-3$ based detectors with increasing distance from a Cf-252 spontaneous fission neutron source is shown in Figure 2. Measurement error bars represent $1-\sigma$ statistical uncertainty, while simulation error bars represent $10 \%$ uncertainty to account for factors, such as electronic dead times, that were not accounted for in the simulations. This figure demonstrates the high-level of agreement between GEANT4 and MCNPX that trended throughout the simulation campaign.

Analysis algorithms for inclusion into GEANT4 simulations were also developed for comparison to MCNPX built in shift register multiplicity features. A full dissemination of the results from this project is currently being assembled into an upcoming journal article.

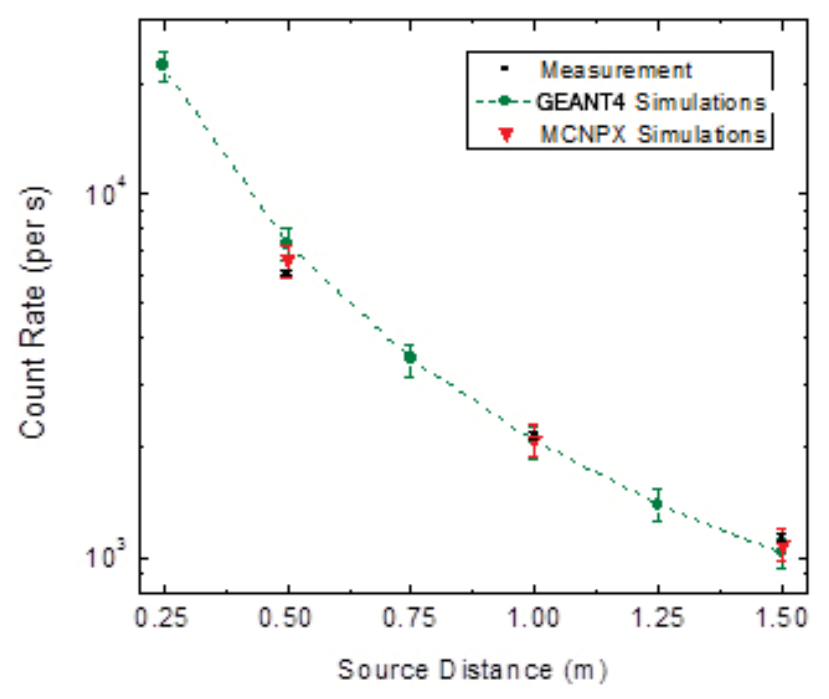

Figure 2. A comparison of GEANT4 and MCNPX simulation results to measurement data. 


\section{Benefits to DOE}

This study is of direct benefit to DOE's national security mission by aiding in the development of new detectors and detection systems for nuclear nonproliferation and counterproliferation measurements related to advanced safeguards, nuclear forensics, emergency response, and nuclear material detection.

\section{Presentations}

Clement, R., "A Nuclear Science Education Course Proposal: Modeling Radiation Transport and Detection in Experimental Apparatus with the GEANT4 Simulation Toolkit," University and Industry Technical Interchange Review Meeting, Oakland, CA, December 6, 2011. 


\title{
13-060 - Metal Fluoride Preparation for AMS Analysis
}

\author{
James Delmore and Gary Groenewold
}

INL researchers are developing methods for novel isotopic ratio measurements to enable U.S. and international organizations to ensure that nuclear energy facilities are operating in compliance with nonproliferation treaty commitments. The planned experimentation within this project is designed to demonstrate concepts for improving the accuracy, detection limit, and analysis costs of measuring ultratrace quantities of minor isotopes in the presence of orders-of-magnitude more abundant major isotopes. In general, the ratio of minor/major isotopes provides a technical measurement basis for verifying treaty compliance. The confidence in these verification measurements increases when the measurements of the ultratrace minor isotopes are not impacted by chemical or detection interferences.

\section{Summary}

Currently, the best approach for overcoming potential measurement interferences is to utilize accelerator mass spectrometry (AMS), which is capable of accurately measuring ratios of minor isotopes at values as low as $10^{-14}$ relative to major isotopes. However, since AMS is routinely applicable to only a few elements of interest to geophysical science, INL's LDRD research is focused on expanding the applicability of AMS to radioisotopes associated with nuclear nonproliferation. To accomplish this, INL is evaluating the conversion of these trace radioisotopes (within an AMS) into fluoroanions using electrospray ionization to improve the efficiency of ion formation, overcome wide kinetic energy distributions, and resolve interferences from the minor oxygen isotopes. INL's research is expected to lead to a deeper understanding of negative ion formation science and new applications for AMS.

In this project, we hypothesized that the AMS measurement limitations associated with oxyanion formation can be overcome by developing microsynthetic approaches for generating metal fluoroanions that are compatible with the source characteristics of AMS instruments. These fluoroanions are expected to have exceptional ion formation efficiencies and are less likely to cause spectral interferences encountered with oxyanions, which are the current method of choice for measuring metal isotope ratios using AMS.

Experimentation demonstrated that metal fluoroanions could be formed by sputtering samples that had been amalgamated into $\mathrm{PbF}_{2}$. Even though this approach proved that the thermodynamics for fluoroanion formation are favorable, it produced undesirably broad kinetic energy distributions and had low efficiency ion formation. Based on these results, INL evaluated the use of electrospray ionization of fluorinating ionic liquids. INL successfully demonstrated $100 \%$ transmission of the sample solution into the mass spectrometer using solutions modified with ionic liquids.

To evaluate the potential of fluorinated ionic liquids, a collaboration was started with Osaka University (Hagiwara and Tsuda) to obtain samples of 1-ethyl-3-methylimidazolium fluorohydrogenate for analysis of its compatibility with radioisotope samples and electrospray ionization mass spectrometry. Initial experiments showed compatibility of 1-ethyl-3-methylimidazolium fluorohydrogenate with mass spectrometer source hardware and demonstrated efficient formation of metal fluoranions for sample introduction (Figure 1). 


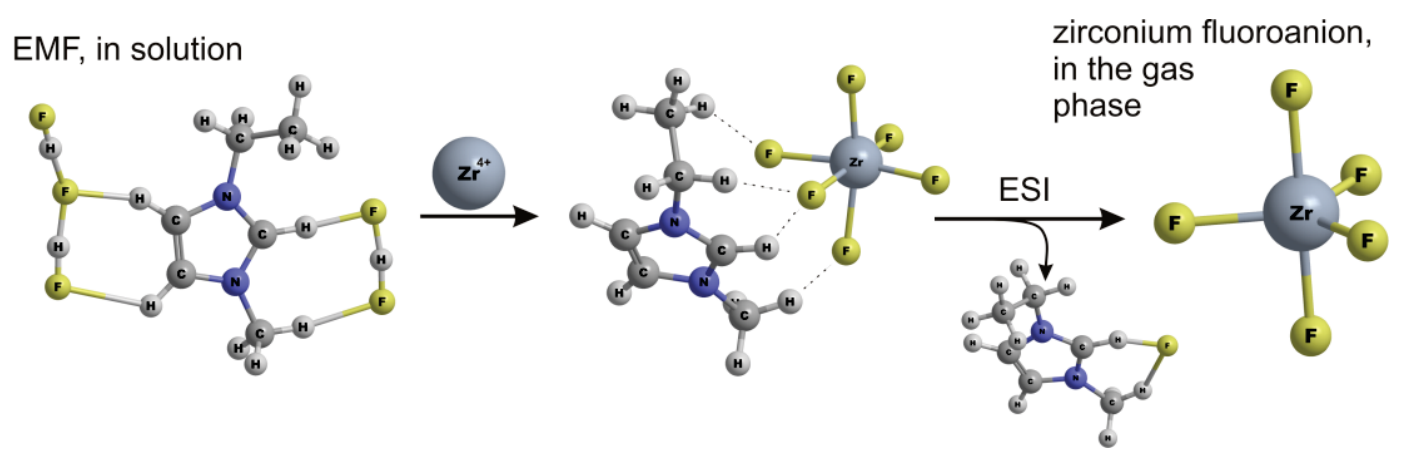

Figure 1. Density functional theory models predict that 1-ethyl-3-methylimidazolium fluorohydrogenate will result in desired metal fluoroanion bonds while avoiding formation of hydrofluoric acid.

The researchers believe that actinide species (like uranyl) can be efficiently (and perhaps easily) converted into fluoroanions. Experimentation during FY 2014 will further evaluate the potential of fluorohydrogenate ionic liquids with AMS measurements. The development of new production schemes for making metal fluoroanions using electrospray ionization holds the promise for a ground-breaking expansion of AMS for making ultratrace isotope ratio measurements. Specifically, the technology will likely result in: (1) measurement of lower concentrations of minor isotopes of elements like uranium (U), (2) reduced molecular background, and (3) ability to measure a wide range of elements like zirconium ( $\mathrm{Zr}$ ), providing opportunities for using new isotopic indicators.

\section{Benefits to DOE}

The development of new ultratrace measurement methods will advance DOE nonproliferation mission objectives and elevate the global recognition of DOE's leadership position in nuclear and radiological science and measurement technologies. Transitioning the experimental results into a more accurate analytical measurement method will enable the U.S. to make more informed nonproliferation policy decisions regarding the nonproliferation status of international nuclear energy programs.

\section{Publications}

Groenewold, G.S., et al., "Fluorohydrogenate Cluster lons in the Gas Phase: Electrospray lonization Mass Spectrometry of the [1-ethyl-3-methylimidazolium $\left.{ }^{+}\right]\left[\mathrm{F}(\mathrm{HF})_{2.3}\right]$ lonic Liquid," J. Phys. Chem. A, 2013, Submitted. 


\title{
13-092 - Fission Product Standard Production
}

\author{
Kevin Carney, Jana Pfeiffer, and Chris McGrath ${ }^{1}$
}

National nonproliferation and nuclear forensics programs rely on the capability to detect fission products at very low levels. The impact of these detection methods is dependent upon the ability to validate results with rigorous quality assurance methods and technologies. This INL research initiative uses INL facilities, capabilities, and university collaborators to build unique national capabilities for the production and qualification of fission product standards to address requirements from the global nuclear security community.

Currently, it is possible to obtain standards of a few fission individual fission products, but these are generally fairly long-lived products that are not commonly used in the community. This research will establish a new source for shorter-lived isotopes, in thermal and fast ratios, as well as individual standards for technique development and validation.

\section{Summary}

During FY 2013, the research team demonstrated the development and qualification of individual fission product standards, the ability to collect fission products on a foil, and partial automation of the separation of individual fission products. The isotopes of interest to be harvested from the spontaneous fission of californium (Cf)-252 are listed in Table 1. A preliminary scheme for the separation of fission products from the fission of Cf-252 was developed on a macro-scale. The schema involved separation of rare earth fission products neodymium $(\mathrm{Nd})$, samarium $(\mathrm{Sm})$, europium $(\mathrm{Eu})$, and terbium $(\mathrm{Tb})$ from yttrium $(\mathrm{Y})$ (Figure 1).

Future research will improve the separation of rare fission products and determine the decontamination factors for Cf-252 and $\mathrm{Cm}-248$. Additional experimentation will test and qualify irradiation methodology for fast spectrum and fissile material Table 1. Isotopes of interest as fission product standards.

\begin{tabular}{|l|c|}
\hline \multicolumn{1}{|c|}{ Isotope } & \multicolumn{1}{|c|}{$\begin{array}{c}\text { Half-life } \\
\text { (days) }\end{array}$} \\
\hline Y-87 (Activation) & 3.35 \\
\hline Y-88 (Activation) & 106.65 \\
\hline Y-91 & 58.5 \\
\hline Ru-103 & 39.27 \\
\hline Te-132 & 3.2 \\
\hline Pm-149 & 2.212 \\
\hline Pm-151 & 1.183 \\
\hline Eu-156 & 15.2 \\
\hline Tb-161 & 6.91 \\
\hline
\end{tabular}
standards. 


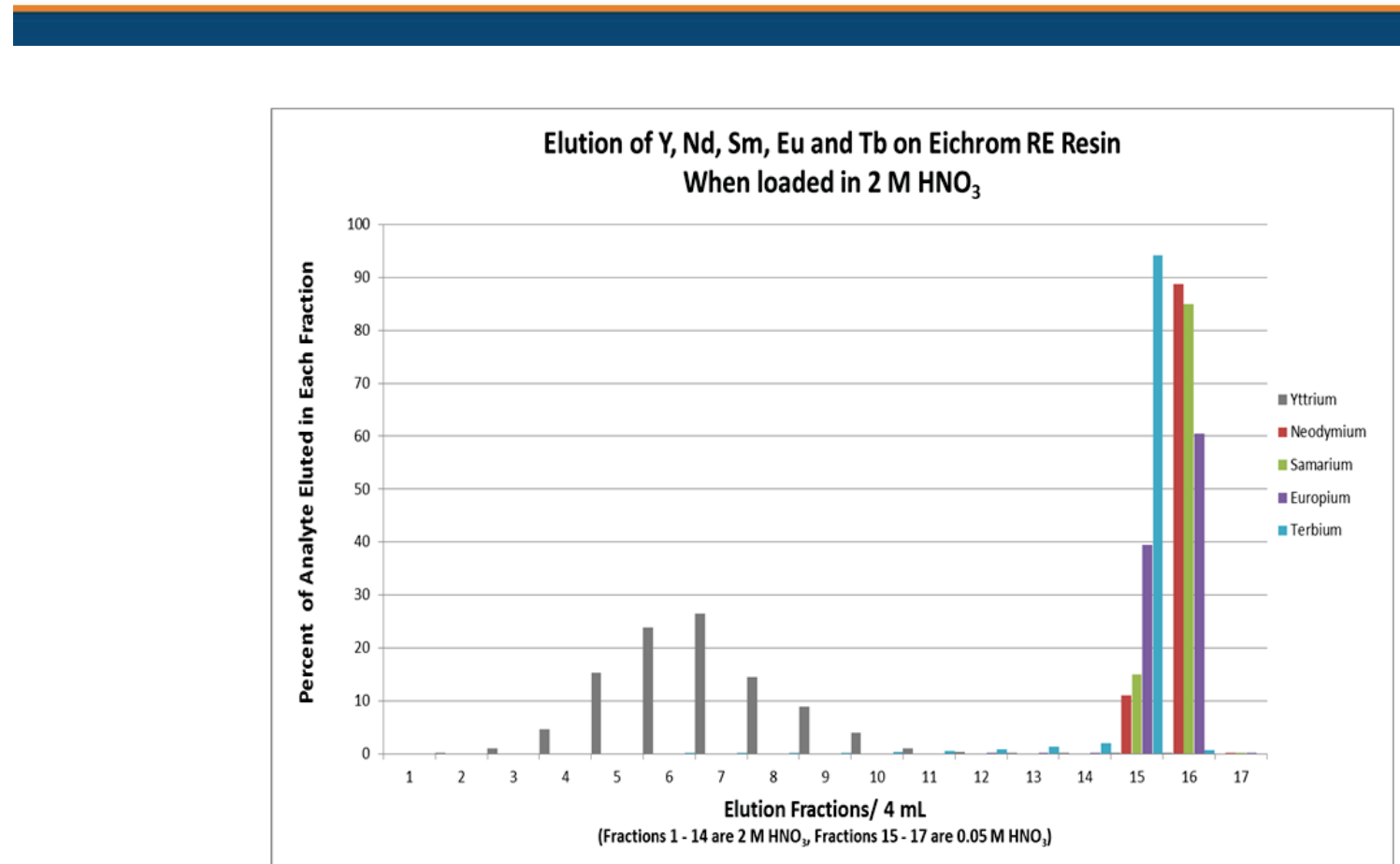

Figure 1. Elution curve of $\mathrm{Y}, \mathrm{Nd}, \mathrm{Sm}, \mathrm{Eu}$, and Tb on Eichrom RE Resin.

\section{Benefits to DOE}

This research will directly enhance the ability of DOE and other national security organizations to achieve nonproliferation and nuclear forensic mission objectives. The availability and use of low-level fission product standards will assure the quality of results from the detection methods used in support of these missions. 


\title{
13-093 - Spectrum Allocation and Communications in Dynamic Spectrum Access Channels
}

\author{
Hussein Moradi, Juan Deaton, and Jose Loera
}

The wireless communications spectrum this nation uses every day is increasingly overwhelmed by wireless communication devices; so much that there have been impacts to emergency response and military operations. These impacts support a Federal Communication Commission prediction that there will be a $300 \mathrm{MHz}$ spectrum deficit by 2014. INL's research in spectrum allocation and Dynamic Spectrum Access (DSA) seeks to solve this growing problem and enable assured communication at all times for public safety, along with commercial, private, and government agencies.

Research has shown that conventional fixed frequency allocations are no longer a viable option because there is not enough spectrum for individual services. Since only $17 \%$ of the allocated spectrum is utilized at a time in certain geographical locations, a robust method for DSA could provide a solution for the spectrum demands. This LDRD is focused on developing technical solutions that enable the sharing of spectrum while supporting information assurance and security for U.S. Government end users.

The research team's Cognitive Media Access Control (cMAC) concept, when developed, will provide a national option for spectrum hole (whitespace) identification for secondary-user channel assignment, control, and spectrum mobility. The objective of this LDRD is to demonstrate the feasibility of the cMAC concept to enable DSA through the use of an underlay control channel based on Filter Bank Multi-carrier Spread Spectrum communications. The INL research team has established collaborations with the University of Utah and the Stevens Institute of Technology.

\section{Summary}

The INL research team identified that there are several research gaps in enabling DSA solutions. Based on these gaps, the cMAC concept (Figure 1) was developed to address DSA requirements for effective spectrum sensing, efficient spectrum mobility, and optimized resource management. The cMAC design includes a baseline spectrum-sensing system, which establishes real-time, fast spectrum sensing in the order of milliseconds-as opposed to seconds when performed by current technologies. This method uses algorithms to predict temporal availabilities to improve spectrum sensing effectiveness. In addition, the project develops a spectrum mobility scheme to maximize usage and minimize interference.

To optimize resource management within the spectrum, cMAC utilizes a scheme for contention-based multiple access and predictive algorithms for pipelined sensing. These innovations enable data transmission up to 10 mbps, while simultaneously allowing time for effective spectrum sensing. 

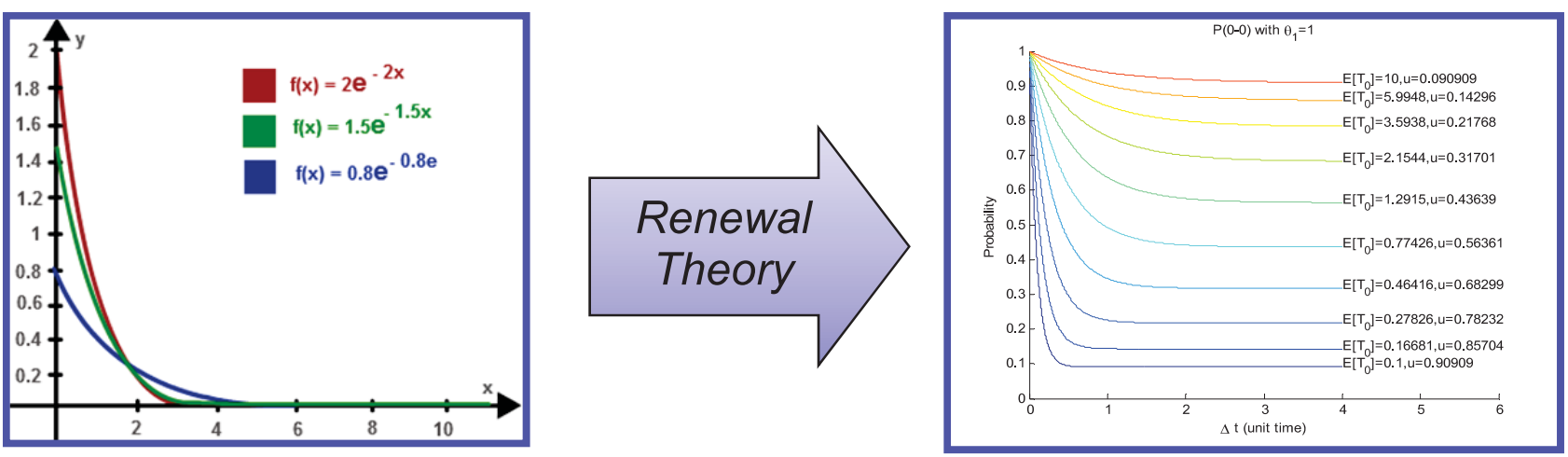

Figure 1. The predictive algorithms used to identify spectrum holes and share channel assignments efficiently.

\section{Benefits to DOE}

This research contributes to DOE's mission by enhancing the security of energy infrastructure and Public Safety communications by developing solutions to implement the U.S. Office of Science and Technology Policy, "Presidential Memorandum-Unleashing Wireless Broadband Revolution." Additionally, this project will benefit federal agencies with operations in the 1755-1850 MHz bands, including the departments of Energy, Defense, Homeland Security, and Justice, as well as the Federal Aviation Administration and National Aeronautics and Space Administration.

\section{Publications}

Wasden, D.L., H. Moradi, and B. Farhang-Boroujeny, "Comparison of Direct Sequence Spread Spectrum Rake Receiver with a Maximum Ratio Combining Multicarrier Spread Spectrum Receiver," IEEE International Conference on Communications 2014, Sydney, Australia, June 10-14, 2014, Submitted, review is in progress.

Deaton, J., et al., "Accelerating Spectrum Sharing Technologies," IEEE Commun. Mag., Vol. 51, 118-122, September 2013.

\section{Presentations}

Moradi, H., "Anti-Jamming LPI-LPD-LPE Wireless Communications System," Wireless Spectrum R\&D (WSRD), Washington, DC, February 5, 2013.

Moradi, H. and R. Subramanian, "DEA Spectrum Communications Research: Video Surveillance," Drug Enforcement Administration (DEA), Idaho Falls, ID, July 29, 2013.

Moradi, H., "National Security /Emergency Preparedness: Technical Execution Discussion," Joint Program Office (JPO), Idaho Falls, ID, June 26, 2013.

Moradi, H., "Anti-Jamming LPI-LPD-LPE Wireless Communications System," USSOCOM, St. Petersburg, FL, June 24, 2013.

Moradi, H., "Anti-Jamming LPI-LPD-LPE Wireless Communications System," USSOCOM, Program Office - PEO SRSE, St. Petersburg, FL, May 3, 2013. 


\title{
13-118 - Geomagnetic Disturbance Field Coupling Measurements on INL Power Grid
}

\author{
Shawn West, Mack Grady, ${ }^{1}$ Scott McBride, Thomas Baldwin, Carol Reid, and Dave Fromme ${ }^{2}$ \\ INL has tested the effects of geomagnetic disturbances (GMDs) caused by solar storms on critical power system \\ components using the laboratory's full-scale power grid. This project measures and correlates geomagnetic \\ storms with the resultant phenomena of Ground Induced Currents (GIC) onto INL's 138kV transmission grid. \\ Because electric utilities are unable to perform these tests on their operating systems, the INL power grid \\ provides a unique opportunity for research and validation. Until INL's LDRD, utility companies have relied upon \\ models and theories to help mitigate the possible effects of solar storms.
}

The project is obtaining GIC measurements from three-phase, alternating-current, high-voltage and mediumvoltage INL transformers, transmission lines, and power infrastructures simultaneously with collocated geomagnetic effect sensors (mass flow sensors). Current-flow sensors have been placed on available transformer phase and ground circuits on the INL transmission line in time to observe the upcoming peak of the solar storm cycle predicted to occur from December 2013 through December 2014. Through the array measurements of electric and magnetic fields, detailed waveforms of the phase voltages, line, and neutral currents will be obtained. Characteristics of the waveforms will be used to develop methods for use by the electric utility industry to detect and protect against grid transformer damage caused by GIC resulting from GMD.

\section{Summary}

To support establishing INL as a leader in understanding and predicting the coupling effects of GIC into a utilityscale grid, this project's researchers developed and installed a GMD/GIC measurement recording system on the INL power grid (see Figures 1 and 2 below). The system measures and correlates GMD effects imposed by solar storms on the INL grid system, allowing INL engineers to understand the phenomena as it affects INL to determine what (if any) damaging effects are possible, and to develop and test mitigations as necessary. The ability to understand, predict, and mitigate GMD/GIC problems on INL's grid will enable assisting the larger-scale utilities in understanding, predicting, and mitigating effects in the national grid system.

\footnotetext{
1. Baylor University

2. Scientific Applications \& Research Associates
} 


\section{NATIONAL \& HOMELAND SECURITY}

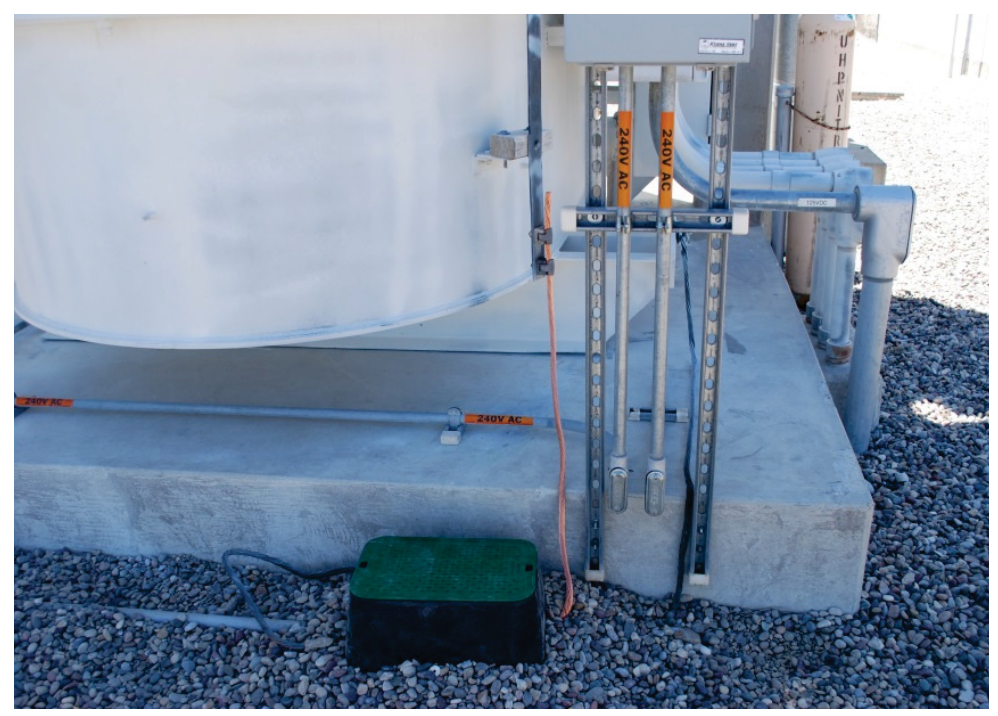

Figure 1. INL Typical INL Transformer - GMD Ground Current Measurement.

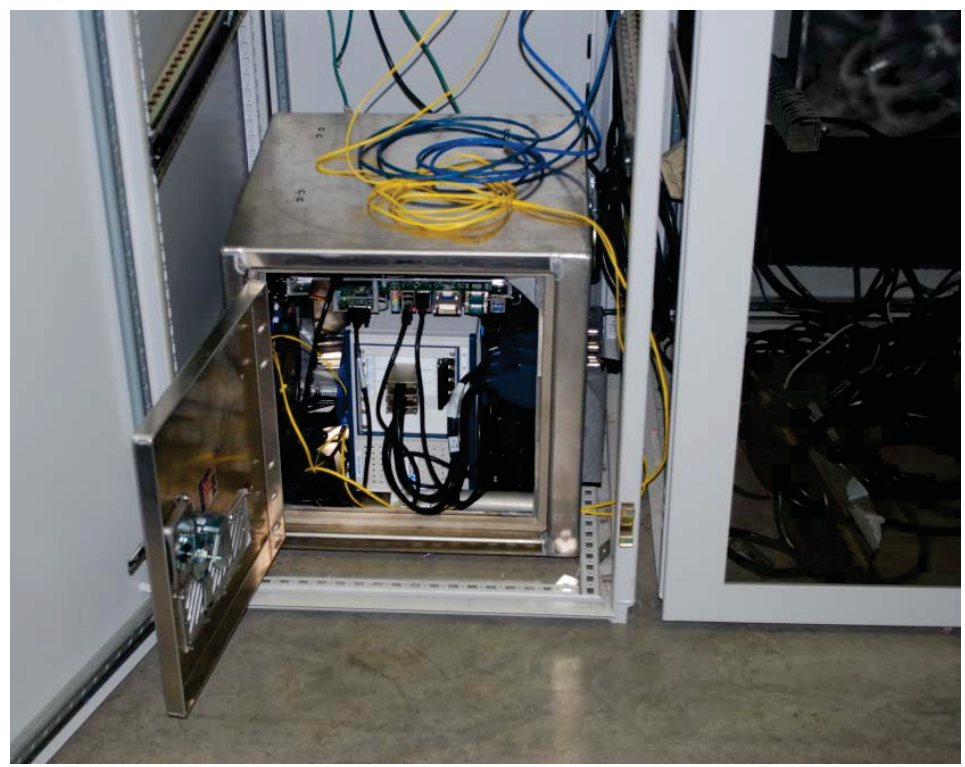

Figure 2. INL Typical Substation GMD Data Acquisition System.

\section{Benefits to DOE}

The resulting technologies of this project will benefit national security by enhancing the reliability and resiliency of energy supplies for our nation's domestic and foreign military installations. This LDRD is developing analysis products that will be deployable to power utilities to assess solar storm impacts to utility systems for critical infrastructure protection. The resulting technology will significantly advance the ability to monitor the electric power grid with benefit to both Microgrid and Smart-Grid research and implementation activities that rely on sensor-based feedback to increase system reliability, efficiency, and resiliency. 


\section{Publications}

West, S., S. McBride, and A. Walker, "INL GMD Measurement System," Report to Industry, In press.

\section{Presentations}

West, S. and A. Walker, "GMD GIC INL DTRA SANDAQ," Idaho Falls, ID, August 2013. 


\section{ENERGY AND ENVIROADENGEAND OTECHNOLOOY}




\title{
11-007 - Integration of Renewable Energy Resources through Energy Storage and SuperGrid
}

\author{
Thomas Baldwin, Mike West, ${ }^{1}$ and David Kelle ${ }^{2}$
}

In 2012, the Assistant Secretary for Energy Efficiency and Renewable Energy, Dr. David Danielson, challenged the renewable energy programs to reach $80 \%$ renewable energy penetration by the year 2050 . As part of this challenge, $40 \%$ of generation would be of the variable power type, such as wind and solar. The technical problem can be simplified as an issue of integration of different types of generation onto an unforgiving electrical grid. A few key research questions spring from this challenge: (1) what is the optimal way of adding variable generation to the grid? (2) how can energy storage and hybrid energy systems (including nuclear energy resources) smooth the power and energy fluctuations for a stable electric grid? and (3) how should the grid evolve to accommodate renewables and hybrid energy generation, energy storage, and new electrical loads such as electric vehicles? The constraints for the challenge include achieving secure, reliable, and resilient energy delivery.

The project aims to: (1) develop the modeling and analysis tools needed to understand the interfacing issues of renewable energy generation to the electrical power grid, (2) determine the impact of placing large-scale energy storage in the power grid to support integration of renewable energy generation, and (3) determine the impact of operating a super-grid on the penetration of renewable energy generation. The LDRD begins with tools and model development, which lead to analysis and the development of best engineering practices. At the end of the LDRD, the conceptual plans for a follow on demonstration project will be developed.

The developed computational tools characterize the dynamic interactions of grid systems where energy storage is used to stabilize the grid. The computational tools support the engineering expertise to analyze and integrate renewable and hybrid energy systems to the electrical grid. The LDRD builds upon the capabilities of INL to address electrical power storage where electrical power may not always be dispatchable and where grid stability and reliability depends on managed load following and supply.

\section{Objectives and Purpose}

The objectives are to identify and study process and grid controls, transmission solutions, and energy storage solutions necessary to couple mixtures of small base-loading constant energy and mid-loading non-dispatchable (variable or intermittent) energy resources to the electric power grid. Three groups of technical solutions will be considered from the electrical perspective. The LDRD will develop control strategies and identify the optimal size and grid location for large-scale energy-storage systems (on the order of $100 \mathrm{MWh}$ or $3.6 \times 1011 \mathrm{~J}$ and larger) for short-term storage (approximately 15 minutes) to address power imbalances and grid instability due to variable or intermittent generation. The work focuses on the electric grid in terms of system performance and the technical challenges with the goal of achieving higher levels of reliability and efficiency in grid performance. The specific focus is on wind and solar energy, the renewable resources with the most potential for significant penetration. Milestones of this effort include acquiring or developing the analysis tools needed to evaluate electric grid control and power plant process control schemes, developing models of grid components and power plants for evaluating and validating new schemes, and identifying the best engineering practices for control philosophies and energy storage sizing, placement, and operating schemes.

1. University of Idaho

2. Florida State University 


\section{Summary}

In the first year, a number of energy storage technologies were evaluated on the basis of engineering sizes and capabilities as well as costs and implementation readiness. Key results indicated that many energy storage technologies work well at small scales ( $<1 \mathrm{MJ})$ and for niche applications. Electric grid applications stress these technologies as energy storage requirements that fall into the gigajoule and terajoule (1 MWhr to $1 \mathrm{GWHr}$ ) range. Technologies that are viable today at these scales are pump-hydroelectric, compressed air, and battery bank systems. Energy density and power density issues remain problematic from many storage technologies. Capital costs for energy systems are also higher than the electricity market is willing to pay.

The characteristics of variable generation were studied. A key aspect of these is the stochastic nature of power from wind and solar production. Understanding the probabilities of supplying power from renewables is necessary for grid integration. The risk of not producing sufficient power is mitigated by expanding the grid control area that supports with spinning reserves. Increasing the penetration of renewables complicates the risk of not producing sufficient power. The concept and quantification of statistical independence of wind and solar resources influences the results of spatial and temporal diversity of renewable resources.

In the second year, the integration of generic variable generation into a simplified grid was studied. Energy storage systems were introduced near the generation and load centers. The costs and benefits of energy storage were assessed. Energy storage demonstrated two important functions. Storage near variable generation can be applied to smooth the energy output to match the electric grid's ability to absorb the power. Energy spillage can be mitigated by sizing the storage capacity. Forced power ramping of conventional thermal power plants due to renewable energy can be reduced with the application of energy storage. Sensitivity analysis has been performed to understand the impact of various levels of renewable penetration into the grid.

In the third year, the concept of fractal power systems architectures was investigated. The architectural framework (see Figure 1) helps to organize the current technical challenges to the grid and the adoption of new classes of "smart" consumer appliances and loads. Fractal grid structures address a fundamental change in philosophy regarding energy structures, such as the recent work in the field of smart grids and micro-grids. The approach unifies existing grid theory and application through developing self-similar energy structures based on scaleinvariant fractal patterns (like patterns in biology). These structures consist of connected, individual cells at scales ranging from Independent System Operators to the level of smart appliances and loads at customer homes. Each cell consists of a transport structure connecting the cell's customers, generation resources, and energy storage systems. The cells are connected to each other via an inter-tie structure that performs the roles of power exchange among cells, sharing of information, and control functions. Due to the nature of these structures, implementation can be distributed spatially as well as temporally. 


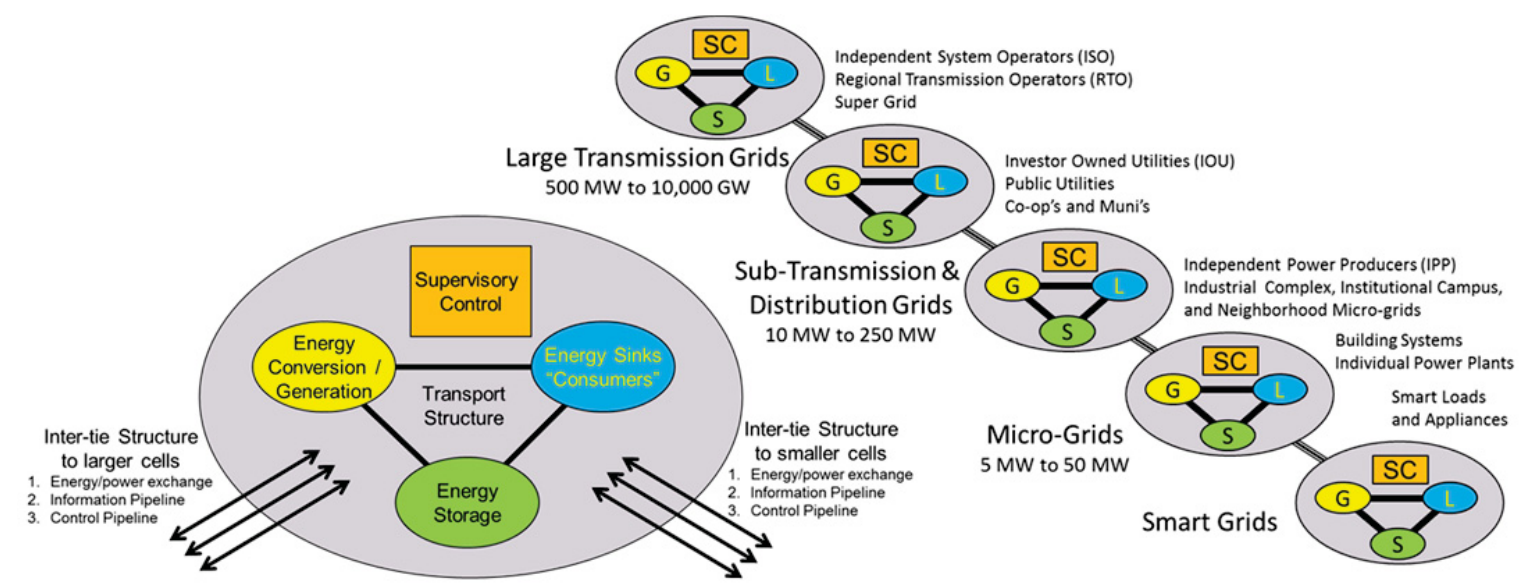

Figure 1. Architectural framework of fractal power systems.

\section{Benefits to DOE}

The DOE Offices of Energy Efficiency and Renewable Energy and Office of Electricity Delivery and Energy Reliability have established a set of mission objectives through the collaborative Grid Tech Team. These objectives have been expressed as a roadmap for the 2030 grid. Key aspects include: (1) the development of the Grid 2030 architecture, (2) development and demonstration of critical technologies such as energy storage and smart controls, (3) accelerating the acceptance of new technologies, (4) strengthening the energy market operations, and (5) building government-education-industrial partnerships.

This effort supports the ongoing R\&D work of integrating renewable and hybrid energy resources by providing engineering tools and architectural solutions for the electric power grid. The results of this work are fundamental to the future operation of electric power systems where $\mathrm{CO}_{2}$ emissions must be regulated and electric vehicles are commonplace. Energy security, reliability, and resiliency will be key characteristics of the new grid. Other outcomes of the project include the buildup of capabilities and expertise in electric power engineering research that support activities in small-modular nuclear, biomass, geothermal, hydro, and wind energy systems.

As the electric power industry is required to move towards greener energy sources and implement a Smart Grid for the benefit of customers, many future technical barriers must be addressed. This work prepares INL with technical expertise and tools to conduct research of interest to the DOE Office of Electricity.

\section{Publications}

Kelle, D. and T. Baldwin, "A Mathematical Foundation for Solving Dynamic Event Location Problems using Wide Area Frequency Measurements," Accepted for publishing at the 2014 Clemson Power System on Smart Grids, Clemson, SC, March 11-14, 2014.

Cordoba, J., D. Kelle, and T. Baldwin, "Fault Locating in Distribution Networks with the Aid of Advanced Metering Infrastructure," Accepted for publishing at the 2014 Clemson Power System on Smart Grids, Clemson, SC, March 11-14, 2014. 
Usman, M.U. and T. Baldwin, "Spinning Reserves and Levelized Cost of Energy in Renewable Energy Integration," Accepted for publishing at the 2014 Clemson Power System on Smart Grids, Clemson, SC, March 11-14, 2014.

Kelle, D. and T. Baldwin, "Fractal Microgrids," Accepted for publishing at the 2014 Clemson Power System on Smart Grids, Clemson, SC, March 11-14, 2014.

\section{Presentations}

West, M. and T. Baldwin, "Energy Storage and SuperGrid Integration," Proceedings of the 2013 IEEE North American Power Symposium, Manhattan, KS, September 22-24, 2013. 


\section{2-093 - Development of Unique Nanomaterials for Selective Replacement of Strategic and Critical Materials}

\section{Robert Fox and Joshua Pak ${ }^{1}$}

Highly luminescent semiconductor nanomaterials, having high quantum-yield and stable thermal performance, are ideal for high power and/or high energy-density applications such as those found in light emitting diodes, laser diodes, and energy-efficient solid-state lighting (SSL). The latest research in SSL phosphor technology (conducted by Dai, Duty, and Hu, and reported in Small) has shown that metal chalcogenides and doped metal chalcogenides are best suited as rare earth phosphor replacements in all three current white light-emitting diode commercial designs. The effort reported here specifically focuses on synthesis of ternary, quaternary, and doped metal chalcogenide quantum dots, arranged in hybrid and core-shell architecture, which are specifically designed to replace rare earth phosphors in SSL applications.

\section{Summary}

During FY 2013, we prepared several Chalcopyrite and Kesterite systems (Table 1), characterized and evaluated their photochemical/photophysical behaviors, and proceeded to construct and test a number of crude lightemitting diodes using synthesized nanoparticles.

\section{Table 1. Various I-III-VI and I-IIIV-VI semiconductors, crystal structures, and their bandgaps.}

\begin{tabular}{|c|l|l|l|}
\hline Entry & \multicolumn{1}{|c|}{ Semiconductor } & \multicolumn{1}{|c|}{ Bandgap } & \multicolumn{1}{c|}{ Crystal Structure } \\
\hline 1 & CulnS & $1.53 \mathrm{eV}$ & Chalcopyrite \\
\hline 2 & $\mathrm{CulnS} / \mathrm{ZnS}$ & $1.53 \mathrm{eV}$ & Chalcopyrite/Core-Shell \\
\hline 3 & $\mathrm{CulnZn} \mathrm{S}_{2+\mathrm{x}}$ & $1.53-3.54 \mathrm{eV}$ & Chalcopyrite/Wurtzite \\
\hline 4 & $\mathrm{AgInS}_{2}$ & $1.87 \mathrm{eV}$ & Orthorhombic \\
\hline 5 & $\mathrm{Ag}_{1-\mathrm{x}} \mathrm{Cu}_{\mathrm{x}} \mathrm{InS}$ & $1.53-1.87 \mathrm{eV}$ & Orthorhombic \\
\hline 6 & $\mathrm{AgInZn}_{2} \mathrm{~S}_{2+x}$ & $1.87-3.54 \mathrm{eV}$ & Orthorhombic/Wurtzite \\
\hline 7 & $\mathrm{Cu}_{2} \mathrm{ZnSnS}_{4}$ & $1.50 \mathrm{eV}$ & Kesterite \\
\hline 8 & $\mathrm{Cu}_{2} \mathrm{ZnSnS}_{4} / \mathrm{ZnS}$ & $1.50 \mathrm{eV}$ & Kesterite/Core-Shell \\
\hline
\end{tabular}

\section{Task 1}

Preparation of I-III-VI Chalcopyrite nanoparticles and related materials: For this task, we prepared several I-III-VI-semiconductor materials (entries 1-6, Table 1) using both single source precursor and multi-source precursor methods. Synthetic methods (microwave and solvothermal decomposition) were optimized from FY 2012 efforts to produce high quality nanoparticles. Materials that were synthesized were characterized using ultraviolet-visible, $x$-ray diffraction, inductively coupled plasma-mass spectroscopy, and high resolutiontransmission electron microscope techniques.

Preparation of CulnS ${ }_{2}$, CulnS $/ 2 n S$ and CulnZn $S_{2} S_{2+x}$ nanoparticles (entries 1-3, Table 1): A multi-source method for preparing CulnS $\mathrm{S}_{2}$ nanoparticles has been explored in order to consistently produce nanoparticles with precise 


\section{ENERGY \& ENVIRONMENT SCIENCE \& TECHNOLOGY}

size and stoichiometric control. The CulnS $S_{2}$ nanoparticles were used as reference particles for comparison with both CulnS $2 / Z n S$ core-shell and CulnZn ${ }_{x} S_{2+x}$ alloy nanoparticles. We have observed a general improvement in photoluminescence behavior of nanoparticles as a function of the addition of a $\mathrm{ZnS}$ shell. Both the thickness of the shell and the method by which the shell is added play a critical role in the associated photoluminescence behavior. Improvements in photoluminescence of nearly $300 \%$ above the reference $\mathrm{CuInS}_{2}$ nanoparticles has been measured as the ZnS shell thickness increases (Figure 1).
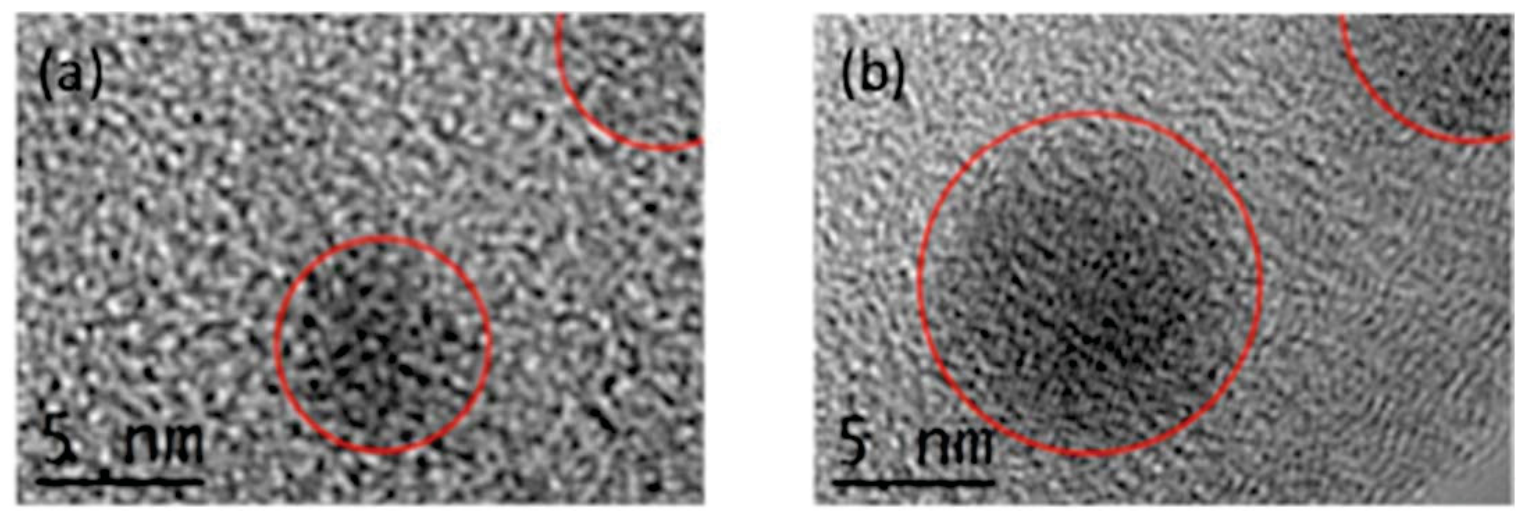

Figure 1. High resolution-transmission electron microscope image of (a) Culn $S_{2}$ and (b) Culn $S_{2} / Z n S$ nanoparticles.

While quantum efficiency could be improved further through post-synthesis thermal treatment and through changes in stoichiometry, the peak quantum efficiency of our core-shell materials has not yet achieved the maximum values found in literature. Further investigation indicates that the technique used to form the shell over the core may be promoting excessive cation exchange between zinc $(\mathrm{Zn})$ in the shell layer and elemental components which comprise the core particle. Infusion of $\mathrm{Zn}$ into the core and changes in core stoichiometry, or crystalline phase, may affect quantum yields. In order to test this hypothesis, we have prepared Culn $Z n_{x} S_{2+x}$ alloy nanoparticles and are currently in the process of evaluating the data.

Our current efforts for this sub-task are focused on improving the crystal quality along with observing distinct coreshell phase boundary formation during particle synthesis and addition of the ZnS shell. New shell application chemistry is being developed to improve core-shell quantum efficiency. Newly applied organic passivating agents, used to isolate core materials and prevent agglomeration, also appear to play a key role in the formation of a distinct $\mathrm{ZnS}$ shell phase around the particle core, without promoting excessive alloying of $\mathrm{Zn}$ with the core.

Preparation of $A g I n S_{2}, \mathrm{Ag}_{1-x} \mathrm{Cu}_{x} \operatorname{InS}_{2}$ and $A g \operatorname{lnZn} \mathrm{n}_{2+x}$ nanoparticles (entries 4-6, Table 1): Another route to increasing core-shell quantum efficiency was developed by substituting silver $(\mathrm{Ag})$ with copper $(\mathrm{Cu})$ in the standard $\mathrm{CulnS}_{2}$ particles. Several Ag containing Chalcopyrite nanoparticles were prepared using both single and muti-source precursor methods. AglnZnS ${ }_{3}$ alloy nanoparticles demonstrated the highest quantum efficiency measured to date in this study (Figure 2). In addition, core-shell particles comprised of AglnZnS ${ }_{3}$ cores and $\mathrm{ZnS}$ shells were prepared and deposited into a crude colloidal quantum dot electroluminescence test device. The particles were found to luminesce upon application of voltage to the device. Along with optimizing device construction, new particles are being synthesized, characterized, and tested to improve luminescence properties. New chemistry to apply a distinct $\mathrm{ZnS}$ shell phase is being investigated. Changing the passivating organics from alkane thiols to amines holds promise for a generation of distinct core-shell phases. 


\section{ENERGY \& ENVIRONMENT SCIENCE \& TECHNOLOGY}

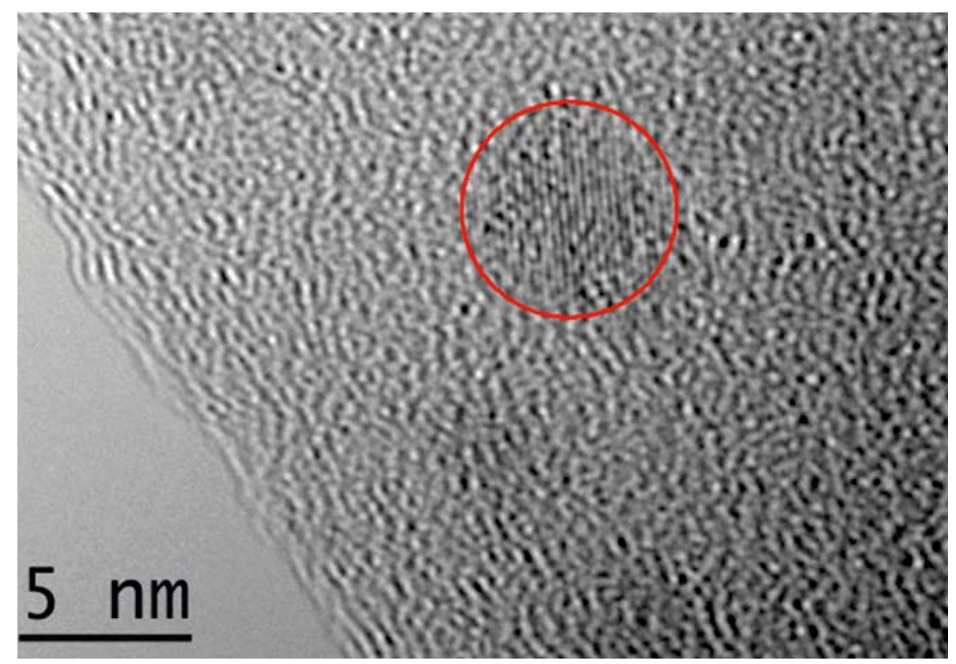

Figure 2. High resolution-transmission electron microscope image of $\mathrm{AgInZnS}$, nanoparticle.

\section{Task 2}

Preparation of I-II-IV-VI Kesterite nanoparticles and related materials (entries 7-8, Table 1): Quaternary Kesterite semiconductor, such as $\mathrm{Cu}_{2} \mathrm{ZnSnS}$ and its related materials, are structurally similar to Chalcopyrite and viewed as alternative to above ternary semiconductor materials. Currently, we are developing a multisource synthetic method which can accommodate alloying of other metal ions to produce related alloy nanoparticles.

\section{Task 3}

Construction of crude electroluminescence test cells and electroluminescence testing of nanomaterial alloys: This task focused on construction of a crude electroluminescence device used for testing colloidal nanoparticle suspensions. Crude devices were constructed from indium tin oxide (ITO) conductive glass having dimensions of roughly $2 \mathrm{~cm} \times 2 \mathrm{~cm}$. The ITO glass was sputter coated with a NiO coating, onto which a nanoparticle suspension was deposited and dried. The particles on the $\mathrm{NiO}$ were then sputter coated with a $\mathrm{ZnO}$ layer, then an aluminumdoped zinc oxide (AZO) layer, and finally with an aluminum (Al) layer. Electrical contacts were made by using alligator clips which attached to the ITO glass and the aluminum. Electrical current was supplied to the glass, causing nanoparticles to luminesce. Electroluminescence occurred as a function of current and nanoparticle type. Data were acquired and compared to fluorescence response and quantum efficiency for each nanoparticle batch.

\section{Benefits to DOE}

This project is focused on synthesis of materials that are useful for replacing lanthanide-based phosphors found in energy-efficient SSL. The development of high-efficiency luminescent materials that are not based on rare earth phosphors is a cross-cutting issue for multiple DOE branches and programs, including the Next Generation Lighting Initiative, Basic Energy Sciences Program, SSL Program, Energy Efficiency and Renewable Energy Building Technologies Program, and Advanced Research Projects Agency - Energy, as well as an upcoming DOE focus area involving replacement, recycle, reduction, and reuse of Energy Critical Elements such as rare earths. Successful synthesis of replacement phosphors for SSL will favorably impact DOE programs and allow for roadmap milestones and performance criteria to be met. 
This project will benefit DOE environmental quality goals and objectives by developing and advancing "green" nanosynthesis technology that is specifically designed to reduce, reuse, recycle, and recover chemicals used in commercial-scale materials manufacturing processes. Successful generation of targeted luminescent materials and successful adaptation of our solvothermal synthesis process to make luminescent materials will provide U.S. industry an advantage by reducing overall environmental liabilities through reduction of hazardous chemicals used in materials synthesis processes. If successful, this work will result in a more rapid advancement of energyefficient SSL into the commercial market by replacing rare earth phosphors with commercially-attractive materials. A more rapid entry of SSL into the U.S. lighting market will reduce U.S. electricity consumption and dependence on non-domestic energy sources. Thus, successful outcome of this LDRD will advance the deployment of secure and sustainable energy systems. 


\title{
12-096 - Isolation and Purification of Critical Metals Using lonic Liquid Based Solvent Extraction
}

\author{
Aaron Wilson, John Klaehn, Eric Dufek, Mark Ogden, Mary Mincher, and Bruce Mincher
}

Dual extractant, nonpolar room temperature ionic liquids (RTIL) are recyclable, may allow reduced processing volumes, avoid problems associated with volatile organic solvents, and allow for novel separation and processing methods targeting strategic and critical materials (S\&C). This project has developed RTIL for the extraction and separation of targeted S\&C that feature a non-polar, anionic extractant and a non-polar, non-coordinating cation. The synthesized materials were generated from existing extractants to avoid costly empirical experiments that produce extractants of unknown quality and behavior. Specifically, this work involved the synthesis and characterization of innovative material RTIL and salting agents, extraction studies, and exploration of electrowinning with such materials.

\section{Summary}

We have produced three dual extractant nonpolar RTIL on a $20 \mathrm{~g}$ scale including: hexadecyltributylphosphate (2,4,4-trimethylpentyl)-dithiophosphinium (HDTBP:Cyanex 301), butyldecyltrihexylphosphate

(2,4,4-trimethylpentyl)-dithiophosphinium (Cyphos:Cyanex 301) butyldecyltrihexylphosphate 4,4,4-trifluoro-1(thiophen-2-yl)butane-1,3-dionate (Cyphos:TTA), betaine bistrifluoromethylsulfonylimide $\left(\mathrm{HBet}\left(\mathrm{Tf}_{2} \mathrm{~N}\right)\right.$ ), and attempted to produce a Octyl(Phenyl)-N,N-Diisobutylcarbonoylmethyl-Phosphine Oxide (CMPO) RTIL. In addition, sodium 4,4,4-trifluoro-1-(thiophen-2-yl)butane-1,3-dionate (NaTTA) was produced as a salting agent. These materials were comprehensively characterized by nuclear magnetic resonance and electrochemically characterized though cyclic voltammetry and other applied potential experiments.

We explored means to enhance electrowinning in RTIL which suffers from mass transfer issues. The methods we developed were described in the Invention Disclosure Record BA-699, "Method to enhance electrodeposition rates in ionic liquids." It was found that the electrochemical window of the extractant RTIL was not sufficient for electrowinning most S\&C. While our electrowinning methods are promising for other applications with RTIL systems featuring wider electrochemical windows, these experiments were not pursued on this LDRD.

The extraction distribution coefficient of the dual extractant nonpolar RTIL was studied using a $10 \mathrm{ppb}$ lanthanide standard containing 13 of the 14 lanthanides, Figure 1. The most novel system which also displayed promising results was the Cyphos:TTA system. However, it was discovered that TTA degraded through hydrolysis to form 2-acetylthiophene and trifluoroacetic acid due to the instability of the deprotonated TTA in aqueous conditions. In an effort to circumvent this issue, Cyphos:HTTA:0.5 $\left(\mathrm{NEt}_{3}\right)$ was prepared. The Cyphos:HTTA:0.5( $\left.\mathrm{NEt}_{3}\right)$ solution also showed promising results, Figure 1, especially when compared to variations on the system, Table 1.

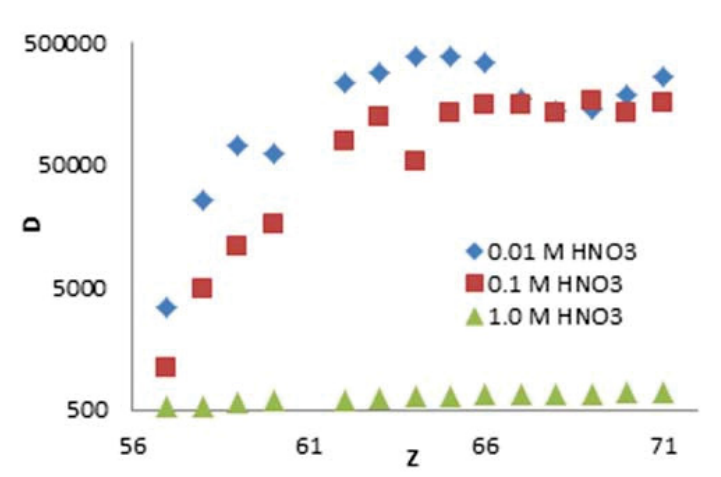

Figure 1. Distribution of $10 \mathrm{ppb}$ nitric salts into the nonaqueous phase (RTIL: Octanol 1:1 by volume). (RTIL =Cyphos:HTTA:0.5[NEt 3$]$.) 
However, even the presence of half a mole equivalent of organic amine resulted in the degradation of the HTTA. The Cyphos:HTTA:0.5( $\left.\mathrm{NEt}_{3}\right)$ solution displayed drastically different distribution coefficients at various points in time after its preparation. It is possible that a Cyphos:HTTA:0.X $\left(\mathrm{NR}_{3}\right)$ solution could be used as effective extractant if prepared fresh before each use and used promptly. This work was not conducted due to funding limitations, but even if the funding was available, serious consideration should be given to the value of exploring an extraction system with such a limited lifespan.

Table 1. Representative set of extraction experiments.

\begin{tabular}{|l|l|l|}
\hline \multicolumn{1}{|c|}{ Extractant } & \multicolumn{1}{c|}{ Solvent } & \multicolumn{1}{c|}{ Result } \\
\hline TTA $\left(\mathrm{PR}_{4}\right)$ & Neat and range of dilutions & Highly variable results. \\
\hline $\mathrm{HTTA}-\left(\mathrm{PR}_{4}\right) \mathrm{Cl}$ & Neat & $\begin{array}{l}0.1-2.0 \mathrm{M} \mathrm{HNO}_{3} \text { No extraction } \\
0.01 \& 0.4 \mathrm{M} \mathrm{HNO}_{3} \text { low general extraction }\end{array}$ \\
\hline $\mathrm{HTTA}-\left(\mathrm{PR}_{4}\right) \mathrm{Cl}$ & $1: 1$ (vol:vol) & $0.01-4.0 \mathrm{M} \mathrm{HNO}_{3}$ No extraction \\
\hline $\mathrm{HTTA}$ & $10 \%$ in octanol & $0.01-4.0 \mathrm{M} \mathrm{HNO}_{3}$ No extraction \\
\hline$\left(\mathrm{PR}_{4}\right) \mathrm{Cl}$ & $10 \%$ in octanol & $0.01-4.0 \mathrm{M} \mathrm{HNO}_{3}$ No extraction \\
\hline 2-acetylthiophene $\left(\mathrm{PR}_{4}\right) \mathrm{Cl}$ & Neat \& 1:1 (vol:vol) & $0.01-4.0 \mathrm{M} \mathrm{HNO}_{3}$ No extraction \\
\hline HTTA-$\left(\mathrm{PR}_{4}\right) \mathrm{Cl}-0.5 \mathrm{NEt}_{3}$ & Neat \& 1:1 (vol:vol) & Results in Figure 1 \\
\hline
\end{tabular}

This project fulfilled all of its proposed tasks and milestones and demonstrated some interesting results. However, the RTIL screening did not lead to a system that displayed consistent and stable behavior. The system which was studied in most detail would require further experiments to characterize the degradation process and how that process affects extraction performance. The value of such research is debatable.

\section{Benefits to DOE}

The importance of S\&C is significant to the United States' economic and defense future. Research into S\&C furthers INL's LDRD mission objectives "Discover, develop, and deploy innovations that advance the critical infrastructure systems" and "Advance the deployment of secure and sustainable energy systems." These are major challenges for mining and recycling S\&C by providing effective extraction and separation of S\&C using remarkably complex systems. 


\title{
12-099 - Designing Algal Biofuel Consortia for Increased Function and Reduced Nutrient Costs
}

\author{
Joni Barnes, Amber Miller, Deborah Newby, and Bill Bauer
}

Microalgae are an excellent platform for multiple applications in biofuel and renewable energy production, as well as $\mathrm{CO}_{2}$ sequestration and organic synthesis. Microalgae are eukaryotic organisms with simple photosynthetic nutrient requirements that grow rapidly and can live in harsh environments. They can provide feedstock for a variety of renewable fuels, including biodiesel, methane, hydrogen, and ethanol, and microalgal biomass can be processed using existing technologies and distribution systems. Currently, to be economical at a large scale, microalgae are frequently cultivated in large open pond systems which are less expensive to build and operate than highly controlled bioreactors. However, due to nutrient availability and unutilized wavelengths of light, open ponds are more susceptible to contamination by undesirable microalgae and bacteria, which leads to decreased productivity and pond population crashes.

In the environment, microbial interactions shape and influence microbial communities and ecosystems. Antagonistic interactions are known to promote diversity in microbial communities, whereas synergistic interactions have been shown to provide nutrients and stability. The principle goal of this project, which was funded for two years, was to assess the use of mixed culture techniques as a means to improve the stability, productivity, and economics of open pond microalgal cultivation. Various cyanobacteria and other photosynthetic organisms can utilize wasted light energy, fix nitrogen, and/or provide growth factors, which, if grown with microalgae, could potentially increase biomass yields and reduce nutrient input costs. In order to construct effective consortia for enhanced biofuels production, these types of organisms must be identified, characterized, and evaluated both under single and mixed culture conditions.

\section{Summary}

In the first year, a culture collection consisting of 24 phototrophic microorganisms was established and is currently available as a resource for biofuels research at INL. The collection includes commercially significant eukaryotic microalgae, a variety of cyanobacteria, and several aerobic anoxygenic phototrophic bacteria (AAnP). These microbial groups represent the major types and combinations of photopigments found in light absorbing microorganisms. In addition, the AAnP members of the collection, which were isolated from INL algal raceway cultures and identified using 16S rRNA sequencing, were found to be close relatives of the AAnP Porphyrobacter. This result corroborated previous metagenomic studies at INL and suggested that the Porphyrobacter group is a common and perhaps important member of stable algal consortia.

As cultures became available, the spectral characteristics of both pure and mixed cyanobacteria and algal cultures were determined using laboratory spectrophotometers and fluorometers. Fluorometry excitation and emission wavelength scans were performed such that light-harvesting pigments from each organism could be resolved, creating a unique fingerprint for specific members of each group. Differences in spectral features were determined using chemometric multi-way data analysis. Results provided evidence that this methodology is a potential platform for the development of a real time co-culture monitoring tool. 


\section{ENERGY \& ENVIRONMENT SCIENCE \& TECHNOLOGY}

In the second year, the project developed a variety of analytical and cultivation tools to monitor and characterize the growth and lipid production of phototrophic microbes including: an improved Nile Red lipid staining technique, a bench-scale photobioreactor system, and a multipurpose modular growth medium for concurrent growth of microalgae and phototrophic bacteria.

Using these tools, we then began building and testing a mixed algal/prokaryotic consortia. Nannochloropsis oceanica, which produces high amounts of lipids and can thrive in both fresh and brackish waters, was selected as the microalgal component in these studies. Porphyrobacter and various cyanobacteria, including Anabaena sp., Gloeocapsa sp. and Synechococcus elongatus, were chosen based on their ability to harvest a broader range of light energy through accessory pigments, fix nitrogen, and/or produce $\mathrm{CO}_{2}$ or other growth medium components to improve system productivity.

Time course growth experiments of single and mixed cultures containing various combinations and amounts of these organisms were conducted in shake flasks and in photobioreactors (Figure 1). Cells were cultivated under aerobic conditions at $25^{\circ} \mathrm{C}$ under controlled light exposure. Flasks were aerated by orbital shaking at $120 \mathrm{rpm}$, whereas the bioreactors were sparged with air at a flow rate of $130 \mathrm{~mL} /$ minute.

To determine optimum growth of Nannochloropsis, three photobioreactors were inoculated with the microalgae and monitored over a four week period. During this time, excellent reproducibility was exhibited with respect to cell growth, nitrate utilization, and lipid production (standard deviation $<1.7 \%$ ). All cultures reached an optical density of 1.5 at $750 \mathrm{~nm}$ by day 15 and, concurrently, had depleted nitrate from the medium. At this time, lipid levels, as measured by Nile Red fluorescence, increased and reached a value of $1.02 \times 10^{-4}$ fluorescence units per cell by day 22 .

Two component culture studies were then conducted using Nannochloropsis plus one of the photosynthetic organisms listed above. Once Nannochloropsis growth was established ( day 4$)$,

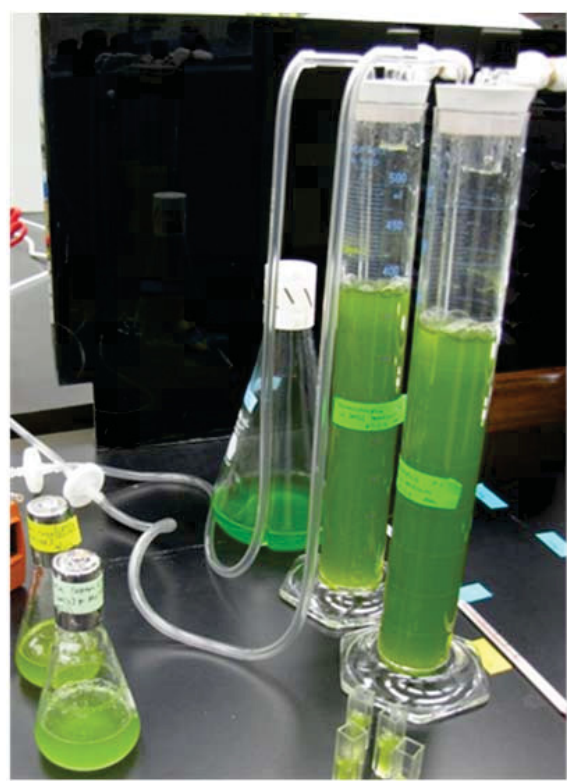

Figure 1. Photobioreactors and shake flasks used for single and mixed culture studies. various volumes (1-20\% of the reactor volume) of the bacterial component were added. Photosynthetic bacteria that accompanied the highest overall growth of Nannochloropsis were selected for further study. As a result, two sets of mixed culture experiments were performed. The first used Nannochloropsis amended with 1\%, 5\%, or $10 \%(\mathrm{v} / \mathrm{v})$ Porphyrobacter, the second used Nannochloropsis with $10 \% \mathrm{~S}$. elongatus. Preliminary data shows that Nannochloropsis amended with $1 \%$ Porphyrobacter had a higher maximum cell density and produced more lipids per cell than Nannochloropsis alone $\left(1.60 \times 10^{-4}\right.$ and $1.20 \times 10^{-4}$ fluorescence units per cell, respectively). In contrast, enhanced growth and/or lipid production by Nannochloropsis was not observed in mixed cultures containing S. elongatus.

Although our work is preliminary and does not account for open pond conditions, these data provide evidence that mixed culture systems can potentially enhance biofuel production and that AAnPs may be important members of a stable biofuel consortia. 


\section{Benefits to DOE}

By developing techniques that enhance algal biofuel production, this work directly supports DOE's mission to secure a sustainable energy future. 


\title{
12-108 - Bakken Hydraulic Fracturing Water Needs and Potential Impacts to the Fox Hills/Hell Creek Aquifer
}

\author{
Mitchell Plummer, Carl Palmer, Hai Huang, Luanjing Guo, A. Jeffrey Sondrup, Jon Reiten, ${ }^{1}$
} Kevin Chandler, ${ }^{1}$ and John Metesh ${ }^{1}$

One of the most important and rapidly expanding developments of fossil fuel resources in the U.S. is the Bakken formation of Eastern Montana and Western North Dakota. Resource extraction from the Bakken formation relies heavily on hydraulic fracturing as a means of creating the fracture space needed to transport tightly constrained oil and gas, a process that requires two to five million gallons of water per well. As development of oil and gas in the Bakken formation moves westward, concerns are increasing that artesian aquifers in Eastern Montana, which have historically supported grazing and agriculture, may be impacted. INL has teamed with the Montana Bureau of Mines and Geology to use numerical simulation to assess the potential impact of water extraction for energy development on other users. This project evaluates water needs for Bakken development in Eastern Montana, characterizes groundwater resources, identifies key areas where development may be water-limited, and seeks to develop an approach for optimizing water usage. Results will help identify strategies for finding water for Bakken development in a manner that limits impact to the sensitive artesian aquifer in Eastern Montana.

\section{Summary}

We have assessed the potential water demand for hydraulic fracturing in Montana from studies of similar development in North Dakota. The number of hydraulic fracturing jobs has been increasing. Available data indicate that the amount of water per hydraulic fracturing job has also been increasing. As a result, water demand for energy development in the Williston basin has greatly increased over the last decade. It is likely that a portion of the water for the Bakken development in Montana will be derived from groundwater sources. One of the more sensitive groundwater resources in Eastern Montana is the confined aquifer of the Fox Hills/Hell Creek formation, which is the primary source of groundwater for stock operations in the area. To assess the potential impact of water withdrawals for hydrofracturing on that aquifer, the Montana Bureau of Mines and Geology has constructed a detailed 3-D groundwater flow model of the aquifer and completed a program of water level monitoring and data collection from existing wells in the aquifer designed to provide historical water level data necessary for establishing historical water potentials. Our preliminary calculations indicated that introduction of new high flow rate wells in the Fox Hills aquifer could lower water levels enough to affect nearby users (Figure 1). When pumped continuously at a rate $(50 \mathrm{gpm})$ that could supply the 2,654 acre-feet per year currently supplied by 35 groundwater pumping stations in North Dakota, for

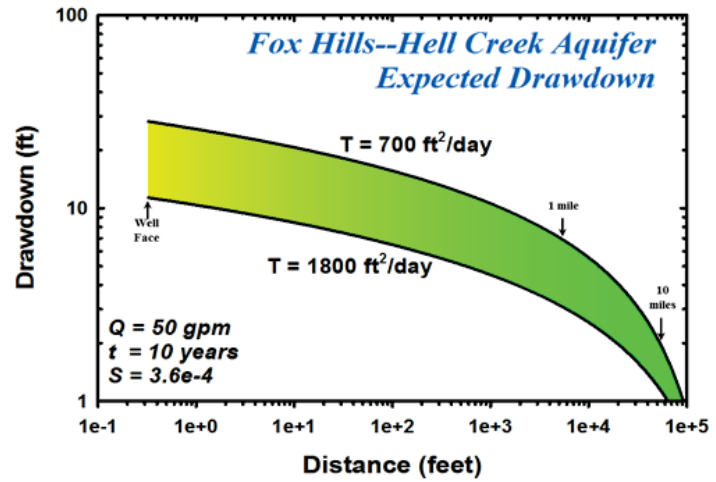

Figure 1. Expected drawdown, after 50 years, around a well pumping 50 gallons per minute (gpm) using the range of hydraulic parameters (transmissivity, $T$, and storativity, S) for the Fox Hills/Hell Creek aquifer system in Eastern Montana. Pumping rate based on reported 2,654 AF/y for 35 groundwater pumping stations in North Dakota. Estimated drawdown at the well ranges from 11 to 28 feet. 
example, drawdowns of up to 10 feet were predicted to extend to approximately a mile from the pumping well. Following development and testing of the 3-D flow model, we were able to examine the likely impact of such pumping using computations that accounted for the variable aquifer thickness, recharge rates, boundary conditions, and other effects that can strongly influence drawdown. Accordingly, we distributed within the 3-D flow model 35 such wells over a 124 square mile area near the center of the likely area of earliest development in Eastern Montana. The superimposed drawdown from such a focused extraction (Figure 2) arrangement suggests that drawdowns of approximately $10 \mathrm{ft}$ would extend to a radial distance of about 15 miles after 10 years. The maximum drawdown near the center of the wells would be about $40 \mathrm{ft}$. Results of such simulations can now be used to test the sensitivity to a variety of hypothetical development scenarios.

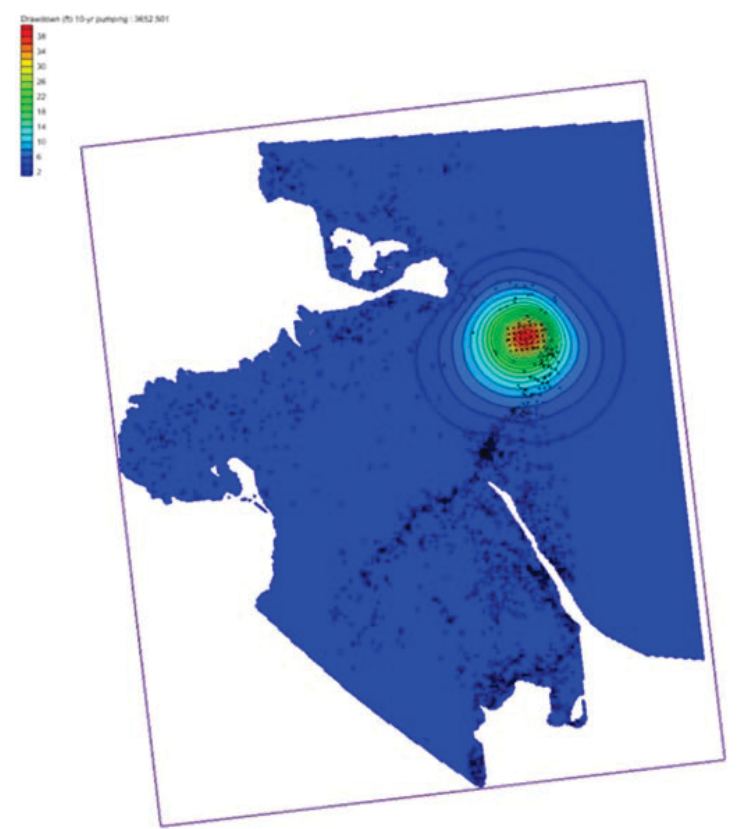

Figure 2. Model predicted drawdown in feet after pumping 35 wells at $50 \mathrm{gpm}$ for 10 years. Wells are shown as black dots. The 35 pumping wells are arranged in a $5 \times 7$ pattern on 4,000 ft centers.

\section{Benefits to DOE}

Water needed for energy developments throughout the arid Mountain West of North America may limit the nation's ability to address energy security issues. Oil and natural gas from tight sand and shale plays an expanding role in the nation's energy future, and hydraulic fracturing is a primary way of accessing these vital resources. Our research, by focusing on the yet to be developed portion of the Williston basin in Eastern Montana, is at the forefront of development activities that are currently exploding in Western North Dakota. We are developing the skills, tools, and resources necessary for analyses of energy projects across the arid western states and Canada. Our research will be directly applicable and useful for state and federal agencies evaluating energy development scenarios and land lease sales and will aid industry in design of drilling plans and optimizing energy developments to minimize impacts to water resources. 


\section{Presentations}

Plummer, M., et al., "Water Need and Availability for Hydraulic Fracturing in the Bakken Formation," Eastern Montana; EPA Technical Workshop - Water Acquisition Modeling: Assessing Impacts through Modeling and Other Means, Arlington, VA, June 4, 2013.

Reiten, J., K. Chandler, and T.R. Wood, "Watering the Bakken Water Quality and Water Quantity Concerns," 2012 Mountain West Water Institute: Ways and Means Workshop, Idaho Falls, ID, May 15-16, 2012.

Reiten, J. and J. Thamke, "Energy Development and Water Needs in the Williston Basin," 2012 Montana Section American Water Resources Association, Anaconda, MT, October 11-12, 2012.

Wood, T.R., et al., "Water Needs for Hydrofracturing the Bakken Formation in Eastern Montana - Data Collection and Model Development," 2012 Mountain West Water Institute: Ways and Means Workshop, Idaho Falls, ID, May 15-16, 2012. 


\section{2-113 - Coupled-Smoothed Particle Hydrodynamics and Discrete Element Model (SPH-DEM) Simulation of Hydraulic Fracturing and Shale Gas Production}

Hai Huang and Milind Deo ${ }^{1}$

A better understanding of the coupling between fluid flow, deformation, and fracturing in shale formations could be used to increase the gas recovery factor and more adequately address environmental concerns associated with hydraulic fracturing. The overall objective of this project is to develop a physics-based, multi-dimensional, coupled flow-transport-geomechanics/fracturing computer model based on the combination of two particle-based methods (Smoothed Particle Hydrodynamics [SPH] and Discrete Element Method [DEM]). The application of the SPH/DEM model can facilitate systematic studies on the nonlinear interactions between fluid flow, proppant transport and settling, and rock failure and deformation at multiple scales, ranging from the core and lab scale to the scale of field hydraulic fractures (hundreds of meters). These studies will fill important gaps in knowledge and fundamental understanding of hydraulic fracturing processes.

\section{Summary}

After two years of the project, key technical milestones have been met, including:

- Developed a parallel DEM code based on Message Passing Interface standard and library, which has been tested and validated on multiple platforms.

- Coupled the DEM code with SPH and the network flow model. The coupled model was applied to a variety of hydraulic fracturing scenarios and generated physics-based hydraulic fracturing patterns that have attracted a lot of attention from industry (see some examples in this report).

- Collaborated with Colorado School of Mines to successfully conduct a laboratory hydraulic fracturing test using a cubic foot granite sample during FY 2013. The data collected will be used to validate the coupled hydraulic fracturing model during FY 2014.

Figure 1 shows the simulated hydraulic fracturing patterns when high-viscosity and low-viscosity fluids are injected into a vertical well. Our model clearly reveals that the low-viscosity fluid creates less dense, more discrete fractures, while high-viscosity fluid creates more complex fracturing patterns involving crack branching due to the complex, strong interactions between fracturing and viscous pressure dissipations.

Figure 2 shows the simulated hydraulic fracturing pattern from a multistage horizontal well bore with multiple initial perforations, which is the key enabling technology for shale gas and oil recovery. Our model correctly resolves the crack-crack interactions via elastic stress and strain field as well as fluid pressure-fracturing coupling. The simulated fracturing patterns completely overturned the widely used industrial hydraulic fracturing simulators, in which cracks are assumed straight and parallel to each other. Our model has generated a lot of attention from the oil and gas industry.

1. University of Utah 


\section{ENERGY \& ENVIRONMENT SCIENCE \& TECHNOLOGY}
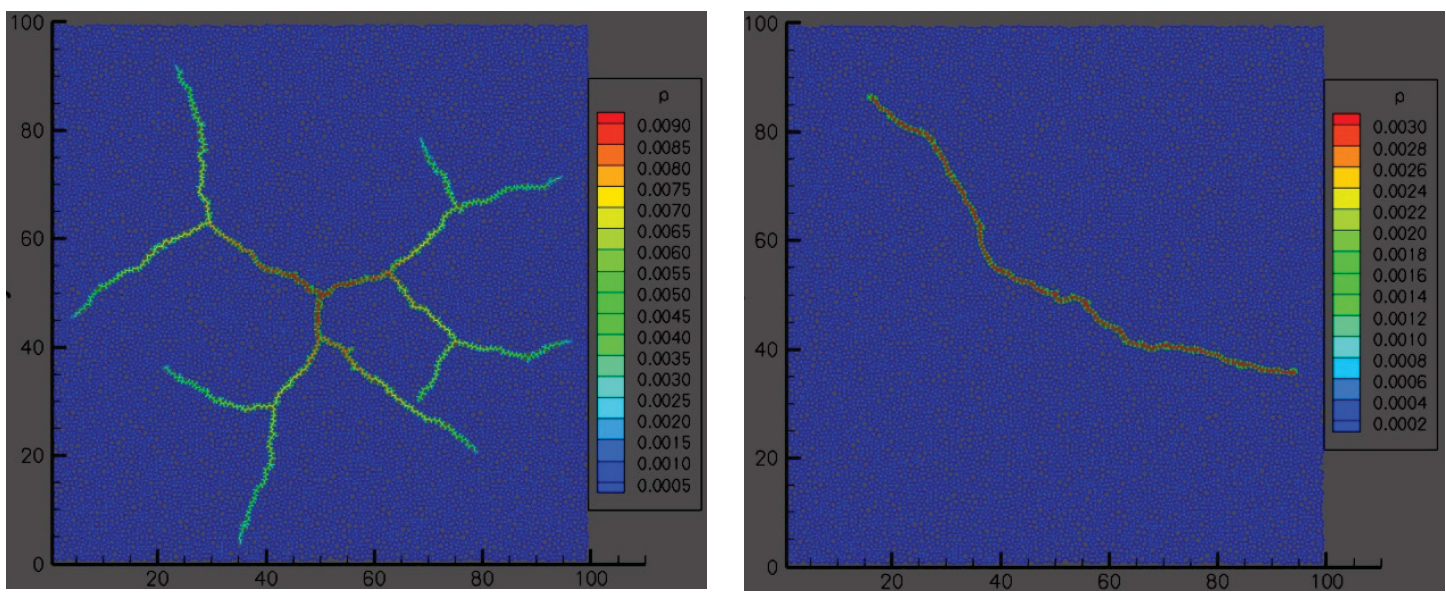

Figure 1. Effect of fluid viscosity on fracturing patterns: (left) high-viscosity and (right) low-viscosity.
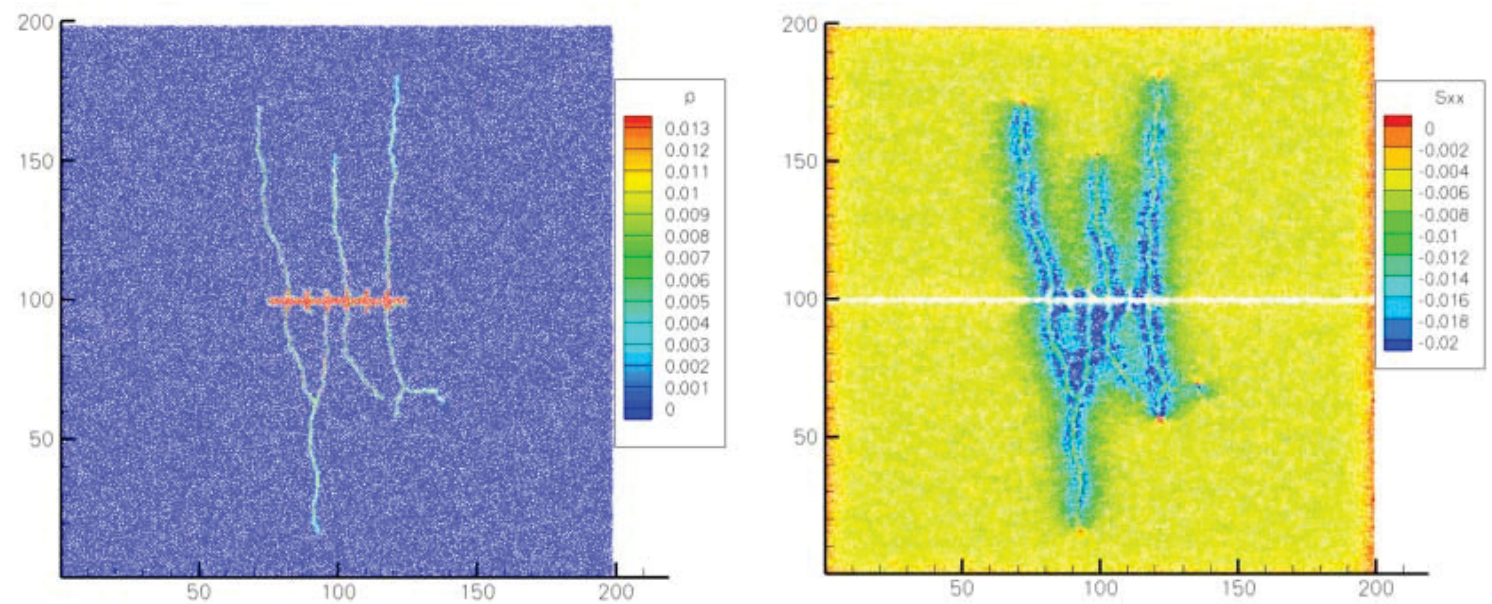

Figure 2. Simulated hydraulic fractures from a multistage horizontal well bore with multiple perforations: (left) fluid pressure field in wellbore and fractures and (right) horizontal stress field.

\section{Benefits to DOE}

The development of the coupled hydraulic fracturing model is of critical importance for the economic and environmentally sustainable production of hydrocarbon fuels from tight rocks (shales) which is directly relevant to DOE's energy security mission. The modeling approach and codes developed in this project will also be readily applicable to a wide range of important subsurface energy/environmental applications at various scales such as enhanced oil and gas recovery from tight source rocks besides shales, thermomechanical fracturing in underground compressed air energy storage, enhanced geothermal system reservoirs, and the geological storage of $\mathrm{CO}_{2}$, chemical waste, and radioactive waste. 


\section{Publications}

Meakin, P., et al., "Shale Gas: Opportunities and Challenges," Environmental Geoscience, 2013, In print.

Huang, H., P. Meakin, and A. Malthe-Sørenssen, "Physics-based Modeling of Interacting Thermal Cracks in Hot Brittle Rocks Subject to Cooling," J. of Geophysical Research-Solid Earth, 2013, In review.

Huang, H., B. Spencer, and J. Hales, "Physics-based Simulation of Thermal Fracturing Process within Uranium Oxide Fuel Pellet," Nuclear Engineering and Design, 2013, In review.

\section{Presentations}

Huang, H., "Physics-based Modeling of Hydraulic Fracture Propagations and Permeability Evolutions During and After Stimulations," AAPG Geosciences Technology Workshop: Geomechanics and Reservoir Characterization of Shales and Carbonates, Baltimore, MD, July 16-17, 2013.

Huang, H., "Simulations of In-situ Production Processes in Reservoir Modeling," University of Utah Unconventional Fuels Conference, Salt Lake City, UT, May 2013.

Huang, H., "Multiphysics Modeling and Simulations of Coupled Thermal-hydro-mechanical-chemical Processes in the Subsurface," University of Utah Unconventional Fuels Conference, Salt Lake City, UT, May 2013.

Huang, H., M. Plummer, and R. Podgorney, "Simulated Evolution of Fractures and Fracture Networks Subject to Thermal Cooling: A Coupled Discrete Element and Heat Conduction Model," in Proceedings of $38^{\text {th }}$ Workshop on Geothermal Reservoir Engineering, Stanford University, Stanford, CA, February 11-13, 2013. 


\title{
12-118 - A Leap Ahead in Energy Storage - An Integrated Approach to an Inorganic High Energy Lithium Battery
}

\author{
Mason Harrup, Frederick Stewart, Eric Dufek, Aaron Wilson, John Klaehn, and Sergiy Sazhin
}

Significant improvements are needed in lithium-ion battery technology, principally in the areas of safety and useful lifetimes, to truly enable widespread adoption of large format batteries for the electrification of the light transportation fleet. The most current research is incremental in nature and seeks to mitigate the shortcomings of current technology. What is instead needed is an entirely different approach — to start fresh with the development of an all inorganic battery. First, there should be a total replacement of the electrolyte - both solvent and salt-for a host of pressing concerns. Chief among these are safety issues of flammability and volatility, too narrow a thermal operating window, and too narrow an electrochemical operating window. A replacement salt must also be found as the current ubiquitous salt $\left(\mathrm{LiPF}_{6}\right)$ degrades, causing damage to all other battery components. Also needed is a complete replacement of the cathode. Current technology employs expensive metal oxides that also exacerbate the safety issues associated with the electrolyte. A replacement that does not use metal oxides and is capable of $5 \mathrm{~V}$ operation is required. In this work, coherent replacements for current technology that overcome these issues have been investigated.

\section{Summary}

This project was proposed to have three principle components: alternative salts, alternative electrolyte solvents, and alternative cathode active materials. After the first year, the alternative salt task was abandoned as the oxocarbon salts that were the focus of this task were found to have insufficient solubility in typical electrolyte solvents to have practical application. The alternative solvents task was continued in year two to continue to find a phosphoranimine molecular architecture that would solve the most pressing problem discovered in year onepoor lithium salt solubility. A new molecular design was discovered that solved the lithium salt solubility problem while retaining the other desired properties. These beneficial properties are non-flammability, to address the serious safety issues with current organic technology, and non-volatility, to address both safety issues and battery lifetime issues. Other beneficial properties of this latest generation phosphoranimine are greatly improved thermal stability and a significantly improved electrochemical stability, particularly at higher voltages that organic electrolytes cannot survive. This opens the door to fabricate higher energy and higher voltage lithium batteries, a goal long held by the DOE and the private battery industry. Lastly, a new synthetic route was devised that makes large-scale phosphoranimine much more practical for ease of transitioning the technology to the private sector. For the alternative cathode material task, the critical advance for cathode design is the hybrid organic-inorganic scaffold that includes the ability to tailor and engineer the material to enhance and optimize desired properties, such as lithium ion uptake capacity and electrical conductivity. The scaffold also serves as the flame resistant structure by which other additives can be included to optimize and target performance. During FY 2013, two distinct chemical pathways appeared most promising. First, a phosphorylation route was employed where a brominated phosphazene precursor was phosphorylated using a chlorophosphate ester. Once attached, the phosphate group was hydrolyzed, followed by lithiation to obtain an active material. The second route that was investigated consisted of two synthetic steps. First, a thiophene ester was formed using a thiophene acid chloride and a hydroxylated phosphazene precursor, followed by complexation of selected metals. Certain metals have a greater utility in cathode due to their oxidation-reduction chemistry. As a model, an iron complex was formed that, when fashioned into a cathode, exhibited electrochemical utility. For all of the new materials formed in this task, characterization was provided by multinuclear Nuclear Magnetic Resonance spectrometry. 


\section{Benefits to DOE}

The project benefited DOE missions by providing an alternative technology to improve the safety and reliability of energy storage devices, in this case lithium-ion batteries. Current batteries lack a proven safety record, have lower voltage capacity, and have a relatively narrow temperature window of operation. The systems studied show promise to solve those problems. Department of Defense is particularly interested in more reliable portable power sources for a wide variety of applications.

\section{Publications}

Harrup, M.K., et al., "Phosphoranimines as Novel Solvents for Lithium-Ion Battery Electrolytes," J. Electrochem. Soc., 2013, In preparation.

Klaehn, J., et al., "Phosphoranimines Designed to Serve as Lithium-Ion Battery Solvents," Phosphorus Sulfur, 2013, In preparation. 


\section{2-120 - Modeling Guided Development of Nanostructured High Energy Electrode Materials for Li-ion Batteries}

Jianguo Yu, Peter Kong, and Michael Glazoff

Nanostructured electrodes in electrochemical cells have improved conductivity and cyclability performance relative to bulk materials. We propose a unique science based strategy (guided by first-principles simulations and thermodynamic modeling of materials) to screen, plasma synthesize, characterize, fabricate, and test advanced nanostructured high energy electrode materials for Li-ion batteries. The inclusion of modeling tasks enables acceleration of new materials development and can help bring INL intellectual property to market within a more competitive timeframe. Our ultimate goal is to have an optimized integrated nanostructure basis for cell design, thus utilizing nanomaterials where they will have the greatest impact toward improving cell performance, stability, and lifetime.

Our objectives for FY 2013 were: (1) modeling: investigate the open circuit voltage and thermal stability of $\mathrm{LiFePO}_{4}(\mathrm{LFP})$ and $\mathrm{Li}_{3} \mathrm{Fe}_{2}\left(\mathrm{PO}_{4}\right)_{3}$ in Li-ion batteries as well as anode materials; and (2) experiment: test plasma synthesis of single phase cathode nanomaterials for $\mathrm{Li}$-ion batteries and develop the process into a single step advanced manufacturing technology.

\section{Summary}

Our accomplishments are summarized as follows:

\section{Modeling:}

1. We investigated the thermal stability of $\mathrm{LiMn}_{1-x} \mathrm{Fe}_{x} \mathrm{PO}_{4}$ at different $x$ values by using a combination of $a b-$ initio calculations and statistical mechanical models. Our results indicated that the thermal stability is significantly enhanced by doping iron $(\mathrm{Fe})$ into $\mathrm{LiMnPO}_{4}$. The stabilized temperatures are 180, 230, and $300^{\circ} \mathrm{C}$, corresponding to $\mathrm{LiMnPO}_{4}, \mathrm{LiMn}_{0.9} \mathrm{Fe}_{0.1} \mathrm{PO}_{4}$, and $\mathrm{LiMn}_{0.8} \mathrm{Fe}_{0.2} \mathrm{PO}_{4}$, respectively.

2. In order to synthesize the anode material $\mathrm{SiC}$, we investigated the relative phase stability of $\alpha-\mathrm{SiC}$ and $\beta$-SiC by using first-principles atomistic thermodynamics calculations. It suggests that to synthesize $\alpha-$ $\mathrm{SiC}$ by plasma, the temperature may need to be higher than $2650 \mathrm{~K}$. This temperature is easily achievable in thermal plasma.

3. We studied the stability of $\mathrm{Si}_{x} \mathrm{C}_{1-x}$ by using first-principles calculations, and our results indicate that $\mathrm{Si}_{x} \mathrm{C}_{1-x}$ may decompose into $\mathrm{C}$ and $\mathrm{SiC}$ if $\mathrm{x}<0.5 \mathrm{Si}$; $\mathrm{Si}$ and $\mathrm{SiC}$ if $\mathrm{x}>0.5$. These modeling results will provide extra useful information for the follow up plasma synthesis process.

4. We studied the open circuit voltage and thermal stability of $\mathrm{Li}_{x} \mathrm{Fe}_{2}\left(\mathrm{PO}_{4}\right)_{3}$ by using first-principles calculations to explore the possibility of mixing LFP and $\mathrm{Li}_{3} \mathrm{Fe}_{2}\left(\mathrm{PO}_{4}\right)_{3}$.

5. We investigated the open circuit voltage and thermal stability of $\mathrm{Li}_{x} \mathrm{Si}_{\mathrm{y}}$ at different $\mathrm{x}$ and $\mathrm{y}$ values by using a combination of ab-initio calculations and statistical mechanical models. 


\section{Experimental:}

1. Using plasma, we synthesized a two phase of nanostructured LFP and $\mathrm{Li}_{3} \mathrm{Fe}_{2}\left(\mathrm{PO}_{4}\right)_{3}$. To test for powder stability in aqueous electrolyte, the plasma generated powder was soaked in water for 24 hours and then dried at $110^{\circ} \mathrm{C}$. No change in powder composition was detected after water soaking and elevated temperature drying, thus indicating that nanomaterials synthesized by plasma are stable.

2. We successfully synthesized a single phase LFP using conventional solid state reaction with the same reactants as the plasma process in one step. Literature reported LFP synthesis is a multiple-step process. The solid state reaction condition will be used for the ongoing plasma reactions to produce a single phase nano LFP.

3. The INL plasma-synthesized, LFP nanopowder is completely spherical, and the particle size ranges from a few $\mathrm{nm}$ to $\sim 300 \mathrm{~nm}$. The majority of particles are significantly below $100 \mathrm{~nm}$.

4. The shapes of Hydro-Quebec carbon coated LFP (C-LFP) powder are irregular, and the particle size ranges from $90 \mathrm{~nm}$ to $830 \mathrm{~nm}$. Due to spherical geometry, the INL plasma nano LFP should have better mass packing density than the Hydro-Quebec C-LFP (i.e., a potential higher gravimetric charge capacity).

5. Due to the spherical geometry and small size of INL plasma LFP nanoparticles, the Li-ion diffusion from the particle interior should be higher than nonspherical particles (i.e., a potential higher volumetric charge capacity).

6. The INL plasma nano LFP may exhibit faster electrode charging and discharging kinetics due to high surface area.

\section{Benefits to DOE}

Our project is central to DOE national security missions. Specifically, it aligns with DOE's energy resources. An important step for the electrification of the nation's personal transportation and for the continued success of the new domestic Li-ion battery manufacturing factories is the development of more cost-effective, long lasting, and abuse-tolerant Li-ion batteries. DOE's continuing R\&D into advanced batteries for transportation offers the possibility of reducing greenhouse gas release and global warming. The techniques developed here will advance the missions of Basic Energy Sciences and the Energy Efficiency \& Renewable Energy's Vehicle Technology Office. DOE's continuing R\&D into advanced batteries for transportation offers the possibility of reducing our dependence on foreign oil and the negative economic impacts of crude oil price fluctuations.

The success of this project will establish a unique experimental, theoretical, and computational capability that will enable acceleration of new advanced materials development. 


\title{
13-024 - Direct Electrochemical Stabilization of Bio-Oils
}

\author{
Tedd Lister and Dayna Daubaras
}

Pyrolysis oils, generated from bio-mass, have great potential to replace petroleum products. However, these oils suffer from chemical instability and must be chemically upgraded for most uses. This one year project initiated the investigation of a unique process using pressurized electrochemical hydrogenation to upgrade and stabilize pyrolysis oils. The specific aim of this concept is to use electrical energy applied at the electrode interface to modify reactive chemicals present in pyrolysis oils and address the low $\mathrm{pH}$, high moisture, and chloride ion content. The goal was to develop a mild method of hydrogenation via electrochemical reduction, where a strongly reducing environment is formed at the cathode surface, enhanced by elevated hydrogen $\left(\mathrm{H}_{2}\right)$ pressure generated inside the reactor. The in-situ generation of $\mathrm{H}_{2}$ coupled with the use of appropriate cell membranes will consume protons and reduce the acidity (and total acid number) of the bio-oil. The process converts reactive carbonyl compounds and stabilizes pyrolysis oil to enable feedstock densification as a liquid intermediate, prior to more robust hydrotreating, to remove most of the remaining oxygen content.

\section{Summary}

The project made significant technical progress over the year of funding. As work developed, two related paths of research were investigated due to the complexity of studying a single process. The first path involved separation and decreasing of the total acid number in bio-oil using a dual membrane cell configured to operate similar to an electrodialysis cell. A unique cell configuration was used where the electrodes were bonded to the membranes to avoid passing current through the bio-oil. Using surrogate mixtures, the $\mathrm{pH}$ was increased to near neutral, and small acid ions (formate and acetate) were removed from the mixture. Experiments demonstrated this function on aqueous fractions of actual pyrolysis oil. It was found that the membrane used was not chemically compatible to raw pyrolysis oil, and leakage across the membrane occurred. The second path of work involved investigating the hydrogenation of organic functional groups using a conventional single membrane cell. Work was performed on both surrogates and pyrolysis oil at ambient pressure. Surrogates such as furfural $\left(\mathrm{C}_{5} \mathrm{H}_{4} \mathrm{O}_{2}\right)$ could be reduced to the corresponding alcohol at ambient pressure. Work on pyrolysis oils proved to be challenging due to problems with analysis. Nuclear magnetic resonance showed some changes in the aldehyde region but not much else could be inferred. Pyrolysis oil is a very complex mixture and presents analysis challenges.

\section{Benefits to DOE}

This work contributes to DOE missions through the advancement of technologies for biomass utilization by enabling use of pyrolysis oil products. The work may enable biomass to replace petroleum products and dependency on foreign sources. It could enable alternative energy by utilizing electricity generated by wind turbines or solar power to create transportation fuels and chemical products. 


\title{
13-027 - Diagnostics of Advanced Energy Storage Materials
}

\author{
Eric Dufek, Michael Glazoff, Lucia Petkovic, and Douglas Akers
}

As new materials for electrochemical energy storage systems such as lithium ( $\mathrm{Li}$ )-ion batteries are developed, means to evaluate the materials need to be advanced. This project aims to develop robust means to evaluate how new materials will perform when included as part of complex electrode structures. The present work uses a host of methods to more accurately predict and evaluate how new materials including electrolytes, binders, and active materials interact. The key emphasis is on the use of methods which provide complimentary data that can be used to effectively distinguish between degradation mechanisms. To date, electrochemical, spectroscopic ellipsometry (SE), scanning electron microscopy, Fourier transform infrared spectroscopy, morphological analysis, and Brunauer-Emmett-Teller surface area analysis have been used to characterize single and multicomponent systems.

The performance of Li-ion batteries is dictated by several factors, including storage material, electrode configuration, composition, and environmental variables, such as temperature. Battery electrodes are often composed of a mixture of a Li storage material, some form of support matrix/binder, and particles of conductive carbon to increase electrical conductivity. The result is an intricate, high porosity, highly tortuous structure that hinders the direct path for $\mathrm{Li}$ to and from storage materials. Factors further complicating the scenario include the need to maintain electronic conductivity, structural changes-such as expansion and contraction of materials based on state-of-charge - growth and dissolution of surface films, and delamination of materials from the current collector. Full evaluation of failure mechanisms, especially for new materials, involves the inclusion of all of these factors, as do methods to gauge how materials will age over extended use.

While all mechanisms are important, no single technique can capture the breadth of information needed for full material evaluation. In the Journal of Solid State Electrochemistry, Amalraj and Aurbach identified the task of evaluating interfaces within energy storage systems as key to advancing technology. The present project is developing a multiplexed interrogation procedure harnessing a combination of in-situ techniques, ex-situ imaging of surfaces, electric signals associated with electrochemical processes, and advanced computational procedures to evaluate interfaces. The techniques developed will be broadly applicable to both anode and cathode electrode systems, including those under development for Li and non-Li energy storage systems. Key objectives of this work are the ability to monitor the growth and dissolution of surface films using a small volume, in-situ SE cell, the ability to identify and model morphological changes which occur in composite electrodes during cycling, and the ability to tie electrical signatures to the processes as a first step toward developing a more thorough understanding of electrode aging mechanisms. 


\section{Summary}

This project aims to involve the use of multiple techniques to more accurately predict and identify how energy storage materials age. To date, investigations looking at the growth of the surface electrolyte interphase (SEI) as a function of electrolyte composition and formation path employ electrochemical and in-situ SE. The SEI is crucial for continued operation of battery systems as it provides stability to thermodynamically unstable systems. A characteristic plot showing the current density associated with SEI formation and the corresponding SEl thickness as determined using SE is shown in Figure 1. The data indicate that as a cell is cycled, slight variations occur within the SEI thickness and some current is required to reform the SEI. This

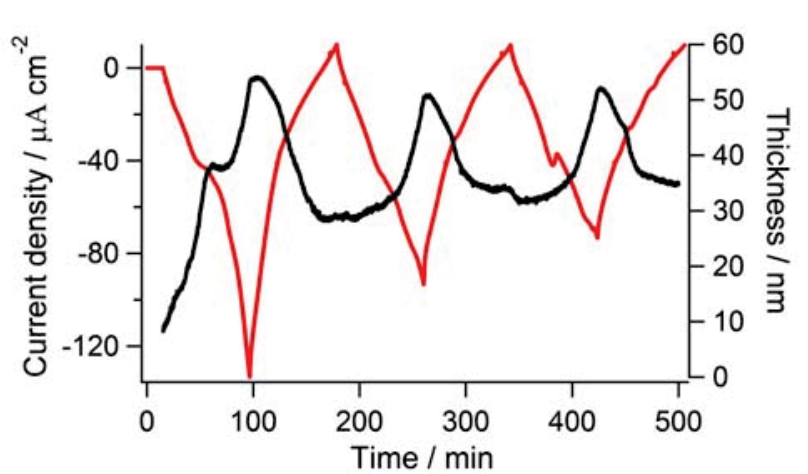

Figure 1. Current density and thickness as determined using SE for a copper (Cu) electrode cycling in battery electrolyte. leads to slight inefficiencies in battery cycling performance. Therefore, minimization of SEI dissolution and regrowth is important to optimize the performance of battery chemistries.

Further, this project has progressed in following the degradation of silicon (Si)-based anodes during cycling and in evaluating the morphological change associated with the degradation of the electrodes after cycling. Additionally, these Si have been evaluated using vibrational spectroscopy and Brunauer-Emmett-Teller measurements to quantify how the surface area of the systems changes during cycling. Future work will focus on combining SE, morphological evaluation, Fourier transform infrared spectroscopy, Brunauer-Emmett-Teller and electrochemical signals to more thoroughly quantify the diverse degradation mechanisms associated with electrode structure and chemistry. By providing information on how structure evolves with cycling, it becomes more plausible to evaluate and model how new electrode systems will behave following extended use and better engineer systems to meet DOE performance metrics.

\section{Benefits to DOE}

The characterization of energy storage interfaces advances the knowledge of electrode performance for energy storage systems. This, in turn, will allow batteries with improved capacity and performance to enter the market. Improved battery capacity and performance support DOE's goal for electrification of the United States vehicle fleet. The diagnostic capabilities also provide knowledge and guidance for the development of advanced energy storage chemistries, including non-Li systems, which minimizes the use of resources while protecting environmental quality. The methods for advanced diagnostics developed under this LDRD advance the science behind energy storage systems by providing a more thorough understanding of complicated battery processes.

\section{Publications}

Dufek, E.J., "Path Dependent Changes in the Optical Properties of the SEI," J. Electrochem. Soc., In preparation.

Dufek, E.J., M.V. Glazoff, and K.L. Gering, "Quantitative Characterization of Si Porous Electrode Morphology Evolution after Several Charge/Discharge Cycles," J. Power Sources or Advanced Energy Materials, In preparation. 


\section{Presentations}

Dufek, E.J. and L.M. Petkovic, "Correlating Electrochemical and Spectroscopic Ellipsometry Signals to Evaluate Energy Storage Interfaces," 224 $4^{\text {th }}$ Meeting of the Electrochemical Society, San Francisco, CA, October 27November 1, 2013.

Dufek, E.J., M.V. Glazoff, and K.L. Gering, "Morphological Analysis for the Evaluation of Electrode Structures," $224^{\text {th }}$ Meeting of the Electrochemical Society, San Francisco, CA, October 27-November 1, 2013. 


\title{
13-045 - Direct Solar Hydrogen Production Using High Temperature Solid Oxide Photoelectrochemical Cells
}

\author{
James O'Brien and Xiaoyu Zhang ${ }^{1}$
}

Hydrogen can be produced directly from solar energy and water using photoelectrochemical cells, a process which has been deemed artificial photosynthesis. The technology currently suffers from low efficiency and materials compatibility issues. The feasibility of a new advanced photocatalytic method for direct solar $\mathrm{H}_{2}$ production from steam at high temperature $\left(\sim 800^{\circ} \mathrm{C}\right)$ using solid oxide photoelectrochemical cells (SOPCs) has been demonstrated as a result of the work performed under this project. In an SOPC, the cathode material of a traditional solid oxide fuel cell is replaced by (or mixed with) a photo-sensitive semiconductor material such as $\mathrm{TiO}_{2}$. The ceramic SOPC operates at high temperature with the $\mathrm{TiO}_{2}$ surface exposed to concentrated solar energy. At room temperature, the magnitude of the bandgap energy of $\mathrm{TiO}_{2}$ is relatively large such that photoelectrochemical excitation of $\mathrm{TiO}_{2}$ requires ultraviolet light. However, as the cell temperature increases, the bandgap energy of $\mathrm{TiO}_{2}$ is reduced, enabling absorption of visible light. In addition, the electrochemical energy required to split $\mathrm{H}_{2} \mathrm{O}$ decreases with temperature. At $800^{\circ} \mathrm{C}, \mathrm{TiO}_{2}$ can absorb visible light to excite electrons to the conduction band, making it suitable for direct solar $\mathrm{H}_{2}$ production. The high-temperature SOPC has several advantages over conventional photoelectrochemical cells. First, since it is operated at high temperature, visible light can be used due to the bandgap narrowing. Second, the thermodynamic $\mathrm{H}_{2}$ production efficiency increases due to the elevated temperature, and activation energies are very low. Third, produced $\mathrm{H}_{2}$ and $\mathrm{O}_{2}$ are physically separated by a solid-state electrolyte. Finally, the $\mathrm{TiO}_{2}$ surface can be directly exposed to solar light without interference from a liquid medium. This concept significantly reduces radiation loss from refraction/reflection effects and attenuation due to scattering from bubble production which is a common problem in conventional aqueous photoelectrochemical cells. In practice, high cell temperatures can be maintained using concentrated solar energy. Concentrated solar light incident on the cells will also result in higher $\mathrm{H}_{2}$ production rates per unit area of cell.

\section{Summary}

Several solid oxide single button cells were fabricated and tested at INL. Testing was performed by heating the cells in a furnace while exposing the photosensitive electrode to high intensity light. The furnace was a cylindrical ceramic fiber furnace capable of operation up to $1000^{\circ} \mathrm{C}$. The top of the furnace was open to allow for light transmission. A solar simulator fitted with a focused mercury $(\mathrm{Hg})$-xenon $(\mathrm{Xe})$ lamp was used as the light source. The cells were based on yttria-stabilized zirconia electrolyte wafers with a platinum $(\mathrm{Pt}) / \mathrm{TiO}_{2}$ electrode on one side and a silver (Ag) mesh counter electrode on the opposite side. Single SOPCs were mounted on top of a ceramic tube with voltage tap wires attached to the top and bottom electrodes, open to air on both sides of the cell. The negative voltage tap was attached to the top $\mathrm{TiO}_{2} / \mathrm{Pt}$ electrode, and the positive voltage tap was attached to the bottom Ag electrode. The ceramic tube was lowered into the furnace until it was resting on an insulation block with the cell positioned at a depth of $\sim 2.5$ in. below the top furnace opening. The solar simulator was powered up to full power $(500 \mathrm{~W})$, aligned, and focused on the top surface of the cell through the opening in the top of the furnace. Data recording was initiated to measure the cell voltage photoresponse. Cell voltage measurements were obtained with the positive voltage tap tied to the top $\mathrm{Pt} / \mathrm{TiO}_{2}$ electrode and the negative

1. Old Dominion University 
voltage tap tied to the bottom Ag mesh electrode. Representative electrical photo-response is shown in Figure 1. The positive cell voltage excursions are associated with the sudden exposure to the high intensity light source. The negative excursions are associated with blockage of the light source. These data indicate an unequivocal photo-response that has the potential for $\mathrm{H}_{2}$ production from steam. The magnitude of the electrical photoresponse of the cells was highly dependent on temperature, with the maximum response of $\sim 100 \mathrm{mV}$ obtained at a cell temperature of $\sim 400^{\circ} \mathrm{C}$ (Figure 2). The reasons for this particular temperature dependence require further investigation. Results of the experimental investigation performed under this LDRD project are promising. Additional work is required to optimize cell and electrode design.

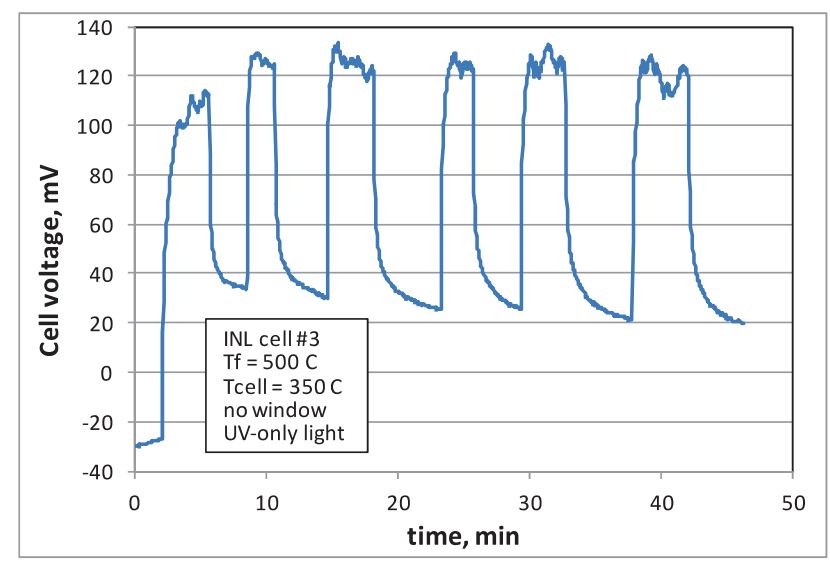

Figure 1. Photoresponse of INL Cell No.3, Tcell $=350^{\circ}$ C, UV mirror, no window in place.

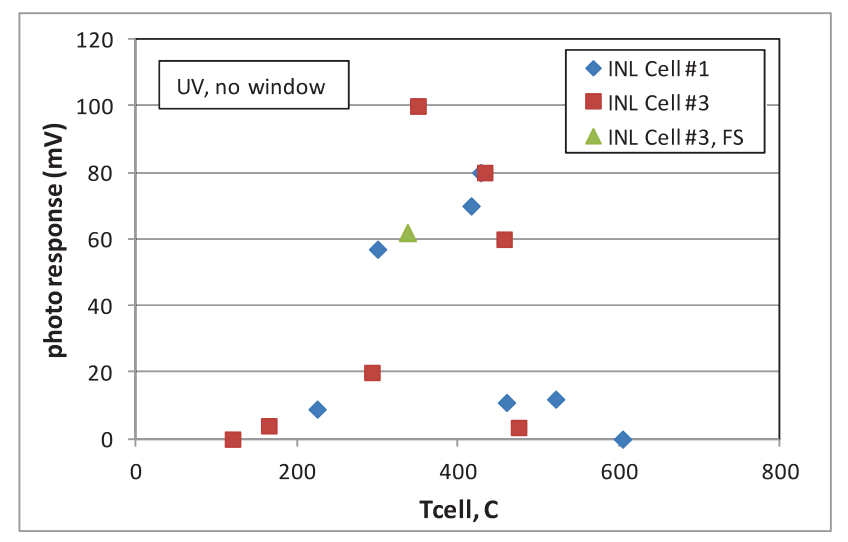

Figure 2. Magnitude of cell voltage photoresponse as a function of temperature.

\section{Benefits to DOE}

Inasmuch as this research has possible application for direct $\mathrm{H}_{2}$ production from renewable solar energy, the ultimate outcome of the research will benefit DOE's energy resources mission. Both $\mathrm{H}_{2}$ production and solar energy applications are prevalent in various DOE research portfolios and are key components of the nation's energy future. Future funding for this work may be pursued with DOE's Basic Energy Sciences program and/or with the National Science Foundation. 


\title{
13-059 - Application of Facilitated Transport Membranes Using Novel Stabilized Metal Complexes
}

\author{
John Klaehn, Aaron Wilson, and Christopher Orme
}

Polymeric facilitated transport membranes can separate olefin (available double bond in unsaturated hydrocarbons) and paraffin (saturated hydrocarbons) gas mixtures using engineered transition metal complexes of silver(I) (Ag[l]) as transport facilitators. By definition, a facilitated transport membrane uses a molecular species that has affinity for a target compound and acts as a shuttle to facilitate transport of the target compound through the polymeric host. Facilitated transport will have a great economic impact on olefin separations, because olefins are needed as feed stocks to the production of plastics and fine chemicals. Olefin production is a multibillion dollar industry that uses several different refinery processes. Typically, olefins are produced by fossil fuel catalytic cracking process; however, this process yields both olefins and paraffins at the same time. Separation of these two products is difficult, because olefins and paraffins have similar molecular sizes and boiling points. The state of the art for the industrial separation of olefins and paraffins utilizes energy intensive cryogenic distillation. It is estimated that 120 trillion Btu/yr are consumed for olefin/paraffin separation in the U.S. As a result, the energy cost makes polymer grade olefins and other industrial raw materials expensive. Our research goal is to provide energy efficient ethylene or propylene separations at near ambient or slightly elevated temperatures by using embedded metal chelate facilitators in polymeric membranes.

Olefin separation has been examined for decades, and it is considered the "holy grail" of gas separations due to the aforementioned issues. The best performance has been achieved by $\mathrm{Ag}(\mathrm{I})$, where the metal ions preferentially complex and facilitate olefin(s) as a gas/vapor through a polymeric membrane. A shortcoming of the previous work is the use of $\mathrm{Ag}(\mathrm{I})$ in a loosely bound or "naked" state in the polymer matrix. Facilitation is lost due to poorly defined coordination spheres and deactivation through oxidation/reduction. To address these issues, a different route to forming transport facilitators is required. Incorporation of metal ions into polymer membranes is a research problem that interfaces both membrane science and coordination chemistry.

\section{Summary}

In FY 2012, our team has successfully synthesized the selected [9]-ane $\mathrm{P}_{3} \mathrm{R}_{3}$ ([9]-ane $\mathrm{P}_{3} \mathrm{R}_{3}=1,4,7-\mathrm{Tri}(\mathrm{R})$-1,4,7-triphosphacyclononane, (Figure 1) metal complex using $\mathrm{Ag}(\mathrm{l})$. The isolated metal complex was proven to be stable towards light, heat, and the atmosphere. Additionally, the $\left[\mathrm{Ag}(\mathrm{I})\left([9]-\mathrm{aneP}_{3} \mathrm{Ph}_{3}\right)(\mathrm{L})_{\mathrm{n}}\right]^{1+}$ complex was stable in aggressive organic solvents, like acetic acid and dimethylsulfoxide $\left(\mathrm{C}_{2} \mathrm{H}_{6} \mathrm{OS}\right)$. INL has submitted a patent application on this ligand for the metal complex for a facilitated transport membrane. Although [9]-ane $\mathrm{P}_{3} \mathrm{R}_{3}$ was an effective ligand, larger yields and improved solubility were needed. Therefore, in

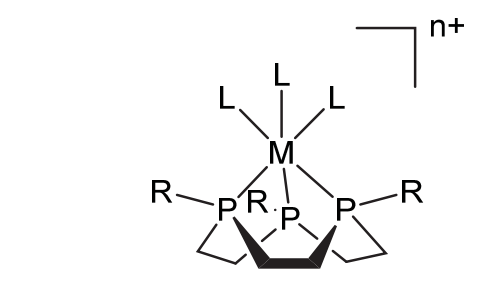

Figure 1. $\left[M\left([9]-a n e P_{3} R_{3}\right)(L)_{3}\right]^{n+}$ FY 2013, commercially available phosphine ligands were employed for better solubility with the polymer to cast polymer membranes. Several different phosphine ligands and metals were used for forming thin, dense, polymeric membranes. 
Two classes of polymers (glassy and rubbery) were used with these tests. Interestingly, the literature does not show a preference to a specific polymer for the olefin/paraffin separation. The dominant transport mechanism for these organic molecules is solubility, thus the polymer membrane must be capable of providing a stable platform that will allow the facilitator to operate without complications. Polymers used in the studies included: polyimides, polyethylene glycol, polydimethylsiloxane, Pebax 2533, and polyphosphazenes. However, as concentrations of the metal salts increased, film formation of dense polymer membranes was difficult. Gas analysis and characterization were not straightforward due to variable film thickness. Therefore, more development was required using simpler $\mathrm{Ag}(\mathrm{I})$ systems before analyzing $\mathrm{Ag}(\mathrm{I})$-phosphine. Thus, the team prepared a series of tests that included several polymers and different Ag salts with varied weight percent

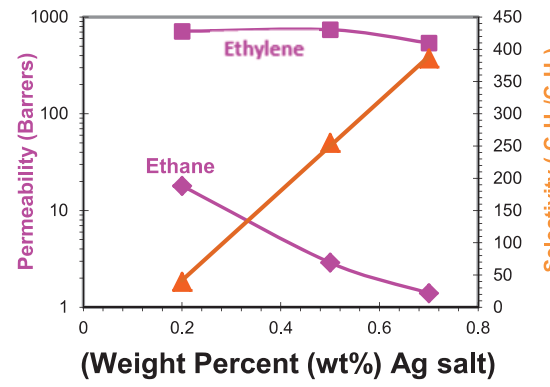

Figure 2. Mixed gas analysis (50:50 ethylene:ethane) using

polydimethylsiloxane membranes with different wt\% AgBF4. compositions. Many provided olefin facilitation, and the best data showed olefin/paraffin gas selectivities of nearly 400 with high olefin throughput ( 1000 barrers), as shown in Figure 2. This is a major success for this project. Despite known issues in film formation, our team found new routes using commercial polymers and Ag complexes which gave these interesting results. The next phase of research would be analyses of the phosphine metal complexes and the metal center's active role in olefin facilitation. The literature does not describe in detail these areas, and INL has found that these items are crucial to olefin facilitation resulting in high-quality separations using these phosphine complexes.

We have analyzed new Ag complexes by multinuclear Nuclear Magnetic Resonance, Fourier Transform Infrared, Raman, thermal analyses, and gas permeability analyses. In the near term, these new data will be published in journal articles and/or future funding opportunities. We have submitted a patent application on this ligand for the metal complex for a facilitated transport membrane.

\section{Tangible Accomplishments (FY 2013)}

- A multi-dentate chelate to $\mathrm{Ag}(\mathrm{I})$ was synthesized and isolated. This novel chelate has excellent stability towards temperature, light, and solvents compared to other $\mathrm{Ag}(\mathrm{I})$ salts.

- New $\mathrm{Ag}(\mathrm{I})$ chelates were successfully mixed with polymer solutions to form thin film membranes. These facilitator-embedded membranes have been analyzed for their preliminary gas separation performance.

- High alkene throughput and excellent olefin selectivities were achieved using a metal complex, which will be published in the future.

- A patent application was sent to the U.S. Patent and Trademark Office.

\section{Benefits to DOE}

DOE, through the Offices of Fossil Energy and Energy Efficiency and Renewable Energy, will benefit from this new capability in improved olefin separation, and this technology also fits into INL's Advanced Manufacturing Platform where specific gas separations are needed. INL is recognized for its contributions to gas separation research using polymer membranes, and this research provides a significant benefit not only to INL and DOE, but also the region and the nation by enabling new economically advantageous, industrially focused chemical separations. Industries this technology benefits include the petrochemical and manufacturing sectors. INL has 
developed many working relationships/collaborations with industry that will lead to development of commercial polymer membranes.

\section{Publications}

Wilson, A.D., et al., "Transition Metal Chelate System for Facilitated Transport," Patent application, June 2012, Submitted.

\section{Presentations}

Klaehn, J.R., A.D. Wilson, and C.J. Orme, "Olefin Gas Separations Using Embedded Transition Metal Chelates in Polymeric Membranes," FY2013 External Review of the Energy and Environment Science \& Technology (EEST) Directorate, Idaho National Laboratory, Idaho Falls, ID, August 2013. 


\section{3-065 - Multi-domain Modeling, Simulation, and Integration Tools for the Dynamic Analysis and Optimization of Hybrid Energy Systems}

Humberto Garcia, Wenbo Du, Amit Mohanty, Farhad Farzbod, and Chris Paredis ${ }^{1}$

This research is establishing scientific and engineering-based computational capabilities (including architectures, environments, constructs, and methods) to design, analyze, optimize, and operate hybrid energy systems (HES). Figure 1 illustrates a hypothetical HES comprising a multiple-input multiple-output system. The development of HES is expected to facilitate meeting national policies regarding high levels of clean energy in our national energy mix, including both renewable and nuclear energy. The research objectives and purpose of this project are summarized as follows:

1. Develop innovative computational capabilities, including modeling, co-simulation, and optimization tools, for designing, operating, and evaluating HES.

2. Develop performance metrics that can be used to offline design and online operate HES as needed by given energy applications.

3. Develop and demonstrate methodologies for the effective deployment of HES that can accommodate high levels of renewable integration.

4. Develop and demonstrate HES configurations that are optimized according to fixed and time-varying requirements and market changes.

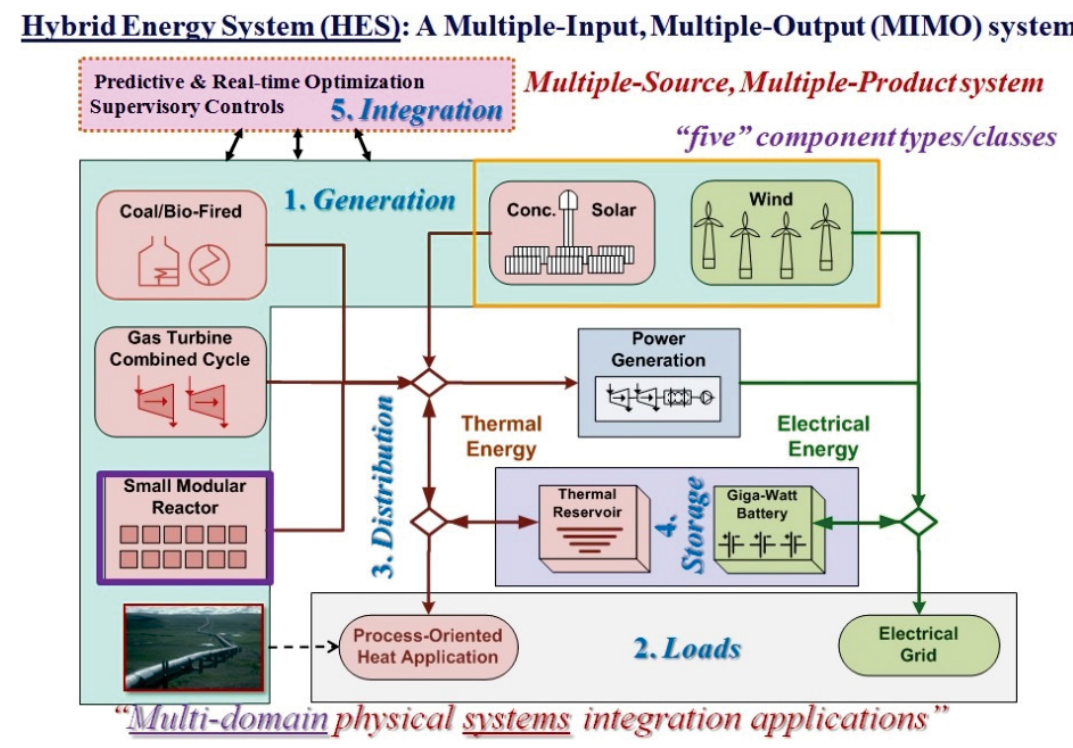

Figure 1. A typical HES and its different component types.

1. Georgia Institute of Technology 


\section{Summary}

In this project, we assume that an HES, when interacting with the electric grid as illustrated in Figure 2, acts as a dynamic energy element that can be used to not only reduce the variability and uncontrollability of renewable generation before connecting it with the grid but also vary output and even flow direction to complement standalone and distributed renewable generation. To better define the computational needs necessary to model, co-simulate, optimize, and operate HES, a detailed HES configuration was identified and designed in detail using typical commercial tools (i.e., ASPEN). The next activity produced a dynamic model using the advanced modeling language Modelica and the Dymola tool. A high level view of this model is shown in Figure 3, with Figure 4 showing a more detailed view of the power subsystem. Other activities included the investigation and implementation of co-simulation environments and experiments involving multiple sub-systems developed in diverse frameworks.

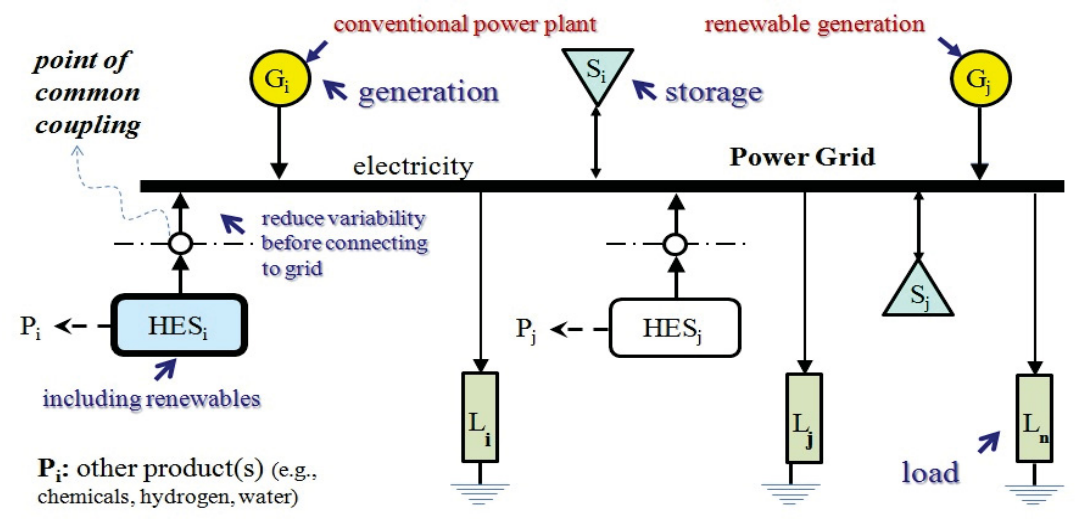

Figure 2. HES interacting with the power grid.

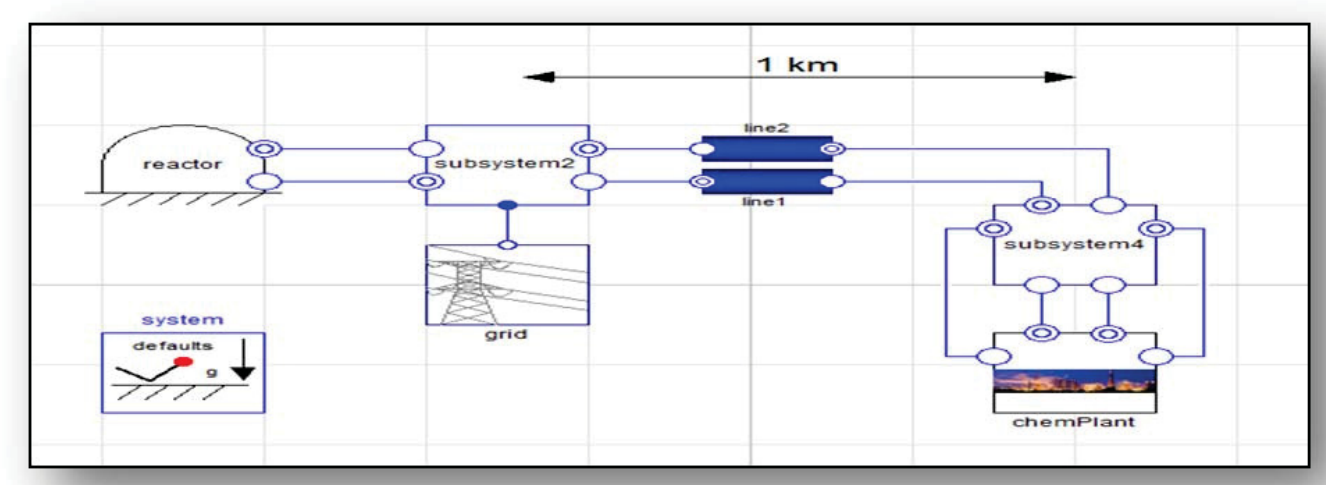

Figure 3. Dynamic model of a HES configuration in Modelica. 


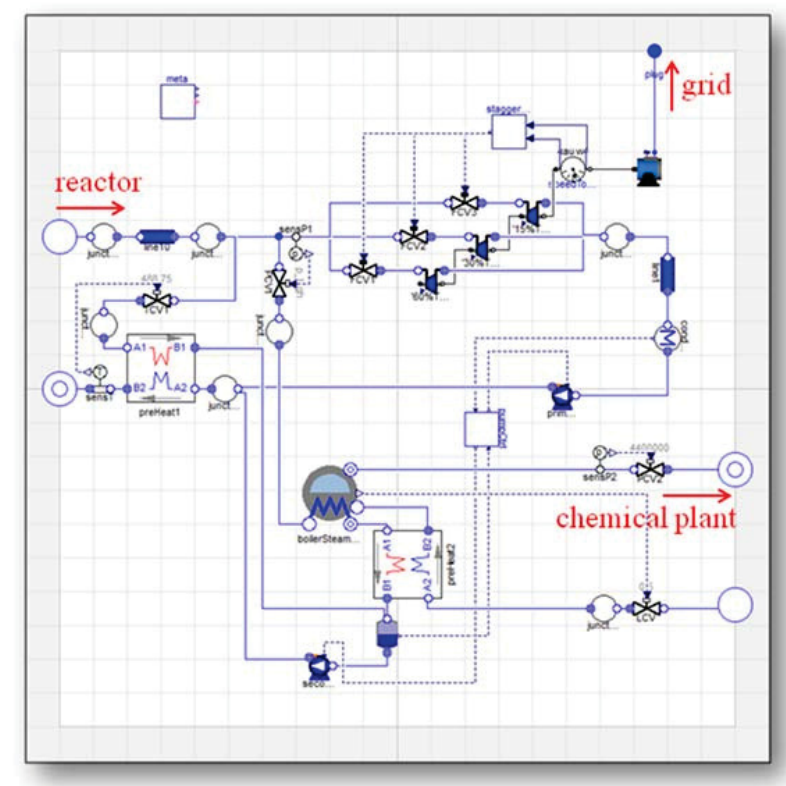

Figure 4. A more detailed view of a power subsystem including turbines, boilers, and distribution of thermal energy.

As an example of the results obtained from the dynamic analyses conducted, Figure 5 plots simulation results for the flow rates in different pieces of equipment, thus demonstrating the key importance of conducting energy analyses within a dynamic context to sufficiently understand the challenges and opportunities raised by HES and its multi-domain and intrinsic dynamic nature.

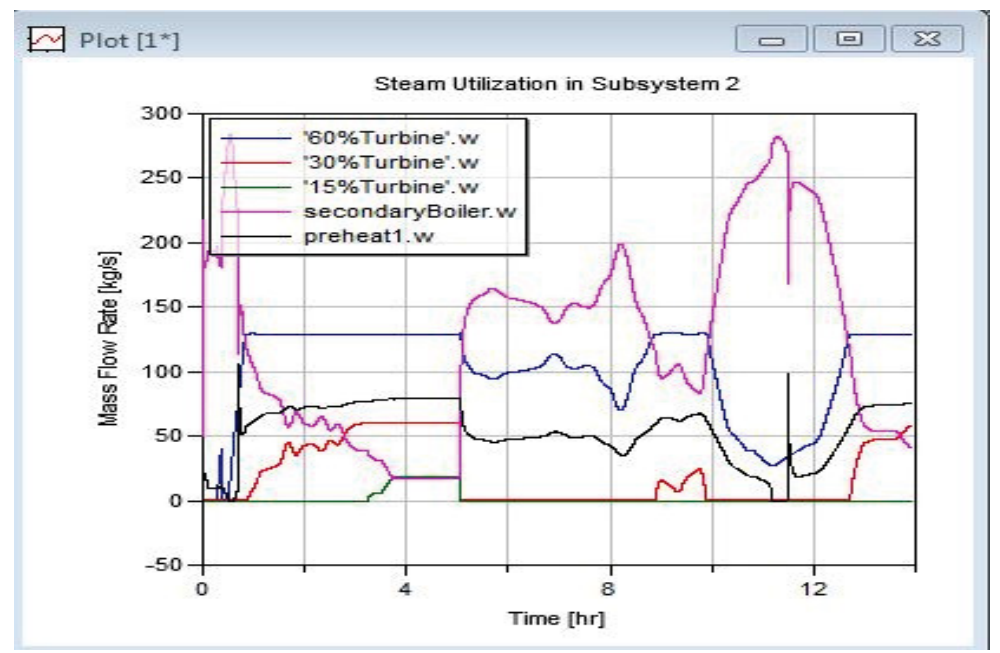

Figure 5. Simulation results showing flow rates in the turbines, secondary boiler, and pre-heater. 
In the process of constructing this dynamic model, numerous modeling constructs and libraries were developed (see Figure 6), including thermo (e.g., heaters, heat exchangers, turbines, boilers, condensers), hydraulic (e.g., pumps, pipes, tanks), renewable (e.g., wind turbines), electrical (e.g., generators, batteries, inventers, capacitors), chemical (e.g., purification, reforming, methanol-gasoline conversion), sensors (e.g., pressure, temperature), and control components (e.g., local controls and supervisors).

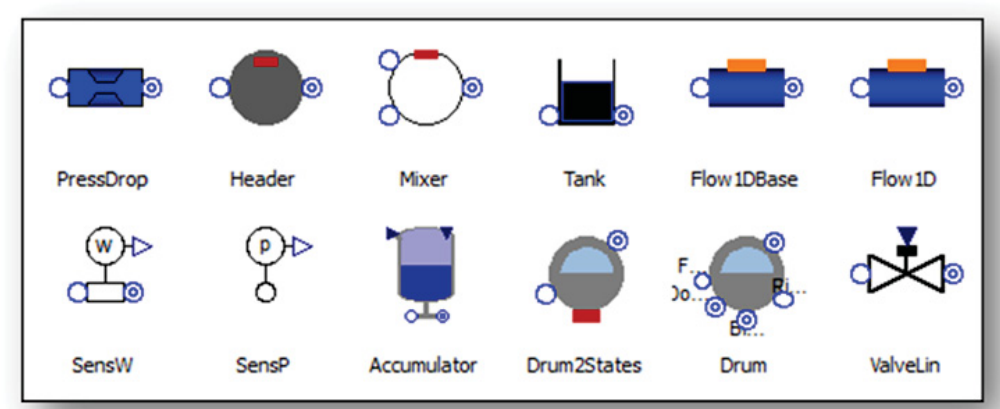

Figure 6. Partial list of models and libraries developed.

\section{Benefits to DOE}

This project will strengthen INL's capability to establish the technical basis and the economic rationale for including HES, and particularly nuclear HES, in the national electric grid as a means of achieving DOE clean energy integration goals. Development of computational capabilities-including those for multi-domain, open modeling, co-simulation, optimization, and controls, would facilitate the design, evaluation, and demonstration of component systems, architectures, and control strategies - and utility-scale analyses of HES and grid integration. This research would be of potential interest to DOE-NE, DOE Energy Efficiency and Renewable Energy, Department of Defense, and the energy-related industry.

\section{Publications}

Du, W., H.E. Garcia, and C. Paredis, "An Optimization Framework for Dynamic Hybrid Energy Systems," submitted to Proc. of the $10^{\text {th }}$ International Modelica Conference 2014 (Modelica 2014), March 10-12, 2014.

Binder, W., C. Paredis, and H.E. Garcia, "Hybrid Energy System Modeling in Modelica," submitted to Proc. of the $10^{\text {th }}$ International Modelica Conference 2014 (Modelica 2014), March 10-12, 2014. 


\title{
13-074 - A Nanobioreactor for Cellulose Degradation Using In Vitro Synthetic Biology
}

\author{
Vicki Thompson, Joni Barnes, Eric Dufek, and James King
}

The emerging discipline of synthetic biology combines biology, chemistry, and engineering to create standardized, modular biological systems with novel or improved function. While most synthetic biology approaches are completed with living cells, the use of cell-free enzyme systems is more appropriate in certain industrial situations. Biotransformations that rely on free enzymes possess high specificity, selectivity, and activity, making them desirable for many industrial processes. These enzyme synthetic pathway biotransformations have distinct benefits: reduced system complexity, reaction specificity without side-product formation, faster reaction rates, and adaptable reaction conditions with no concern for cell viability or unintended release of genetically modified organisms. However, most enzymes are not optimal in industrial processes due to stability issues, slow processivity of multi-step, multi-enzyme reactions, and/or lack of adsorption onto insoluble substrates.

Recent research has demonstrated that immobilization of single enzymes on nanoparticles can overcome enzyme shortcomings. Furthermore, process economics often necessitates recovery and re-use of biocatalysts. Immobilizing enzymes is a widely accepted method to overcome all of these limitations, and in recent work, different single enzymes have been immobilized onto nanoparticles to investigate their usefulness as supports. In this work, an in vitro synthetic biology approach was used to prove that multiple enzymes consisting of the key cellulose degrading enzymes can be immobilized onto single nanoparticles and that this novel approach will improve both the activity and/or stability of each enzyme and the overall system. If successful, this system will have application in the production of biofuels from biomass. The cellulose degrading enzymes currently used are one of the most expensive components in the conversion of biomass to biofuels primarily due to long term instability and the inability to recycle them.

\section{Summary}

Endo-glucanase, exo-glucanase, and $\beta$-glucosidase are three key enzymes involved in the breakdown of cellulose to free glucose. Hence, these types of enzymes were chosen for immobilization. Our approach was to obtain commercially available enzymes, immobilize and characterize each single enzyme, and then construct and test multi-enzyme immobilization.

The procedure we used to immobilize enzymes onto gold $(\mathrm{Au})$ nanoparticles is a multi-step process that is based on proven chemistries. First, the particles were incubated with $\mathrm{N}$-succinimidyl-(heptaethylene glycol) ester disulfide, which reacts with Au to form an n-hydroxy succinimide terminated monolayer on the surface of the particle. Next, enzyme was added, which reacts via primary amines on the exterior of the enzyme molecule to form amide linkages with the $n$-hydroxy succinimide moieties. Finally, bovine serum albumin was added as a blocking and stabilization agent, and the particles were washed in buffer to remove residual enzyme. Following immobilization, ultraviolet-visible absorbance spectra was collected to determine the relative concentration of particles, and dynamic light scattering measurements were taken to evaluate short- and long-term particle stability. After initial preparation, the lack of change in average particle diameter, as measured using dynamic light scattering, indicated that no significant aggregation or particle break up was occurring. 
Enzyme-nanoparticle activity was screened using para-nitrophenyl based chromogenic substrates (4-nitrophenyl$\beta$-D-cellobioside and 4-nitphenyl- $\beta$-glucopyranoside) in a microplate format. Screens were run at $50^{\circ} \mathrm{C}$ and $\mathrm{pH} 5.0$ for 45 minutes; subsequently, the amount of para-nitrophenyl released was determined by measuring absorbance at $420 \mathrm{~nm}$ and quantified using a 4-nitrophenol based standard curve.

Enzyme synergy was measured using carboxymethylcellulose or cellobiose as substrates and glucose released by the enzymes ( $\beta$-glucosidase and/or cellobiohydrolase) were quantified using a commercial kit (D-Glucose Hexokinase Assay, Megazyme Intl., Wicklow, Ireland). The glucose detection kit uses hexose kinase with the cofactor adenosine triphosphate, coupled to glucose phosphate dehydrogenase and its cofactor nicotinamide adenine dinucleotide phosphate + , to monitor formation of nicotinamide adenine dinucleotide phosphate hydrogen, which exhibits absorbance at $340 \mathrm{~nm}$. Glucose standards were provided in the kit for sample quantification from a standard curve.

Cellulase from Trichoderma reesei (Sigma-Aldrich), which is an endo-glucanase, was buffer exchanged into a $50 \mathrm{mM}$ sodium acetate $\left(\mathrm{NaCH}_{3} \mathrm{COO}\right)$ buffer at $\mathrm{pH} 5$ and immobilized on Au nanoparticles. Initial activity of the immobilized enzyme and the average particle size were determined and then monitored over time to assess stability. In this study, enzyme activity (normalized absorbance of 0.6 ) was stable for over 63 days, and during this time, the initial average particle diameter $(\sim 80 \mathrm{~nm})$ remained unchanged.

Next, cellobiohydrolase from T. longibrachiatum (Megazyme), an exo-glucanase, was prepared and immobilized in the same manner as the cellulase; however, no activity was detected once the protein was immobilized. Hence, Dtur CelA, a different cellobiohydrolase enzyme (Lucigen) that exhibits dual endo and exo-glucanase activities, was evaluated. However, during preparation of the enzyme, it precipitated in the $\mathrm{pH} 5 \mathrm{NaCH}_{3} \mathrm{COO}$ buffer. It was determined that precipitation could be avoided if we used a $50 \mathrm{mM}, \mathrm{pH} 7$ phosphate suspension buffer and then immobilized the protein. Although this approach worked and activity was observed, we would not be able to co-immobilize these enzymes to construct the multi-enzyme nanoparticles since their $\mathrm{pH}$ ranges were incompatible. It was then necessary to find a set of enzymes with compatible $\mathrm{pH}$ ranges. Because the activity of T. reesei cellulase was lost at $\mathrm{pH} 7$ and Dtur CelA has both endo and exo-glucanase activity, we chose to obtain a Thermotoga maritima $\beta$-glucosidase (Megazyme) with activity at $\mathrm{pH} 7$.

We demonstrated that this $\beta$-glucosidase could be successfully immobilized using the optimum conditions determined for Dtur CelA and then began mixed enzyme immobilization studies. To determine the amounts of each enzyme needed for maximum hydrolysis yield and synergy, the ratio of the enzymes during immobilization was varied. Enzyme activity data indicated a 95\% Dtur CelA 5\% $\beta$-glucosidase mixture gave the highest activity levels. Although low levels of activity on both carboxymethylcellulose and cellobiose were measured by the Dtur CelA- $\beta$-glucosidase-nanoparticles, data indicated that $\beta$-glucosidase played the major role and that Dtur CelA activity was too low to allow accurate synergy measurements.

\section{Benefits to DOE}

This work supports the DOE mission of energy resources and will help establish U.S. leadership in clean energy technologies. This proof of concept establishes new capabilities at INL and expands our existing expertise in the areas of industrial enzymes, biofuels, nanomaterials, and biomass research. 


\title{
13-079 - Diverse Biological Factories for Sustainable Manufacturing
}

\author{
Dayna Daubaras
}

Creating "Diverse Biological Factories" is an effort to support sustainable manufacturing, identified as a key area of innovative manufacturing within the DOE-Energy Efficiency and Renewable Energy Advanced Manufacturing Office. We are creating unique cellular factories to facilitate manufacturing processes for energy efficient production of commodity chemicals, fine chemicals, biofuels, or biological products. Biological systems have diverse catalytic capabilities to efficiently convert central metabolic intermediates to potential products. However, only a few host systems are available for genetic optimization and large scale production under a limited set of conditions. The focus of "Diverse Biological Factories" is to identify and evaluate diverse hosts that function under extreme conditions ( $\mathrm{pH}$, temperature, pressure) to facilitate industrial processes.

\section{Summary}

To date, we have evaluated a host system which has an efficient metabolic pathway for the production of hydrocarbon molecules. We chose to target an extreme halophile from the Archaeal kingdom which uses the "mevalonate pathway" for biosynthesis of isoprenoid compounds. It is believed that this pathway is not subject to feedback inhibition, and so it will have a higher flux of intermediates toward the desired isoprenoid products. This halophile can inherently use the inexpensive carbon (C) source glycerol while growing in high saline environments and at slightly elevated temperatures. Glycerol is not only cheap but is also a waste product of other industrial processes, and so using it as a $\mathrm{C}$ source for a bioprocess is advantageous. The unique growth environment that this Archaeal strain has adapted itself to can be useful to prevent contamination of large scale processes. Eliminating the need for sterile conditions in a scale-up process can significantly reduce the energy required for maintaining that sterility, affording an advantage over current scale-up processes.

As per the first year goals, we have successfully introduced several different versions of a particular synthetic catalyst into this host. We chose to target a valuable 5-C molecule as our target product. Isoprene is a building block currently produced from petroleum products, as a co-product to the generation of other valuable petroleum products. There is high demand for pure isoprene as a building block to polymer rubbers used for a variety of products, one being vehicle tires. Pure isoprene production is an energy intensive process dependent upon the petroleum industry. In order to facilitate the creation of an alternative source for isoprene, we desired to create a bioprocess which removes that dependence on petroleum and reduces the energy required for such processes.

We have synthesized two plant isoprene synthase genes and introduced them into the Archeal host on extrachromosomal elements, as well as placed them on the host chromosome. We chose a targeted location on the chromosome to disrupt carotenoid biosynthesis in an effort to divert intermediates of the mevalonate pathway toward the production of isoprene. We have successfully interfered with a gene that created a colorimetric system for identification of genetic modifications, an important step required for rapid screening. The interchangeable genetic modifications we created are versatile enough to easily replace the current synthetic catalyst, isoprene synthase, with other optimized or more valuable catalysts. In the second year, we will evaluate the production levels of isoprene, as well as the impact on the central metabolism of the modified strain. Ultimately, optimization of production through manipulation of growth conditions or further strain modifications may be required to obtain the desired products. 


\section{Benefits to DOE}

Ultimately, having diverse sets of organisms which can easily be modified for scale-up industrial purposes can improve the efficient use of energy, thus decreasing dependence on foreign oil. Many biological processes are considered more environmentally friendly due to the decreased use of energy in scale-up processes, the reduction in $\mathrm{CO}_{2}$ emissions compared to chemical processes, and the value added back to a primary process by using otherwise "waste" products as biological substrates for growth. Reducing our dependence on foreign oil by generating hydrocarbon compounds through biological processes can be invaluable to national energy security. 


\title{
13-100 - An Efficient System for the Production and Harvesting of Lipids from Photosynthetic Microbes
}

\author{
Vicki Thompson, David Reed, Jeffrey Lacey, Amber Miller, and Heather Silverman
}

Production of biodiesel from algal lipids is attractive since it only requires sunlight and atmospheric $\mathrm{CO}_{2}$ as energy and carbon sources, respectively. In addition, algae can be grown on land not suitable for crops using wastewater, and they do not compete as a food source. However, because algae are very dilute, harvesting and dewatering poses serious economic challenges, representing up to $30 \%$ of the total biodiesel production costs, as explained by Brennan and Owende in Renewable Sustainable Energy Reviews. Additionally, the DOE 2010 National Algal Biofuels Technology Roadmap identified algae harvesting and dewatering as one of four critical research thrust areas, since current technologies are expensive, energy intensive, and difficult to scale up.

One promising harvesting and dewatering technology is algae flotation. Once the algae are floating on the pond surface, they can be easily harvested using low cost and efficient skimmers that are commonly used in the oil, mining, and food industries. Unfortunately, algae do not naturally float, and entrainment with microbubbles is required to induce flotation. With current technology, microbubble production is expensive and energy intensive because it requires a high pressure air source. A potential solution to this lies in a discovery that some bacteria have the ability to produce hollow protein structures (gas vesicles) that can fill with air from ambient air diffusion. Bacteria may use these structures to alter their buoyancy for vertical movement in water to find nutrients, light, etc. This project examined genetically engineering bacterial algae (cyanobacteria) with genes to produce gas vesicles. These genes were also inducible so that when the cyanobacteria had accumulated sufficient lipids, gas vesicle formation could be induced and result in flotation of the cyanobacteria. Our research for the first year of the project focused primarily on the cyanobacteria Synechococcus elongatus PCC 7942, one of the highest lipid yielding strains known, as demonstrated by Quintana et al. in 2011.

\section{Summary}

Gas vesicles occur naturally in some types of cyanobacteria (although not PCC 7942); however, the functions and the need for many of those genes are not well understood. There is also considerable variation amongst the occurrence, number, and variation of these genes, thus it is difficult to identify which genes would be essential for gas vesicle production. In 1998, Li et al. were able to confer buoyancy to $E$. coli, a bacterium normally lacking gas vesicles, by transferring 11 genes from another bacterium, Bacillus megaterium. We hypothesized that the 11 genes from B. megaterium might be sufficient to allow PCC 7942 to produce gas vesicles.

The 5646 base pair $B$. megaterium gene cluster was polymerase chain reaction (PCR) amplified with a high fidelity polymerase, cloned into a broad host range vector (containing an antibiotic selectable marker, sites for integration into the PCC 7942 genome, a constitutive cyanobacteria promoter, and a ribosomal binding site), and transformed into an $E$. coli host strain. The resulting plasmid DNA was purified from the host strain, and the gene insert was sequenced. The plasmid DNA was transformed into PCC 7942, cells were plated on antibiotic containing agar plates, and isolated antibiotic resistant colonies were restreaked. PCC 7942 cells with putative integrations of the gene cluster were grown under varying conditions of nickel (Ni) (inducer) and light and analyzed via phase-contrast microscopy, differential interference contrast (DIC) microscopy, buoyancy testing, transmission electron microscopy (TEM), and conventional PCR. Ten putative transformants were archived for future research studies. 
Samples were collected at various time-points during growth and examined for the presence of gas vesicle formation using both phase-contract and DIC microscopy. In addition, samples were prepared for buoyancy testing via a test tube method similar to the one used by $\mathrm{Li}$ and Cannon. Culture flotation at the meniscus was monitored for indication of successful gas vesicle production in the transformants. Promising PCC 7942 samples were prepared and subsequently visualized and analyzed by TEM at INL. The ten putative PCC 7942 clones were lysed for genomic DNA and screened for the presence of the gene cluster. Initial analysis by PCR indicated that the $5 \mathrm{ft}$ end of the gene cluster was present. Results suggest that $\mathrm{Ni}$ is initially inhibitory to the growth of PCC 7942; however, the organism was able to rebound and regain its normal growth pattern. All transformants grew similarly under both high and low light conditions. Phase-contrast and DIC microscopy revealed healthy cell cultures under all growth conditions; however, definitive identification of gas vesicles was not confirmed. TEM analysis with resolution from 2-500 $\mathrm{nm}$ showed structures (Figure 1) that may be gas vesicles and possibly clusters, known as gas vacuoles, and are similar to structures observed by Li and Cannon in E. coli expressing the same gene cluster.

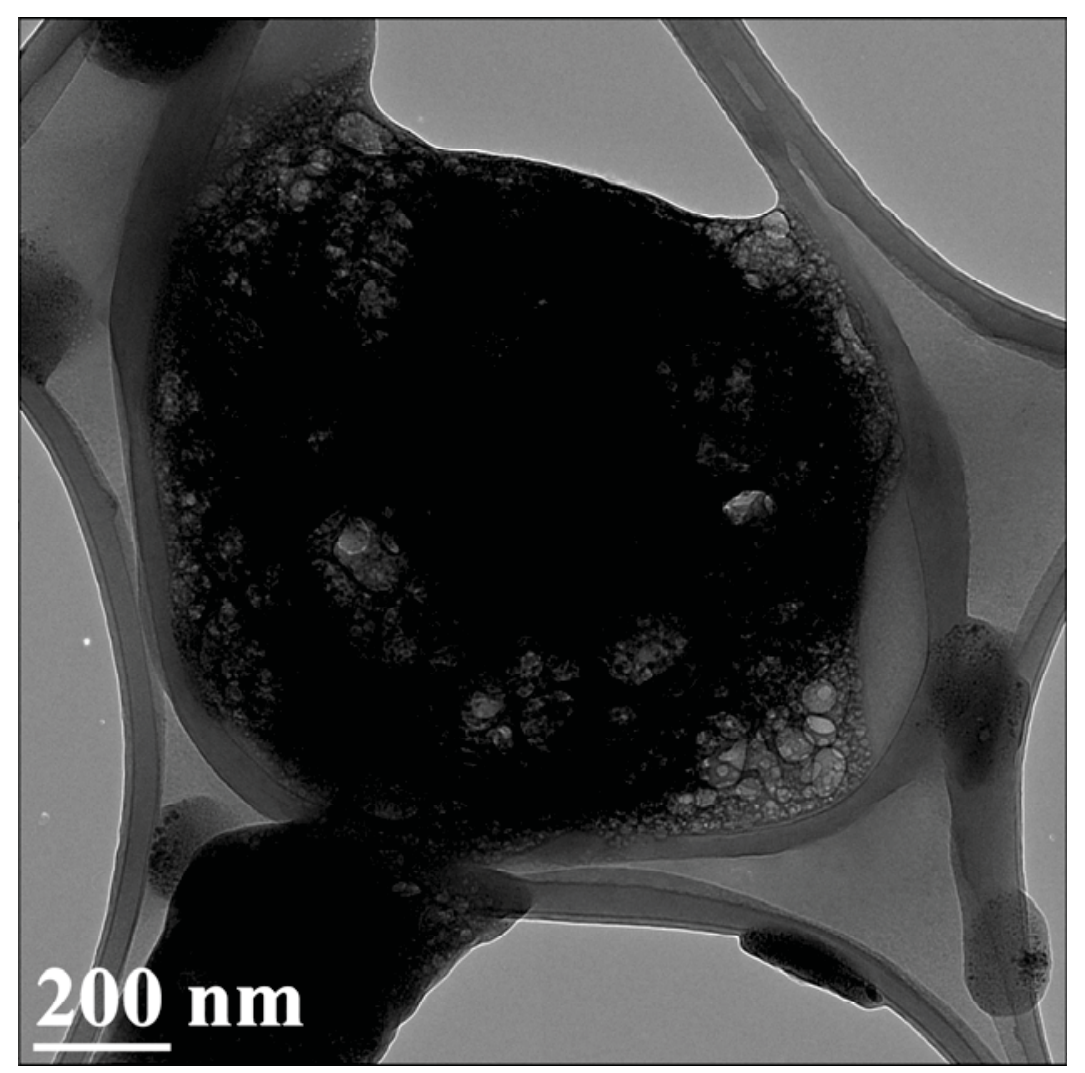

Figure 1. TEM analysis with resolution from 2-500 $\mathrm{nm}$.

During this project, 11 genes responsible for gas vesicle formation in $B$. megaterium were successfully inserted into Synechococcus elongatus PCC 7942. Initial evidence shows that these genes are being expressed in the altered PCC 7942 strain, although further verification is still required. Based upon this, we have developed a path forward that will substantially reduce both cost and energy consumption for harvesting and dewatering of algae for biodiesel production. 


\section{Benefits to DOE}

This work is a novel harvesting and dewatering strategy that can either be used directly with lipid producing cyanobacteria or adapted for use in bioenergy and general industrial processes. As such, this work directly supports DOE's National Algal Biofuels Roadmap for work on cyanobacteria development, in particular, novel strategies to decrease costs and energy consumption for harvesting and dewatering of algae. DOE Office of Science Biological and Environmental Research strives to understand how microorganisms can be manipulated so their processes can be harnessed to make useful products. This manipulation starts with an understanding of the organism's genetic code and associated capabilities and how the code can be modified to make the organism more useful. This work supports this mission by better understanding how to engineer the genetic code of lipid producing cyanobacteria to make them and their lipids easier to harvest. This technology also meets DOE's energy mission and will be both disruptive and innovative, and as such, supports the goals of DOE's Advanced Research Projects Agency-Energy program. 


\title{
13-114 - Multiphase Fluid Trapping in Propped Hydraulic Fracture
}

\author{
Earl Mattson and Mitchell Plummer
}

Hydraulic fracturing of shale reservoirs for natural gas production has created public concern about potential contamination of drinking water from the fracturing process, the handling and disposal of returned fluids, and the amount of water used in the process. Minimization of water usage for hydraulic fracturing would benefit both the environment and the gas industry. At present, there does not appear to be any clear agreement within the industry as to whether the most important damage that hinders gas removal is via alteration of fracture proppant conductivity or via alteration of the formation directly adjacent to the fracture, explained by Shaoul et al. in 2011. This is in part due to the difficulty of identifying unique characteristics in either the flowback water or the gas rate decline in these two instances. While this proposal focuses primarily on water in the proppant pack, we will also consider potential impacts to the matrix interface in our numerical simulations by examining how a hydraulic fracture de-saturates and how liquids remaining in the proppant affect gas production.

The general approach we have taken is to refine our initial conceptual model for fluid entrapment in propped fractures in shale-gas reservoirs. Next, we have implemented this conceptual model in a numerical code and compared simulation results to previously conducted laboratory experiments by our industrial collaborator that provided key information about the distribution of water saturation in propped fractures. Currently, we are conducting new hydraulic fracture-scale water saturation experiments using INL's 2-meter geocentrifuge which will allow us to physically scale experiments in proportion to the acceleration applied to experimental fractures.

\section{Summary}

A new fluid dynamic model of a proppant filled fracture has been constructed and used to guide geocentrifuge experiments. This two-phase flow model simulates water drainage from an initially water-filled 1-mm-wide hydraulic fracture with a sand proppant due to gas egress from the sides of the fracture. Figure 1 (on next page) illustrates one wing of a vertical fracture where the extraction well is located at the middle of the left-hand side. The color represents water saturation (blue is all water, whereas red represents dry conditions). The contours are the non-wetting phase gas pressure. The streamlines represent the water flow pathways, whereas the arrows represent the gas flow pathways. In Figure 1, six different times were selected to illustrate the evolution of the water-gas flow pathways. The simulations indicate that the water and gas flow pathways do not coincide, suggesting that different portions of the fracture are important at different times during the drainage.

Simulation validation experiments are being conducted at the INL 2-meter centrifuge facility. A 2-D geocentrifuge experiment has been built to conduct scaled flow and transport experiment of gas movement in a water-filled hydraulic fracture (see Figure 1). The centrifuge offers the advantage of scaling the fracture height to simulate more realistic field conditions. Initial testing has confirmed operation of the test cell. Details of the construction can be seen in INL/MIS-13-29817. 


\section{ENERGY \& ENVIRONMENT SCIENCE \& TECHNOLOGY}
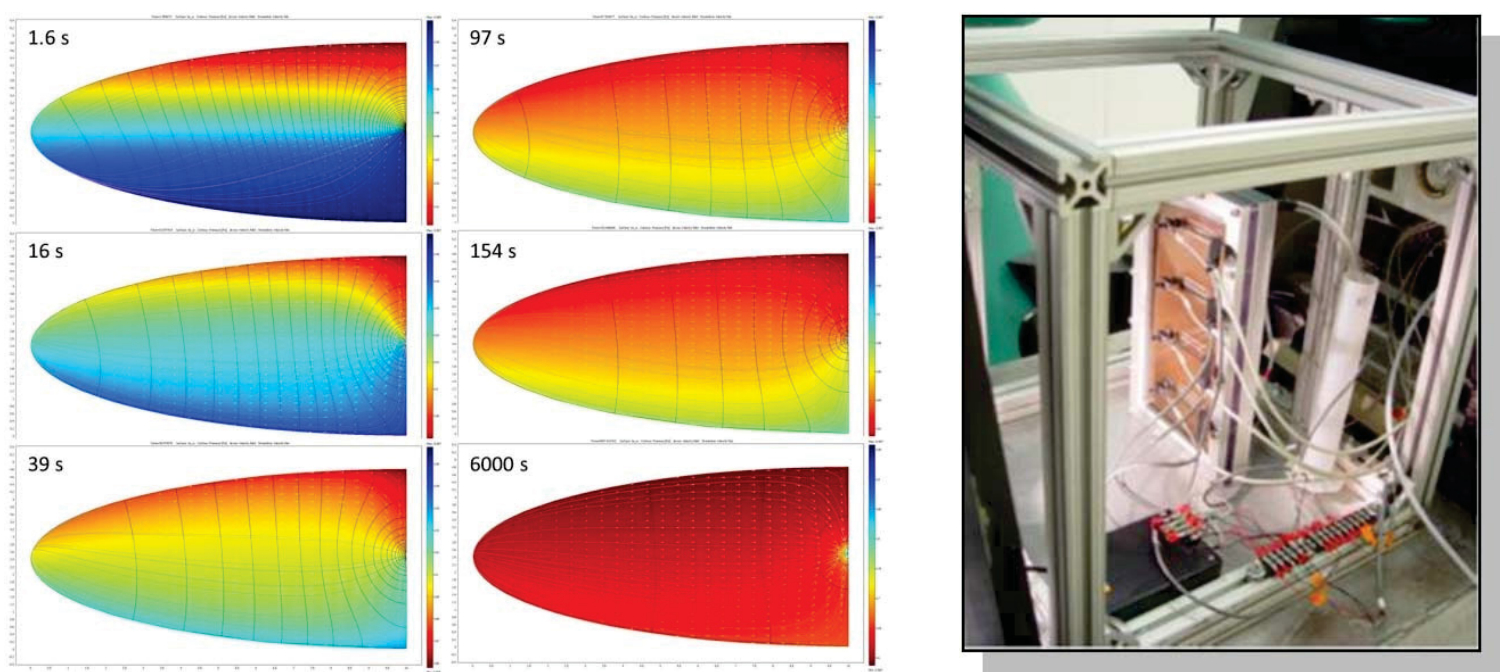

Figure 1. Simulated modeling results for a simulated hydraulic fracture and the experimental hydraulic fracture drainage testing cell on the INL geocentrifuge.

As part of this LDRD's project outreach to industry, INL has been accepted as a guest member in Stim-Labs industrial proppant consortium. We attended two of the consortium meetings and presented some initial results to the members (see Presentations section). An abstract has been submitted to the Society of Professional Engineers with our industrial collaborators (Stim-Labs, Inc.) on evaluating the flow field of water in the standard Cooke testing cell using digital elevation modeling (see Presentations section).

A University of Idaho mechanical engineer summer intern, Nathan Petersen, was funded under this LDRD and was awarded one of the University of Idaho's CAES Energy Scholarships from the INL's CAES for Idaho students interested in performing energy-related research. Nathan also presented his LDRD work at the annual INL intern poster competition. His poster and presentation were awarded runner-up in two of the categories: best technical content and best oral presentation.

Activities to address engineering solutions to gas/water interaction in a hydraulic fracture will be addressed in FY 2014 with the modeling and experimental tools that were developed in FY 2013. Additional modeling and experiments are being planned with our industrial collaborators to address potential engineering solutions.

\section{Benefits to DOE}

Efforts funded under this LDRD project support the DOE national security missions by providing a better understanding and possible solutions to increasing natural gas recovery from tight reservoirs.

\section{Publications}

Mattson, E., H. Huang, and M. Conway, "Discrete Element Modeling Results of Proppant Rearrangement in the Cooke Conductivity Cell," SPE (Society of Petroleum Engineering), Hydraulic Fracturing Symposium, February 2014, Abstract accepted, paper/presentation pending. 


\section{Presentations}

Frash, L., et al., "Laboratory Measurement of Critical State Hydraulic Fracture Geometry," 2014 ARMA (American Rock Mechanics Association), Abstract submitted.

Petersen, N. and E. Mattson, "Geo-Centrifuge Experimental Apparatus Design," INL Student Intern Poster presentation and report, CAES, Idaho Falls, ID, August 2013.

Mattson, E. and M. Plummer, "Proppant Fluid Drainage Studies Using a Geocentrifuge," Stim Labs Proppant Consortium Meeting, Rhodent CA, July 19, 2013. 


\section{3-122 - Examination of the Impacts on Water Resources from Commercialization of Fossil Energy Development in an Arid Western Basin Using Integrated Hydrologic Modeling}

John Koudelka

The goal of this research is to understand the impact on water resources from commercialization of fossil energy in the Uintah Basin and provide options for mitigating these impacts using an integrated hydrologic modeling system. The system takes advantage of advanced computational frameworks, including High Performance Computing and web-based computing.

\section{Summary}

For the first FY of funding, the planned work was to develop the scenario as well as begin model development. The funding for this work was not initiated until April of 2013 and was terminated at the end of FY 2013. The technical progress that was completed was development of the Uintah Basin scenario, including multiple datasets and model setup. The model was being run to develop a water balance for the Uintah Basin with quantifiable acre-feet in subsurface and surface water at annual and monthly timesteps. It was being run on an average water year (2000), a dry year (2010), and a wet year (2002). Work was started on tuning the model parameters (mainly soil parameters) to achieve appropriate and accurate flow and storage values at the close of FY 2013.

\section{Benefits to DOE}

This work supports INL and DOE interests in minimizing water resource impacts associated with energy development and advanced computational modeling capabilities. 


\section{INSTITUTE FOR

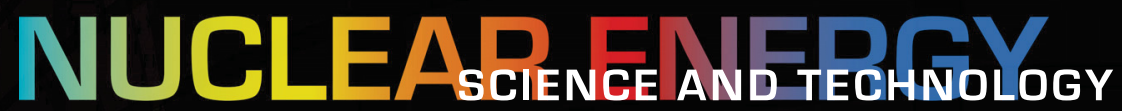




\title{
11-006 - Supercritical Fluid Extraction of Actinides from TRISO Reactor Fuels
}

\author{
Bruce Mincher and Chien Wai ${ }^{1}$
}

Methods for the treatment and/or reprocessing of used tristructural isotropic (TRISO) fuels from Very High Temperature Reactors have not been developed. This project examined the feasibility of using supercritical (sc)$\mathrm{CO}_{2}$ technology for extracting actinides from the used TRISO fuels of Very High Temperature Reactors. Sc- $\mathrm{CO}_{2}$ is effective for removing lanthanides and actinides directly from solid and densely packed materials, such as crushed TRISO fuel, because it possesses both gas-like and liquid-like properties; therefore, it is capable of penetrating porous materials and dissolving constituents in a solid matrix. This creates the possibility for actinide/lanthanide separations without the need to dissolve the fuel in acid. The possibility of shattering the graphite/SiC layers of the TRISO fuel by ultrasound $\mathrm{CO}_{2}$ cavitation was also investigated.

\section{Summary}

The dissolution of $\mathrm{UO}_{2}$ in sc- $\mathrm{CO}_{2}$ with a tributyl phosphate-nitric acid $\left(\mathrm{HNO}_{3}\right)$ complex was already a wellestablished technology. Our results indicate that, once the $\mathrm{SiC}$ layer is fractured, uranium $(U)$ is also readily extracted from a TRISO matrix this way. Further, $U$ is partitioned from neptunium ( $\mathrm{Np}$ ), plutonium ( $\mathrm{Pu}$ ), and americium $(\mathrm{Am})$ by sc- $\mathrm{CO}_{2}$ extraction using tributyl phosphate amended with the appropriate reducing and/or complexing agents. These results suggest that a counter-current separation scheme for isolation of $\mathrm{U}$ from $\mathrm{Np}$, $\mathrm{Pu}$, and Am from TRISO fuels using sc- $\mathrm{CO}_{2}$ is feasible. This indicates that actinides dissolved and co-extracted from a mixture of their oxides in $\mathrm{CO}_{2}$ can be selectively stripped using dilute $\mathrm{HNO}_{3}$ solutions amended with appropriate complexing and/or reducing agents. These actinide separation results have been summarized in a manuscript currently undergoing review prior to submission to a peer-review journal.

An important advantage of the sc- $\mathrm{CO}_{2}$ process is that it may be possible to fracture the SiC layer in an in-situ fashion in the extraction vessel using ultrasound. This would obviate the need for an inherently radiologically dirty crushing step during TRISO reprocessing. We used ultrasound cavitation of liquid $\mathrm{CO}_{2}$ to crack the graphite layer of the TRISO and also destroyed the glass beaker containing the fuel sample in this work. However, we did not demonstrate cracking of the $\mathrm{SiC}$. It is likely that use of a higher power ultrasound probe could do so and should be investigated in future work.

\section{Benefits to DOE}

Reprocessing of used nuclear fuel will extend the $U$ supply and mitigate environmental issues to enhance the sustainability of nuclear power generation for DOE. This research was a pioneering study using a non-traditional extraction technique to explore the feasibility of extracting and separating actinides from the TRISO fuels. The results suggest a new extraction technique using much lower volumes of acids and ligands and a non-hazardous diluent. This provides a reprocessing option that could have a big impact on the development of environmentally sustainable processes for managing spent fuels from Very High Temperature Reactors.

1. University of Idaho 


\section{Presentations}

Wai, C.M., B.J. Mincher, and D.L. Quach, "Dissolution of Metal Oxides and Separation of Uranium from Lanthanides and Actinides in Supercritical Carbon Dioxide," Global 2013, International Nuclear Fuel Cycle Conference, Salt Lake City, UT, September 29-October 3, 2013.

Quach, D.L., B.J. Mincher, and C.M. Wai, "Development of an Ultrasound Assisted Supercritical Fluid Extraction Technique for Reprocessing TRISO Fuel: Separation of Uranium from Actinides and Lanthanides," $37^{\text {th }}$ Actinide Separations Conference, Spokane, WA, June 24-27, 2013. 


\title{
11-041 - In-situ Micro-raman Spectroscopy and Modeling of Breakaway Oxidation of Zircaloy Cladding
}

\author{
Xianming "David" Bai and Haitham El Kadiri ${ }^{1}$
}

Zirconium $(\mathrm{Zr}$ ) based Zircaloys are widely used as the fuel cladding materials in light water reactors. Corrosion is a primary degradation mechanism of these alloys. During corrosion, the thermally grown oxide (TGO) scale on Zircaloy acts as a protective layer for further oxidation of the metal. Past research has suggested that crack development in TGO scale due to internal stresses has strong detrimental effects on the adherence and protectiveness of the TGO coating, and thus, drives breakaway oxidation. Cracks may induce the layer to spall off, allowing hydrogen to easily penetrate and reach the metal, thereby forming brittle hydrides and reducing the adherence of the TGO. This project aims at predicting the threshold of breakaway oxidation for Zircaloy-4. Previously, knowledge gaps have resided in the understanding and modeling of stress generation and crack patterning in the oxide scale, and in their relation to the kinetics and transformation in the oxide layer. These challenges have motivated the research objectives of this project.

\section{Summary}

Originally, this project was launched based on the widely admitted hypothesis that breakaway oxidation is directly associated with the scale deterioration due to tetragonal-to-monoclinic transformation stresses and oxidation stresses. However, in addition to Micro-Raman spectroscopy and phase field (PF) modeling, some other experimental and modeling efforts were performed. These included the use of atomic force microscopy, optical microscopy, and Eshleby's based micromechanical calculations. These techniques revealed that breakaway oxidation is due to the change of the sign of the circumferential stress (from compressive to tensile) at the free surface of the oxide scale. This tensile stress triggers radial cracks that catastrophically propagate toward the interface. Thus, corrosive elements such as oxygen and hydrogen penetrate readily through the oxide scale and thereby reach the metallic substrate.

Stresses affect the corrosion of $\mathrm{Zr}$ alloys significantly because the volume expansion during the $\mathrm{Zr}$ metal to oxide transformation is about $56 \%$. The evolution of the circumferential stress depends on the oxide thickness and the associated tetragonal to monoclinic phase transformations during oxide growth. To understand how the stress evolution affects the oxidation behavior, three PF models have been developed in this work:

Model 1-Stress-dependent oxidation model responsible for stress generation in the metal and oxide scale. This model is used to describe the oxide growth kinetics and the oxidation stresses.

Model 2-Stress-dependent model for phase transformation from tetragonal to monoclinic phase transformation. This model is used to describe phase transformation induced stresses in the oxide. In addition, a precipitation model was also developed.

Model 3-Stress-dependent model for interfacial roughening due to the biaxial oxidation stresses (Model 1) and phase transformation stresses (Model 2).

1. Mississippi State University 
To our knowledge, these mechanisms have not been addressed in the literature, which may explain why the breakaway oxidation was not understood and predicted. One research objective of this project is to investigate the stress evolution during the tetragonal-monoclinic transformation. This transformation impacts thermal barrier coating techniques as well as nuclear fuel performance modeling. Our PF model was developed based on the martensitic twinning mechanisms previously identified in literature via transmission electron microscopy and atomic force microscopy analyses. This task was successful as we reproduced the experimental results of the kinetics and morphologies reported in literature. For instance, Figure 1(a)-(b) provides a comparison of monoclinic twins in a tetragonal matrix between our PF simulation results and the transmission electron microscopy results reported in literature. The model is further extended to study stress-induced tetragonal to monoclinic transformation in zirconia and its effect on transformation toughening (Figure 1[c]). In addition, this model is applied to study the transformation plasticity and shape memory effects in tetragonal zirconia polycrystals (Figure 1[d]-[f]). Furthermore, we completed the Micro-Raman results with new atomic force microscopy analyses to describe in detail the transformation kinetics and morphologies that occur in the oxide scale.
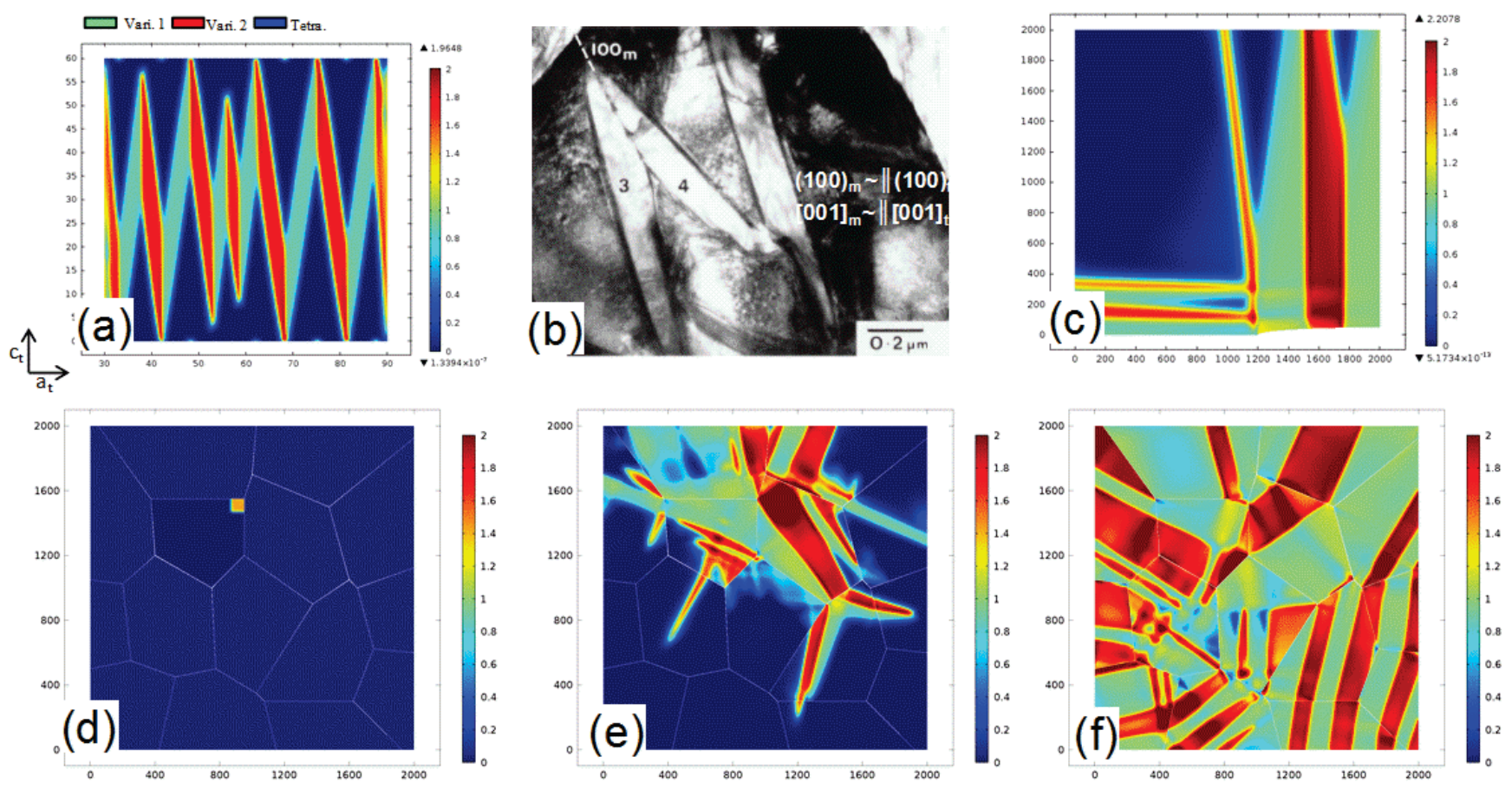

Figure 1. Some highlights of PF modeling of phase transformation between tetragonal and monoclinic phases in zirconia. (a) Tetragonal to monoclinic phase transformation predicted by our model; (b) Transmission electron microscopy image of the phase transformation in literature; (c) PF modeling of the nucleation and evolution of monoclinic phase in a cracked tetragonal zirconia single crystal under a tension stress; ( $d$-f) PF modeling of the evolution of monoclinic embryo in a tetragonal zirconia polycrystal at $0 \mu s, 0.37 \mu s$, and $0.5 \mu s$, respectively. 


\section{Benefits to DOE}

Accurately predicting breakaway will ultimately help the evaluation of extending the service life of the current fleet of nuclear reactors, reduce down times that increase the cost of energy production, and refine designs of the next generation of nuclear reactors for DOE. The developed PF models of phase transition and oxidation of Zircaloy and the micromechanical model of crack patterning are necessary to predict breakaway oxidation thresholds. These experimentally validated models would benefit the safe and secure management of nuclear materials and radioactive waste. A broader impact will be on the future development of experimentally-validated, predictive tools to characterize solid state phase transformation in various material alloys in a variety of environments.

\section{Publications}

Mamivand, M., M. Asle Zaeem, and H. El Kadiri, "Phase Field Modeling of Stress Induced Tetragonal to Monoclinic Transformation in Zirconia and its Effect on Transformation Toughening," Acta Mater., Submitted.

Asle Zaeem, M. and H. El Kadiri, "Experimental and Phase-Field Studies of Oxidation Mechanisms of Zirconium Alloys," Acta Mater., Submitted.

Mamivand, M., M. Asle Zaeem, and H. El Kadiri, "A Phase Field Model for Transformation Plasticity and Shape Memory Effects in Tetragonal Zirconia Polycrystals," Int. J. Plasticity, Submitted.

Mamivand, M., M. Asle Zaeem, and H. El Kadiri, "A Review on Phase Field Modeling of Martensitic Phase Transformation," Comp. Mater. Sci., Vol. 77, 304-311, September 2013.

Mamivand, M., et al., "Phase Field Modeling of Tetragonal to Monoclinic Phase Transformation in Zirconia," Acta Mater., Vol. 61, 5223-5235, August 2013.

El Kadiri, H., et al., "Transformations and Cracks in Zirconia Films Leading to Breakaway Oxidation of Zircaloy," Acta Mater., Vol. 61, 3923-3935, June 2013.

Asle Zaeem, M., et al., "Effects of Internal Stresses and Intermediate Phases on the Coarsening of Coherent Precipitates: A Phase-Field Study," Curr. Appl. Phys., Vol. 12, 570-580, March 2012.

\section{Presentations}

Mamivand, M., M. Asle Zaeem, and H. El Kadiri, "Phase Field Modeling of Tetragonal to Monoclinic Phase Transformation at Zirconium Oxide," TMS Annual Meeting \& Exhibition, San Antonio, TX, 2013.

Asle Zaeem, M., H. El Kadiri, and M. Horstemeyer, "Phase-Field Simulation and Experimental Studies of Oxidation of Zirconium," TMS Annual Meeting \& Exhibition, Orlando, FL, 2012. 


\title{
11-043 - Characterization of Nanomechanical Response of Material to Determine Key Variables in Stress Corrosion Cracking
}

\author{
John Jackson, William Gerberich, ${ }^{1}$ Andre Mkhoyan, ${ }^{1}$ and Eric Hintsala ${ }^{1}$
}

Although stress corrosion cracking (SCC) has been a known issue for many decades, the mechanisms responsible for SCC initiation and growth are complex and not well understood. The goal of this project is to broadly investigate the primary factors contributing to SCC in reactor systems through the use of nanomechanical testing. Much of the background data on cold work, fatigue, creep, and SCC of austenitic alloys suggest that near surface nucleation of dislocations is critical to the initiation of damage leading to SCC. The idea is to use nanopillars fabricated using a Focused lon Beam (FIB) to provide a test bed for chemo-mechanical mapping of crack initiation and propagation mechanisms. This project is a proof-of-principle study using austenitic stainless steel and iron ( $\mathrm{Fe}$ )-nickel $(\mathrm{Ni})$ thin films to demonstrate the sensitivity of material response in nano-pillar deformation experiments to the measurement of fundamental parameters that may affect SCC. Ultimately, through the use of this type of testing, we hope to reduce the number of experimental time-steps required to reach a conclusion about which mechanistic phenomena are most logical to pursue in detail to provide a better mechanistic understanding of SCC. Understanding of the mechanistic basis by which SCC occurs may lead to development of SCC resistant replacement materials for light water reactors or better SCC mitigation processes.

\section{Summary}

Refinement of the ion milling technique for production of geometrically consistent and location relevant (with respect to material characteristics) nano-pillars consumed a large part of the first year of this project. This work included milling an analog material (silicon [Si]) to refine system parameters, followed by milling of 304 stainless steel pillars. A tribo-indenter was used to obtain baseline material properties for the 304 stainless steel nano-pillars and to determine the correct aspect ratio and geometry to give optimally consistent data. A material of interest to the nuclear industry (Nitronic 50) was then obtained and cold worked to nominally $20 \%(0.2 \mathrm{in} / \mathrm{in}$ plastic strain). The cold work is known to increase the material susceptibility to SCC; this material will be used to study the fundamental differences between materials that lead to SCC. Efforts during the first year also resulted in production of thin films for Transmission Electron Microscopy studies to determine grain boundary characteristics of the 304 stainless steel and the cold worked Nitronic 50.

The second year of effort for this project produced stress-strain data for nano-pillars of multiple sizes, ranging from $300 \mathrm{~nm}$ to $1600 \mathrm{~nm}$. A size effect exhibited by the nano-pillars of Nitronic 50 material was noted as the yield strength increased with decreasing size. Additionally, load drops that were attributed to either grain misorientation or twin boundary stoppage in larger volumes were noted to increase in magnitude as pillar size decreased. Grain boundary triple points were identified as optimal locations for ensuing studies on hydrogen charging due to enhanced local plasticity.

Year two of this study also involved development of an improved method for manufacturing nano-pillars via a ladder base with a multiple sub-millings method. Additionally, a protective platinum cap was employed to improve the loaded surface of the nano-pillars. These two improvements served to improve aspect ratio for the pillars

1. University of Minnesota 
(thereby improving accuracy of stress and strain calculations) and improve contact of the nano-indenter with the top of the pillars. Following these improvements in nano-pillar production, two methods were tested for hydrogen charging. First, an electrochemical process was attempted; this process was deemed unacceptable due to development of a thick oxide film that completely occluded the nano-pillar. The second method utilized gas phase hydrogen flowing over the pillar under high temperatures. This method proved successful in charging the Nitronic 50 pillars, and mechanical properties were subsequently measured as a function of hydrogen charging. It was found that hydrogen charging of 1,600-nm-diameter nano-pillars resulted in an approximate doubling of yield strength. Additionally, formation of a brittle surface layer on the pillars was noted; examination of this surface layer is ongoing.

The third and final year of the project was focused on refining techniques for hydrogen charging and on modification of an existing model for prediction of the ductile to brittle transition temperature shift in metal as a function of hydrogen content. Utilizing $\mathrm{Fe}-3 \% \mathrm{Si}$, a material known to be highly susceptible to hydrogen embrittlement, a model that had historically been used to predict the ductile to brittle transition temperature in semiconductor materials was adapted for use with metals. A similar adaptation may be applied to Nitronic 50 or other reactor-relevant materials in future research.

Some success had been realized when utilizing gas phase hydrogen charging previously, but a thin oxide layer remained that affected nano-pillar strength measurements; plasma charging was investigated as an improved alternate, and pillars of $\mathrm{Fe}-3 \% \mathrm{Si}$ were successfully charged with hydrogen late in this final year. Although success was achieved somewhat late and results on Nitronic 50 were not obtained, the project did prove that this method is feasible and laid the foundation for future research.

As this LDRD project has reached its three year mark, the promising results provided by the project will need to be continued with funding from a different source. Plans are underway to submit a proposal based on these results to the DOE-NE University Program Office. The foundation laid by this LDRD project should provide an excellent springboard for continuing to progress toward understanding the fundamental mechanisms contributing to SCC and SCC initiation.

\section{Benefits to DOE}

Work on fundamental issues in crack growth has recently been funded by DOE's Office of Science, and further interest is anticipated. The need for a quantitative, predictive SCC model is well understood by the nuclear community and the wider materials community. This project directly addresses this need, if successful, and will contribute to the fundamental understanding of material failure in operating nuclear reactors. This will allow decisions to be made on re-licensing of the current nuclear reactor fleet as well as planning for future nuclear reactor design.

\section{Publications}

Hintsala, E., et al., "The Hardening Sequence in Dislocation Free Nano-Volumes: A Back-Stress Mediated Effective Stress for Dislocation Nucleation," In preparation.

Hintsala, E., et al., "Fracture Transitions in Irons and Steels: Strain Rate and Environmental Effects," 2013, To be submitted. 
Wagner, A.J., et al., "The Role of Back Stress in Small Volume Strengths," Appl. Phys. Lett., 2013, To be submitted.

Gerberich, W.W., et al., "Hydrogen Effects on the Brittleness Transition in BCC Iron," 2012 International Hydrogen Conference on Hydrogen-Materials Interactions, Materials Park: ASM International, 2013 (Accepted).

Hintsala, E., et al., "In-Situ TEM Compression of MgO Nanocubes," Microscopy and Microanalysis Abstracts, Indianapolis, IN, August 4-8, 2013, Accepted poster.

Gerberich, W.W. and D.D. Stauffer, "Damage Mechanisms, Historical," Gaseous Hydrogen Embrittlement of High Performance Materials, Cambridge: Woodhead Publishing, U.K., In press.

Gerberich, W.W., et al., "The Brittleness Transition Due to Scale,” J. Mater. Res., Vol. 27,552-561, 2012.

\section{Presentations}

Gerberich, W.W., et al., "Hydrogen Effects on Brittleness in Fe-Base Systems," Quantitative Micro-Nano 4, Toronto, Canada, June 4, 2013.

Gerberich, W.W. and E. Hintsala, "Hydrogen's Ubiquity: Fixating, Facilitating, Fracturing, Flip-Flopping," 2012 International Hydrogen Conference, Jackson Hole, WY, September 9-12, 2012. 


\title{
12-007 - Uncertainty Quantification of Safety Codes Using a Bayesian Approach with Data from Separate- and Integral- Effects Tests
}

\author{
Robert Youngblood, Jacopo Buongiorno, ${ }^{1}$ and Joseph Yurko ${ }^{1}$
}

Large-scale system codes for simulation of facility safety performance may contain parameters whose values are not understood well enough to be very accurate in the simulation, thus limiting confidence in the results of the simulation. In order to be able to use the results of these simulation codes with confidence, it is important to learn as much as we can about the appropriate values of these parameters. New information from tests or operating experience is incorporated into safety codes by a process known as "calibration," which reduces uncertainty in the output of the safety code and thereby improves its support for decision-making.

Modern analysis capabilities potentiate very significant improvements on classical ways of doing calibration, and this project aims to accomplish some of those improvements. Key innovations have come from development of safety code surrogate (code emulator) model construction algorithms and Bayesian model selection.

A surrogate is needed because the multivariate nature of the problem (the need to adjust multiple uncertain parameters at once to fit multiple pieces of new information) makes calibration very computation-intensive in principle. Some investigators foresee a time when supercomputing will solve this problem, but, presently, this capability is not on the horizon. Currently, use of a fast surrogate makes certain calibration processes feasible that otherwise would not be (simulated annealing, Gibbs sampling) and additionally may make it much easier to use gradient information in the calibration process.

\section{Summary}

Work in the first year has focused on how best to construct the safety code surrogate. Construction of surrogates has been demonstrated for many years now, but usually for prediction of single-variable outcomes (such as a peak value of temperature within a time history), not typically for entire time histories at once. Moreover, most traditional surrogates do not provide uncertainty information along with their predictions, but the Gaussianprocess-based code surrogates do. This will improve the soundness of the code calibration process. At this point, Mr. Yurko has demonstrated the capability to develop Gaussian- process-based code surrogates for entire time histories, and is conversant with fairly leading-edge Gaussian-process technology, including "warping" (building the Gaussian process model on a transformation of the original data, which allows the Gaussian process model to do a better job in some cases) and function factorization (constructing a complicated surrogate as a product of factors that are each built on a subspace of the whole problem).

At this writing, Mr. Yurko has demonstrated calibration of Reactor Excursion and Leak Analysis Program (RELAP) parameters for the models describing flow past the wire wrapped fuel elements in Experimental Breeder Reactor (EBR) II and is currently in the process of performing the RELAP runs necessary to train the emulator needed for calibration of models describing integral effects tests. This should be completed shortly; Mr. Yurko's thesis will then be finalized and defended within a short time.

1. Massachusetts Institute of Technology 


\section{Benefits to DOE}

The DOE-NE mission relies essentially on using large-scale, system-level simulations to support decision-making for both safety and operational purposes. For example, deployment of new reactor technologies (such as small modular reactors) will need new simulation capability, and some issues in sustainability of existing light water reactors call for improved simulation as well. This project will lead to a significant improvement in the state of the art of calibrating these simulations so that they better predict reality. The techniques will benefit any technology making use of system-scale simulations in its decision-making, such as National Aeronautics and Space Administration.

\section{Presentations}

Yurko, J.P. and J. Buongiorno, "Quantitative Phenomena Identification and Ranking Table (QPIRT) for Bayesian Uncertainty Quantification," Proceedings of ICAPP '12, Chicago, IL, June 24-28, 2012. 


\title{
12-026 - Protectiveness and Stability of the Zirconium Oxide in Early-Phase Corrosion of Zirconium Alloys Predictive Relations to Surface Structure and Composition
}

\author{
Xianming "David" Bai and Bilge Yildiz"
}

Current and next-generation nuclear reactors require materials to perform reliably under increasingly extreme environments. Of particular importance is the water-side corrosion of the cladding materials (zirconium [Zr] alloys) which can lead to cladding failure. The corrosion depends on many factors such as microstructural features (e.g., grain boundaries [GBs]), alloy elements (e.g., niobium [Nb]), stresses, and radiation. To selectively design materials to suppress corrosion, a fundamental understanding of each unit process that governs the corrosion kinetics is needed. This project aims at gaining a better understanding and better control of the protectiveness and stability of $\mathrm{ZrO}_{2}$ films formed on the $\mathrm{Zr}$ alloy surfaces during their corrosion at the atomic level using integrated state-of-the-art surface-sensitive experimental and computational approaches. Three main controlling parameters are investigated-alloying elements in the $\mathrm{Zr}$ matrix, microstructure of the $\mathrm{Zr}$ surface, and stresses.

\section{Summary}

In our experiments, we performed in-situ angle resolved $x$-ray photoelectron spectroscopy (XPS) and synchrotron XPS on both pure $\mathrm{Zr}$ and $\mathrm{Zr}-2.5 \% \mathrm{Nb}$ alloy to characterize the properties of the surface regions during early-stage oxidation. Understanding these characteristics is important because they impact the protectiveness of the new oxide. Some key findings and accomplishments from this work are summarized as follows. First, Nb substitution of $\mathrm{Zr}$ is suggested to provide a better corrosion resistance than pure $\mathrm{Zr}$. However, the chemical and structural reasons behind this behavior are not clear. Using angle resolved XPS analysis, we found that a more stoichiometric $\mathrm{Zr}$ oxide layer is formed on the $\mathrm{Zr}-2.5 \% \mathrm{Nb}$, with respect to that on the pure $\mathrm{Zr}$, indicating that $\mathrm{Nb}$ reduces the oxygen vacancy content and slows down oxygen transport through the oxide, thereby providing better protectiveness against corrosion. The chemical content analysis showed $\mathrm{Nb}$ segregation near the oxide surface during oxidation (Figure 1a), supporting our computational predictions (see below). Second, a microscopic understanding of the initial oxidation kinetics and the oxidation state of $\mathrm{Zr}$ at the early-stage corrosion is an open question. We have used synchrotron XPS to analyze the charge states of $\mathrm{Zr}$ in the oxide when $\mathrm{Zr}$ (0001) and (10-10) surfaces were oxidized at different temperatures (100K, 300K, 500K). The data demonstrated directly for the first time the formation of $\mathrm{Zr}$ sub-valence states during oxidation, answering a long-debated question of whether $\mathrm{Zr} 1+, 2+$, and 3+ charge states are able to form at the metal-oxide interface (Figure 1b).

Complimentary density-functional-theory based calculations were performed to study the impact of $\mathrm{Nb}$ on the defect equilibria of tetragonal $\mathrm{ZrO}_{2}\left(\mathrm{t}-\mathrm{ZrO}_{2}\right)$ which in turn impacts the corrosion resistance of $\mathrm{Zr}$ alloys. The previously computed zero-Kelvin formation energies of $\mathrm{Nb}$ defects are extended to finite-temperature free energies to construct the Kröger-Vink diagram of $\mathrm{Nb}$-doped $\mathrm{t}-\mathrm{ZrO}_{2}$ at $1500 \mathrm{~K}$ (Figure 1c). Our modeling revealed: (1) $\mathrm{Nb}$ is predominantly present in the form of a substitutional defect at a $\mathrm{Zr}$ site with the oxidation state $5+;$ (2) $\mathrm{Nb}$ tends to be present in oxygen-rich regions such as the oxide/environment interface, consistent with our XPS experiments (Figure 1a); and (3) the highest impact of $\mathrm{Nb}$ on the defect equilibria takes place near the oxide/environment interface. Compared to pure $\mathrm{t}-\mathrm{ZrO}_{2}, \mathrm{Nb}$-doped $\mathrm{t}-\mathrm{ZrO}_{2}$ has two to three times more free

1. Massachusetts Institute of Technology 
electrons and five times less oxygen vacancies. These $\mathrm{Nb}$-induced changes may result in competing effects on corrosion resistance and hydrogen pickup.

To study how strains/stresses affect the oxygen transport in $\mathrm{t}-\mathrm{ZrO}_{2}$ (and therefore the corrosion kinetics), temperature accelerated dynamics and molecular dynamics simulations were conducted. When a $\mathrm{Zr}$ metal transforms to a $\mathrm{ZrO}_{2}$, the volume expansion is about $56 \%$, which induces significant growth strains/stresses. Our results (Figure 1d) show that under compressive strains, the oxygen defect diffusivities decreases significantly, particularly for interstitials. At small tensile strains, the diffusivities increase moderately. At large tensile strains, the diffusivities decrease again. These results suggest that compressive stresses may help improve the corrosion resistance and vice versa. The effects of microstructural features, such as $\mathrm{GBs}$ on oxygen transport in $\mathrm{t}-\mathrm{ZrO}_{2}$, are also studied with atomistic simulations. The oxygen defect diffusion in a few GBs was studied (Figure 1e). Compared to a bulk oxide, the oxygen vacancy diffusivities (the slopes of the mean square displacements in Figure 1e) at these GBs are smaller than that in a bulk $\mathrm{ZrO}_{2}$. To further quantify the GB effects, the oxygen defect migration energies at some GBs are symmetrically studied, and an example is shown in Figure $1 \mathrm{f}$. Clearly, the oxygen defect migration barrier at a GB can be much higher than in the bulk, suggesting that GBs may not be fast oxygen transport paths, which is counter-intuitive. In these calculations, the defect formation energies were not considered, so the conclusion is more relevant to the scenario of radiation-accelerated corrosion in which a highconcentration of defects is produced by irradiation.
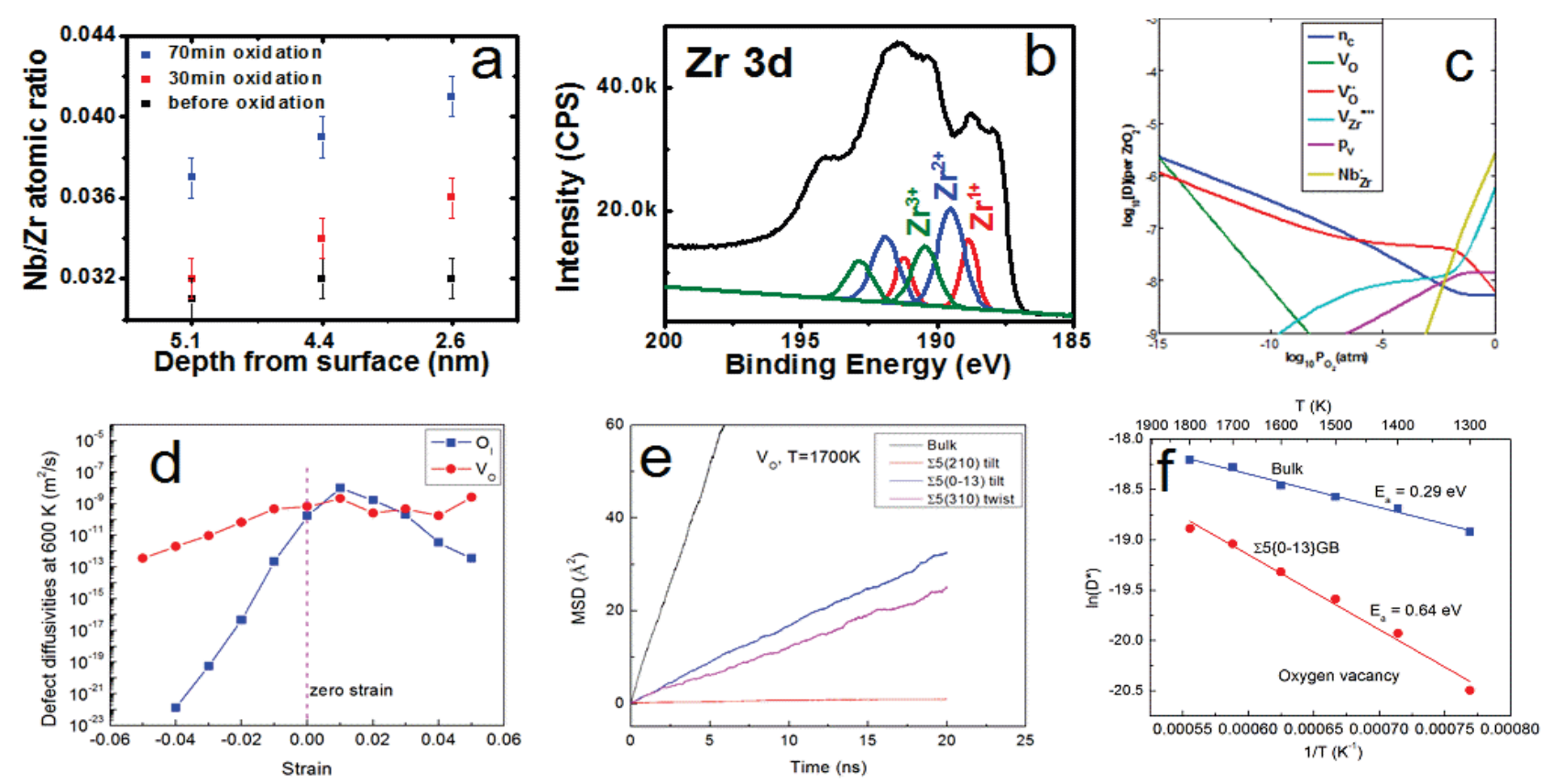

Figure 1. Highlights of some experimental and theoretical results of oxidation in Zr and Zr alloys: (a) Angle resolved XPS measurement of $\mathrm{Nb} / \mathrm{Zr}$ ratio in the surface oxide. Nb tends to segregate to the surface after oxidation; (b) Zr 3d XPS spectrum. The sub-oxide charge states were identified; (c) Density-functional-theory calculation of Kröger-Vink diagram of the point defects in a Nb-doped $t-\mathrm{ZrO}_{2}$ at $1500 \mathrm{~K}$; (d) Temperature accelerated dynamics simulation of the strain effects on oxygen defect diffusivities in $t-\mathrm{ZrO}_{2} ;$ (e) Molecular dynamics simulations of oxygen vacancy diffusion in three GBs and in bulk; and ( $f$ ) Comparison of the oxygen vacancy migration barrier in bulk and in a GB. The result shows defect migration in a GB is slower than in a bulk $t-\mathrm{ZrO}_{2}$. 


\section{Benefits to DOE}

Our work will contribute to DOE's leading role on basic science research of materials behavior and performance in extreme environments. This work will help guide the design of corrosion-resistant $\mathrm{Zr}$ alloys for cladding and improve nuclear energy safety and economics while reducing the carbon footprint of the energy sector.

\section{Publications}

Bai, X., Y. Zhang, and M.R. Tonks, "Strain Effects on Oxygen Transport in Tetragonal Zirconium Dioxide," Phys. Chem. Chem. Phys., Vol. 15, pp. 19438-19449, 2013.

Bai, X., Y. Zhang, and M.R. Tonks, "Atomistic Studies of Cation Transport in Tetragonal $\mathrm{ZrO}_{2}$ During Zirconium Corrosion," Proceedings for the Third International Workshop on Structural Materials for Innovative Nuclear Systems, Idaho Falls, ID, October 7-10, 2013, Submitted.

Otgonbaatar, U., et al., "The Effect of Niobium on the Defect Chemistry and Corrosion Kinetics of Tetragonal $\mathrm{ZrO}_{2}$ : A Density Functional Theory Study," In preparation.

Ma, W., et al., "Effect of Niobium on the Initial Oxidation of Zirconium Based Alloys," In preparation.

Otgonbaatar, U., The Effect of Niobium on the Defect Chemistry and Corrosion Kinetics of Tetragonal ZrO ${ }_{2}: A$ Density Functional Theory Study, M.S. Thesis, Massachusetts Institute of Technology, 2013.

\section{Presentations}

Bai, X., "Computational Studies of Oxygen and Zirconium Diffusion in Tetragonal $\mathrm{ZrO}_{2}$ Related to Zirconium Corrosion," 2012 Computer Simulations of Radiation Effects in Solids, Santa Fe, NM, June 2012.

Bai, X., "Computational Studies of Oxygen Transport along Grain Boundaries during Zirconium Corrosion," 2013 TMS Annual Meeting, San Antonio, TX, March 2013.

Otgonbaatar, U., M. Youssef, and B. Yildiz, "Effect of Niobium on the Defect Chemistry and Corrosion Kinetics of Tetragonal $\mathrm{ZrO}_{2}$," Third International Workshop on Structural Materials for Innovative Nuclear Systems, Idaho Falls, ID, October 2013.

Ma, W., et al., "Effect of Niobium on the Initial Oxidation of Zirconium Based Alloys," Third International Workshop on Structural Materials for Innovative Nuclear Systems, Idaho Falls, ID, October 2013.

Bai, X., "Atomistic Studies of the Effects of Strain and Grain Boundary Character on Oxygen Transport during Zirconium Corrosion," Third International Workshop on Structural Materials for Innovative Nuclear Systems, Idaho Falls, ID, October 2013. 


\title{
12-043 - Science-Based Simulation Model of Human Performance for Human Reliability Analysis
}

\author{
Ronald Boring, Carol Smidts, ${ }^{1}$ and Ali Mosleh $^{2}$
}

To date, much of human reliability analysis (HRA) has been developed as a series of methods to address requirements of probabilistic risk assessment (PRA). This PRA emphasis has resulted in a piecemeal collection of methods and practices that, in many cases, lack a scrutable scientific underpinning. This approach has also left HRA ill-prepared to address current issues such as beyond-design-basis accidents. The current project addresses this shortcoming by improving two fundamental areas of HRA: (1) the data basis by developing an approach to collect human performance data at Ohio State University (OSU) and INL control room simulator facilities, and (2) the ability to model dynamic phenomena by expanding the Information-Decision-Action-Crew (IDAC) dynamic simulation engine at the University of Maryland (UMD).

\section{Summary}

A prerequisite for empirical human performance data collection and dynamic simulation is a realistic control room scenario. Nuclear power plants are operated from main control rooms at the plant. In order to train operators for proper decision making and action at the plant, every commercial plant internationally is required to have a control room simulator. Scenarios are developed by researchers and trainers to ensure operators have a broad exposure to different plant upset conditions. These conditions occur rarely; thus, training ensures operators are adequately prepared to respond to such conditions. INL reviewed abnormal operating scenarios for potential use in INL's and OSU's simulators and for modeling in UMD's IDAC. A set of factors was reviewed for guiding the selection, including the applicability across different reactor types and designs, speed of the accident progression, potential for operator misdiagnosis, mix of cognitive and physical responses, and availability of emergency operating procedures. This process resulted in the selection of small loss of coolant accident (LOCA) and steam generator tube rupture (SGTR) as initiators. The research team developed training for students at OSU and conducted a series of experiments using student operators for the small LOCA and SGTR scenarios. These scenarios were modeled using IDAC at UMD.

Both INL and OSU have previously completed significant work on deploying their respective control room simulators for research applications. In order to have a common platform for research, both INL and OSU have purchased licenses to GSE Systems Inc.'s Generic Pressurized Water Reactor, which has recently been installed at both locations. The Generic Pressurized Water Reactor is modeled after an actual plant in operation, and INL and OSU attended LOCA and SGTR training scenarios with actual crews at the reference plant. These data served as an informal baseline to compare against student performance. Additionally, INL reviewed documented crew runs through SGTRs conducted by Halden Reactor Project in Norway, which allowed a comparison of performance for student operators vs. professional reactor operators. Compared to professional reactor operators, student operators:

- Showed greater susceptibility to issues with finding correct information in the panels

- Exhibited more difficulty successfully diagnosing issues in a timely manner

\footnotetext{
1. Ohio State University
}

2. University of Maryland 


\section{INSTITUTE FOR NUCLEAR ENERGY SCIENCE \& TECHNOLOGY}

- Experienced greater challenges with three-way communication.

Nonetheless, the student operators demonstrated similar paths in working through the procedures and showed similar levels of stress to their professional counterparts. The study demonstrated there is merit in using student operators to gather human performance data for nuclear applications.

The IDAC model is an important component of this research that provides a tool that can dynamically predict operator performance in changing situations. The UMD team completed its evaluation of the optimum path for integrating the IDAC simulation model with the INL and OSU simulators, considering practical issues (e.g., the scale of changes in the code and level of knowledge transfer between UMD and other team members), short-term needs of the project, and extent of capabilities needed for any future expansion of the scope. The research team determined that the optimum path would be to link the IDAC platform to an available reactor design similar to the Generic Pressurized Water Reactor. In this context, IDAC serves as a "virtual operator" that makes decisions and acts upon simulator parameters. UMD provided a walkthrough of the IDAC architecture, features, programming environments, sample input files, and the communication protocol between the IDAC operator model and RELAP5 thermalhydraulic code. To support the interaction of the IDAC virtual operator with the INL and OSU simulators, UMD developed a sample virtual operator that followed the emergency operating procedures associated with the small LOCA and SGTR scenarios. The IDAC virtual operator closely predicted the performance of the professional reactor operators, and decreasing the skill of the virtual operators approached performance of the student operators.

The joint OSU-INL-UMD project generated several key accomplishments, including:

- Development of the metrics for measuring operator performance

- First-of-a-kind use of students in operator performance studies

- Development of a model of virtual operators for testing a much wider range of situations than is possible in current static HRA models.

The project was able to serve as the primary research funding source for four graduate students:

- Rachel Benish, Ph.D. candidate in nuclear engineering at OSU

- Nan Wang, Ph.D. candidate in nuclear engineering at OSU

- Rajesh Sundaramurthi, M.S. candidate in nuclear engineering at OSU

- Yuandan Li, Ph.D. candidate in reliability engineering at UMD

\section{Benefits to DOE}

This project firmly plants INL (and, as a result, DOE) at the forefront of dynamic HRA research and provides INL the opportunity to develop a joint testbed for validating more complex HRA models such as IDAC. Currently, HRA and, to a large extent, related PRA activities at INL are focused on traditional static models. Static PRA and HRA models are the mainstay of current risk and reliability programmatic work, but already the long-term utility of such approaches is being called into question. These static models capture only a snapshot of plant and operator performance, but the models cannot readily be adapted to respond to complex event evolutions, including those 
that might be found in severe accidents. The need to model events dynamically and to understand the human impact on the successful resolution or negative escalation of events is crucial to the future of risk and reliability.

Because this need is becoming increasingly vital, the opportunity for follow-on funding from entities like the U.S. Nuclear Regulatory Commission is significant. In addition, dynamic HRA has been an area of considerable interest to newcomers to HRA like aerospace and the oil industry, which have multiple phases of operation and have found little utility in static, single-phase models. The proof-of-concept of tying a dynamic HRA simulation engine with empirical data sources in this project sets the stage for other industries that are waiting for clear demonstrations before investing in HRA.

\section{Publications}

Boring, R., R. Benish, and C. Smidts, "A Comparison of Student and Professional Reactor Operator Performance," Probabilistic Safety Assessment and Management (PSAM 12) Conference, Honolulu, HI, June 22-27, 2014, Submitted.

Azarkhil, M. and A. Mosleh, "A Simulation Approach to Investigate the Impact of Team Characteristics on Operating Crew Performance," International Topical Meeting on Probabilistic Safety Assessment and Analysis, Columbia, SC, September 22-26, 2013.

Benish, R., et al., "A Pilot Experiment for Science-Based Human Reliability Analysis Validation," International Topical Meeting on Probabilistic Safety Assessment and Analysis, Columbia, SC, September 22-26, 2013.

Li, Y. and A. Mosleh, "Simulating Nuclear Power Plant Operators' Use of Knowledge in Situation Awareness," International Topical Meeting on Probabilistic Safety Assessment and Analysis, Columbia, SC, September 2226, 2013.

Sundaramurthi, R. and C. Smidts, "Human Reliability Modeling for Next Generation System Codes," Ann. Nucl. Energy, Vol. 52, 137-156, February 2013.

Gupta, A., et al., "Validating THERP: An Approach to Experimentally Validating the Human Error Prediction Rates in the THERP Tables," American Nuclear Society Winter Meeting, San Diego, CA, November 11-15, 2012.

Boring, R., et al., "Microworlds, Simulators, and Simulation: Framework for a Benchmark of Human Reliability Data Sources," Joint Probabilistic Safety Assessment and Management and European Safety and Reliability Conference, Helsinki, Finland, June 25-29, 2012.

Mosleh, A., et al., "A Model-Based Human Reliability Analysis Methodology," Joint Probabilistic Safety Assessment and Management and European Safety and Reliability Conference, Helsinki, Finland, June 2529, 2012.

Sundaramurthi, R., Human Reliability Modeling for Next Generation System Codes, M.S. Thesis, Ohio State University, 2011. 


\section{Presentations}

Azarkhil, M. and A. Mosleh, "A Simulation Approach to Investigate the Impact of Team Characteristics on Operating Crew Performance," International Topical Meeting on Probabilistic Safety Assessment and Analysis, Columbia, SC, September 22-26, 2013.

Benish, R., et al., "A Pilot Experiment for Science-Based Human Reliability Analysis Validation," International Topical Meeting on Probabilistic Safety Assessment and Analysis, Columbia, SC, September 22-26, 2013.

$\mathrm{Li}, \mathrm{Y}$. and A. Mosleh, "Simulating Nuclear Power Plant Operators' Use of Knowledge in Situation Awareness," International Topical Meeting on Probabilistic Safety Assessment and Analysis, Columbia, SC, September 22$26,2013$.

Boring, R., et al., "Microworlds, Simulators, and Simulation: Framework for a Benchmark of Human Reliability Data Sources," Joint Probabilistic Safety Assessment and Management and European Safety and Reliability Conference, Helsinki, Finland, June 25-29, 2012.

$\mathrm{Li}, \mathrm{Y}$. and A. Mosleh, "Simulation of Nuclear Power Plant Operators Reasoning Process for Situation Assessment in ADS-IDAC Dynamic PRA Platform," Joint Probabilistic Safety Assessment and Management and European Safety and Reliability Conference, Helsinki, Finland, June 25-29, 2012.

Mosleh, A., "Delivering on the Promise: PRA, Real Decisions, and Real Events," Joint Probabilistic Safety Assessment and Management and European Safety and Reliability Conference, Helsinki, Finland, June 2529, 2012.

Mosleh, A., et al., "A Model-Based Human Reliability Analysis Methodology," Joint Probabilistic Safety Assessment and Management and European Safety and Reliability Conference, Helsinki, Finland, June 2529, 2012. 


\title{
12-056 - Recovery of Precious Metals from Used Nuclear Fuel and Electronic Waste Using Sulfur-Impregnated Nanoscaffolds
}

\author{
Peter Zalupski and Linda Nazar ${ }^{1}$
}

In an article published in Nature Materials, Ji, Lee, and Nazar defined ordered mesoporous carbons (OMC) as nanostructured carbon materials characterized by a hexagonal, porous molecular framework. The most attractive property of such materials is their porosity of mesoscale dimensions. The diameters of typical pores of mesoporous materials range between 2-50 nanometers. As such, a mesoporous framework may be envisioned as the architecture constructed from nano-sized building blocks. Mesoporous carbons are engineered agglomerates of carbon nanotubes held together by small carbon nanofibers. They exhibit a uniform pore diameter, high pore volume, as well as high channel permeability. Such mesoporous assembly may span $\sim 100$ channels (i.e., carbon nanotubes of 6-7 nanometer diameters) interlocked into hexagonal unit cells by carbon nanofibers to create wide 3-4 nanometer channel voids. Such porous agglomerates, when coated with molecular sulfur, exhibit attractive affinity for precious metals such as platinum $(\mathrm{Pt})$, gold $(\mathrm{Au})$, and palladium $(\mathrm{Pd})$, as demonstrated by Xiulei, Evers, Lee, and Nazar in Chemical Communications. This LDRD project evaluates the use of the new nanosized materials-mesoporous carbons-in hydrometallurgical recovery of precious metals from aqueous acidic waste streams.

\section{Summary}

Sulfur-impregnated nanoscaffolds (SINs) adsorb Au from acidic chloride mixtures very effectively. $\mathrm{Au}, \mathrm{Pd}$ and $\mathrm{Pt}$ adsorption studies, presented in Figure 1, illustrate that the uptake of these elements continues well into the highly acidic conditions. The adsorption studies revealed that the sulfur coating has no influence on its uptake from highly acidic media. The adsorption of noble metals shown in Figure 1 is very similar for OMC and SIN. The comparison of the efficiency of the observed Au sorption with a study reported by Pangeni and his coauthors in Green Chemistry give the mesoporous carbons a competitive edge over many biopolymer-based adsorbent materials.

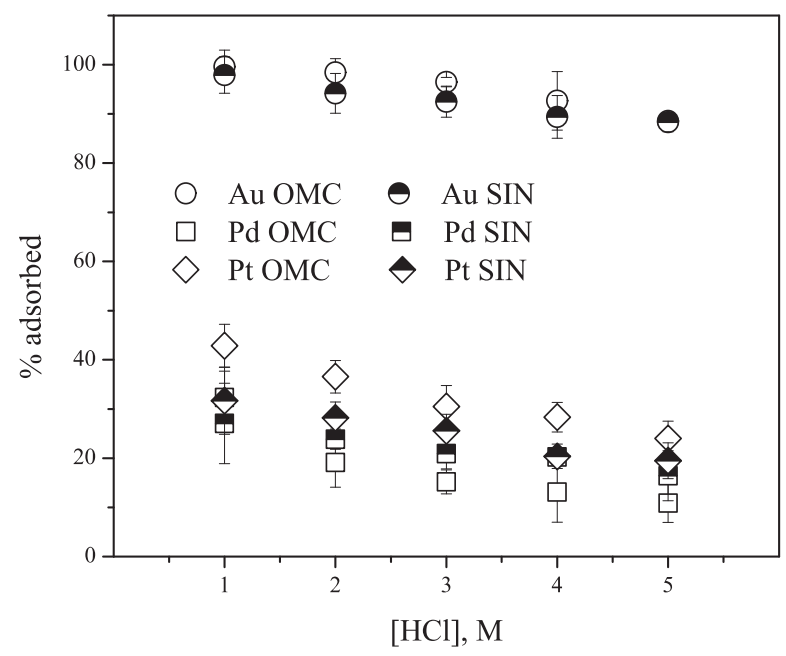

A demonstration has been carried out where personal computer and cell phone circuit boards were leached in aqua regia mixtures (three parts $\mathrm{HCl}$, one part $\mathrm{HNO}_{3}$ ). OMC and SIN materials were

Figure 1. Adsorption of Au, Pd and Pt on OMC and SIN from hydrochloric acid solutions. Conditions: Volume of solution $=10 \mathrm{~mL}$, concentration of each metal $=1.3 \mathrm{mM}$, dry weight of adsorbent $=10 \mathrm{mg}$, shaking time $=18 \mathrm{hr}$. contacted with the three fold dilutions of those leacheates. Figure 2 shows a sorption efficiency histogram for the elements found in the aqua regia leach of computer scrap. The leach mixture contained 22 ppm Pd, 170 ppm silver (Ag), 154 ppm Au, 892 ppt copper (Cu),

1. University of Waterloo, Canada 
3.9 ppt nickel (Ni), 1.9 ppt zinc ( $\mathrm{Zn}), 60 \mathrm{ppm}$ lead (Pb), $586 \mathrm{ppm}$ of iron (Fe), and $324 \mathrm{ppm}$ tin (Sn). Very promising sorption of Au from such complex elemental cocktails was observed. The uptake of other precious metals may be enhanced by building inorganic-organic hybrid systems. The sorption of $\mathrm{Sn}$ is the only competitive equilibrium. This is an important advantage of SIN material as other adsorbents available on the market (ex: SAMMS) attract other hazardous soft metals such as $\mathrm{Pb}$ and cadmium (Cd).

This project supported the internship of Guy Dutech—a French visiting scientist—and the graduate studies of Kaitlin Town from the University of Waterloo, Canada.

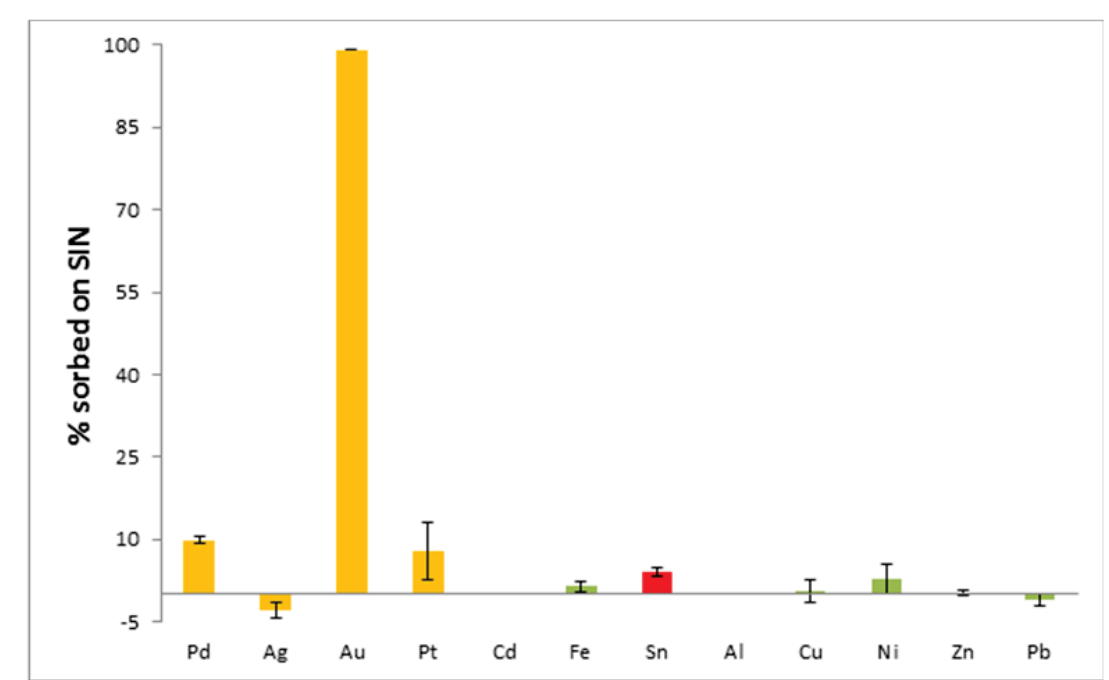

Figure 2. Sorption efficiency histogram for SIN from the diluted aqua regia leach of personal computer circuit boards. Conditions: Volume of solution $=10 \mathrm{~mL}$, concentration of acid $\sim 5 \mathrm{M}$, dry weight of adsorbent $=30 \mathrm{mg}$, shaking time $=18 \mathrm{hr}$.

\section{Benefits to DOE}

This project is contributing to a growing global movement, which, if sustained, will ease the challenges of supporting our society for years to come. The Special Section of the August 10, 2012 issue of Science has been devoted to the challenges associated with the growing production of consumer byproducts. Entitled "Working with Waste," the series of articles highlight the importance of focusing on the recovery of valuable materials. This LDRD project cultivates the belief that our waste may indeed be called treasure instead of trash. 


\title{
12-059 - Chemistry and Flowsheet Development for Lanthanide Separations
}

\author{
R. Scott Herbst, Peter Zalupski, and Mikael Nilsson ${ }^{1}$
}

The lanthanide (LN) series consists of the 15 elements with atomic numbers 57 through 71 (lanthanum [La] through lutetium [Lu]), all occurring naturally with the exception of promethium (Pm). The term rare earth elements (REE) is often used interchangeably with "LNs," but typically include chemically similar yttrium $(Y)$ and scandium (Sc); this convention is adopted in this report. The exceptionally similar chemical characteristics of the REE are attributed to their existence in the characteristic and very stable trivalent $(+3)$ oxidation state. This universal preference for the +3 oxidation state, and the notable similarity in atomic radii (size), results in great difficulties during the chemical separation and concomitant purification of these elements. Existing INL expertise in LN chemistry and flowsheet development, coupled with resources and equipment developed for nuclear fuel cycle applications, was leveraged to develop and test efficient solvent extraction flowsheets for the separation, purification, and concentration of the individual REE from each other. The goal is to design and test flowsheets that will significantly improve process economics by utilizing one or more of the following principles: increased throughputs, improved separation factors, reducing chemical costs/inventories, reducing waste costs, or reducing the conversion costs for products.

Many of the REE have experienced substantial increases in "high tech" uses and enable varying technologies ranging from super magnets to electric vehicles, lighting, and gasoline production. Over the past two decades, China's low mining and milling costs, concomitant with increasingly stringent environmental regulations in other countries, have resulted in the current situation where fully $97 \%$ of the world's production and supply of REE comes from China. To exacerbate the issue, Chinese limitations on REE export quotas have left international demand far above supply, resulting in dramatic price increases or even eliminating availability all together. Limited availability and increased international competition for the world's available supply has resulted in growing concern, to the point that several of the REE are now considered "strategic and critical materials" in the United States.

It is noteworthy that elevated interest in REE processing and economics at the INL level, at the DOE level, and at the industrial level were not fortuitously superimposed events; rather this set of events provides testimony of an urgent national sense to address shortfalls in REE supply.

\section{Summary}

During the first year of this project, an initial investment was made in procuring many of the REE in the form of high purity $(99+\%)$ nitrate salts for use as feed stocks for the separation experiments. Additionally, samples of radioactive isotopes of cesium $(\mathrm{Ce}), \mathrm{Y}$, gadolinium $(\mathrm{Gd})$, and holmium $(\mathrm{Ho})$ were also obtained for determination of distribution ratios in a variety of solvent extraction tests. These materials provide a convenient analytical means to determine extraction behavior of individual elements from a mixture of REE- Ce, Y, Gd, and Ho that can be analyzed simultaneously and directly from solutions containing mixtures of the four elements. The radiometric analysis method is also very accurate and sensitive and provides quick analytical turnaround for scoping tests. Additionally, a Mettler-Toledo titration system provides a rapid analytical means to evaluate REE concentrations

1. University of California, Irvine 
in liquid solutions and can be used to evaluate single element concentrations of the REE by colorimetric complexation titration using a phototrode. This method has also proven valuable for the rapid evaluation of REE behavior in experimental scoping studies and in a non-radiological environment.

These scoping studies were completed during the second year of this project on multiple rare earth extractants in nitrate media. Laboratory scale evaluations were performed on three organophosphorous extractants (Cyanex-923, Cyanex-272 and Cyanex-572) supplied by Cytec Industries and also on di(2-ethyl hexyl) phosphoric and trialkyl phosphine oxide. Each of these extractants were evaluated for acid dependency, diluent effects, synergistic effects, metal loading, optimal stripping conditions, and separation of the individual REEs from each other. The choice of organic diluents for these extractants had a dramatic impact on the separation ability. In fact, improvements with di(2-ethyl hexyl) phosphoric, which is a well-known REE separative agent, are possible with a simple change in the organic diluent used. Cyanex-923 in Isopar-L diluent was found to have the highest extraction efficiency for the $\mathrm{LNs}$ at a nitric acid concentration of $0.1 \mathrm{M}$. However, selectivity between the individual elements tested was low. In addition to extraction data, a low acidity stripping condition of the Cyanex-923 provided promising results that may prove to reduce the chemical consumption of traditional flowsheets.

Cyanex-572 in Exxsol D80 diluent showed a lower extraction efficiency than the Cyanex-923, but it demonstrated a much higher selectivity for the heavier rare earths (i.e., Ho and erbium $[E r]$ ) and $Y$ and may in fact increase individual REE separation factors. Cyanex-272 resulted in the lowest extraction efficiency and is no longer being considered as an option. There were no synergistic effects observed with any of the extractants tested. The data obtained from these tests provide the necessary information to build a process model and to develop a flowsheet for testing in the 30 stage centrifugal contactor pilot plant in FY 2014.

The completion of these scoping studies was supported by a chemical engineering undergraduate student from New Mexico Tech (Adam Martinez).

\section{Benefits to DOE}

Several of the REE are considered strategic for certain DOE clean energy technologies and Department of Defense applications. The outcomes of this LDRD project have the potential to impact economics and supply of these "critical materials" in a favorable manner. 


\title{
12-077 - Observation of Zirconium Oxidation at Atomic Level Using Non-linear Optical Spectroscopy
}

\author{
Marat Khafizov, Kenan Gundogdu, ${ }^{1}$ and Izabela Szlufarska ${ }^{2}$
}

Understanding of the atomic level mechanisms underlying the initial oxidation kinetics of zirconium ( $\mathrm{Zr}$ ) metal will benefit further development of $\mathrm{Zr}$ alloys as a cladding material in nuclear fuels. We seek an integrated approach that brings together advanced spectroscopic techniques and state-of-the-art first principle calculations. We implement Second Harmonic Generation (SHG) spectroscopy (a nonlinear optics technique), which provides bond specific information about chemical and structural properties of interfaces. We use this technique to study the formation of an initial oxide layer on the surface of single crystal $\mathrm{Zr}$ metal. In conjunction with the SHG experiments, we perform ab initio calculations based on the density functional theory (DFT). These calculations are highly accurate and particularly beneficial in discovering preferential bonding of oxygen $(O)$ in $\mathrm{Zr}$ alloys as a function of applied strain. In addition, advanced, time-dependent DFT methods are used to quantify hyperpolarizabilities of $\mathrm{Zr}-\mathrm{O}$ bonds. The results of those calculations are applied to interpret the SHG spectra.

\section{Summary}

On the modeling side, we finished the DFT studies of the effect of surface strain on $\mathrm{O}$ adsorption on $\mathrm{Zr}$ (0001). It was demonstrated that both surface strain and interactions between $\mathrm{O}$ adsorbates influence the adsorption process. The $\mathrm{O}$ binding to $\mathrm{Zr}$ becomes stronger as the strain changes from compressive to tensile. When $\mathrm{O}$ coverage is low and the $O$ interactions are negligible, surface, face-centered cubic sites are the most stable for $O$ binding. At high $\mathrm{O}$ surface coverage and under compression, octahedral sites between second and third $\mathrm{Zr}$ layers become most favorable because the interactions between adsorbates are weakened by positive charge screening. Calculations with both the single-layer adsorption model and multiple-layer adsorption model demonstrate that compressive strain at the $\mathrm{Zr}$ /oxide interface will provide a thermodynamic driving force for $\mathrm{O}$ to incorporate from the surface into the bulk of $Z r$.

Efforts have also been put into investigating the structure and energy of interfaces between $Z r$ metal and its suboxide phases, $\mathrm{ZrO}_{\mathrm{x}}$. The $\mathrm{Zr}-\mathrm{O}$ system is comprised of a variety of ordered $\mathrm{ZrO}_{\mathrm{x}}$ obtained by dissolving $\mathrm{O}$ into hexagonal close-packed $\mathrm{Zr}$. Previous DFT calculations predicted three major ground states, $\mathrm{ZrO}_{1 / 6}, \mathrm{ZrO}_{1 / 3}$, and $\mathrm{ZrO}_{1 / 2}$, all of which have been found experimentally. It still remains unknown how these suboxides nucleate and grow and what the effects of the suboxides precipitates on the oxidation process are. To answer the above questions, the first step is to study the interfaces between $\mathrm{Zr}$ suboxides and O-Zr solutions at many concentrations. Such study requires a large number of calculations of systems consisting of more than one thousand atoms. To achieve this goal, we evaluated different simulation methods (including molecular dynamics, DFT, Monte Carlo, etc.) and determined the best method to be the combination of DFT and cluster expansion (CE). In the CE technique, effective cluster interactions between pair clusters, triplets, and quadruplets are fitted based on the DFT energies, and these interaction terms form the system Hamiltonian. The CE technique makes it possible to do massive calculations within reasonable time and accuracy. We adopted the Cluster Assisted Statistical Mechanics code for our CE calculation. We have now modified the inputs for the codes to construct random $\mathrm{Zr}$-O solutions, and we calculated energies of prismatic interfaces between $\mathrm{Zr}$-O solution and three major

1. North Carolina State University

2. University of Wisconsin-Madison 
suboxides $\left(\mathrm{ZrO}_{1 / 6}, \mathrm{ZrO}_{1 / 3}\right.$, and $\left.\mathrm{ZrO}_{1 / 2}\right)$. Our preliminary calculations show that among the three phases, $\mathrm{ZrO}{ }_{1 / 3}$ has the lowest positive interfacial energy at the $O$ concentration range of $30 \%-40 \%$, which indicates the lowest nucleation barrier and fastest nucleation rate for this specific phase. This result agrees well with previous experiments showing the existence of $\mathrm{ZrO}_{1 / 3}$ at the advancing metal/oxide interface. Specifically, experiments using transmission electron microscopy and microbeam synchrotron radiation diffraction identified the formation of $\mathrm{ZrO}_{1 / 3}$ ahead of the advancing metal/oxide interface during $\mathrm{Zr}$ oxidation.

On the experimental side, a chemical cleaning method for removing the oxide from the $\mathrm{Zr}$ surfaces has been successfully tested and adopted as the starting point of our experiments. An SHG experimental technique has been utilized to investigate bonding at the $\mathrm{Zr}-\mathrm{ZrO}$ interface in a freshly oxidized $\mathrm{Zr}(0001)$ surface. We found that this interface gives rise to a rotationally anisotropic SHG, suggesting the presence of a well-ordered interface with three types of bonds. A bond model was developed to fit our experimental data. Figure 1(a-b) shows the experimental geometry and the assumed atomic structural organization at the $\mathrm{Zr} / \mathrm{ZrO}$ interface. Figure 1 (c-d) displays the resulting SHG data and bond model simulation for $\mathrm{p}(\mathrm{s})$ - polarized excitation and emission.
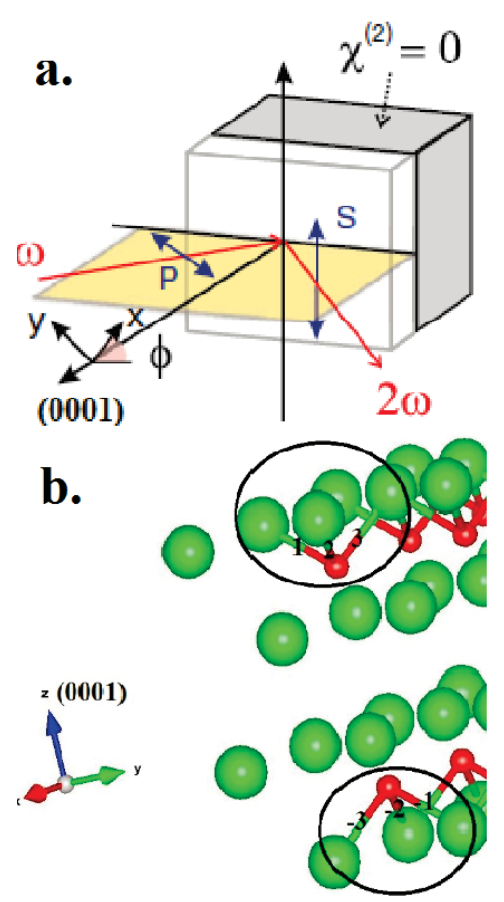

C.
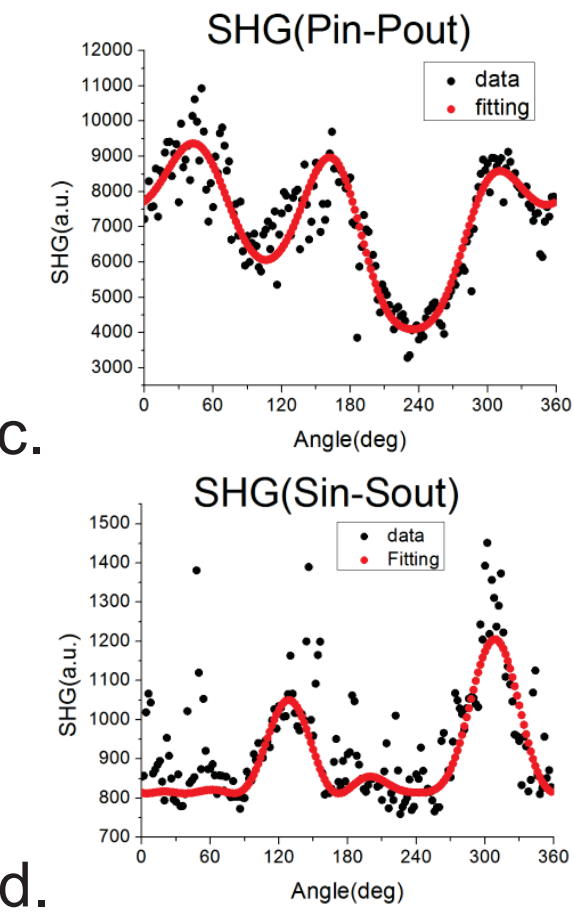

Figure 1. a. Experimental geometry ( $\omega$ is input primary beam, and $2 \omega$ is output $S H G)$. P and S refer to the polarization state of the excitation and radiation. b. 1, 2, 3 bonds near the first Zr layer and -1, -2, -3 bonds near $2^{\text {nd }} \mathrm{Zr}$ for bond model simulation. c. SHG under Pin-Pout geometry with fitting. d. SHG under Sin-Sout geometry with fitting.

In order to study adsorption of $\mathrm{O}$ onto the $\mathrm{Zr}$ surface, experiments need to be performed in ultrahigh vacuum conditions to obtain an oxide-free surface and to control the reaction rate. We upgraded an existing ultrahigh vacuum chamber for the purpose of our studies. The upgrade involved repair and modification of the Auger Electron Spectroscopy, annealing, and argon ion etching equipment and sample mount, which enable various experiments with minimal change in the setup, all located inside the chamber. We developed a recipe for dry 
removal of the oxide layer by a sequential annealing and ion etching method. We monitor the resulting oxide thickness by Auger Electron Spectroscopy experiments. Below are the experimental data for before etching, two hours etching, and 10 hours etching (Figure 2). It is clear that etching successfully removes the surface hydro carbide contamination, and etching reduces the oxide peak at $500 \mathrm{eV}$. We are currently optimizing this etching process in order to completely remove the oxide layer.
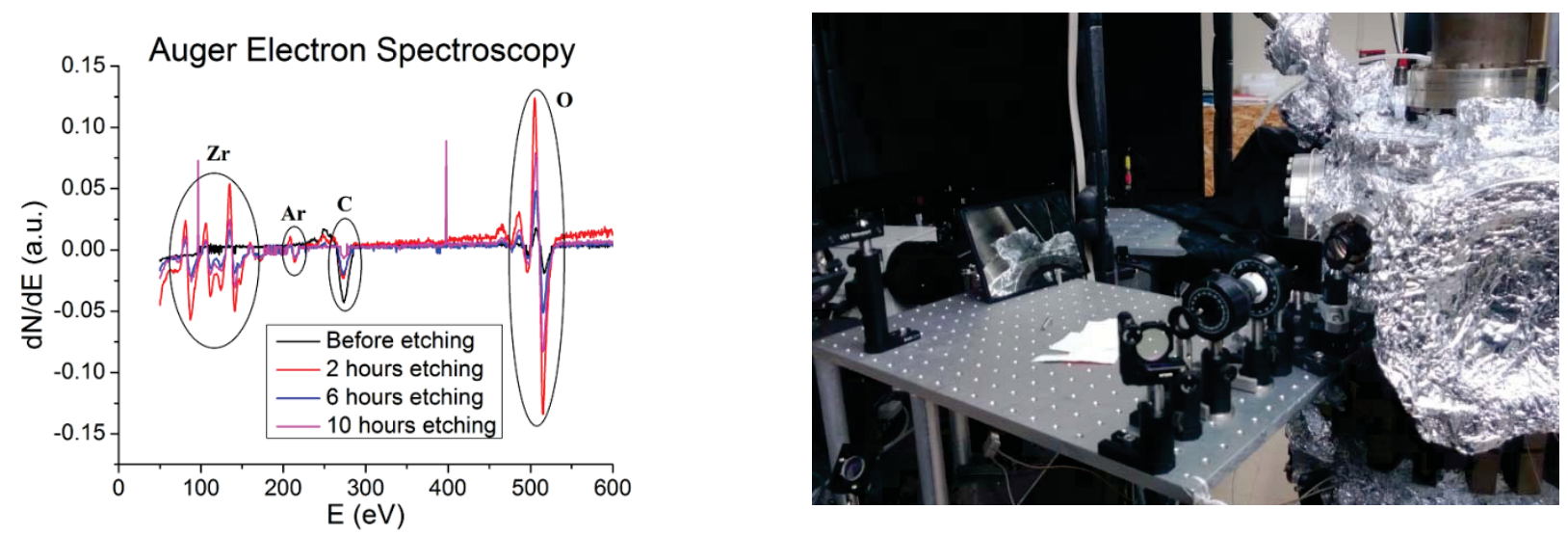

Figure 2. Auger electron spectroscopy after different times of ion etchings (as etching time increases, $\mathrm{Zr}$ peak/O-peak increases). Optical set-up in chamber.

\section{Benefits to DOE}

The results of this work further the understanding of degradation mechanisms of nuclear fuel at the fundamental level. The results also provide important experimental data for modeling efforts. This work establishes an additional experimental capability for performing studies of corrosion interfaces, and it could further be expanded to interfacial chemistry studies relevant to DOE-NE.

\section{Publications}

Wang, X., M. Khafizov, and I. Szlufarska, "Effect of Surface Strain on Oxygen Adsorption on Zr (0001) Surface," J. Nucl. Mater., Vol. 445, 1-6, 2014. 


\title{
12-090 - Development of Emissivity-tuned, Robust Encapsulated Radioisotope Heat Sources for an Advanced Radioisotope Thermo-Photovoltaic (RTPV) Power System
}

\author{
Carl Stoots, Robert O'Brien, and Troy Howe
}

Radioisotope thermo-photovoltaic (RTPV) systems work by using a radioisotope heat source to raise the temperature of a material to sufficient levels to radiate light, which can then be captured by a photovoltaic cell and produce electricity. RTPV systems are useful from a space exploration viewpoint because they can provide power for extended amounts of time and have no moving parts, reducing the risk of failure. An effective RTPV system needs to reach high temperatures and have a good spectral emission profile to maximize energy conversion. Higher temperature means more of the emitted spectrum falls in the higher energy photon range, so minimizing conductive and convective losses allows for more useful photons to be emitted. The emission profile can also be changed based on materials which are known as "selective emitters," or materials that do not emit radiation in a blackbody curve. Instead, they have peaks at certain wavelengths, and, when multiplied by the blackbody emission curve at that temperature, their emission profiles can change. Finding the correct temperature to match an emission curve to the photovoltaic conversion curve can maximize the efficiency of the system. The purpose of this project was to construct a simulation for a RTPV power source that would allow measurement of temperature and emissivity properties of the solid fuel emitter.

\section{Summary}

To achieve the project's goals, it was necessary to develop a testing device capable of heating samples to high temperatures and measuring the emissive spectrum of the material. The desired temperature range was determined to be between 1000-1500K, which would allow for the emissivity spectrum to be tuned over a range of temperatures and the peak of the emission curve optimized to the photovoltaic cell. Maintaining power levels equivalent to the power levels provided by radioisotope fuel sources was also necessary, so the heating method had to be one that could have the power levels monitored. $\mathrm{PuO}_{2}$ provides $345 \mathrm{w} / \mathrm{kg}$, and, when added to a solid fuel matrix, the specific power of the fuel source is reduced to $160 \mathrm{w} / \mathrm{kg}$. The power source and sample must be capable of matching these power levels.

Testing was performed by fabricating a tantalum target and heating it with a $\mathrm{CO}_{2}$ laser in a vacuum. The tantalum target was easily machined and surface features such as reflectivity or roughness could be maintained. The target was hollow and fitted with a small $(3.5 \mathrm{~mm})$ hole in one side to absorb the laser as a blackbody cavity. The $\mathrm{CO}_{2}$ laser provided a clear power input level that could be changed to match the levels of an appropriate radioisotope. The vacuum chamber removed the convective cooling factor, and thermal insulation reduced the conductive factor, allowing for the temperatures of the sample to rise. Thermocouples were fed through to test temperature, and fiber optic cables fed into the chamber allowed for a spectrometer to take readings and provide emissive spectra.

A large portion of testing was dedicated to maximizing the temperature of the sample. To accomplish this, tests were performed with different mounting structures and insulation. The final design was determined to be a tungsten (W) wire suspension system held in place by zirconia insulation beads secured in a steel block. The maximum temperature achieved was $970 \mathrm{~K}$, just short of the desired temperature of $1000 \mathrm{~K}$. According to computer-aided engineering models used to simulate the experiment, this was due to the total emissivity value of 
the surface increasing with temperature. Without the ability to limit the emissivity value, heat could escape much more rapidly and reduce temperatures. Maintaining maximum temperatures for extended periods of time (see Figure 1) provided challenges as well, as outgassing of insulation began to cause oxidation on the sample's surface.

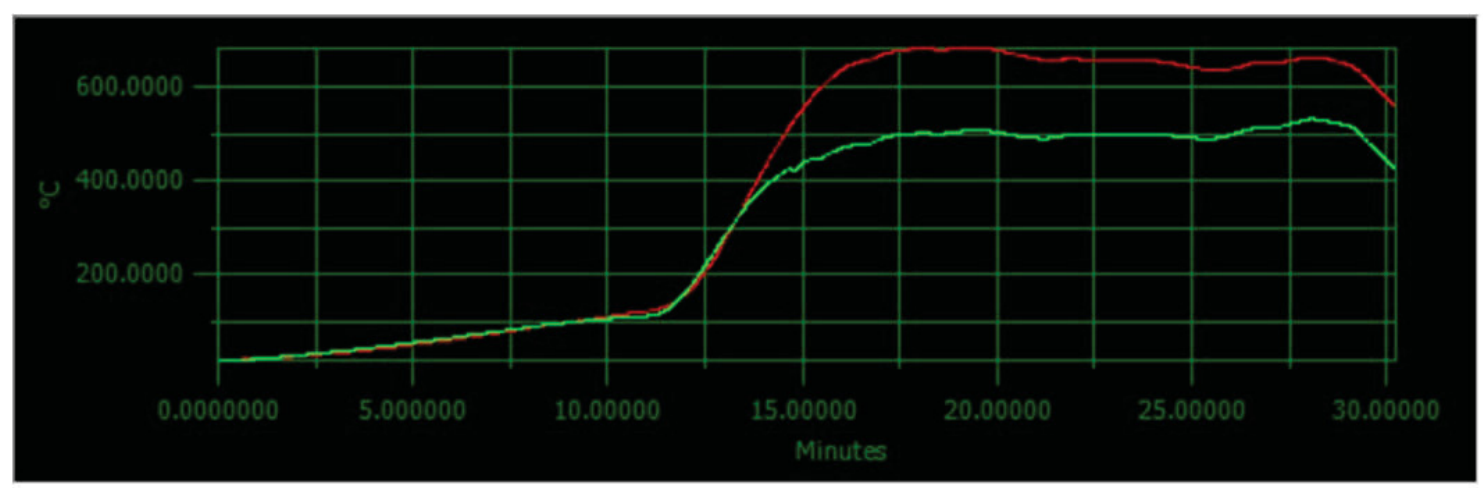

Figure 1. Plot showing temperature and time for experiment.

The laser-based experiment accurately emulated $\mathrm{PuO}_{2}$ fuel source and showed what progress needs to be made to RTPV emitters. Continued testing would require emissivity limiting methods, such as reflective insulation or full surface selective emitters.

Emissivity measurements were taken by spectrometer, but to acquire data over a wide range of temperatures, a higher maximum temperature must be met. To achieve this, it was determined that a small heater could be inserted into the tantalum target and the power levels increased. This would remove the ability to measure power input levels accurately but would provide spectral data as a function of temperature. By the end of the year, a 200 watt heating unit had been used, but, due to view factor losses, the maximum temperature did not increase. 500 watt units are available but not yet testable.

During the summer of 2013 , the RTPV technology was adapted to a nuclear reactor using thermo-photovoltaic conversion instead of a fluid-driven power cycle. The work involved exploring large-scale power production, long distance view factor considerations, structural considerations, and ultra high temperature efficiencies.

\section{Benefits to DOE}

This project provided insight into the factors necessary for improvement for RTPV devices, theoretical designs for high power systems, and the capability to simulate radioisotope fuel sources in a vacuum. This information will help extended-mission radioisotope power systems (such as those utilized by DOE) improve and function at higher efficiencies in space and on earth. 


\title{
13-095 - Development and Validation of a Societal-Risk Goal for Nuclear Power Plant Safety
}

\author{
Robert Youngblood, Vicki Bier, ${ }^{1}$ and Michael Corradini ${ }^{1}$
}

Events at Fukushima have had very serious consequences, but those consequences have, for the most part, not involved actual dose to humans. Rather, the consequences have involved evacuation and land interdiction. While radiological exposure to the public has been minimal, the disruptive effect of the protective actions has been quite serious. This outcome points to a shortcoming of existing Nuclear Regulatory Commission (NRC) safety goals, which focus on dose consequences to individuals, but do not address disruptive effects of significant accidents. A more comprehensive set of safety goals would help to focus regulatory priorities on the desirability of preventing a need for evacuation rather than effectively relying on evacuation as if it were an engineered safety barrier.

Correspondingly, this project is evaluating the social disruption effects from severe reactor accidents as a basis for developing a societal-risk goal for nuclear power plants. The work is considering both health effects and nonhealth concerns like property damage and land interdiction. To achieve our goal, we are generating and using data on accident consequences at various reactor sites in the U.S. to assess the implications of concern about societal risk for nuclear-power regulation.

\section{Summary}

The project began by studying the possible societal disruptions that could occur from nuclear-power accidents, considering both past accidents and potential future accident scenarios. One important finding is that an improved societal-risk goal for nuclear power plants should ideally account for not only anticipated health effects but also the number of people that would need to be evacuated in the event of an accident. This would be an important addition to traditional safety-goal policy for two reasons. First, the number of people to be evacuated appears to be a good proxy for the level of societal disruption from an accident while still being relatively straightforward to compute in an objective manner. Second, health effects and evacuation trade off directly against each other. For example, even a large accident could have negligible health effects if the number of people evacuated was sufficiently large, but that does not mean it would cause negligible societal disruption. Thus, the ultimate purpose of the project is to enhance traditional NRC safety-goal policy through development of a societal-risk goal that places a limit on the aggregate extent of disruption that could result from a severe accident.

One student participating in the project has completed his Ph.D. and is currently on faculty at the Air Force Institute of Technology. He is continuing to collaborate with the project on an unfunded basis. We have also collaborated with a number of individuals active in emergency planning at the NRC and participated in a workshop on assessment of the risk of extreme floods to pursue commonalities in the assessment and management of disruptive severe accidents. 


\section{Benefits to DOE}

The work has already generated insights into possible improvements in evacuation policy for large accidents (e.g., by extending emergency-planning zones beyond 10 miles if needed). Similar methods, such as using stateof-the-art dispersion models and geographic-information systems, should be applicable to emergency planning in other areas as well (e.g., chemical emergencies and military planning). Better modeling of societal disruption (rather than relying on relatively crude proxies, such as number of fatalities) is a particularly novel aspect of the work. Additionally, the work may shed interesting light on siting decision-making for novel reactor technologies, such as Small Modular Reactors, whose severe accident behavior should be very different. 


\section{3-105 - Micro/Nano Scale AFM-based Thermal Conductivity Measurement and Atomistic Modeling for Oxide Fuel: the Effects of Grain Boundary, Fission Gas, and Radiation Damage}

Jian Gan, Yong Yang, ${ }^{1}$ Heng Ban, ${ }^{2}$ Aleksandr Chernatynskiy, ${ }^{1}$ and Xianming "David” Bai

By using a bottom-up approach, the effects of particularly tailored microstructures and controlled radiationdamage on materials' thermal conductivity can be individually evaluated and modeled. The complementary atomistic modeling of the thermal transport is conducted to compare the results directly with experiments. The proposed research aims to study the microstructure and radiation effects on the thermal conductivity of oxide fuel using a novel Atomic Force Microscopy (AFM)-based measurement technique and complementary computer modeling. Specifically, the following objectives are being pursued:

1. Determine grain boundary (GB) thermal resistance for different types of GBs in undamaged materials to correlate specific GB structure with thermal transport.

2. Investigate the effect of fission gas implantation on thermal resistance of GBs and the effect of different types of GBs on fission gas segregation.

3. Determine thermal conductivity degradation caused by radiation damage at different irradiation temperatures.

\section{Summary}

Modeling efforts were focused on how GB character affects thermal transport in $\mathrm{CeO}_{2}$. As a first step, an assessment of empirical potentials was conducted to determine which is the most suitable for modeling both thermal transport and GB structures. Both bulk thermal transport properties and GB structures were investigated with different potentials. Comparison with the experimental data for these properties shows that the Gotte 2007 potential is the best. With this potential, the thermal conductance of GBs (both twist [University of Florida] and tilts [INL] with a wide range of misorientation angles) was investigated. Results indicated that GB conductance correlates strongly with the GB energy and misorientation angle: thus, low-angle GBs exhibit much higher conductance than high-angle ones. Note that exact atomic structure of the most GBs is not known experimentally. Moreover, for a given misorientation, different relative positions of the neighboring grains might generate multiple different low-energy structures; such a map of the GB energy dependence on relative positions of the grains is known as a $\gamma$-surface. We are currently performing $\gamma$-surface study for a specific GB and analyzing how different structures affect thermal transport. The initiation of the development of the potential for the $\mathrm{CeO}_{2}-\mathrm{Xe}$ interactions is ongoing.

One of the experimental research efforts was focused on the sample preparation needed in order to achieve a desired grain size and potentially representative types of GBs. Both the Spark Plasma Sintering and conventional furnace sintering methods were tried. A $1700^{\circ} \mathrm{C}$ tube furnace was procured, and the high temperature sintering process protocol for producing controlled grain structure of $\mathrm{CeO}_{2}$ has since been established. The sintered samples will be further subject to noble gas implantation and heavy ion irradiation. For thermal resistance 
measurements using the Atomic Force Microscopy-based technique (see Figure 1), many test measurements have been taken to explore thermal signal characteristics of the glass-pipette thermal probes. Problems with the system's feedback and software have been identified and solved. Meanwhile, electro-thermal finite element modeling of the probe has been done to facilitate the interpreting of experiment results.

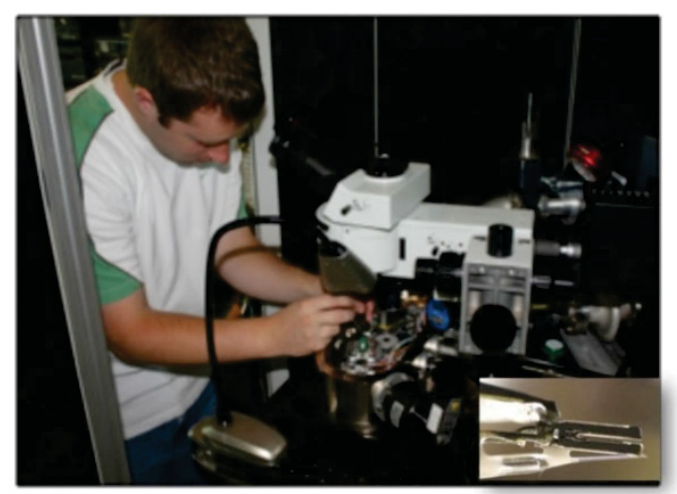

Ph.D.student working on AFM equipped with glass-pipette thermal probes

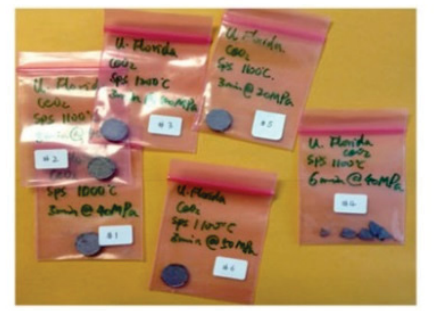

$\mathrm{CeO}_{2}$ pellets produced using SPS

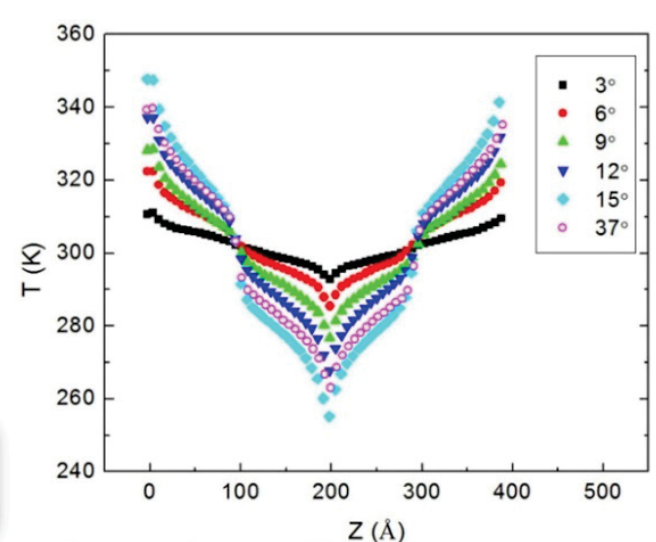

Temperature profiles across different tilt GBs of different anqles

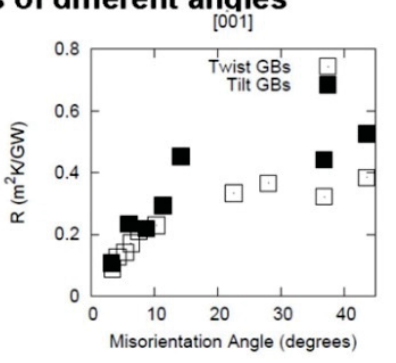

Grain boundary resistance for twist and tilt GBs of different angles

Figure 1. Project-specific images including GB plots.

\section{Benefits to DOE}

The fundamental study will contribute to DOE's leading role on basic science research of materials behavior and performance in extreme environments. The light water reactors' parameters, such as nominal power level and fuel temperature margin to failure, have been limited by the poor thermal conductivity of oxide fuel and the associated large temperature gradient. A better understanding of thermal conductivity for oxide fuel during operation will have an important impact on developing advanced light water reactor fuels with improved thermal physical properties, which can enhance the safety and performance of commercial nuclear fuels.

\section{Presentations}

Bai, X., A. Chernatynskiy, and J. Gan, "Misorientation-Dependence of Grain Boundary Thermal Resistance in $\mathrm{CeO}_{2}$," 2014 TMS Annual Meeting \& Exhibition, San Diego, CA, February 16-20, 2014, Accepted.

$\mathrm{Wu}, \mathrm{Y}$., et al., "Correlation between Thermal Conductivity and Microstructural Evolutions in $\mathrm{CeO}_{2}$ Upon Radiation and Fission Gas Implantation," 2014 TMS Annual Meeting \& Exhibition, San Diego, CA, February 16-20, 2014, Accepted. 


\title{
13-106 - Building Organic-Inorganic Hybrid Materials to Protect Metal Ion Sequestering Agents from Radiation-Induced Oxidative Damage
}

\author{
Peter Zalupski, Bruce Mincher, Michael Schultz, ${ }^{1}$ and Gary Buettner ${ }^{1}$
}

Radiolytic damage incurred to solvent extraction reagents during the process of hydrometallurgical recovery of actinides from used nuclear fuel has obstructed aqueous separations for decades. This LDRD project supports the back-end of nuclear fuel cycle through studies of the "shielding" properties of mesoporous carbon nanostructures, which will be utilized as encapsulating hosts for the metal ion complexing reagents illustrated in Figure 1. The inorganic (carbon) - organic (complexant) hybrid materials are tested for their ability to alleviate the damaging influence of free radicals produced when the energy of radioactive decay is deposited in matter. In short, the project seeks antioxidizing benefits of such hybrid capsules.

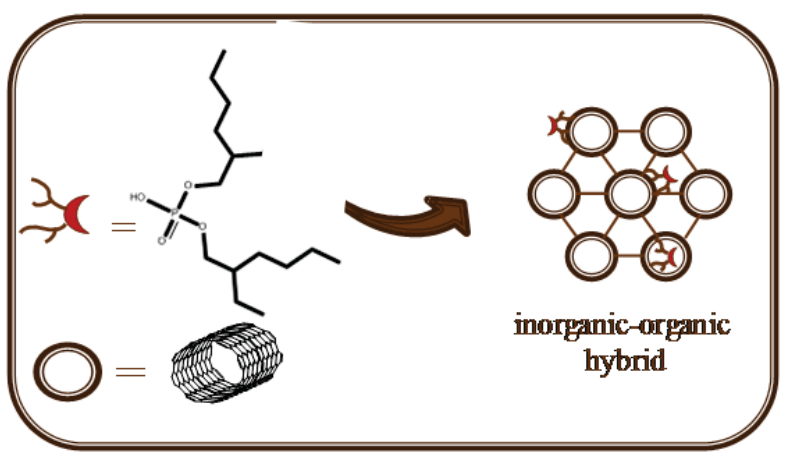

Figure 1. Schematic representation of an organicinorganic hybrid material where the metal complexing organic molecule is di(2-ethylhexyl) phosphoric acid and the inorganic capsule is ordered mesoporous carbon.

\section{Summary}

A synthetic recipe for the preparation of organic-inorganic hybrid materials has been developed. A variety of hybrid materials has been prepared. Encapsulated complexants have been shown to maintain their metal coordination properties. The engineered hybrids complex metals much more efficiently, relative to analogous conventional solvent extraction systems.

The first fully characterized hybrid system consisting of di(2-ethylhexyl) phosphoric acid (HDEHP) inside the ordered mesoporous carbon has been irradiated using a Co-60 gamma ray source. Figure 2 shows the sorption of an americium (Am) metal ion by the irradiated hybrid as compared to analogous conventional solvent extraction system where HDEHP is not shielded from damaging effects of gamma-induced free radicals. 


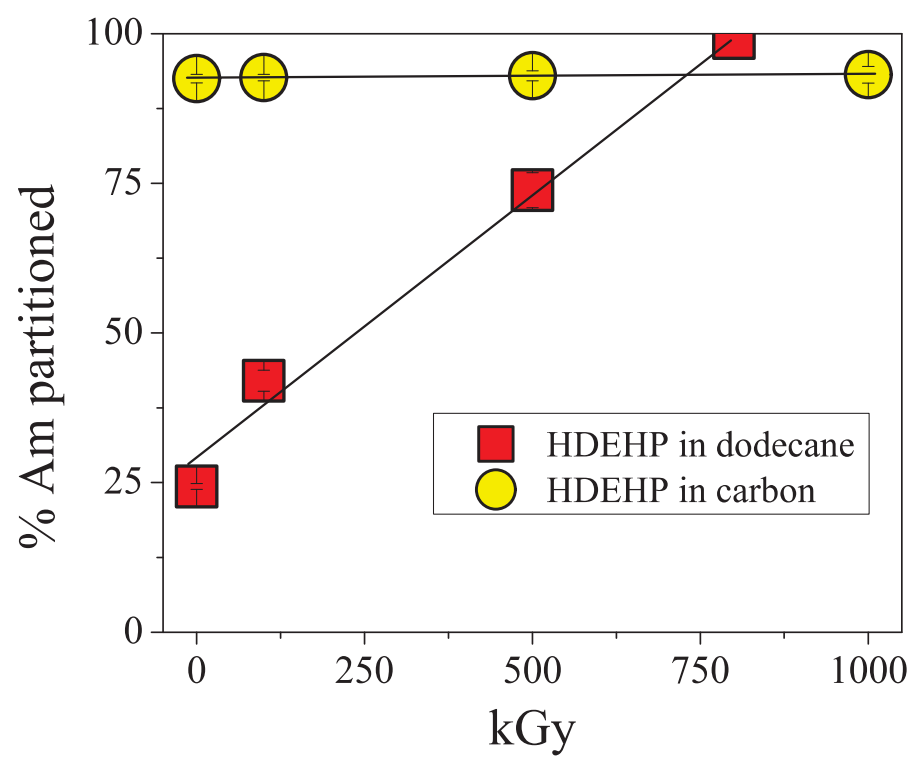

Figure 2. Effect of radiation dose on the partitioning of Am from $0.1 \mathrm{MHNO}_{3}$ using two HDEHP containing systems.

Figure 2 summarizes the results of the irradiation study, proving the scientific concept proposed by this LDRD project. The characteristics of Am uptake by the hybrid material did not change throughout all the received doses, proving that HDEHP is not affected by gamma radiation when encapsulated by mesoporous carbon. At the same time, liquid-liquid partitioning of Am in a conventional solvent extraction system changes drastically, illustrating the effects of gamma-induced degradation of HDEHP molecule.

This project supported the graduate studies of Andrew Knight from the University of lowa.

\section{Benefits to DOE}

This project supports DOE's energy resources mission by conducting the feasibility studies on closing the nuclear fuel cycle, where valuable materials locked inside used nuclear fuel are recovered and reused through further burning of fissionable isotopes. Hydrometallurgical recovery of actinides from dissolved nuclear fuel is challenged by the radiolytic degradation of reagents used in aqueous separations systems. If protected, such reagents may function more effectively, improving the economics of such methodologies. 


\title{
13-107 - Application of Chemical Heat Pumps for Temperature Amplification in Nuclear Hybrid Energy Systems for Synthetic Transportation Fuel Production
}

\author{
Piyush Sabharwall, Daniel Wendt, and Vivek Utgikar ${ }^{1}$
}

Nuclear energy, the key to the long-term energy security and sustainability of the carbon (C)-free energy future of the nation, can be integrated into non-electric power uses through nuclear hybrid energy systems (NHESs). NHESs based on conventional light water reactors (LWRs) require temperature amplification through technologies such as chemical heat pumps (ChHPs) for applications requiring temperatures higher than the outlet temperature of LWRs. The purpose of the project was to investigate and develop ChHPs for the production of synthetic transportation fuels. The following objectives were defined for this research:

1. Conduct a literature review, evaluation, and down selection of candidate ChHP systems that would be applicable to upgrade the thermal energy produced by a small modular LWR.

2. Complete thermodynamic calculations and preliminary economic feasibility assessments of the candidate ChHP systems.

3. Provided there is justification to proceed, setup a lab-scale test prototype and collect data to verify the thermodynamic and heat transfer mechanisms of the ChHP unit operations.

4. Complete a plant design and economics study to evaluate an integrated system consisting of a nuclear reactor (current LWRs or advanced small modular reactors based on the LWR design), a ChHP, and a selected fuels production plant, such as a biomass-conversion system, to produce 10,000 barrels per day of liquid transportation fuel.

\section{Summary}

The project was initiated in January 2013 and concluded on September 30, 2013. The activities conducted include a literature survey of ChHPs, identification of candidate ChHP and suitable biomass/C resources for the synthesis of the transportation fuels, thermodynamic analysis of the system, and definition of the systems for further thermodynamic, economic, and environmental analysis. These activities correspond to objectives one and two stated above. The planned activities for the second and third year of the project included experimental work and detailed techno-economic-environmental analysis, as stated in objectives three and four.

The literature search indicated that a reversible hydration/dehydration cycle based on $\mathrm{Ca}(\mathrm{OH})_{2} / \mathrm{CaO}$ was the most suited ChHP to provide the desired temperature amplification. The key characteristics of this ChHP are: (1) high thermal energy storage density (over $1250 \mathrm{~kJ} / \mathrm{kg}$ ), (2) high output temperature potential $(773 \mathrm{~K}+)$, (3) fast kinetics, (4) good reaction reversibility and durability, (5) no toxicity (good reactant corrosion resistance), (6) abundant and low cost $\mathrm{CaO}$ resources, (7) the hydration/dehydration reaction permits use of evaporators/condensers for low temperature reactors (simple and low cost method of collecting, storing, and regenerating mobile phase; eliminates additional packed bed batch mode only reactor; simplified process control and operation; improved heat and mass transfer characteristics), and (8) does not release any contaminating gases. 
Preliminary thermodynamic efficiency analysis was conducted for this ChHP. The maximum theoretical efficiency of the system was found to be $\sim 72 \%$.

The literature search also indicated poplar to be an attractive biomass material for conversion into transportation fuel. The three potential alternatives examined included: poplar (Populus), pine (Pinus) and corn. Corn has received attention due to its potential for conversion to ethanol; however, it is eliminated from consideration here due to its value as a food crop. Pine and poplar are both softwoods that grow rapidly; both are also hardy, adaptable to most U.S. environments, grow naturally, and require little maintenance. Pine, due to the nature of the current genetic modification science, does not grow in a limbless or easily accessed (by equipment) variety. Poplar trees have been the focus of several scientific studies and grow in several modified formats. Poplars are also able to be grown in closer proximity and produce larger yields per acre-year of chipped material. Essentially, poplar can be grown and harvested cyclically in a small space with high and sustainable yields. Poplar is an attractive alternative due to its potential for $\mathrm{C}$-neutrality, low energy requirements, and fast growth. Willow and poplar act as symbiotic growth enhancers, with poplar supporting an aggressive growth cycle that allows for a three-year plant-harvest cycle that can be repeated up to seven times before another more rejuvenating product needs to be planted. Willow is the sister product of poplar, supporting an active growth cycle that rejuvenates the earth in preparation for the poplar cycles to begin again. Genetically modified poplar is proposed to be used as the base biomass material, with minor willow constituents during off-peak cycles.

It is recommended that further research be undertaken on this ChHP to complete the thermodynamic analysis and determine the feasibility through bench- and pilot-scale experimentation.

\section{Benefits to DOE}

Utilizing nuclear reactors to produce synthetic transportation fuels will lessen the nation's dependence on imported petroleum products and thus enhance DOE's mission of energy security. The project will also enhance environmental quality as the fuels will be synthesized from domestic biomass, reducing $C$ emissions. Nuclear reactors are ideally suited to provide baseload electric power; however, integration of the nuclear energy in process heat and other non-electric power uses, such as manufacture of synthetic transportation fuels, requires the development of NHESs. NHESs are ideally suited to enhance the nation's energy security while minimizing emission concerns. The potential applications of nuclear reactors for non-power generation applications range from low temperature operations, such as desalination, to high temperature coal gasification and hydrogen production. Production of synthetic transportation fuels is another of these applications that requires higher temperatures than what are possible from the conventional LWRs that will be the primary energy sources for the first generation NHESs. The project addresses the crucial need for temperature amplification technologies to bridge this temperature gap.

\section{Publications}

Wendt, D.S., P. Sabharwall, and V. Utgikar, "Light Water Reactor Temperature Upgrading Using a Chemical Heat Transformer," Proceedings of the ASME 2013 Summer Heat Transfer Conference, Minneapolis, MN, July 14-19, 2013. 


\section{Presentations}

Utgikar, V., "Chemical Heat Pump Systems for Temperature Amplification for Light Water Reactors," INEST NHES Workshop, Idaho Falls, ID, July 2013.

Wendt, D., P. Sabharwall, and V. Utgikar, "Technologies for Upgrading Light Water Reactor Outlet Temperature," ASME Summer 2013 Heat Transfer Conference, Minneapolis, MN, July 14-19, 2013. 


\title{
13-110 - Nuclear-Renewables-Oil Shale Hybrid Energy System
}

\author{
Michael McKellar, Charles Forsberg, ${ }^{1}$ and Daniel Curtis $^{1}$
}

The oil shale deposits of Colorado, Utah, and Wyoming are the largest potential sources of oil on earth; however, they contain no ready-to-recover oil. In-situ heating of the oil shale is required to break down kerogen to produce a light crude oil and hydrocarbon gases. A quarter of the energy value of the recovered crude oil and gases would have to be burnt to operate the process, resulting in large greenhouse gas releases relative to conventional sources of oil. A strategy is proposed to produce the oil with the lowest greenhouse footprint per liter of product and enable large-scale renewables on the western grid. Nuclear reactors operate at base-load conditions. At times of high electricity prices, electricity is sold to the grid. This provides a reasonable cost source of electricity to replace fossil generating plants that provide variable electricity with major reductions of $\mathrm{CO}_{2}$ releases. At times of low electricity prices, nuclear heat and external electricity is used to convert oil shale to oil and hydrocarbon gases. The system operates like a multi-gigawatt storage battery on the grid that produces oil. That capability allows the grid to use more renewables without creating major challenges in providing backup power. In other words, the objective of this project is to develop and evaluate the viability of a nuclear-renewable shale oil system.

\section{Summary}

Alternative system designs were developed for different reactor types. With a light water reactor, the reactor provides preheating of the oil shale to between 210 and $240^{\circ} \mathrm{C}$. Electric heating of steam at times of low electricity demand/prices is used to raise the oil shale to its final temperature. With high-temperature reactors, the full required steam temperatures can be reached with the reactor steam being used for either electricity production or shale oil production. Preliminary revenue estimates for different operating modes have been calculated using the average hourly prices of electricity in California—the largest local market.

An initial assessment of deployment strategies for light water reactors was completed. Development of oil shale resources implies many tens of small modular reactors (up to $1000 \mathrm{MWt}$ each). The large scale deployment in small areas allows serial manufacture and shared services, such as spent fuel management and security. This implies the potential for substantially lower costs per unit installed capacity than would exist if such reactors were deployed by utilities.

Assessments of reductions in greenhouse emissions using nuclear energy have been initiated. There are two sources of savings. First, nuclear energy reduces greenhouse gas emissions per liter of gasoline or diesel that is produced. Second, combining variable electricity and shale oil production can eliminate the use of fossil power plants for variable electricity production resulting in added reductions in greenhouse gas emissions.

\section{Benefits to DOE}

The project reduces the emissions of greenhouse gasses, provides a much deeper penetration of renewable energies into the electric grid, extracts fossil fuels from a very large source, and provides a large market for the use of small modular reactors; all of which directly benefit the DOE national security missions. 


\section{Presentations}

Forsberg, C. and D. Curtis, "Integrating Nuclear Renewable Shale-Oil Systems: Low-Greenhouse-Footprint Liquid Fuels and Variable Electricity," Presentation at Idaho National Laboratory, Idaho Falls, ID, July 23, 2013.

Curtis, D. and C. Forsberg, "Light-Water Reactor Arrays for Production of Shale Oil and Variable Electricity," Transactions of the American Nuclear Society Annual Meeting, Atlanta, GA, June 16-20, 2013.

Kadak, C., C. Curtis, and C. Forsberg, "Integrated Nuclear Shale Oil System: Lowest $\mathrm{CO}_{2}$-Emission Fossil-Liquid Fuel and Variable Electricity," International Oil Shale Symposium 2013, Tallinn Estonia, June 10-13, 2013. Curtis, D., "Nuclear Shale Oil Development Reactor Arrays and System Effects," American Nuclear Society 2013 Student Conference, Boston, MA, April 4-6, 2013.

Curtis, D. and C. Forsberg, "Light-Water Reactor Renewable Shale-Oil Systems for Variable Electricity Production and Liquid Fuels," Transactions of the American Nuclear Society, San Diego, CA, November 11-15, 2012. 


\section{3-112 - High-Temperature Nuclear Co-Electrolysis for Spacecraft Cabin Air Revitalization \& Liquid Fuel Production}

\section{Carl Stoots and Robert O'Brien}

For manned spaceflight and surface habitation under planetary exploration missions, efficient long duration life support is essential. Most fundamentally, metabolic $\mathrm{CO}_{2}$ must be removed from the cabin air. Additionally, metabolic water vapor must also be removed for humidity control. The integrated use of low mass Solid Oxide Electrolysis Cell and stack technology in conjunction with space or surface fission power reactors is studied here for cabin atmospheric revitalization applications via the co-electrolysis of $\mathrm{CO}_{2}$ and $\mathrm{H}_{2} \mathrm{O}$. The resultant products of co-electrolysis are syngas $\left(\mathrm{H}_{2}+\mathrm{CO}\right)$ and $\mathrm{O}_{2}$, which are naturally separated via the Solid Oxide Electrolysis Cells. Syngas is a highly valuable commodity that may be used to produce water via hydrogenation of methanol $\left(\mathrm{CH}_{4} \mathrm{O}\right)$ and other liquid fuels. The efficiency of co-electrolysis is enhanced by operation of Solid Oxide Electrolysis Cells at a high temperature $\left(\sim 850^{\circ} \mathrm{C}\right)$. Abundant nuclear process heat and electrical power generated by a small fission power plant or a bi-modal nuclear thermal rocket may be used to significantly enhance the overall thermal-toproduct efficiency (Figure 1). The overall co-electrolysis reaction considered is presented in Equation (1) below.

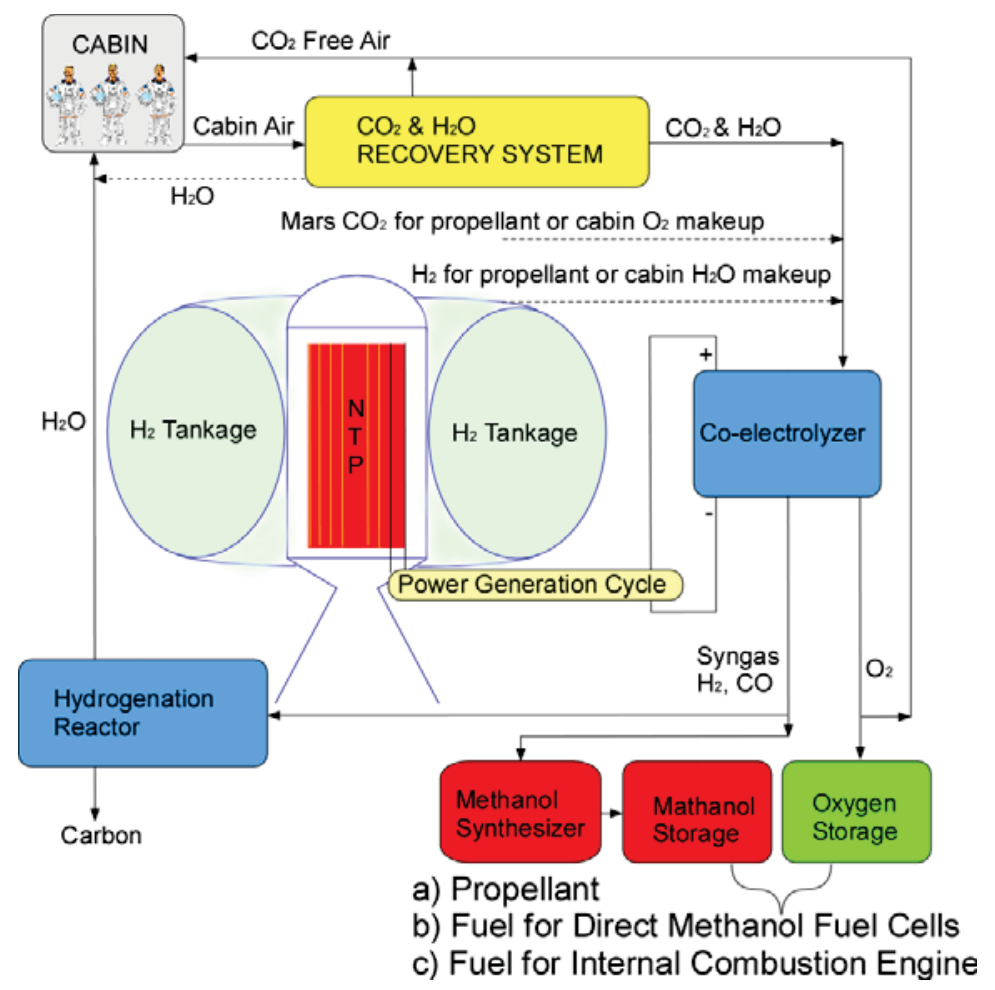

Figure 1. Schematic of a concept coupled system for cabin air revitalization and fuel production via high temperature nuclear co-electrolysis operated under a manned Mars surface exploration mission.

$\mathrm{CO}_{2}+\mathrm{H}_{2} \mathrm{O} \rightarrow \mathrm{H}_{2}+\mathrm{CO}+\mathrm{O}_{2}$ 
The National Aeronautics and Space Administration (NASA) is already highly interested in the INL/Center for Space Nuclear Research co-electrolysis concept. While current state-of-the-art NASA life support systems can successfully remove and store $\mathrm{CO}_{2}$ from cabin air, NASA currently seeks suitable technologies and systems that can efficiently close the loop in cabin air revitalization, minimize supplemental life support tankage, store energy, and produce return propellants and liquid fuels for surface operations. Preliminary paper studies produced by R.C. O'Brien, C.M. Stoots, and M.E. McKellar regarding space nuclear co-electrolysis have generated substantial interest within NASA. This LDRD research project started the development and demonstration of the co-electrolysis of $\mathrm{CO}_{2}$ and $\mathrm{H}_{2} \mathrm{O}$ on a flight scale experimental test skid that will be operated in collaboration with NASA Ames Research Center and NASA Johnson Space Flight Center. Metabolic $\mathrm{CO}_{2}$ and $\mathrm{H}_{2} \mathrm{O}$ capture and compression technologies developed at NASA Ames were to be integrated with the co-electrolysis test skid in addition to an INL/Center for Space Nuclear Research built hydrogenation system during second and third year testing. The overall aim is to demonstrate the technology readiness level $3 / 4$ at the end of the proposed project.

\section{Summary}

The project duration was one year. Detailed systems design work has been performed and is largely complete for an International Space Station implementation of high temperature co-electrolysis test skid. A surplus International Space Station rack was requested and was being delivered before cancellation of the project. A detailed computational fluid dynamics model of the International Space Station co-electrolysis system, including a novel way of maintaining solid oxide stack temperature in a loss-of-power event, was developed and analyzed. Computational fluid dynamics results show that the radioisotope heaters (Invention Disclosure Record to be submitted) in a zero gravity environment (no convective heat transfer) allow the solid oxide stacks to survive lossof-power events. This is a very important, technology-enabling development. Since the co-electrolysis stacks are needed for life support, robustness is an important attribute.

NASA Ames Research Center and NASA Johnson Space Flight Center have been following INL developments closely. It is believed that this concept of integrated radioisotope heaters could elevate the technology to a higher technology readiness level and, to a large extent, resolve concerns NASA has about stack restart after power loss.

\section{Benefits to DOE}

Co-electrolysis for space applications has been shown theoretically to improve energy efficiency at lower payload mass. It can greatly simplify the existing NASA cabin air revitalization system and can enable in-situ resource utilization via the Martian atmosphere for return propellant and liquid fuel production. These factors benefit NASA space exploration missions. 


\section{CENTER FOR}




\title{
11-065 - Development and Demonstration Project for National Fuel Efficiency
}

\author{
David Gertman, Milos Manic, ${ }^{1}$ and David Bruemmer $^{2}$
}

According to the U.S. Energy Information Administration, the U.S. transportation sector (including cars, trucks, buses, and tractor-trailers) consumes more than 200 billion gallons of fossil fuel each year. There are over 2.5 million heavy trucks on the highways, and while medium and heavy trucks represent only $4 \%$ of all vehicles, they collectively use more than $20 \%$ of all fuel. One category of heavy trucks, long haul heavy duty trailers, uses over 22 billion gallons of fuel annually. These vehicles are critical to our economy and transport equipment, supplies, and other goods hundreds of millions of miles back and forth across the country each year. Even a small increase in fuel efficiency for these vehicles results in tremendous cost savings as well as reductions in emissions.

Much effort has been expended in development of gas or diesel/electric hybrid vehicles both in conversion of existing vehicles and in development of new designs for vehicles that use compressed natural gas. While these efforts are yielding reductions in petroleum usage, new fuel efficiency optimization software can be applied to help human operators improve their driving efficiency and enable them to more fully benefit from existing and new technologies with minimum cash investments. This is of critical importance for many large fleet operators where purchasing new vehicles and making modifications can be cost prohibitive.

At a minimum, fleet efficiency has three components. These are the driver, the vehicle-including the fuel type and safety features - and the environment, which includes terrain features, road and traffic conditions, and connected vehicle technology. It is widely recognized that improvements in human behavior can significantly improve fuel efficiency. A 2004 study by Truck Maker International found that improvements in driver behavior could yield significant improvements in fuel efficiency (up to 30\%) without making changes to the vehicle hardware or software. Other studies have similar findings. Rapid acceleration, improper speed selection for conditions, unnecessary and aggressive braking, excessive idling, and poor route selection are among the human factors recognized to reduce fuel economy. Of the resources that can be optimized by fleet operators, none is more valuable (or variable) than the human operator. Consequently, implementation of an inexpensive system that helps drivers understand their behavior can reduce petroleum consumption, save fleet operators millions of dollars, and contribute to environmental and national security benefits.

To date, much of the effort in improving fuel efficiency has centered on improving fuel blends and alternative fuels, improving transmission and engine efficiency, and the use of composite materials to reduce weight. To a lesser extent, there has been some research conducted to evaluate the effects of aggressive acceleration and aggressive braking on the part of drivers. There has not been research on the development of tools, especially for motor coaches, that could be used to develop a predictive means to aid drivers.

The current project addresses this need by improving the current display technology available to drivers. It provides real time analysis and predictive speed control information based on change in driving slope and elevation provided by Global Positioning System information in coordination with a model of best driver

1. University of Idaho

2. 5D Robotics 
performance. The research approach we determined was to develop a best driver algorithm (developed through data mining of real driver information), make real-time comparisons to engine data, and to present this information to the driver in a graphic user interface (GUI). The emphasis is to cue the motor coach driver to accelerate or decelerate based on changes in road slope, in advance of these elevation changes. This project entailed the use of INL buses, drivers, and INL big shop master technicians in conjunction with INL human factors, University of Idaho data mining, and 5D Robotics real-time sensor information management strategies.

\section{Summary}

The execution of this project has led to the development of successful software architecture, including algorithm development and real time data management, leading to an initial functional prototype system for driver efficiency. In order to achieve this, we needed to develop an approach to obtain real time engine data from a fixed driving route and make comparisons of motor coach performance to an optimized fuel efficiency driving model. The fixed routes driven at INL allow for easy comparisons between drivers and evaluation of the software tool. Decoding engine data in real time for the purpose of making comparisons to a model can be daunting. Software was written to decode the engine data without swamping the system and this information was successfully compared to a historical model of optimum performance. A GUI was developed by graduate students at the University of Idaho, Department of Computer Science to present this information to drivers. Additionally, to aid in software GUI prototype development, a bus simulation-the VirtualBus based on real route data-was developed. Different prompting schemes were evaluated for realism and the degree of effort required to use them. Realism of the exercise was obtained by collecting the data from fixed INL site bus routes driven thousands of times per year. At the close of the project, we were able to provide a prototype that allowed drivers to see suggested speeds and receive feedback on efficiency of acceleration and deceleration.

A number of technical innovations were demonstrated:

- Addition of on-board intelligence/software architecture

- Communication with the vehicle diagnostic (sensor) system

- Ability to process on- and off-board data (e.g., Global Positioning System data)

- Application of advanced computational intelligence techniques to sensor data

- Abstraction of real-time sensor feedback (e.g., vacuum, speed, fuel use rate, battery status)

- Calculation and update of optimal efficiency for terrain in real time

- Integration of game-style low-user workload GUI

- System promptings for optimal speeds and fuel efficiency on fixed routes

- Utilization of commercially available laptops, tablets, and cabling.

A sample of data logging from our simulation work is presented in Figure 1. 


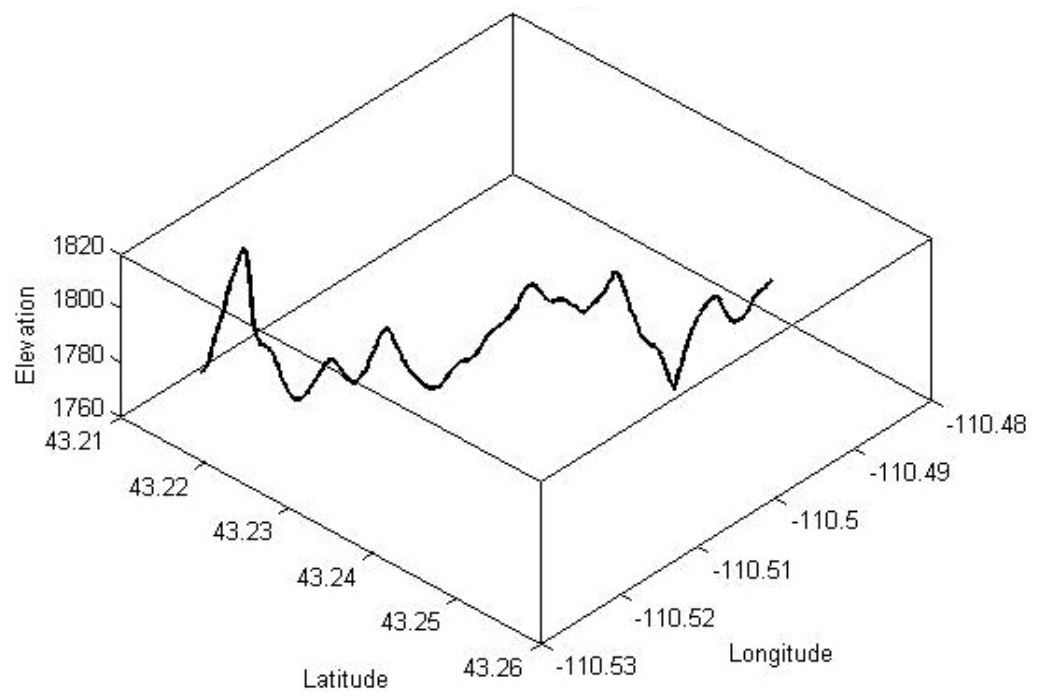

Figure 1. Data logging for INL motor coaches as a function of elevation.

These data are representative of the type of changes in elevation associated with certain route characteristics around the INL site. Gas pedal displacement, another variable constituting part of the model, is presented in Figure 2. The pedal displacement and fuel rate usage have been sampled from a test using an INL motor coach. In support of software development, field experiments were conducted in FY 2013. Pedal position, elevation, and efficiency data were compared for driver prompting and non-prompting conditions.

The driver efficiency system configuration is presented in Figure 3.

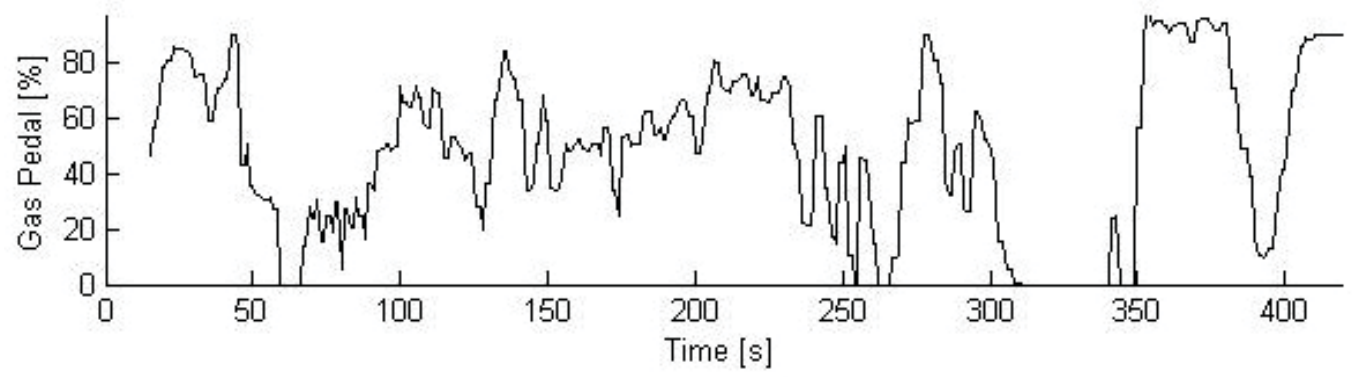

Figure 2. Gas pedal displacement (source: simulated data).

The essential elements of this configuration include the common area network bus (used to extract engine parameter data), the NEXIQ Universal Serial Bus data link hardware and software package (for streaming the engine data), the 5D ICE-Application Programming Interface (that manages real time stream of engine data for comparison with the efficiency model data mined from historical data), and the parser program (that takes the comparison data and uses it as input to the driver GUI display - VBus virtual dashboard). The data acquisition model uses a comma separated value format that allows for large data sets to be developed over time that can be mined for model refinement. 


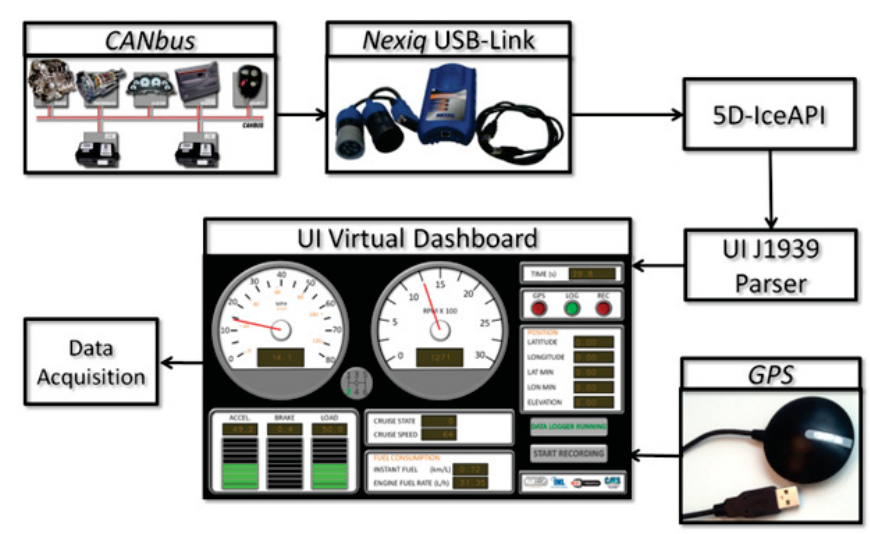

Figure 3. Driver efficiency data collection and presentation system configuration.

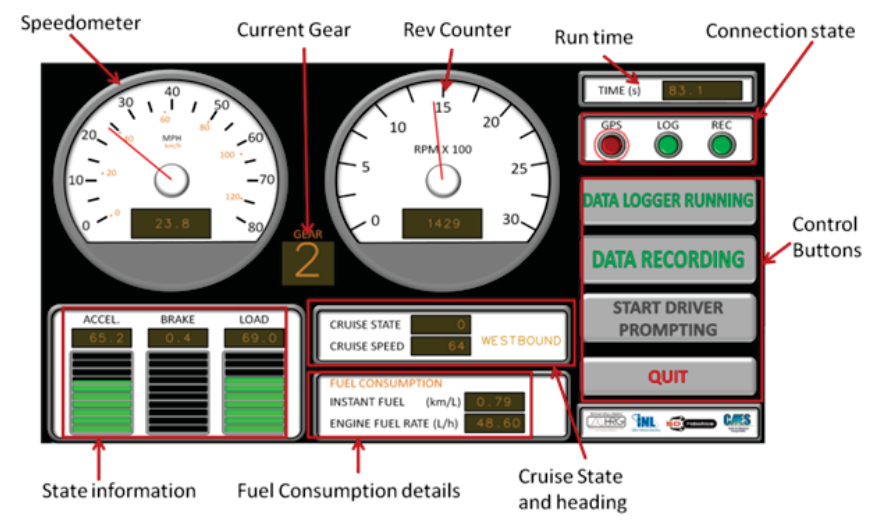

Figure 4. Dashboard GUI for fuel efficiency.

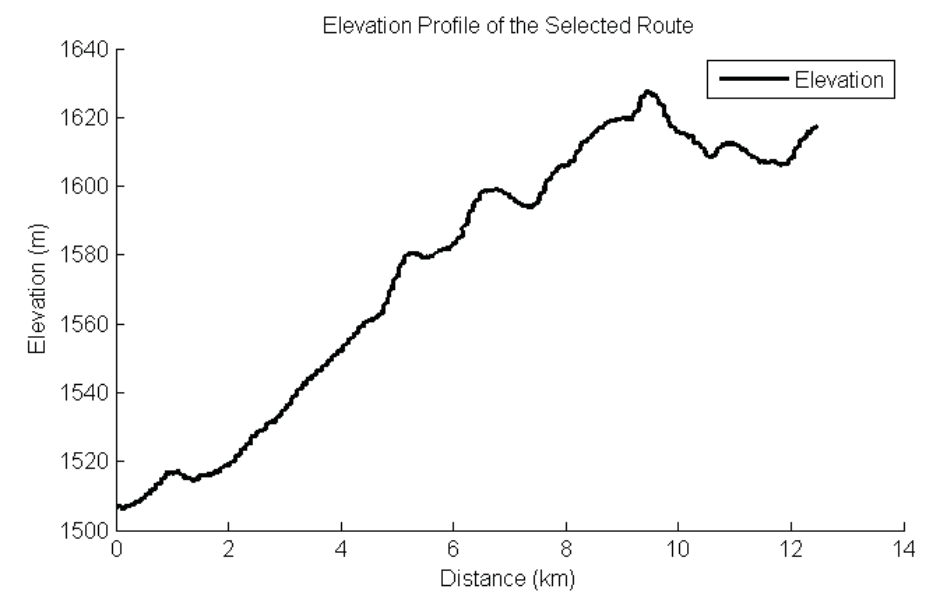

Figure 5. Elevation profile for prototype system data collection.
Figure 4 presents the experimenter's control panel used on the bus to engage the driver efficiency prompting system. Only the speedometer, rev counter, and state information are available to the motor coach driver. Not shown in the diagram is the color coding scheme that appears on the driver speedometer for accelerating or decelerating. When the driver needs to accelerate, a pale blue wedge appears over the speedometer. When the optimal efficiency speed for that Global Positioning System location is reached the wedge disappears. The same is true if the driver is going too slowly. In this case, the pale blue wedge appears below the speedometer needle and disappears when the optimal speed is reached.

Many of the fixed routes at the Site used to take workers to and from town are 50 miles or more. In order to more fully test the advantage of the prototype efficiency system, an elevation profile from the existing INL bus route was selected. The rise in elevation of the course of 10 miles was from 1510 to 1630 feet, enough to present a fair test of the system. Figure 5 presents this test information.

Finally, the prompting screen location presenting optimal speed is shown in Figure 6. 


\section{CENTER FOR ADVANCED ENERGY STUDIES}

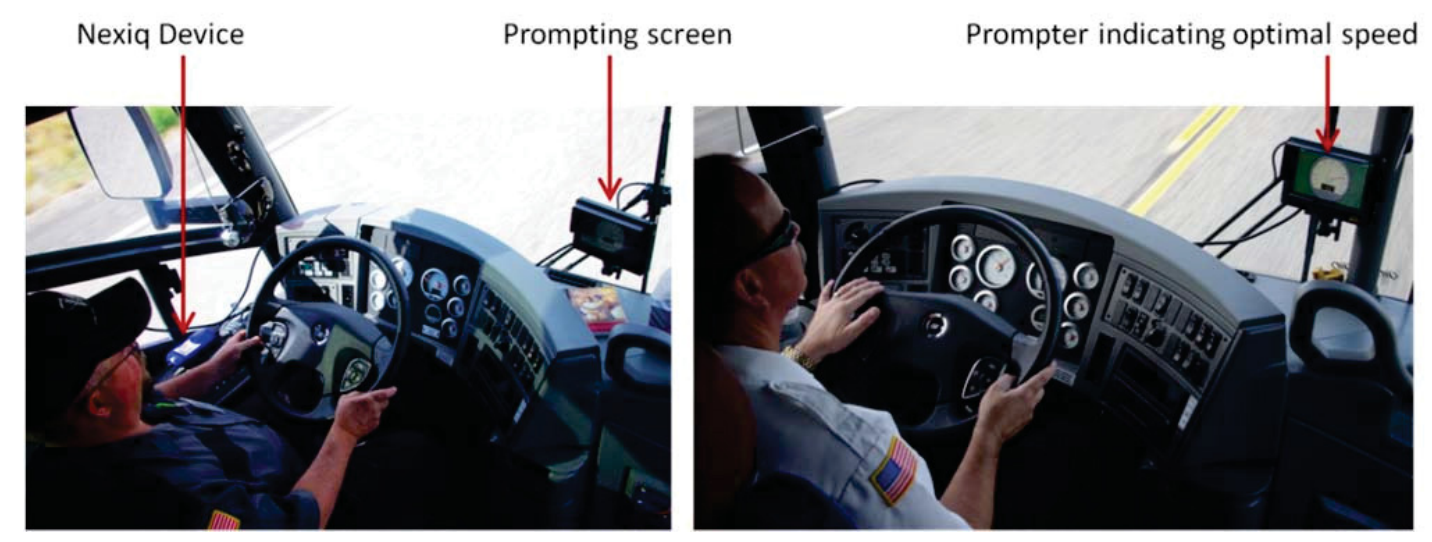

Figure 6. Prompting system installation on an INL Integrated Transportation Services D-series motorcoach (September 2013).

Over three years, this modest project was able to serve as the primary research funding source for five University of Idaho graduate students:

- Ondre Linda, Ph.D. in Computer Sciences (graduated 2012)

- Joel Hewitt, Ph.D. candidate in Computer Sciences

- Dumidu Wijayasekara, Ph.D. candidate in Computer Sciences

- Kasun Amarasinghe, Ph.D. candidate in Computer Sciences

- Jordan Holmberg, M.S. candidate in Human Factors and Neurosciences.

\section{Benefits to DOE}

This LDRD project in fleet fuel efficiency contributes to DOE's mission to "continually develop cost-effective energy-saving solutions that help make our country run better through increased efficiency - promoting better plants, manufacturing processes, and products; more efficient new homes and improved older homes and other solutions to enhance the buildings in which we work, shop, and lead our everyday lives." Results from this project have captured the attention of Motor Coach Industries, the Idaho Department of Transportation, and the DENSO Corporation-suppliers to U.S. General Motors. Funding entities, like the U.S. Department of Transportation and the U.S. Department of Defense would benefit from this technology. Because of its potential to reduce the amount of fuel used, the proof of concept demonstrated in this project is also expected to be of interest to U.S. commercial motor coach fleets.

\section{Presentations}

Linda, O. and M. Manic, "Improving Vehicle Fleet Fuel Economy via Learning Fuel Efficient Driving Behavior," in Proceedings of the 2012 IEEE International Conference on Human System Interaction, IEEE, Perth, Australia, June 6-8, 2012.

Pearson, T. and D. Gertman, "Making Every Driver Your Best Driver: Modifying Behavior to Improve Fuel Efficiency," Federal Fleet, Louisville, KY, June 26-30, 2012. 


\title{
11-066 - Targeted Energy Management Toolset for Comfort and Savings Based on Advanced Computational Intelligence Techniques
}

\author{
Craig Rieger, Milos Manic, ${ }^{1}$ Kevin Van Den Wymelenberg, ${ }^{1}$ and John Gardner ${ }^{2}$
}

Changes in energy management can lead to significant cost reductions. In addition to optimizing existing Energy Management Systems (EMS), this project is working on a resilient and secure toolset to proactively maintain a safe level of operational normalcy in response to anomalies and malicious actions. This toolset will integrate advanced sensor systems with advanced computational intelligence (e.g., neural networks and fuzzy arithmetic) to enable effective energy management while minimizing negative human comfort consequences and promoting improved occupant satisfaction. Preliminary work suggests that energy cost reductions above ten percent are easily achievable. In addition to optimizing existing EMS, the proposed toolset will be both resilient and secure in order to proactively maintain a safe level of operational normalcy in response to anomalies and malicious actions. The main technical advantage of this work is the development of a "bolt-on" toolset that brings advanced computational power to existing EMS in a secure and resilient manner (Figure 1). Novel features include the integration and modeling of human comfort/occupancy and energy usage data to enable building managers to achieve both energy efficiency and optimal human comfort.

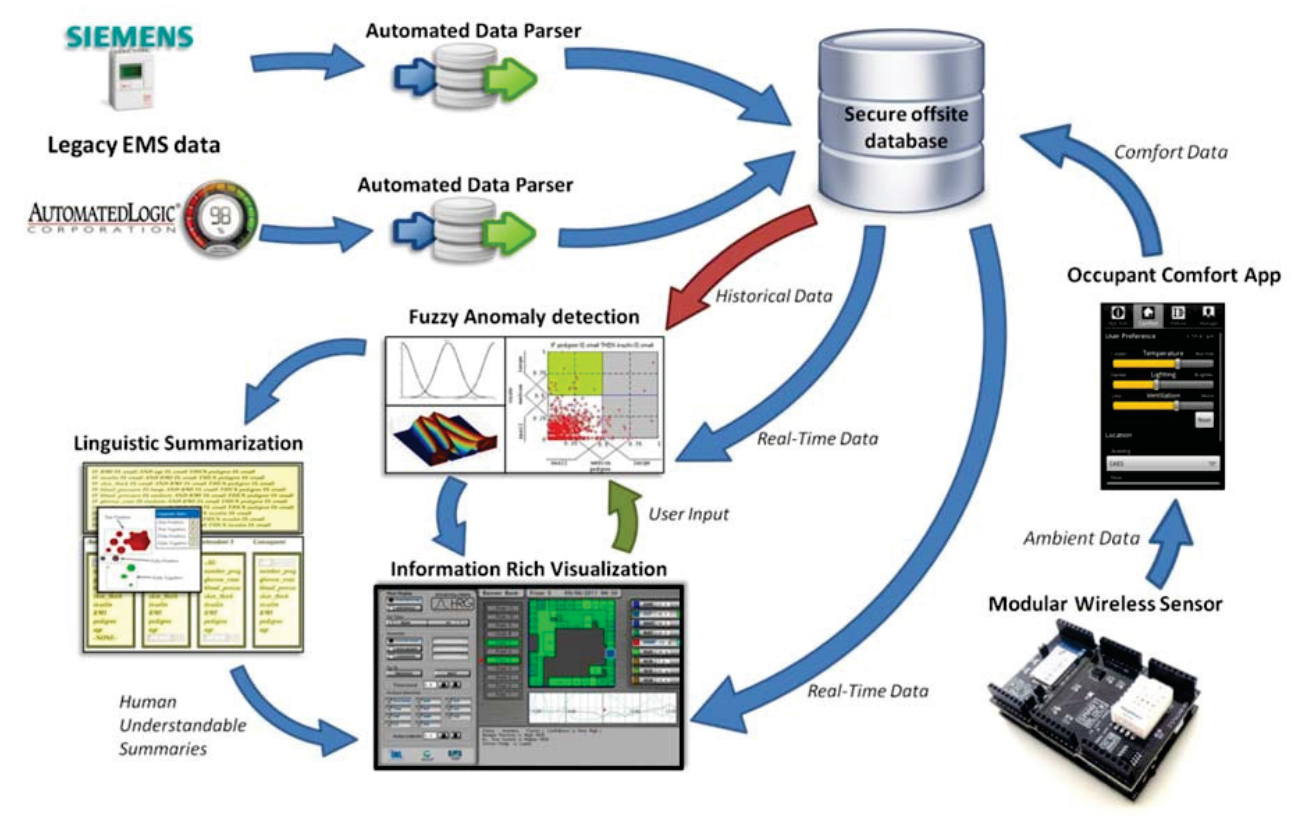

Figure 1. Implemented architecture.

1. University of Idaho

2. Boise State University 


\section{Summary}

Three buildings were selected for evaluation on this project: Banner Bank and the Mobilization and Training Equipment Site facilities in Boise, Idaho, and the Center for Advanced Energy Studies in Idaho Falls, Idaho. INL's Utility Building 4 (UB4) facility will be used for additional testing and evaluation. Within these facilities, individual types of occupancies have been selected and EMS data have been provided by the building managers for evaluation. Supplemental wireless systems were installed and remotely transmit $\mathrm{CO}_{2}$, radiant temperature, air temperature, occupancy, and ambient light. An interview survey was also performed for the building occupants of the Center for Advanced Energy Studies and UB4 to provide the primary mechanism of correlating human comfort needs within occupied spaces.

In addition, a scalable Bluetooth sensor pack that includes temperature, humidity, and ambient light has been created (Figure 2) and updated from a component based system to a surface mount version. This system can be coupled with a human comfort feedback application (Figure 3) - developed for an Android-based smart phone-to record individual perceptions against baseline data.
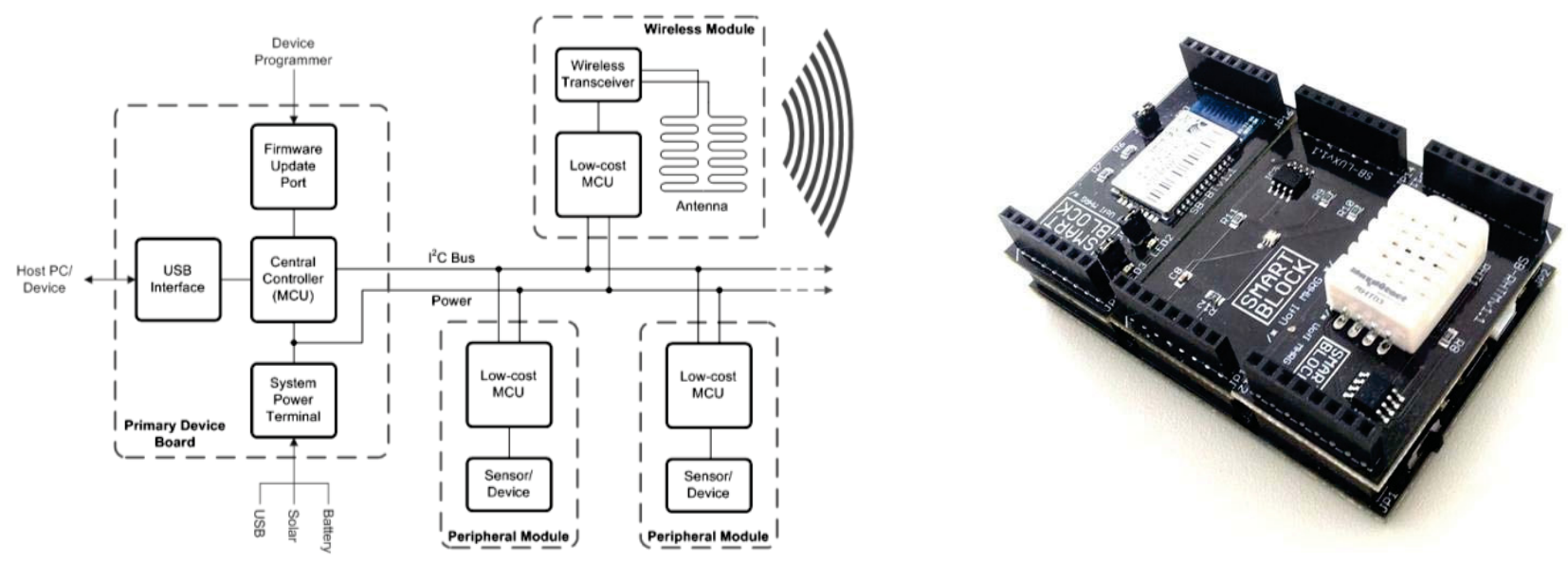

Figure 2. Scalable Bluetooth Sensor Design and Sensor Prototype.
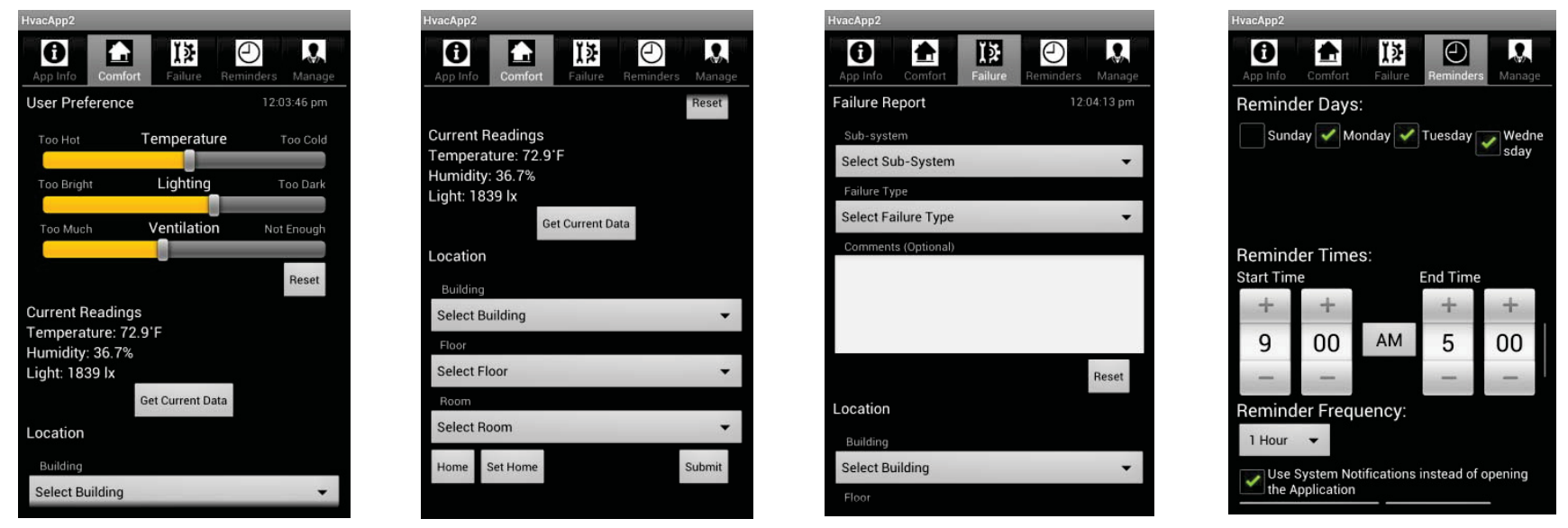

Figure 3. Human Comfort Feedback Application. 
The initial design of the analysis and visualization tool has been developed based on Banner Bank and Mobilization and Training Equipment Site EMS data and is implemented in both web and native android (Figure 4) versions. This has been extended to include UB4 and the Center for Advanced Energy Studies EMS data and supplemental wireless sensors added by the project team. The display provides spatial and temporal representation of individual physical parameters and allows individual thresholds to be established to characterize normal behaviors and flag anomalies. The implementation includes a fuzzy logic design that allows preestablished (comfort, effiiciency, blended), as well as building manager-designed, rule sets to tailor the desired anomalies and patterns in the building for single or multiple physical parameters. A separate evaluation to show connectivity between individual physical parameters within the data was also performed using self-organizing maps which could be integrated within the analysis and visualization tool as desired. Several demonstrations of the tool were performed to building stakeholders as well as potential commerical entities, including Fujitsu Laboratories of America and Idaho Power.

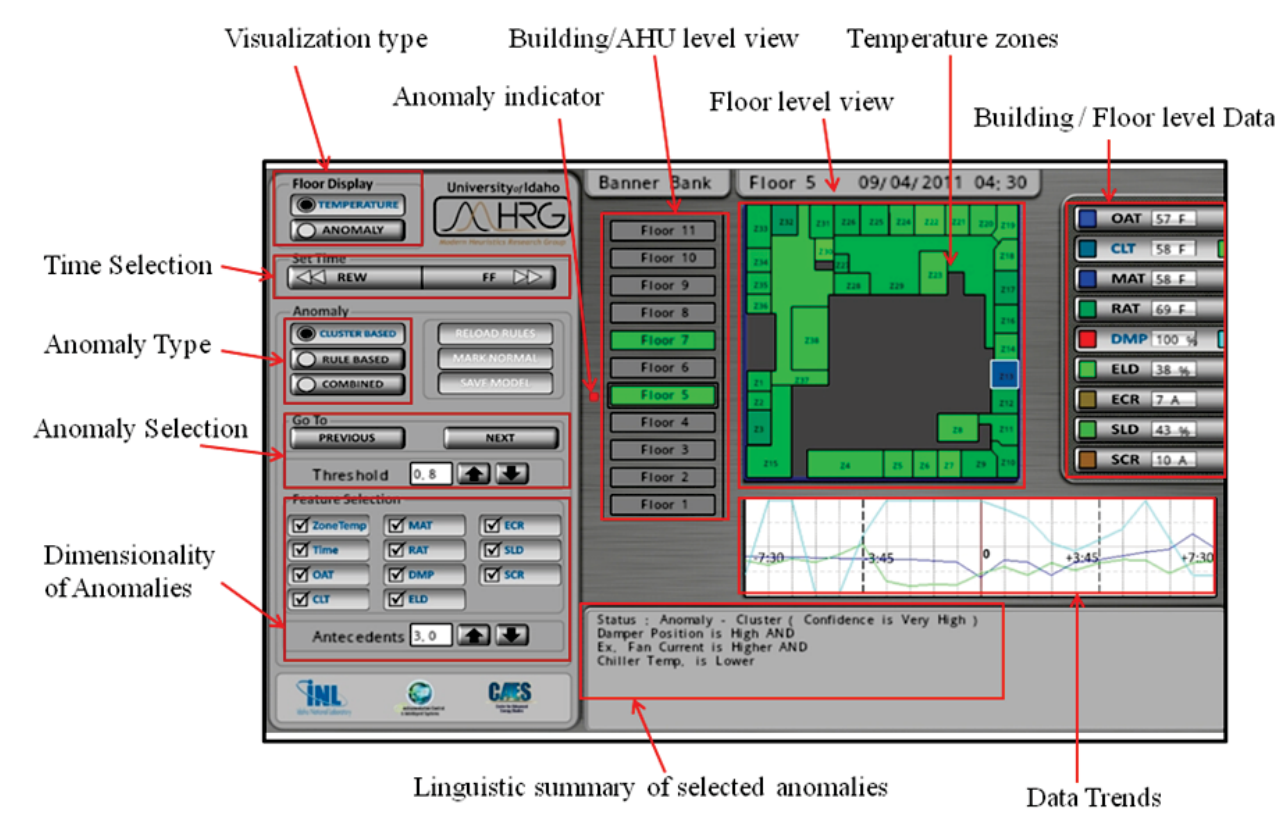

Figure 4. Analysis and visualization tool.

\section{Benefits to DOE}

A key barrier to the widespread adoption of existing energy efficiency technologies is the low return on investment realized by users. The proposed technical advance will contribute to DOE's efforts to reduce dependence on energy imports through energy efficiency by enabling the more effective use of existing EMS via a bolt-on toolkit. Through an innovative and multidisciplinary approach that integrates human comfort principles with advanced real-time visualization and control tools, an actionable information tool will be provided for building managers.

Disciplinary perspectives were brought in from a diverse set of University of Idaho, Boise State University, and INL researchers in mechanical engineering, architecture, computer science, electrical engineering, and human factors. The team benefited from individual research in resilient controls and energy efficiency applied toward a holistic, human-enabled approach to intuitive design of energy efficiency tools. Several graduate students and a 
postdoc have been participating in the development of the toolset, mentoring a new generation of individuals that recognize the value of multidisciplinary involvement in addressing multifaceted issues such as energy efficiency.

\section{Publications}

Duarte, C., K. Van Den Wymelenberg, and C. Rieger, "Revealing Occupancy Patterns in an Office Building through the Use of Occupancy Sensor Data," Energ. Buildings, 2013, Accepted.

Wijayasekara, D., M. Manic, and C. Rieger, "Fuzzy Linguistic Knowledge Based Behavior Extraction for Building Energy Management Systems," $6^{\text {th }}$ International Symposium on Resilient Control Systems, San Francisco, CA, August 13-15, 2013.

Gardner, J., et al., "Understanding Flow of Energy in Buildings Using Modal Analysis Methodology," $7^{\text {th }}$ International Conference on Energy Sustainability \& $11^{\text {th }}$ Fuel Cell Science, Engineering and Technology Conference, Minneapolis, MN, July 14-19, 2013.

Duarte, C., K. Van Den Wymelenberg, and C. Rieger, "Revealing Occupancy Patterns in Office Buildings through the Use of Annual Occupancy Sensor Data," 2013 ASHRAE Annual Conference, Denver, CO, June 22-26, 2013.

Linda, O., et al., "Computational Intelligence Based Anomaly Detection for Building Energy Management Systems," $5^{\text {th }}$ International Symposium on Resilient Control Systems, Salt Lake City, UT, August 14-16, 2012.

Hewlett, J., et al., "WESBES: A Wireless Embedded Sensor for Improving Human Comfort Metrics Using Temporospatially Correlated Data," $5^{\text {th }}$ International Symposium on Resilient Control Systems, Salt Lake City, UT, August 14-16, 2012.

\section{Presentations}

Duarte, C., et al., "Prioritizing and Visualizing Energy Management and Control System Data to Provide Actionable Information for Building Operators," Western Energy Policy Research Conference, Boise, ID, August 25-26, 2012.

Van Den Wymelenberg, K., "Energy Security and Resilient Control Systems," Idaho National Laboratory and the University of Southern California's Information Sciences Institute sponsored, November 15-16 2011. 


\title{
12-086 - Alert/Alarm Dashboard
}

\author{
Ronald Boring, David Gertman, and Brian Dyre ${ }^{1}$
}

While full-scale, high-fidelity control room simulators exist for research on displays and control systems and training of operators, their utility is limited by high demand on technical staff and expensive facilities that greatly increase overhead costs. Research with such simulators also requires a pool of trained operators-whose time is very constrained-to act as research participants. These factors are significant barriers to conducting efficient empirical research on novel display and control systems or providing training that does not necessitate full-scale, high-fidelity simulation. To help solve this problem and others, this research project advances theoretical and applied knowledge with regard to alarm systems at nuclear power plants (NPPs). The focus of the research is to develop first principles of alarm management that will help establish the technical basis for the design of new alarm systems at NPPs. This project covers two steps toward improved alarm systems.

1. Evaluation of alarm management system technologies. INL and the University of Idaho (UI) have reviewed various alarm management system principles and technologies and are working to deploy them on a simulator. In addition, the research team has developed a plan for testing operator performance with these systems.

2. Deployment of a microworld simulator model for NPP process control. A microworld simulator represents a simplified form of the process control system that may be tailored to less skilled operators. For research, more efficient use of limited resources could be realized by using these lower-fidelity "microworld" simulations, allowing DOE researchers and their collaborators to focus on particular display and control issues (e.g., decision making and prioritization of actions in response to alarms) using a low-cost simulation that mimics the perceptual, cognitive, and action processes of real-world process control in a more tightly-constrained microworld environment. An additional advantage of the microworld environments is that ideas for novel displays and controls can be examined in a simplified context that does not necessarily require trained operators to act as participants. Novel display and control concepts can be tested quickly and efficiently using the microworld, and those concepts that show promise can then be further tested using full-scale, high-fidelity simulation with trained operators. In addition, the flexibility of the microworld can provide trainees a view of the internal dynamics of a process control system much more efficiently than using a full-scale, high-fidelity, control room simulation.

\section{Summary}

\section{Alarm Technologies}

INL has worked closely with UI to build a research team focused on alarm research. This included key INL staff engaged in the Nuclear Science and Technology Division as well as faculty and students at UI. This bridging crucially aligns a research university in Idaho with the research needs of industry and puts DOE at the forefront of alarm research.

1. University of Idaho 
The joint INL-UI research team conducted a preliminary study to review two types of alarm strategies: traditional two-state alarms (i.e., those alarms that are either on or off) versus three-state alarm technologies (i.e., those alarms that display an intermediate value between on and off to signify a "possible" alarm). This research addressed how alarm systems can increase operator performance within NPP operations. Sixty university students ( 26 female, 34 male) from UI participated in this initial experiment. Participants ranged from ages 18 to 41. The participants used an early version of the microworld developed for this project to control water level, flow, and temperature of a simple control process. In concurrence with the process control task, participants executed a secondary task in which they performed fault diagnosis by monitoring alarms and indicators. This study found evidence that three-state alarms may assist NPP operators in appropriately allocating attention and thereby may increase the effectiveness and efficiency of plant operations. The implementation of three-state alarms within NPPs may have important practical applications as operators systematically monitor numerous indicators to diagnose critical faults. The effectiveness of plant operations may improve as three-state alarm advisories guide operators more appropriately through emergency operating procedures. By using the advisories to guide them, operators could give more time and attention to those procedures corresponding to faults that have high magnitude and/or probability of occurring. The initial experiment only looked at one element of alarms in a control room setting.

\section{Microworld Development}

A large portion of the research to date has centered on developing a flexible and scalable microworld simulation environment by UI and INL staff. UI was responsible for developing the code, while INL staff provided specifications and technical guidance on the implementation. The development approach is loosely based on previous microworld simulators used for research purposes, such as the University of Toronto's Dual Reservoir System Simulation (DURESS). The DURESS simulator presents operators (or research participants) with a simple process control task wherein the operator must control valves, pumps, and heaters to ensure a given output flow and temperature. System faults can be introduced that require the operators to engage in troubleshooting and fault diagnosis. DURESS was specifically designed to examine how ecological interface displays might aid in these processes with one particular task — controlling the flow rate and temperature of the output water flow. The joint UI-INL microworld simulator could be used similarly, but has been designed to be much more flexible and include components and processes that are of more direct interest to nuclear power control systems.

Two related software tools, a visual Microworld Scenario Editor and a Microworld Scenario Simulator (the player), have been developed for training and research using Oracle's platform-independent Java language. The Editor provides a visual tool in which a variety of system components can be visually created and connected using a drag-and-drop visual interface. Figure 1 shows an example of a simple interconnected set of pumps, pipes, valves, and reservoirs designed in the Microworld Scenario Editor. 


\section{CENTER FOR ADVANCED ENERGY STUDIES}

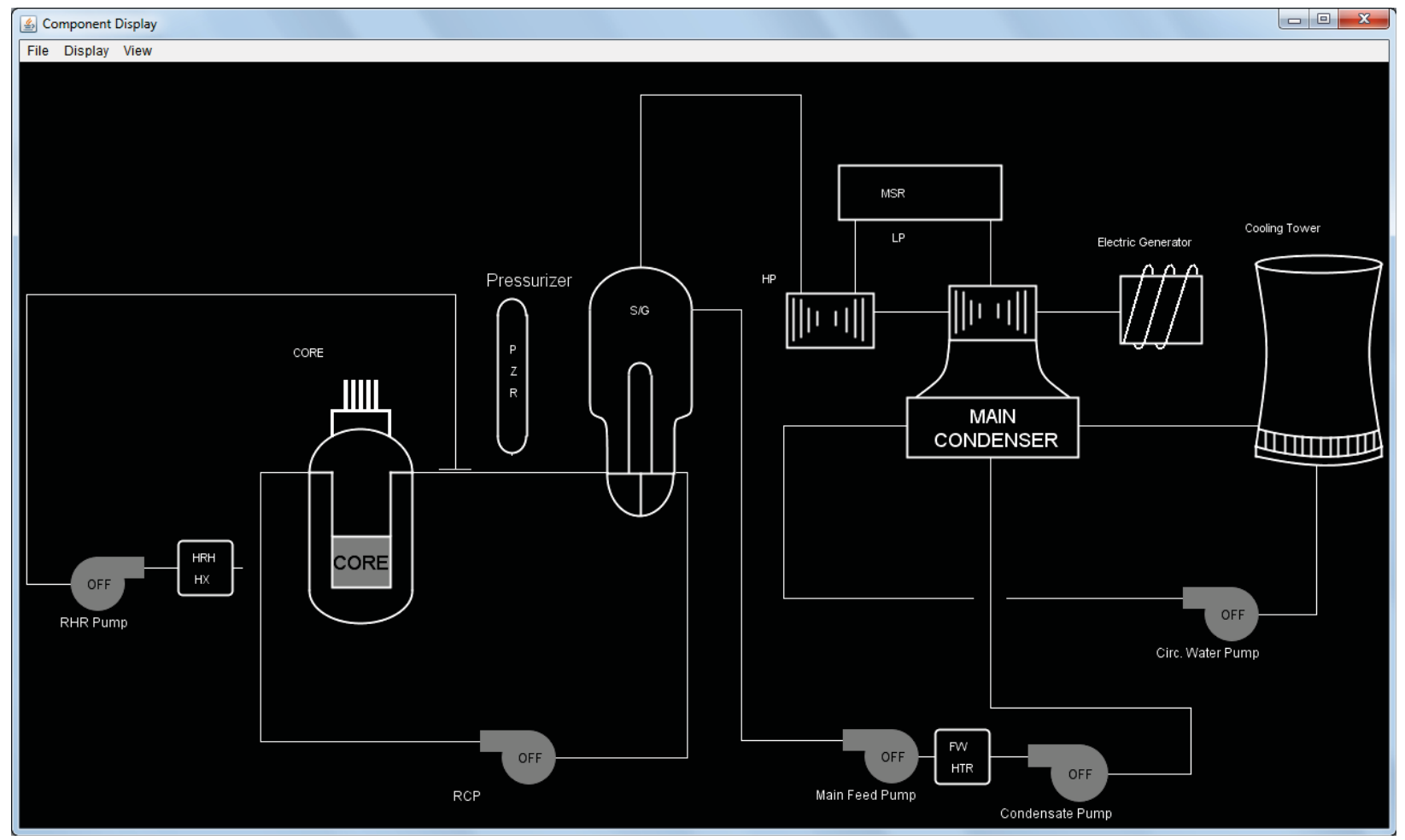

Figure 1. A simple process control microworld shown in the editor window.

Each component is created by selecting it from the menu, then clicking in the display area to place the component. Once placed, the components can be linked, moved, resized, and deleted by right-clicking on them and selecting these commands from a pop-up menu. System components particular to a pressurized water reactor in a NPP are also available as illustrated in Figure 1. While the current objects are customized for nuclear processes, the tool set is flexible and can be used for other process control applications like chemical manufacturing or oil and gas production and distribution. It is also possible to configure the objects to represent a grid, which makes future applications such as smart grids an easy extension.

A configuration editing window can also be opened by selecting the "config" command from the pop-up menu that appears when a component is right-clicked. The component configuration window allows the simulation designer to set parameter values, parameter and variable displays, parameter controls, set-point alarms of various types, and disturbances (e.g., failures such as external repeating disturbances defined by various waveforms). These can be set independently for all components.

The displays for system variables and the displays and controls for component parameters selected in the component configuration window can be visually arranged within their own dedicated display window using a drag-and-drop interface. Tiled alarm panels can be created by turning on the alarms and creating set points in the component configuration window. These alarm tiles are displayed in a specialized alarms window. Functionality currently under development will allow scenario designers the option of generating alarm lists and for custom displays and controls to embed alarm states. 
Simulation designers can use the visual interface to layout system components and configure some of the simpler components (pipes, valves, and reservoirs) with controls, displays, and alarms. NPP-specific components and the disturbance configuration components were refined in FY 2013. Developed scenarios can be saved as Extensible Markup Language-formatted files and reopened. The Microworld Scenario Simulator can read in these scenario files and display the simpler system components in a visually dynamic display (e.g., reservoirs fill and flush, pumps turn on, and alarms change state based on set points being exceeded). Once a simulation scenario has been created it can be played using the Microworld Scenario Simulator. A typical scenario would display the system overview screen, the displays and controls screen, and the alarms screen, which the designer can place on different physical screens depending on the hardware used to run the simulator.

The Scenario Simulator will provide logging of all system component variables, parameters, and user inputs, affording both offline analysis and variable-speed playback of operator interactions with the simulation. We plan to also eventually include the capability for the simulator to interface with software controlling physiological measures such as eye tracking, skin conductance, respiration, heart rate, and electroencophalography. A dedicated data analysis tool could also be added in the future to facilitate data collection.

The project was able to serve as the funding source for four graduate students:

- Austin Ragsdale, M.S. candidate in psychology at University of Idaho

- Jordan Holmberg, M.S. candidate in psychology at University of Idaho

- Roger Lew, Ph.D. candidate in neuroscience at University of Idaho

- Tom Ulrich, Ph.D. candidate in neuroscience at University of Idaho.

\section{Benefits to DOE}

DOE currently uses control rooms to control generation, transmission, and distribution of power sources. Each control room features alarm systems. While the focus of this research is primarily on alarm systems in NPPs, this project is developing a set of alarm research methods that can be used across energy sectors to produce more effective alarms. This research has the opportunity to establish a core competence within DOE in alarm research for energy applications. It provides a pipeline of competent student researchers from the university setting who can support such research needs in the future.

While INL maintains a full-scope reconfigurable simulator for human factors studies of operators, in practice, this facility has proven costly to set up for research. The present research develops and validates a processequivalent simulator at UI that can be used at INL for first principles research. The availability of a processequivalent microworld simulation significantly increases the frequency and variety of empirical human factors studies that INL can complete.

\section{Publications}

Ragsdale, A., R. Lew, and R. Boring, "Differences in Trust between Two Types of Alarms and Events within a Nuclear Power Plant Control Simulation," Human Performance in Extreme Environments, In press. 
Dyre, B.P., et al., "A Microworld Simulator for Process Control Research and Training," Proceedings of the Human Factors and Ergonomics Society $57^{\text {th }}$ Annual Meeting, San Diego, CA, September 30-October 4, 2013.

Ragsdale, A., et al., "Fault Diagnosis with Multi-state Alarms in a Nuclear Power Control Simulator," Proceedings of the Human Factors and Ergonomics Society $56^{\text {th }}$ Annual Meeting, Boston, MA, October 22-26, 2012.

Boring, R.L. and J.J. Persensky, "Hybrid Alarm Systems: Combining Spatial Alarms and Alarm Lists for Optimized Control Room Operation," $8^{\text {th }}$ International Topical Meeting on Nuclear Power Plant Instrumentation, Control, and Human-Machine Interface Technologies, San Diego, CA, July 22-26, 2012.

Ragsdale, A., et al., "Alarm Strategy and Complexity: Predictions of Operator Response," $8^{\text {th }}$ International Topical Meeting on Nuclear Power Plant Instrumentation, Control, and Human-Machine Interface Technologies, San Diego, CA, July 22-26, 2012.

Boring, R., et al., "Microworlds, Simulators, and Simulation: Framework for a Benchmark of Human Reliability Data Sources," Joint Probabilistic Safety Assessment and Management and European Safety and Reliability Conference, Scandic Marina Congress Center, Helsinki, Finland, June 25-29, 2012.

Ragsdale, A., Nuclear Power Plant Operator Performance When Systems Vary in Alarm Type, Alarm Accuracy, and Information Type, M.S. Thesis, University of Idaho, 2012.

\section{Presentations}

Dyre, B.P., et al., "A Microworld Simulator for Process Control Research and Training," Presented at the Human Factors and Ergonomics Society $57^{\text {th }}$ Annual Meeting, San Diego, CA, September 30-October 4, 2013.

Ragsdale, A., R. Lew, and R. Boring, "Differences in Trust between Two Types of Alarms and Events within a Nuclear Power Plant Control Simulation," Presented at Resilience Week, San Francisco, CA, August 13-15, 2013.

Ragsdale, A., et al., "Fault Diagnosis with Multi-state Alarms in a Nuclear Power Control Simulator," Presented at the Human Factors and Ergonomics Society $56^{\text {th }}$ Annual Meeting, Boston, MA, October 22-26, 2012.

Boring, R.L. and J.J. Persensky, "Hybrid Alarm Systems: Combining Spatial Alarms and Alarm Lists for Optimized Control Room Operation," Presented at $8^{\text {th }}$ International Topical Meeting on Nuclear Power Plant Instrumentation, Control, and Human-Machine Interface Technologies, San Diego, CA, July 22-26, 2012.

Ragsdale, A., et al., "Alarm Strategy and Complexity: Predictions of Operators Response," Presented at $8^{\text {th }}$ International Topical Meeting on Nuclear Power Plant Instrumentation, Control, and Human-Machine Interface Technologies, San Diego, CA, July 22-26, 2012.

Boring, R., et al., "Microworlds, Simulators, and Simulation: Framework for a Benchmark of Human Reliability Data Sources," Presented at Joint Probabilistic Safety Assessment and Management and European Safety and Reliability Conference, Helsinki, Finland, June 25-29, 2012. 


\title{
13-011 - Integrated Approach to Algal Biofuel, Bio-power, and Agricultural Waste Management
}

\author{
Deborah Newby, Erik Coats, ${ }^{1}$ Kevin Feris, ${ }^{2}$ and Armando McDonald ${ }^{1}$
}

Over nine million dairy cows in the U.S. generate $>226$ billion $\mathrm{kg}$ of wet manure and produce $>5.8$ billion $\mathrm{kg}$ of $\mathrm{CO}_{2}$ equivalents annually. In the anaerobic digestion (AD) process, animal manure is treated by natural bacteria in the absence of oxygen to produce methane-rich biogas that can be captured and used for the production of electricity or heat. Integrated with $A D$, residuals (upstream/downstream) can be separated and used for various purposes, including fertilizer, animal bedding, polyhydroxyalkanoates (PHAs, which are biological, biodegradable thermoplastics with significant commercial value), and algae cultivation, as shown in the process flow diagram below (Figure 1).

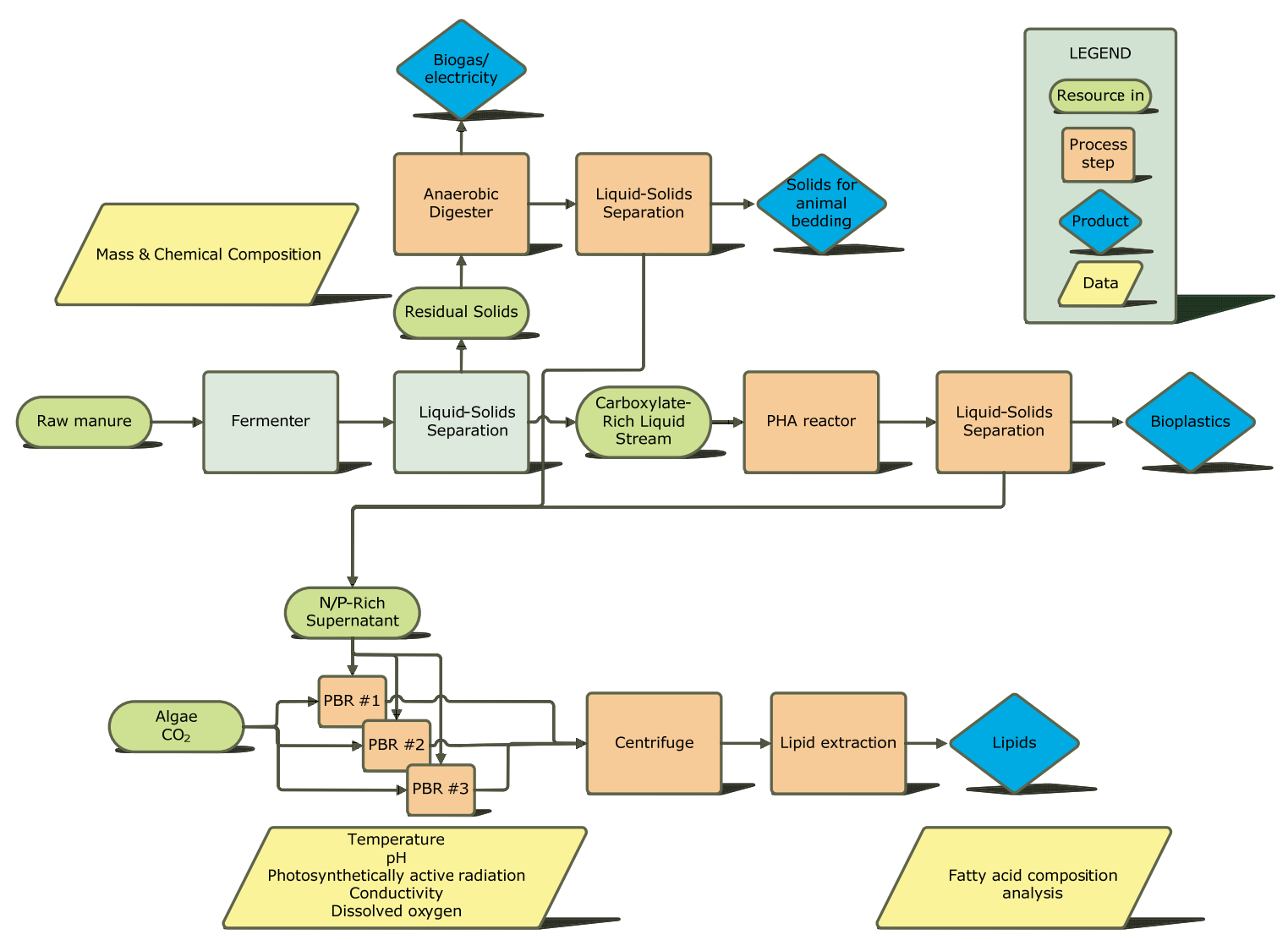

Figure 1. Process flow diagram for the integrated AD-Algal operations.

While $A D$ is a mature technology, its growth is stunted due to poor economics and the need to further mitigate release of emissions (e.g., nitrogen $[\mathrm{N}]$, phosphorus $[\mathrm{P}], \mathrm{CO}_{2}$ ) to the environment. While these nutrients are problematic for the dairy industry, they are of significant value for large-scale cultivation of algal biomass for 
biofuels. The goal of this project is to build upon and scale up prior work that has established the effectiveness of deploying a novel two-stage AD system coupled with an open algal photobioreactor to produce multiple valueadded commodities (bio-power, biofuels, PHA, etc.). This research differs from a conventional algal biofuel approach in that we incorporate a strategy to minimize costly nutrient import and reduce water use by connecting algal production to dairy wastewater treatment. Outcomes of this work will simultaneously enhance AD-Algal economics while producing valuable end-products and reducing nutrient emissions from animal waste management systems. Coupling the generation of additional product streams to an established industry that demands enhanced waste/emissions management will facilitate enhanced AD-Algae biofuel adoption by addressing key barriers, as well as enhancing commercial viability, and deployment.

Specific project tasks are as follows:

Task 1a Design, construct, deploy, and test a scaled up algal production system.

Task 1b Quantify algal biomass production and characterize lipid/biomass quality and nutrient levels.

Task 1c Identify and address how the quality of PHA and AD effluent influences algal production.

Task 2a Assess the influence of spatial-temporal variability of algal community structure on algal biomass quality.

Task $2 \mathrm{~b} \quad$ Assess the viability of residual algal biomass as a fermenter or AD substrate to improve PHA and/or methane production.

Task 3 Produce the next generation of bio-product innovators and system operators by integrating undergraduate and graduate training and work force development via collaborative student training between INL and university collaborators.

\section{Summary}

Significant progress has been made on Tasks 1 and 2 during year one. Three 100L capacity algal cultivation systems have been constructed in the Boise State greenhouses. Initial control experiments, development of continuous operation and maintenance protocols, and monitoring systems for process chemistry are being established, and 30-day experimental trials for continuous operation are being initiated (Task 1a). Algal biomass characterization is underway (Task $1 \mathrm{~b}$ ). PHA and AD reactors, supplied with dairy manure-based substrate, are being continuously operated in Dr. Coats' lab to provide Dr. Feris with PHA and AD effluent for his algal production investigations. The investigations are also being used to establish baseline operations as a future contrast with algal-augmented operations (Task 1c). Investigations into photochemical modification of AD and PHA wastewater have progressed well, and a draft manuscript has been prepared, as well as an intellectual property disclosure submitted (Task 1c). Work during year two will begin to apply these findings to the larger scale, continuously operated photobioreactors (Tasks 1 and 2). Characterizations of photobioreactor spatial heterogeneity have been initiated and will be continued during year two (Task 2a). Baseline data is being collected on the performance of fermentation, AD, and PHA production systems in preparation for ultimate augmentation with algal biomass provided by Dr. Feris (Task $2 b$ ). A fermentation potential test (Task $2 b$ ) has been completed as an initial assessment toward enhancing volatile fatty acid production with a combined algal- 
manure matrix. Data analysis is ongoing, which will lead to a more comprehensive experimental design. One student has graduated with a master's in biological and agricultural engineering from the University of Idaho (Maxine Prior, July 2013), and one new master's student has been recruited to Boise State University (Jeric Harper, started June 2013) (Task 3). One master's graduate student (Ed Stowe), who started in January 2013, is performing fermentation/AD studies with Dr. Coats (shown in Figure 2).

\section{Benefits to DOE}

Algal biomass has significant potential as a biofuel feedstock, and could displace fossil fuels, thereby reducing U.S. dependence on foreign oil. The displacement of fossil fuels with biofuels generated using algae reduces overall fossil fuel emissions. Animal waste is a large source of greenhouse gas emissions and produces excess nutrients $(\mathrm{N}, \mathrm{P})$ that can impair the environment. This research will transform dairy waste from a problem into a resource that can be used to produce electricity and provide the nutrients for algal production. AD of all the waste from U.S. dairy cows would generate enough electricity to power $\sim 1.3$ million homes per year; comparatively, a $500 \mathrm{MW}$ coal-fired power plant (typical size) can power $\sim 0.36$ million homes per year.

This research addresses barriers to wider-scale $A D$ deployment, which is presently under-used due to un-optimized systems. New knowledge on a more

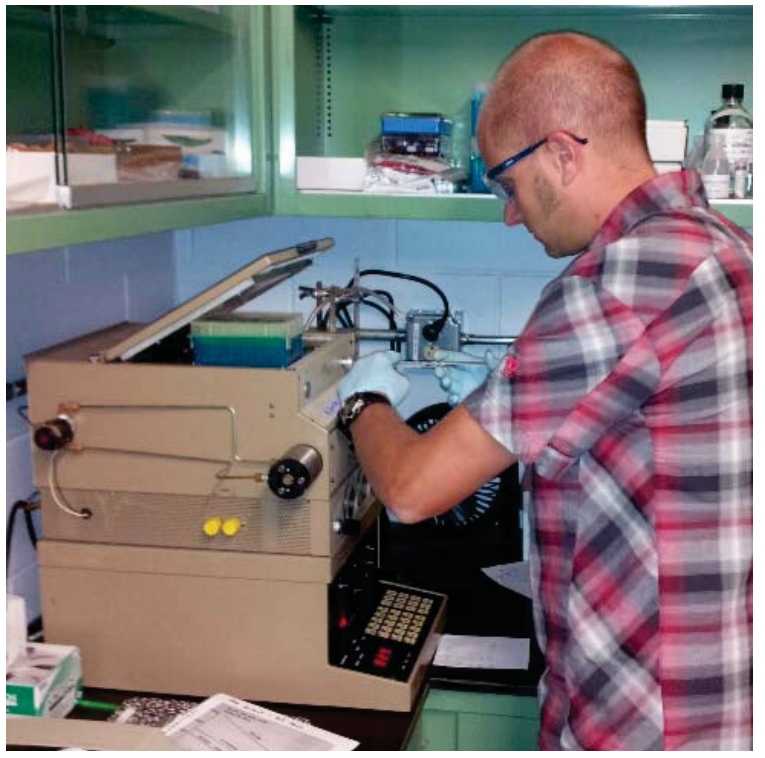

Figure 2. Ed Stowe, University of Idaho graduate student (a) analyzing biogas on gas chromatograph/thermal conductivity and (b) processing samples from a manure AD/fermenter system.

comprehensive and integrated waste-to-fuels process is needed by the dairy/waste processing industry to improve our understanding of biological processes for processing organic matter. Improving the economics of algal systems by using waste streams as a nutrient source could also advance algal technologies, thereby making broader implementation of commercial scale algal cultivation for fuels more economically feasible. Further, this effort will provide the basis to build strong industrial partnerships by taking this technology from bench-scale to the demonstration level. By integrating education and work force development, skilled bio-product innovators and future system operators will be trained.

\section{Publications}

Prior, M., et al., "Algal Productivity in Dairy Manure Wastewaters: Polyhydroxyalkanote Effluent Exceeds Anaerobic Digester Effluent as an Algal Cultivation Medium," Algal Research, In preparation.

Prior, M., et al., "Ultraviolet Radiation Pre-treatment Modified Dairy Wastewater, Improving its Utility as a Medium for Algal Cultivation," Algal Research, In preparation. 


\section{Presentations}

Coats, E., and E. Stowe, "Investigating a Novel 3-Stage Anaerobic Digestion System to Enhance Resource Recovery from Residual Organic Matter," Water Environment Federation Biosolids and Residuals Conference, Austin, TX, March 2014, Submitted.

Prior, M., et al., "Ultraviolet Radiation Pre-treatment Modifies Dairy Wastewater, Improving Its Utility as a Medium for Algal Cultivation," $3^{\text {rd }}$ International Conference on Algal Biomass, Biofuels and Bioproducts, Toronto, Canada, June 16-19, 2013.

Coats, E., "Integrated 2-Stage Anaerobic Digestion to Reduce Dairy GHG Emissions," $7^{\text {th }}$ Annual EPA AgStar Conference, Indianapolis, IN, June 11, 2013.

Feris, K., "Nutrient Sequestration Using Algae with AD Systems," $7^{\text {th }}$ Annual EPA AgStar Conference, Indianapolis, IN, June 11, 2013. 


\title{
13-033 - Magnetic Separation Nanotechnology for Spent Nuclear Fuel Recycle
}

\author{
Leigh Martin, You Qiang, ${ }^{1}$ and Yaqaio $\mathrm{Wu}^{2}$
}

Due to their unique properties, nanoparticles are central to a variety of exciting applications. The goal of this project is to understand the usefulness of functionalized magnetic nanoparticles (MNPs) in used nuclear fuel separations, in particular minor actinide $\left(\mathrm{An}^{3+}\right) /$ lanthanide $\left(\mathrm{Ln}^{3+}\right)$ separations. The project will characterize the physical and chemical properties of the ligand-functionalized MNPs (size, shape, aggregation, magnetization, etc.) using electron microscopy, transmission electron microscopy (TEM), vibrating sample magnetometer, Localized Electron Atom Probe, and simple solid liquid partitioning experiments. In later years of this project, the radiation stability of these separations reagents will be investigated.

\section{Summary}

The University of Idaho collaborators have focused on two areas of research in FY 2013: (1) Sorption/Stripping Cycles, and (2) computational fluid dynamics (CFD) simulations of the magnetic separations device. As the University cannot use radioactive materials, they have investigated cadmium (Cd) as a surrogate for the "soft" actinide elements. One of the most important features for used fuel separations technologies is the reusability of the reagents utilized in the process. As such, these sorption/stripping cycles are important to demonstrating reagent reusability. Using $\mathrm{HNO}_{3}$ and $\mathrm{HCl}$, stripping kinetics tests for the MNP's functionalized with diethylenetriaminepentaascetic acid (DTPA) were initially conducted, and $99 \%$ of the Cd could be recovered in five minutes or less of contact time. By adding an additional stripping step, all of the Cd could be recovered from the MNPs. Once it had been identified that stripping would occur, sorption/stripping cycle tests were performed. For each cycle, $10 \mathrm{mg}$ of DTPA-MNP were contacted with $10 \mathrm{~mL}$ of $4 \mathrm{ppm} \mathrm{Cd}$ solution at $\mathrm{pH} 5$ for 1 hour at $25^{\circ} \mathrm{C}$. After sorption, the supernatant was removed for $\mathrm{Cd}$ analysis to calculate the sorption efficiency, while the DTPAMNP went to the stripping process and was then reused for another sorption cycle. It was found that $\sim 90 \%$ of $\mathrm{Cd}$ was recovered by the first stripping step. Overall, $\sim 98 \%$ of $\mathrm{Cd}$ was recovered by stripping twice with $0.1 \mathrm{M} \mathrm{HNO}_{3}$ and $\sim 93 \%$ by $1 \mathrm{M} \mathrm{HCl}$. The differences between the $\mathrm{HNO}_{3}$ stripping and $\mathrm{HCl}$ stripping occurred in the second stripping. In addition, no decrease in sorption efficiency was observed during five sorption cycles. Instead, the sorption efficiency increased significantly from $90 \%$ in cycle 1 to $100 \%$ in cycle 2 and remained in a high level in the following cycles. It was hypothesized that the increase in sorption efficiency was caused by the acid activation of the ion binding sites during the stripping process.

In order to get a better understanding of the magnetic separation process and the interaction between magnetic, hydrodynamic, gravitational, and inter-particle forces, we are developing a mathematical model using commercial CFD software, Fluent, to simulate the separation behavior of the magnetic particles in a magnetic field. A 2-D, two-phase, Eulerian model has been developed to predict the flow profile as well as the concentration profile of the magnetic particles in the separator. The Navier-Stokes equations are used to predict the flow profile for the liquid phase. A preliminary user defined function based on these equations has been developed for the source term for the momentum balance equation of magnetic particles to account for the force due to the magnetic field.

1. University of Idaho

2. Boise State University 
The CFD simulation has the same magnetic configuration and boundary conditions with the magnetic separation experiment setup (see inset in Figure 1). CFD results will be compared with experimental data as shown in Figure 1.

Collaborators at Boise State University have used facilities at the CAES building to characterize the MNPs using TEM. Nano-sized magnetic powders of core-shell Fe/Fe-oxide (sample No. 1) and Feoxide (sample No. 2) were characterized by using the TEM technique. Figure 2a is a bright-field TEM image of sample No. 1 taken at a lower magnification that contains many particles. This image reveals that the size of the powder particles varies from less than $10 \mathrm{~nm}$ to about $50 \mathrm{~nm}$. Particles over $10 \mathrm{~nm}$ clearly show a core-shell structure, as seen in high-resolution TEM (HRTEM) images in Figure 2b. In the $20 \mathrm{~nm}$ particle, the Fe-oxide shell is about $5 \mathrm{~nm}$ thick and the Fe core is about $10 \mathrm{~nm}$ in diameter.

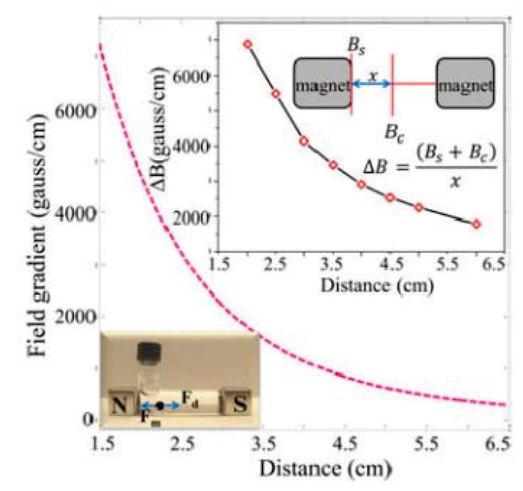

Figure 1. The calculated and measured (inset) magnetic field gradient $(\triangle B)$ along the symmetry axis of a cubic $N d-B$ magnet as a function of the distance from the magnet pole face (inset). Lattice fringes can be clearly seen in these HRTEM images. The same sample was also investigated under the scanning TEM mode with high angle annular dark field detector (Zcontrast) and energy dispersive $x$-ray spectrometry (EDS) techniques. The results further confirm that the powder particles have a core-shell Fe/Fe-oxide structure.

The size of the Fe-oxide (sample No. 2) powder particles varies from less than $10 \mathrm{~nm}$ to about $30 \mathrm{~nm}$ (Figure 2b). HRTEM images (Figure 2c) of several particles show continuous lattice fringes inside these particles, which indicates a one phase particle. Scanning TEM High Angle Annular Dark Field Z-contrast imaging and EDS analysis confirm the TEM results.

a.

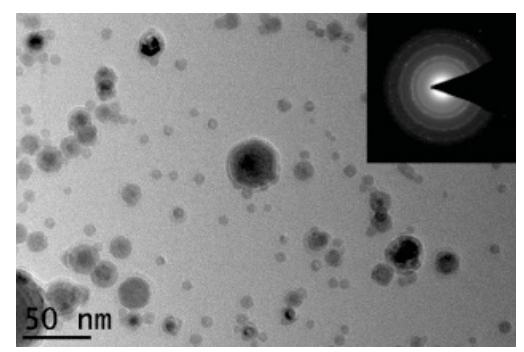

b.

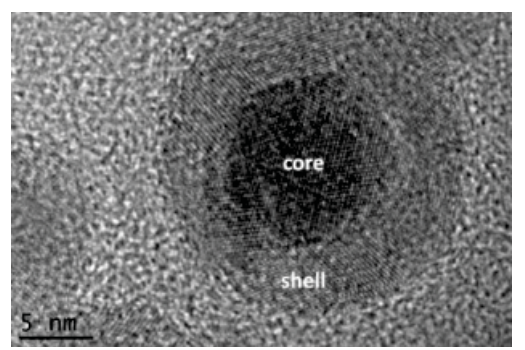

c.

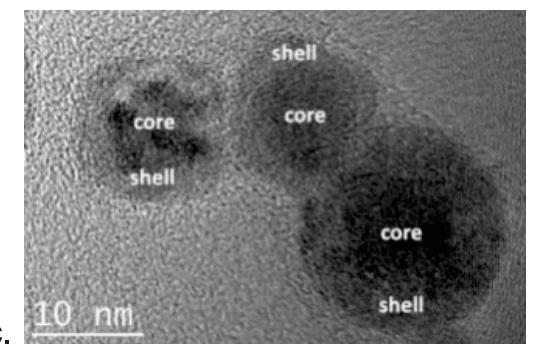

Figures 2(a-c). Bright-field TEM image (2a) and HRTEM images (2b-2c) showing fine structure of the core shell Fe/Fe-oxide (sample No. 1) particles.

At INL, spectroscopic studies were initiated to investigate the sorption and recovery efficiency of selected lanthanide elements to the DTPA-MNPs. Ultraviolet-visible and fluorescence spectroscopies were employed for this purpose; these techniques allowed the researchers to identify if the lanthanide elements could be removed from solution at macro scale. Using these techniques, both sorption and recovery of the lanthanides were successfully demonstrated, leading to a significantly cheaper technique than inductively coupled plasma-mass spectroscopy to study these processes. However, though we have not been able to use these techniques to obtain spectra of the metal ions bound to the MNPs, this would allow researchers to identify the coordination environment of the metal ions, which may be important for future development of more effective ligands to bind the metal ions from a solution. In addition to these studies, radiotracer experiments have been conducted to study 
the sorption of $\mathrm{Am}^{3+}$ and $\mathrm{Eu}^{3+}$ when both are present in the same solution. Distribution coefficients $\left(\mathrm{K}_{d} \mathrm{~s}\right)$ have been calculated for each of these elements and separation factors have been determined. The calculated $\mathrm{K}_{d}$ values show that $\mathrm{Am}^{3+}$ binds more strongly than $\mathrm{Eu}^{3+}$; however, the separation factors are not yet high enough for an efficient separation (Table 1).

Table 1. $\mathrm{Am}^{3+} / \mathrm{Eu}^{3+}$ separation factor through the course of a 90 minute experiment.

\begin{tabular}{|c|c|c|c|c|c|c|c|c|c|c|}
\hline Time/min & 0 & 10 & 20 & 30 & 40 & 51 & 61 & 71 & 81 & 91 \\
\hline $\begin{array}{l}\text { Separation factor: } \\
\left({ }^{241} \mathrm{Am} \mathrm{K}_{\mathrm{d}}{ }^{154} \mathrm{Eu} \mathrm{K}_{\mathrm{d}}\right)\end{array}$ & 0 & 1.31 & 1.3 & 1.27 & 1.27 & 1.33 & 1.22 & 1.31 & 1.32 & 1.27 \\
\hline
\end{tabular}

\section{Benefits to DOE}

The study of MNPs for minor actinide/lanthanide separations is providing otherwise unavailable data on the longterm performance of these materials in separations applications. This work is contributing towards the design of an advanced fuel cycle that is proliferation resistant, improving DOE's nuclear security mission.

\section{Publications}

Kaur, M., et al., "Conjugates of Magnetic Nanoparticle - Actinide Specific Chelator for Radioactive Waste Separation," Envir. Sci. Tech., 2013, 47 (21), pp. 11942-11959.

Kaur, M., H. Zhang, and Y. Qiang, "Magnetic Separation Dynamics of Colloidal Magnetic Nanoparticles," Magnetics Letters, IEEE, Vol. 4, August 19, 2013, doi:10.1109/LMAG.2013.2271744.

Kaur, M., et al., "Separation Nanotechnology of Diethylenetriamine-pentaacetic Acid Bonded Magnetic Nanoparticles for Spent Nuclear Fuel," Nano Energy, Vol. 2, 124-132, January 2013.

\section{Presentations}

Kaur, M., et al., "Characterization of Magnetic Fe-Fe Oxide Core-Shell Nanoparticles," TMS 2014, San Diego, CA, February 16-20, 2014, Accepted.

Qiang, Y., et al., "Magnetic Separation - Advanced Nanotechnology for Future Nuclear Fuel Recycle," American Nuclear Society Global 2013, Salt Lake City, UT, September 29-October 3, 2013.

Zhang, H., M. Kaur, and Y. Qiang, "Magnetic Nanosorbents for Fast Separation of Radioactive Waste," American Nuclear Society Global 2013, Salt Lake City, UT, September 29-October 3, 2013. 


\section{3-035 - Development of New Molten Salt Sensor Technology for Application to Safeguarding Pyroprocessing}

Ken Bateman, Michael Simpson, ${ }^{1}$ Kerry Allahar, ${ }^{2}$ Darryl Butt, ${ }^{2}$ Supathorn Phongikaroon, ${ }^{3}$ Robert Hoover, ${ }^{3}$ and Michael Shaltry ${ }^{3}$

There is an increasing need for DOE to safeguard its pyroprocessing facilities. Current state-of-the-art sensors are insufficient to provide real-time process monitoring of electrorefiners suitable to prevent diversion of fissile nuclear materials. This project involves the experimental investigation of various sensor technologies for application in monitoring the state of molten salt used for electrorefining in a pyroprocessing operation. In the first year, focus has been on preliminary investigation of electrical impedance spectroscopy (EIS) along with a survey of different spectroscopic methods that may be particularly useful for this application.

\section{Summary}

EIS has been identified as a promising method to yield real-time molten salt composition information, including identification of salt species and quantitative determination of their concentrations. The efforts for the first year included EIS experimentation on several systems and the analysis of EIS data associated with data sets obtained by students and research staff from the group of Professor Phongikaroon of the University of Idaho. The efforts of the first year provided valuable insights into the experimentation and analysis which would be used in the future work.

Initial EIS experiments of the project were conducted on the $\mathrm{LaCl}_{3}$ system at $500^{\circ} \mathrm{C}$ together with cyclic voltammetry in December 2012. EIS and cyclic voltammetry experiments were also conducted on $\mathrm{UCl}_{3}$ and $\mathrm{ZrCl}_{4}$ systems by graduate students of Professor Phongikaroon at CAES in the radiochemistry laboratory. The results of these three systems were analyzed, and the findings were presented at the TMS 2013 meeting in San Antonio in March 2013. The analysis involved using a modeling technique to quantify the spectra (a signature) that is associated with a system at a given applied potential bias. The bias potential was used to promote a reduction reaction such that the spectrum was associated with that particular reduction reaction. The signature of an EIS response was given by a series of time constants and resistances. The analysis yielded different time constants for the different reactions, namely $\mathrm{U}^{3+} /$ uranium (U), $\mathrm{La}^{3+} /$ lanthanum (La), and $\mathrm{Zr}^{4+} / \mathrm{Zr}^{2+}$, demonstrating that differentiation of species by their EIS response was possible. Quantification of EIS data for the $\mathrm{ZrCl}_{4}$ system with different concentrations of $\mathrm{ZrCl}_{4}$ indicated that the resistance values were sensitive to this variable. These results demonstrated the feasibility of the EIS analysis for characterizing $\mathrm{LiCl}-\mathrm{KCl}$ systems with one added salt.

EIS experiments were conducted in May and June of 2013 that involved the $\mathrm{LaCl}_{3}$ system at three concentrations. Also investigated was the $\mathrm{LaCl}_{3}-\mathrm{CeCl}_{3}$ system at three concentrations of $\mathrm{CeCl}_{3}$. Compositional changes in the $\mathrm{LaCl}_{3}$ and $\mathrm{LaCl}_{3}-\mathrm{CeCl}_{3}$ systems were tracked using parameters obtained from the modeling technique. Analysis of the data associated with the $\mathrm{LaCl}_{3}$ system was performed with the intent of yielding a manuscript. However, it was determined that further experimentation on the $\mathrm{LaCl}_{3}$ system was required with EIS experimentation planned for September 2013, but it was not conducted. Analysis of the data for the $\mathrm{LaCl}_{3}-\mathrm{CeCl}_{3}$ system demonstrated that the

3. University of Idaho 
close proximity of the reduction potentials for species may lead to simultaneous deposition with the EIS measurement being significantly influenced. This was supported by cyclic voltammetry.

EIS experimentation was conducted on systems that involved the heterogeneous reduction of species, $\mathrm{La}^{3+}$ to $\mathrm{La}^{-}$ and $\mathrm{Ce}^{3+}$ to $\mathrm{Ce}$. With this in mind, EIS was used to investigate the $\mathrm{LiCl}-\mathrm{KCl}-\mathrm{SmCl}_{3}$ system which involved the $\mathrm{Sm}^{3+}$ to $\mathrm{Sm}^{2+}$ reduction reaction involving soluble species. Experiments were performed to investigate the influences of applied potential, $\mathrm{SmCl}_{3}$ concentration, and temperature on the EIS response of the system. The consistency of the data and the informative analysis has led to the data and analysis of the $\mathrm{SmCl}_{3}$ system being drafted into a manuscript. The methodology and the mathematical modeling approach are provided in the manuscript. The results demonstrated that the polarization resistance at the redox potential could be used to track to concentration changes in the eutectic. The results of the $\mathrm{SmCl}_{3}$ system are to be submitted for presentation at an international pyroprocessing conference during 2014.

The experimentation planned for the first quarter of 2014 include investigation of the $\mathrm{EuCl}_{3}$ system examining the influences of applied potential, concentration and temperature on the $\mathrm{Eu}^{3+} / \mathrm{Eu}^{2+}$ redox reaction. A second manuscript is planned where the $\mathrm{EuCl}_{3} \mathrm{EIS}$ would be presented along with the comparison of the EIS responses of the $\mathrm{SmCl}_{3}$ and $\mathrm{EuCl}_{3}$ systems. The EIS response of the $\mathrm{SmCl}_{3}-\mathrm{EuCl}_{3}$ binary system would be investigated in the second quarter of 2014 with the EIS data associated with the individual systems being used as benchmarks. The investigation of the $\mathrm{LaCl}_{3}-\mathrm{CeCl}_{3}$ binary system that was conducted in 2013 was unsuccessful and it is hoped that the investigation of reactions involving soluble species would be more definitive.

The third quarter of 2014 will be used to investigate the $\mathrm{UCl}_{3}$ system where the influences of applied potential, concentration and temperature on the EIS response will be determined. The last quarter of 2014 will be used to investigate the binary $\mathrm{UCl}_{3}-\mathrm{SmCl}_{3}$ system. The research approach planned for 2014 was built on the insights gained during 2013 involving the $\mathrm{LaCl}_{3}$ and $\mathrm{LaCl}_{3}-\mathrm{CeCl}_{2}$ systems. There is much to understand about the EIS response of the lanthanides in the eutectic. Important findings were that soluble-soluble species reactions at the redox potential yield consistent data down to a low frequency of $0.01 \mathrm{~Hz}$. The leap to binary systems is included in the 2014 plan based on limited EIS data obtained for $\mathrm{UCl}_{3}-\mathrm{EuCl}_{3}$ during a chemical reduction experiment. The demonstration of compositional tracking in a binary system by EIS responses would represent a significant contribution to the state of molten salt electrochemistry.

Developing a procedure for species identification requires EIS experimentation on many systems from which a database of EIS responses can be constructed. Currently, we have compiled EIS data associated with $\mathrm{ZrCl}_{4}$, $\mathrm{LaCl}_{3}, \mathrm{SmCl}_{3}, \mathrm{UCl}_{3}, \mathrm{LaCl}_{3}-\mathrm{CeCl}_{2}, \mathrm{LaCl}_{3}-\mathrm{EuCl}_{3}$, and $\mathrm{UCl}_{3}-\mathrm{EuCl}_{3}$ systems and will add more detailed $\mathrm{EIS}$ for these and other species during the course of the project.

Additionally, ultraviolet-visible spectroscopy is being examined for potential application to real time monitoring of molten salt used in pyroprocessing operations. Initially, the focus of this study is on molten $\mathrm{LiCl}$ which is used for reducing oxide fuel to metals which can then be electrorefined. A labscale molten-salt furnace coupled with a ultraviolet-visible system is being assembled at the University of Utah this year. Initial investigations will include study of the effect of $\mathrm{CsCl}$ and $\mathrm{SrCl}_{2}$ concentrations in the salt on the ultraviolet-visible spectra. If successful, this will be extended to detecting the presence of uranium chloride in the salt, which is seen as an important capability to safeguard a pyroprocessing facility. 


\section{Benefits to DOE}

DOE's national security mission is to enhance nuclear security through defense, non-proliferation, and environmental efforts. This project directly contributes to the proliferation need. There is currently insufficient technology to prevent proliferation of nuclear material from pyroprocessing-based fuel reprocessing systems. If successful, this project will deliver key sensor technologies that are anticipated to contribute greatly to solving this problem.

\section{Presentations}

Allahar, K., et al., "Electrochemical Impedance Spectroscopy of $\mathrm{LaCl}_{3}, \mathrm{ZrCl}_{4}$, and $\mathrm{UCl}_{3}$ in $\mathrm{Molten} \mathrm{LiCl}-\mathrm{KCl}$ Eutectic," TMS 2013, San Antonio, TX, March 3-7, 2013.

Simpson, M., et al., "Sensor Technology for Real Time Monitoring of Molten Salt Electrolytes during Nuclear Fuel Electrorefining," TMS 2013, San Antonio, TX, March 3-7, 2013. 


\title{
13-068 - Cooling In Fractured Geothermal Reservoirs: Analyses of Long-Term Cooling In Typical Geothermal Reservoirs and Application to Geothermal Resource Potential Assessments
}

\author{
Mitchell Plummer, Jerry Fairley, ${ }^{1}$ John Welhan, ${ }^{1}$ Michael McCurry, ${ }^{2}$ Roy Mink, ${ }^{3}$ and Ian Warren ${ }^{4}$ \\ Engineered geothermal systems could provide new clean energy sources from resources that were previously \\ difficult or impossible to access. However, the success of such systems is dependent on the ability to assess \\ geothermal reservoir potential. This project aims to use a combination of established analytical and numerical \\ modeling techniques to improve understanding of long-term cooling in geothermal reservoirs. The primary task \\ objectives designed to achieve that goal are:
}

1. Develop a set of software tools that can be used to examine and predict the long-term thermal evolution and energy productivity of real and hypothetical geothermal reservoirs

2. Conduct analyses of available data describing different geothermal reservoirs to describe long-term productivity under typical operational constraints.

The former task is partly aimed at developing a software tool that the broader geothermal community can use to make reasonable assessments of geothermal potential based on flow and heat transport constraints, which are not considered in commonly used 'heat-in-place' estimates of geothermal potential. It also provides a tool for geothermal students involved in this project to make preliminary estimates of cooling behavior in more complicated systems, and thereby aids in development and testing of numerical simulations of such systems. The latter effort focuses on analysis of geothermal potential in Idaho and development of improved methods of assessing cooling behavior using field data from Idaho. The effort leverages INL's extensive experience in geothermal tracer development to demonstrate how more sophisticated analysis of pump test-and tracer test data-can provide a better estimate of long-term cooling induced by injection wells and considers the geothermal potential of sites in southern Idaho using a combination of analytical and numerical simulation methods.

\section{Summary}

This year's efforts included the following activities in support of project goals:

- Using a combination of analytical solutions and numerical modeling, Dr. Plummer demonstrated how cold water injection in a geothermal reservoir is likely to impact well injectivity over time and how pressure response in a well may be used to make inferences about hydraulic properties critical to predicting long-term cooling behavior. Results were presented at the 2013 Geothermal Resources Council Annual Meeting, where it received a "GRC Best Presentation Award," at the 2013 Geological Society of America annual meeting and at a CAES Geofluids Colloquium.

1. University of Idaho

2. Idaho State University

3. Mink GeoHydro, Inc.

4. U.S. Geothermal, Inc. 
- To promote better understanding of long-term cooling behavior in fractured reservoirs, Dr. Plummer collaborated with a summer intern from Boise State University to develop a MATLAB interface to a set of semi-analytical solutions to flow and heat transport equations for fracture flow. That geophysics student, Timothy Ronan, successfully completed most of the interface development, learned fundamentals of fluid flow and heat transfer, and presented his work at the Summer Internship Poster Session. Tim was selected as a Boise State University CAES Energy Scholar for his work.

- To support the CAES mission to investigate the geothermal potential of Southern Idaho, INL staff and summer interns worked with collaborators Welhan and McCurry to test and install a distributed temperature sensing optical fiber cable in a borehole in the Blackfoot Volcanic field. That temperature monitoring system will be used to look for the signal of a hypothesized fluid flow system carrying heat away from an area of high geothermal potential. Results should help determine the geothermal potential of that area and its vertical and horizontal distribution.

- Hypotheses to explain the unusual temperature distribution in the Blackfoot Volcanic field are now being tested using numerical simulations conducted at INL and the University of Idaho using a geothermal reservoir simulator based on INL's MOOSE. Preliminary findings suggest that a conduction-dominated flow regime would not produce the observed temperature distribution, suggesting that a blind hydrothermal system may supply heat to the relatively high-temperature brines found in parts of the field.

- Collaborator, Jerry Fairley, at the University of Idaho, supported a masters level graduate student, Alex Moody, who spent part of the summer term with Dr. Plummer at INL. Mr. Moody assisted Mr. Ronan with development of the MATLAB code to calculate temperature evolution in a fractured rock reservoir and used that program to make preliminary calculations describing the geothermal potential of the Snake River Plain. Mr. Moody presented his work in a poster at the 2013 Geological Society of America Annual Meeting in Denver, Colorado.

- The project team collected reference material necessary to summarize long-term thermal decline in hydrogeothermal reservoirs and tracer data that has been used to predict local thermal decline rates. Dr. Plummer reanalyzed data from one of those tracer tests to demonstrate how the traditional analysis approach likely underestimates actual thermal decline rate. As that type of reanalysis should be of significant interest to commercial reservoir operators, the team plans to have a 2014 summer intern repeat that analysis, using several other publically available data sets, for presentation at the Geothermal Resources Council 2014 meeting.

\section{Benefits to DOE}

Development of engineered geothermal systems could play a substantial role in DOE's mission to bring new clean energy sources to market. Such development requires, however, that we have appropriate tools for understanding and assessing reservoir potential. This project aims to provide a tool for rapid assessment of the relationships between fluid flow and basic reservoir characteristics in fractured geothermal reservoirs. Such tools are needed as a reference base for understanding and application of more sophisticated reservoir simulators. This project would fill a critical gap in the industry's ability to understand and predict the effects of heat exchange in fractured reservoirs. 


\section{Publications}

Plummer, M.A., "Insights into Cold Water Injection Stimulation Effects through Analytical Solutions to Flow and Heat Transport," Geothermal Transactions, 2013, Accepted.

\section{Presentations}

Plummer, M.A., "Insights into Cold Water Injection Stimulation Effects through Analytical Solutions to Flow and Heat Transport," Geothermal Resources Council Annual Meeting, Las Vegas, NV, September 29-October 2, 2013.

Moody, A.C., J.P. Fairley, and M.A. Plummer, "Theoretical Fracturing and Power Production from LowPermeability Tuffs in the Snake River Plain, Southern Idaho," 2013 GSA Annual Meeting, Denver, CO, October 27-30, 2013.

Plummer, M.A., K. Smith, and R.K. Podgorney, "Cold Water Injection Effects in Fractured Reservoirs (2013)," 2013 GSA Annual Meeting, Denver, CO, October 27-30, 2013. 


\section{CENTER FOR

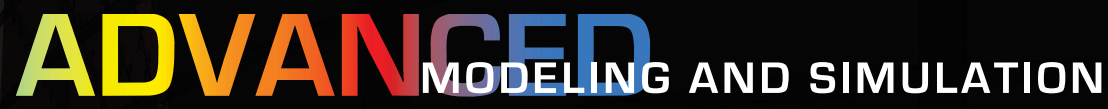




\title{
10-031 - Fickian and Thermal Diffusion in Nuclear Materials from Linear Response Theory and Multiscale Simulations
}

\author{
Jianguo Yu and Jacob Eapen ${ }^{1}$
}

Modeling and simulation are important tools for solving difficult scientific problems and improving efficiency in complex processes. The central objective of this project is to develop new statistical-mechanical models and longtime atomistic simulation methods to determine complex diffusive properties in nuclear materials. Specifically, the project will develop state-of-the-art methods to compute Fickian and thermal diffusivities in $\mathrm{UO}_{2}$ and metallic systems under different thermodynamic conditions.

\section{Summary}

The traditional molecular dynamics (MD) method is impractical beyond the nanosecond time scale, so it has limitations in accessing the slow thermo-diffusion processes in nuclear materials that occur on time scales of microseconds and above. Accelerated MD—a term introduced by Voter and co-workers at the Los Alamos National Laboratory—is a technique for extending the traditional MD method for accessing longer time scales.

We have implemented a theoretical method which computes the thermal diffusion ratio (Soret coefficient) using the transition state or rate theory. In 2011-2012, we used Temperature Accelerated Dynamics-a member of the accelerated MD family - to compute the thermal diffusion ratio for point defects in $\mathrm{UO}_{2}$. In 2012-2013, as outlined in the original proposal, we have computed the thermal diffusion ratio for point defects in molybdenum (Mo), which is representative of a metallic system.

The interaction between atoms in Mo is described using an embedded atom method potential on the basis of good agreement with various experimental data. Next, Temperature Accelerated Dynamics is employed to calculate the energy barriers for vacancy and interstitial migration for different temperatures. Using the elementary theory outlined by Mortimer and Eyring, we have computed the thermal diffusion factor for equi-molar vacancy and interstitial defect concentrations. It is observed that the thermal diffusion factor is negative, unlike that in $\mathrm{UO}_{2}$, and increases with increasing temperature. The thermal diffusion factor is portrayed in Figure 1.

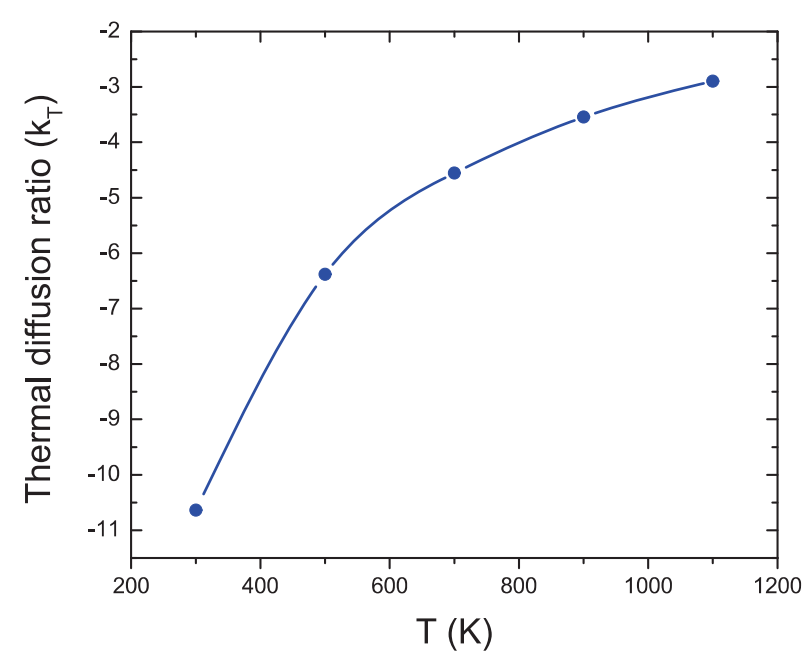

Figure 1. Thermal diffusion ratio (kT) for an equi-molar mixture of vacancies and interstitials at different temperatures for Mo. In contrast to Mo, the thermal diffusion ratio in $\mathrm{UO} 2$ is positive.

1. North Carolina State University 


\section{Benefits to DOE}

Our project is central to DOE's nuclear energy missions. For example, performance optimization and lifetime extension of the current fleet of light water reactors require an understanding of the nuclear fuel performance and material degradation in operating regimes where there is little to no observational data. Modeling and simulation at the atomistic level can help to accelerate this understanding. Atomistic scale determination of thermal properties of metallic systems and uranium oxide fuels will benefit many DOE mission areas.

\section{Publications}

Nandi, P., X. Mei, and J. Eapen, "Thermodiffusion in Nuclear Fuel," to be submitted to J. Appl. Phys., In preparation.

\section{Presentations}

Eapen, J., "Thermodiffusion in $\mathrm{UO}_{2}$," Fall MRS Meeting, Boston, MA, November 25-30, 2012. 


\title{
11-074 - Uncertainty Analysis for Multiscale Models of Nuclear Fuel Performance
}

\author{
John Peterson, Don Estep, ${ }^{1}$ and Simon Tavener $^{1}$
}

Developing nuclear fuel cycles that optimize uranium $(U)$ utilization and energy generation, minimize waste and proliferation risk, and increase safety is a principal priority of DOE-NE. Modeling and simulation can help to reduce the cost and time required to develop new nuclear fuel forms and to understand the behavior of fuel at high burn-up. There is scant experimental data for the new fuel forms and performance regimes under consideration, which brings the validity of existing empirical models into question. Consequently, a first principles approach to determining properties of materials under radiation is being pursued. This approach uses atomistic calculations to construct a mesoscale description of microstructural evolution of irradiated materials and then upscales that description to determine material properties that can then be used in engineering-scale models of nuclear fuel performance.

Such models present very significant challenges for mathematical and computational analysis. This project was aimed at addressing some key issues having to do with reliable uncertainty analysis of multiscale simulations of nuclear fuel performance. Key goals of the project included: consistent mathematical formulation of the coupling stochastic mesoscale and macroscale models, analysis of iterative solution of coupled stochastic models, a-posteriori error analysis for numerical simulations of coupled multiscale models, and finally developing adaptive computational strategies based on error estimates. In addition to accounting for the stochastic behavior of uncertain parameters typical of current approaches to uncertainty analyses, the work accounted for numerical error incurred in formulation of the deterministic problem as well as approximation and sampling errors in the stochastic problem.

\section{Summary}

In the first year of the project, the Colorado State University team focused on acquiring necessary background in fuel performance models and INL codes and implementing and testing the proposed macroscale and mesoscale coupling method in a testbed code in MATLAB. In addition to attending the International Summer School on the Evolution of Microstructural Defects on In-Reactor Material Response at INL, the postdoc, Newton, carried out a systematic study of background materials related to the modeling of fuel performance. The team developed and implemented a mesoscale and macroscale modeling method using an intermediate numerical transfer scale, devised a fixed point iteration for the coupling, and studied the convergence. This was done in the context of a simplified stationary model with nonlinear elliptic problems for each scale. Newton began implementation of the $\mathrm{C}++$ testbed code. We studied the target mesoscale model application code, MARMOT, in preparation for an initial set of uncertainty analyses and to develop the expertise needed to make modifications to suit our methods. Finally, we continued the ongoing a-posteriori error analysis for numerical solution of coupled multiscale problems.

In the second year, the team turned to deriving a rigorous mathematical foundation for the coupling of the macroscale and mesoscale components of stochastic multiscale models of fuel performance that includes convergence of the required iteration. The rigorous mathematical foundation has two parts: a theory of solution of

1. Colorado State University 


\section{CENTER FOR ADVANCED MODELING \& SIMULATION}

a system of coupled stochastic models and the theory of a mesoscale "layer" that serves for the upscaling and downscaling between the macro and microscales. We formulated the coupled stochastic system as a system of integral equations for the probability distributions of the coupling variables and proved a general theorem about an iterative solution process for the system, akin to a fixed point result. At the same time, we evolved the testbed code to become a MOOSE/BISON compliant $\mathrm{C}++$ code and incorporated the Portable, Extensible Toolkit for Scientific Computation into the code to increase efficiency.

Because the typical Cahn-Hilliard mesoscale model presents very significant computational challenges, we developed a Cahn-Allen stochastic microscale model for use as a surrogate. We discovered parameter value ranges that provide for mesocale layer behavior, which is a close mimic of the more complicated Cahn-Hilliard model.

We also continued ongoing efforts in a-posteriori error analysis of coupled multiphysics simulations, focusing on the effects of incomplete iteration in the solution process.

Finally, we began work on uncertainty analysis in BISON and MARMOT by developing a prototype MOOSE Executioner that can serve to coordinate execution of BISON and MARMOT during uncertainty analysis.

In the third year, we continued research on several projects initiated in the first two years, and we initiated research on another project goal.

We also worked on significantly improving time accuracy and computational efficiency in MARMOT in order to support statistical sensitivity analysis. To achieve this, we adapted the time stepping methodology used in SUNDIALS and then improved the time step selection mechanism and solver efficiency.

The team continued the development of a mathematical analysis of the coupled integral equation for probability distributions arising in coupled stochastic models representing engineering and mesoscales (devising iterative solution algorithms to solve the coupled system and comparing the iterative approaches to the standard "samplebased" Monte Carlo simulation) and continued the analysis of convergence of iterations. This raised interesting questions about the effects of incomplete iteration in nonlinear solvers on the output of sensitivity analysis. Using a finite number of iterations means the domain and range of the "finite iterated solution" are different than those for a fully iterated solution, which affects the probability measure structure. We are currently analyzing the effects of incomplete iteration in a nonlinear solver for a coupled system of models on the samples used in a stochastic sensitivity analysis.

The team also made significant progress in developing a rigorously justified systematic approach to constructing upscaled stochastic models of unresolved microscale behavior. The general problem is the "upscaling a microscale" continuous function appearing as a parameter in a macroscale differential equation when the microscale behavior is not resolved. To address this problem, essentially, we approximate the microscale function using a class of stochastic splines whose parameter distributions are chosen to provide a representation of the unresolved microscale behavior. This is significantly different than the common approaches that basically use an uninformed stochastic representation. Our approach can also incorporate the case that the microscale behavior is itself stochastic.

We also continued ongoing efforts in a-posteriori error analysis of coupled multiphysics simulations, focusing on the effects of incomplete iteration in the solution process. 


\section{CENTER FOR ADVANCED MODELING \& SIMULATION}

In addition to accepted and submitted research manuscripts, we have developed several sets of notes describing the mathematical work that we intend to use as a basis for research publications in the future.

\section{Benefits to DOE}

Multiscale simulation of the performance of nuclear fuel supports several programmatic objectives in DOE-NE in areas such as light water reactor sustainability and alternative fuel cycles. The new analysis capabilities that we developed in this project will ultimately lead to improved predictive capabilities for multiscale models of nuclear fuels by creating a mathematically sound framework for coupling stochastic models at different scales and estimating the error in coupled multiscale model simulations. These mathematical tools and frameworks are directly applicable to other research areas of interest at INL, including carbon sequestration and unconventional fossil fuel recovery, as well as the Advanced Simulation Capability for Environmental Management program in the DOE Office of Environmental Management, the broader mission of the DOE national security missions, and any other federal agencies dealing with simulation of multiscale systems.

\section{Publications}

Estep, D., et al., "A Posteriori Analysis of an Iterative Multi-Discretization Method for Reaction-Diffusion Systems," Comput. Method. Appl. M., 2013, Accepted.

Arbogast, T., et al., "A-posteriori Error Estimates for Mixed Finite Element and Finite Volume Methods for Problems Coupled through a Boundary with Non-matching Grids," IMA J. Numer. Anal., 2013, Accepted.

Estep, D. and S. Lee, "Adaptive Error Control for an Elliptic Optimization Problem," Appl. Anal., Vol. 92, 14341448, July 1, 2013.

Butler, T. and D. Estep, "A Numerical Method for Solving a Stochastic Inverse Problem for Parameters," Ann. Nucl. Energy, Vol. 52, 86-94, February 2013.

Keyes, D., et al., "Multiphysics Simulations: Challenges and Opportunities," Int. J. High Perform. C., Vol. 27, 4-83, February 2013.

Estep, D., M. Holst, and A. Malqvist, "Nonparametric Density Estimation for Randomly Perturbed Elliptic Problems III: Convergence, Complexity, and Generalizations," Journal of Applied Mathematics and Computing, Vol. 38, 367-387, 2012.

Butler, T., D. Estep, and J. Sandelin, "A Computational Measure Theoretic Approach to Inverse Sensitivity Problems II: A Posteriori Error Analysis," SIAM J. Numer. Anal., Vol. 50, 22-45, 2012.

Estep, D., V. Ginting, and S. Tavener, "A Posteriori Analysis of Multirate Numerical Method for Ordinary Differential Equations," Comput. Method. Appl. M., Vol. 223, 10-27, 2012.

Burch, N., D. Estep, and J. Hoeting, "Parameter Estimation and Directional Leverage with Applications in Differential Equations," Metrika, Vol. 75, 895-911, October 2011, Submitted.

Sheehan, B., et al., "The Interaction of Iteration Error and Stability for Time Dependent Linear Partial Differential Equations Coupled through an Interface," Numer. Algorithms, 2013, Submitted. 
Collins, J., D. Estep, and S. Tavener, "A Posteriori Error Estimates for Explicit Time Integration Methods," BIT, 2012, In revision.

Carey, V., D. Estep, and S. Tavener, "A Posteriori Analysis and Adaptive Error Control for Multiscale Operator Decomposition Solution of Elliptic Systems II: Fully Coupled Systems," International Journal of Numerical Methods in Engineering, 2011, In revision.

\section{Presentations}

Pernice, M. and S. Tavener, "Quantification in CASL," Uncertainty Quantification at SAMSI, July 2013.

Estep, D., "Validation and Uncertainty," SAMSI Uncertainty Quantification Transition Workshop, May 2012.

Tavener, S., "Multiscale Modeling and Uncertainty Quantification for Nuclear Fuel Performance," Nottingham University, England, November 2011.

Estep, D. and M. Pernice, "Multiscale Modeling and Uncertainty Quantification for Nuclear Fuel Performance," ICiS Workshop on Multiphysics Simulations: Challenges and Opportunities, Park City, UT, August 2011. 


\title{
11-076 - Exploratory Nuclear Reactor Safety Analysis and Visualization via Integrated Topological and Geometric Techniques
}

\author{
Diego Mandelli, Valerio Pascucci, ${ }^{1}$ and Bei Wang ${ }^{1}$
}

A recent trend in the nuclear power engineering field is the implementation of heavily computational and timeconsuming codes for the design and safety analysis of nuclear plants. In particular, the new generation of system analysis codes aims to embrace several phenomena, such as thermo-hydraulic and system dynamics as well as uncertainty quantification and sensitivity analyses associated with these phenomena. The use of dynamic probabilistic risk assessment (PRA) methodologies allows a systematic approach to uncertainty quantification. Dynamic methodologies account for possible coupling between triggered or stochastic events through explicit consideration of the time element in system evolution, often through the use of dynamic system models (simulators), and are also capable of propagating epistemic and aleatory uncertainties. Major challenges in using dynamic methodologies are: (a) the heavier computational cost, and (b) the large amount of data that needs to be analyzed. This LDRD project aims to improve furthermore dynamic PRA methodologies by tackling the two challenges mentioned above using: (a) adaptive sampling techniques to reduce computational cost of the analysis, and (b) topology-based methodologies to interactively analyze and visualize high-dimensional data and extract risk-informed insights. First, we employ topology-based, adaptive sampling algorithms that aim to infer or predict simulation outcome and determine the coordinates in the input space of the next sample that maximize the amount of information that can be gained. Such methodologies can be used to both explore and exploit the input space. The latter one is especially used for safety analysis scopes to focus samples along the limit surface (i.e., the boundaries in the input space between system failure and system success). Second, we present a software tool that is designed to analyze and visualize high-dimensional data and, in particular, the datasets generated by dynamic methodologies. Our tool includes a host of various analysis and visualization capabilities. We first perform topological analysis to identify the underlying topological structure of the dataset. Statistical information is then summarized and linked to the topological structures extracted from the data, allowing the users to identify correlations between timing/sequencing of events and simulation outcome. From an uncertainty quantification point of view, such a tool allows the user to identify and consequently rank variables based on their correlations with simulation outcome. Such software becomes even more effective for the analysis of complex systems such as nuclear power plants where the number of variables is very large.

\section{Summary}

We developed an approach that maps the uncertainty quantification problem in large-scale nuclear simulations to the analysis of functions in high-dimensional spaces. We introduced techniques that couple topology and geometry in the analysis and visualization of nuclear datasets, especially those generated by dynamic PRA methodologies.

First, we developed a software tool that provides scientists and domain experts with an interactive analysis and visualization environment for understanding the structures of high-dimensional nuclear simulation data. We model each large-scale nuclear simulation dataset as a high-dimensional scalar function defined over a discrete sample of the domain; from such topological models we: (a) provide structural analysis at multiple scales and provide

1. University of Utah 


\section{CENTER FOR ADVANCED MODELING \& SIMULATION}

insight into the relationship between the input parameters and the output, and (b) enable exploratory analysis for users where we help the users differentiate features from noise through multi-scale analysis on an interactive platform, based on domain knowledge and data characterization. Our analysis is performed by exploiting the topological and geometric properties of the domain, building statistical models based on its topological segmentations, and providing interactive visual interfaces to facilitate such explorations. The visual interfaces provided by the system are highly interactive and tightly integrated, providing users with the ability to explore various aspects of the datasets for both analysis and visualization purposes. We provide a user's guide to our software tool by highlighting its analysis and visualization capabilities. We demonstrate the usability of our tool for several nuclear simulation datasets, including the one generated for the Risk Informed Safety Margin Characterization project analysis of a Boiling Water Reactor station blackout case study.

Second, we developed a range of topologically driven adaptive sampling techniques aimed at understanding the space of all possible simulation outcomes: the response surface. In this respect, we focused on developing topology-driven scoring functions. We have explored sampling strategies from the context of what is necessary for reconstructing the topological features of the response surface. We have used topological information to guide the selection of sampled points from the candidate set. Topology-driven scoring functions for adaptive sampling selectively choose topologically informative points that would be useful in improving the underlying surrogate model in an iterative fashion. We analyzed the specific use case of identifying the limit surface (i.e., the boundaries in the simulation space between system failure and system success). We explore several techniques for adaptively sampling the parameter space in order to reconstruct the limit surface. We focused forward learning a global model of the entire simulation space using prediction models or neighborhood graphs and extracting the limit surface as an iso-surface of the global model. We estimated the limit surface (i.e., boundaries between system failure and system success) by sampling in the neighborhood of the current estimate based on topological segmentations obtained locally. Our techniques draw inspiration from the topological structure known as the Morse-Smale complex. We highlight the advantages and disadvantages of using a global prediction model versus local topological view of the simulation space, comparing several different strategies for adaptive sampling in both contexts. One of the most interesting models we propose attempts to marry the two by obtaining a coarse global representation using prediction models and a detailed local representation based on topology. Our methods are validated on several analytical test functions as well as applied to the analysis of the limit surface for a simplified Pressurized Water Reactor during an accident scenario.

Third, via our integrated system, we allow the end users to perform both end state analyses and transient analyses that apply to their time evolution data to classify the set of nuclear scenarios. We focus on topologybased clustering algorithms. We apply our methodologies on a dataset generated by a dynamic PRA methodology for a sodium ( $\mathrm{Na}$ )-cooled fast reactor during an aircraft crashing scenario, as well as a Boiling Water Reactor station blackout case study. We demonstrate that these techniques offer complementary views of the data that help illuminate key features that may be otherwise hidden using a single methodology.

\section{Benefits to DOE}

The deliverables will be directly employed within existing PRA codes (e.g., RAVEN) in order to satisfy the needs of many DOE-NE programs. The new generation of safety analysis codes (e.g., RELAP-7) will incorporate a new series of algorithms and dynamic PRA methodologies (e.g., RAVEN) that are able to model system dynamics, human interaction, and digital control systems and perform uncertainty quantification and sensitivity analysis. This process may result in large amounts of data generated that can be difficult to analyze and, from a user point of view, might be difficult to assess with regard to the main contributors of risk and most relevant trends. This project 
applies cutting edge methodologies and algorithms to safety analysis codes and data for nuclear systems in order to: analyze the simulations generated by generic safety analysis codes in an intuitive graphical form, identify correlations between sequence and timing of events, and identify system characteristics and limitations using adaptive sampling techniques. These new techniques for visual analysis of uncertainty provide a unique capability that helps safety analysts to more fully explore the high-dimensional landscape of uncertainties in a simulation. Moreover, we also focused on the development of adaptive sampling techniques that will help in exploring failure regions of the simulation without oversampling the space and, thus, greatly reduce the computational time of such analyses.

\section{Publications}

Maljovec, D., et al., "Exploration of High-Dimensional Scalar Function for Nuclear Reactor Safety Analysis and Visualization," Proceedings International Conference on Mathematics and Computational Methods Applied to Nuclear Science \& Engineering (M\&C), 712-723, 2013.

Maljovec, D., et al., "Adaptive Sampling with Topological Scores," Working with Uncertainty Workshop at IEEE VisWeek, 2011, International Journal for Uncertainty Quantification, Vol. 3, 119-141, 2013.

Wang, B., et al., "Branching and Circular Features in High Dimensional Data," IEEE T. Vis. Comput. Gr., Vol. 17, 1902-1911, 2011.

Maljovec, D., et al., "Exploration of High-Dimensional Scalar Function for Nuclear Reactor Safety Analysis and Visualization," Under preparation for the J. Nucl. Eng. Des.

Maljovec, D., et al., "Adaptive Sampling Algorithms for Probabilistic Risk Assessment of Nuclear Simulations," Under preparation for submission to the Journal of Reliability Engineering \& System Safety.

Maljovec, D., et al., "Analyze Dynamic Probabilistic Risk Assessment Data through Topology-Based Clustering," Under preparation for submission to the Journal of Reliability Engineering \& System Safety.

\section{Presentations}

Wang, B., D. Maljovec, and D. Mandelli, "Workshop on Topological Data Analysis and Visualization for LargeScale and High-Dimensional Science Discovery," Topical Meeting on Probabilistic Safety Assessment and Analysis (PSA), Columbia, SC, September 22-26, 2013. Organizers: Bei Wang, Dan Maljovec, Diego Mandelli, Valerio Pascucci and Peer-Timo Bremer.

Maljovec, D., et al., "Adaptive Sampling Algorithms for Probabilistic Risk Assessment of Nuclear Simulations," International Topical Meeting on Probabilistic Safety Assessment and Analysis (PSA), Columbia, SC, September 22-26, 2013.

Maljovec, D., et al., "Analyze Dynamic Probabilistic Risk Assessment Data through Topology-Based Clustering," International Topical Meeting on Probabilistic Safety Assessment and Analysis (PSA), Columbia, SC, September 22-26, 2013. 
Maljovec, D., et al., "Exploration of High-Dimensional Scalar Function for Nuclear Reactor Safety Analysis and Visualization," International Conference on Mathematics and Computational Methods Applied to Nuclear Science \& Engineering (M\&C), Sun Valley, ID, May 5-9, 2013.

Wang, B., et al., "Adaptive Sampling with Topological Scores," SAMSI Uncertainty Quantification Transition Workshop, Durham, NC, May 21-23, 2012.

Wang, B., et al., "Adaptive Sampling with Topological Scores," Fields Institute for the Thematic Program on Discrete Geometry, Toronto, Canada, Workshop on Computational Topology (November 7-11) and Workshop on Sphere Arrangements (November 14-18), 2011.

Maljovec, D., et al., "Adaptive Sampling with Topological Scores," Working with Uncertainty Workshop at IEEE VisWeek 2011, Providence, RI, October 24, 2011.

Summa, B., et al., "Branching and Circular Features in High Dimensional Data," IEEE Visualization Conference, Providence, RI, October 23-28, 2011. 


\section{INSTRUMENTATION, CONTROLInAt MleENT SvStems}




\title{
11-046 - Known Secure Sensor Measurements for Detecting Unauthorized Process Manipulation and Falsification of State
}

\author{
Miles McQueen, Milos Manic, ${ }^{1}$ Linda Ondrej, ${ }^{1}$ Kameshwar Poolla, ${ }^{2}$ and Annarita Giani ${ }^{3}$
}

This research is an investigation of a low-cost, low-false alarm, and reliable mechanism for detecting manipulation of critical physical processes and falsification of system state in critical infrastructure systems. This detection mechanism can enable system operators to more quickly react to malicious manipulation of critical infrastructure systems, thereby preventing an attack. We call this novel mechanism Known Secure Sensor Measurements (KSSM). The method moves beyond analysis of network traffic and host-based state information and uses physical measurements of the process being controlled to detect falsification of state. KSSM is intended to be incorporated into the design of new, resilient, cost-effective critical infrastructure and associated control systems. Perhaps more importantly, KSSM can also be included in incremental upgrades of already installed infrastructures and systems for enhanced resilience. KSSM is based on "known secure," i.e., tamper proof, physical measurements for assessing the likelihood of a cyber attack and is intended to demonstrate a practical approach to creating, transmitting, and using the known secure measurements for detection.

\section{Summary}

We assume that the attacker can compromise any of the components in the information layer of a control system, without being detected, as long as the attacker does not modify the sensor signals being transmitted back to the controller and the control room. KSSM is not designed to detect the system process exceeding its operational performance envelope; normal system monitoring is expected to detect that situation. Rather, KSSM is designed to reliably detect any attempt to falsify system state through manipulation of one or more of the sensor measurements being reported back to the control room. As a case study, we applied the KSSM concept to the issue of detecting data integrity attacks on the power grid through optimal placement of Phasor Measurement Units (PMU).

In 2013, we continued our work on quantifying cyber-attack consequences. We developed a formal mathematical model of calculating worst case consequence of an unobservable data integrity attack and developed algorithms for solving the models (under various assumptions). Following the work of the previous years we improved the upper bound on the number of PMUs necessary to protect the grid against attacks.

We also started an investigation on techniques to detect faults to relay systems. If a false relay measurement has been detected, then it is necessary to localize this measurement in order to determine the true state of the system. We proposed a computationally fast algorithm that localizes a relay measurement attack. Our method can check 1000 single relay faults in 2-3 minutes and multiple relay faults.

As another task in FY 2013, we focused on solving the problem of optimal selection and placement of PMUs in the power grid. We introduced interactive features to the produced simulation tool and algorithms so that a better understanding could be gained on different "what if" scenarios. We also introduced additional criteria to be used in identifying optimal placement. 
The new interactive features of the tool enable users to change decision-making criteria "on the fly." For example, users can decide whether or not to use Zero Injection Busses (ZIBs) to change the priority of criteria (security becomes more important than the observability). The tool enables users to change the structure of the power grid by installing more PMUs, siting new base stations, or installing communication lines.

The first criterion introduced was the consideration of ZIBs. A ZIB is a bus where no current is injected to the system (Figure 1). Using ZIB, the state of adjacent buses can be estimated. Using this estimation, the number of PMUs required for complete coverage can be reduced. However, replacing this estimation with measurements from a PMU can increase the robustness of the solution. The interactive elements enable the comparison of the two solutions. Figure 2(a) and Figure 2(b) show solutions using ZIB estimation and using actual measurements instead of estimation, respectively.

The second criterion introduced was placement and cost associated with installing communication lines. Secure, high-speed communication between PMUs is essential for their operation. Therefore, the additional enhancements incorporated into the algorithm consider the cost of required communication lines. This solution assumes communication lines installed between buses in the power grid (a conservative estimation). Figure $3 a$ and Figure $3 \mathrm{~b}$ show two different solutions for the same power grid using the same number of PMUs, where the solution in Figure 3b has a lower communication line cost.

\section{Benefits to DOE}

This research is establishing scientific and engineering based techniques for improved and reliable detection of cyber attacks against infrastructures and their associated control system processes, addressing DOE's national security missions. The techniques are based on control system specific "known secure" physical measurements, which are not available in computer-based detection mechanisms and have not been generally incorporated into infrastructure control system cyber security attack detection tools. The research has focused on multicriteria/multi-stage optimal placement of PMUs within the power grid to detect an attacker's falsification of system state. Initial algorithms for PMU placement have been created and validated with simulations on small and
Figure 1. ZIB and the bus where the state can be estimated using the ZIB for the IEEE 14 bus problem.

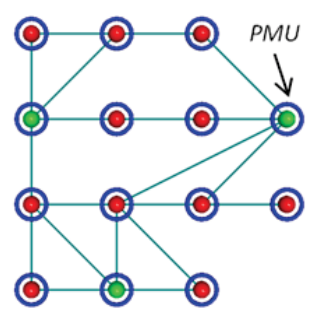

(a) Using ZIB estimation (3PMUs)

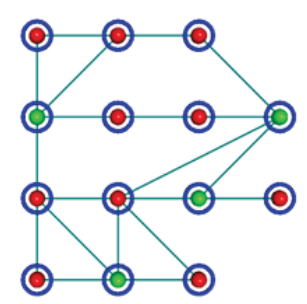

(b) Using actual measurements (4PMUs)
Figure 2(a-b). (a) Solutions for optimal placement of known secure sensors in the power grid using ZIB estimation and (b) actual measurements.

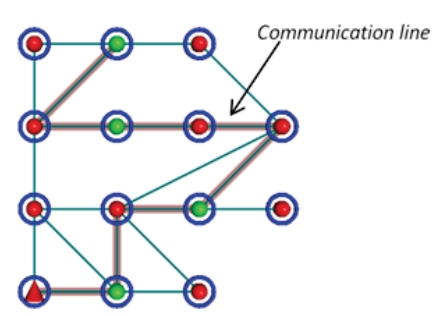

(a) Solution 1

Number of PMUs: 4 Number of communication lines: 8

Length: 8.8

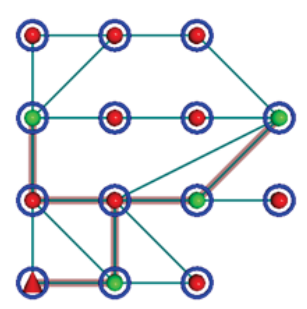

(b) Solution 2 Number of PMUs: 4 Number of communication lines: 6 Length: 6.4
Figure 3. Comparison of two solutions considering communication lines for the same problem using the same number of PMUs for full coverage where (a) has 8 communication lines and (b) has 6 communication lines. 
medium sized grids. More general infrastructure control systems and their corresponding infrastructures have also been investigated and the feasibility of the core KSSM concepts evaluated using initial simulations.

\section{Publications}

Linda, O., M. Manic, and M. McQueen, "Optimal Placement of Phasor Management Units Using Memetic Algorithms," IEEE PES Innovative Smart Grid Technologies Conference, In preparation.

Giani, A., et al., "Smart Grid Data Integrity Attacks," IEEE Transactions on Smart Grid, Vol. 4, 1244-1253, September 2013.

Giani, A., et al., "Known Secure Sensor Measurements Concept and Its Application for Critical Infrastructure Systems," Optimization and Security Challenges in Smart Power Grids, Berlin: Springer Verlag, July 31, 2013.

Linda, O., et al., "Multi-Criteria Based Staging of Optimal PMU Placement Using Fuzzy Weighted Average," Proceedings of IEEE International Symposium on Industrial Electronics, Taipei, Taiwan, May 28-31, 2013.

Linda, O., M. Manic, and M. McQueen, "Improving Control System Cyber-State Awareness Using Known Secure Sensor Measurements," $7^{\text {th }}$ International Workshop on Critical Information Infrastructures Security, Lillehammer, Norway, September 2012.

Giani, A., et al., "Metrics for Assessment of Smart Grid Data Integrity Attacks," IEEE Power \& Energy Society General Meeting, San Diego, CA, July 2012. 


\title{
12-071 - Battery Resilient Monitoring and Control
}

\author{
Jon Christophersen and Humberto Garcia
}

Batteries and other energy storage devices are becoming increasingly important for several industries, including automotive, electric utilities, and military applications. Increased performance requirements, combined with the high costs of energy storage devices, emphasize the need for improved battery management systems that accurately assess the overall state-of-health $(\mathrm{SOH})$ and remaining useful life. In this LDRD effort, a prototype battery $\mathrm{SOH}$ metric was developed based on a combination of passive and active measurements using novel sensor technology to demonstrate the ability to extend the life of batteries. In this way, INL seeks to advance battery storage technologies to support our nation's energy needs.

Impedance spectroscopy has been emphasized as a valuable tool for battery modeling and accurate life estimation. INL, in collaboration with Montana Tech of the University of Montana, has developed a novel sensor technology that can rapidly acquire impedance spectroscopy measurements of energy storage devices for online applications. Studies at INL have been conducted to examine the effectiveness for long-term impact of this novel impedance sensor. The acquired test data provide an opportunity to develop unique $\mathrm{SOH}$ assessment techniques.

\section{Summary}

The first year of experimentation focused on developing the framework for the $\mathrm{SOH}$ assessment architecture using the in-situ rapid impedance spectrum measurement technique. Also during FY 2012, we focused on the prototype $\mathrm{SOH}$ architecture based on cells that were aged from fresh conditions to end of useful life while periodically subjected to rapid impedance measurements under both no-load and load conditions.

During FY 2013, we further refined the SOH architecture to enable the development of smart algorithms for directly estimating state-of-charge (SOC) based on impedance spectral measurements. Accurate SOC identification is a dynamic requirement for improved power management, whereas accurate $\mathrm{SOH}$ estimation is primarily for increased battery longevity, executing predictive maintenance schedules, and accommodating aging when computing battery parameters. These battery parameters, when measured online, will support optimized battery life and usage under various applications. This principle was demonstrated after assessing new test data that included impedance spectra as a function of SOC for enhanced health and life estimations.

In FY 2013, the focus was primarily on developing an SOC assessment architecture that used rapid impedance measurement data over a broad range of SOC and aging conditions. First, a preprocessing filter was developed to examine the existing test data and determine if noisy measurements that were corrupted by the aging prototype hardware systems should be completely rejected or smoothed. This was followed by curve fitting using equivalent circuit models to extract features of interest and identify various critical parameters, such as the ohmic resistance $\left(R_{O}\right)$, charge transfer resistance $\left(R_{C T}\right)$ and the maximum imaginary resistance $\left(X_{\max }\right)$ at the semicircle peak. Since the measured impedance spectra grow as a function of both $\mathrm{SOH}$ and $\mathrm{SOC}$, knowledge about $\mathrm{SOH}$ is critical for successful SOC estimation. Assessment of the resulting parameter data at a full charge condition was first completed to assess $\mathrm{SOH}$ compared with standard aging markers (i.e., capacity, available power, pulse resistance). The data driven input-output mapping trained with $R_{C T}$ and $X_{\max }$ for fresh cells (i.e., $S O H=1$ ) is shown in Figure 1. These results clearly demonstrate that both $R_{C T}$ and $X_{\max }$ are affected by SOC conditions and 
could be used as indicators for online SOC estimation. Models were then developed to estimate SOC from an impedance spectrum at given $\mathrm{SOH}$ conditions.

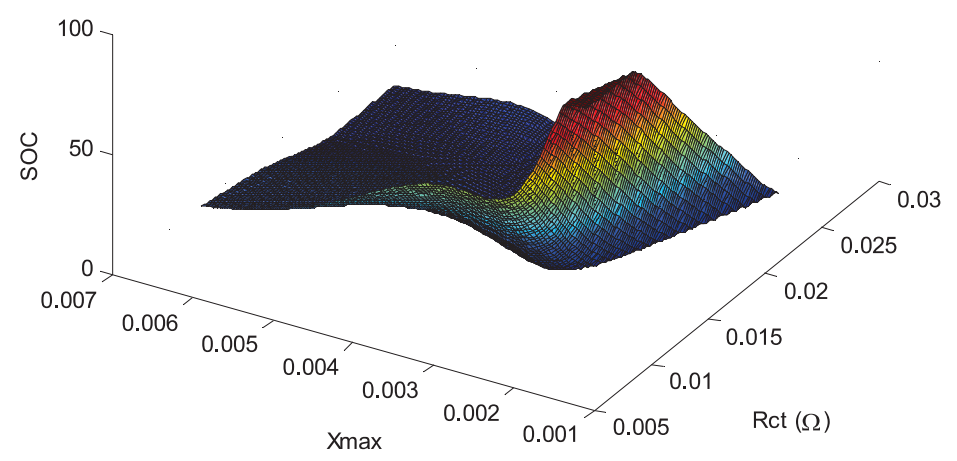

Figure 1. Data driven input-output mapping.

The estimated SOC compares well with the measured SOC for these commercially-available lithium-ion cells as a function of $\mathrm{SOH}$, as demonstrated in Figure 2. Charge transfer resistance, used in this figure, is primarily influenced by kinetic reactions at the electrodes. These data also indicate that SOC can be successfully determined from rapid impedance spectra measurements once the $\mathrm{SOH}$ has been properly determined. These results, combined with the efforts from FY 2012, form the prototype SOH assessment architecture that can be useful for a number of different applications, including automotive, military, utilities, etc.

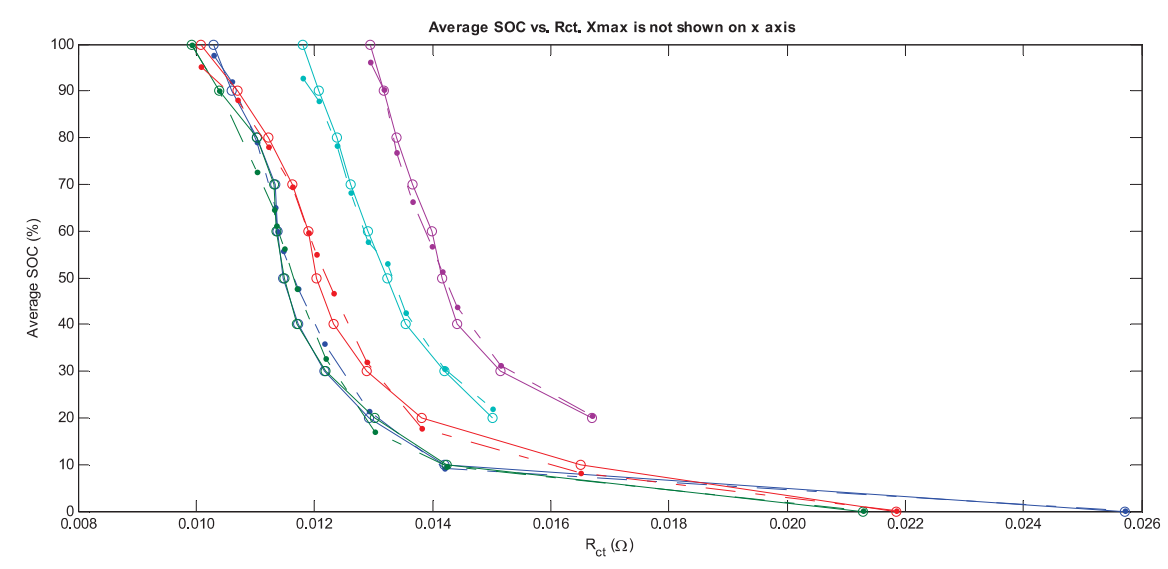

Figure 2. Average SOC vs. $R_{C T}$ as a function of $\mathrm{SOH}$. 


\section{Benefits to DOE}

DOE will benefit from this research through the advancement of battery energy storage technologies, a key component of the national energy strategy. Knowledge of battery $\mathrm{SOH}$ and remaining useful life is critical for optimized energy usage, control, and management. Developing a more complete SOH metric will allow battery management systems to perform real-time decisions more intelligently using the developed sensor technology and online condition monitoring methodology, including diagnostics and prognostics to better predict battery $\mathrm{SOH}$ and remaining useful life.

\section{Presentations}

Christophersen, J.P. and H. Garcia, "Battery Resilient Monitoring and Control," ICIS External Review Committee, San Francisco, CA, August 12, 2013.

Christophersen, J.P., "Battery Life Estimations for Offline and Online Applications," Advanced Automotive Battery Conference, Pasadena, CA, February 5, 2013. 


\section{2-082 - Resilient Monitoring, Adaptation, and Control (ReMAC) System}

Humberto Garcia, Wen-Chiao Lin, Semyon Meerkov, ${ }^{1}$ Kris Villez, ${ }^{2}$ and Maruthi Ravichandran ${ }^{3}$

The Resilient Monitoring, Adaption, and Control (ReMAC) system purpose is to prevent catastrophic consequences of a cyber attack against critical infrastructure systems and increase the security of those systems. This research is establishing scientific- and engineering-based techniques to monitor and control critical infrastructure systems during malicious attacks in such a way that most sensors, actuators, and other physical assets can adapt and remain stable despite adverse conditions. The research objectives and purpose of this project are:

1. Develop innovative arrangements and its constituents for implementing resilient systems

2. Develop performance metrics, such as information quality, assessment quality, control quality, and measure of resiliency, that can be used online to reconfigure monitoring and control systems as needed based on assessed cyber and physical health

3. Develop and demonstrate methodologies for the effective deployment of resilient systems that can accommodate natural and malicious disturbances to both the monitored system and its information and control infrastructures

4. Develop and demonstrate resilient approaches that drive data needs, and select monitoring and control strategies according to time-varying cyber and physical requirements and assessment estimations.

\section{Summary}

In this project, we assume a set of sensors and actuators is used to observe and control an industrial process plant where there may be natural or malicious disturbances to the sensors, actuators, and the plant. Previously developed monitoring technologies are used to collect and interpret sensor observations for diagnosing plant health, while a supervisory controller is used to select the most appropriate controllers based on monitoring reports. Integration of these technologies results in a preliminary structure of the experimental ReMAC system. At this stage of the project, the monitored and controlled plant is emulated by INL's Machine Condition Monitoring (MCM) testbed in a Hardware-in-the-Loop setup, mimicking a cooling loop of a chemical reactor that includes a cold water supply, valve subsystems, and a simulated reactor subsystem (Figure 1). To demonstrate the efficacy of the ReMAC system, experiments were completed using various scenarios for physical anomalies (e.g., valve stuck or pipe congestion) and cyber attacks (e.g., introducing bias to sensor signals). As shown in Figure 2, the MCM testbed experiments showed that ReMAC is able to correctly assess system health within desired decision periods and consequently select appropriate controllers for acceptable system operations under diverse attack situations. 


\section{INSTRUMENTATION, CONTROL AND INTELLIGENT SYSTEMS}

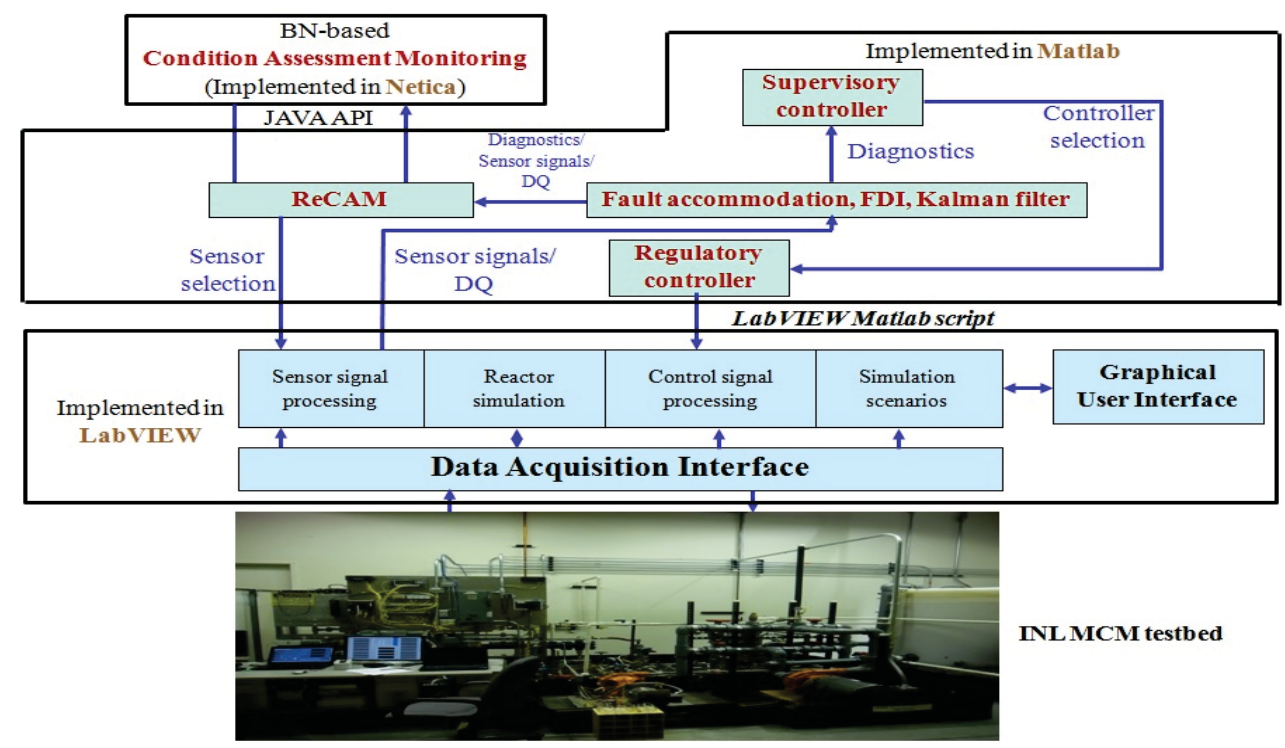

Figure 1. Application of ReMAC system to INL MCM testbed.
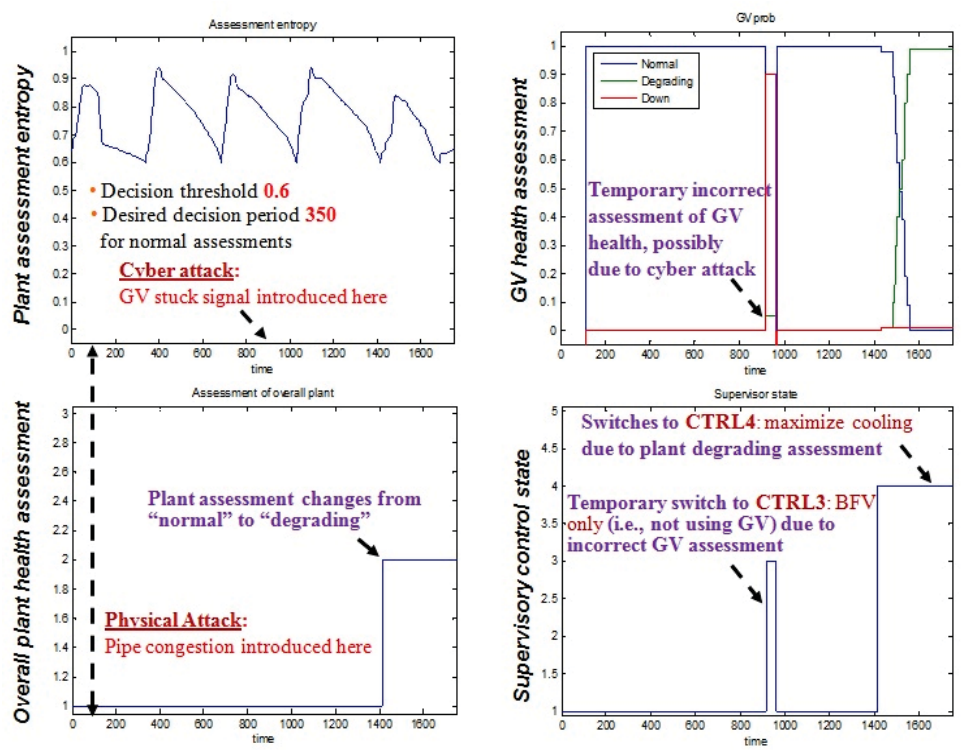

Figure 2. Demonstration of ReMAC system using INL MCM testbed.

\section{Benefits to DOE}

This project provides a path to addressing DOE's and the nation's need to prevent catastrophic consequences under compromising events to enhance energy security and safety. This research is establishing scientific- and engineering-based techniques for resilient monitoring and control of critical infrastructures under natural and malicious perturbation to sensors, actuators, and physical assets. As shown throughout the project, the developed monitoring and control system is applicable to various large systems with multiple unit operations such as energy systems (e.g., power plants) where a large amount of sensors and actuators are deployed and may be attacked. 


\section{Publications}

Garcia, H.E., S.M. Meerkov, and M.T. Ravichandran, "Combating Curse of Dimensionality in Resilient Plant Monitoring Systems: Overlapping Decomposition and Knowledge Fusion," Proceedings of the $19^{\text {th }}$ World Congress of the International Federation of Automatic Control, Cape Town, South Africa, August 24-29, 2014, Submitted.

Garcia, H.E., et al., "Resilient Plant Monitoring System: Design, Analysis, and Performance Evaluation," Proceedings of the IEEE $52^{\text {nd }}$ Conference on Decision and Control, Firenze, Italy, December 10-13, 2013, Accepted.

Lin, W.C. and H.E. Garcia, "Inclusion of Game-Theoretic Formulations for Resilient Condition Assessment Monitoring," Proceedings of the $6^{\text {th }}$ International Symposium on Resilient Control Systems, San Francisco, CA, August 13-15, 2013, pp. 96-103.

Garcia, H.E., et al., "Resilient Monitoring System for Boiler/Turbine Plant," Proceedings of the $6^{\text {th }}$ International Symposium on Resilient Control Systems, San Francisco, CA, August 13-15, 2013, pp. 104-110.

Garcia, H.E., et al., "Resilient Monitoring Systems: Architecture, Design, and Application to Boiler/Turbine Plant," IEEE T. Cybernetics, 2013, Submitted.

Garcia, H.E., S.M. Meerkov, and M.T. Ravichandran, "Resilient Monitoring System for Power Plant Design Addressing Curse of Dimensionality and Performance Analysis," IEEE Power Systems, 2013.

Garcia, H.E., et al., "Resilient Condition Assessment Monitoring for Cyber-Physical Systems," Automatica, 2013.

Lin, W.C., K.R. Kris, and H.E. Garcia, "Experimental Validation of a Resilient Monitoring and Control System," J. Process Contr., 2013, Submitted.

\section{Presentations}

Garcia, H.E., W.C. Lin, and S.M. Meerkov, "A Resilient Condition Assessment Monitoring System," Proceedings of the $5^{\text {th }}$ International Symposium on Resilient Control Systems, Salt Lake City, UT, August 14-16, 2012, pp. 98-105.

Garcia, H.E., et al., "Data Quality Assessment: Modeling and Application in Resilient Monitoring Systems," Proceedings of the $5^{\text {th }}$ International Symposium on Resilient Control Systems, Salt Lake City, UT, August 14-16, 2012, pp. 124-129.

Garcia, H.E., et al., "Resilient Monitoring System: Design and Performance Analysis," Proceedings of the $4^{\text {th }}$ International Symposium on Resilient Control Systems, Boise, ID, August 9-11, 2011, pp. 61-68. 


\title{
12-089 - Multi-agent Analysis to Characterize Complex Infrastructure Interdependencies
}

\author{
Craig Rieger, Milos Manic, ${ }^{1}$ Ronald Boring, Thomas Baldwin, Jonathan Butts, ${ }^{2}$ and John Doyle ${ }^{3}$
}

Microgrid is a common term used to define an electrical generation and distribution system for a smaller local grid, such as on a university campus or military base. Due to the nature of these systems and scale they are applied to, there will be very limited ability to recover the costs of supporting a staff to monitor and control them. Near full autonomy is therefore expected to reduce the human involvement necessary and self-diagnose problems when they occur. Unfortunately, modern control systems do not provide this capability, but are dependent on experts for configuration, implementation, and maintenance.

However, through the resilient control system approach taken in this effort, a framework that can support greater autonomy is being researched. This framework is notionally called a hierarchical, multiagent dynamic system (HMADS). The HMADS framework provides an adaptive, semi-autonomous mechanism for response to rapidly changing conditions with multiple complex interdependencies. Figure 1 provides a high level depiction of the HMADS and associated complexities for this project.

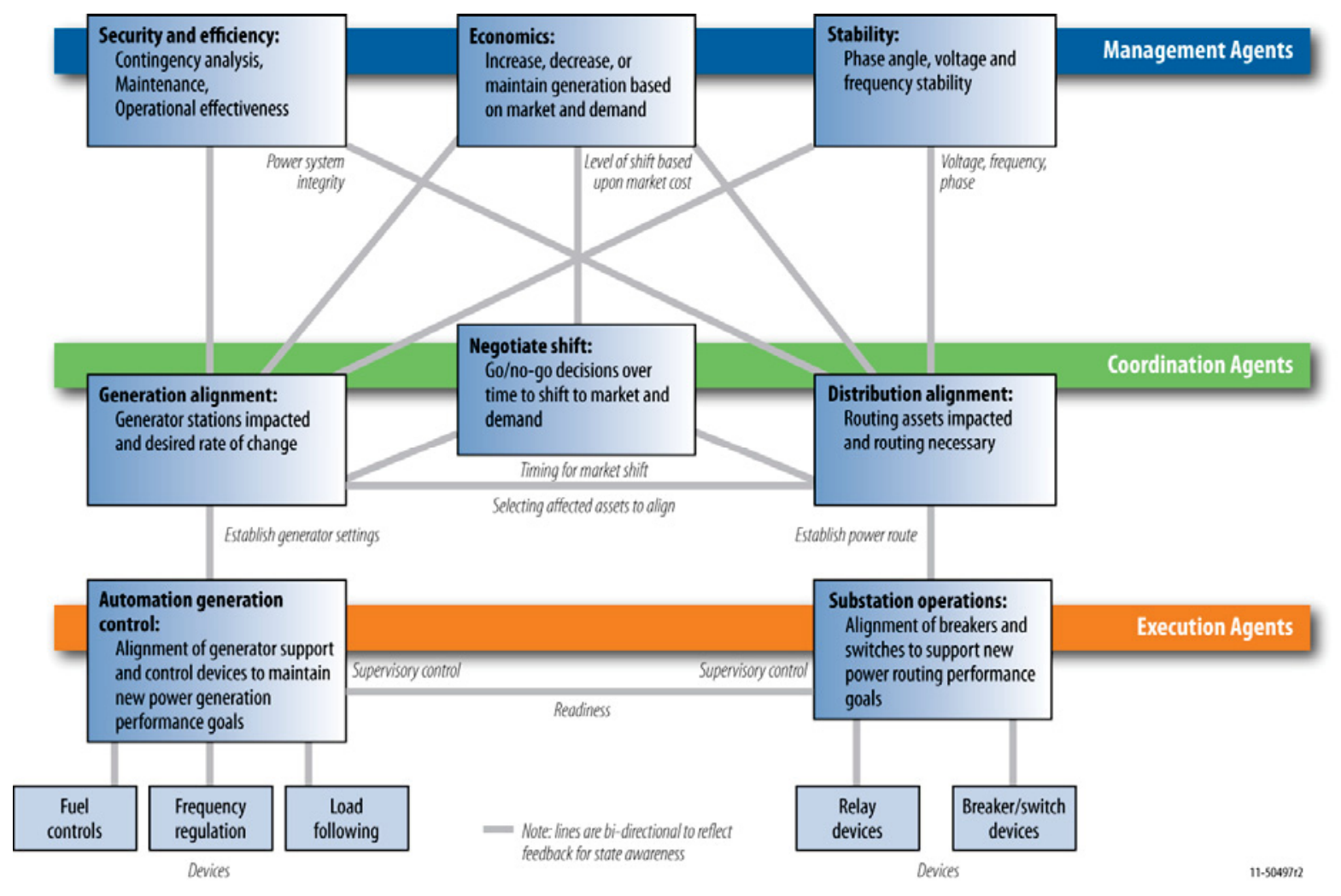

Figure 1. Multi-agent framework.

1. University of Idaho

2. Air Force Institute of Technology

3. California Institute of Technology 


\section{INSTRUMENTATION, CONTROL AND INTELLIGENT SYSTEMS}

Developing a multi-agent design first requires the decomposition of the philosophy of operation (command and control) from subject matter experts and leadership into requirements. As these requirements are human-based, the use of computational intelligence tools, fuzzy logic, and Bayesian belief networks, will be used to codify the management and coordination (agent) layers depicted in Figure 1. Effectively, the management layer is associated with the management policy for operating, such as determining when to perform market shifts. This is directly applicable to fuzzy logic, as leadership goals can be established and deconflicted within the membership functions and if/then rulebase. Bayesian design is applied to the coordination layer, which interprets the policy and realigns assets to direct this policy to the generators and substations. The resulting architecture ties to the control devices that adjust the power output of the generators and direct the power to the loads in the execution layer.

While this project is focused on microgrids, the developed HMADS provides a generalized framework for full autonomy with other critical infrastructure control systems. In addition, because most of the Instrumentation, Control and Intelligent Systems Distinctive Signature projects have a cyber security component, the Air Force Institute of Technology will be researching application of cyber security to the HMADS.

\section{Summary}

To develop an appropriate structure for testing of the HMADS design, a microgrid simulation (Figure 2) was developed by the University of Idaho. This computer-aided simulation allows different variations of a microgridand as a result, the effectiveness of the Management and Control Layers of the HMADS—-to be evaluated. Candidates for the fuzzy agent design of the Management and Control Layer have been developed, namely governing stability, efficiency, and economics, as depicted in Figure 3. The resulting microgrid simulation will also be used for other projects within the Instrumentation, Control and Intelligent Systems signature as a common reference, such as for use in evaluating human performance of grid systems with varying levels of autonomy.

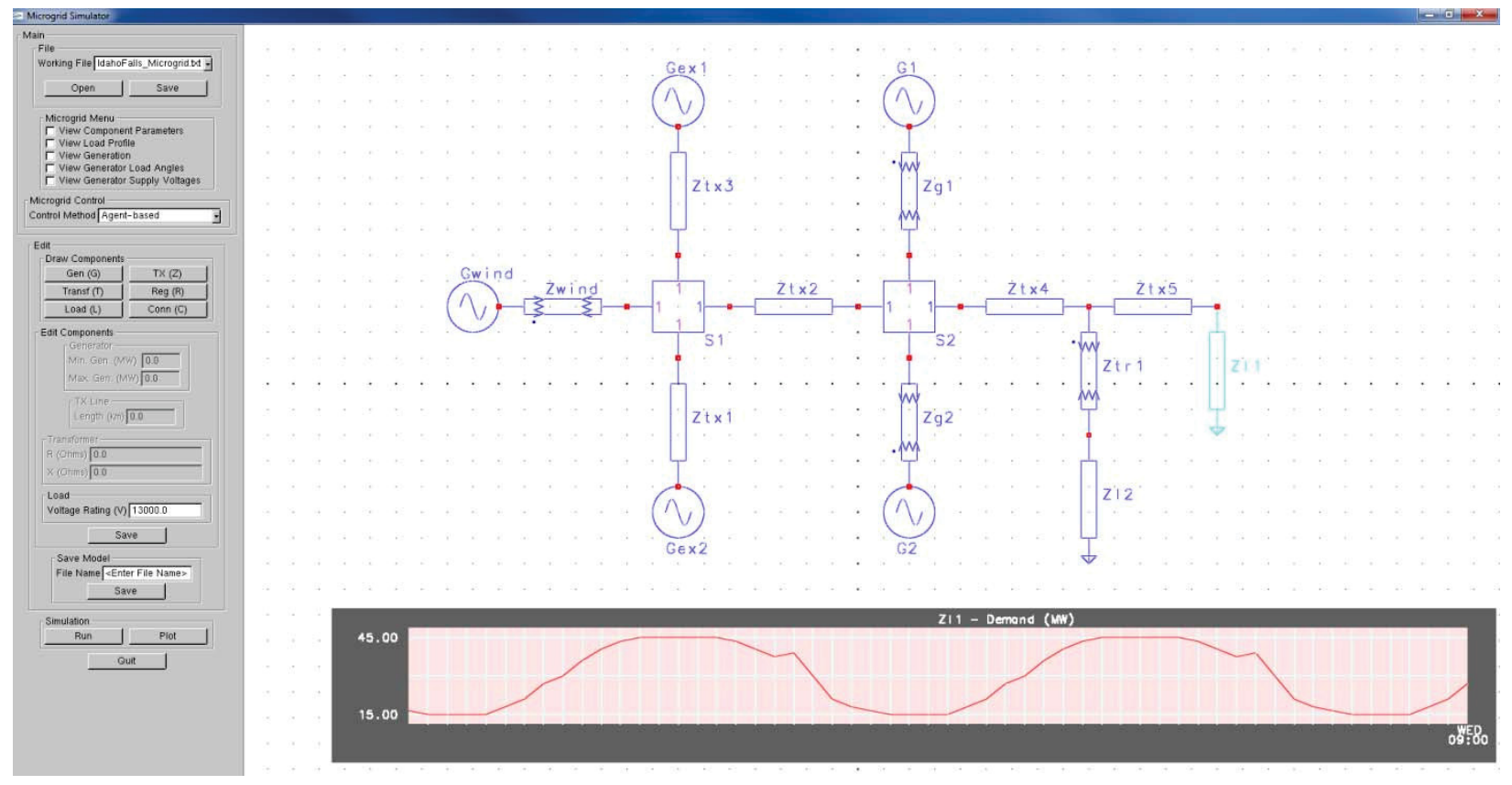

Figure 2. Microgrid Simulation Interactive Design Interface. 


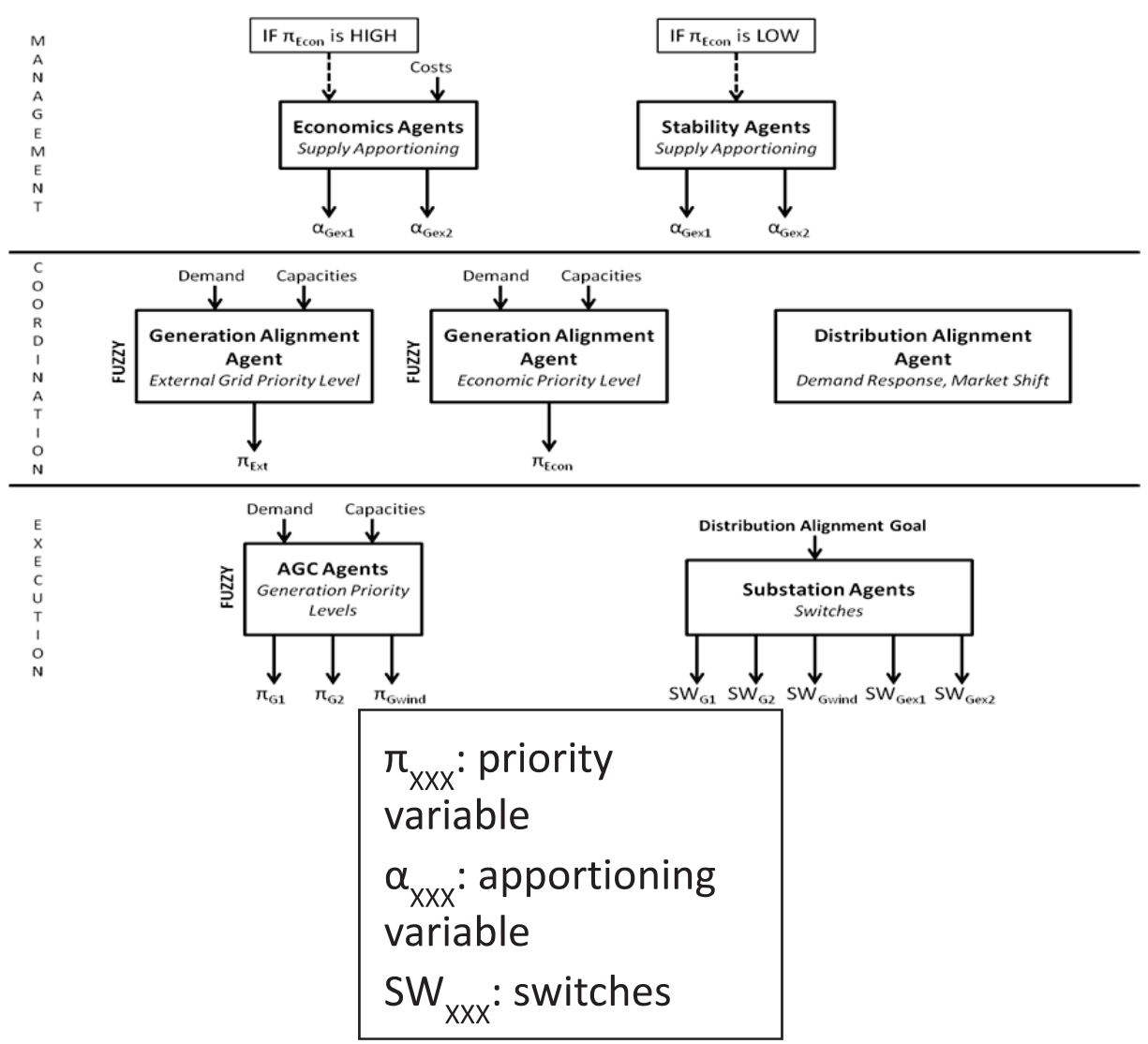

Figure 3. Multi-agent Design for Fuzzy Agent Strategy.

As a first step in the evaluation of a HMADS, fuzzy logic was directly applied to the agents shown in Figure 3 . This first step provided clear feedback on the performance of the fuzzy logic aspect before integrating the Bayesian aspect for the coordination layer. Several scenarios of this agent design were applied using this HMADS design, where variables included market shifts (economics) along with types and numbers of generators (stability) using the agent definitions below:

- Distribution Alignment Agents

- Demand Response based on available resources.

- Generation Alignment Agents

- Sets the priority on external grids to meet the demand $\left(\pi_{E x t}\right)$

- Sets the priority of economic reception of power from external grids $\left(\pi_{E c o n}\right)$.

- AGC Agents

- Sets the supply priority levels for each of the local generations $\left(\pi_{\mathrm{G} 1}, \Pi_{\mathrm{G} 2}, \Pi_{\mathrm{G} \text { wind }}\right)$.

- Economics Agents - IF $\pi_{E c o n}$ is HIGH

- Cost based apportioning of supply from external grids $\left(\alpha_{\mathrm{Gex} 1}, \alpha_{\mathrm{Gex} 2}\right)$. 
- Stability Agents - IF $\pi_{E c o n}$ is LOW

- Apportions the supply from the external grids $\left(\alpha_{\mathrm{Gex} 1}, \alpha_{\mathrm{Gex} 2}\right)$.

The scenarios were applied using a test grid that was designed fundamentally to represent the hydro-power grid of the local municipality. However, additional wind generation was added to provide more diversity of generation and a better understanding of the effectiveness of the fuzzy agents. The representation of this grid is shown in Figure 4. For all the economics and stability scenarios applied, it was recognized that the agent based logic performed effectively.

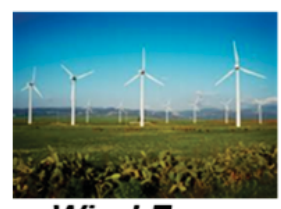

Wind Farm

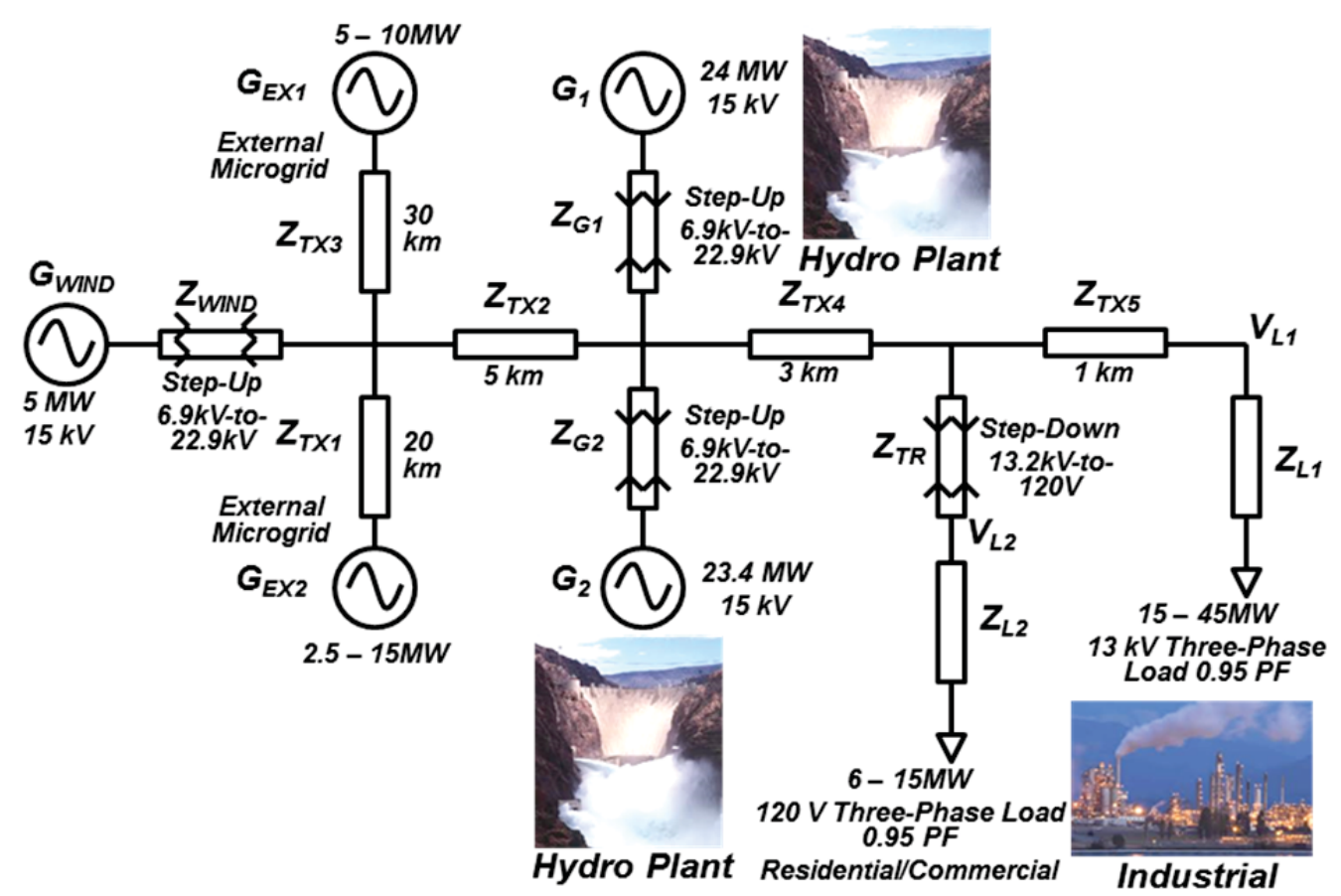

Figure 4. Scenario Grid for Testing Agent Strategy.

\section{Benefits to DOE}

This work supports DOE's mission of energy security through improved use of energy resources. The multifaceted nature of our energy infrastructure and the associated interdependencies will only get more complex with the implementation of digital technologies, such as designs targeted to automate the Smart Grid. While some of these advances can actually lead to more resilience, a true holistic and multi-disciplinary approach to control system design has not been taken. Specifically, more automation is implemented, but the necessary modeling of the associated complexity has not been demonstrated. This research promises to provide this modeling aspect as a real world Microgrid example, which can then further vet the underlying framework proposed. In addition, cyber threat poses a new paradigm for dynamic control systems. Therefore, cyber awareness is a key component of resilient control system research, and the actions of the malicious intruder are a design factor in this effort. 


\section{Presentations}

Ravishankar, U., M. Manic, and C. Rieger, "A Micro-Grid Simulator Tool (SGridSim) Using Effective Node-toNode Complex Impedance (EN2NCI) Models," $20136^{\text {th }}$ International Symposium on Resilient Control Systems, San Francisco, CA, August 13-15, 2013.

Rieger, C., and Q. Zhu, "A Hierarchical Multi-Agent Dynamical System Architecture for Resilient Control Systems," $20136^{\text {th }}$ International Symposium on Resilient Control Systems, San Francisco, CA, August 13-15, 2013.

Rieger, C., K. Moore, and T. Baldwin, "Resilient Control Systems: A Multi-Agent Dynamic Systems Perspective," 2013 IEEE International Conference on Electro/Information Technology, Rapid City, SD, May 9-11, 2013.

Rieger, C. and K. Villex, "Resilient Control System Execution Agent (ReCoSEA)," $20125^{\text {th }}$ International Symposium on Resilient Control Systems, Salt Lake City, UT, August 14-16, 2012.

Rieger, C., Q. Zhu, and T. Başar, "Agent-Based Cyber Control Strategy Design for Resilient Control Systems: Concepts, Architecture and Methodologies," $20125^{\text {th }}$ International Symposium on Resilient Control Systems, Salt Lake City, UT, August 14-16, 2012. 


\section{2-121 - State Awareness for Smart Grid Electrical Load Balancing, Distribution, and Security}

Victor Walker, Ronald Boring, and Timothy McJunkin

Increasingly networked and complicated control systems create correspondingly increased control/human interdependencies and security risks. Providing the most essential information needed (state awareness) to human operators during a natural or malicious event will enable these operators to respond calmly and efficiently. This LDRD improves human interaction with critical and potentially compromised systems, particularly Smart Grids, enabling the system to remain resilient and secure.

Every control system contains an element of human interaction.

Often the most impactful component of a control system is how the humans in the system respond and act. Their understanding of the current state of the system and their response to that system will have a large impact on the ability of a system to respond to changing environments. The resilience of the human/controller system is related to three major aspects of state awareness: first, how the system and the human understand the goals of the system, secondly how the human and system understand how the current state might impact the overall system

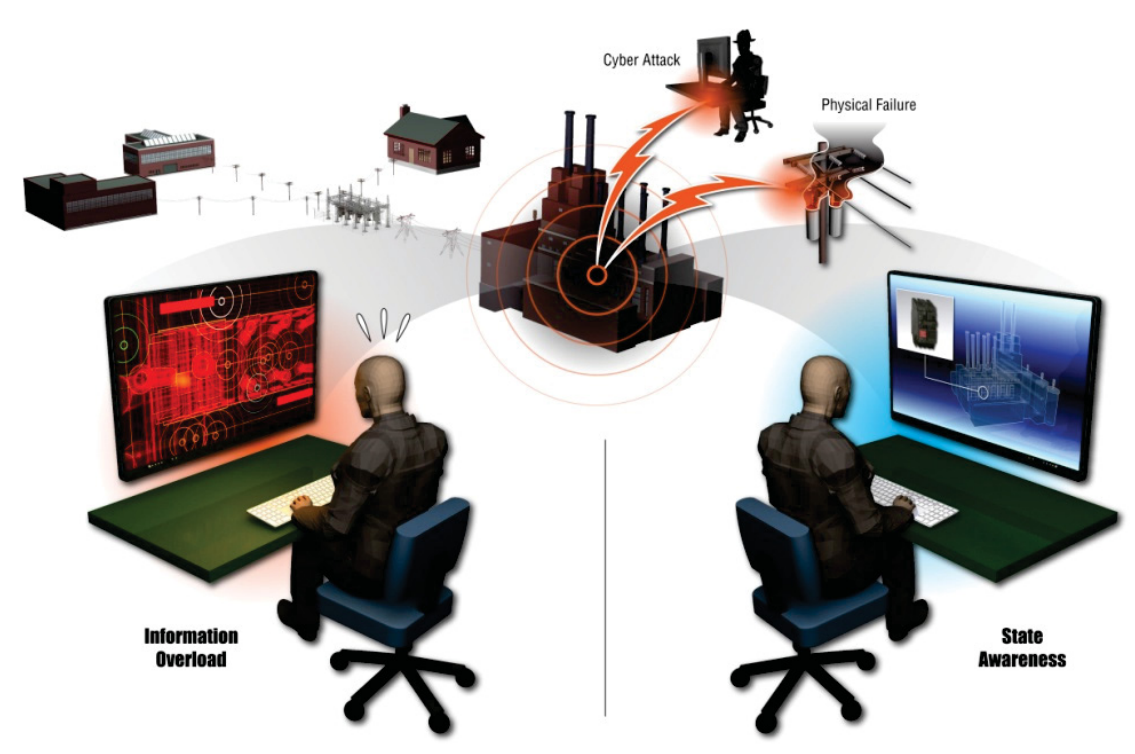

Figure 1. The objective of this project is to improve human interaction with critical and potentially compromised systems, particularly Smart Grids, enabling the system to remain resilient and secure.

goals, and finally how the system and humans can determine the potential actions and their impact on the system's goals.

This project focuses on science and methods for improving control system interactions with human operators. The tasks identified included defining metrics and measures for state awareness, designing the infrastructure and models to better identify state and goal states, creating agents and models for intelligent interactions with the human operators, and performing actual evaluations with human operators to determine the effectiveness of the different models in improving state awareness and decision making.

\section{Summary}

The project addresses critical components in learning about state awareness and designing resilient interactions with users. The project is developing and documenting base-concepts around a robust architecture, creating a language for objectives and goal states, developing methods for impact determination, developing concepts for 


\section{INSTRUMENTATION, CONTROL AND INTELLIGENT SYSTEMS}

visualization paradigms, creating definitions of state awareness metrics, and creating a framework for testing of the technologies and concepts.

The robust architecture designed by the team was based around the concepts of a physiological control architecture. Based on observations of the control systems in the human body and other natural forces, we developed an overarching control system framework that implemented three layers of integrated control. The lowest Stability Layers allow for localized control using negative feedback loops and supporting loops. These layers react to thresholds set at higher levels to make localized decisions. The Intention Layer represents the goal states and the impacts the current system state has on the goal state. And finally, in the Cognition Layer, the control system shapes a tailored state-awareness picture for the operator awareness and response.

As a part of the structure of the architecture and the testing system, the team created methods for articulating the goals of the system in terms of data elements and for using those data elements to determine the impacts of fluctuations in the goal state. These systems feed the Intention Layer and help determine what visualization is available to the Cognition Layer.

The team identified three methods of visualization for the Cognition Layer that were to be tested and evaluated in relation to presenting state awareness. First was a geographic/connectivity method that focuses on relationships between elements and highlights changes in a connected picture of the overall state. The second was the Impact Visualization method that focuses on the goal state determined by the user and system, and then references the potential impacts of value changes in terms of the goal state. The third method offers dynamic information scaling that utilizes an agent observing the user and applies a model of their cognition to highlight and emphasize elements key to their needs.

To prepare for testing these visualization methods, the team worked with Idaho State University to create a system for interacting with an electrical grid model and dynamically adjusting and modifying the grid model with a realistic interface. We also extended and modeled a user interface system to allow for observation of user attention based upon their mouse or touch movement and the identifying of data sources and types for each element of the interface. We developed an interface language for the interaction and we began work on the observation and statistical agents.

The team identified several potential methods for evaluating state awareness and for quantifying the sense of "team cognition," or the ability of the intelligent controllers and human operators/decision makers to share the understanding of the current state, impact, and decision possibilities for the system. In the current plan, we have proposed to use a five phase approach, representing the attention paid to each data element and the response to events. These are gathered, weighted, and compared to system response to determine joint cognition. The principles and methods identified and developed will influence interface design and methodologies for complex system interactions in many different environments.

\section{Benefits to DOE}

This project will benefit DOE's energy security mission by providing ways to help operators of critical infrastructure systems to better understand their state and to respond appropriately to any natural occurring or malicious event, such as a cyber attack. Experimental results will assist system operators in making more relevant and appropriate decisions when overloaded with information and to more quickly resolve issues - thus increasing overall system reliability and resilience. 


\section{Presentations}

Walker, V., "ICIS Distinctive Signature annual review," August 12, 2013.

Walker, V., "ICIS Distinctive Signature annual review," August 13, 2012. 


\title{
13-120 - Improved Control System Resilience via Secure Machine Learning
}

\author{
Miles McQueen, Timothy McJunkin, Denis Vollmer, Anthony Joseph, ${ }^{1}$ and Doug Tygar ${ }^{1}$
}

In many ways, current intrusion detection systems are not ideally suited for resilience. First, these systems look at historical patterns, whether signature-based or anomaly-based, which are not necessarily predictive of what may be seen in the future from an intelligent adversary's attack. Second, these detection methods are not foolproof and invariably require unfortunate tradeoffs between false positives and false negatives. Lastly, applying restrictions on traffic flow as the result of a detected threat may end up limiting functionality of a control system's communications for no valid reason and may even be used by an attacker in a denial-of-service attack. The goal of this Secure Machine Learning LDRD is to develop a set of technologies that attempt to address these shortcomings in order to improve control system resilience. This research establishes an engineering- and experimentally-based framework for improved and reliable detection of cyber attacks against control systems in critical infrastructure (e.g., the power grid). University of California at Berkeley and Idaho State University are research partners on this project.

\section{Summary}

This project formally kicked off in April of 2013 with a meeting held at the University of California at Berkeley. Since then, the University of California at Berkeley has established a strong working relationship with two private repositories of attack information: VirusTotal and McAfee. The repository data is currently undergoing analyses to identify attacks on machine learning algorithms. It is already clear that these attacks are, in fact, occurring.

During this same timeframe, the INL focused on enhancement of a testbed in CAES to enable testing and validation of the intended new and improved Secure Machine Learning techniques. An enhanced Secure Machine Learning testbed, representing coupled critical infrastructure, was designed and implemented. The testbed is comprised of several control systems tied together through a common power infrastructure with background network traffic provided by the previously developed wireless testbed. The testbed includes a communication network on which an intrusion detection system or Intelligent Cyber Sensor may be inserted or attached as a layer of defense against attacks. In implementation, the new machine learning algorithms will reside in this device.

The control systems incorporated into the testbed are shown in Figure 1. The power infrastructure part of the platform has been developed in cooperation with Idaho State University's Energy Systems and Education Center. Grid in a Box provides outputs to the loads on the system with relays that may allow them to be subject to demand response. The testbed is also comprised of generation sources, including battery storage units. An Allen Bradley programmable logic controller controls the system with relays and generation based on feedback from current transformers and voltage sensors. The Grid in a Box is interconnected to other control systems as the power source for the actuators and controllers.

1. University of California, Berkeley 


\section{INSTRUMENTATION, CONTROL AND INTELLIGENT SYSTEMS}

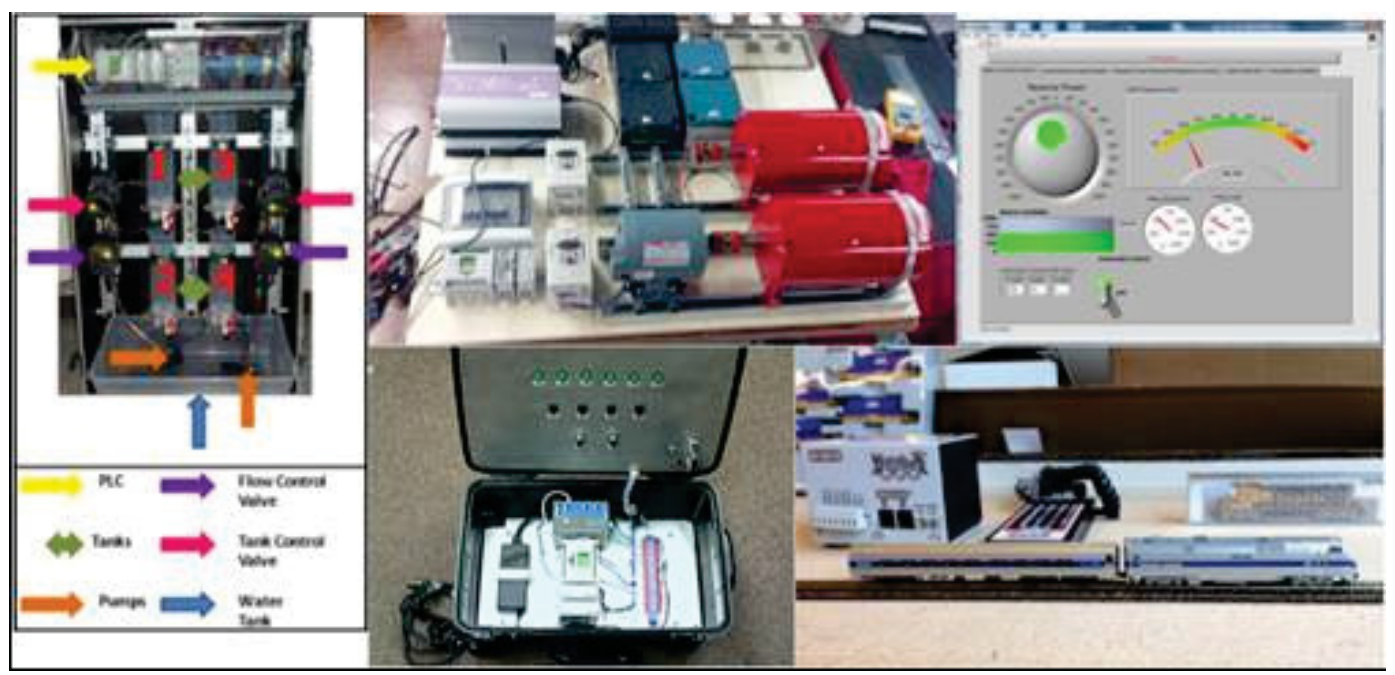

Figure 1. A collage of the enhancements to the testbed for machine learning security experiments include: (clockwise from top left) four tank control, Grid in a Box prototype, control screen for Grid in a Box, the scale model layout of train, and the environmental control system.

A dual-loop, track scale model train was also constructed with two engines and controllers that allow the individual engines to be commanded and track-switching semaphores to switch the trains from one loop to another. The basic concept allows for trains to nominally share the track through scheduled and supervised control and provides the opportunity to illustrate an attack on the control or the grid powering the train, causing a loss of service or more dramatic issues (e.g., a crash or derailment) should attacks be successful.

Other control components of the testbed include an environmental control system designed to maintain temperature of a volume of air (representing the heating/cooling system of a building) and a four-pump control system previously used in the University Resilient Control System Challenge.

Each control system in the testbed has connectivity to a network with many computation devices attached. This provides some similarity to Supervisory Control and Data Acquisition devices that are interconnected to a wider network for efficiency and convenience in supervisory control.

\section{Benefits to DOE}

This research supports DOE's nuclear security mission by establishing an engineering- and experimentally-based framework for improved and reliable detection of cyber attacks against control systems in critical infrastructure (e.g., the power grid). In particular, the framework will be based on game theoretic approaches and include advanced machine learning algorithms for attack detection. The enhanced integrated detection system will be beneficial in protecting the nation's critical infrastructure against cyber attacks.

\section{Presentations}

Tygar, J.D., "Adversarial Machine Learning," Resilience Week 2013, San Francisco, CA, August 13-15, 2013. 


\section{Author Index}

A

Akers, Douglas, 18, 139

Allahar, Kerry, 224

Andrs, David, 71

\section{B}

Bakas, Michael, 90, 91

Baldwin, Thomas, 111, 115, 251

Ban, Heng, 191

Barnes, Joni, 125, 151

Bateman, Ken, 224

Bauer, Bill, 125

Bier, Vicki, 189

Biner, S. Bulent, 49, 61, 67

Boring, Ronald, 176, 212, 251, 256

Bruemmer, David, 203

Buettner, Gary, 193

Buongiorno, Jacopo, 171

Burgett, Eric, 39, 77

Butt, Darryl, 224

Butts, Jonathan, 251

\section{C}

Cannon, W. Roger, 13

Cantonwine, Paul, 18

Cárdenas, Edna, 98

Carney, Kevin, 107

Carroll, Laura, 61

Carroll, Mark, 42, 61

Castle, Brian, 102

Chandler, Kevin, 128

Chernatynskiy, Aleksandr, 191

Chichester, David, 37, 87

Christophersen, Jon, 245

Chu, Henry, 90, 94

Clement, Ryan, 96, 102

Coats, Erik, 217

Cole, James, 35

Condit, Reston, 100

Corradini, Michael, 189

Crawford, Anthony, 2
Cristian Rabiti, 73

Curtis, Daniel, 198

Czerwinski, Ken, 6

D

Daniels, Michael, 100

Daubaras, Dayna, 138, 153

Deaton, Juan, 109

DeHart, Mark, 73

Delmore, James, 105

Deo, Milind, 131

Derr, Kurt, 92

Dolbow, John, 69

Doyle, John, 251

Drigert, Mark, 18

Du, Wenbo, 147

Dufek, Eric, 52, 123, 134, 139, 151

Dyre, Brian, 212

E

Eapen, Jacob, 231

El Kadiri, Haitham, 165

Elias, Gracy, 63

Estep, Don, 233

F

Fairley, Jerry, 227, 228

Farzbod, Farhad, 147

Feris, Kevin, 217

Fielding, Randall, 35

Forsberg, Charles, 198

Fox, Robert, 119

Fromme, Dave, 111

G

Gan, Jian, 80, 191

Ganapol, Barry, 21, 73

Garcia, Humberto, 147, 245, 248

Gardner, John, 208

Gaston, Derek, 67, 69, 71, 72

Gerberich, William, 168 
Gertman, David, 203, 212

Giani, Annarita, 242

Glazoff, Michael, 136, 139

Gorman, Brian, 28

Gougar, Hans, 44

Grady, Mack, 111

Groenewold, Gary, 63, 105

Gundogdu, Kenan, 184

Guo, Luanjing, 128

H

Hales, Jason, 61, 69

Harrup, Mason, 134

Henriksen, Emily, 151

Henriksen, James, 125

Herbst, R. Scott, 182

Hill, Tony, 18, 39, 77

Hintsala, Eric, 168

Hiromoto, Robert, 65

Hongbin Zhang, 73

Hoover, Robert, 224

Howe, Troy, 187

Huang, Hai, 69, 128, 131

Hunt, Alan, 98

\section{J}

Jackson, John, 168

Jewell, Keith, 39, 77

Johnson, James, 37, 87

Joseph, Anthony, 259

K

Karpeev, Dmitry, 67, 68, 71

Kelle, David, 115

Kennedy, Rory, 80

Khafizov, Marat, 184

King, James, 151

Kinlaw, Mathew, 98

Klaehn, John, 123, 134, 144

Klein, Ross, 42

Kohse, Gordon, 57

Kong, Peter, 136

Koudelka, John, 161
L

Lacy, Jeffrey, 90

Ladefian, Robert, 42

Langhorst, Benjamin, 90, 94

Larsen, Jason, 83

Lessing, Paul, 13

Lin, Wen-Chiao, 248

Linda, Ondrej, 242

Lister, Tedd, 138

Loera, Jose, 92, 109

M

Mandelli, Diego, 237, 239

Manic, Milos, 83, 203, 208, 242, 251

Mariani, Robert, 35

Martin, Leigh, 9, 221

Martineau, Richard, 21, 73, 80

Mattson, Earl, 158

McBride, Scott, 111

McCurry, Michael, 227

McDonald, Armando, 217

McGrath, Chris, 107

McJunkin, Timothy, 256, 259

McKellar, Michael, 198

McQueen, Miles, 83, 242, 259

Meerkov, Semyon, 248

Metesh, John, 128

Meyer, Mitch, 80

Miller, Amber, 125, 155

Millett, Paul, 49

Mincher, Bruce, 63, 123, 163, 193

Mincher, Elias, 63, 123, 163, 193

Mincher, Mary, 123

Mink, Roy, 227

Mkhoyan, Andre, 168

Mohanty, Amit, 147

Molitor, Jeff, 2

Moradi, Hussein, 109

Morel, Jim, 73

Mosleh, Ali, 176

$\mathbf{N}$

Natrajan, Louise, 9

Nazar, Linda, 180 
Newby, Deborah, 125, 217

s

Nielsen, Joseph, 65

Nilsson, Mikael, 182

0

O'Brien, James, 142

O'Brien, Robert, 90, 187, 200

Ogden, Mark, 123

Okuniewski, Maria, 18

Orme, Christopher, 144

Sabharwall, Piyush, 31, 195

Sazhin, Sergiy, 134

Schultz, Michael, 193

Sharrad, Clint, 9

Silverman, Heather, 155

Simpson, Michael, 224

Sintay, Stephen, 28

Smidts, Carol, 176

Smith, Barton, 31

Smith, Kord, 21, 73

Solstad, Steinar, 57

Pak, Joshua, 119

Palmer, Carl, 128

Pantoya, Michelle, 100

Paredis, Chris, 147

Pascucci, Valerio, 237, 239

Paviet-Hartmann, Patricia, 6

Permann, Cody, 71

Peterson, John, 71, 233

Sondrup, A. Jeffrey, 128

Spencer, Benjamin, 69

Stanek, Chris, 80

Stewart, Frederick, 134

Stoots, Carl, 31, 187, 200

Szlufarska, Izabela, 184

Petkovic, Lucia, 139

Pfeiffer, Jana, 107

Phongikaroon, Supathorn, 224

Plummer, Mitchell, 128, 158, 227

Poolla, Kameshwar, 242

Pope, Michael, 44

\section{$\mathbf{Q}$}

Qiang, You, 221

$\mathbf{R}$

Rabiti, Cristian, 73

Ramirez, Sam, 92

Rasmussen, Nikki, 100

Ravichandran, Maruthi, 248

Reed, David, 155

Reid, Carol, 111

Reiten, Jon, 128

Rempe, Joy, 57

Richard Martineau, 21, 73, 80

Riddle, Catherine, 6

Rieger, Craig, 208, 251

Ryskamp, John, 44

W

Wai, Chien, 163

Walker, Victor, 256

Tavener, Simon, 233

Teague, Melissa, 28, 54

Teysseyre, Sebastien, 57

Thompson, Scott, 87, 98, 102

Thompson, Vicki, 151, 155

Thornton, Katsuyo, 67

Tokuhiro, Akira, 65

Tonks, Michael, 28, 49, 61, 67

Tripathy, Prabhat, 52

Tygar, Doug, 259

Utgikar, Vivek, 195

Van Den Wymelenberg, Kevin, 208

Villez, Kris, 248

Vlachos, Pavlos, 31

Vollmer, Denis, 259

U

V 


\section{AUTHOR INDEX}

Wang, Bei, 237, 239

$\mathbf{Y}$

Wang, Yaqi, 73, 74

Warren, Ian, 227

Watson, Scott, 37, 87

Welhan, John, 227

Wellman, Lawrence, 83

Wendt, Daniel, 195

West, James, 87

West, Mike, 115

West, Shawn, 111

Wijayasekara, Dumidu, 83, 207

Williamson, Richard, 67

Wilson, Aaron, 123, 134, 144

Wright, Jason, 83

Wright, Richard, 42

$\mathrm{Wu}$, Yaqaio, 221

Yang, Yong, 191

Yildiz, Bilge, 173

Youngblood, Robert, 171, 189

Yu, Jianguo, 136, 231

Yurko, Joseph, 171

Z

Zagula, Thomas, 90

Zalupski, Peter, 180, 182, 193

Zhang, Hongbin, 73

Zhang, Xiaoyu, 142

Zhang, Yongfeng, 49, 67 
APPENDICES 


\section{Appendix A}

\section{Commonly Used Acronyms}

The following acronyms are used in many of the project descriptions.

\begin{tabular}{ll} 
ATR & Advanced Test Reactor \\
BIGHORN & Neutronic Solver for Prismatic Geometry Computer Code \\
CAES & Center for Advanced Energy Studies \\
CAVETM & Computer Assisted Virtual Environment \\
CAMS & Center for Advanced Modeling and Simulation \\
CRADA & Cooperative Research and Development Agreement \\
DOE-NE & Department of Energy Office of Nuclear Energy \\
FY & fiscal year \\
INL & Idaho National Laboratory \\
IRC & Idaho National Laboratory Research Center \\
LDRD & Laboratory Directed Research and Development \\
MARMOT & Mesoscale Model Application Computer Code \\
MFC & Materials and Fuels Complex \\
MOOSE & Multiphysics Object Oriented Simulation Environment \\
NSUF & National Scientific User Facility \\
PI & Principal Investigator \\
R\&D & Research and Development \\
RELAP5 & Thermal-Hydraulic Analysis Code \\
\hline
\end{tabular}




\section{Appendix B}

\section{Relevance to Major National Programs}

\begin{tabular}{|c|c|c|c|c|c|c|c|c|c|c|c|c|c|}
\hline $\begin{array}{l}\text { Tracking } \\
\text { No. }\end{array}$ & PI Name & Title & 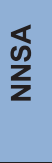 & ш & $\sum_{\boldsymbol{W}}$ & 岀 & u & ш & $\frac{\alpha}{z}$ & $\sum_{\substack{\frac{1}{u} \\
0}}$ & 亗 & 壳 & $\Sigma$ \\
\hline $10-031$ & Jianguo Yu & $\begin{array}{l}\text { Fickian and Thermal Diffusion in Nuclear } \\
\text { Materials from Linear Response Theory } \\
\text { and Multiscale Simulations }\end{array}$ & & & & & $S$ & $\mathrm{P}$ & & $S$ & & & \\
\hline $11-000$ & Anthony Crawford & $\begin{array}{l}\text { Haptic Tele-Operated End Effector for } \\
\text { Hazardous Environments }\end{array}$ & $S$ & & $S$ & & & $\mathrm{P}$ & $S$ & $S$ & & $S$ & \\
\hline $11-005$ & Catherine Riddle & $\begin{array}{l}\text { Speciation Behavior of Americium } \\
\text { Oxidation States for the Separation of } \\
\text { Americium from Curium in Nuclear } \\
\text { Processing }\end{array}$ & & $S$ & & & & $\mathrm{P}$ & & & & & \\
\hline $11-006$ & Mincher, Bruce & $\begin{array}{l}\text { Supercritical Fluid Extraction of Actinides } \\
\text { from TRISO Reactor Fuels }\end{array}$ & & & S & & & $\mathrm{P}$ & & $S$ & & & \\
\hline $11-007$ & Baldwin, Thomas & $\begin{array}{l}\text { Integration of Renewable Energy } \\
\text { Resources Through Energy Storage and } \\
\text { Supergrid }\end{array}$ & & $P$ & & & & & & & $S$ & & \\
\hline $11-023$ & Leigh Martin & $\begin{array}{l}\text { Development of a simplified soft donor } \\
\text { technique for trivalent lanthanide actinide } \\
\text { separations }\end{array}$ & & & $S$ & & $S$ & $\mathrm{P}$ & & $S$ & & & S \\
\hline $11-041$ & Bai, Xianming & $\begin{array}{l}\text { In situ Micro-raman Spectroscopy and } \\
\text { Modeling of Breakaway Oxidation of } \\
\text { Zircaloy Cladding }\end{array}$ & $S$ & $S$ & $S$ & & $S$ & $\mathrm{P}$ & $S$ & $S$ & & $S$ & \\
\hline $11-042$ & Miles McQueen & $\begin{array}{l}\text { Experimental Cyber Security - Vulnerability } \\
\text { Prediction, Discovery, and Mitigation }\end{array}$ & & & & & $S$ & $\mathrm{~S}$ & & & $P$ & & \\
\hline $11-043$ & Jackson, John & $\begin{array}{l}\text { Characterization of Nanomechanical } \\
\text { Response of Material to Determine Key } \\
\text { Variables in Stress Corrosion Cracking }\end{array}$ & & & & & $S$ & $\mathrm{P}$ & $S$ & & & & \\
\hline $11-046$ & Miles McQueen & $\begin{array}{l}\text { Known Secure Sensor Measurements for } \\
\text { Detection Unauthorized Process } \\
\text { Manipulation and Falsification of State }\end{array}$ & & & & & $S$ & S & & & $P$ & & \\
\hline $11-051$ & Paul Lessing & $\begin{array}{l}\text { Alternative Processing for Improved UO2 } \\
\text { Based Fuels }\end{array}$ & & & & & & $\mathrm{P}$ & & & & & \\
\hline $11-055$ & Doug Akers & $\begin{array}{l}\text { Spatially Resolved Positron Analysis of } \\
\text { Hydride Formation in LWR Fuels with GE - } \\
\text { Gloval Nuclear Nuclear Fuels }\end{array}$ & & & & & & $\mathrm{P}$ & & $S$ & & & \\
\hline $11-059$ & David Chichester & $\begin{array}{l}\text { Unconventional Radiation Detection } \\
\text { Methods for Nuclear Nonproliferation, } \\
\text { Safeguards, and Treaty Verification }\end{array}$ & $P$ & & & & S & S & & & & & \\
\hline $11-060$ & Henry Chu & $\begin{array}{l}\text { SMC Advanced Armor Materials and } \\
\text { Systems R\&D }\end{array}$ & $S$ & & & & $P$ & S & & & & & \\
\hline $11-061$ & Rich Martineau & $\begin{array}{l}\text { Advanced Modeling and Simulation } \\
\text { Concepts for ATR }\end{array}$ & & & & & & $\mathrm{P}$ & S & & & & \\
\hline $11-065$ & Gertman, David & $\begin{array}{l}\text { Development and Demonstration for } \\
\text { National Fuel Efficiency }\end{array}$ & & S & & $S$ & & & & & & $\mathrm{P}$ & \\
\hline $11-066$ & Rieger, Craig & $\begin{array}{l}\text { Targeted Energy Management Toolset for } \\
\text { Comfort and Savings Based on Advanced } \\
\text { Computational Intelligence Techniques }\end{array}$ & & $P$ & & $S$ & & & & & S & & \\
\hline
\end{tabular}




\begin{tabular}{|c|c|c|c|c|c|c|c|c|c|c|c|c|c|}
\hline $\begin{array}{l}\text { Tracking } \\
\text { No. }\end{array}$ & PI Name & Title & 选 & 岃 & $\sum_{\text {W }}$ & ш & U & ш & $\frac{\alpha}{z}$ & $\sum_{\substack{\alpha \\
0}}$ & 亗 & 意 & $\Sigma$ \\
\hline $11-074$ & Peterson, J.W. & $\begin{array}{l}\text { Uncertainty Analysis for Multiscale Models } \\
\text { of Nuclear Fuel Performance }\end{array}$ & $S$ & & $S$ & $S$ & $S$ & $\mathrm{P}$ & & & & & \\
\hline $11-076$ & Diego Mandelli & $\begin{array}{l}\text { Exploratory Nuclear Reactor Safety } \\
\text { Analysis and Visualization via Integrated } \\
\text { Topological and Geometric Techniques }\end{array}$ & $S$ & & & $S$ & $S$ & $\mathrm{P}$ & & & & & \\
\hline $12-001$ & Kurt Derr & $\begin{array}{l}\text { Long Term Evolution Wireless Cyber } \\
\text { Security }\end{array}$ & $S$ & & & & $P$ & $S$ & & & $S$ & & \\
\hline $12-007$ & Robert Youngblood & $\begin{array}{l}\text { Uncertainty Quantification of Safety Codes } \\
\text { using a Bayesian Approach with Data from } \\
\text { Separate- and Integral-Effects Tests }\end{array}$ & & & & & & $\mathrm{P}$ & $S$ & & & & \\
\hline $12-017$ & Benjamin Langhorst & $\begin{array}{l}\text { Polymer Components in Laminate Armor } \\
\text { Systems for Ballistic Protection }\end{array}$ & & & & & & & & & & $P$ & \\
\hline $12-026$ & $\begin{array}{l}\text { Xianming "David" } \\
\text { Bai }\end{array}$ & $\begin{array}{l}\text { Protectiveness and Stability of the } \\
\text { Zirconium Oxide in Early-Phase Corrosion } \\
\text { of Zirconium Alloys - Predictive Relations } \\
\text { to Surface Structure and Composition }\end{array}$ & & & & & $S$ & $\mathrm{P}$ & & & & & \\
\hline $12-031$ & Melissa Teague & $\begin{array}{l}\text { 2-D and 3-D EBSD Technique } \\
\text { Development and Microstructure } \\
\text { Reconstruction for Phase Field } \\
\text { Microstructure Evolution Models }\end{array}$ & & & & & & $\mathrm{P}$ & & $S$ & & & \\
\hline $12-043$ & Ronald Boring & $\begin{array}{l}\text { Science-Based Simulation Model of } \\
\text { Human Performance for Human Reliability } \\
\text { Analysis }\end{array}$ & & & & & & $\mathrm{P}$ & $S$ & & & & \\
\hline $12-045$ & Piyush Sabharwall & $\begin{array}{l}\text { Development of an Uncertainty } \\
\text { Quantification Method for MIR Facility }\end{array}$ & & $S$ & $S$ & $S$ & S & $\mathrm{P}$ & & & & & $S$ \\
\hline $12-050$ & Ryan Clement & $\begin{array}{l}\text { Cross Section Representations for Nuclear } \\
\text { Nonproliferation }\end{array}$ & $\mathrm{P}$ & & & & $S$ & $S$ & & & & & \\
\hline $12-054$ & Mathew Kinlaw & $\begin{array}{l}\text { Treaty Verification with Photo-Induced } \\
\text { Prompt Signatures }\end{array}$ & $\mathrm{P}$ & & & & & & & & & & \\
\hline $12-056$ & Peter Zalupski & $\begin{array}{l}\text { Recovery of Precious Metals from Used } \\
\text { Nuclear Fuel and Electronic Waste Using } \\
\text { Sulfur-Impregnated Nanoscaffolds }\end{array}$ & & & $S$ & & & $\mathrm{P}$ & & & & & \\
\hline $12-059$ & R. Scott Herbst & $\begin{array}{l}\text { Chemistry and Flowsheet Development for } \\
\text { Lanthanide Separations }\end{array}$ & & $S$ & $S$ & $S$ & $\mathrm{~S}$ & $\mathrm{P}$ & $S$ & & & & \\
\hline $12-061$ & Robert Mariani & $\begin{array}{l}\text { Development of a High Burn up Zr-Based } \\
\text { Fuel Cladding for LWRs }\end{array}$ & & & & & $S$ & $\mathrm{P}$ & & & & & \\
\hline $12-062$ & David Chichester & $\begin{array}{l}\text { Development of Diamond Based Sensors } \\
\text { for User Facility Capabilities at INL } \\
\text { Advanced Test Reactor }\end{array}$ & $S$ & & & & $S$ & $\mathrm{P}$ & $S$ & & & & \\
\hline $12-066$ & Michael Daniels & Electronic Energetic Initiation & & & & & $\mathrm{P}$ & & & & & & \\
\hline $12-070$ & J. Keith Jewell & $\begin{array}{l}\text { Separate Effects Fuels Development } \\
\text { Capability }\end{array}$ & $\mathrm{s}$ & $\mathrm{S}$ & & & $\mathrm{S}$ & $\mathrm{P}$ & & & & & \\
\hline $12-071$ & Jon Cristophersen & Battery Resilient Monitoring and Control & & $\mathrm{P}$ & & & & & & & S & $S$ & \\
\hline $12-073$ & Scott Thompson & $\begin{array}{l}\text { A Nonproliferation Focused Study and } \\
\text { Comparison of the GEANT4 Simulation } \\
\text { Toolkit and MCNP Functionality, Accuracy, } \\
\text { and Development Needs }\end{array}$ & $\mathrm{P}$ & & & & & & & & & & \\
\hline $12-077$ & Marat Khafizov & $\begin{array}{l}\text { Observation of Zirconium Oxidation at } \\
\text { Atomic Level Using Non-linear Optical } \\
\text { Spectroscopy }\end{array}$ & & & & & $S$ & $\mathrm{P}$ & & & & & \\
\hline
\end{tabular}




\begin{tabular}{|c|c|c|c|c|c|c|c|c|c|c|c|c|c|}
\hline $\begin{array}{l}\text { Tracking } \\
\text { No. }\end{array}$ & PI Name & Title & 选 & 岃 & 岀 & ш & U & ш & $\frac{\mathscr{\alpha}}{z}$ & $\sum_{\substack{\alpha \\
0}}$ & 山 & 甹 & $\sum$ \\
\hline $12-082$ & Humberto Garcia & $\begin{array}{l}\text { Resilient Monitoring, Adaptation, and } \\
\text { Control (ReMAC) System }\end{array}$ & $S$ & $P$ & & $S$ & $S$ & $S$ & & & $S$ & $S$ & \\
\hline $12-086$ & Ronald Boring & Alert/Alarm Dashboard & & & & & $\mathrm{s}$ & $\mathrm{P}$ & & & $S$ & & \\
\hline $12-089$ & Craig Rieger & $\begin{array}{l}\text { Multi-agent Analysis to Characterize } \\
\text { Complex Infrastructure Interdependencies }\end{array}$ & $\mathrm{s}$ & $\mathrm{s}$ & & & $\mathrm{P}$ & $S$ & & & $S$ & & \\
\hline $12-090$ & Dr. Carl M. Stoots & $\begin{array}{l}\text { Development of Emissivity-tuned, Robust } \\
\text { Encapsulated Radioisotope Heat Sources } \\
\text { for an Advanced Radioisotope Thermo- } \\
\text { Photovoltaic (RTPV) Power System }\end{array}$ & & & & & $\mathrm{S}$ & $P$ & & & & & \\
\hline $12-093$ & Robert Fox & $\begin{array}{l}\text { Development of Unique Nanomaterials for } \\
\text { Selective Replacement of Strategic and } \\
\text { Critical Materials }\end{array}$ & & $P$ & & & $\mathrm{~S}$ & & & & & & \\
\hline $12-096$ & Aaron Wilson & $\begin{array}{l}\text { Isolation and Purification of Critical Metals } \\
\text { Using lonic Liquid Based Solvent } \\
\text { Extraction }\end{array}$ & & $P$ & $S$ & & & $S$ & & & & & \\
\hline $12-099$ & Joni Barnes & $\begin{array}{l}\text { Designing Algal Biofuel Consortia for } \\
\text { Increased Function and Reduced Nutrient } \\
\text { Costs }\end{array}$ & & $P$ & & & $\mathrm{~S}$ & & & & & & \\
\hline $12-108$ & Mitchell Plummer & $\begin{array}{l}\text { Bakken Hydraulic Fracturing Water Needs } \\
\text { and Potential Impacts to the Fox Hills/Hell } \\
\text { Creek Aquifer }\end{array}$ & & & $S$ & $\mathrm{P}$ & $\mathrm{S}$ & & & & & $S$ & \\
\hline $12-112$ & Mark Carroll & $\begin{array}{l}\text { Reactor Coolant Pump Alternate Can } \\
\text { Material Development Program }\end{array}$ & $\mathrm{s}$ & $\mathrm{s}$ & & & & $P$ & $s$ & & $S$ & & \\
\hline $12-113$ & Hai Huang & $\begin{array}{l}\text { Coupled-Smoothed Particle } \\
\text { Hydrodynamics and Discrete Element } \\
\text { Model (SPH-DEM) Simulation of Hydraulic } \\
\text { Fracturing and Shale Gas Production }\end{array}$ & & & $S$ & $P$ & $S$ & & & $S$ & & $S$ & \\
\hline $12-117$ & Hans Gougar & $\begin{array}{l}\text { Feasibility of a Broad Application Test } \\
\text { Reactor Using LEU }\end{array}$ & & $\mathrm{s}$ & & & & $\mathrm{P}$ & & & & & \\
\hline $12-118$ & Mason Harrup & $\begin{array}{l}\text { A Leap Ahead in Energy Storage - An } \\
\text { Integrated Approach to an Inorganic High } \\
\text { Energy Lithium Battery }\end{array}$ & & $P$ & & & & & & & $S$ & & \\
\hline $12-120$ & Jiango Yu & $\begin{array}{l}\text { Modeling Guided Development of } \\
\text { Nanostructured High Energy Electrode } \\
\text { Materials for Li-ion Batteries }\end{array}$ & & $P$ & & & $\mathrm{~S}$ & & & & & & \\
\hline $12-121$ & Victor Walker & $\begin{array}{l}\text { State Awareness for Smart Grid Electrical } \\
\text { Load Balancing, Distribution, and Security }\end{array}$ & & & & & & $S$ & & & $P$ & & \\
\hline $13-011$ & Deborah Newby & $\begin{array}{l}\text { Integrated Approach to Algal Biofuel, } \\
\text { Bio-power, and Agricultural Waste } \\
\text { Management }\end{array}$ & & $P$ & & & & & & & & & \\
\hline $13-013$ & Bulent S. Biner & $\begin{array}{l}\text { Development a Micromechanistic Phase- } \\
\text { Field Modeling Approach for Life } \\
\text { Estimation and Risk Assessment of } \\
\text { Reactor Pressure Vessels }\end{array}$ & & & & & $S$ & $\mathrm{P}$ & $S$ & & & & \\
\hline $13-014$ & Prabhat Tripathy & $\begin{array}{l}\text { Aluminum Electroplating from Low } \\
\text { Temperature Molten Bromide Salts }\end{array}$ & & $P$ & $S$ & & & & & & & & \\
\hline $13-023$ & Melissa Teague & $\begin{array}{l}\text { Characterization and Modeling of High } \\
\text { Burn-up Mixed Oxide Fuel }\end{array}$ & & & & & & $P$ & & & & & \\
\hline $13-024$ & Tedd Lister & $\begin{array}{l}\text { Direct Electrochemical Stabilization of Bio- } \\
\text { Oils }\end{array}$ & & $P$ & & $s$ & & & & & & & \\
\hline
\end{tabular}




\begin{tabular}{|c|c|c|c|c|c|c|c|c|c|c|c|c|}
\hline $\begin{array}{l}\text { Tracking } \\
\text { No. }\end{array}$ & PI Name & Title & 交 & 岀 & $\sum_{\text {U }}$ & 虫 & U & $\mathbf{z}$ & $\frac{\mathscr{\alpha}}{z}$ & 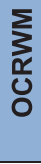 & 岁 & \\
\hline $13-027$ & Eric Dufek & $\begin{array}{l}\text { Diagnostics of Advanced Energy Storage } \\
\text { Materials }\end{array}$ & & $P$ & & & S & & & & & \\
\hline $13-029$ & Joe Palmer & $\begin{array}{l}\text { In-Pile Detection of Crack Growth in the } \\
\text { ATR }\end{array}$ & & & & & & $\mathrm{P}$ & $S$ & & & \\
\hline $13-032$ & Michael Tonks & $\begin{array}{l}\text { Experimental and Computational Analysis } \\
\text { of Hydride Microstructures in Zirconium in } \\
\text { Dry Storage Conditions }\end{array}$ & & & & & $S$ & $\mathrm{P}$ & & $S$ & & \\
\hline $13-033$ & Leigh Martin & $\begin{array}{l}\text { Magnetic Separation Nanotechnology for } \\
\text { Spent Nuclear Fuel Recycle }\end{array}$ & & & & & & $\mathrm{P}$ & & & & \\
\hline $13-035$ & Ken Bateman & $\begin{array}{l}\text { Development of New Molten Salt Sensor } \\
\text { Technology for Application to } \\
\text { Safeguarding Pyroprocessing }\end{array}$ & $\mathrm{P}$ & & & & & $S$ & & & & \\
\hline $13-039$ & Gary Groenewold & $\begin{array}{l}\text { Induction Based Fluidics Mass } \\
\text { Spectrometry for Characterizing } \\
\text { Radioactive Extraction Solvents }\end{array}$ & & & & & $S$ & $\mathrm{P}$ & & & & \\
\hline $13-040$ & Joseph Nielsen & $\begin{array}{l}\text { Epistemic Uncertainty Quantification in } \\
\text { Dynamic Probabilistic Risk Assessment }\end{array}$ & & & & & & $P$ & $\mathrm{~S}$ & $S$ & & \\
\hline $13-045$ & James O'Brien & $\begin{array}{l}\text { Direct Solar Hydrogen Production Using } \\
\text { High Temperature Solid Oxide } \\
\text { Photoelectrochemical Cells }\end{array}$ & & $P$ & & & $S$ & & & & & \\
\hline $13-050$ & Michael Tonks & $\begin{array}{l}\text { Concurrent Atomistic to Macroscale } \\
\text { Modeling of Materials Under Irradiation } \\
\text { Using the Phase Field Crystal Model }\end{array}$ & $S$ & & & & $S$ & $\mathrm{P}$ & $\mathrm{s}$ & $S$ & & \\
\hline 13-059 & John Klaehn & $\begin{array}{l}\text { Application of Facilitated Transport } \\
\text { Membranes Using Novel Stabilized Metal } \\
\text { Complexes }\end{array}$ & & $S$ & $S$ & $P$ & & $\mathrm{~S}$ & & & & \\
\hline $13-060$ & James Delmore & $\begin{array}{l}\text { Metal Fluoride Preparation for AMS } \\
\text { Analysis }\end{array}$ & $\mathrm{P}$ & & & & & & & & & \\
\hline $13-065$ & Humberto Garcia & $\begin{array}{l}\text { Multi-domain Modeling, Simulation, and } \\
\text { Integration Tools for the Dynamic Analysis } \\
\text { and Optimization of Hybrid Energy } \\
\text { Systems }\end{array}$ & & $P$ & & $S$ & $S$ & $S$ & & & S & \\
\hline $13-068$ & Mitchell Plummer & $\begin{array}{l}\text { Cooling in Fractured Geothermal } \\
\text { Reservoirs: Analyses of Long-Term } \\
\text { Cooling in Typical Geothermal Reservoirs } \\
\text { and Application to Geothermal Resource } \\
\text { Potential Assessments }\end{array}$ & & $P$ & & & $S$ & & & & & \\
\hline $13-071$ & Benjamin Spencer & $\begin{array}{l}\text { Advanced Fracture Modeling for Nuclear } \\
\text { Fuel }\end{array}$ & & & & & & $P$ & & & & \\
\hline $13-074$ & Vicki Thompson & $\begin{array}{l}\text { A Nanobioreactor for Cellulose } \\
\text { Degradation Using In Vitro Synthetic } \\
\text { Biology }\end{array}$ & & $P$ & & & $S$ & & & & & \\
\hline $13-079$ & Dayna Daubaras & $\begin{array}{l}\text { Diverse Biological Factories for } \\
\text { Sustainable Manufacturing }\end{array}$ & & $P$ & & & $S$ & & & & & \\
\hline $13-092$ & Kevin Carney & Fission Product Standard Production & $\mathrm{P}$ & & & & & & & & & \\
\hline $13-093$ & Hussein Moradi & $\begin{array}{l}\text { Spectrum Allocation and Communications } \\
\text { in Dynamic Spectrum Access Channels }\end{array}$ & $S$ & & & & $S$ & $S$ & & & $P$ & \\
\hline 13-095 & Robert Youngblood & $\begin{array}{l}\text { Development and Validation of a Societal- } \\
\text { Risk Goal for Nuclear Power Plant Safety }\end{array}$ & $S$ & & & & & $\mathrm{P}$ & & $S$ & & \\
\hline $13-097$ & Derek Gaston & $\begin{array}{l}\text { MOOSE Capability Extension In Support } \\
\text { of Full Core Modeling }\end{array}$ & & & $S$ & & $S$ & $\mathrm{P}$ & $\mathrm{s}$ & $S$ & & \\
\hline
\end{tabular}




\begin{tabular}{|c|c|c|c|c|c|c|c|c|c|c|c|c|c|}
\hline $\begin{array}{l}\text { Tracking } \\
\text { No. }\end{array}$ & PI Name & Title & $\begin{array}{l}\varangle \\
\sum \\
z\end{array}$ & ш & 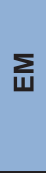 & 岌 & U & ш & $\frac{\alpha}{z}$ & $\sum_{0}^{\infty}$ & 岁 & エ & $\sum$ \\
\hline $13-100$ & Vicki Thompson & $\begin{array}{l}\text { An Efficient System for the Production and } \\
\text { Harvesting of Lipids from Photosynthetic } \\
\text { Microbes }\end{array}$ & & $\mathrm{P}$ & & & & & & & & & \\
\hline 13-105 & Gan, Jian & $\begin{array}{l}\text { Micro/Nano Scale AFM-based Thermal } \\
\text { Conductivity Measurement and Atomistic } \\
\text { Modeling for Oxide Fuel: the Effects of } \\
\text { Grain Boundary, Fission Gas and } \\
\text { Radiation Damage }\end{array}$ & & & & & & $\mathrm{P}$ & & & & & \\
\hline $13-106$ & Peter Zalupski & $\begin{array}{l}\text { Building Organic-Inorganic Hybrid } \\
\text { Materials to Protect Metal Ion } \\
\text { Sequestering Agents from Radiation- } \\
\text { Induced Oxidative Damage }\end{array}$ & $\mathrm{S}$ & & $\mathrm{S}$ & & & $\mathrm{P}$ & & $\mathrm{S}$ & & & \\
\hline 13-107 & Piyush Sabharwall & $\begin{array}{l}\text { Application of Chemical Heat Pumps for } \\
\text { Temperature Amplification in Nuclear } \\
\text { Hybrid Energy Systems for Synthetic } \\
\text { Transportation Fuel Production }\end{array}$ & & $S$ & $S$ & & $\mathrm{~S}$ & $\mathrm{P}$ & & & $\mathrm{S}$ & & \\
\hline $13-110$ & Michael McKellar & $\begin{array}{l}\text { Nuclear-Renewables-Oil Shale Hybrid } \\
\text { Energy System }\end{array}$ & & $S$ & & $\mathrm{~S}$ & & $\mathrm{P}$ & & & S & & \\
\hline $13-112$ & Carl Stoots & $\begin{array}{l}\text { High-Temperature Nuclear Co-Electrolysis } \\
\text { for Spacecraft Cabin Air Revitilization \& } \\
\text { Liquid Fuel Production }\end{array}$ & & $\mathrm{S}$ & & & $\mathrm{P}$ & $\mathrm{S}$ & $\mathrm{S}$ & & & & \\
\hline $13-114$ & Earl Mattson & $\begin{array}{l}\text { Multiphase Fluid Trapping in Propped } \\
\text { Hydraulic Fractures }\end{array}$ & & & $\mathrm{S}$ & $\mathrm{P}$ & $\mathrm{S}$ & & & & & & \\
\hline $13-115$ & Mark DeHart & $\begin{array}{l}\text { Multi-scale Full Core Reactor Physics } \\
\text { Simulation }\end{array}$ & & & & & & $\mathrm{P}$ & $\mathrm{S}$ & & & & \\
\hline $13-118$ & Shawn West & $\begin{array}{l}\text { Geomagnetic Disturbance Field Coupling } \\
\text { Measurements on INL Power Grid }\end{array}$ & $\mathrm{S}$ & & & & & & & & $\mathrm{P}$ & & \\
\hline $13-120$ & Miles McQueen & $\begin{array}{l}\text { Improved Control System Resilience via } \\
\text { Secure Machine Learning }\end{array}$ & $\mathrm{S}$ & & & & $\mathrm{S}$ & $\mathrm{S}$ & & & $\mathrm{P}$ & & \\
\hline $13-121$ & Tony Hill & $\begin{array}{l}\text { Advanced In-Situ Measurement } \\
\text { Techniques in TREAT }\end{array}$ & $S$ & & $S$ & & $\mathrm{~S}$ & $\mathrm{P}$ & & & & & \\
\hline $13-122$ & John Koudelka & $\begin{array}{l}\text { Examination of the Impacts on Water } \\
\text { Resources from Commercialization of } \\
\text { Fossil Energy Development in an Arid } \\
\text { Western Basin Using Integrated } \\
\text { Hydrologic Modeling }\end{array}$ & & $\mathrm{P}$ & $\mathrm{S}$ & S & $\mathrm{S}$ & $\mathrm{S}$ & $\mathrm{S}$ & & $\mathrm{S}$ & $\mathrm{S}$ & $\mathrm{S}$ \\
\hline $13-123$ & Rory Kennedy & $\begin{array}{l}\text { Experimental Verification of Predicted } \\
\text { Chemical Transmutation of Cesium-137 to } \\
\text { Barium-137 via Beta Decay in CsCl } \\
\text { Resulting in the Radioparagenetic } \\
\text { Formation of Rocksalt BaCl }\end{array}$ & $\mathrm{S}$ & & & & & $\mathrm{P}$ & & & & & \\
\hline
\end{tabular}

